$$
\text { OCDO- } 9800 / 950
$$

\title{
COOLSIDE WASTE MANAGEMENT DEMONSTRATION \\ OCDO GRANT AGREEMENT NO. CDO/D-902-9 \\ FINAL REPORT
}

October 1997

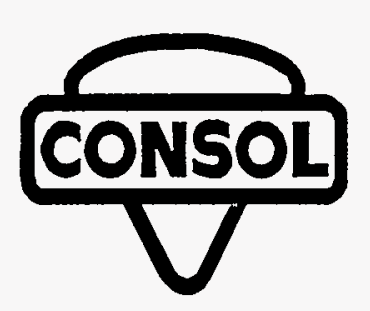

Milton Wu (412) 854-6708

Richard A. Winschel (412) 854-6683

CONSOL Inc.

Research \& Development

4000 Brownsville Road

Library, PA 15129

MASTER

This report contains no trade secret information

This project was funded in part by the Ohio Coal Development Office, Department of Development, State of Ohio 


\section{DISCLAIMER}

Portions of this document may be illegible electronic image products. Images are produced from the best available original document. 


\section{TABLE OF CONTENTS}

Page

EXECUTIVE SUMMARY

Major Conclusions $\quad 2$

Aggregates from FBC Waste

Aggregates from Coolside and LIMB Wastes 3

FBC Waste as a Chemical Admix for Road Base Stabilization 3

Properties of Coolside Waste Important for Landfill Placement $\quad 4$

Phase $1 \quad 5$

Phase $2 \quad 10$

Phase $3 \quad 10$

$\begin{array}{ll}\text { Publications and Presentations } & 10\end{array}$

$\begin{array}{ll}\text { INTRODUCTION } & 12\end{array}$

TECHNICAL DISCUSSION 13

Phase $1 \quad 13$

Phase $2 \quad 16$

Phase $3 \quad 20$

MARKETING/COMMERCIALIZATION DISCUSSION 20

$\begin{array}{lr}\text { FINAL BUDGET SUMMATION } & 21\end{array}$

LIST OF TABLES

Page

Schedule S - Total Project Expenditures by Co-Sponsor $\quad 22$

Schedule T - OCDO Expenditures $\quad 23$

\section{APPENDICES}

Appendix 1 - The Landfill Disposal Characteristics of Coolside Waste

Appendix 2 - Investigation of the Utilization Potential of Coolside, LIMB, and FBC Waste as Construction Materials

Appendix 3 - Pilot Plant Production of Coolside Waste Samples 


\section{COOLSIDE WASTE MANAGEMENT DEMONSTRATION OCDO GRANT AGREEMENT No. CDO/D-902-9}

FINAL REPORT

\section{EXECUTIVE SUMMARY}

The objectives of this project were to evaluate the potential utilization in road construction of wastes produced from the Coolside, LIMB (limestone injection multi-stage burner) and FBC (fluidized-bed combustion) processes, and to specify criteria for landfill disposal of waste from the Coolside process. These three processes are considered to be clean coal technologies. The Coolside process involves injecting an aqueous slurry of hydrated lime into the ductwork downstream of the air preheater in a coal-fired boiler. The hydrated lime captures sulfur dioxide from the flue gas producing anhydrous calcium sulfite and calcium sulfate, which are collected along with the unused hydrated lime and fly ash. The LIMB process involves injection of lime or hydrated lime directly into the furnace to capture sulfur dioxide. The waste consists principally of anhydrous calcium sulfate, lime, and fly ash. Both processes were demonstrated successfully at the Edgewater Station of Ohio Edison in Lorrain, $\mathrm{OH}$, from 1989 to 1992 . Circulating fluidized-bed combustion (FBC) is a commercial technology which combines steam generation with $\mathrm{SO}_{2}$ control by burning coal in a circulating bed of limestone. The waste, chemically similar to LIMB waste, is produced by bleed-off of the bed material and by collection of the flue dust. All three processes produce a dry solid waste, which must either be used or disposed of and managed to ensure environmental compliance and economic feasibility. The project was completed in June 1996. The major conclusions are summarized below.

\section{MAJOR CONCLUSIONS}

\section{Aggregates from FBC Waste}

High-strength abrasion-resistant synthetic aggregates were produced from FBC waste using the CONSOL pelletization/aggregate production technology. The synthetic aggregates produced meet all specifications (unit weight, soundness index, LA abrasion index and size distribution) for use as AASHTO Class A aggregate in road construction. 
The addition of pulverized coal (p. c.) fly ash to the waste improved the aggregate durability.

A bituminous concrete test patch was constructed to demonstrate the feasibility of using FBC waste aggregates as Class $A$ aggregate in surface-wearing course for road construction. A flexible bituminous concrete test patch was constructed with standard paving equipment in April 1995 with 1.5 ton synthetic aggregates made from a $65 / 35$ blend of FBC waste and p. c. fly ash, an equal quantity of crushed limestone (Class A aggregate), and about $6 \%$ asphalt. The heavy truck traffic and the high permeability of the open-graded mix design made this a severe test of the aggregate durability. Inspections revealed no visible degradation of the aggregate or the asphalt mix after 13 months of service. Monthly analyses of core samples showed that no degradation of either the FBC waste aggregate or the limestone aggregate between June 1995 and May 1996. Thus, all available evidence indicates that the FBC waste aggregates are suitable for use in bituminous concrete paving.

\section{Aggregates from Coolside and LIMB Wastes}

High-strength, abrasion-resistant synthetic aggregates were made from Coolside and LIMB wastes. Although the aggregates fail to meet certain specifications as road aggregates, they pass several important ASTM C-331 specifications as lightweight aggregates (unit weight, clay lumps, staining, grain size) for use in concrete masonry units. Both Coolside and LIMB waste aggregate have potential for use as coarse lightweight aggregates in concrete masonry units.

\section{FBC Waste as a Chemical Admix for Road Base Stabilization}

The utilization potential of unconsolidated FBC waste as a chemical admix in road base stabilization was evaluated. Mixing $8 \%$ hydrated FBC waste with an Ohio soil of marginal quality as road base increased the strength and load-bearing capacity of the soil enough to make it suitable for use in road base construction. However, the FBC waste/soil specimen swelled slowly while it was submerged in water. Slow swelling of the admixture would require special precautions to be taken for its use in road base stabilization. 
Laboratory swelling tests to evaluate the swell potential should be conducted before using FBC waste or any alkaline FGD waste as a replacement of hydrated lime in road base stabilization.

\section{Properties of Coolside Waste Important for Landfill Placement}

Coolside waste is a fine silt material; $95 \mathrm{wt} \%$ is finer than 200 mesh $(75 \mu \mathrm{m})$. It is nonplastic and has a lower specific gravity than that of typical soils.

The wetted Coolside waste has cementitious properties. When wetted and compacted, it develops high unconfined compressive strength and low permeability. For example, Coolside waste compacted at 95\% maximum dry bulk density (ASTM D698-91, standard Proctor test) with near optimum moisture content (simulating a typical landfill operation) develops unconfined compressive strength of $2000 \mathrm{psi}$ and permeability of $1 \times 10^{-6} \mathrm{~cm} / \mathrm{sec}$ after aging. The strength is similar to that of a low-strength concrete. The cementitious properties are caused by the formation of the mineral ettringite in the compacted waste.

The Coolside waste compacted at $95 \%$ maximum dry bulk density with near optimum moisture content has a high angle of friction, high cohesion and high load bearing capacity. This indicates that the material could be used as a construction material. However, compacted Coolside waste swells (up to $23 \%$ ), when submerged in water. Mineralogic characterization indicated that the swelling was related to the formation of the mineral ettringite in the compacted waste. Aging the compacted waste before submerging it in water, and adding a static load, greatly reduced the potential for swell. Swelling could have an impact on the utilization of the material for some engineering application.

Field lysimeter tests, conducted from October 1992 to May 1996, showed that the geotechnical properties and leachate characteristics of Coolside waste are affected by the degree of compaction to which the waste is subjected during placement. Compaction of the waste (e.g., at $95 \%$ maximum dry bulk density with optimum moisture content) greatly increased its strength, reduced its permeability, and reduced the initial release rates of

most leachate species. However, compaction caused the leachate to have a higher $\mathrm{pH}$, 
which, in turn, increased the leachability of certain trace elements that form alkalinesoluble oxyanions (e.g., As, V, Se). The leachates from the well-compacted waste had relatively high Se and As concentrations for the first year, which may be of environmental concern. Therefore, even though laboratory TCLP leaching test results indicated that Coolside waste is non-hazardous, precautions (such as containment or treatment of the relatively high Se and As concentrations in the leachate) should be considered before landfilling of Coolside waste.

Mineralogical characterization of the Coolside wastes excavated from field lysimeters after $31 / 2$ years of natural weathering indicates that the reaction of the calcium-containing minerals in the waste with groundwater $\mathrm{CO}_{2}$ reduced the strength and increased the permeability of the waste, but only when the waste was not sufficiently compacted. Thus, sufficient compaction promotes the long-term stability of landfilled Coolside waste.

Although laboratory specimens of compacted Coolside waste undergo long-term swelling when immersed in water, no significant swelling of the Coolside waste or pressure change occurred in the field lysimeters during the $31 / 2$ year test period.

Long-term laboratory column lysimeter tests produced leachates with major and trace elemental concentrations of the same order of magnitude as produced by the field lysimeters. Therefore, it appears that long-term column leaching tests could replace the more costly field lysimeter tests in future evaluations.

\section{PHASE 1}

Phase 1 of the project was directed toward obtaining data useful for the design of environmentally safe and economically sound landfills for disposal of Coolside waste. The characterization was conducted on numerous waste samples produced at the Ohio Edison Edgewater Station and at the CONSOL Coolside pilot plant. The Edgewater Coolside wastes were collected from the ESP hopper during the process demonstration. The pilot plant Coolside wastes were manufactured for this project. Coolside waste was characterized via chemical, physical, geotechnical, and mineralogical analyses and tests. 
Coolside waste is a fine silt material; $95 \mathrm{wt} \%$ is finer than 200 mesh $(75 \mu \mathrm{m})$. The waste is composed of spent sorbent $\left(\mathrm{CaSO}_{3}\right.$ and $\left.\mathrm{CaSO}_{4}\right)$, unused sorbent $\left(\mathrm{Ca}(\mathrm{OH})_{2}\right)$, and coal fly ash $\left(\mathrm{SiO}_{2}, \mathrm{Al}_{2} \mathrm{O}_{3}, \mathrm{Fe}_{2} \mathrm{O}_{3}\right.$, etc.). The wetted waste has cementitious properties and can develop high unconfined compressive strength and low permeability after adequate compaction. For example, Coolside waste compacted at $95 \%$ maximum dry bulk density (ASTM D698-91, Proctor test) with near optimum moisture content (simulating a typical landfill operation) develops unconfined compressive strength of $2000 \mathrm{psi}$ and permeability of $1 \times 10^{-6} \mathrm{~cm} / \mathrm{sec}$ after aging. The strength is similar to that of a low-strength concrete. The cementitious properties are caused largely by the formation of the mineral ettringite from various components of the compacted waste. The compacted waste also has high angle of friction, cohesion, and load bearing capacity. However, the compacted waste swells (up to $23 \%$ ) when submerged in water as a result of the formation of ettringite. Aging the remolded specimen before submerging them in water and adding a static load greatly reduces the potential for swell. Swelling could have an impact on the utilization of the material for some engineering application.

Three large-scale test landfill cells (field lysimeters) were filled in the autumn of 1992 with Coolside waste at three degrees of compaction (loose fill, low, and high compaction) and one cell was filled with fly ash for comparison. The Coolside waste was collected from the Ohio Edison Edgewater Station during the Coolside process demonstration. In November 1992, the field lysimeters were monitored to determine moisture, temperature, and carbon dioxide $\left(\mathrm{CO}_{2}\right)$ uptake profiles, pressure changes, and leachate characteristics. Tests in laboratory column lysimeters were conducted simultaneously for comparison. The field lysimeters were excavated in May 1996 to determine the geotechnical properties and mineralogy of the waste after $31 / 2$ years of monitoring during natural weathering. The field lysimeter test results show that the geotechnical properties and leachate characteristics of Coolside waste are affected by the degree of compaction (loose fill, low, and high compaction) to which the waste is subjected during placement. The loose fill was uncompacted waste which contained the optimum moisture content. The high compaction fill was waste compacted at $95 \%$ of maximum dry bulk density (standard Proctor) with optimum moisture content. The low compaction fill was waste compacted to an 
intermediate degree. Coolside waste in the high compaction fill field lysimeter developed high compressive strength (average 1,629 psi), low permeability coefficient (average $2.2 \times 10^{-6} \mathrm{~cm} / \mathrm{sec}$ ), and had lower leachate concentrations and slower release of $\mathrm{Ca}, \mathrm{Na}$, $\mathrm{Cl}, \mathrm{K}$ and $\mathrm{SO}_{4}^{-2}$. Overall, compacting the Coolside waste at $95 \%$ maximum dry bulk density with optimum moisture content greatly increased the strength, and reduced the permeability and the initial release of highly soluble salts. However, the leachate $\mathrm{pH}$ remained high and, as a result of high leachate $\mathrm{pH}$, the leachability of certain trace elements that form alkaline-soluble oxyanions (such as $\mathrm{As}, \mathrm{V}$, and $\mathrm{Se}$ ) also increased with increasing compaction. The relatively high $\mathrm{Se}$ and $\mathrm{As}$ concentrations in the leachate produced in the first year from the high compaction fill may be of environmental concern. Precautions, such as containment or treatment of the relatively high Se and As concentrations in the leachate, should be considered before landfilling of Coolside waste. During the $31 / 2$ year test period, no significant swelling of the Coolside waste or pressure change occurred in the field lysimeters. The reaction of groundwater $\mathrm{CO}_{2}$ with the calcium-bearing ettringite and gypsum resulted in low strength and high permeability in the loose and low compaction lysimeters. Thus, sufficient compaction promotes the long-term stability of landfilled Coolside waste.

Long-term (two to three years) laboratory column lysimeter tests produced leachates with major and trace element concentrations of the same order of magnitude as produced by the field lysimeters. Therefore, it appears that the long-term column leaching test could replace the more costly field lysimeter work in future evaluations.

Phase 1 progressed as planned, except that the field lysimeter leaching study was delayed from the original schedule because of an eleven-month delay in obtaining National Environmental Act Policy (NEPA) approval from DOE. The study was extended by an additional year (ending June 1996) to complete the $31 / 2$ year field lysimeter study. 


\section{PHASE 2}

Task 1 of Phase 2, road construction testing, was concentrated on evaluating the use of hydrated FBC waste (unconsolidated) as a chemical admix in road base stabilization. The FBC waste was collected from the ash silo of a circulating fluidized-bed combustor firing Pittsburgh seam coal (same as Ohio No. 8). The waste included fly ash and bed-drained ash, and it is composed of spent sorbent $\left(\mathrm{CaSO}_{4}\right)$, unused sorbent $(\mathrm{CaO})$, and fly ash $\left(\mathrm{SiO}_{2}, \mathrm{Al}_{2} \mathrm{O}_{3}\right.$, and $\mathrm{Fe}_{2} \mathrm{O}_{3}$, etc.). The $\mathrm{FBC}$ waste was hydrated to convert $\mathrm{CaO}$ to $\mathrm{Ca}(\mathrm{OH})_{2}$ before testing. Mixing $8 \%$ hydrated FBC waste with an Ohio soil of marginal quality as road base increased its unconfined compressive strength and load bearing capacity, and made the soil suitable for use in road base construction in terms of strength and load bearing capacity. However, the specimen swelled slowly while it was submerged in water. Slow swelling of the admixture would require that special precautions be taken for use of this material in road base stabilization. Laboratory tests to evaluate the swell potential should be conducted before using FBC waste or any alkaline sulfate-containing FGD waste as a replacement for hydrated lime in road base stabilization.

Task 2 of Phase 2, waste pelletization research, was concentrated on developing, testing, and demonstrating FBC waste pellets for use as synthetic aggregates in the road construction. A pelletization technology was developed to make synthetic aggregates from FBC waste and dry FGD wastes from the Coolside and LIMB (limestone injection multistage burner) processes. The FBC and Coolside wastes used in Task 2 of Phase 2 were collected in the same ways as discussed previously. The LIMB wastes were collected from the ESP hopper during the Edgewater process demonstration. The LIMB waste is composed of spent sorbent $\left(\mathrm{CaSO}_{4}\right)$ unused sorbent $(\mathrm{CaO})$, and fly ash $\left(\mathrm{SiO}_{2}, \mathrm{Al}_{2} \mathrm{O}_{3}\right.$, and $\mathrm{Fe}_{2} \mathrm{O}_{3}$, etc.). High-strength synthetic aggregates with low abrasion index were produced from FBC waste. The synthetic aggregates meet all specifications (unit weight, soundness index, LA abrasion index and size distribution) for use as AASHTO Class A aggregate in surface-wearing course for road construction. However, the aggregates produced with the original formulation degraded when they were fully immersed in water during long-term natural weathering and laboratory freeze/thaw tests. Characterization of the degraded aggregates indicated that the slow hydration of anhydrite $\left(\mathrm{CaSO}_{4}\right)$ to gypsum 
$\left(\mathrm{CaSO}_{4} \cdot 2 \mathrm{H}_{2} \mathrm{O}\right)$ was the major cause for the degradation. Durability was improved by blending the FBC waste with p.c. fly ash (from the Ohio Edison Mansfield Station) to reduce gypsum formation and by coating the aggregate with asphalt to increase water resistance. A flexible bituminous concrete test patch was constructed in April 1995 with 1.5 ton synthetic aggregates made from a $65 / 35$ blend of $F B C$ waste and p.c. fly ash. Equal quantities of crushed limestone (commercial Class A aggregate) were blended with the synthetic aggregates to produce a large quantity of bituminous concrete and to provide an internal standard for evaluating the performance of the synthetic aggregate. About $6.5 \mathrm{wt} \% \mathrm{AC}-20$ asphalt was mixed with the aggregates. The heavy truck traffic and the high permeability of the open-graded mix design made this a severe test of the aggregate durability. The bituminous concrete was used to pave a large surface area (12' $\times 35^{\prime} \times$ 1.5-2") for the test. Standard paving equipment was used. The evaluation was completed on May 30, 1996. Inspections revealed no visible degradation of the aggregate or the pavement after 13 months of service. Monthly analyses of core samples showed no degradation of either the FBC waste aggregate or the limestone aggregate between June 1995 and May 1996. This test demonstrated the technical feasibility of using synthetic aggregates made from $\mathrm{FBC}$ waste as Class $\mathrm{A}$ aggregate in the construction of asphalt surface wearing course.

High-strength synthetic aggregates with low abrasion indices also were made from Coolside and LIMB wastes. The Coolside wastes were obtained from the Ohio Edison Edgewater Station and from the CONSOL Coolside pilot plant. The LIMB waste was obtained from the Ohio Edison Edgewater Station. Although synthetic aggregates made from Coolside and LIMB waste fail to meet the unit weight and sodium sulfate soundness index specifications of road aggregate, they pass the ASTM C-331 specifications of lightweight aggregates for concrete masonry units. Both Coolside and LIMB waste aggregates have potential for use as coarse lightweight aggregates in concrete masonry units. The addition of fly ash to Coolside and LIMB wastes during aggregate preparation further improves the aggregate properties. 


\section{PHASE 3}

Three $500 \mathrm{lb}$ Coolside waste samples and a fourth sample of about $20 \mathrm{lb}$ were produced under different process conditions (once-through, recycled, and advanced modes) at the CONSOL Coolside pilot plant. These samples were used to evaluate the impact of process variables on waste properties and on disposal and utilization in Phases 1 and 2. The samples were produced at conditions that simulate Coolside process operations on a unit burning medium-sulfur Ohio coal.

\section{PUBLICATIONS AND PRESENTATIONS}

1. Wu, M. M.; Winschel, R. A.; Burke, F. P.; Robl, T. "Coolside and LIMB By-Product Utilization and Management", Spring Forum of the Ohio Alliance for the Environment, Management Options for Combustion By-Products: Waste or Asset?, Columbus, $\mathrm{OH}$, June 1991.

2. Winschel, R. A.; Wu, M. M.; Burke, F. P. "Coolside/LIMB/FBC Waste Management", DOE Duct Injection Technology Working Group Meeting, Columbus, OH, April 2829, 1992.

3. Bosart, S. J.; Brown, T. D. "Management of Solid Wastes from the Coolside Process", Ninth Annual International Pittsburgh Coal Conference, Pittsburgh, PA, October 1992.

4. Hopkins, T. C.; Wu, M. M.; Winschel, R. A.; Robl, T. L. "The Ohio Coal Development Office Coolside Waste Management Demonstration Project", Tenth International Symposium on Coal Ash Use and Disposal, Orlando, FL, January 1993.

5. Hopkins, T. C.; Wu, M. M.; Winschel, R. A.; Robl, T. L. "The Ohio Coal Development Office Coolside Waste Management Demonstration Project", Tenth International Symposium on Coal Ash Use and Disposal, Orlando, FL, January 1993.

6. Graham, U. M.; Wu, M. M.; Robl, T. L.; Hopkins, T. C., "Mineralogical Transformations of Ettringite in Concrete Derived from Dry-FGD By-Products", Tenth Annual International Pittsburgh Coal Conference", Pittsburgh, PA, September 1993.

7. Hopkins, T. C.; Beckham, T. L.; Wu, M. M. "Swelling Characteristics of Coolside and FBC By-Products", Tenth Annual International Pittsburgh Coal Conference", Pittsburgh, PA, September 1993. 
8. Lafferty, C. J.; Robl, T. L.; Schram, W. H.; Yewell, M. E. "Elemental Release Characteristics of Dry Flue Gas Desulfurization By-Products", Tenth Annual International Pittsburgh Coal Conference, Pittsburgh, PA, September 1993.

9. Beckham, T. L.; Hopkins, T. C. "Techniques Used in Placement of Coolside ByProduct in Field Lysimeters" Tenth Annual International Pittsburgh Coal Conference, Pittsburgh, PA, September 1993.

10. Wong, A. S.; Thomas, G. A.; Schram, W. H.; Givens, E. N.; Robertson, J. D. "Characterization of By-Products from the Coolside Process", Tenth Annual International Pittsburgh Coal Conference, Pittsburgh, PA, September 1993.

11. Wu, M. M.; Winschel, R. A.; Wasson, G. E.; Yuran, P. J. " Properties of Synthetic Aggregates Made from FBC and Dry FGD By-Products", Eleventh International Symposium on Coal Ash Use and Management, Orlando, FL, January 1995.

12. Wu, M. M.; Winschel, R. A.; Wasson, G. E., "Composition Effects on Durability of Aggregates Made from Coal Combustion By-Products", Twelfth Annual International Pittsburgh Coal Conference, Pittsburgh, PA, September 1995.

13. Winschel, R. A.; Wu, M. M.; Burke, F. P. "Synthetic Aggregates From Coal-Fired Fluidized-Bed Combustion Residues", Eighth International Conference on Coal Science, Oviedo, Spain, September 1995.

14. Wu, M. M.; Winschel, R. A.; Wasson, G. E. " Composition Effects on Durability of Aggregates Made from Coal Combustion By-Products", 1995 International Ash Utilization Symposium, Lexington, Kentucky, October 1995.

15. Winschel, R. A.; Wu, M. M. "Use of Aggregate Produced from Coal Fired Fluidized Bed Combustion Residues as a Component in Bituminous Concrete", Ninth International Coal Conference on Coal Science, Essen, Germany, September 1997. 


\section{INTRODUCTION}

Waste management is an important cost element to determine the commercial feasibility of the Coolside and LIMB flue gas desulfurization processes. Coolside process involves injection of hydrated lime downstream of the air preheater and the LIMB process involves injection of limestone or hydrated lime into the furnace. The Coolside and LIMB sorbent injection processes were demonstrated successfully at the Ohio Edison Edgewater Station in Lorain, Ohio, from 1989 to 1992, as part of the DOE Clean Coal Technology (CCT). Fluidized-bed combustion (FBC) is a commercial technology which combines steam generation with $\mathrm{SO}_{2}$ control. All of these processes produce a dry solid waste which must be managed to ensure environmental compliance and economic feasibility. The grant agreement, entitled "Coolside Waste Management Demonstration", was awarded by OCDO to CONSOL in July 1991 to conduct an in-depth study of certain aspects of Coolside/ LIMB/FBC waste management. The grant agreement was coordinated with a companion project entitled "Coolside Waste Management Research" that was awarded by the U.S. Department of Energy Morgantown Energy Technology Center (DOE/METC) to the University of Kentucky (UK) under a separate contract (DE-AC21-91MC28162).

The overall objectives of this grant agreement were to specify criteria for landfill disposal of waste from the Coolside process and to evaluate the potential utilization of Coolside, LIMB, and FBC wastes in road construction. Improved options for disposal and utilization of the wastes can reduce the cost of these $\mathrm{SO}_{2}$ control technologies and benefit Ohio's high-sulfur coal industry.

The project was divided into three phases. Phase 1 was to identify the landfill disposal characteristics of Coolside waste. The Phase 1 study was conducted by the University of Kentucky Center for Applied Energy Research (UKICAER) and Kentucky Transportation Center (UK/KTC). DOE and the University of Kentucky provided funding for Phase 1. Phase 2 evaluated the utilization potential of pelletized and unconsolidated FBC, Coolside, and LIMB wastes as highway and construction materials. UKIKTC evaluated the use of the unconsolidated wastes as road base stabilization material in highway construction. 
CONSOL R\&D evaluated the use of pelletized FBC, Coolside, and LIMB wastes as synthetic aggregates for highway and other construction applications. In Phase 3, CONSOL R\&D generated additional waste samples from the CONSOL Coolside pilot plant for use in the Phase 1 and Phase 2 studies. OCDO, CONSOL, and UK provided funding for Phases 2 and 3.

\section{TECHNICAL DISCUSSION}

Highlighted program results are discussed below, ordered according to project phase. Thorough discussions of each project phase are included in the attached appendices. Appendix 1 is the Phase 1 milestone report, "Coolside Waste Management Research", by the University of Kentucky. Appendix 2 is the Phase 2 milestone report, "Investigation of the Utilization Potential of Coolside, LIMB and FBC wastes as Construction Materials", by CONSOL R\&D and the University of Kentucky. Appendix 3 is the Phase 3 milestone report, "Pilot Plant Production of Coolside Waste", by CONSOL R\&D.

\section{PHASE 1}

The Phase 1 study included the determination of chemical, physical, mineralogical, and geotechnical properties and the leachability of Coolside waste. Geotechnical properties and leachability of the waste were examined extensively in both laboratory and field lysimeter studies. The characterization was conducted on numerous waste samples produced at the Ohio Edgewater Station and at the CONSOL Coolside pilot plant. The Edgewater Coolside wastes were collected from the ESP hopper during the process demonstration. The pilot plant Coolside wastes were collected from the baghouse under different process conditions (once-through, recycle, and advanced modes). The laboratory study included the classification of the waste as an engineering material and determining its strength, permeability, and swelling properties as functions of compaction and aging time. The field study included the controlled emplacement of the waste with varying degrees of compaction in $8 \mathrm{ft} \times 8 \mathrm{ft} \times 10 \mathrm{ft}$ test landfill cells, or field lysimeters. The field lysimeters were monitored for $3 \frac{1}{2}$ years of natural weathering (from 1992 to 1996). The geotechnical properties and mineralogy of the wastes were determined to evaluate the 
changes caused by the natural weathering. The laboratory leachability study of the Coolside waste included determination of the leaching characteristics under column leaching and batch extraction (TCLP) conditions. The field leachability study of the waste included determination of the elemental release profiles from the field lysimeters over three and a half year period.

Laboratory test results indicate that Coolside waste is a fine silt material, 95 wt $\%$ of which has particle sizes finer than 200 mesh $(75 \mu \mathrm{m})$. The wetted Coolside waste has cementitious properties caused by the formation of the mineral ettringite, $\left(\mathrm{Ca}_{3} \mathrm{Al}_{2}\left(\mathrm{SO}_{4}\right)_{2}(\mathrm{OH})_{12} \cdot 24 \mathrm{H}_{2} \mathrm{O}\right)$. The essential components for the ettringite formation are $\mathrm{Ca}(\mathrm{OH})_{2}$ (unused sorbent) and $\mathrm{SO}_{3}{ }^{2-}$ or $\mathrm{SO}_{4}{ }^{2-}$ (spent sorbent), soluble $\mathrm{Al}$ (from the fly ash component in the waste) and high $\mathrm{pH}(>10)$. With adequate compaction, the wetted waste can develop high unconfined compressive strength and low permeability. The strength increased and permeability decreased with increasing compaction. Coolside waste compacted at 95\% maximum dry bulk density (ASTM D698-91 Proctor test) with optimum moisture content developed unconfined compressive strength up to 2000 psi and permeability as low as $1 \times 10^{-6} \mathrm{~cm} / \mathrm{sec}$ after aging. 95\% Proctor compaction simulates a typical landfill disposal operation. The strength is similar to that of a low-strength concrete. The compacted Coolside waste has a high angle of friction, high cohesion, and high loadbearing capacity. The angle of friction, cohesion, and California Bearing Ratio (CBR) increased with aging. These properties make Coolside waste suitable for landfill disposal and for use as an engineering material in construction. However, laboratory tests show that the remolded Coolside waste has potential to swell (up to $23 \%$ ) when submerged in water. Mineralogic characterization indicated that the swelling was related to the mineral ettringite in the compacted waste. Aging the remolded specimens before submerging them in water and adding a surcharge (or a static load) greatly reduced the swell potential. Swelling could affect detrimentally the utilization of the material for some engineering applications.

Three field lysimeters were filled in the autumn of 1992 with Coolside waste at three degrees of compaction (loose fill, low compaction and high compaction) and one lysimeter 
was filled with fly ash for comparison. The required densities and moisture were selected based on laboratory testing results. The Coolside waste was collected from the Ohio Edison Edgewater Station. From November 1992 through May 1996, the field lysimeters were monitored to determine moisture, temperature and carbon dioxide $\left(\mathrm{CO}_{2}\right)$ uptake profiles, pressure changes, and leachate characteristics. Laboratory column lysimeter tests were conducted simultaneously for comparison. The field lysimeters were excavated in May 1996 to determine the geotechnical properties and mineralogy of the waste after $31 / 2$ years of natural weathering. The field lysimeter test results show that Coolside waste leachate characteristics, geotechnical properties and mineralogy are affected by the degree of compaction (loose fill, low compaction and high compaction) used during their initial installation. The loose fill was uncompacted waste, which had an average dry density of $44 \mathrm{lb} / \mathrm{ft}^{3}$ and an initial moisture content of $37.5 \%$. The high compaction fill had an average dry bulk density of $66{\mathrm{lb} / \mathrm{ft}^{3}}^{3}$ (i.e., $95 \%$ of maximum standard Proctor dry bulk density) and an initial moisture content of $37.0 \%$. The low compaction fill was mildly compacted waste, which had an average dry bulk density of $49 \mathrm{lb} / \mathrm{ft}^{3}$ and an initial moisture content of $38.9 \%$. With increasing compaction in the field lysimeters, the unconfined compressive strength and leachate $\mathrm{pH}$ increased, and the permeability and leachability of major elements $\left(\mathrm{Ca}, \mathrm{Na}, \mathrm{Cl}, \mathrm{K}_{,} \mathrm{SO}_{4}^{-2}\right)$ decreased. The formation and transformation of minerals in the field lysimeters were affected by the compaction. Coolside waste in the high compaction field lysimeter developed high compressive strength (average 1,629 psi) and a low permeability coefficient (average $2.2 \times 10^{-6} \mathrm{~cm} / \mathrm{sec}$ ). It also had lower concentrations of $\mathrm{Ca}, \mathrm{Na}, \mathrm{Cl}, \mathrm{K}$ and $\mathrm{SO}_{4}^{-2}$ in the leachate and higher leachate $\mathrm{pH}$ (12.3 in the first year). In contrast, Coolside waste in the loose fill field lysimeter developed low compressive strength (average $24.1 \mathrm{psi}$ ) and a high permeability coefficient (average 1.6 $\times 10^{-4} \mathrm{~cm} / \mathrm{sec}$ ). The low compaction fill field lysimeter developed unconfined compressive strength of average $162 \mathrm{psi}$ and a permeability coefficient of $4.6 \times 10^{-4} \mathrm{~cm} / \mathrm{sec}$. In the first year, the leachate concentrations of $\mathrm{Na}, \mathrm{Cl}, \mathrm{K}$ and $\mathrm{SO}_{4}^{-2}$ were higher by a factor of 3 to 4 , and the concentration of $\mathrm{Ca}$ was higher by a factor of $>10$ in the loose fill and low compaction field lysimeters than in the high compaction field lysimeter. Mineralogic characterization indicates that $\mathrm{CO}_{2}$ uptake affects the geotechnical properties and stability of Coolside waste. In the loose fill and low compaction field lysimeters, calcium carbonate 
was formed at the expense of ettringite and gypsum throughout the lysimeters. This may be the cause of the low unconfined compressive strength and high permeability coefficients in these two lysimeters. During the $3 \frac{1}{2}$ year test period, no significant swelling of Coolside waste or pressure change occurred in the field lysimeters.

Compacting the Coolside waste at $95 \%$ maximum dry bulk density with optimum moisture content greatly enhanced the strength, reduced the permeability and the initial release rate of highly soluble salts, and improved its resistance to weathering. Due to the high leachate $\mathrm{pH}$, the leachability of trace elements that form oxyanions, specifically $\mathrm{As}$, Se and $\mathrm{V}$ (which form $\mathrm{AsO}_{4}^{-2}, \mathrm{SeO}_{4}^{-2}$ and $\mathrm{VO}_{4}^{-2}$ ), also increased in the high compaction field lysimeter. The field lysimeter studies show that the release of relatively high As (up to $5 \mathrm{ppm}$ ) and Se (up to $1 \mathrm{ppm}$ ) concentrations in the first year could be of environmental concern, even though the TCLP leaching tests indicate that Coolside waste is nonhazardous for landfill application under RCRA regulations. The RCRA allowable limits of As and Se in TCLP leachate are $5 \mathrm{ppm}$ and $1 \mathrm{ppm}$, respectively. Precautions, such as leachate containment or treatment, should be considered before disposing of Coolside waste.

Long-term laboratory column lysimeter tests produced leachates with major and trace elemental concentrations generally of the same order of magnitude as were produced by the field lysimeters. Long-term column leaching tests may be considered as replacements for field lysimeter tests. The data obtained are useful for the design of environmentally safe and economically sound landfills for the disposal of Coolside waste.

More detailed discussion of Phase 1 test procedures and results appear in Appendix 1.

\section{PHASE 2}

The Phase 2 was divided into two tasks. Task 1 was an evaluation of the utilization of hydrated FBC waste as a chemical admix in road base stabilization and an evaluation of Coolside and FBC wastes as road base materials. The development and demonstration 
of pelletization technology to produce synthetic aggregates from FBC, Coolside, and LIMB wastes and evaluating the synthetic aggregates for road and other construction applications. A large quantity of FBC waste aggregates was produced and used to construct a flexible bituminous test patch. The test patch was monitored for more than one year to evaluate the performance of FBC waste aggregates under heavy traffic and natural weathering conditions.

The potential for using unconsolidated FBC waste as a chemical admix in road base stabilization was evaluated by UKJKTC. The FBC waste was collected from the ash silo of a circulating fluidized-bed combustor firing a Pittsburgh seam coal (same as Ohio No. 8). The FBC ash included both fly ash and bed-drain ash, and it was hydrated at CONSOL R\&D before testing. An Ohio soil that was considered to be of "marginal" quality as a road base was used as the baseline for the road base stabilization work. Mixing $8 \%$ hydrated FBC waste with the marginal Ohio soil increased the unconfined compressive strength from 21 psi to about 100 psi when the mixture was compacted at $95 \%$ maximum Proctor dry bulk density with optimum moisture addition. The load bearing capacity, as indicated by the California bearing ratio or $\mathrm{CBR}$, doubled after $8 \%$ hydrated FBC waste was added. These improvements made the soil suitable for use in road base construction, in terms of strength and bearing capacity. However, laboratory tests indicated that the soil and hydrated FBC waste blend swelled about $9 \%$ over time when submerged in water. The untreated soil swelled only about $4 \%$ under the same conditions. The $9 \%$ swell could be detrimental for use of the blend in road base stabilization, if swell occurred after the road surface was applied. The slow swelling potential of the admixture would require special precautions for its use in road base stabilization. Laboratory swelling tests should be conducted before using the FBC waste as a replacement for hydrated lime in road base construction.

UK/KTC contacted the Ohio Department of Transportation (ODOT) in spring 1993 for assistance in identifying a site suitable to demonstrate the use of hydrated FBC waste as a chemical admix for road base stabilization. Although DOT does not routinely stabilize road base with chemical additives, they identified a potential site: the pavement of 
portions of 1-275 in the Cincinnati area was scheduled to be removed, the subgrade modified, and a new pavement was to be placed in summer 1993. Due to the uncertainty of the FBC waste swelling characteristics and the high traffic volume, this site was not selected for a field demonstration. Follow-up testing would be very costly on a high traffic volume interstate highway, and would be beyond the budget of this project. Therefore, the road base stabilization study was limited to the laboratory testing, and the field trial was not conducted.

The load bearing capacity of $100 \%$ Coolside waste and the long-term swell of $100 \%$ Coolside waste and $100 \%$ FBC waste were determined to evaluate the feasibility of using either waste as a road base construction material. The Coolside wastes included waste samples collected from the Ohio Edison Edgewater Demonstration program and from the CONSOL pilot plant (Phase 3 program). The FBC wastes evaluated included as-is and hydrated waste samples. The load-bearing capacity was determined by the California bearing ratio (CBR) test. Coolside wastes compacted at $95 \%$ maximum Proctor dry bulk density or higher with near optimum moisture content had high CBRs ranging from 6 to 144. Material with a CBR of 6 or greater has sufficient load-bearing capacity for use in road base construction. However, the compacted Coolside waste swelled up to $30 \%$ over time. FBC waste compacted at $95 \%$ maximum Proctor dry bulk density with optimum moisture content swelled up to $40 \%$ over time. Such high swelling could cause pavement to heave or buckle. With Coolside and FBC wastes, aging the remolded specimen before submerging in water and adding a surcharge weight greatly reduced the swell potential. Swelling potential could limit the utilization of FBC and Coolside wastes as road base materials. The tendency for swelling should be determined and appropriate precautions should be taken before the use of these wastes in construction.

A pelletization technology was developed to make synthetic aggregates from $\mathrm{FBC}$ or dry FGD wastes produced by the Coolside and LIMB processes. The sources of the FBC and Coolside wastes were discussed previously. The LIMB wastes were collected from the ESP hopper during the Ohio Edison Edgewater process demonstration. High-strength synthetic aggregates with low abrasion index were produced from FBC waste. The 
synthetic aggregates produced meet all specifications (unit weight, soundness index, Los Angeles abrasion index and size distribution) for use as a AASHTO Class A aggregate in surface-wearing course for road construction. Although not required by specification, additional tests were conducted to evaluate the effects of natural weathering and freeze thaw on pellet properties. The aggregates degraded when they were fully immersed in water during long-term natural weathering and laboratory freeze/thaw tests. Characterization of the degraded aggregates indicated that the slow hydration of anhydrite $\left(\mathrm{CaSO}_{4}\right)$ to gypsum $\left(\mathrm{CaSO}_{4} \cdot 2 \mathrm{H}_{2} \mathrm{O}\right)$ was the major cause for the degradation. Durability was improved by blending FBC waste with pulverized coal (p.c.) fly ash (from the Ohio Edison Mansfield Station) to reduce the gypsum formation and by coating the aggregate with asphalt to increase the water resistance. A flexible bituminous test patch (12' $\times 35^{\prime}$ $\times 1.5-2 ")$ was constructed in April 1995 with 1.5 ton synthetic aggregates made from a $65 / 35$ blend of FBC waste and p.c. fly ash as half of the coarse aggregates. This test demonstrated the use of these materials for road construction under heavy traffic and natural weathering conditions. Class $\mathrm{A}$ crushed limestone was used as half the aggregates to provide an internal standard for evaluating aggregate performance. About $6.5 \mathrm{wt} \% \mathrm{AC}$ 20 asphalt was mixed with the aggregates. The test patch was constructed with standard equipment. The heavy truck traffic on the selected road and the high permeability of the open-graded mix design made this a severe test of aggregate durability. The evaluation ended on May 30, 1996. Inspections revealed no visible degradation of the patch or aggregate after 13 months of service. Monthly analysis of core samples showed no degradation of either the $\mathrm{FBC}$ waste aggregate or the limestone aggregates between June 1995 and May 1996. This test convincingly demonstrated the potential for use of synthetic aggregates made from $\mathrm{FBC}$ waste as Class $\mathrm{A}$ aggregate in construction of asphalt surface wearing course.

High-strength synthetic aggregates with low abrasion indices were also made from Coolside and LIMB wastes. The Coolside wastes were obtained from the Ohio Edison Edgewater Station during the Coolside process demonstration and from the CONSOL Coolside pilot plant. The LIMB waste was obtained from the Ohio Edison Edgewater Station during the LIMB process demonstration. Synthetic aggregates made from Coolside 
and LIMB wastes failed to meet the unit weight and sodium sulfate soundness index specifications as road aggregate. However, they met many ASTM specifications as coarse lightweight aggregates. (Not all ASTM lightweight aggregate specification properties were evaluated.) Test results show that both aggregates have potential for use as coarse lightweight aggregates in concrete masonry units. The addition of fly ash to Coolside and LIMB wastes during aggregate preparation further improved the aggregate properties.

More detailed discussion concerning the test procedures and results of Phase 2 appear in Appendix 2.

\section{PHASE 3}

Three Coolside waste samples of about $500 \mathrm{lb}$ each and a fourth sample of about $20 \mathrm{lb}$ were produced under different process conditions (once-through, recycled and Advanced modes) at the CONSOL Coolside pilot plant. These samples were used to determine the impact of process variables on waste properties and on disposal and utilization options in Phases 1 and 2. The samples were produced at conditions that simulate operation of the Coolside process installed on a unit burning medium-sulfur Ohio coal. More detailed discussion concerning the pilot plant run procedures and test results in Phase 3 appear in Appendix 3.

\section{MARKETING/COMMERCIALIZATION DISCUSSION}

The Clean Air Act Amendments (CAAA) of 1990 favor high-performance FGD systems with over $90 \% \mathrm{SO}_{2}$ removal efficiency. Although the Coolside and LIMB processes were demonstrated as low-capital-cost $\mathrm{SO}_{2}$ abatement technologies, their intermediate $\mathrm{SO}_{2}$ removal performance ( $50 \%$ to $70 \%$ ) limited the commercial application of these processes. Coal-fired fluidized-bed combustion (FBC) failed to make a substantial penetration into Ohio or into the market area of Ohio coal. These developments limit the current usefulness of the pelletization technology developed here for promoting Ohio high-sulfur coal use. The pelletization technology could be used to produce synthetic aggregates from dry FGD process wastes, if these processes are ever deployed in Ohio. 
By far, wet scrubbers are and will continue to be the most important means of controlling $\mathrm{SO}_{2}$ emissions from coal-fired electric utility plants in Ohio and the market area of Ohio coal. Waste management costs increase the cost of producing power in scrubbed units, especially in power plants burning high-sulfur coal. Utilization of FGD sludge could reduce the waste management cost and could benefit Ohio's high-sulfur coal industry. The pelletization technology that was developed for producing synthetic aggregates from FBC and dry FGD (Coolside and LIMB) wastes should be evaluated for use in producing synthetic aggregates from wet FGD sludge generated in Ohio. The product should be tested for use in road and structural construction. The huge aggregate market provides a promising opportunity for high-volume use of FGD materials.

\section{FINAL BUDGET SUMMATION}

A table, Schedule $S$, details the total project budget by major category and contributions of all co-sponsors. A table, Schedule $T$, details by line items the expenditure of OCDO funds on this project. 


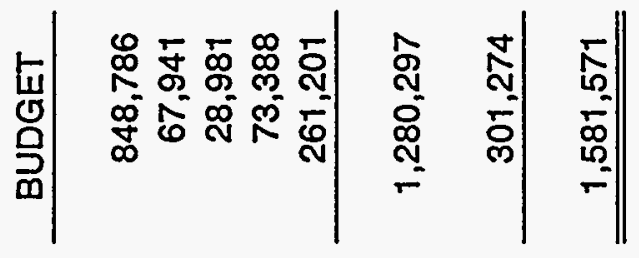

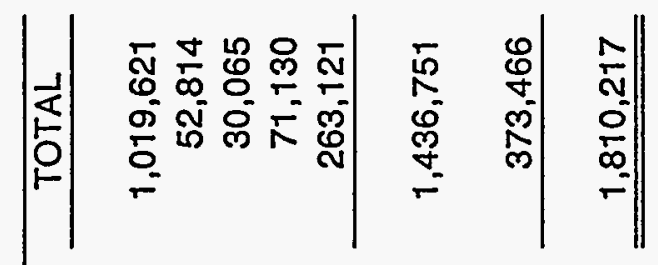

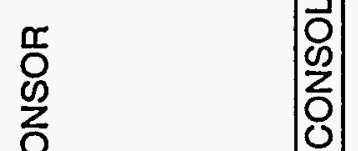

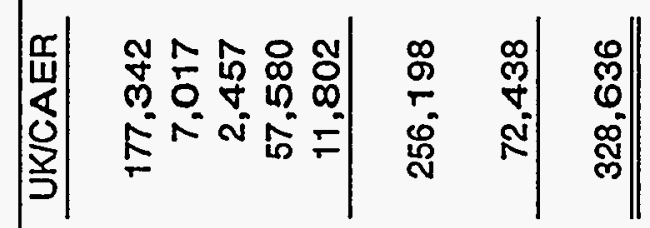

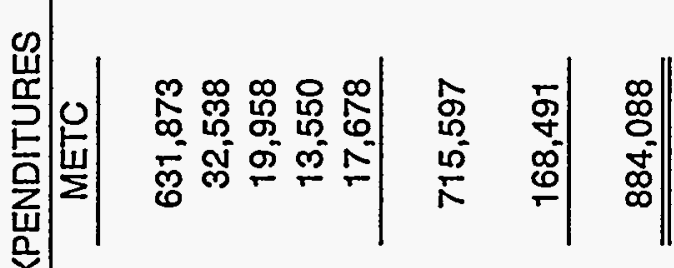

迹

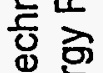

Ф

केष

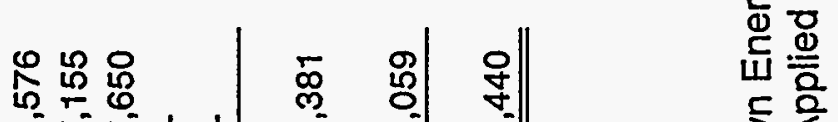

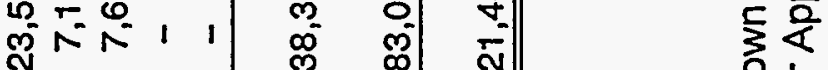

은

造 $\quad \sum_{0}^{0}$

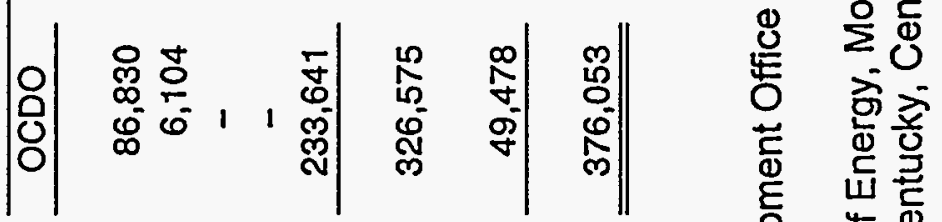

ז

品

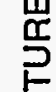

은

\begin{tabular}{l|ll|l|l|l|l|l|l|l|}
$\stackrel{m}{N}$ & $\infty$ & $\mathbb{N}$
\end{tabular}

绕

๘岕号

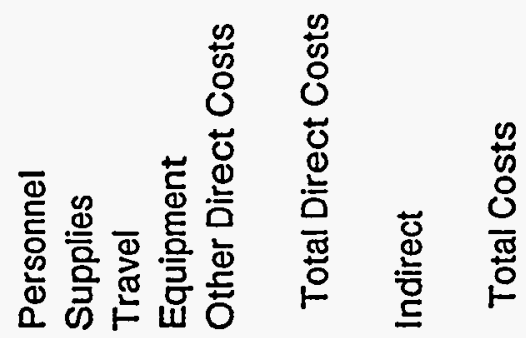

山

苛造

虽安

殅它苍

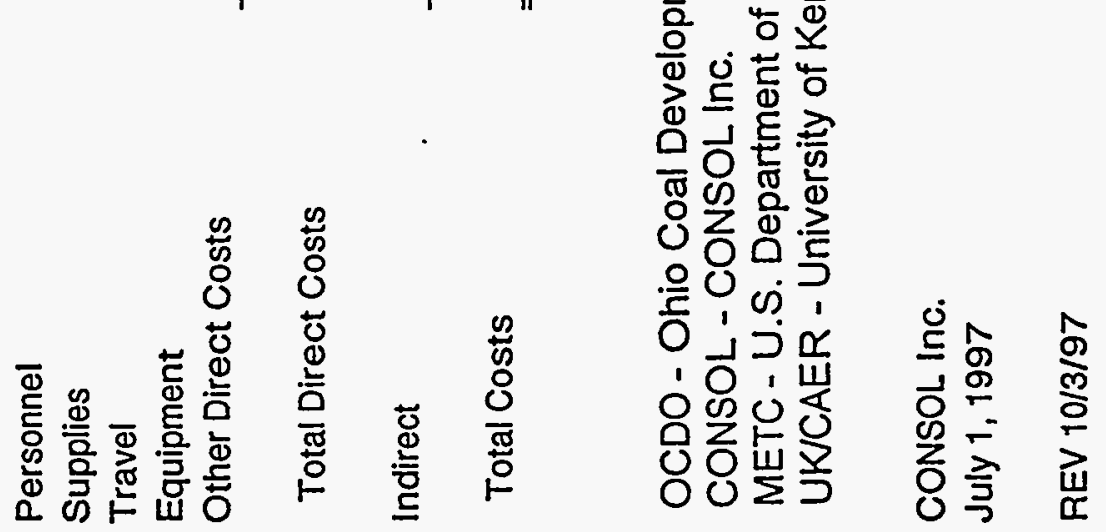


SCHEDULE T

OCDO EXPENDITURES

CONTRACT CDO/D-902-9

\begin{tabular}{lrr} 
& \multicolumn{1}{r}{ OCDO } & BUDGET \\
\cline { 2 - 2 } Salaries & 67,204 & 63,318 \\
Fringe Benefits & 19,626 & 13,930 \\
Travel & 0 & 0 \\
Equipment & 0 & 4,250 \\
Supplies & 6,104 & 7,250 \\
Contractual & 233,641 & \\
Total Direct & 326,575 & 322,642 \\
Indirect & 49,478 & 53,664 \\
\hline & & \\
Total Cost & 376,053 & 376,054 \\
\hline
\end{tabular}

CONSOL Inc.

July 1,1997 


\section{APPENDIX 1}




\title{
COOLSIDE WASTE MANAGEMENT DEMONSTRATION OCDO GRANT AGREEMENT NO. CDO/D-902-9
}

\author{
Milestone Report - Phase 1 \\ The Landfill Disposal Characteristics of Coolside Waste
}

February 1997

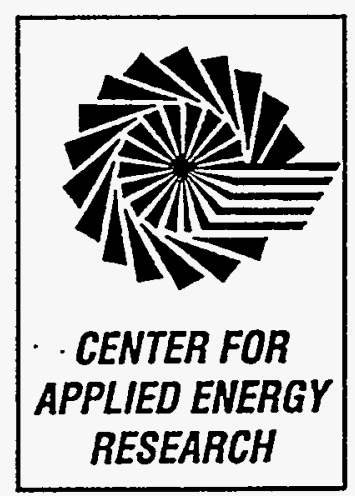

\section{UNIVERSITY OF KENTUCKY}

Center for Applied Energy Research

3572 Iron Works Pike

Lexington, KY 40511

T. L. Robl 606-257-0272

U. M. Graham 606-257-0299

T. C. Hopkins 606-257-4513

T. Beckham 606-257-4513

D. N. Taulbee 606-257-0238

W. K. Adkins 606-257-0263 


\section{Coolside Waste Management Research}

Contract No: DE-AC21-91MC28162 Final Report

Center for Applied Energy Research

University of Kentucky 3572 Iron Works Pike

Lexington, KY 40511-8433 
Table of Contents

COOLSIDE WASTE MANAGEMENT RESEARCH, Final Report, Executive Summary ........................................................................

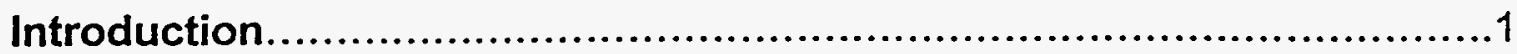

Project Summary ..................................................................

Background and Methodology...........................................1

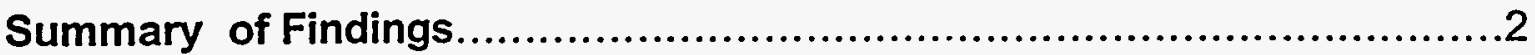

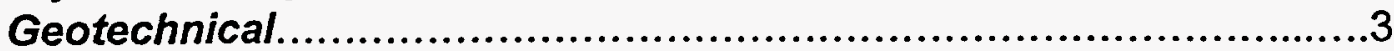

Laboratory Results.....................................................

Field Results............................................................3

Geochemical Results.......................................................4

CHAPTER 1. INTRODUCTION AND PROJECT BACKGROUND................7

Description of the Technology and Statement of Objectives...................7

Project Objectives..............................................................

Discription of Coolside Technology ......................................7.

Process Description..........................................................8

The Edgewater Demonstration............................................10

Coolside Waste Sample Collection......................................11

Lysimeter Description and Instrumentation.............................13

Filling Operations.............................................................14 14

Laboratory Compaction for Field Emplacement ........................21

\section{CHAPTER 2. MINERALOGIC INVESTIGATION OF THE COOLSIDE}

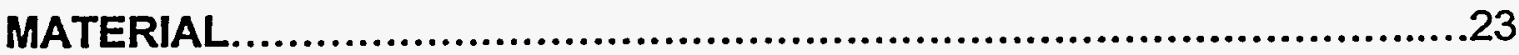

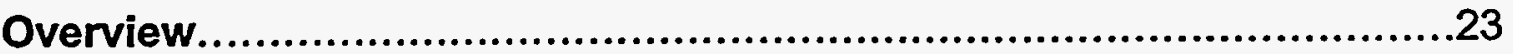

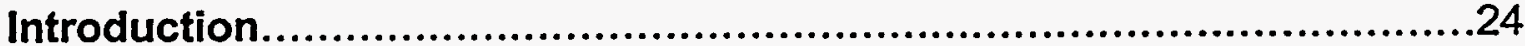

Analytical Methods......................................................25

Important Mineral Phases................................................26

Glassy Phase...........................................................26

Sillicon Dioxide............................................................27

Mullite....................................................................27

Portlandite........................................................27

Anhydrite.........................................................28

Gypsum/Hemihydrite..............................................28

Ettringite..........................................................29

Calcium Carbonate...................................................29

Hannebachite .........................................................30 
Series I: Field Lysimeter Study

Series II: Laboratory Lysimeter Study ....................................53

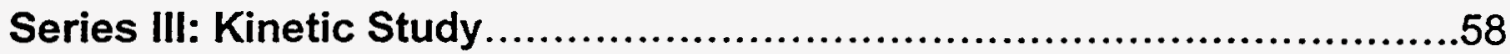

Series IV: Pelletization of FBC Material...........................................71

CHAPTER 3: THE PHYSICAL AND GEOTECHNICAL CHARACTERISTICS OF THE COOLSIDE MATERIALS ...................................................75

The Characteristics of the Coolside Materials as Emplaced in the Field....75 Geotechnical Characteristics of the Materials As Emplaced...........75

Density and Moisture Content..........................................75

Pressure, Temperature, and Swell Measurements...................8.82

Collection and Testing of Specimens.................................84

Field and Laboratory Tests on Specimens Collected

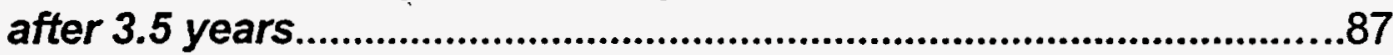

Geotechnical Laboratory Test Program......................................97

Index Properties and Classification.......................................97

Moisture-Density Relations................................................97

Recommendations........................................................101

Structural Properties Tests..............................................102

Remolding Procedure.............................................102

Equations...........................................................103

Compaction Equipment and Method.................................106

Procedure...........................................................107

Statistical Analysis...............................................112

Triaxial Strength Properties............................................113

Unconsolidated-Undrained (UU) Triaxial Compression Tests.....113

Unconfined Compression Tests.........................................114

California Bearing Ratio..............................................116

Permeability............................................................121

Swelling Characteristics Of the Coolside Ash.........................125

Sources Of Materials For Swelling Tests And Index Properties.. 125

Swell Testing Procedures.............................................128

Test Results and Analysis.........................................129

CASE STUDY......................................................... 141

The Utilization of Coolside and Other FGD Materials in Highway

Embankments 
Summary.

Background.

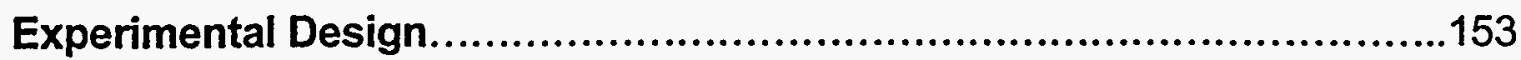

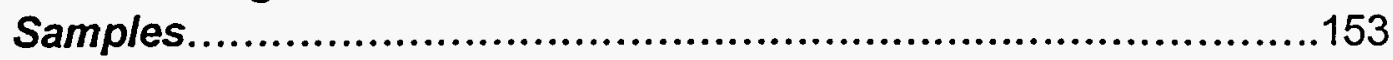

Laboratory-Lysimeter Description......................................155

Lysimeter Packing Procedures..........................................155

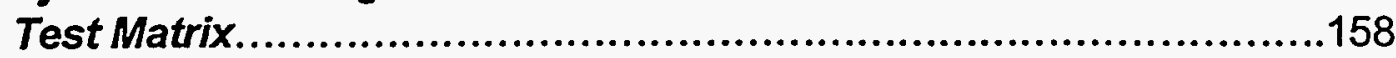

Compaction............................................................158

Fixed Feed and Rain Simulation......................................158

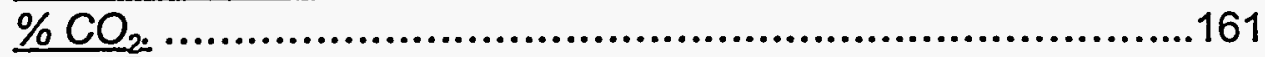

Prehydration Water.....................................................161

Dry Weight................................................................162

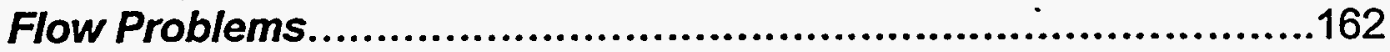

Leachate Analysis.........................................................162

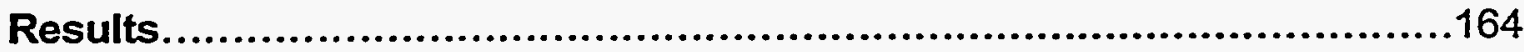

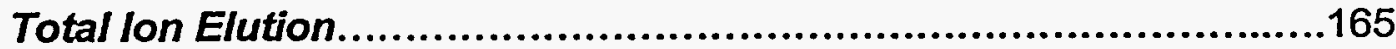

TCLP Extraction Data....................................................166

Demonstration Run \#3..................................................169

$\mathrm{CO}_{2}$ Effects. .......................................................183

Prehydration Effects. .......................................................192

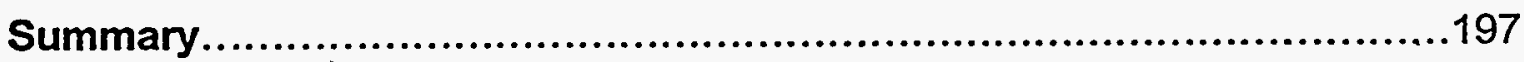

CHAPTER 5. CHEMISTRY OF THE FIELD LEACHATES......................199

Sample Collection and Analytical Methodology...............................199

Analytical Protocol.............................................................199

Sample Collection Methodology.......................................200

Chemistry of the Leachates Collected from the Field Lysimeters...........202

Leachate Composition.....................................................202

Major lons. .............................................................203

Minor and Trace lons.....................................................211

Carbon Dioxide. ................................................221

Factors Affecting Leachate Chemistry.................................224

Early Reactions. ...................................................224

Longer Term Weathering Reactions..................................224

Material Compaction and Leachate Chemistry. .....................227 


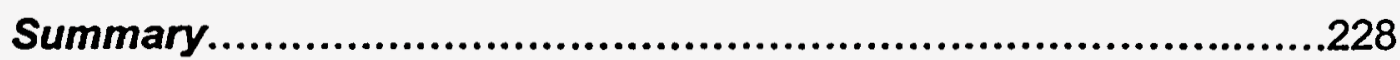

CHAPTER 6. THERMODYNAMIC DATA BASE............................230

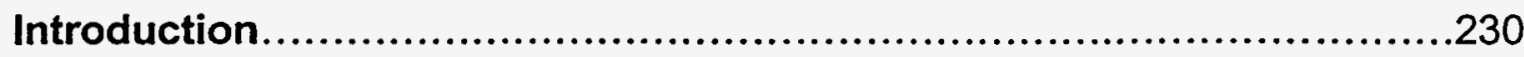

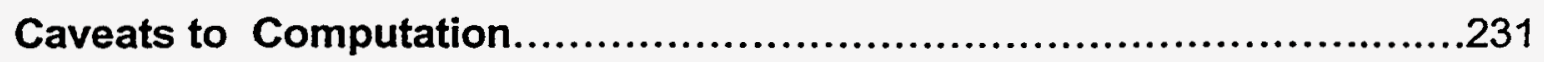

Preliminary Investigations..............................................233

Selection of Primary Mineral Forms and lonic Species and Results.......234

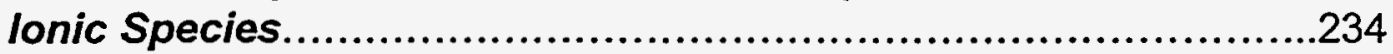

Mineral Forms.........................................................236

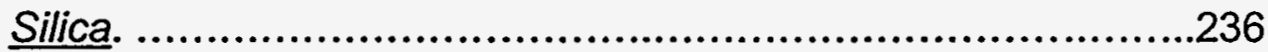

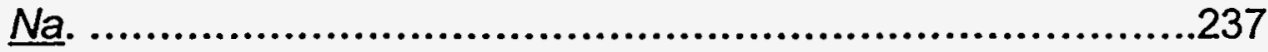

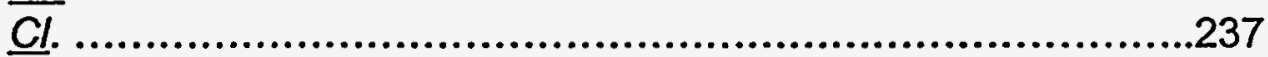

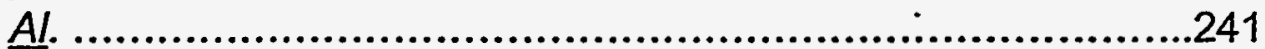

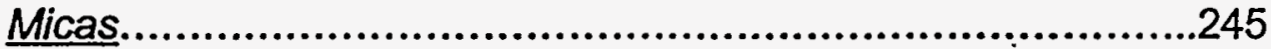

Feldspars. .......................................................245

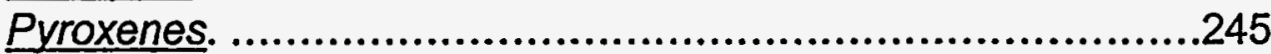

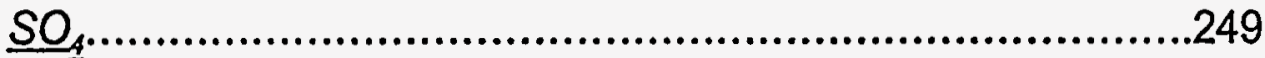

Ettringite............................................................252

Ca and Mg. Carbonates and Hydroxides...........................252

Minor Species Carriers...................................................259

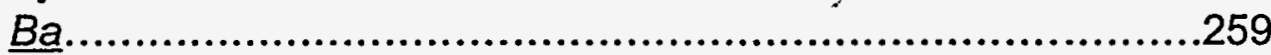

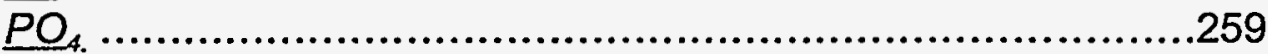

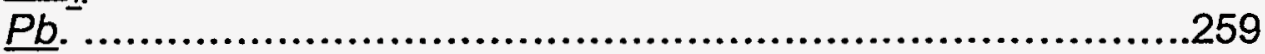

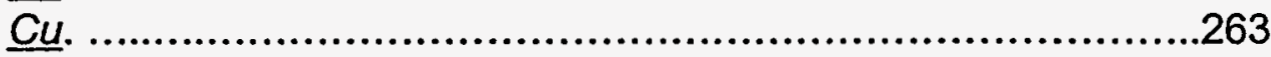

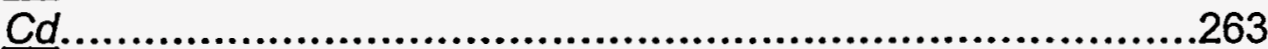

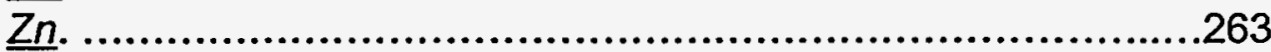

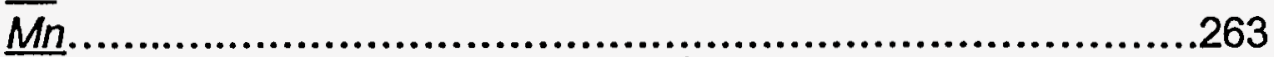

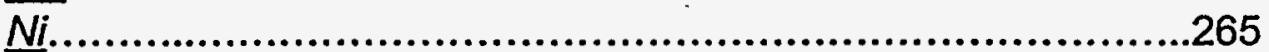

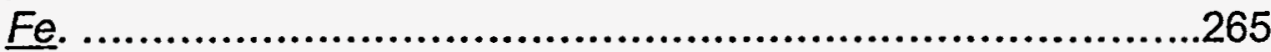

Investigation of Initial pH Dip, Field Lysimeters 1 and 2...................265 


\section{COOLSIDE WASTE MANAGEMENT RESEARCH \\ Final Report \\ Executive Summary}

\section{Introduction}

This final report summarizes the important aspects of the project, but it does not present all of the data that was produced. Further details may be found in the monthly and quarterly reports that were filed with the Morgantown Energy Technology Center. This report is organized into six chapters which present the important conclusions of the principal areas of investigation. A summary of the more significant observations follows.

\section{Project Summary}

\section{Background and Methodology}

This study was initiated during a successful test of the Coolside flue gas desulfurization technology at Ohio Edison's Edgewater generating station in 1991. Coolside is a lime duct injection technology which is installed on the : downstream side of the last heat exchanger (Chapter 1). As tested by Ohio Edison, it also employs an alkali reagent, in this case $\mathrm{NaOH}$, to enhance sulfur capture.

The overall goal of the this study was to develop sufficient chemical and physical data to insure the environmentally safe disposal of the material. A related project conducted in conjunction with CONSOL Research and Development investigated the utilization potential of the material. Two areas were chosen for 
particular emphasis. These are the elemental release or leaching characteristics of the material and the geotechnical characteristics of the material.

The methodology and approach for the geotechnical work (Chapter 3) included the laboratory classification of the waste as an engineering material and laboratory investigations of the its strength and swell properties as a function of compaction. Field studies included the controlled emplacement of the material under varying degrees of compaction. During the fill the geotechnical properties of the materials were measured. The lysimeters were excavated at the end of the project and the physical properties of the materials were again measured to determine the changes which had taken place.

The leaching properties of the material investigated included the determination of the chemistry of the leachate under laboratory column leaching and batch extraction (TCLP) conditions (Chapter 3). The leachates from the field lysimeters were also collected and analyzed over a three and a half year period (Chapter 5).

The geotechnical and geochemical properties of the materials are dependent upon complex mineral solution reactions. In an attempt to determine the overall controls on the system, detailed mineralogical determinations were made of the materials (Chapter 2). Also, investigations were made into the state of the thermodynamic data available. A publicly available database and computer program was obtained from the United States Geological Survey and modified for this project (Chapter 6).

\section{Summary of Findings}

\section{Geotechnical}


Laboratory Results. Ninety-five percent (95\%) of the Coolside material passed a 200 mesh sieve ( -75 micron) and had a specific gravity of $\sim 2.5$. Index properties tests indicated that the Coolside materials were non-plastic and classified as ML (silt) in the Unified Classification System and A-4 under the ASSHTO System.

The Coolside material has the potential to be an excellent engineering material. Specimens compacted to $95 \%$ of maximum dry density $\left(\sim 1120 \mathrm{~kg} / \mathrm{m}^{3}\right.$ or $\sim 70$ $\left.\mathrm{lbs} / \mathrm{ft}^{3}\right)$ and with optimum moisture (36.5\%) were found to develop unconfined compressive strengths of between 1,000 psi and 2,500 psi $(\sim 6,900$ to $\sim 17,200$ $\mathrm{kPa})$. These strengths approach some types of concrete and greatly exceed natural soils under comparable conditions. The compacted Coolside materials achieved permeabilities from $4 \times 10^{-5}$ to $3 \times 10^{-6} \mathrm{~cm} / \mathrm{sec}$ (low to very low) in the lab.

The Coolside materials exhibited unusual swell properties. Submerged molded specimens which were unaged and unconditioned had total swell which ranged from $10 \%$ to $23 \%$. Aging the samples before submerging and the addition of surcharging greatly reduced the amount of swell to values within the range of typical soils. Swelling could have an impact on the utilization of the materials for some engineering applications.

Field Results. The field lysimeters were filled at three differing levels of compaction. Lysimeter L1 was loose filled (i.e. uncompacted) and had average dry density and moisture content of $706 \mathrm{~kg} / \mathrm{m}^{3}\left(44.1 \mathrm{lbs} / \mathrm{ft}^{3}\right)$ and $37.5 \%$, respectively. Lysimeter $L 2$ was compacted to the density designed to simulate the compactive efforts of a D9 bulldozer (117.3 kPa or $\sim 17 \mathrm{psi})$. Dry density and moisture contents averaged $788 \mathrm{~kg} / \mathrm{m}^{3}\left(49.2 \mathrm{lbs} / \mathrm{ft}^{3}\right)$ and $38.9 \%$ in this lysimeter. The third lysimeter ( $L-3$ ) was filled with Coolside material compacted near $95 \%$ of standard maximum dry density and optimum moisture content. Average 
values of dry density and moisture content were $1060 \mathrm{~kg} / \mathrm{m}^{3}\left(66.2 \mathrm{lbs} / \mathrm{ft}^{3}\right)$ and $37.0 \%$, respectively.

Upon excavation, after 3.5 years of weathering, the materials were excavated and their geotechnical properties determined. The average strengths of the materials in the three lysimeters were $44.1,46.4$ and 1,629 psi for the $L 1, L 2$ and L3 lysimeters respectively. Average permeabilities were $1.55 \times 10^{-4}, 4.6 \times 10^{-4}$ and $2.2 \times 10^{-6}$ for $L 1, L 2$, and $L 3$ respectively. Thus, compaction had an overarching effect on the physical properties of the material. Achieving optimum compaction resulted in a fill which had substantial strength (layers of L3 achieved compressive strengths as high as 2,600 psi) and greatly reduced permeability (layers with permeabilities as low as $10^{-8}$ to $10^{-9} \mathrm{~cm} / \mathrm{sec}$ were measured).

\section{Geochemical Results}

The Coolside material as received was composed of quartz $\left(\mathrm{SiO}_{2}\right)$, mullite $\left(\mathrm{Al}_{6} \mathrm{Si}_{2} \mathrm{O}_{13}\right)$, portlandite $\left.\left(\mathrm{Ca}(\mathrm{OH})_{2}\right)\right)$, calcite $\left(\mathrm{CaCO}_{3}\right)$, hannebachite $\left(\mathrm{CaSO}_{3}\right.$ $\left.0.5 \mathrm{H}_{2} \mathrm{O}\right)$ and minor anhydrite $\left(\mathrm{CaSO}_{4}\right)$. A glassy phase is also present in the raw Coolside material and typically consists of spherical Si-Al fly ash particles. Upon hydration ettringite $\left(\mathrm{Ca}_{6} \mathrm{Al} 2\left(\mathrm{SO}_{4}\right)_{3} \mathrm{OH}_{12} 26 \mathrm{H}_{2} \mathrm{O}\right)$, the principal cementitious mineral in the system rapidly forms,

$$
6 \mathrm{Ca}^{+2}+2 \mathrm{Al}^{+3}+3 \mathrm{SO}_{4}^{-2}+12 \mathrm{OH}^{-}+26 \mathrm{H}_{2} \mathrm{O}=\mathrm{Ca}_{6} \mathrm{Al}_{2}\left(\mathrm{SO}_{4}\right)_{3} \mathrm{OH}_{12} \cdot 26 \mathrm{H}_{2} \mathrm{O}
$$

The state of compaction of the materials was found to have a strong impact on the chemistry of the leachates. Two distinct patterns emerged over the study. Lysimeters L1 and L2 initially had much higher elemental concentrations compared to $\mathrm{L} 3$ which was compacted to optimum density. Sodium, $\mathrm{Cl}, \mathrm{K}$ and sulfate were all higher in concentration by factors of 3 to 4 and $\mathrm{Ca}$ by a factor of 
more than 10 in $L 1$ and $L 2$ leachates. The longer term elemental release pattern showed a more rapid decline in elemental concentrations in the $\mathrm{L1}$ and $\mathrm{L2}$ leachates. For example, $\mathrm{Na}$ in $\mathrm{L} 1$ dropped from an average value of 15,767 ppm--which is approaching the concentration of a brine--the first year, to an average value of $1,637 \mathrm{ppm}$ the second year, and $604 \mathrm{ppm}$ the third. The L3 leachates declined in concentration at a lower rate, with an average of 5,480 ppm the first year followed by averages of $2,302 \mathrm{ppm}$ and 1,436 ppm the second and third year.

The leaching pattern for minor and trace elements did not necessarily show a similar pattern to that of the major elements. For example, Al and Si increased in concentration during the second year of the study in the $L 1$ and $L 2$ leachates as a function of $\mathrm{pH}$ of the leachates which increased over time. The average $\mathrm{pH}$ of the L1, L2 and L3 leachates was 9.7, 10.4, and 12.3 during the first year of collection. Most transition metals are insoluble under these conditions and were not detected. However, elements which can form oxyanionic complexes such as $\mathrm{Mo}\left(\mathrm{MoO}_{4}^{-2}\right)$, Se $\left(\mathrm{SeO}_{4}^{-2}\right), \mathrm{As}\left(\mathrm{AsO}_{4}^{-3}\right)$ and $\mathrm{V}\left(\mathrm{VO}_{4}^{-3}\right)$ were found in measurable and sometimes significant concentration (e.g. Mo reached maximum concentrations $>100 \mathrm{ppm}$ in several samples the first year).

Carbon dioxide concentrations in the soil gases of the lysimeters were monitored in the second and third year of the study. In general, the highest $\mathrm{CO}_{2}$ concentrations were reached during the summer and the lowest during midwinter, as a function of respiration. The highest concentration recorded was $3.2 \%(32,000 \mathrm{ppm})$, well above that of atmospheric concentration ( $350 \mathrm{ppm})$. Soil gas profiles gave clear and conclusive evidence of the highly reactive nature of the Coolside materials with respect to $\mathrm{CO}_{2}$. At pH's above $\sim 10 \mathrm{CO}_{2}$ reacts directly with hydroxide and forms carbonate ion,

$$
\mathrm{CO}_{2}+2 \mathrm{OH}^{-}=\mathrm{HCO}_{3}^{-}=\mathrm{H}^{+} \mathrm{CO}_{3}^{-2}
$$


The thermodynamic analysis of the chemical composition of the leachates indicated that they are all supersaturated with respect to calcite and undersaturated with respect to gypsum and hannebachite. This, combined with direct mineralogical evidence, indicates that minerals are both dissolving and precipitating. The most important long term weather reactions include the precipitation of calcium carbonate and the dissolution of sulfites and sulfates, primarily hannebachite in this case, summarized as follows:

$$
\mathrm{CaSO}_{3} .1 / 2 \mathrm{H}_{2} \mathrm{O}+1 / 2 \mathrm{O}_{2}+\mathrm{CO}_{3}^{-2}=\mathrm{CaCO}_{3}+\mathrm{SO}_{4}^{-2}+1 / 2 \mathrm{H}_{2} \mathrm{O}
$$

Both the mineralogical and thermodynamic data indicate that ettringite will also. break down over time. However this does not appear to proceed until the sulfate is largely exhausted. Thus, in effect, the sulfate acts as a buffer to extend the stability of the ettringite.

Elements of environmental concern which were found in concentrations exceeding RCRA limits included Se (RCRA limit $1 \mathrm{ppm}$ ) and As (RCRA limit 5 $\mathrm{ppm}$ ). Some of the leachates from the L3 lysimeter reached concentrations as high as $19 \mathrm{ppm}$ As during the first year of the study. The average concentration of Se for all of the leachates from the Coolside materials for the first year of study exceeded the $1 \mathrm{ppm}$ limit.

Batch leaching extractions following US EPA protocols were not relatable to elemental concentrations as observed in the field. Long term column leaching experiments produced leachates with elemental concentrations of the same magnitude as produced by the field lysimeters. This included similar As and $\mathrm{Se}$ concentrations as found in the field leachates. 


\section{CHAPTER 1. INTRODUCTION AND PROJECT BACKGROUND}

\section{Description of the Technology and Statement of Objectives}

\section{Project Objectives}

The primary objective of the study was to produce sufficient chemical and geotechnical information to assure that the disposal of Coolside flue gas desulfurization (FGD) material can be conducted in an environmentally safe manner. More specifically, our objectives were to determine the elemental release or "leaching" characteristics as well as changes in the physical characteristics of the materials over time.

The leaching research included both field lysimeter studies and laboratory research, including standard and column extraction methods. The geotechnical research also included field and laboratory work. The field research focused on the testing of the materials as emplaced in the lysimeter and after 3.5 years of leaching. The laboratory work included standard methodology to classify the material's engineering properties, and tests to measure its performance under various conditions of moisture and compaction. Two areas of special emphasis included the measurement of the swelling characteristics of the material and its mineralogy.

\section{Description of Coolside Technology}

Coolside is a dry flue gas desulfurization (FGD) technology for removal of $\mathrm{SO}_{2}$ in coal fired power plants. The technology was designed as a retrofit to existing power plants by CONSOL Research and Development. 
Coolside is an attractive option for retrofit $\mathrm{SO}_{2}$ reduction for plants which have limited boiler life or where capital cost or space limitations are important factors. The capital investiture is modest compared to wet scrubbing technologies, as all that is required is duct work modification and the installation of a reagent delivery system. Because the technology utilizes the existing hardware, it is ideal for installations which have space limitations. The technology operates on the tail end of the power generating system, where malfunctions in the $\mathrm{SO}_{2}$ scrubber technology are unlikely to cause disruptions of the operation of the power plant. Coolside can be coupled with burner lime injection (BLI) technology to provide higher overall calcium utilization and $\mathrm{SO}_{2}$ reductions than can be achieved by either alone.

Process Description. Coolside is similar to other dry scrubbing technologies such as Dravo's HALT process (Hydrate Addition at Low Temperatures). ${ }^{1}$ in these processes hydrated lime is pneumatically injected dry into the power plant duct work on the "cool side" of the air preheater (Figure 1-1). The flue gas stream is then humidified to within about 20 to $30^{\circ} \mathrm{F}$ of adiabatic saturation by spraying atomized water. This also reduces the flue gas temperature.

Humidification of the flue gas is critical to the process as it greatly increases $\mathrm{SO}_{2}$ sulfur capture efficiency by activating the sorbent. Sulfur capture has been shown to increase linearly with relative humidity and to be strongly affected by the presence of water droplets. ${ }^{2}$ Humidification enhances the performance of the electrostatic precipitators (ESP) by reducing the resistivity of the ash.

The Coolside process can be operated with the addition of a "promoter", such as $\mathrm{NaOH}$ or $\mathrm{Na}_{2} \mathrm{CO}_{3}$, to the water spray to enhance $\mathrm{SO}_{2}$ capture efficiency. Increased capture efficiency may be due to several factors including: 1) additional sulfur capture by the promoter itself; 2) the modification of the surface of the sorbent by the promoter; 3 ) increasing the basicity of the sorbent with the 


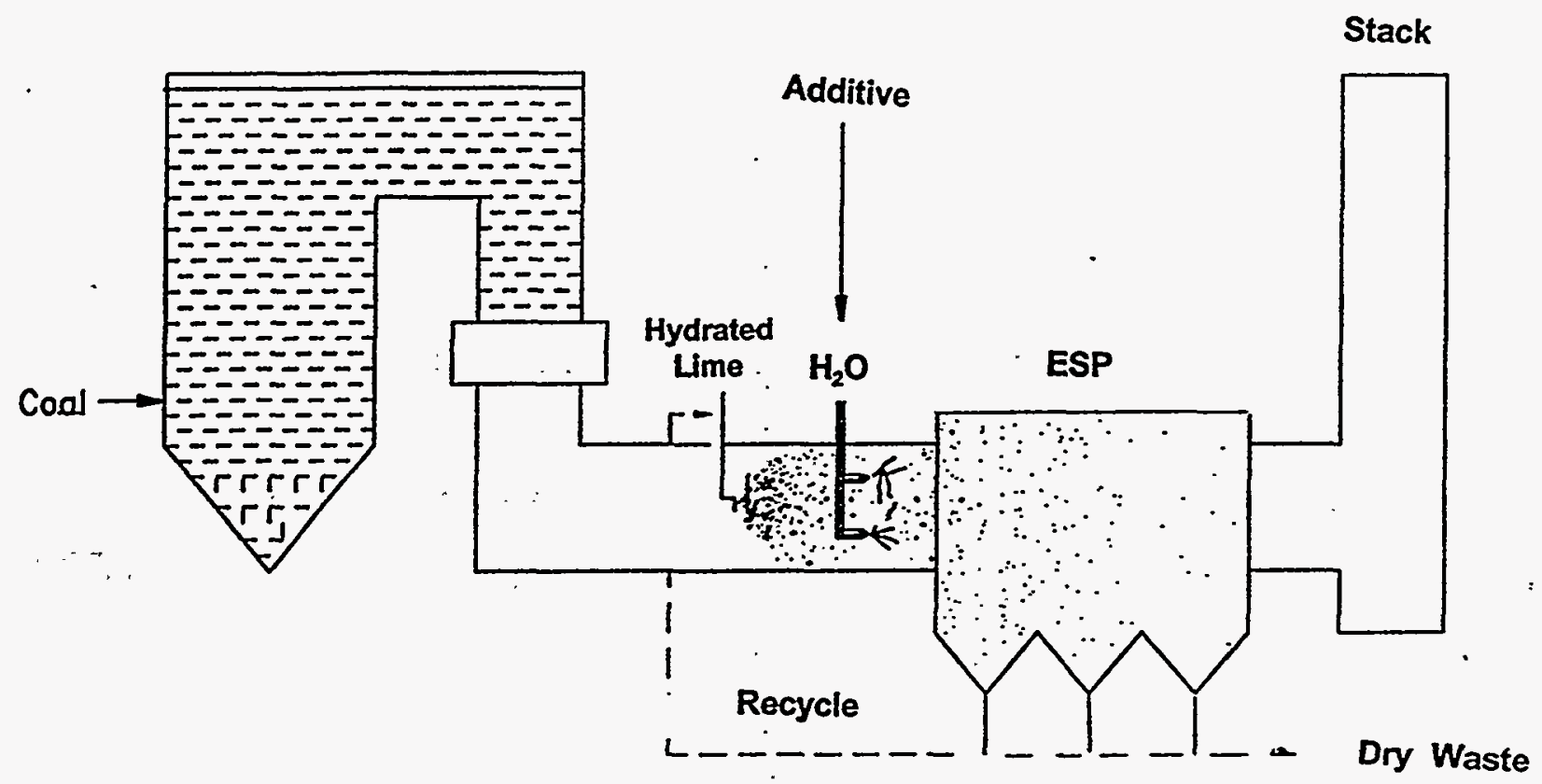

Figure 1-1. Schematic diagram of Coolside process. 
promotion of acid-base $\mathrm{SO}_{2}$ capture reactions, and; 4) increasing or retaining moisture at the sorbent surface, of importance for deliquescent promoters such as $\mathrm{NaOH}^{3}$ In general sodium based compounds have been found to be the most effective promoters, although chloride salts have also been shown to be effective if added during lime hydration.

Calcium utilization efficiency can also be increased by recycling the ash from the ESP. The recycled sorbent has been shown to be almost as effective as fresh material in absorbing $\mathrm{SO}_{2}$. Recycling can increase calcium utilization from approximately $40 \%$ to $50 \%$ and improve the overall process costs. ${ }^{4}$

Coolside can be combined with lime or limestone injection (BLI) to achieve even greater $\mathrm{SO}_{2}$ reductions. In combined Coolside-BLI application the sulfated lime, fly ash and unreacted calcined lime from BLI pass through the Coolside humidification zone where a promoter may also be added. This results in sorbent reactivation and further $\mathrm{SO}_{2}$ reduction. The ash is collected in an ESP and can also be slaked and recycled to the burner for improved calcium utilization. BLI alone results in low calcium utilization, typically about $20 \%$, and sulfur captures of approximately $50 \%$. Humidification results in lime reactivation, increasing the overall $\mathrm{SO}_{2}$ removal to $65 \%$. The injection of the promoter results in further $\mathrm{SO}_{2}$ removal, up to about $80 \%$.

\section{The Edgewater Demonstration}

A demonstration of the Coolside technology was successfully proposed in 1986 under the first Clean Coal Solicitation. The actual demonstration included the participation of the Ohio Coal Development Office, Babcox and Wilcox Co., Ohio Edison and the Consolidation Coal Co., in addition to the U.S. DoE. 
The demonstration was conducted at Ohio Edison's Edgewater Power Plant, located in Lorain, Ohio, on the $105 \mathrm{MW} \# 13$ Utility boiler. The test lasted from July of 1989 through February of 1990 and had two primary goals. The first was to achieve overall $\mathrm{SO}_{2}$ reductions of $30 \%, 50 \%$ and $70 \%$. The second was to achieve reliable process operations at $20^{\circ} \mathrm{F}$ and $25^{\circ} \mathrm{F}$, approaching adiabatic saturation. The demonstration was considered successful, as the earlier pilot plant results were reproduced and the suitability to scale-up of the technology was proven. ${ }^{5}$ Problems encountered during the tests indicated that improvements in the humidifier design and the ESP and ash removal systems needed to be made.

Coolside Waste Sample Collection. Personnel from the Center for Applied Energy Research of the University of Kentucky spent the last two weeks of the demonstration in residence at the Edgewater Plant collecting solid waste for the process. The sample collection was conducted in response to an invitation by the U.S. DoE. The samples were loaded pneumatically into 55 gallon fiber barrels from the No. 2 ESP (the No. 1 unit was out of service). Each barrel was capped and tightly sealed with duct tape and stored indoors. The samples, totaling 65 tons in weight, were transported to Lexington in covered vans and since that date have been warehoused indoors at a commercial facility. This material serves as the focus for this proposal.

The solid waste produced by the Coolside process is similar to other types of dry scrubber waste, such as that from BLI and LSD (Lime Spray Dryer), in that it is composed primarily of coal fly ash and partially sulfated lime. These solids are very high in calcium compared to conventional fly ash (Table 1-1). ${ }^{6}$

Coolside solids differ from BLI materials in that they are produced at relatively low temperatures, and the residual lime remains hydrated. Coolside waste differs from that of other low temperature scrubbing technologies due to the 
Table 1-1 Comparison of elemental composition between Coolside material and fly ash (FA) used in the field lysimeters.

\begin{tabular}{|r|r|r|r|}
\hline & Coolside & & PCC FA \\
\hline Element & Avg & St. Dev. & Avg \\
\hline $\mathrm{Wt} \%$ & & & \\
\hline $\mathrm{Al}, \%$ & 10.56 & 1.65 & 17.4 \\
\hline $\mathrm{Ca}, \%$ & 9.39 & 1.69 & 0.85 \\
\hline $\mathrm{Fe}, \%$ & 6.29 & 0.91 & 7.99 \\
\hline $\mathrm{K}, \%$ & 1.35 & 0.15 & 2.17 \\
\hline $\mathrm{Na}, \%$ & 1.11 & 0.18 & 0.225 \\
\hline $\mathrm{Ti}, \%$ & 0.38 & 0.05 & 0.88 \\
\hline $\mathrm{ppm}$ & & & \\
\hline $\mathrm{As}$ & 219 & 31 & 108 \\
\hline $\mathrm{Ba}$ & 428 & 190 & 1805 \\
\hline $\mathrm{Br}$ & 113 & 51 & 142 \\
\hline $\mathrm{Cr}$ & 138 & 19 & 176 \\
\hline $\mathrm{Cu}$ & 79 & 8 & 172 \\
\hline $\mathrm{F}$ & 450 & 95 & 68 \\
\hline $\mathrm{Ga}$ & 36 & 6 & 58 \\
\hline $\mathrm{Ge}$ & 47 & 8 & 39 \\
\hline $\mathrm{Li}$ & 86 & 27 & 188 \\
\hline $\mathrm{Mn}$ & 116 & 20 & 136 \\
\hline $\mathrm{Mo}$ & 29 & 6 & 25 \\
\hline $\mathrm{Ni}$ & 73 & 10 & 76 \\
\hline $\mathrm{Pb}$ & 112 & 32 & 93 \\
\hline $\mathrm{Rb}$ & 97 & 10 & 632 \\
\hline $\mathrm{Se}$ & 12 & 4 & 3 \\
\hline $\mathrm{Sr}$ & 318 & 32 & 98 \\
\hline $\mathrm{V}$ & 186 & 14 & 241 \\
\hline $\mathrm{Y}$ & 62 & 8 & 376 \\
\hline $\mathrm{Zn}$ & 281 & 35 & 149 \\
\hline $\mathrm{Zr}$ & 175 & 29 & 38 \\
\hline & & & \\
\hline & $\mathrm{N}=263$ & & $\mathrm{~N}=2$ \\
\hline
\end{tabular}


presence of significant quantities of sodium compounds derived from the sorbent activator. In addition to fly ash, the other major compounds present in Coolside waste include $\mathrm{Ca}(\mathrm{OH})_{2}, \mathrm{CaSO}_{3}, \mathrm{CaSO}_{4}$, with minor amounts of $\mathrm{Na}_{2} \mathrm{SO}_{4}, \mathrm{Na}_{2} \mathrm{SO}_{3}$ and $\mathrm{CaCO}_{3}$.

The samples for the study were collected by the CAER during the pilot demonstration of the Coolside technology at Ohio Edison's Edgewater Power Station. Three "samples" consisting of a series of barrels during specified intervals were collected. The first and third of these (Coolside No. 1 and No. 3) were utilized in the field work.

An aliquot of each barrel of material was recovered at the sample storage . warehouse and was analyzed for total sulfur. This data will be used to identify individual barrels with compositions which are not representative of that feasibly produced under commercial conditions.

- The second sample was clearly lower in sulfur than either the first or third. It is not considered to be representative of a material which would be produced by a commercial application and will not be used. The three samples averaged $1.94 \%, 1.67 \%$ and $1.94 \%$ sulfur, respectively $\left(4.8 \%, 4.2 \%, 4.8 \%\right.$ as $\left.\mathrm{SO}_{2}\right)$. The samples collected later in the test were also more variable. Standard deviations of $0.23,0.36$ and $0.36(1 \mathrm{~F})$ were calculated for the three samples, respectively. The sulfur data confirmed the information received from the pilot plant operators. For example, during the final sample collection day the system was not operating effectively early in the afternoon. However when load conditions dropped, system efficiency improved, reaching a peak late at night.

\section{Lysimeter Description and Instrumentation}

Four concrete lysimeters located at the University of Kentucky's Environmental Field Research Station in Montgomery County, Kentucky were used to study 
field behaviors of material generated from the Coolside process. These are compared to a commercially available Class F fly-ash. The four lysimeters surround a central chamber used for leachate collection and instrumentation. Each individual lysimeter is $2.4 \mathrm{~m}(8 \mathrm{ft}$ ) by $2.4 \mathrm{~m}(8 \mathrm{ft})$ by $3 \mathrm{~m}(10 \mathrm{ft}$ ) deep (Figure 1-2). The tops of the lysimeters are flush with the ground surface. The inner walls, adjacent to the central access chamber, are fitted with knock-outs at various depths to allow installation of leachate transport tubes and access to instrumentation.

Pressure cells were mounted in Lysimeter 3 (L-3 in Figure 1-2) at depths of 4 and 6 feet from the surface. A view of one of the pressure cells is shown in Figure 1-3. An additional pressure cell was installed in Lysimeter 4 at the midpoint of the fly ash fill. Before filling, a stainless steel tube was placed in the center of each lysimeter to allow access for a nuclear moisture probe. The probe has been used to monitor changes in moisture under natural weathering conditions. Thermocouples (right cable in Figure 1-3) were placed at various depths in the lysimeters to measure temperature differences which might occur from hydration or mineralogical phase changes. Settlement (swell) platforms were placed at the soil-Coolside interfaces and the soil-fly ash interface to measure vertical movements of the materials.

\section{Filling Operations}

The material was transported to the site in barrels, as shown in Figure 1-4. Enough material was mixed with water to construct one lift. Each lift ranged from 10.2 to $15.2 \mathrm{~cm}$ ( 4 to $6 \mathrm{in}$ ) in thickness. Usually about five barrels of Coolside residue was sufficient to make one lift. To avoid weighing each individual barrel, a correlation was established to determine the weight. The distance from the top of the barrel to the surface of the Coolside residue was measured for several barrels. The barrels were then weighed on a portable field scale. A correlation 


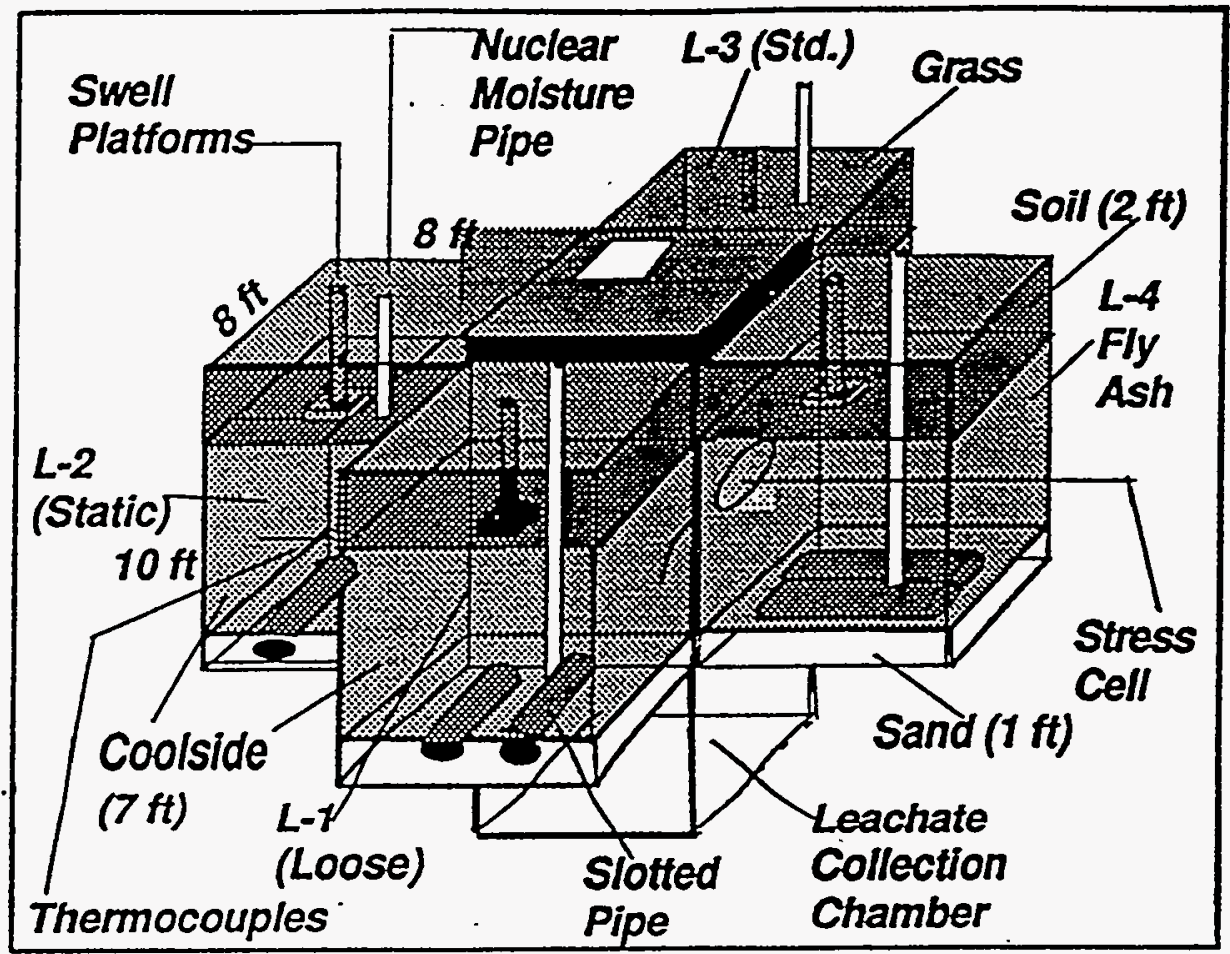

Figure 1-2. Schematic of field lysimeters.

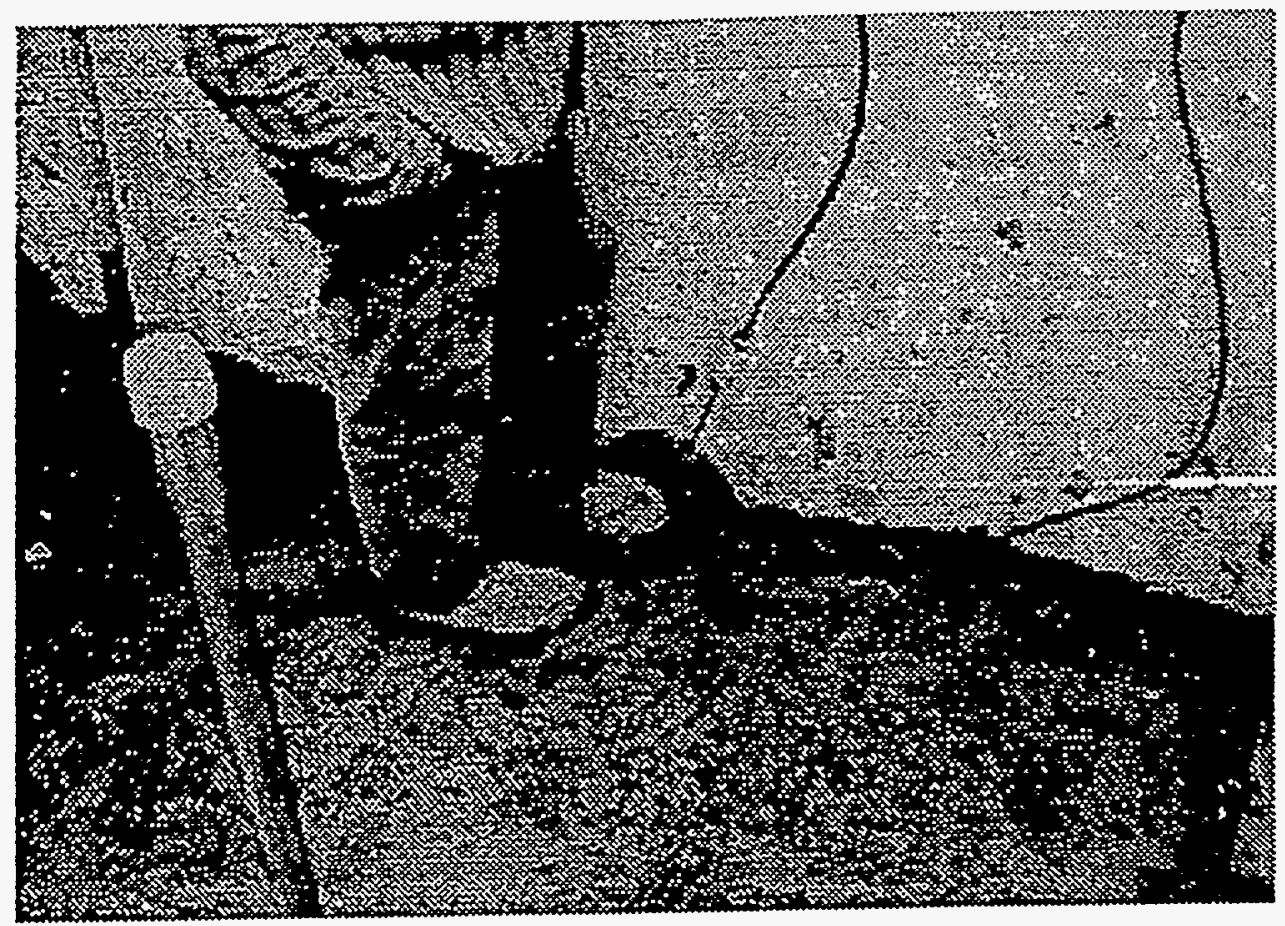

Figure 1-3. View of pressure cell and thermocouple placed in Lysimeter 3. 


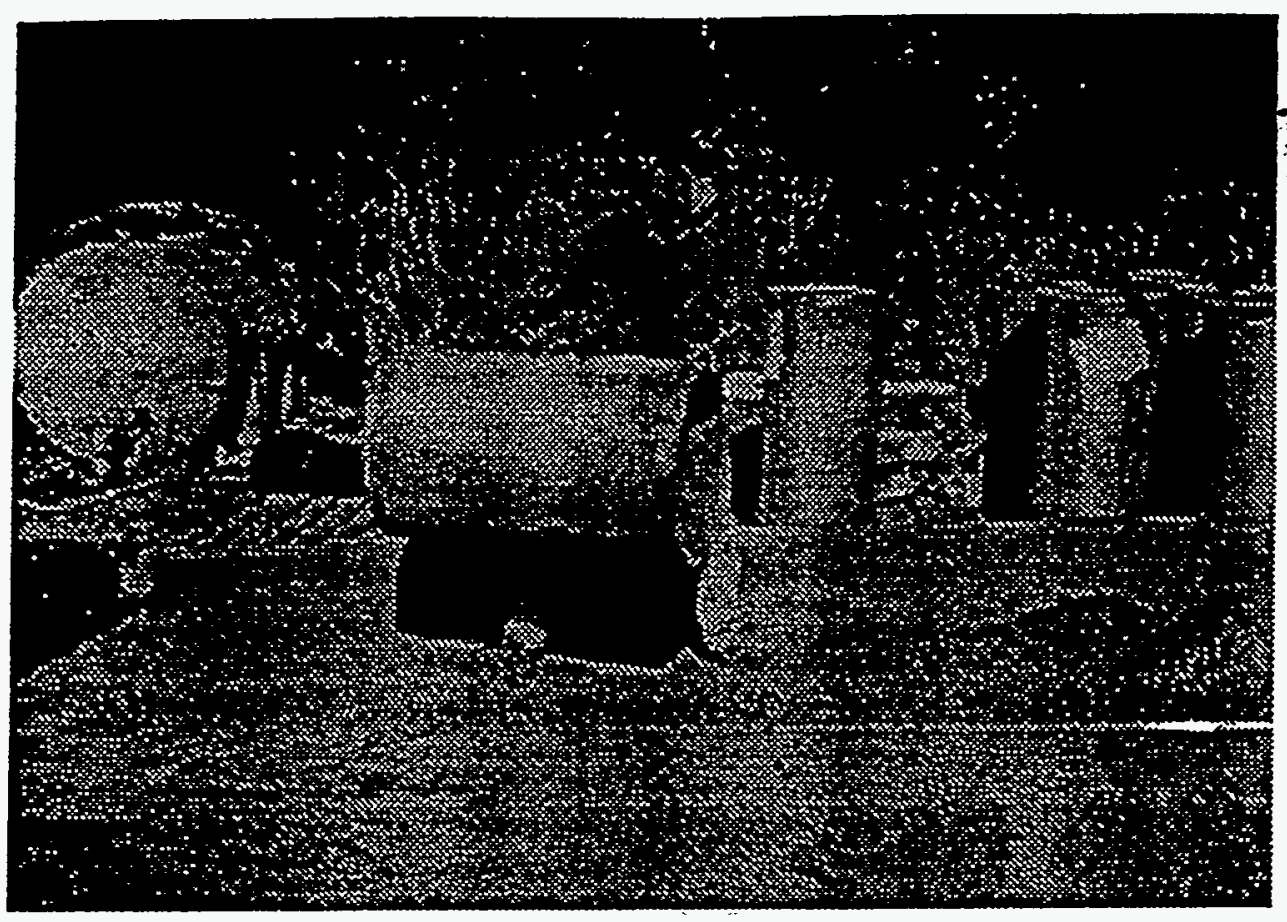

Figure 1-4. View of the barrels used to transport the Coolside ash to the lysimeter site and concrete mixing pad.

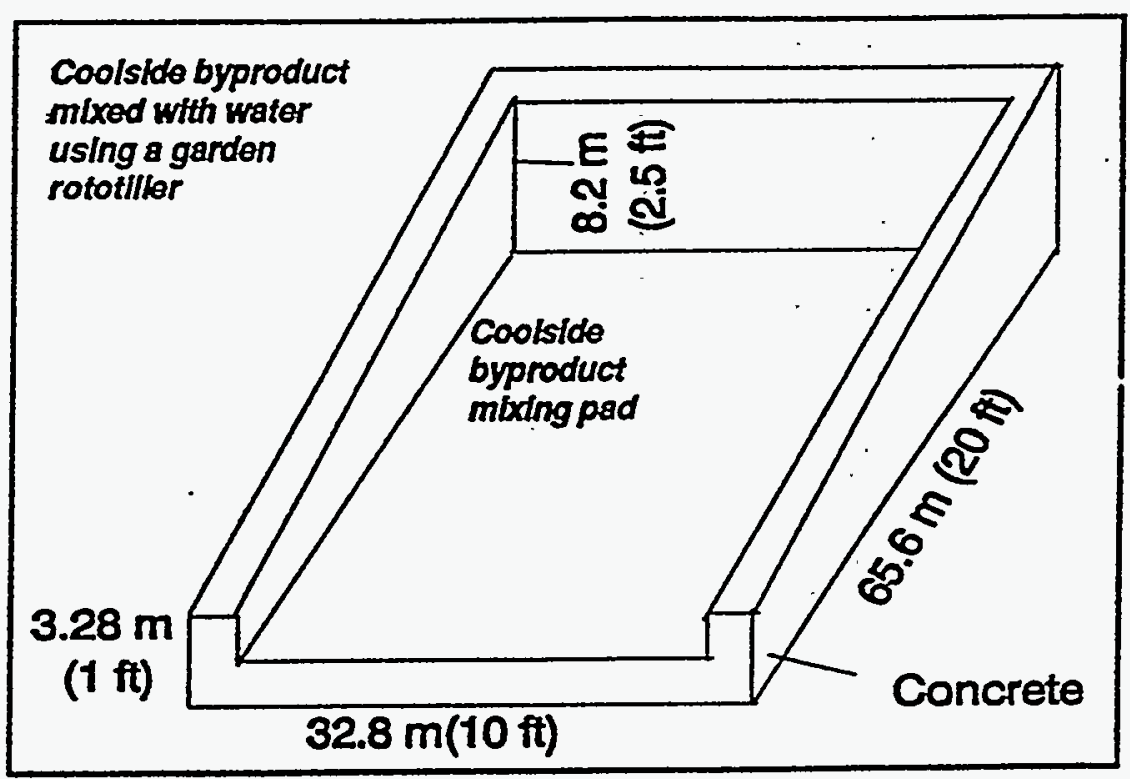

Figure 1-5. Configuration and dimensions of Coolside concrete mixing pad. 
was established whereby the weight of the material could be determined by simply measuring the distance from the top of the barrel to the surface of the material. This procedure worked very well in determining the weight of the material because of the uniform particle size and density of the material. The average weight of each barrel was approximately $112 \mathrm{~kg}$ ( $250 \mathrm{lbs})$. The amount of water needed to reach the desired moisture content was then calculated. Each lift consisted of approximately $572 \mathrm{~kg}(1,250 \mathrm{lbs})$ of dry Coolside material. The residue was placed on a concrete mixing pad, as shown in Figures 1-5 and 1-6, which was built with three side curbs to contain the materials on the pad while mixing. Water from a calibrated tank, Figure 1-7, was then added to sufficiently moisten the material to control dust. The water for mixing with the Coolside ash was transported to the site using the truck-mounted tank shown in Figure 1-8. The mixture was blended with a rear tine tiller, as shown in Figure 16. Water was continually added until the desired moisture content was reached. A front end loader was used to place the mixture into the lysimeter, Figure 1-9. The front end of the concrete pad did not contain a side curb so that the front end loader was free to move onto the pad to load the mixed materials, back out, and place the mixed ash in the lysimeters.

A $30.5 \mathrm{~cm}$ (1- $\mathrm{ft}$ ) layer of Ottawa sand was placed in the bottom of each lysimeter (Figure 1-2) to improve leachate collection. Three leachate transport tubes (Figure 1-10) were embedded in the sand. Lysimeters were filled with $2.1 \mathrm{~m} \mathrm{(7}$ $\mathrm{ft}$ ) of Coolside materials. Lysimeter 4 was filled with $2.1 \mathrm{~m}(7 \mathrm{ft})$ of fly ash. Leachate collection tubes were also placed at the soil-Coolside interfaces and the fly ash-soil interface and at various depths in the four lysimeters. A native silty clay was loosely placed, to facilitate the downward movement of rainfall, on the residue ash in the top $0.6 \mathrm{~m}(2 \mathrm{ft})$ of each lysimeter.

Moisture and density measurements were obtained from drive-tube and sandcone tests. A nuclear moisture-density gauge was also used. Results obtained from the nuclear gauge were used for comparison and calibration purposes and 


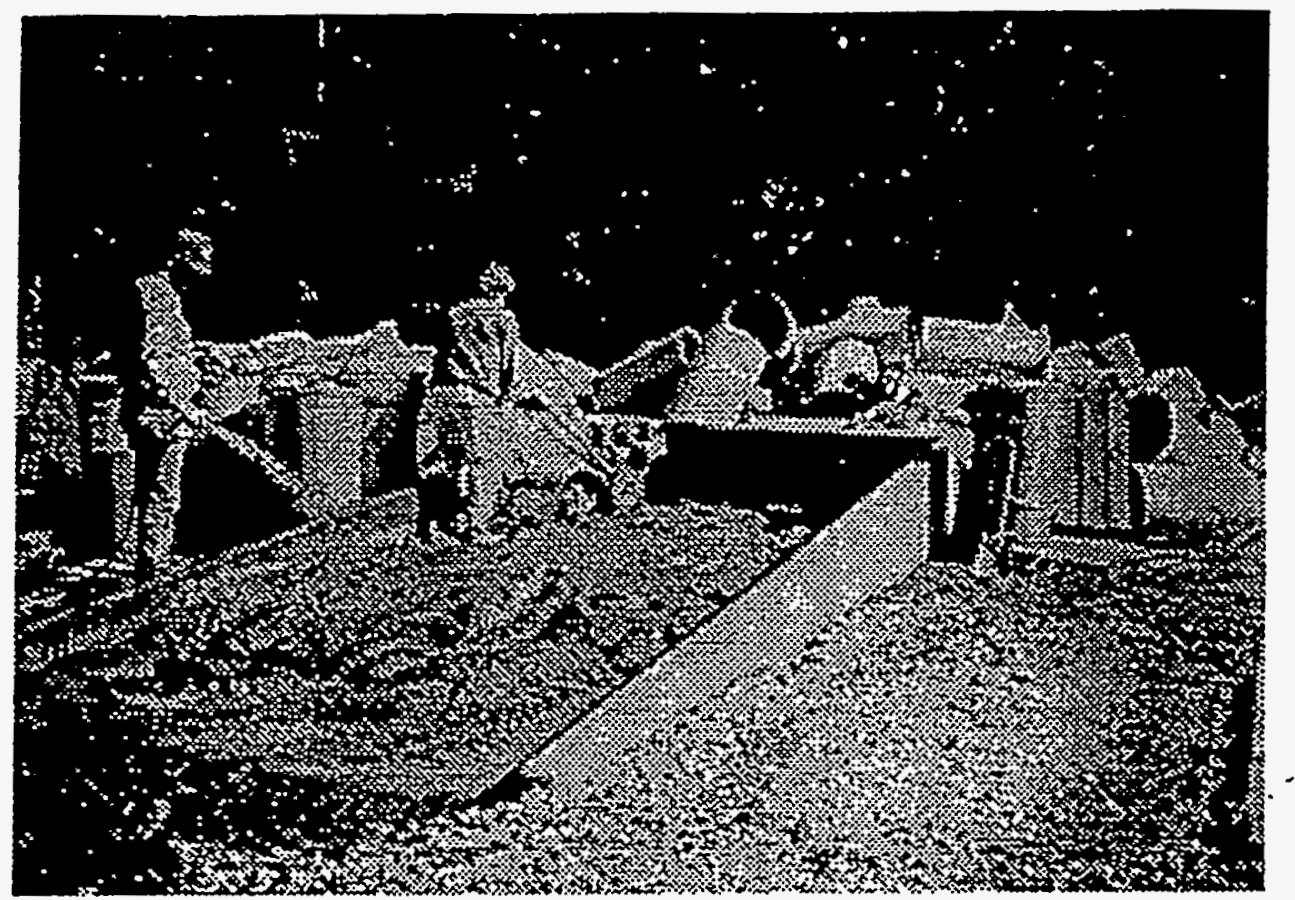

Figure 1-6. View of concrete mixing pad, Coolside ash, watering, and rototiller.

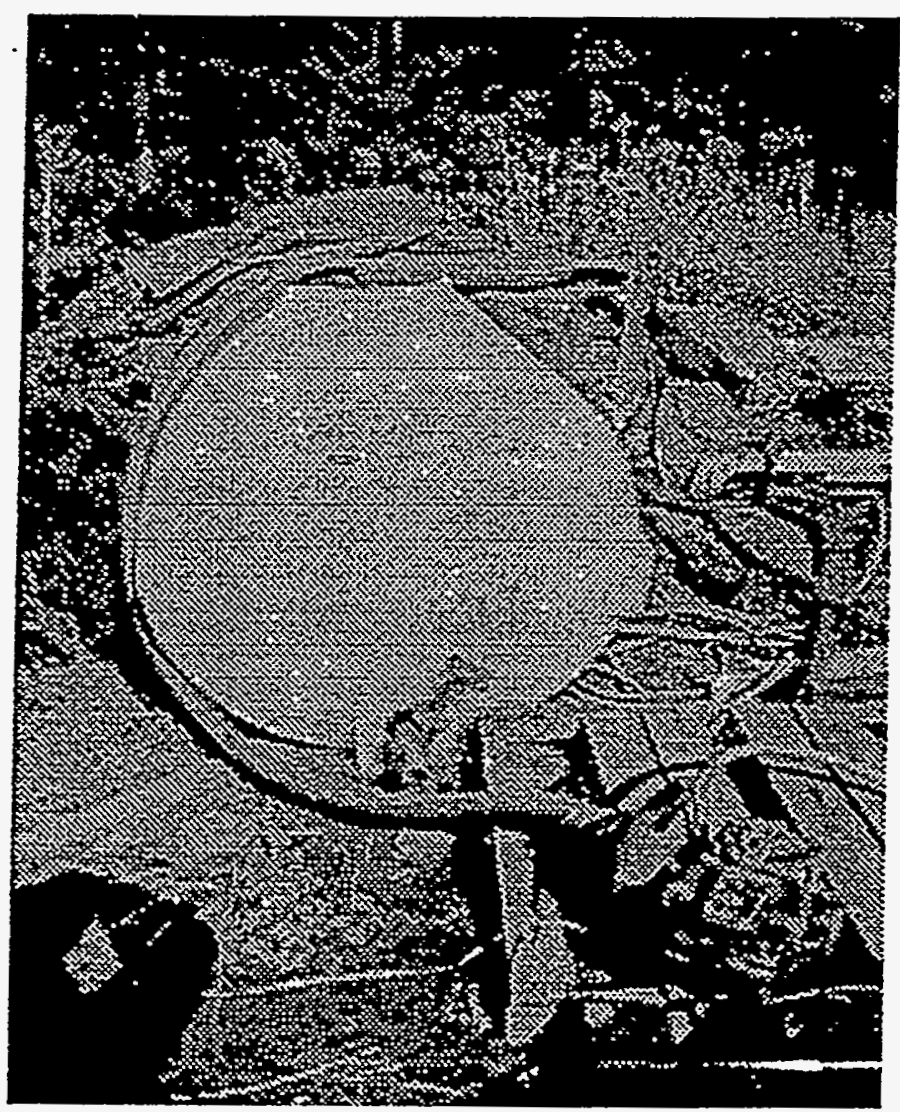

Figure 1-7. View of calibrated water tank. 


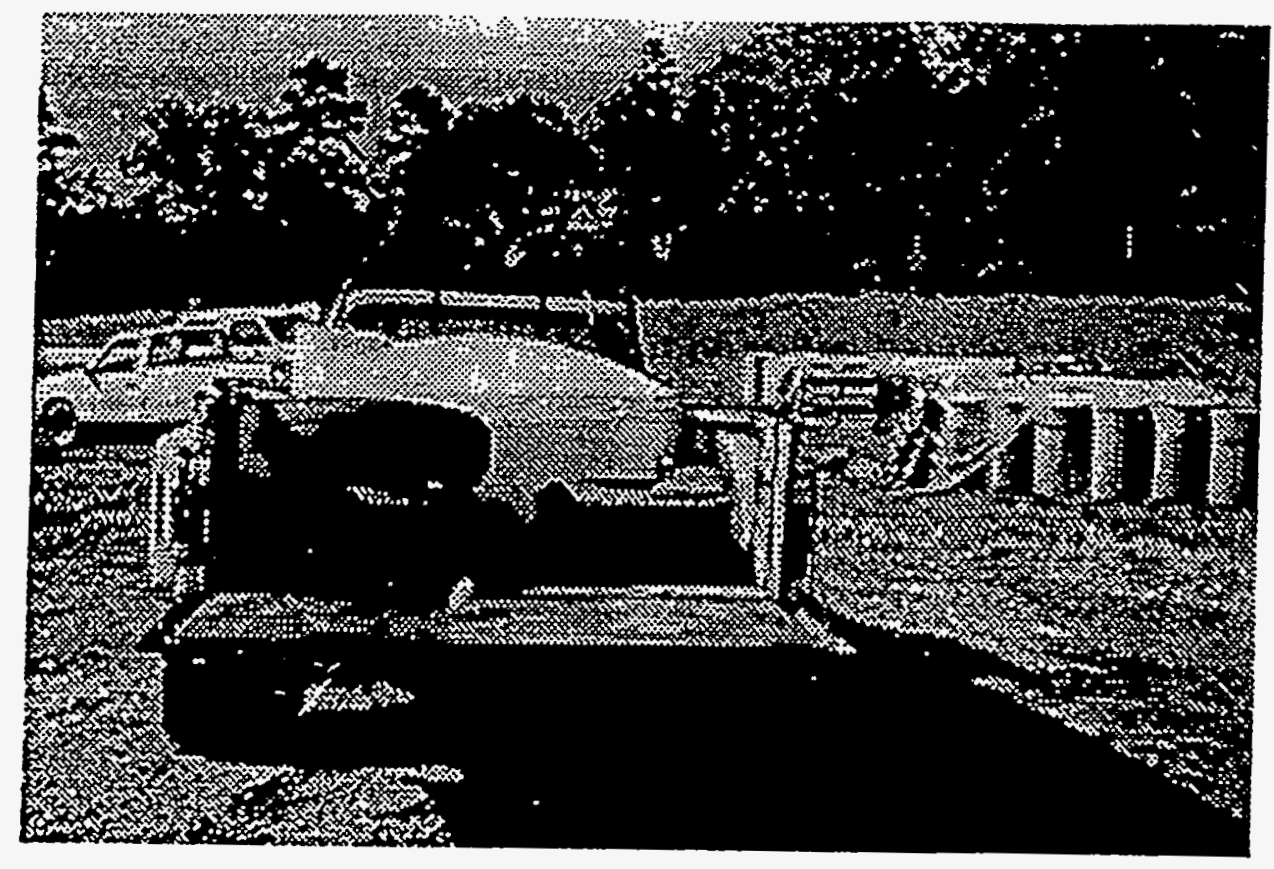

Figure 1-8. View of truck-mounted plastic tank used to transport water to the site.

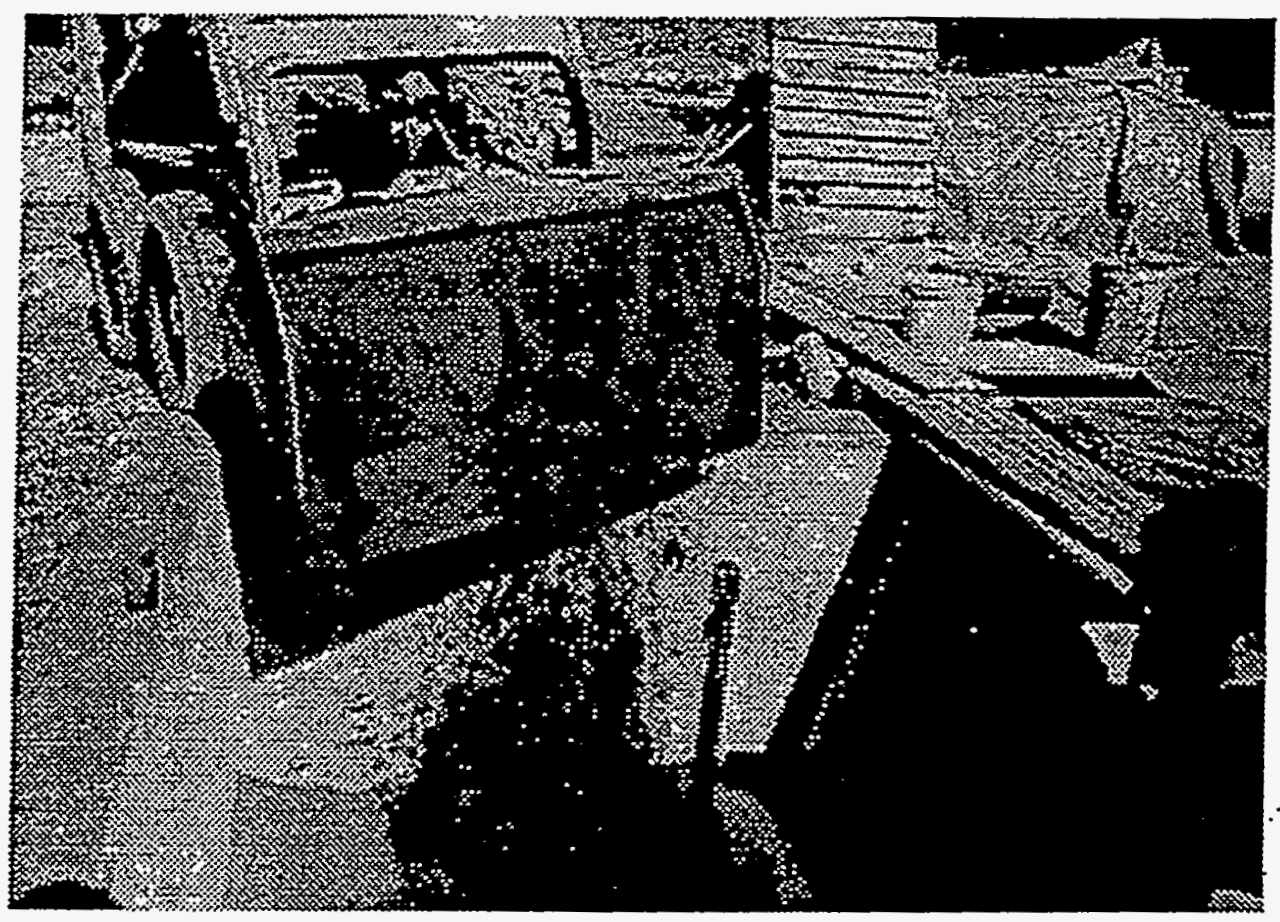

Figure 1-9. View of front end loader used to transport barrels of Coolside to the mixing pad and to place the Coolside ash mixture into the lysimeters. 


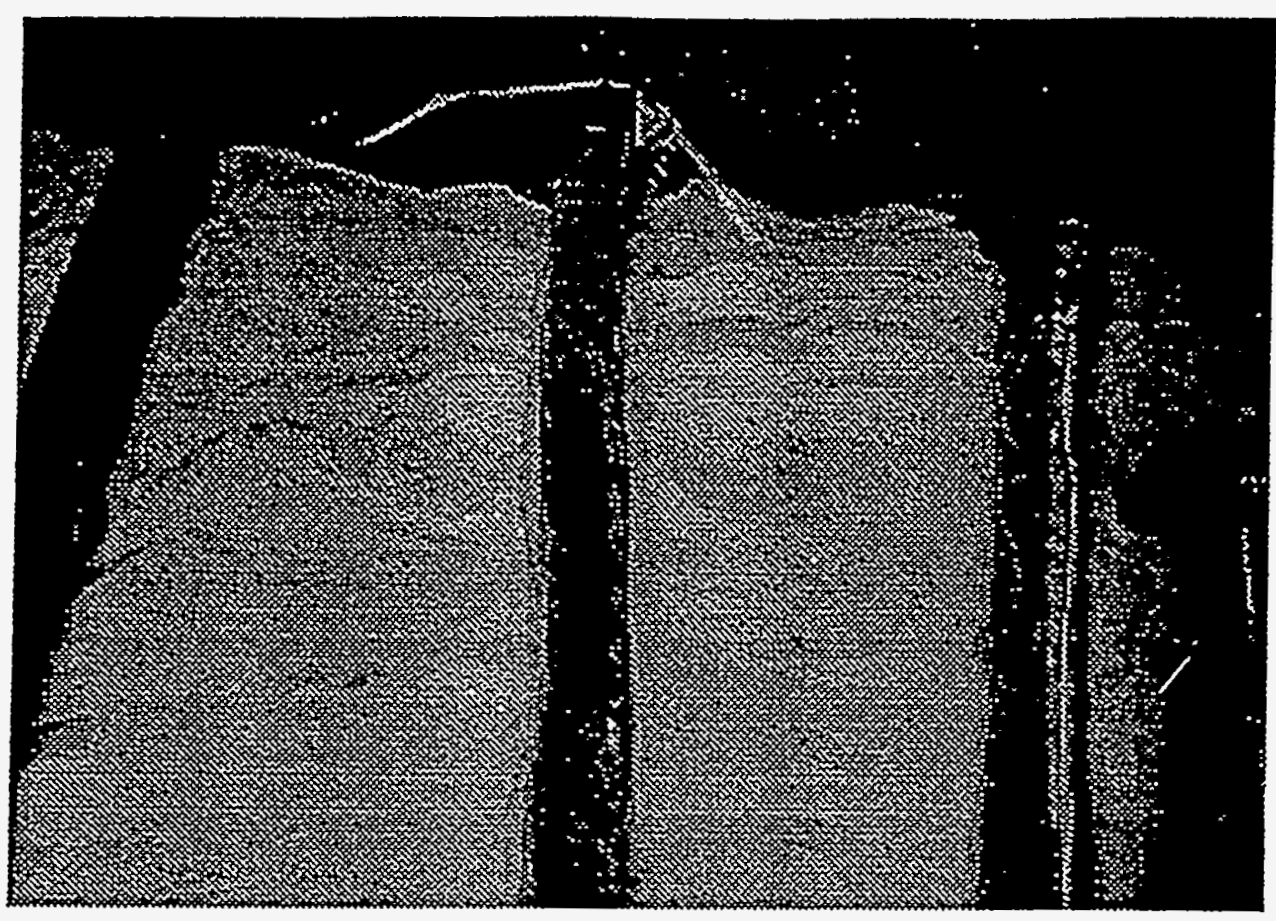

Figure 1-10. View of leachate collection tubes and the Ottawa sand used at the bottom of the lysimeters.

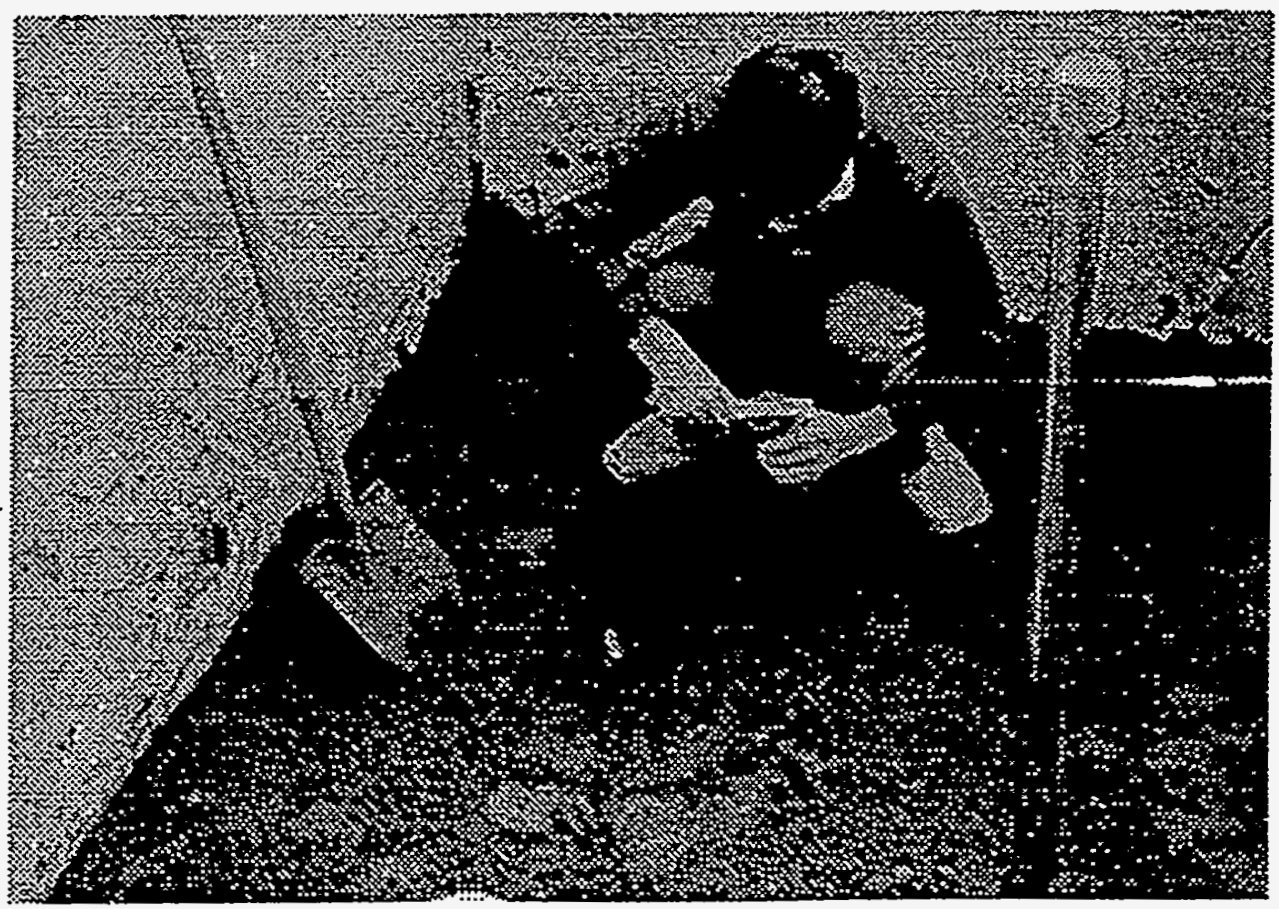

Figure 1-11. Drive sampling tube used to obtain specimens for checking dry density and moisture content of each lift. 
were not used to determine actual density and moisture conditions. Because of the fine-grained nature of the materials, the drive-tube sampler (Figure 1-11) technique provided the most reliable means of measuring the dry density and the moisture content of each lift.

Compacting ash and FGD materials conserves landfill space while increasing disposal costs. In placing the materials, different compactive efforts were used in an attempt to simulate different compactive states of the Coolside by-product as it might exist in a landfill.

\section{Laboratory Compaction for Field Emplacement}

The filling of the field lysimeters was preceded by extensive laboratory studies. Different laboratory compactive energies were used to examine the effects of increased compactive effort. Laboratory moisture-density tests were performed at modified, standard, and low energy compaction. Compactive energies of these tests were $376,754,71,885$, and $14,430 \mathrm{~kg}-\mathrm{m} / \mathrm{m}^{3}(56,246,12,374$, and $4,050 \mathrm{ft}-\mathrm{lbs}_{\mathrm{f}} \mathrm{ft}^{3}$ ), respectively. A low energy compaction procedure was devised to examine engineering properties of lightly compacted materials. The test is similar to standard methods, except a lighter $(0.835 \mathrm{~kg}$, or $1.84 \mathrm{lbs}$.) rammer is used. Dry density increased as the compactive effort was increased. Values ranged from $576 \mathrm{~kg} / \mathrm{m}^{3}$ (36 lbs/ $\left./ \mathrm{ft}^{3}\right)$--loose state-- to $1251 \mathrm{~kg} / \mathrm{m}^{3}\left(78.1 \mathrm{lbs} / \mathrm{ft}^{3}\right)$-modified compaction. When the compactive energy was increased above the low energy compactive energy, the dry density increased from $1079 \mathrm{~kg} / \mathrm{m}^{3}(65.5$ $\left.\mathrm{lbs} / \mathrm{ft}^{3}\right)$ to $1251 \mathrm{~kg} / \mathrm{m}^{3}\left(78.1 \mathrm{lbs} / \mathrm{ft}^{3}\right)$. The maximum dry densities obtained from standard (ASTM D698) and modified compaction (ASTM D1557) were only $6.4 \%$ and $19.2 \%$, respectively, greater than the maximum dry density obtained from the low energy test, although the compactive energies were 3.1 and 13.9 times larger than the low energy compactive effort. Consequently, efforts to compact the Coolside material to a dry density greater than maximum dry density 
obtained from standard or low energy compaction would probably be uneconomical. However, the economics would depend on such variables as land cost, equipment cost, and haul distances. Moreover, the economics of environmental impacts would play a major role in formulating a field compaction scheme. Optimum moisture content decreased as compactive energy increased. Optimum moisture contents for low energy, standard, and modified compactions were $45.0,36.5$, and $28.5 \%$, respectively.

References

1. Yoon, H., F.W. Theodore, F.P. Burke, B.J. Koch, 1986, Low Capital Cost, Retrofit $\mathrm{SO}_{2}$ Control Technologies for High Sulfur Coal Applications. Reprint, Air Pollution Control Association, 79th Annual Meeting, Paper No. 86-47.5, $21 \mathrm{p}$.

2. Stouffer, M.R., H. Yoon, and F. P. Burke, 1989, An Investigation of the Mechanisms of Flue Gas Desulfurization by In-Duct Dry Sorbent Injection. I\&EC Research, 28, p. 20-27.

3. Yoon, H., J.A. Withum, W.A. Rosenhoover and F.P. Burke, 1986, Sorbent Improvement and Computer Modeling Studies for Coolside Desulfurization. Presented at EPA/EPRI Joint Symposium on Dry $\mathrm{SO}_{2}$ and Simultaneous $\mathrm{SO}_{2} / \mathrm{NO}_{x}$ Control Technologies, Raleigh, N.C., 20 p.

4. Withum, J.A., W.A. Rosenhoover and H. Yoon, 1988, A Pilot Plant Study of the Coolside Desulfurization Process With Sorbent Recycle. Proceedings, 5th Annual Pittsburgh Coal Conference, Pittsburgh, PA, p. 84-96.

5. Kanary, D.A., R.M. Statnick, H. Yoon, D.C. McCoy, J.A. Withum and G.A. Kudlac, 1990, Coolside Process Demonstration at the Ohio Edison Company Edgewater Plant Unit 4 - Boiler 13. Proceedings, $1990 \mathrm{SO}_{2}$ Control Symposium, EPRI and U.S. EPA, Session 7A, V 3, New Orleans, LA, 19p.

6. Wu, M.M., and R.A. Winschel, 1988, Coolside Waste Management Studies-Final Report. U.S. DoE Cooperative Agreement No. DE-FC22-87PC79798, $78 \mathrm{p}$. 


\section{CHAPTER 2. MINERALOGIC INVESTIGATION OF THE COOLSIDE MATERIAL}

\section{Overview}

The mineralogical study of the Coolside material was aimed at a comprehensive understanding of the material's impact on the environment. Of particular importance was the question of how soon after wetting the powderous Coolside material would turn into a hardened crystalline or semi-crystalline matrix, and whether it would gain concrete-like properties. After solidification of the materials the next stage was to determine how severely the Coolside material would undergo weathering reactions.

The main emphasis of this part of the research program, therefore, included short- and long-term monitoring of the material's overall physical and chemical transformations, both of which are governed by mineral growth, dissolution, precipitation and disintegration reactions. In the beginning, wetting of the Coolside material causes reactions to form hydrated phases, either by crystallographically bonding or physically adsorbing portions of the added moisture. The excess moisture can either be contained as pore fluids, and the $\mathrm{pH}$ of the pore fluids controls the formation of new crystals as well as the dissolution of others, or, it contributes to the leachate phase. The excess moisture also plays a major role in the transport of dissolved ions and dissolved carbon dioxide from the atmosphere which mineralize within available pore space. The degree of compaction of the Coolside materials restricts the accessibility of moisture and controls the leaching characteristics by affecting the rate of fluid migration through the Coolside materials. The ionic strength of the solutions (amount of dissolved ions) affects the precipitation of the solid phases. Hydration reactions are responsible for the bulk of the expansive properties of the materials, including strength gain, and have been found to be affected by the degree of compaction of the materials. 


\section{Introduction}

To gain a comprehensive understanding of the individual mineral reactions that affect the Coolside material, including permeability, leaching potential and stability, the mineralogical study was sub-divided into four series of investigations with distinct objectives.

$\begin{array}{ll}\text { Series I: } & \text { Field Lysimeter Study } \\ \text { Series II: } & \text { Column Lysimeter Study } \\ \text { Series III: } & \text { Kinetic Study } \\ \text { Series IV: } & \text { Pelletization of FBC Material }\end{array}$

The investigations focused on a field demonstration (Series I) using field lysimeters to monitor changes in the mineralogy of the Coolside material as a function of different packing densities (degree of static loading) and moisture content. Additional moisture was controlled by the seasonal rain water influx. The field lysimeters were sampled on four different occasions within the field testing period. Results from the field demonstration are then compared to those obtained from controlled laboratory column lysimeter tests (Series II). The laboratory columns received controlled amounts of moisture over the testing period. Top, middle and bottom zones of individual laboratory lysimeters were collected at the end of the test period and subjected to a thorough analysis of new mineral growth or decay.

In order to gain a better understanding of the mineralogical transformations that occur during hydration of the Coolside material, a kinetic study (Series III) was conducted in which the material was submerged in distilled water, within a sealed container, and the crystalline phases were monitored soon after hydration as well as over an extended time period. Although these conditions do not reproduce those in the field lysimeters, the experiments provide information on mineral stability relations in a closed system, and assist in predicting reactions that would occur under other conditions. 
Finally, a pelletization study was conducted as part of the Coolside testing program (Series IV). The FBC material was received from Wormser Development Company. The pelletization study aimed at understanding the engineering properties of a selected FBC waste material using a disc pelletizer, controlled moisture content, carefully chosen additives and curing of the materials in a humidified atmosphere at elevated temperatures. The optimum pelletization conditions were determined and discussed by Dr. Milton Wu at Consol. The mineralogical processes involved in the curing, aging and weathering of the pelletized materials were subjected to a thorough testing at CAER as part of the Coolside program and the results are summarized in this report. The mineralogical findings of the pelletization project are expected to aid in the overall understanding of the long-term stability of the materials, but also help determine new applications such as high volume usage.

\section{Analytical Methods}

The materials were analyzed using $x$-ray diffraction $(X R D)$ methods. The XRD methods depend on the measurement of the intensities of suitable $X$-ray diffraction lines combined with a calibration from a single compound standard. Mineral phases were typically detected, if present, in excess of 5 volume percent within the sample material. The XRD spectra were obtained with a Philips APD $3500 \mathrm{X}$-ray diffractometer, using Cu-K radiation $(1.5418 \mathrm{~nm})$, a scan speed of two counts/second, an increment of $0.1^{\circ}$ over a 2 range from 7 to $40^{\circ}$. Mineral phases that were not abundantly present within the sample materials to be detected with the XRD method could be investigated using a scanning electron microscope (SEM) with energy dispersive $x$-ray capabilities for chemical determinations. Crystallization of mineral compounds may occur within small voids and can be observed in situ using the SEM application. Additional sample identification was based on crystal morphology. The SEM analyses were conducted using a Hitachi S-2700 SEM ( $\times 10^{4}$ magnification) after gold-coating of the samples. 
Important Mineral Phases

Because the following discussions on the fate of the Coolside material focus on specific mineral phases that are either present in the raw Coolside material or formed as a result of the aging and weathering process, an overview of the dominant phases is presented first. Particular emphasis is placed on the mineral formation/decay process, including the crystal morphologies and XRD profiles.

The predominant mineral phases that are observed in the raw or aged Coolside material include:

$\begin{array}{ll}\text { Silicon Dioxide: } & \mathrm{SiO}_{2} \\ \text { Mullite: } & \mathrm{Al}_{6} \mathrm{Si}_{2} \mathrm{O}_{13} \\ \text { Portlandite: } & \mathrm{Ca}(\mathrm{OH})_{2} \\ \text { Anhydrite: } & \mathrm{CaSO}_{4} \\ \text { Ettringite: } & \mathrm{Ca}_{6} \mathrm{Al}_{2}\left(\mathrm{SO}_{4}\right)_{3} \mathrm{OH}_{12} 26 \mathrm{H}_{2} \mathrm{O} \\ \text { Calcium Carbonate: } & \mathrm{CaCO}_{3} \\ \text { Hannebachite: } & \mathrm{CaSO}_{3} 0_{5.5 H_{2} \mathrm{O}}\end{array}$

Glassy Phase. A glassy phase is present in the raw Coolside material and typically consists of spherical Si-Al fly ash particles. The amount of glassy particles diminishes in the aged samples as the amorphous phase partially dissolves and contributes to new mineral growth. SEM investigations indicate that the glassy particles are often frothy in appearance. Their relatively high surface areas promote the occurrence of dissolution at a faster rate compared to a dense glass phase. In contrast to the glassy particles which are part of the raw Coolside material, a Si-Al gel phase as well as a Ca-Si-Al gel phase develops in the aged materials forming a binder between mineral grains. Although the gel phase has not enough symmetry on a molecular level to produce characteristic XRD peaks for identification, the gels are "semi-oriented" and have a higher density compared to the frothy glass phase. 
Silicon Dioxide. Pure silicon dioxide occurs in the form of hexagonal quartz crystals. Quartz is a chemically very inert substance at ordinary temperatures and behaves as an indifferent material. In the XRD analyses quartz may be viewed as an internal standard. The non-crystalline silicon dioxide (glassy component) is less dense compared to the crystalline modification and readily participates in early hydration reactions. The differences in reactivity are therefore not due to chemical but rather physical conditions based on texture and porosity. Under alkaline conditions which are favored in the hydrated Coolside materials, the solubility of $\mathrm{SiO}_{2}$ becomes enhanced because of the formation of monomeric and multimeric silicates. The solubility of $\mathrm{SiO}_{2}$ can be characterized by the following equilibria:

$$
\begin{array}{ll}
\mathrm{SiO}_{2} \text { (quartz) }+\mathrm{H}_{2} \mathrm{O}=\mathrm{Si}(\mathrm{OH})_{4} & \log \mathrm{K}=-3.70 \\
\mathrm{SiO}_{2} \text { (amorphous silica) }+2 \mathrm{H}_{2} \mathrm{O}=\mathrm{Si}(\mathrm{OH})_{4} & \log \mathrm{K}=-2.70 \\
\mathrm{Si}(\mathrm{OH})_{4}=\mathrm{SiO}(\mathrm{OH})_{3^{-}}+\mathrm{H}^{+} & \log \mathrm{K}=-9.46
\end{array}
$$

Mullite. Mullite is a stable form of the system $\mathrm{Al}_{2} \mathrm{O}_{3} \mathrm{SiO}_{3}$. The crystals form from a cooling liquid which controls their composition. Mullite melts congruently at $1850^{\circ} \mathrm{C}$. Like crystalline $\mathrm{SiO}_{2}$, the mullite crystals are very erosion resistant and have only minor affects on the mineral transformations that are important to the Coolside material. The composition of mullite approximates to $3 \mathrm{Al}_{2} \mathrm{O}_{3} \cdot 2 \mathrm{SiO}_{2}$ with the formula being $\mathrm{Al}_{6} \mathrm{Si}_{2} \mathrm{O}_{13}$. The mullite crystals can be identified by their characteristic $x$-ray peaks, but are also easily recognized by their very distinctive needle-like morphology using SEM applications.

Portlandite. Portlandite is a calcium hydroxy hydrate phase which forms by the hydration of lime by water. If crystallized slowly, as in the hydration of portland cement, it forms large, well developed hexagonal crystals with a typically platy appearance. In the Coolside materials, morphologically well developed portlandite crystals are rare but identification is readily achieved using XRD methods. In the presence of excess water, portlandite reacts with $\mathrm{CO}_{2}$ to form calcium carbonate. 
$\mathrm{Ca}(\mathrm{OH})_{2}+\mathrm{CO}_{2}=\mathrm{CaCO}_{3}+\mathrm{H}_{2} \mathrm{O}$

Anhydrite. Anhydrite is a calcium sulfate mineral, $\mathrm{CaSO}_{4}$. Anhydrite occurs in the raw Coolside material as a result of the sulfur removal process. Depending on the temperature of the process, the anhydrite crystals may be present in a more or less reactive form, distinguishing the soluble and insoluble anhydrite. Upon reaction with water anhydrite is converted to gypsum, $\mathrm{CaSO}_{4} \cdot 2 \mathrm{H}_{2} \mathrm{O}$. The transformation is associated with a 60 percent increase in molar volume. The anhydrite crystals do not simply adsorb the water molecules into their structure, thereby forming gypsum. The process involves a dissolution-reprecipitation reaction. Upon dissolution of the anhydrite crystals, $\mathrm{Ca}$ and $\mathrm{SO}_{4}$ ions are liberated. Nucleation and growth of gypsum occurs secondarily.

Gypsum/Hemihydrite. The hydration of anydrite may occur in different steps. The transformation point of gypsum to hemihydrite $\left(\mathrm{CaSO}_{4} 0.5 \mathrm{H}_{2} \mathrm{O}\right)$ in water is $97^{\circ} \mathrm{C}$, and therefore, the hemihydrite is typically present in temperature cured samples (FBC pellets), rather than in the lysimeters. The hemihydrite occurs as needle-like crystals which can be distinguished from gypsum using XRD application or using SEM based on the pseudotrigonal symmetry of the needles. Compared to anhydrite, gypsum is much less soluble (at $\left.25^{\circ} \mathrm{C}, 1 \mathrm{~atm}\right)$. For comparison, the equilibrium constants for the anhydrite - gypsum conversion are presented hereof.

$$
\begin{array}{ll}
\mathrm{CaSO}_{4} 2 \mathrm{H}_{2} \mathrm{O}(\mathrm{s})=\mathrm{Ca}^{2+}+\mathrm{SO}_{4}^{2-}+2 \mathrm{H}_{2} \mathrm{O} & \text { LogKs gypsum }=-4.60 \\
\mathrm{Ca}^{2+}+\mathrm{SO}_{4}{ }^{2-}=\mathrm{CaSO}_{4}(\mathrm{~s}) & \text { LogKs anhydrite }=+4.38
\end{array}
$$

$$
\mathrm{CaSO}_{4} 2 \mathrm{H}_{2} \mathrm{O}(\mathrm{s})=\mathrm{CaSO}_{4}(\mathrm{~s})+2 \mathrm{H}_{2} \mathrm{O} \quad \mathrm{Log} \mathrm{K} \quad=-0.22
$$


The gypsum - anhydrite stability fields are temperature dependent. At ordinary temperatures gypsum is more stable compared to anhydrite. Above $42^{\circ} \mathrm{C}$ anhydrite becomes more stable instead.

Ettringite. Ettringite is both an individual mineral $\left(\mathrm{Ca}_{6} \mathrm{Al}_{2}\left(\mathrm{SO}_{4}\right)_{3}\left(\mathrm{OH}_{12}\right) 26 \mathrm{H}_{2} \mathrm{O}\right)$, and also a group name for a series of analogous isostructural compounds. Ettringite crystals require a substrate surface to nucleate. They incorporate aluminum from the glass phase, and hydroxyl ions into their lattices, thereby causing portlandite to continue to dissolve. Nucleation occurs on precursor surfaces which need to be enriched in $\mathrm{Si}, \mathrm{Al}, \mathrm{Ca}$ or $\mathrm{S}$. Ideal substrate surfaces in the Coolside materials would be the Si-Al glassy phases, portlandite and anhydrite/gypsum surfaces. Ettringite is commonly observed in the SEM observations to have crystallized on the glassy fly ash spheres. The morphology of the ettringite crystals. is that of an elongated hexagonal rod which makes the ettringite appear needle shaped. The needles usually coat the fly ash surfaces giving the impression of spiky billiard balls. The trigonal-hexagonal unit cell of the mineral consists of columns of composition $\left(\mathrm{Ca}_{6}\left[\mathrm{Al}(\mathrm{OH})_{6}\right]_{2} 24 \mathrm{H}_{2} \mathrm{O}\right)^{6+}$ which are aligned along the crystallographic caxis. These columns are accompanied by channels of composition $\left\{\left(\mathrm{SO}_{4}\right)_{3} 2 \mathrm{H}_{2} \mathrm{O}\right\}^{-6}$. Within that structure the Al ions are octahedrally surrounded by $\mathrm{OH}$ ions. The sulfate ion, on the other hand, occurs in tetrahedral orientation. Within the natural and experimental systems, sulfate may be substituted by the sulfite ion. Crystallization of 1 mole of ettringite, theoretically, consumes 6 moles of calcium and 3 moles of sulfate/sulfite while incorporating 26 moles of water.

Calcium Carbonate. Calcium carbonate $\left(\mathrm{CaCO}_{3}\right)$ precipitates from $\mathrm{Ca}(\mathrm{OH})_{2}$ solution by $\mathrm{CO}_{2}$. The crystals are typically idiomorphic, which is viewed using SEM applications. Factors that are important in the formation (and dissolution) of calcium carbonate are low supersaturation and poor mechanical agitation. These conditions are preferably present in the field and lab lysimeters. The introduction of atmospheric carbon dioxide into the system could cause portlandite dissolution via the following reactions in which portlandite is a buffer: 


$$
\begin{aligned}
& \mathrm{CO}_{2}+\mathrm{OH}-=\mathrm{HCO}_{3-} \\
& \mathrm{HCO}_{3-}=\mathrm{H}^{+}+\mathrm{CO}_{3}^{2-} \\
& \mathrm{Ca}(\mathrm{OH})_{2}=\mathrm{CaOH}^{+}+\mathrm{OH}_{-}^{-} \\
& \mathrm{CaOH}^{+}=\mathrm{Ca}^{2+}+\mathrm{OH}_{-}^{-}
\end{aligned}
$$

Hannebachite. The calcium sulfite hydroxide $\left(\mathrm{CaSO}_{3} 0.5 \mathrm{H}_{2} \mathrm{O}\right)$ is the major source of sulfite for the formation of ettringite. Hannebachite's solubility causes the $\left[\mathrm{Ca}^{2+}\right] /[\mathrm{OH}-]$ ratio to increase in the solution. Portlandite which is the most soluble calcium-bearing mineral $\left(K_{\mathrm{sp}}=10^{-5.3}\right)$ continues to dissolve when hannebachite goes into solution ([Ca $\left.{ }^{2+}\right]\left[\mathrm{SO}_{3}{ }^{2-}\right]=10^{-7.0} ; \mathrm{K}_{\mathrm{sp}}$ of hannebachite).

\section{Mineralogical Makeup of the "Raw" Coolside Material}

The mineralogical composition of the "raw" Coolside material was determined using XRD application. The mineralogical analysis of the bulk "raw" Coolside material indicates the material is composed principally of unreacted $\mathrm{Ca}(\mathrm{OH})_{2}$, crystalline $\mathrm{SiO}_{2}$ (quartz), mullite $\left(\mathrm{Al}_{6} \mathrm{Si}_{2} \mathrm{O}_{13}\right)$ and $\mathrm{x}$-ray amorphous $\mathrm{Al}-\mathrm{Si}$-Fe oxide/hydroxide. No ettringite occurs in the raw mixture. The crystalline sulfate that was identified by XRD was gypsum $\left(\mathrm{CaSO}_{4}\right.$ $\left.2 \mathrm{H}_{2} \mathrm{O}\right)$ and anhydrite $\left(\mathrm{CaSO}_{4}\right)$.

\section{Series I: Field Lysimeter Study}

This study comprises the mineralogical findings of the four field lysimeters from the time of sample emplacement (April 1992) till the end of the monitoring period (April 1996). A 
summary of the field lysimeter conditions is presented with the results of the corresponding XRD analyses.

The field lysimeters 1-3 contain Coolside material with different degrees of compaction. The changes in mineralogy were monitored as a function of lysimeter depth and compared for lysimeters with different degrees of compaction. At three different time intervals throughout the study, core samples were taken and mineral phases were identified by reference to the JCPDS powder diffraction files. The main objective was to identify how much ettringite and calcium carbonate formed in the lysimeters upon reaction of the Coolside waste with seasonal rain water and to establish a depth profile for the reactions. Ettringite was of importance as it would contribute to the strength development within the materials. Calcium carbonate was studied as a weathering indicator. The drill cores obtained during the first field testing in March of 1994 are summarized first. The profiles of the drill core samples are shown in Figure 2-1. The drill cores, all of which are characterized by a 2 " diameter, were taken two feet below the soil interface and extended to four feet depth. Material was collected from the drill cores at two inches apart and subjected to XRD analyses. The results indicated that, independent from the packing densities, the amounts of ettringite present in the core samples increased with increasing lysimeter depth (Figures 2-2; 2-3; 2-4). Ettringite was observed in the SEM study of the materials to have formed both on mineral surfaces and within available pore space (Figure 2-5). The SEM study further indicated that at a greater depth, more pores were infilled by ettringite crystals. The ettringite crystals that formed a dense layer appear more prismlike, while those that nucleated and grew on mineral surfaces and fly ash surfaces were characterized by a more needle-like morphology. These findings suggest that the ettringite morphology may be controlled by the confining pressure (Figure 2-6).

Near the soil interface for all field lysimeters the majority of the mineral transformations could be identified as calcium carbonate reactions. At greater depth, independent of the confining pressure, only very little calcium carbonate was observed in the XRD analyses, suggesting that the migration rates of $\mathrm{CO}_{2}$ within the lysimeters is only minor. 


\section{DRILL-CORES \\ Experimental Field Lysimeters Montgomery County, KY}
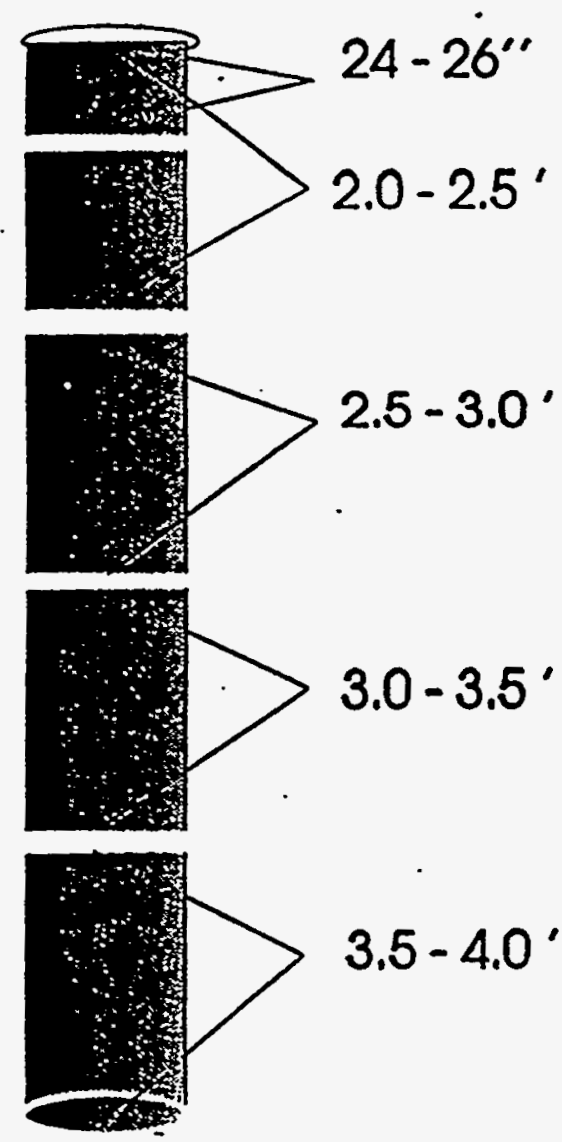

$L-1$

$3.5-4.0^{\prime}$ $3.5-4.0$
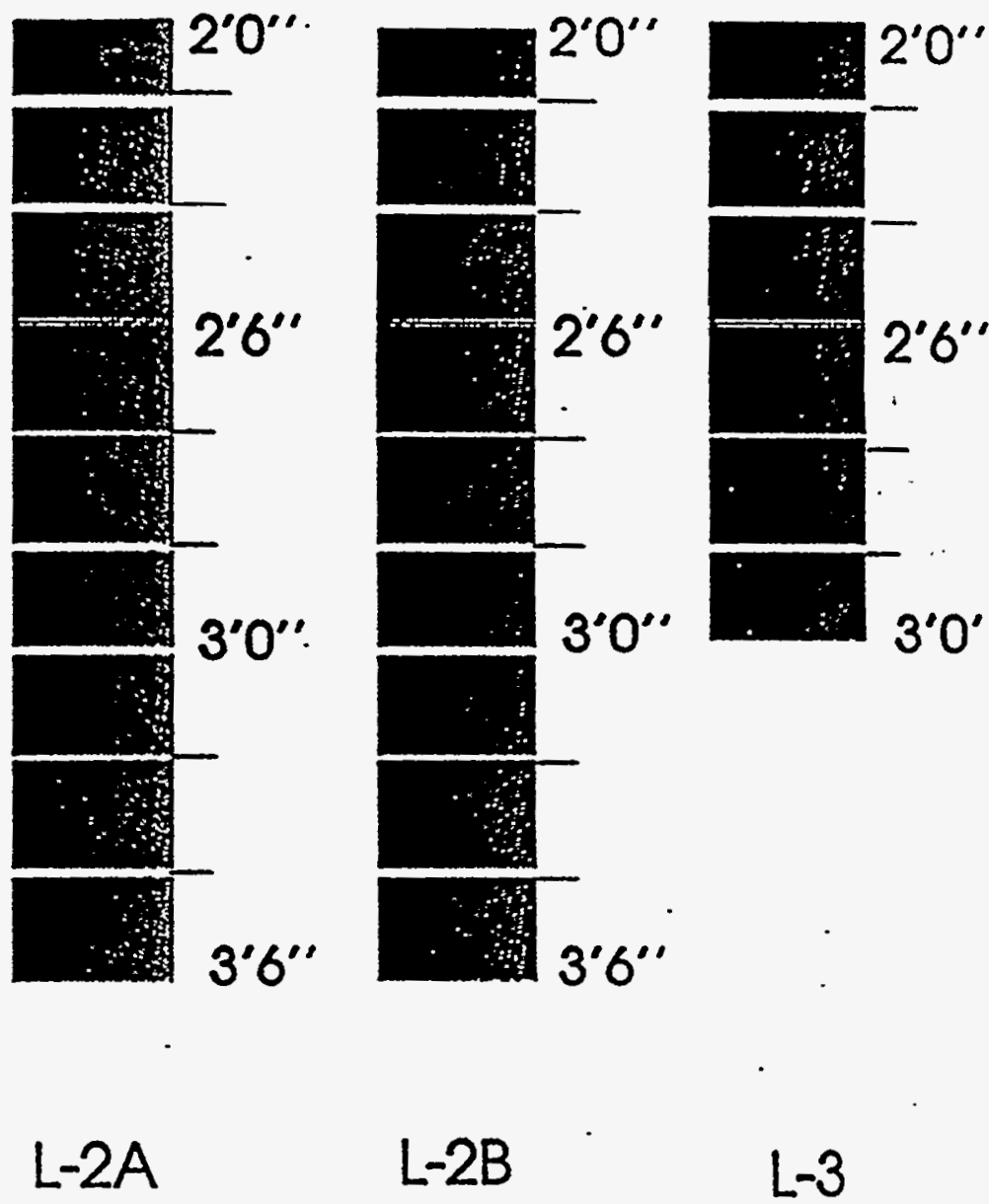

$L-2 B$

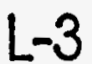

Figure 2-1. llustrated are drill core profiles for experimental field lysimeters in Montgomery County, KY. L1 represents Lysimeter 1 with the lowest packing density of Coolside material. Samples for XRD analyses were taken at the intervals shown in the figure. Two cores were obtained for Lysimeter 2 which are represented by $L-2 A$ and $L$ $2 B$. The drill core obtained from Lysimeter 3 is represented by $L-3$ and ranged only to a depth of three feet. 
Lysimeter $12^{\prime} 0^{\prime \prime}-22^{\prime \prime}$

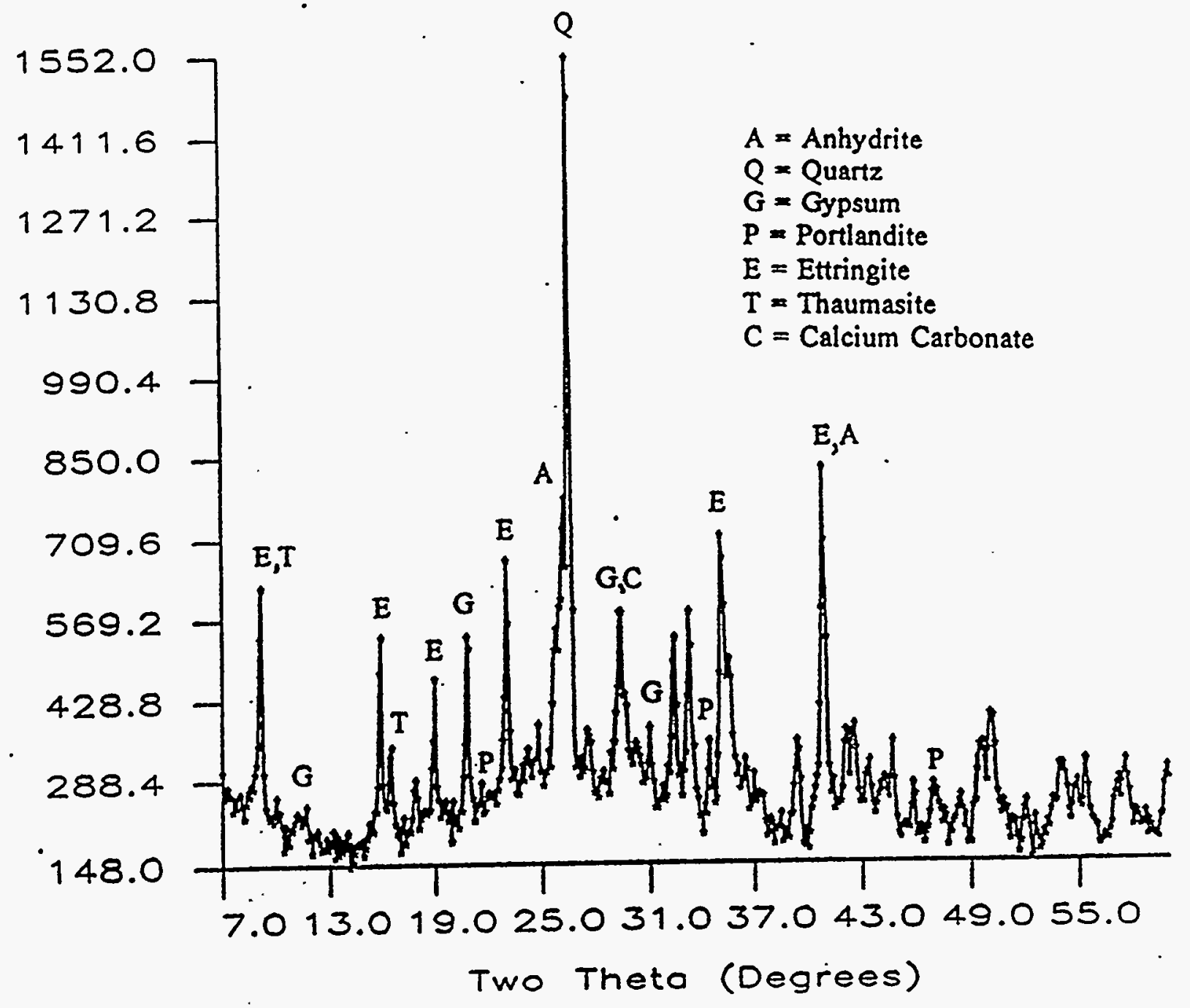

Figure 2-2a. XRD analysis of drill core obtained from Lysimeter 1 at $2^{\prime} 0^{\prime \prime}-22^{\prime \prime}$ depth. Major mineral constituents are indicated in the legend and by their corresponding peaks. Ettringite $(E)$ was abundantly present. 
Lysimeter $13^{\prime} 6^{\prime \prime}-4^{\prime} 0^{\prime \prime}$

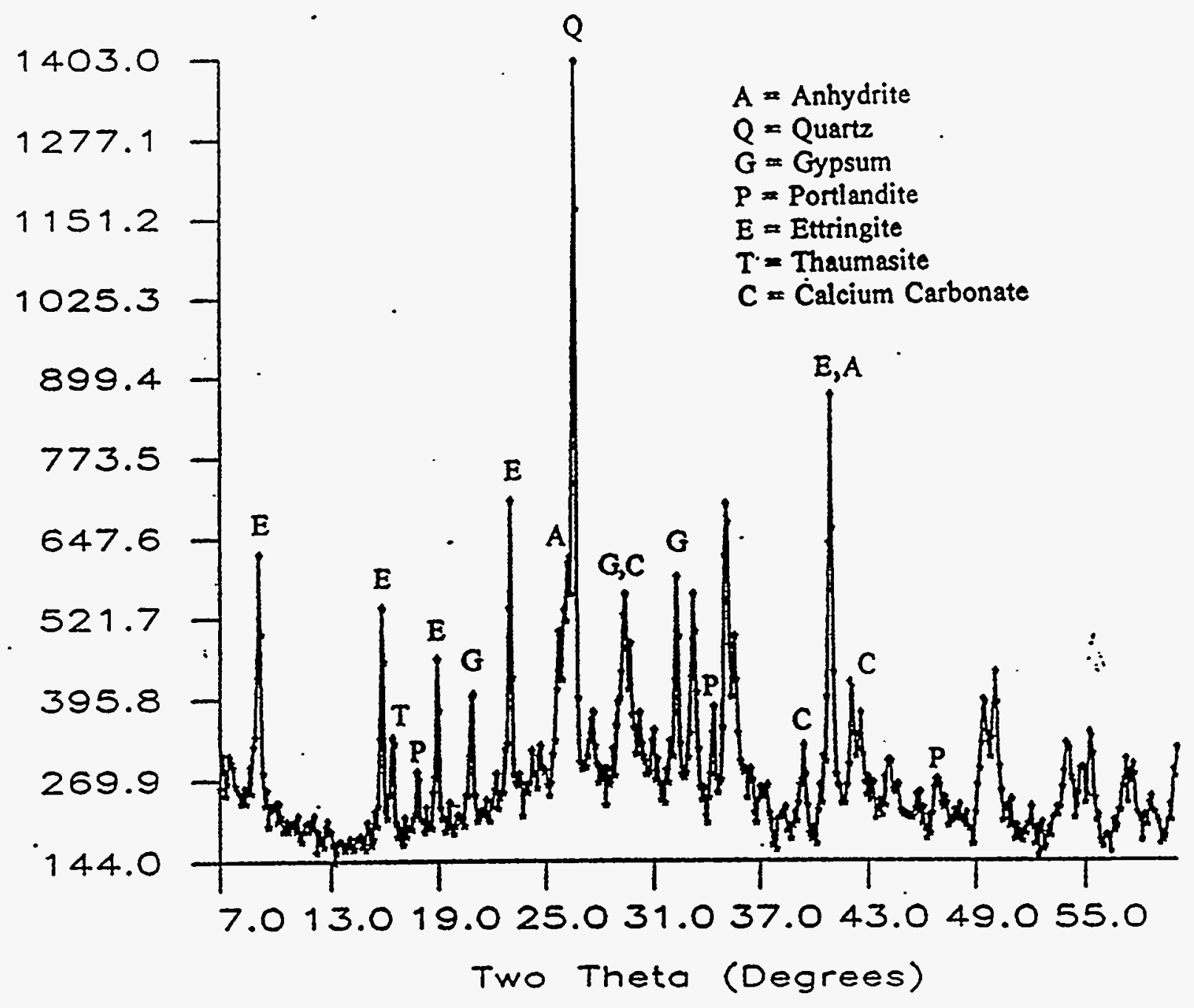

Figure 2-2b. XRD analysis of sample material from same drill core as in 2-2a, but at greater lysimeter depth $\left(3^{\prime} 6^{n}-4^{\prime} 0^{\prime \prime}\right)$. Ettringite $(E)$ is abundantly present. 


\section{Ettringite in Field Lysimeter-1}

\section{Depth Profile}

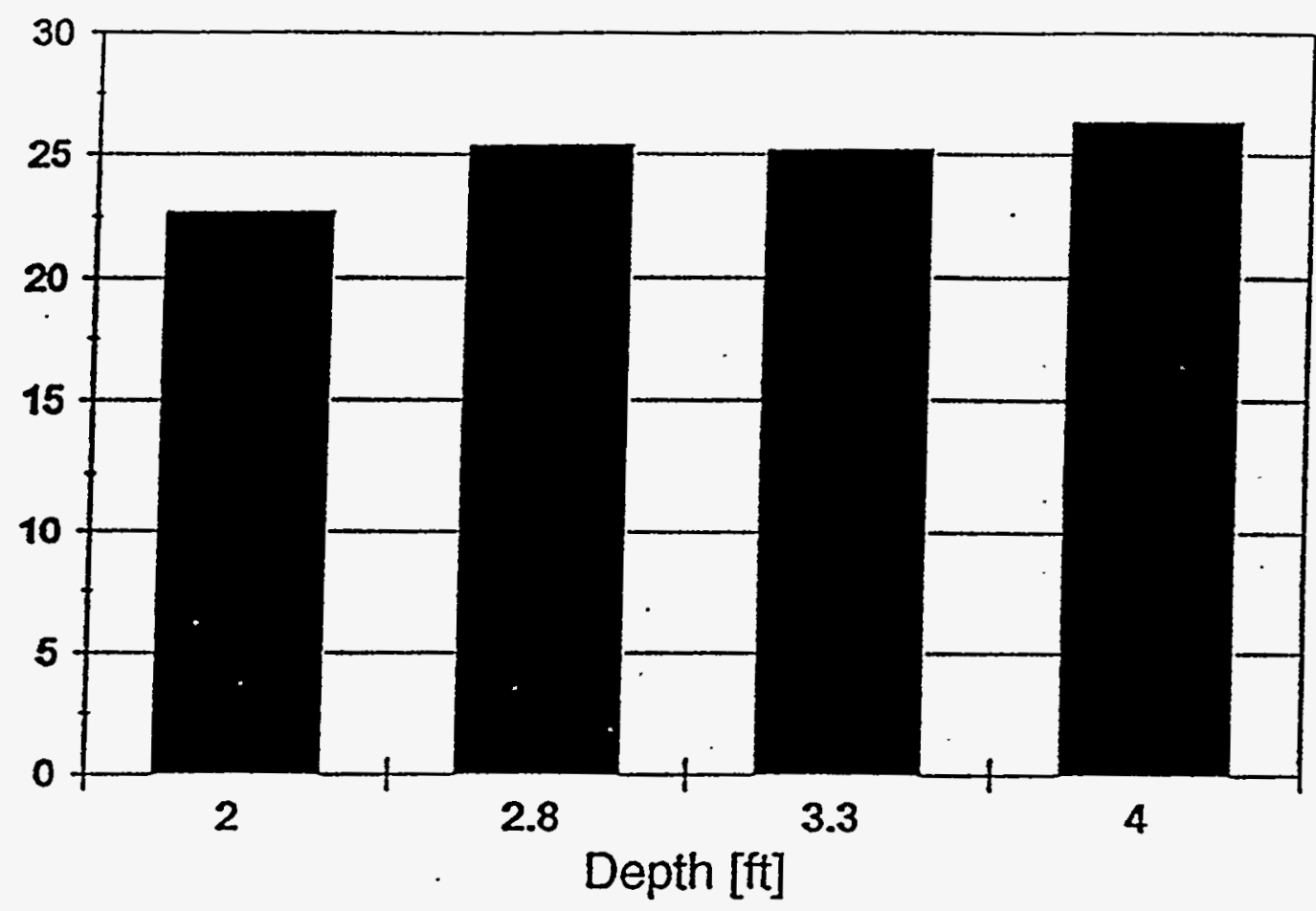

\section{\% Ettringite in $L-1$ relative to Quartz}

Figure 2-2c. Ettringite abundance relative to Quartz in Field Lysimeter 1 is illustrated for 4 different depth profiles. Ettringite seems to increase with increasing lysimeter depth. 
Lysimeter 2A $24^{\prime \prime}$

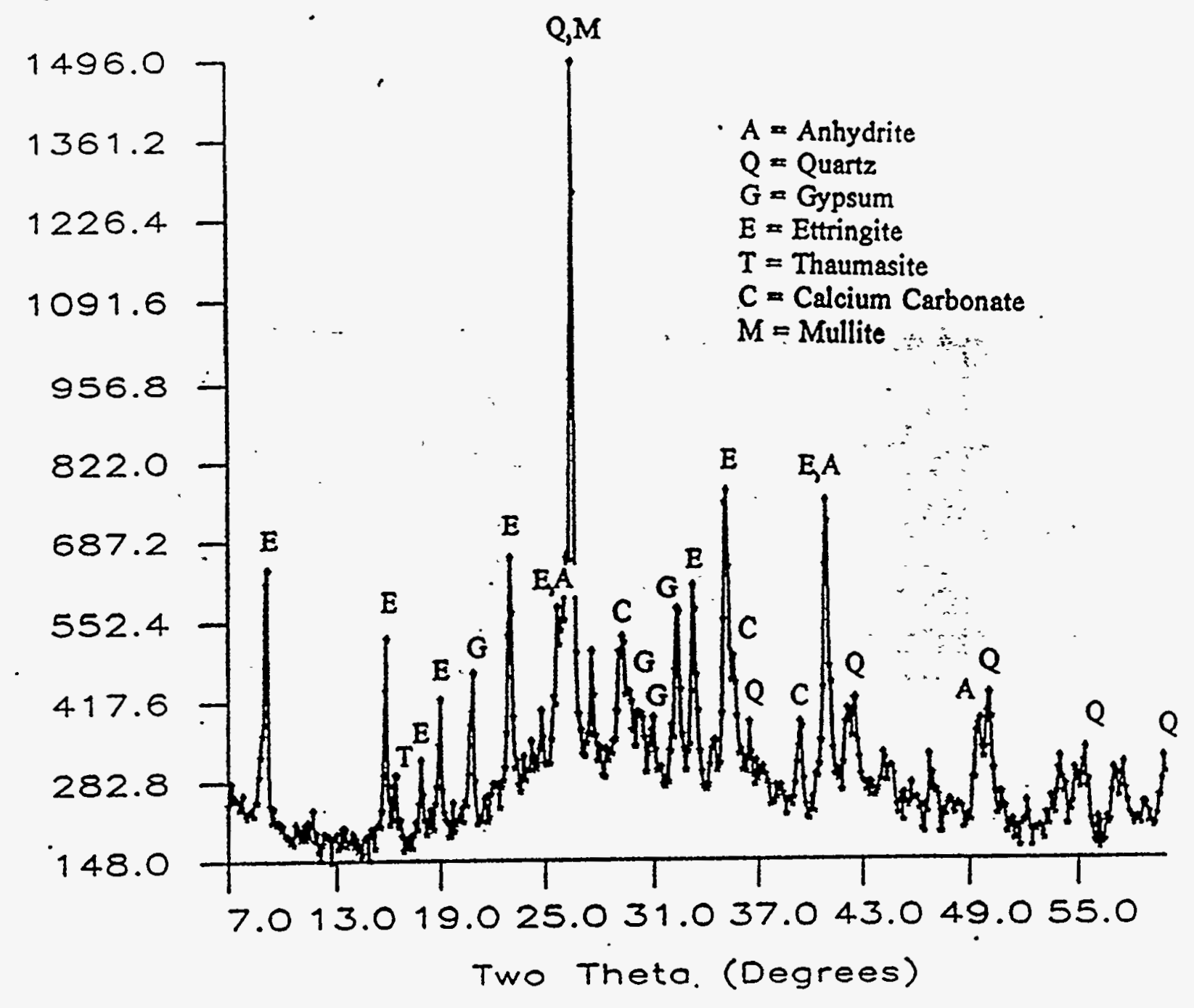

Figure 2-3a. XRD analyses of drill core obtained from Lysimeter 2A at 2'4" depth. Major mineral constituents are indicated in the legend and by their corresponding peaks. Ettringite $(E)$ was abundantly present: 
Lysimeter 2A 3'6"

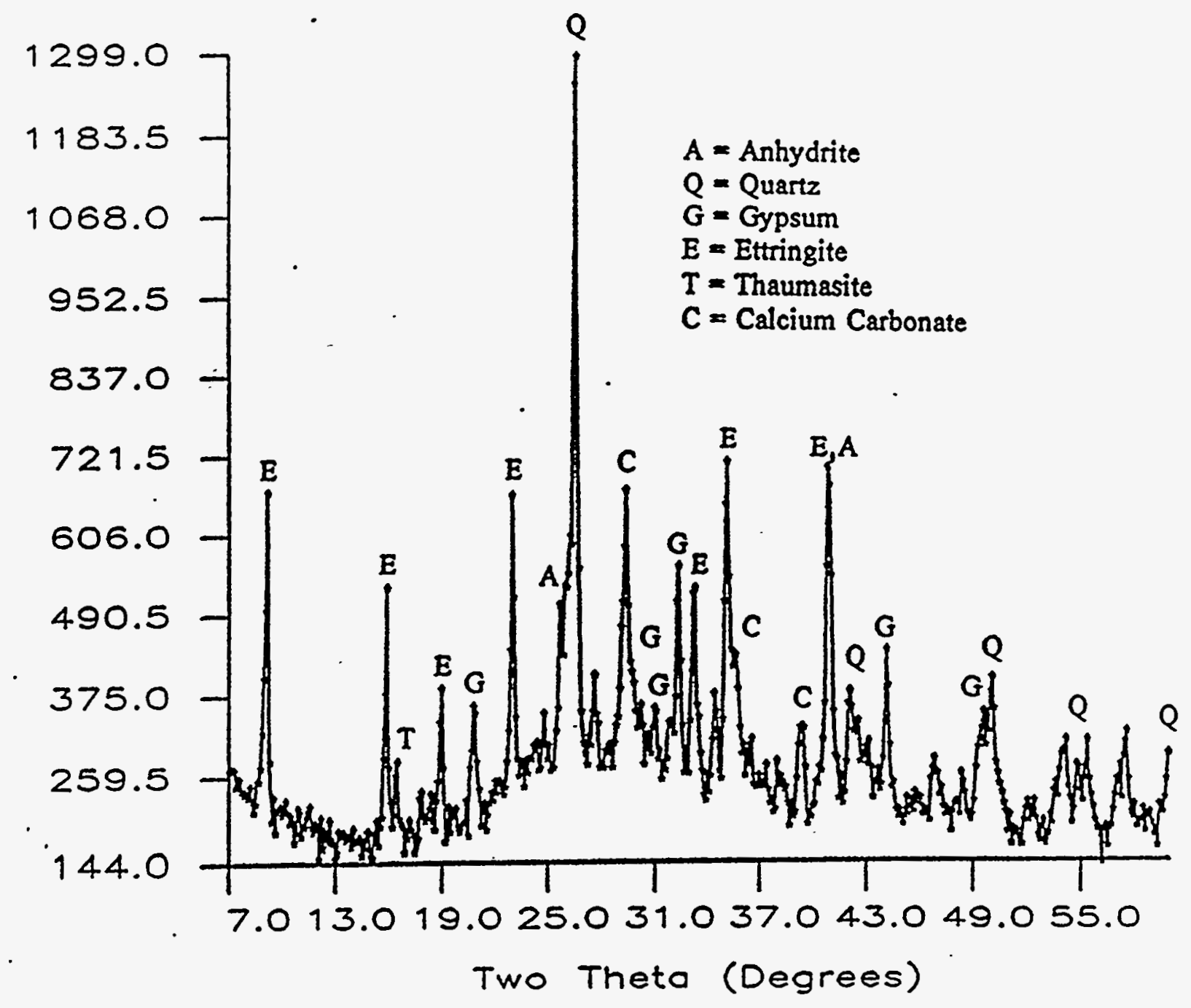

Figure 2-3b. XRD analysis of sample material from same drill core as in 2-3a, but at greater lysimeter depth $\left(3^{\prime} 6^{\prime \prime}\right)$. Ettringite $(E)$ is abundantly present. 


\section{Ettringite in Lysimeter-2A \\ Depth Profile}

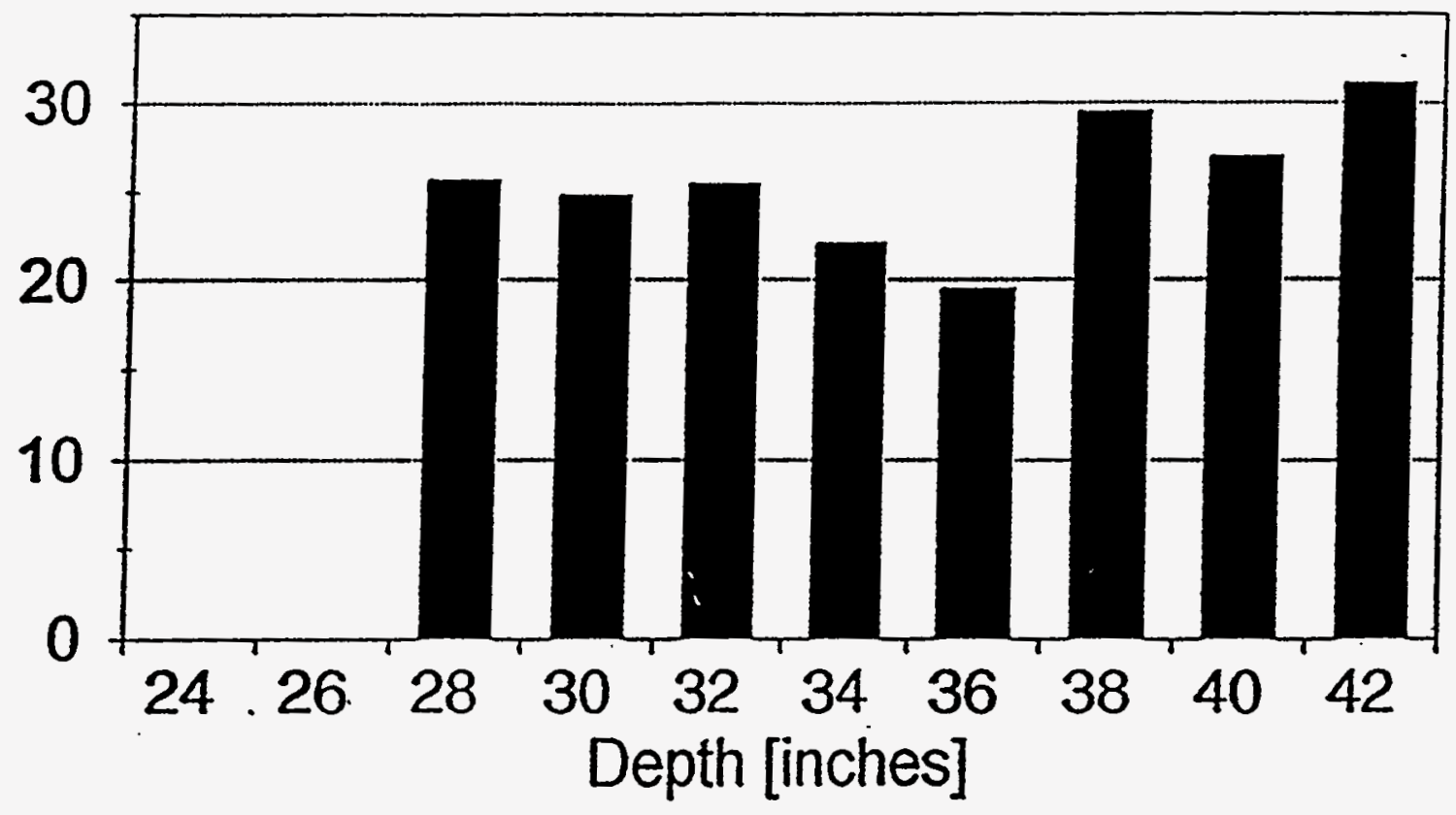

\section{$\%$ Ettringite relative to Quartz}

Figure 2-3c. Ettringite abundance relative to quartz in Field Lysimeter $2 A$ is illustrated for 8 different depth profiles. Ettringite abundance fluctuates with increasing lysimeter depth. 


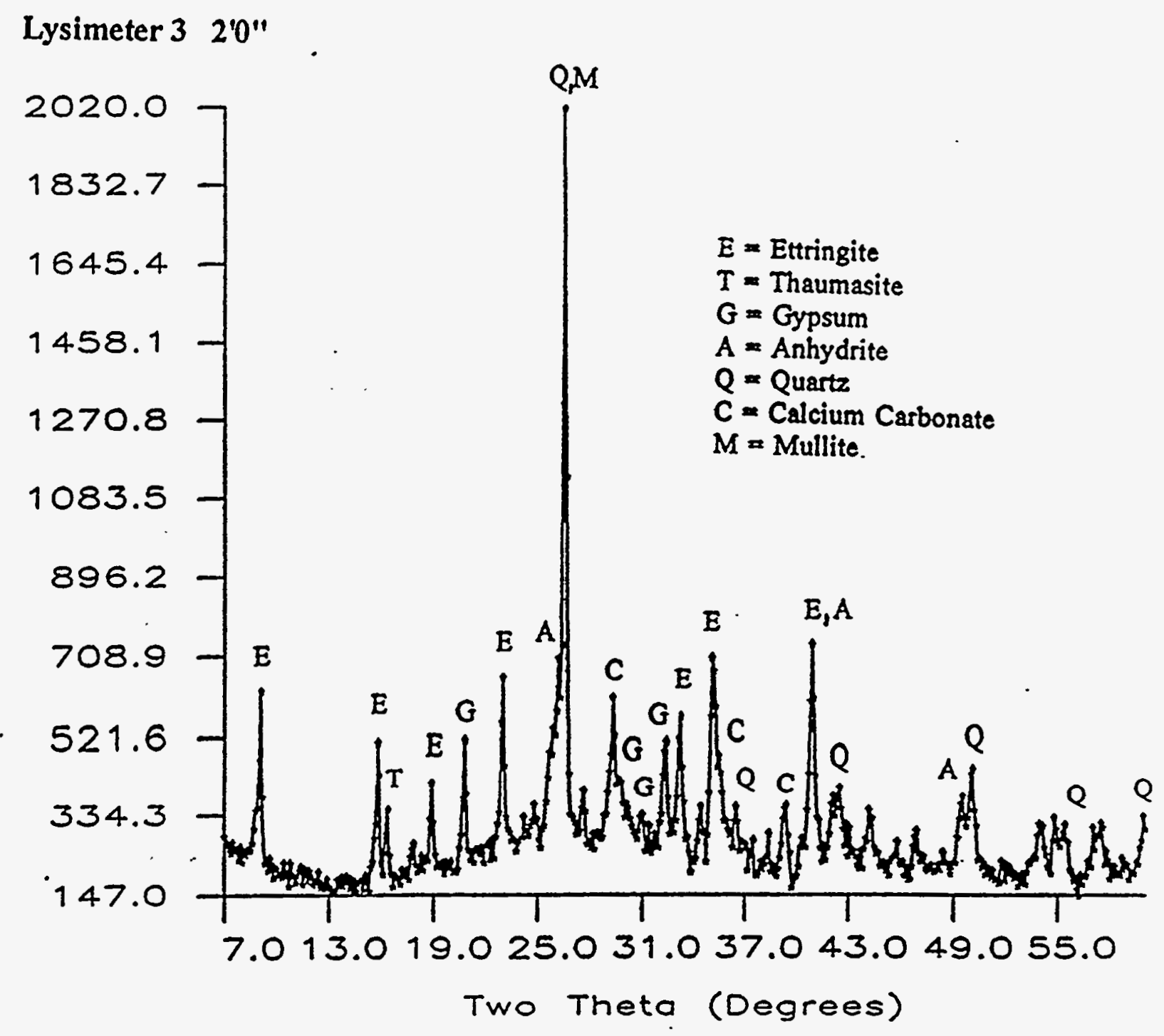

Figure 2-4a. XRD analyses of drill core obtained from Lysimeter 3 at 2'0" depth. Major mineral constituents are indicated in the legend and by their corresponding peaks. Ettringite $(E)$ was abundantly present. 
Lysimeter 3 30"

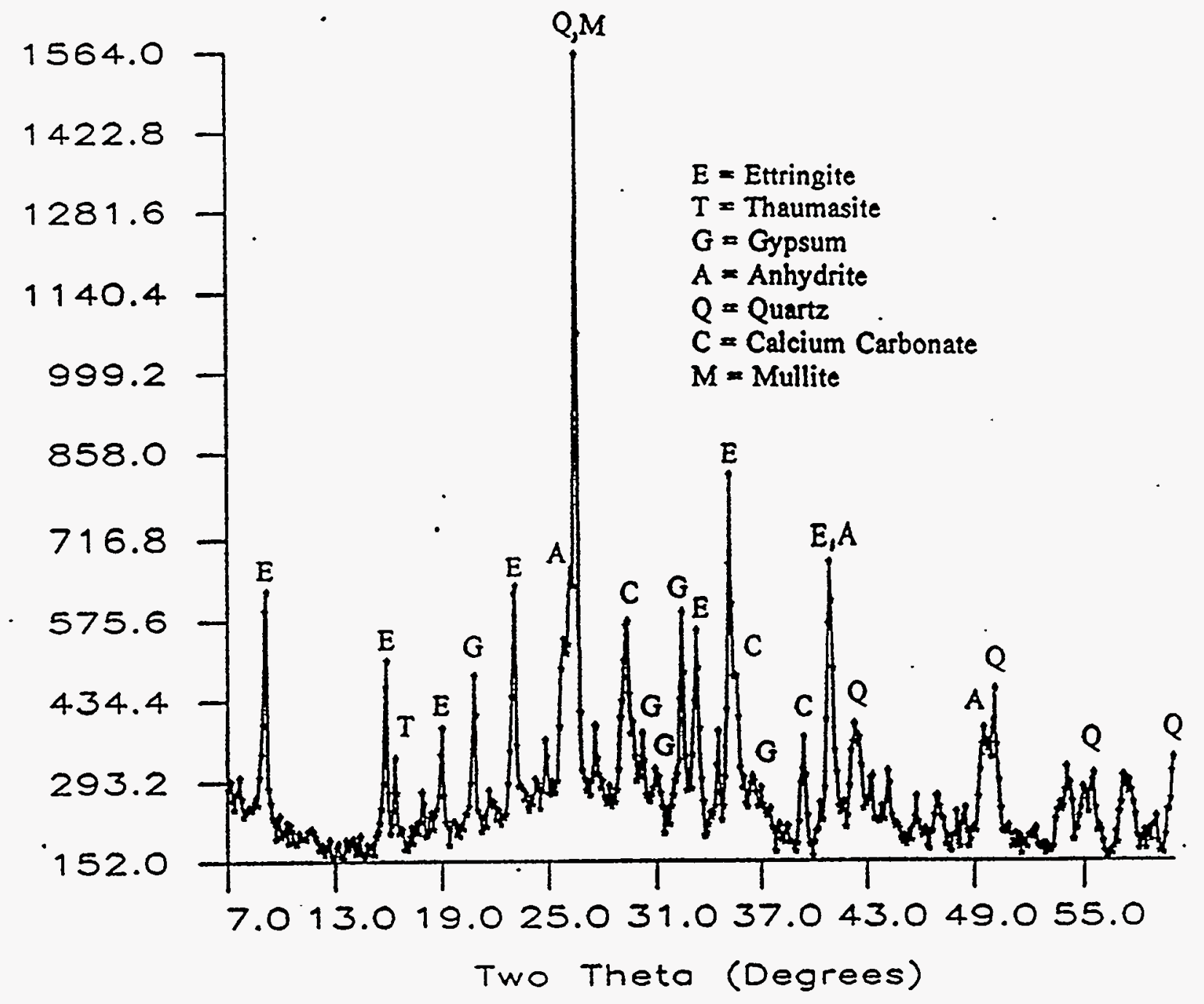

Figure 2-4b. XRD analysis of sample material from same drill core as in 2-4a, but at greater lysimeter depth $\left(3^{\prime} 0^{\prime \prime}\right)$. Ettringite $(E)$ is abundantly present. 


\section{Ettringite in Field Lysimeter L-3 \\ Depth Profile}

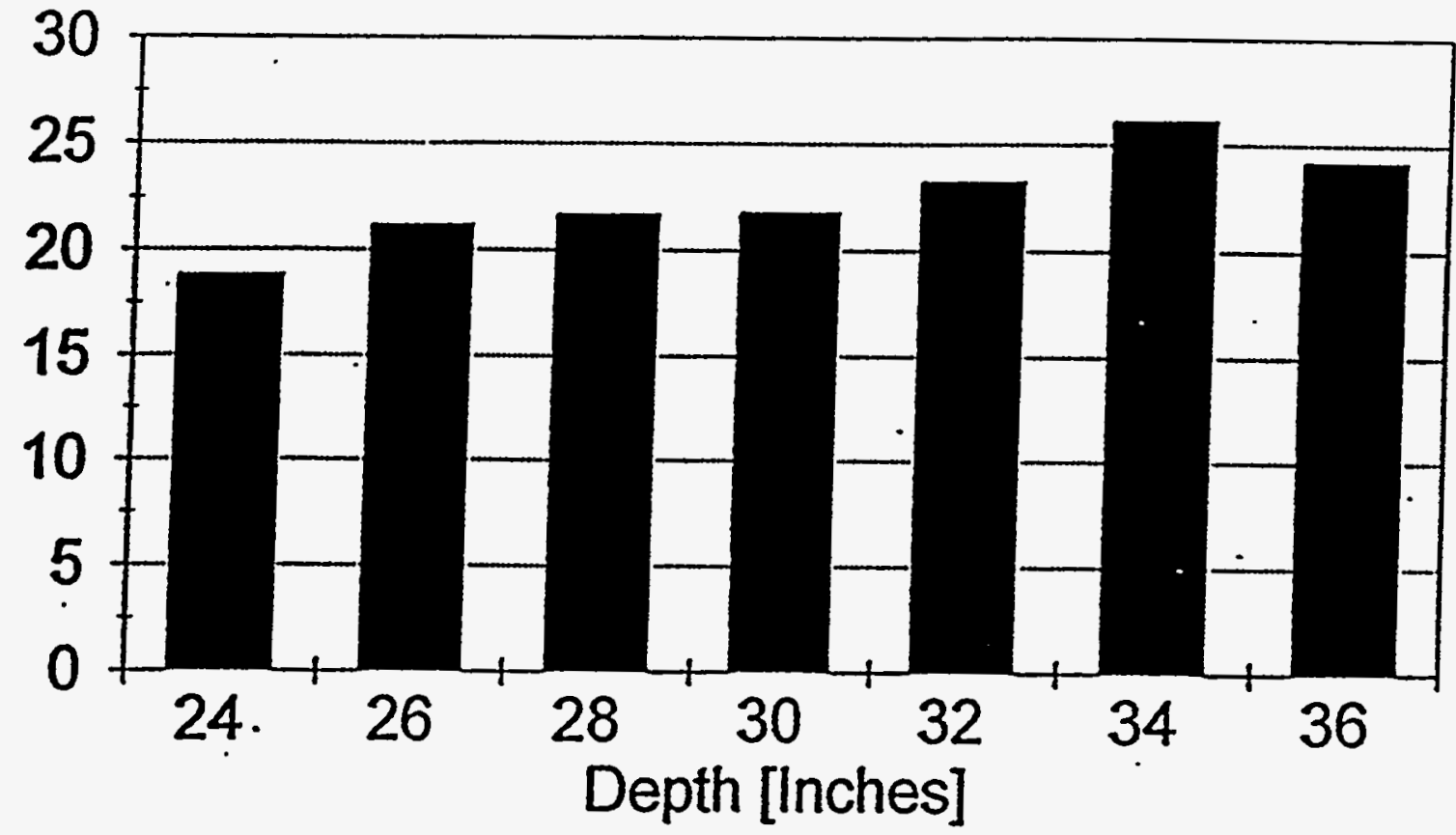

\section{- Ettringite in L-3 rel. to Quartz}

Figure 2-4c. Ettringite abundance relative to quartz in Field Lysimeter 3 is illustrated for 7 different depth profiles. Ettringite abundance appears to increase with increasing lysimeter depth with minor fluctuations at the bottom of the core. 


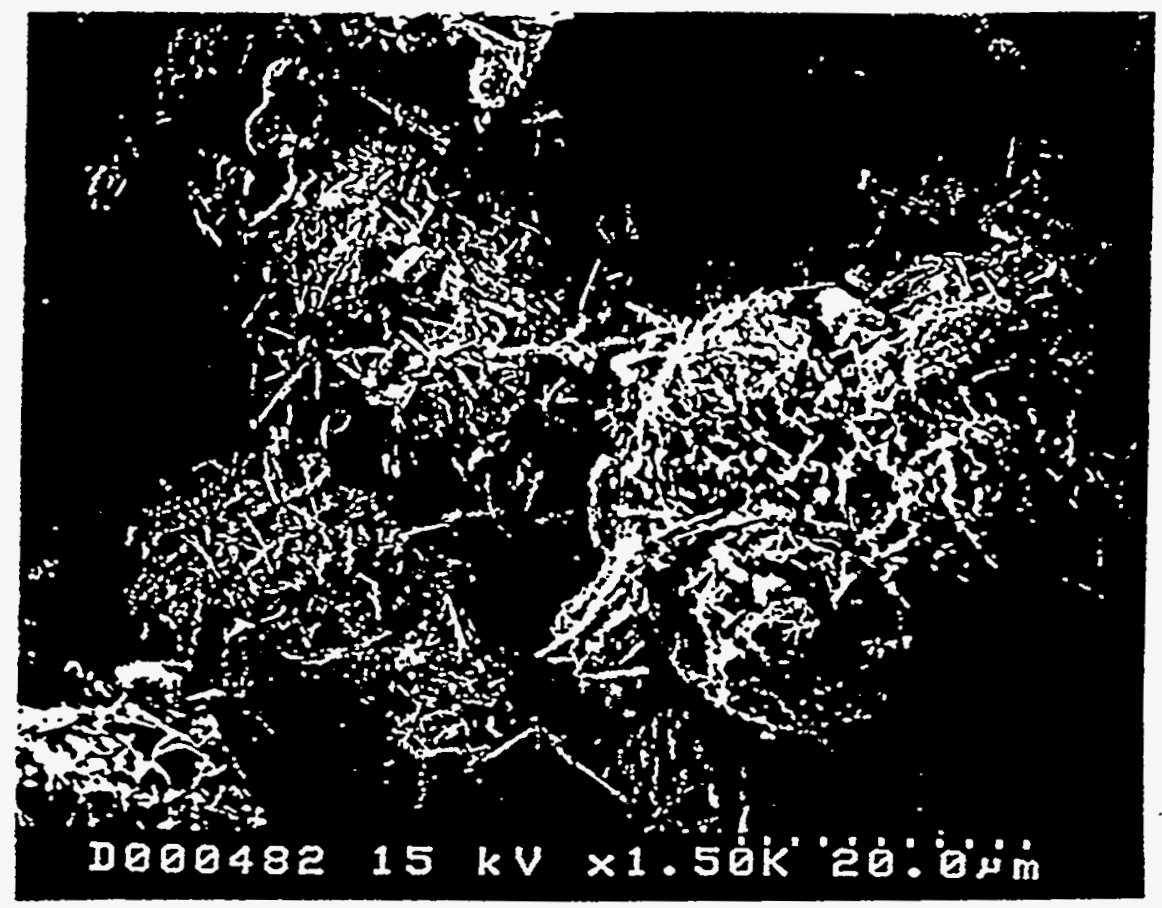

Figure 2-5a. Illustration of ettringite needles overgrowing fly ash surfaces. The ettringite needles form a network of interlinking crystals. Pore space has not yet been infilled by crystal growth.

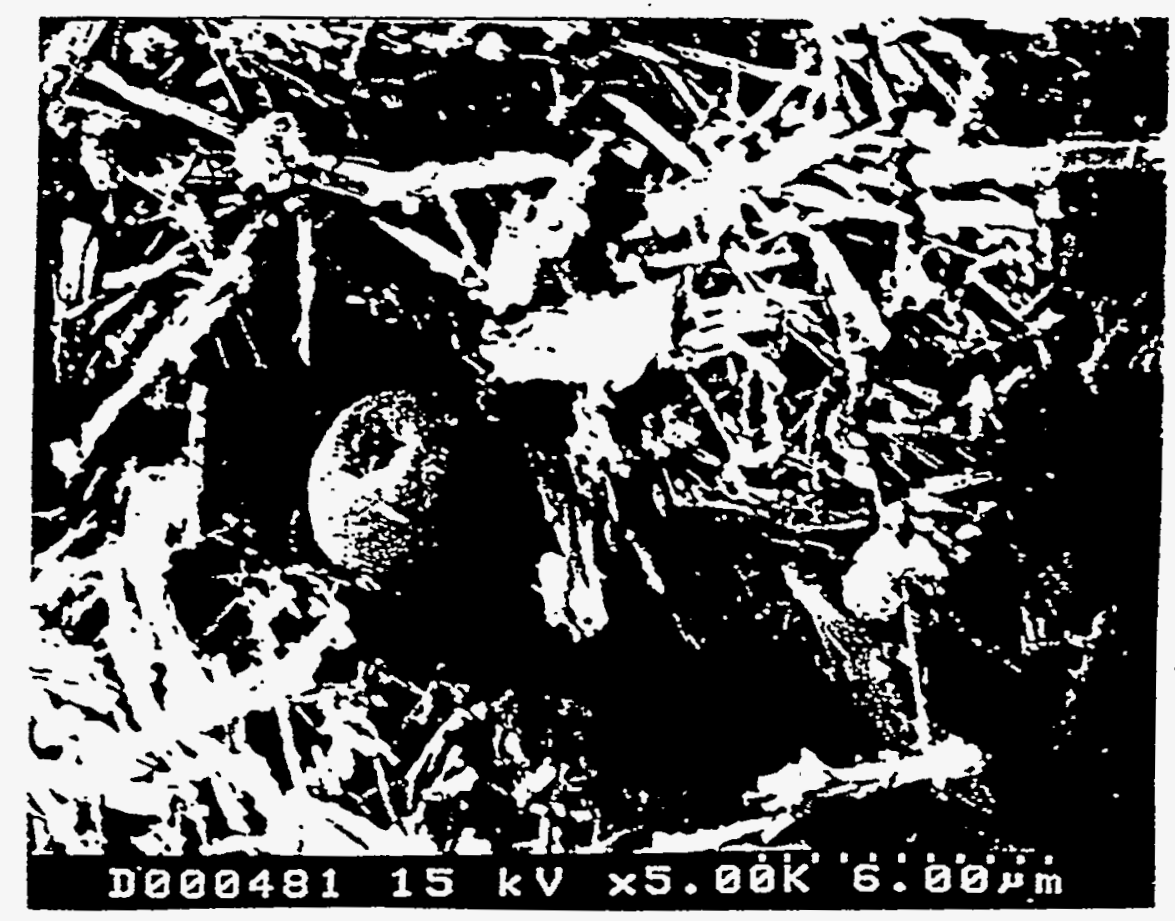

Figure 2-5b. Dense crystal growth of ettringite needles with only minimal pore space. 


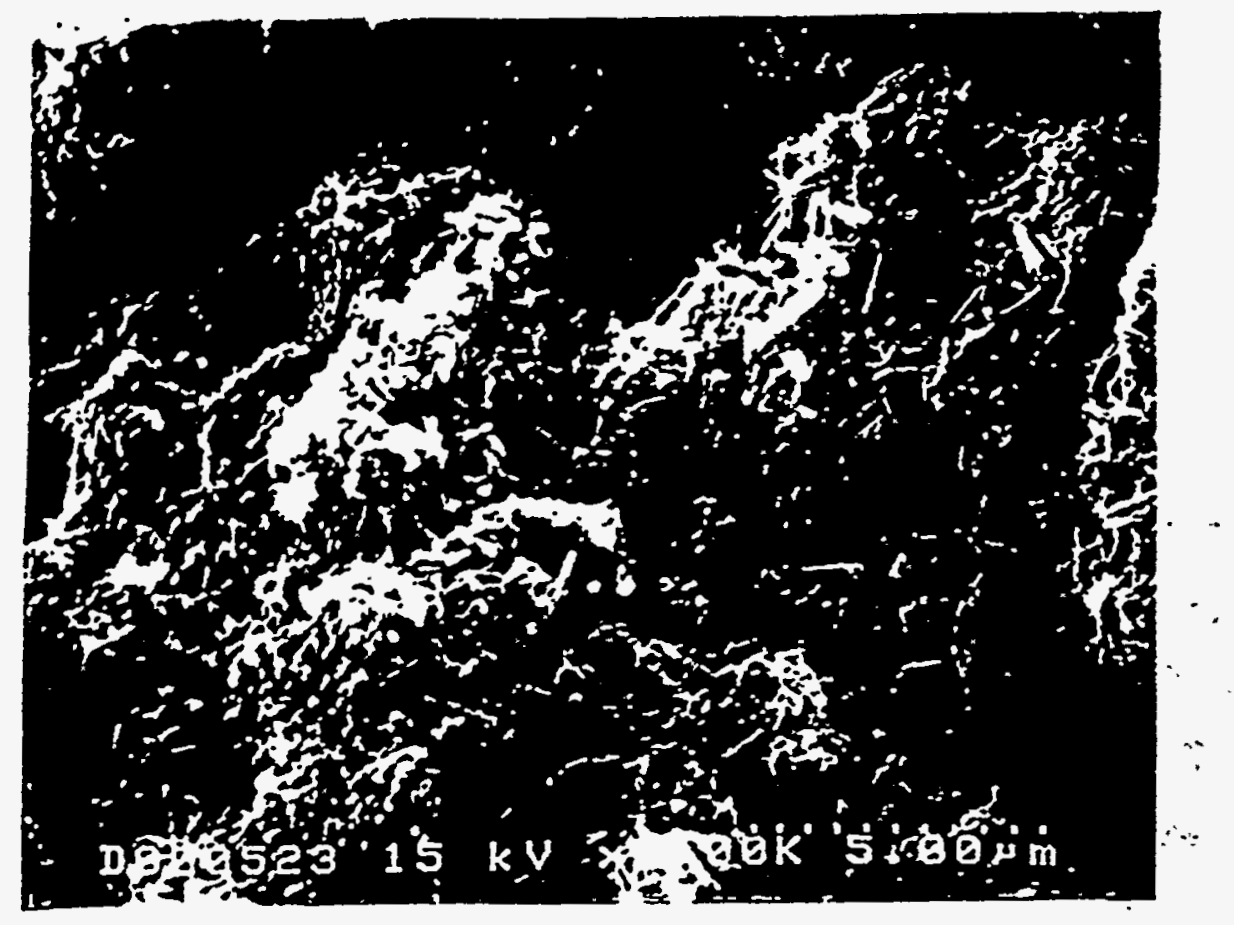

Figure 2-6. SEM illustration of platy ettringite crystals inside the densely packed Lysimeter 3. 
A continuation of the field lysimeter tests occurred during the report period of October 1 , 1995 - October 31, 1995. Additional data obtained during this sampling period supported earlier results and indicated that mineralogical transformations of the Coolside materials continued in the lysimeters as a function of available moisture, aging and static loading. Ettringite was observed to continue to be the dominant product in all lysimeters (1-3) packed with the Coolside material (Figure 2-7; 2-8; 2-9). Lysimeter 1 with the least compaction showed a recognizable difference in ettringite abundance from top to bottom of the drill cores. Ettringite needles were more abundant in the lower zones (Figure 2-7). In comparison the more densly packed Lysimeters 2 and 3 did not have distinguishable differences in ettringite abundance within the top, middle and bottom zones of the drill cores (Figure $2-8 ; 2-9$ ). Trends in gypsum availability within the lysimeters suggest that a rapid leaching of gypsum must have taken place at all layers of the lysimeter cores (Figures $2-7 ; 2-8 ; 2-9$ ). It is expected that gypsum continues to be present in the deeper zones that were out of reach during drilling.

In lysimeters with higher degrees of static loading (static loading increases in the order of Lysimeter 1 to 3 ), minerals are forced to grow within available pore space. This minimizes perk and causes leaching rates to slow down. Leaching rates, in turn, control the dissolution/reprecipitation of minerals in the lysimeter zones. Calcium carbonate is a mobile phase and its presence strongly depends on the perk within the systems. Calcium carbonate is enriched in the top layer within the drill core taken from Lysimeter 1 and less abundant in the deeper zones (Figure 2-7). In the denser packed lysimeters (2 and 3), the calcium carbonate abundance was approximately the same for the top, middle and bottom zones of the drill cores obtained (Figure 2-8; 2-9).

The mineralogy obtained during XRD analyses of the second set of drill cores was summarized as follows: 
Loose compaction in Lysimeter $1\left(33 \mathrm{lb} / \mathrm{f}^{3}\right)$ corresponds to an overall increase in ettringite abundance with lysimeter depth (Figure 2-7) and a significant calcium carbonate enrichment in the top zone.

A compaction of $41 \mathrm{lb} / \mathrm{f}^{3}$ in Lysimeter 2 corresponds to a minor increase in ettringite abundance with increasing lysimeter depth. Calcium carbonate is preferentially present at the top zone, but the variation with increasing lysimeter depth is less pronounced compared to the loosely packed Lysimeter 1 (Figure 2-8).

This study observed only a minor variation in ettringite and calcium carbonate abundance with increasing depth in Lysimeter 3 with the highest compaction of 66 $\mathrm{Ib} / \mathrm{f}^{3}$ (Figure 2-9).

Because the Lysimeter 4 (reference lysimeter) with fly ash has no calcium that could react with $\mathrm{CO}_{2}$, the XRD analyses revealed no calcium carbonate or ettringite (Figure 2-10).

- The results suggest, that mineral growth is strongly controlled by the perk of the solutions downwards. The high degree of compaction restricted perk in Lysimeter 3 and minimized mineral transformations. The depletion of ettringite in the upper zone of the least compacted Lysimeter 1 in conjunction with a calcium carbonate enrichment may be caused by rapid leaching and ion migration through large void spaces and channel ways. Ion migration causes depletion in the upper lysimeter zone of $\mathrm{Al}$ and $\mathrm{SO}_{4}$. As a result, ettringite becomes more soluble and starts to disintegrate. Recarbonization (formation of calcium carbonate) occurs in areas with high $\mathrm{pCO}_{2}$ and lower $\mathrm{pH}$ regimes. Fluctuations in $\mathrm{pH}$ are governed by the influx of rain water. Because calcium hydroxyhydrate is only a minor constituent in the aged lysimeters and gypsum almost entirely restricted to the lower lysimeter zones, ettringite becomes the dominant calcium bearing mineral phase. When dissolved $\mathrm{CO}_{2}$ is mixed into the lysimeter zones by means of incoming rain water, the $\mathrm{pH}$ drops below the stability of ettringite crystals and calcium carbonate forms by reaction with Ca ions that are provided in the dissolution of ettringite needles. In contrast to the loosely 


\section{Lysimeter \#1}

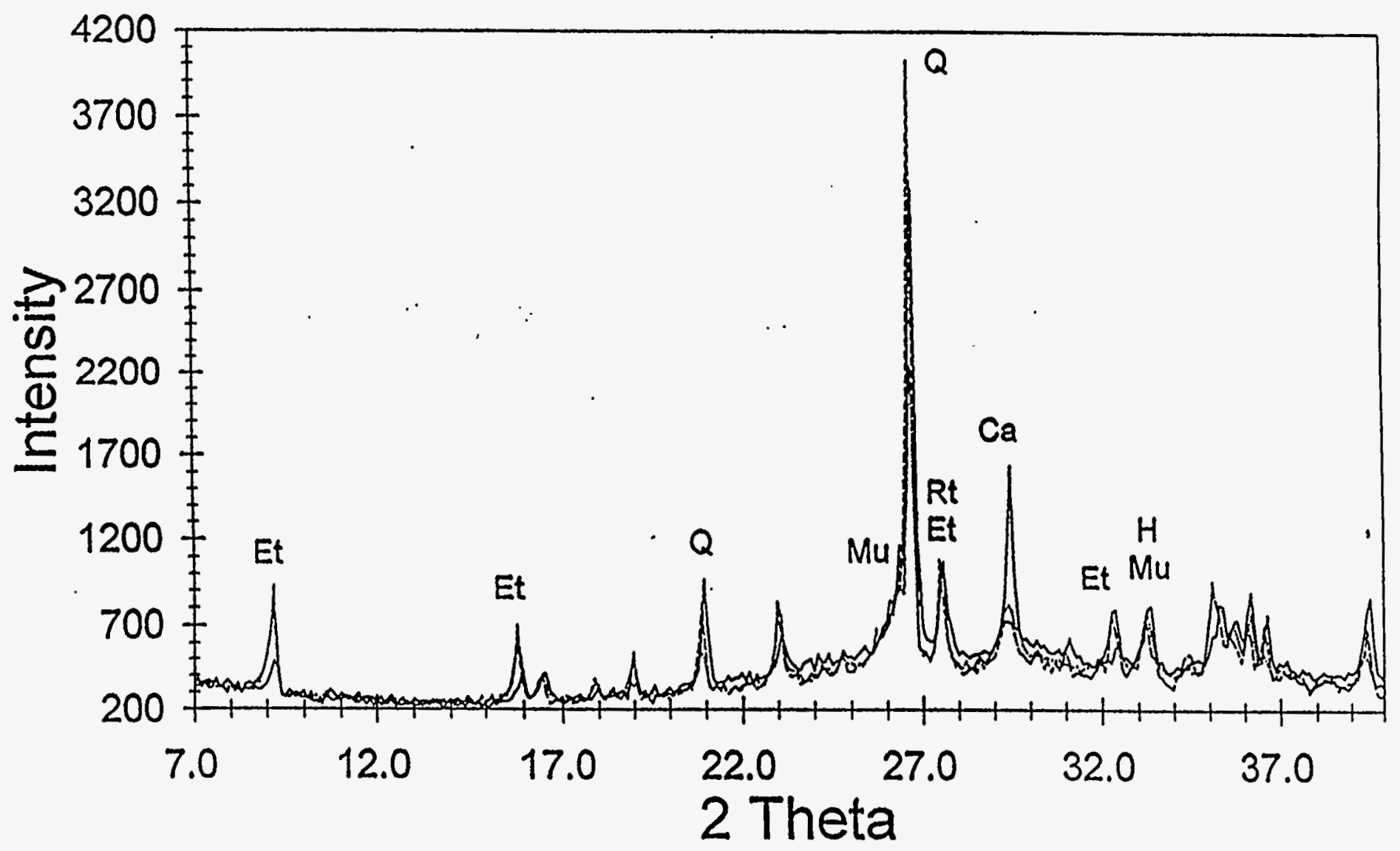

$$
\text { - top } 3^{\prime \prime} \cdot-\cdot-6-8^{\prime \prime}-\text { - }^{\prime \prime}
$$

Figure 2-7. XRD analysis of Coolside material from Lysimeter 1 after second drilling. Figure includes data for samples from $3^{\prime \prime}$ (top), 6-8", and 18" depths. ( $E t=$ ettringite; $Q$ $=$ quartz; $\mathrm{Mu}=$ mullite; $\mathrm{Rt}=$ rutile (internal standard); $\mathrm{Ca}=$ calcium carbonate; $\mathrm{H}=$ hematite). Ettringite is abundantly present in the sample. 


\section{Lysimeter \#2}

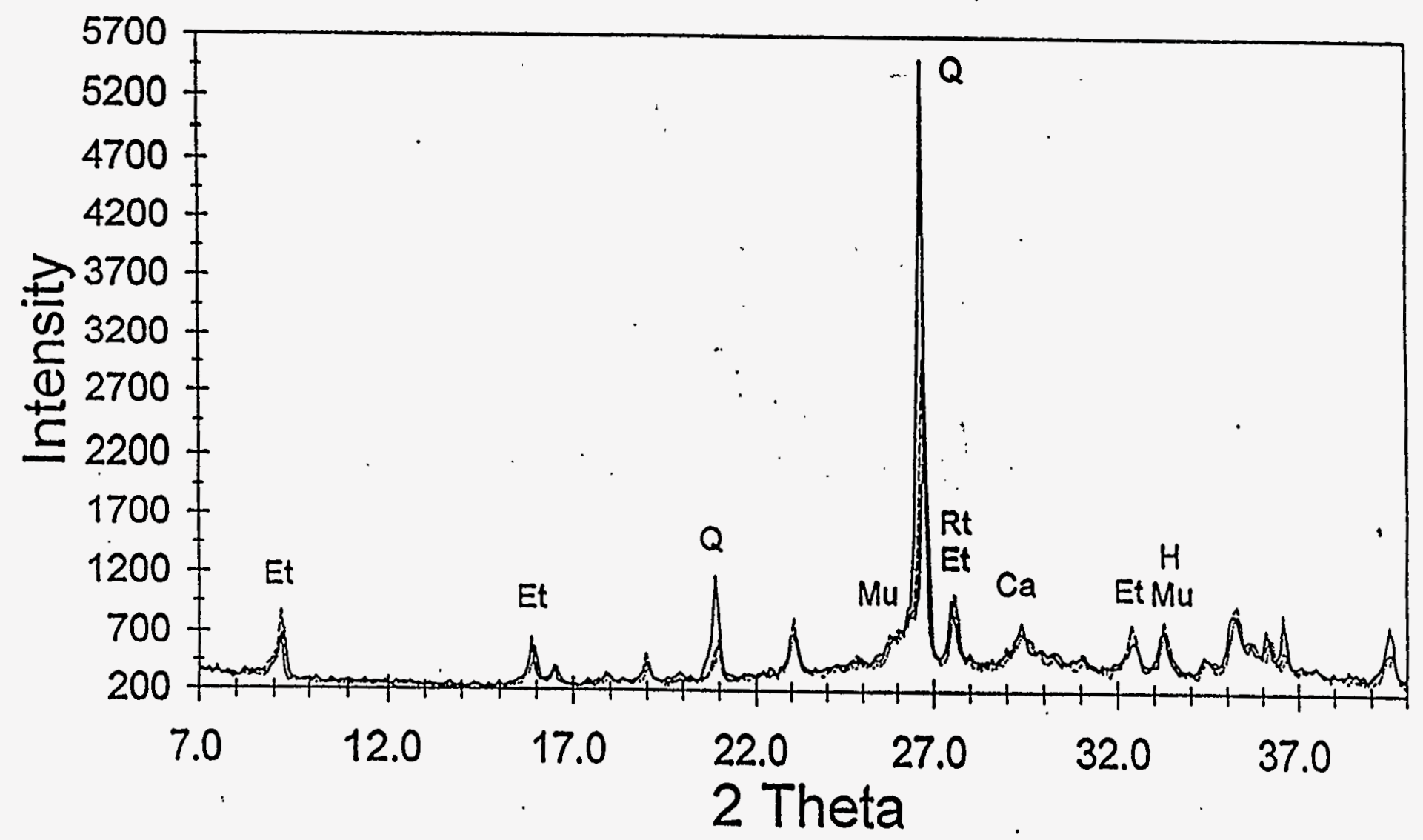

$$
\text { top 3" -.-6-8" -16-18" }
$$

Figure 2-8. XRD analysis of Coolside material from Lysimeter 2 after second drilling. Figure includes data for samples from $3^{\prime \prime}$ (top), 6-8", and 16-18" depths. ( $E t=$ ettringite; $\mathrm{Q}=$ quartz; $\mathrm{Mu}=$ mullite; $\mathrm{Rt}=$ rutile (internal standard); $\mathrm{Ca}=$ calcium carbonate; $\mathrm{H}=$ hematite). Ettringite is present in all three layers of the core. 


\section{Lysimeter \#3}
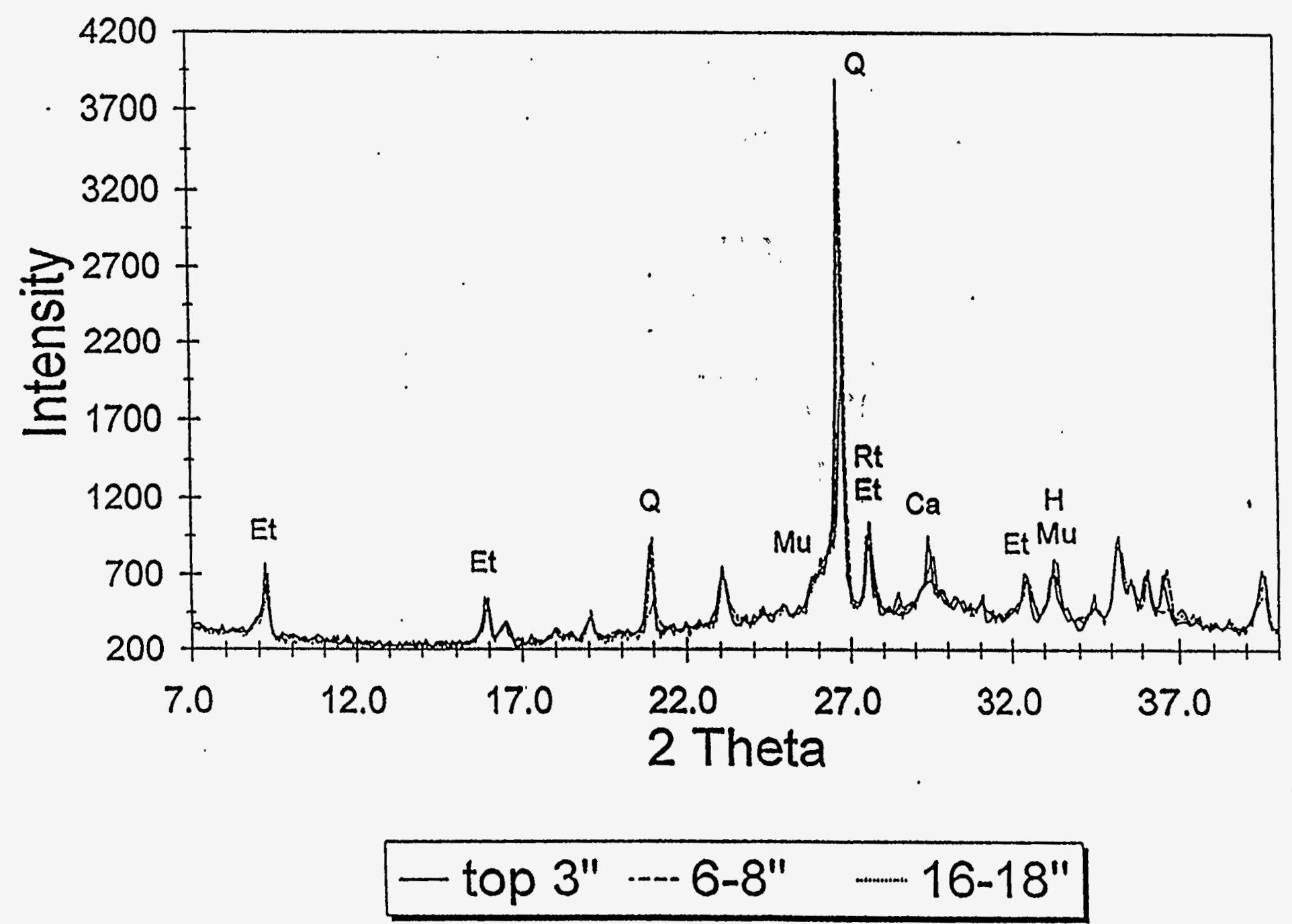

Figure 2-9. XRD analysis of Coolside material from Lysimeter 3 after second drilling. Figure includes data for samples from $3^{\prime \prime}$ (top), 6-8", and 18" depth. (Et = ettringite; $Q=$ quartz; $\mathrm{Mu}=$ mullite; $\mathrm{Rt}=$ rutile (internal standard); $\mathrm{Ca}=$ calcium carbonate; $\mathrm{H}=$ hematite). Ettringite abundance is identical for the three layers. 


\section{Lysimeter \#4}

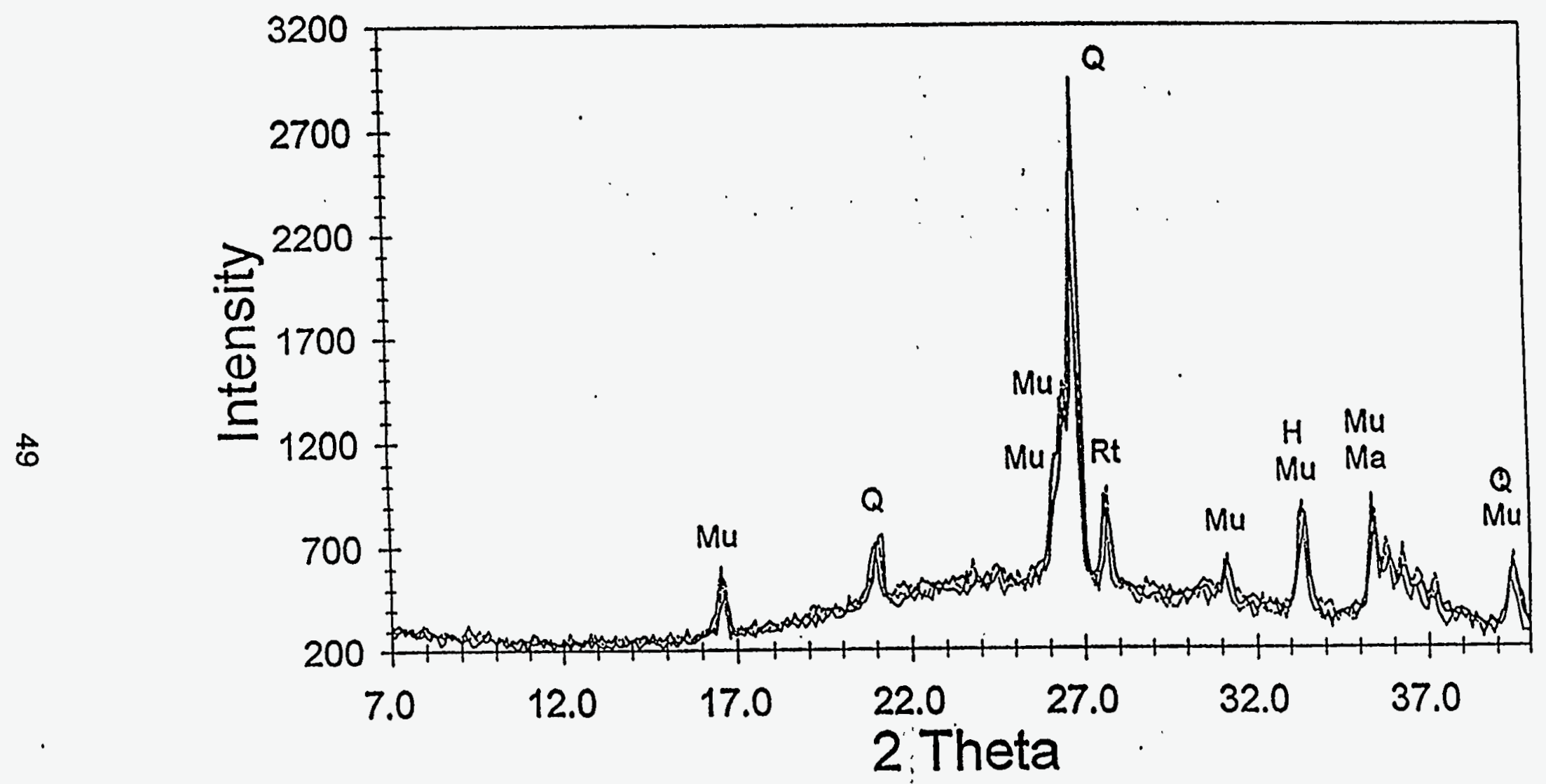

$$
\text { -top 3" -.- 6-8" -16-18" }
$$

Figure 2-10. XRD analysis of material from the reference Lysimeter 4. 
compacted Lysimeter 1, Lysimeters 2 and 3 show minor or no increase in ettringite abundance with lysimeter depth. These findings are in accordance with the earlier suggested mechanism of ion migration by slower flowing fluids through more densly packed lysimeters. The longer residence times of the fluids in voids may lead to mineral precipitation which further enhances the compaction and density of the lysimeters, thereby reducing the mineral migration rates even more.

The third and final core sampling of the field lysimeters occurred in April of 1996. XRD analyses of the cored materials indicated that mineralogical transformations continued in the field lysimeters as a function of available moisture, aging and static loading of the Coolside materials. As indicated in the introduction, one of the major objectives of the Coolside study was to investigate the formation and transformation of minerals during long-term exposure. Throughout the testing period ettringite, gypsum and calcium carbonate are the dominant phases in Lysimeters 1-3, but their abundances vary with time. The XRD results for the final Coolside field samples obtained during the most recently taken lysimeter cores are summarized in Figures 2-11 and 2-12. Results indicate that the ettringite which was still abundantly present in the earlier cores, now is completely leached from the top, middle and bottom parts of the cores. Similar trends are observed for the gypsum distribution within the lysimeters. Calcium carbonate is now the most prominent phase and is present throughout all core samples and in all depth profiles. Within instrumental errors, no differences were observed for Lysimeters 1 and 2 . Lysimeter 3 was cored, but only one mixed sample rather than top, middle and bottom samples was available due to difficulties during drilling.

The mineralogical observations indicate that in Lysimeters 1 and 2 essentially all of the sulfate-bearing phases, including ettringite and gypsum had been dissolved out. The XRD pattern for Lysimeter 1 with the least compaction showed no differences for the top, middle and bottom zones. The same results were obtained for the Lysimeter 2 with higher compaction of the Coolside materials. Mullite, quartz, calcium carbonate and hematite are the minerals that correspond to the XRD peaks in Figures 2-11 and 2-12 for.all:zones of 


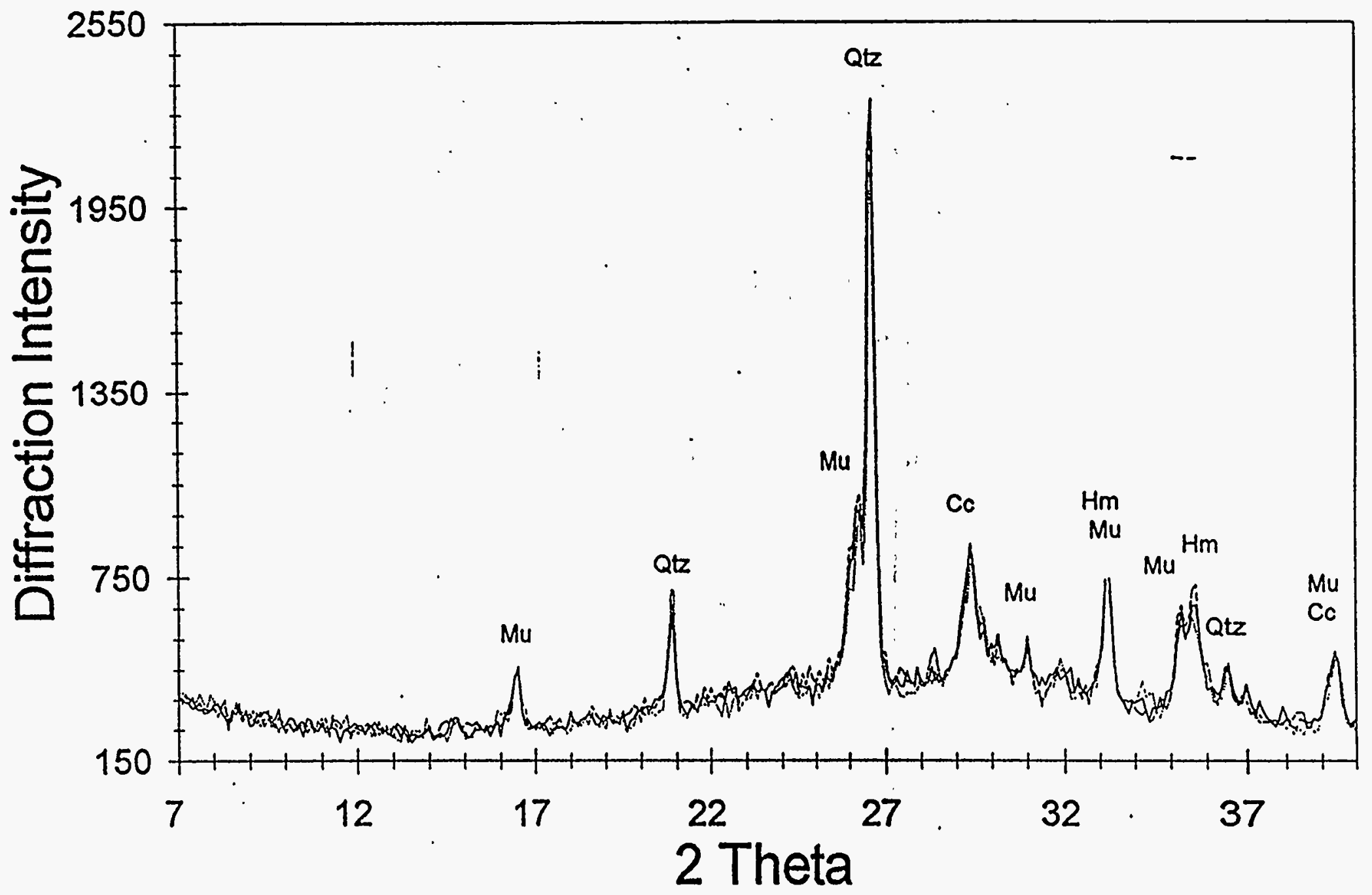

$$
\text { - Lys1 top -.--- Lys1 mid - Lys1 bott }
$$

Figure 2-11. XRD analysis of final drilling material obtained from Lysimeter 1. 


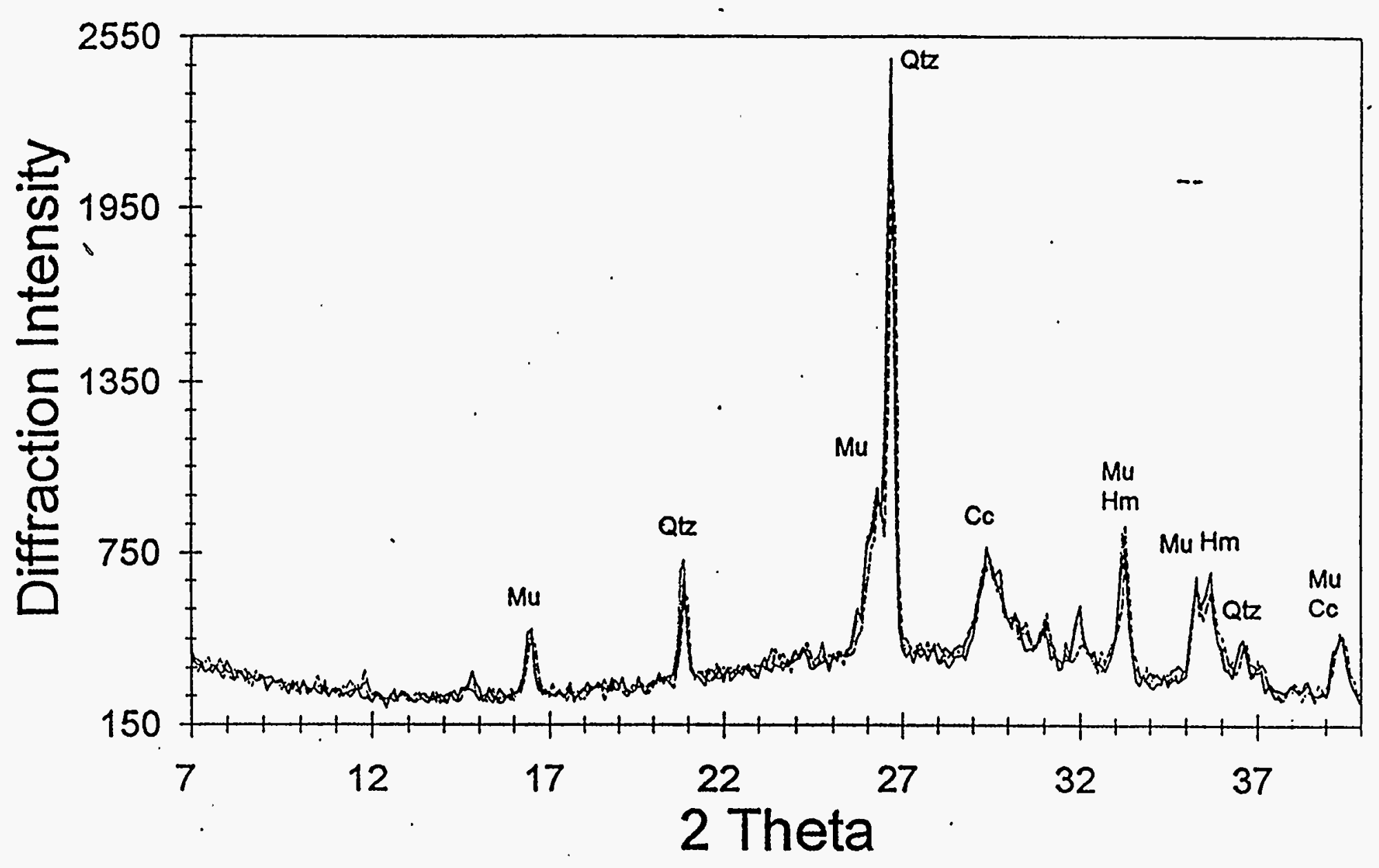

Figure 2-12. XRD analysis of final drilling material obtained from Lysimeter 2. 
the Lysimeters 1 and 2. Differences in packing densities affected mineralogical changes while leaching progressed. When the final drilling was performed, leaching in Lysimeters 1 and 2 had progressed to what may be considered a steady state mineral composition. Minerals include those with very low solubilities such as quartz and mullite. Calcium carbonate, which is less soluble than gypsum under the conditions in the lysimeters, formed at the expense of gypsum and ettringite. The decrease in sulfate concentration in the lysimeters is concomitant with an increase in carbonate concentration. Although ettringite was found to be relatively stable within the weathering lysimeter zones as indicated by the results of the second drill core analyses, long-term leaching causes ettringite disintegration and massive recarbonization.

\section{Series II: Laboratory Lysimeter Study}

The laboratory lysimeter study was aimed at investigating the elemental release characteristics of the Coolside materials. A preliminary study using a variety of lysimeter conditions, packing densities, and fluid throughput, set the stage for the final testing phase. Although the earlier study supplied information on mineral transformations and dissolution patterns, particluarly the early formation of ettringite throughout the lysimeter columns, the leaching rates were extremely slow in the compacted columns and, therefore, leaching characteristics did not resemble closely enough the flow patterns in the field lysimeters where incoming seasonal rainwater was briskly transported to lower lysimeter zones.

The final testing phase is described in detail in Chapter IV: Laboratory-Lysimeter Studies. This section of the Coolside study focuses on the mineralogical changes within the top, middle and bottom zones of the laboratory lysimeters to better understand the effects of fluid migration on new mineral growth. 
The laboratory columns that were sampled for the XRD study of the aged Coolside material are listed in Table 2-1. A discussion of the lysimeter characteristics is presented in great detail in Chapter IV.

Table 2-1

\begin{tabular}{|l|l|l|l|l|l|}
\hline SAMPLE ID & RUN \# & $\% \mathrm{CO}_{2}$ & MOISTURE & COMPACTION & XRD Analyses \\
\hline LC1 & CS Run \#3 & $0 \%$ & Fixed Feed & Loose & Figure 2-13 \\
\hline LC2 & CS Run \#3 & $2.5 \%$ & Fixed Feed & Loose & Figure 2-14 \\
\hline LC3 & CS Run \#3 & $0 \%$ & Rain Simulation & Loose & Figure 2-15 \\
\hline LC4 & CS Run \#3 & $2.5 \%$ & Rain Simulation & Loose & Figure 2-16 \\
\hline LC5 & CS Run \#3 & $0 \%$ & Fixed Feed & Proctor. & Figure 2-17 \\
\hline LC6 & CS Run \#3 & $2.5 \%$ & Fixed Feed & Proctor & Figure 2-18 \\
\hline LC7 & 1000 & $2.5 \%$ & Fixed Feed & Loose & Figure 2-19 \\
& Series & & & & \\
\hline LC8 & CS Run \#1 & $2.5 \%$ & Fixed Feed & Loose & Figure 2-20 \\
\hline
\end{tabular}

A comparison of the XRD analyses of top, middle and bottom samples from LC1 with $0 \%$ $\mathrm{CO}_{2}$, fixed feed and loose compaction (Figure 2-13) showed no significant differences for any minerals within the aged materials. In contrast, samples obtained from LC2 with $2.5 \%$ $\mathrm{CO}_{2}$ addition had removed ettringite from the top layer, enriched gypsum in the middle zone and formed significant amounts of calcium carbonate in all layers, with the top layer showing the highest concentration (Figure 2-14).

A comparison of the XRD data for Coolside Run \#3 with rain simulation for samples LC3 which had loose compaction and $0 \% \mathrm{CO}_{2}$ indicates that ettringite abundance increased downwards in the column lysimeter while gypsum appeared to be preferentially present in the top zone of the lysimeter (Figure 2-15). Calcium carbonate was predominantly observed in the top layer, suggesting $\mathrm{CO}_{2}$ uptake from the atmosphere. 


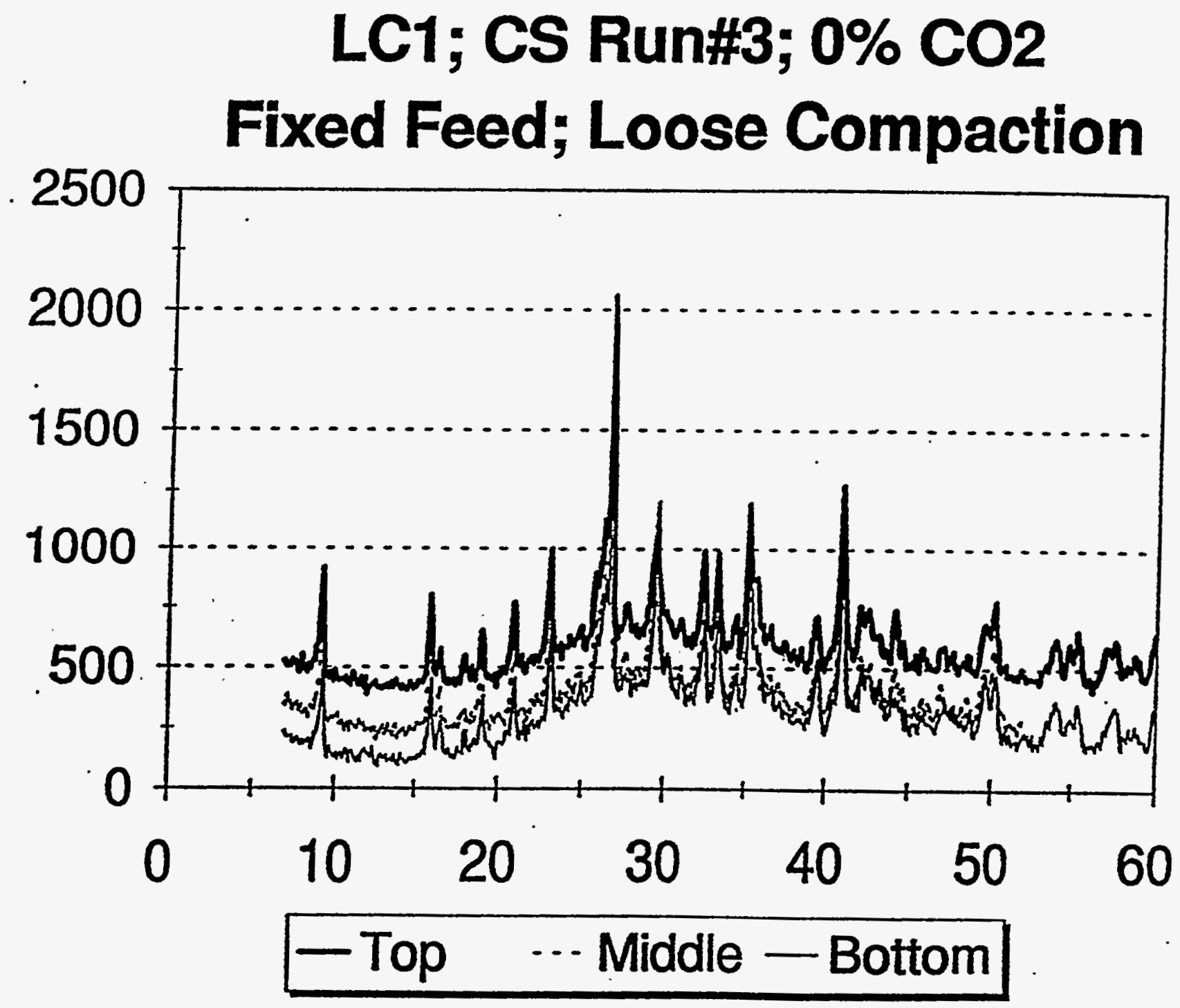

Figure 2-13. XRD analysis of Coolside Run $\# 3$ after $0 \% \mathrm{CO}_{2}$ was added to a fixed feed through the loose compacted Column LC1. The ettringite peak is present at the top, middle and bottom samples ( 9.22 theta). 


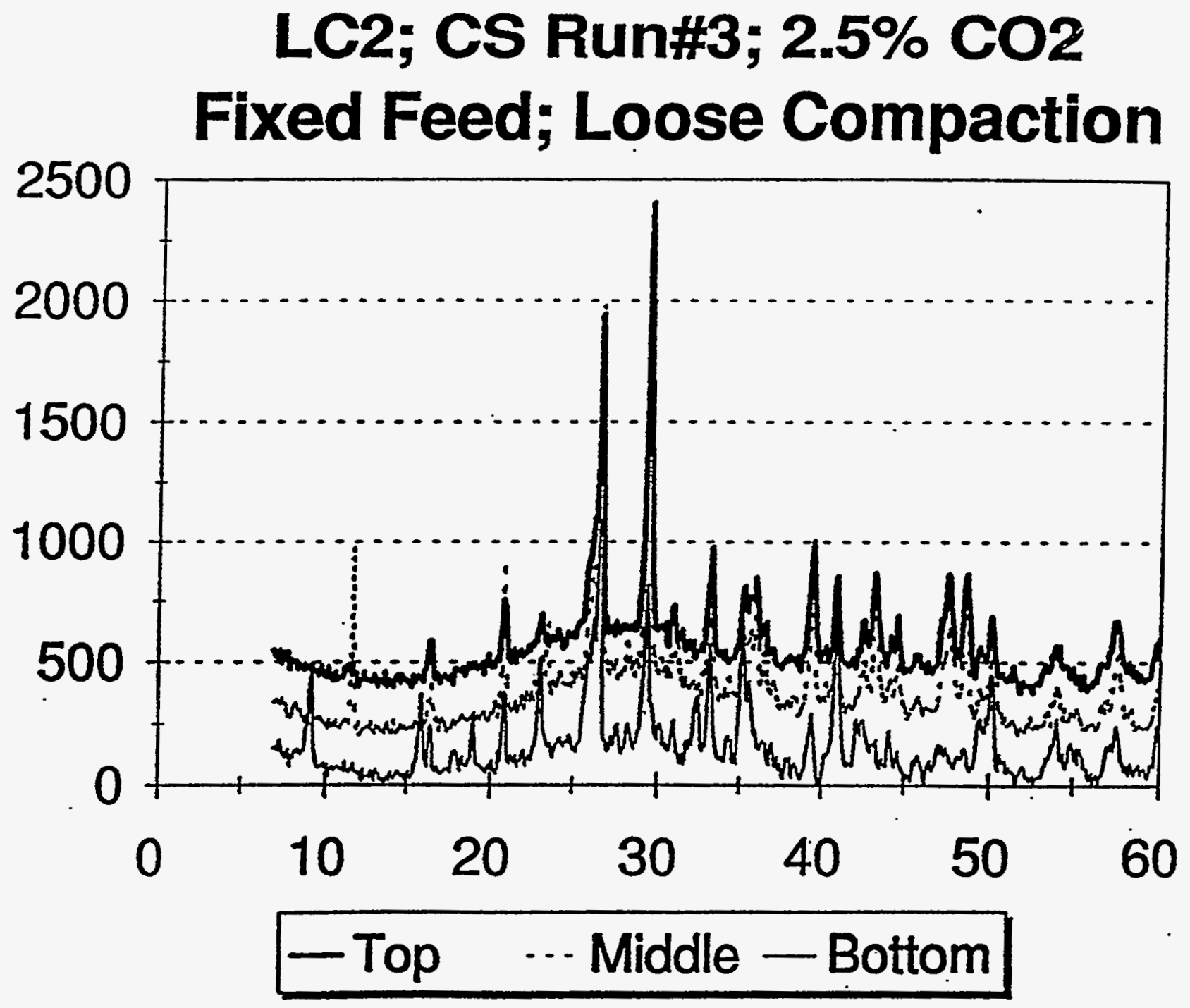

Figure 2-14. XRD analysis of Coolside Run \#3 after $2.5 \% \mathrm{CO}_{2}$ was added to a fixed feed through the loose compacted Column LC2. The ettringite peak is present at the bottom sample (9.22 theta). 


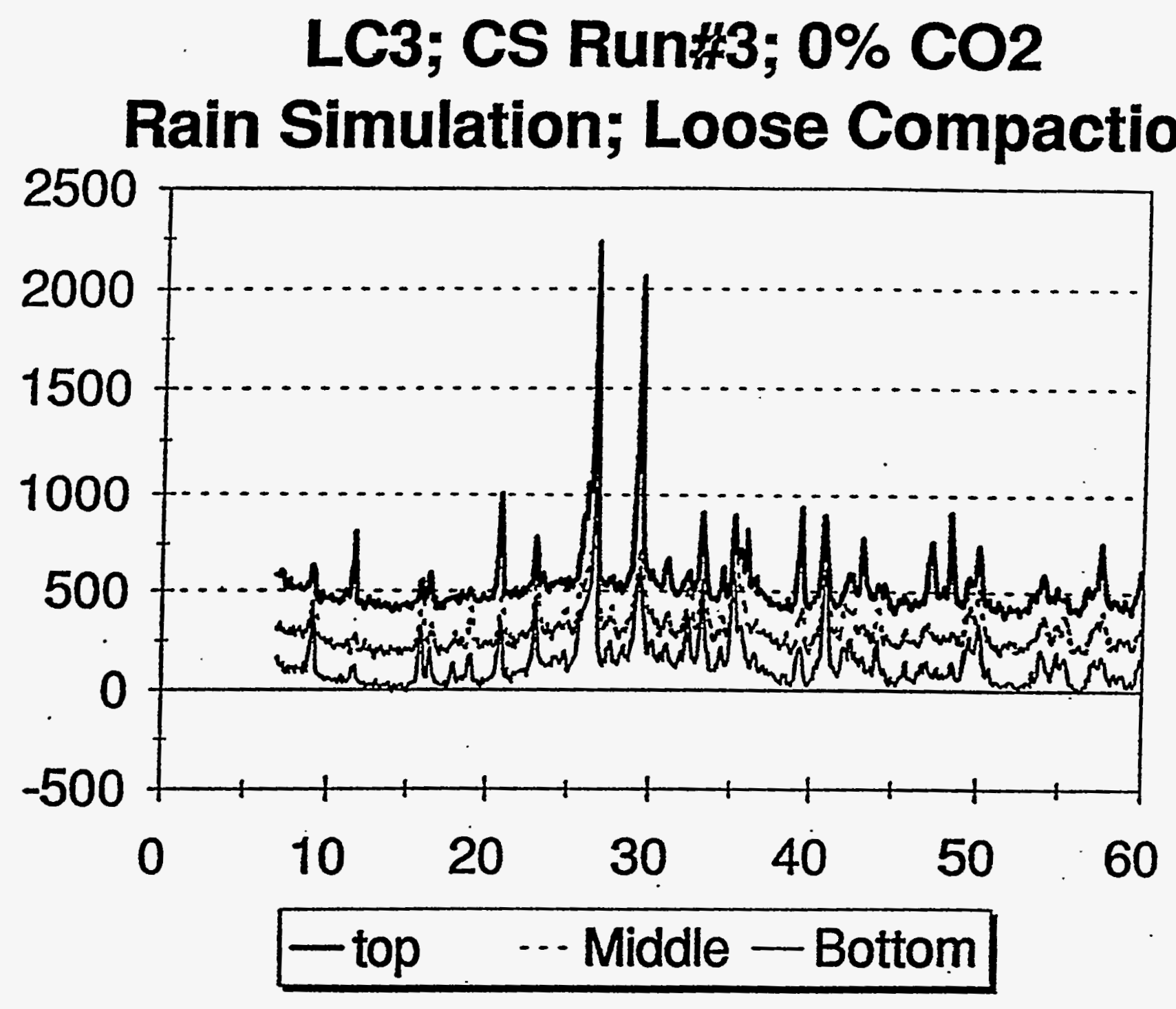

Figure 2-15. XRD analysis of Coolside Run \#3 after $0 \% \mathrm{CO}_{2}$ was added to a rain simulation through the loose compacted Column LC3. The ettringite peak is present at the top, middle and bottom samples ( 9.22 theta) and is most prominent at the bottom layer. 
Sample LC4, which had the same conditions as LC3 with the exception of a $2.5 \%$ addition of $\mathrm{CO}_{2}$ indicated minor differences in the XRD patterns obtained. Addition of $\mathrm{CO}_{2}$ caused ettringite in the top layer to be completely removed. The amount of gypsum in all zones of LC4 was significantly less in the sample without $\mathrm{CO}_{2}$ addition (Figure 2-16).

Samples with proctor rather than loose compaction for the Coolside Run \#3 material showed no recognizable differences for the materials that had $0 \%$ (LC5; Figure 2-17) or $2.5 \%$ (LC6; Figure 2-18) $\mathrm{CO}_{2}$ additions. A comparison of the top, middle and bottom zones for both columns LC5 and LC6 suggest that ettringite was not removed from the top layer as was the case for all loosely compacted columns. Gypsum was absent from all layers in both LC5 and LC6 columns. The relative amounts of calcium carbonate in the compacted columns did not vary significantly for top, middle and bottom zones. .

Coolside material from the 1000 Series was placed in column LC7 using $2.5 \% \mathrm{CO}_{2}$ additions, fixed feed and loose compaction. A comparison of the XRD results for top, middle and bottom zones for LC7 indicated that ettringite had been removed from the top layer while calcium carbonate was preferentially enriched in the top layer (Figure 2-19).

Cooside material from Run \#1 was placed in column LC8 using $2.5 \% \mathrm{CO}_{2}$ additions, fixed feed and loose compaction. The most striking variation in mineral composition between top, middle and bottom zones was the presence of gypsum in the top layer of the column while no gypsum was recognized in the XRD patterns for either middle or bottom zones of LC8 (Figure 2-20). Calcium carbonate was preferentially present in the top zone which is similar in all columns that had additions of $\mathrm{CO}_{2}$.

\section{Series III: Kinetic Study}

In order to gain a better understanding of the mineralogical transformations that occur during hydration of the Coolside FGD material, a laboratory study was completed in which the material was submerged in distilled water, within a sealed container, and the crystalline 


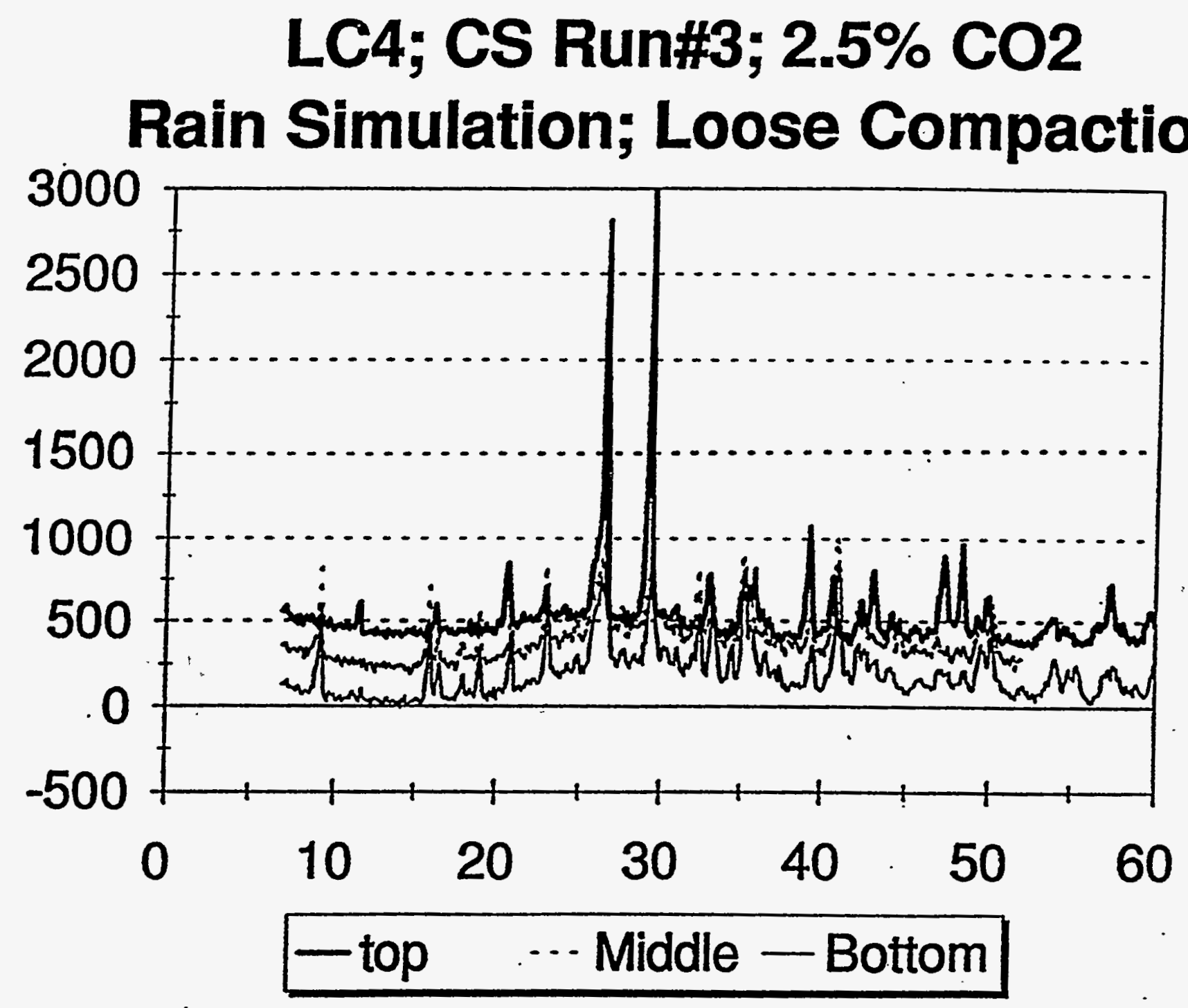

Figure 2-16. XRD analysis of Coolside Run $\# 3$ after $2.5 \% \mathrm{CO}_{2}$ was added to a rain simulation through the loose compacted Column LC4. The ettringite peak is present at the middle and bottom samples (9.2 ? theta), but was more intense for the bottom layer. 


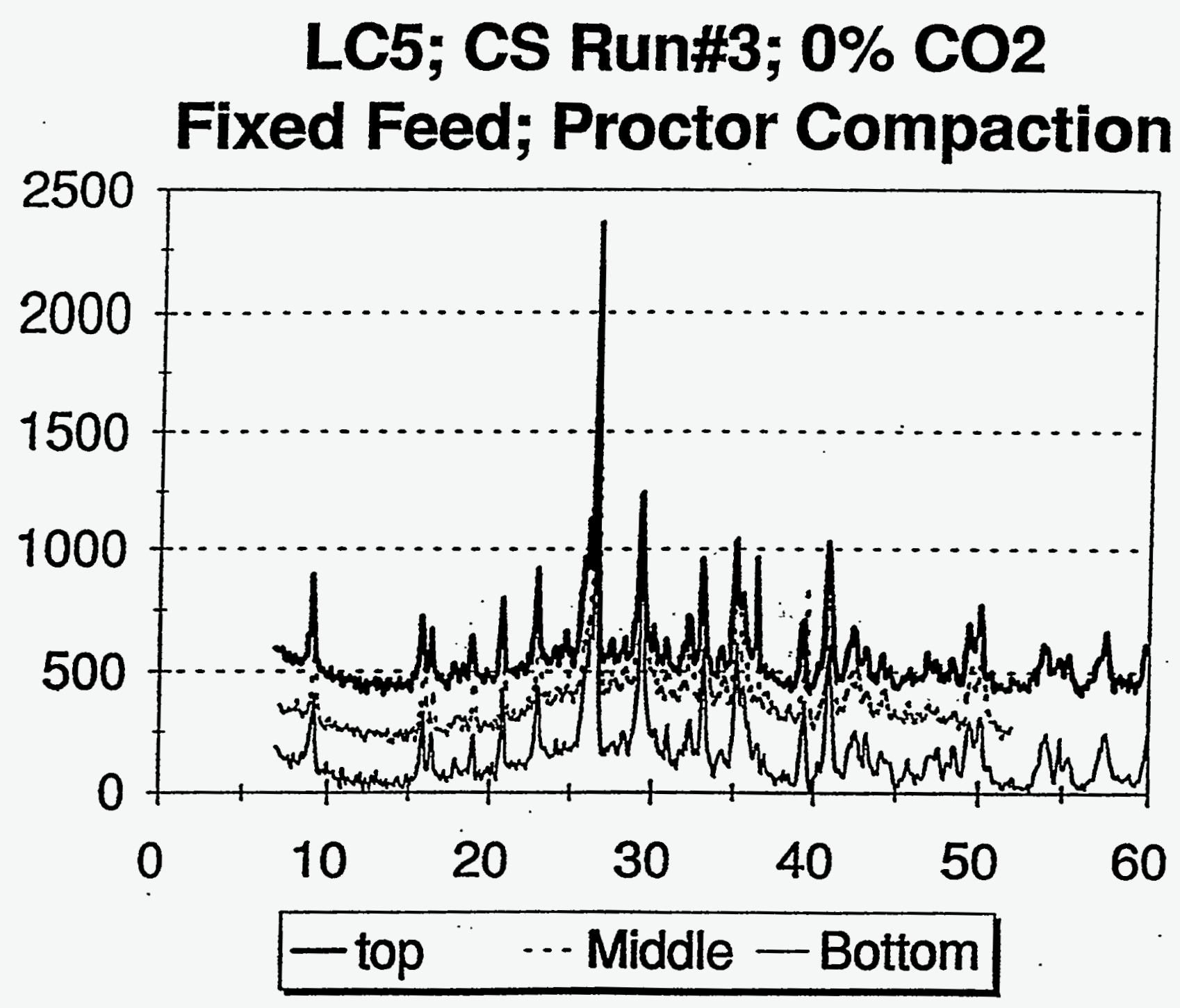

Figure 2-17. XRD analysis of Coolside Run \#3 after $0 \% \mathrm{CO}_{2}$ was added to a fixed feed through the proctor compacted Column LC5. The ettringite peak is present at the top, middle and bottom samples (9.2 2 theta) at very similar intensities. 


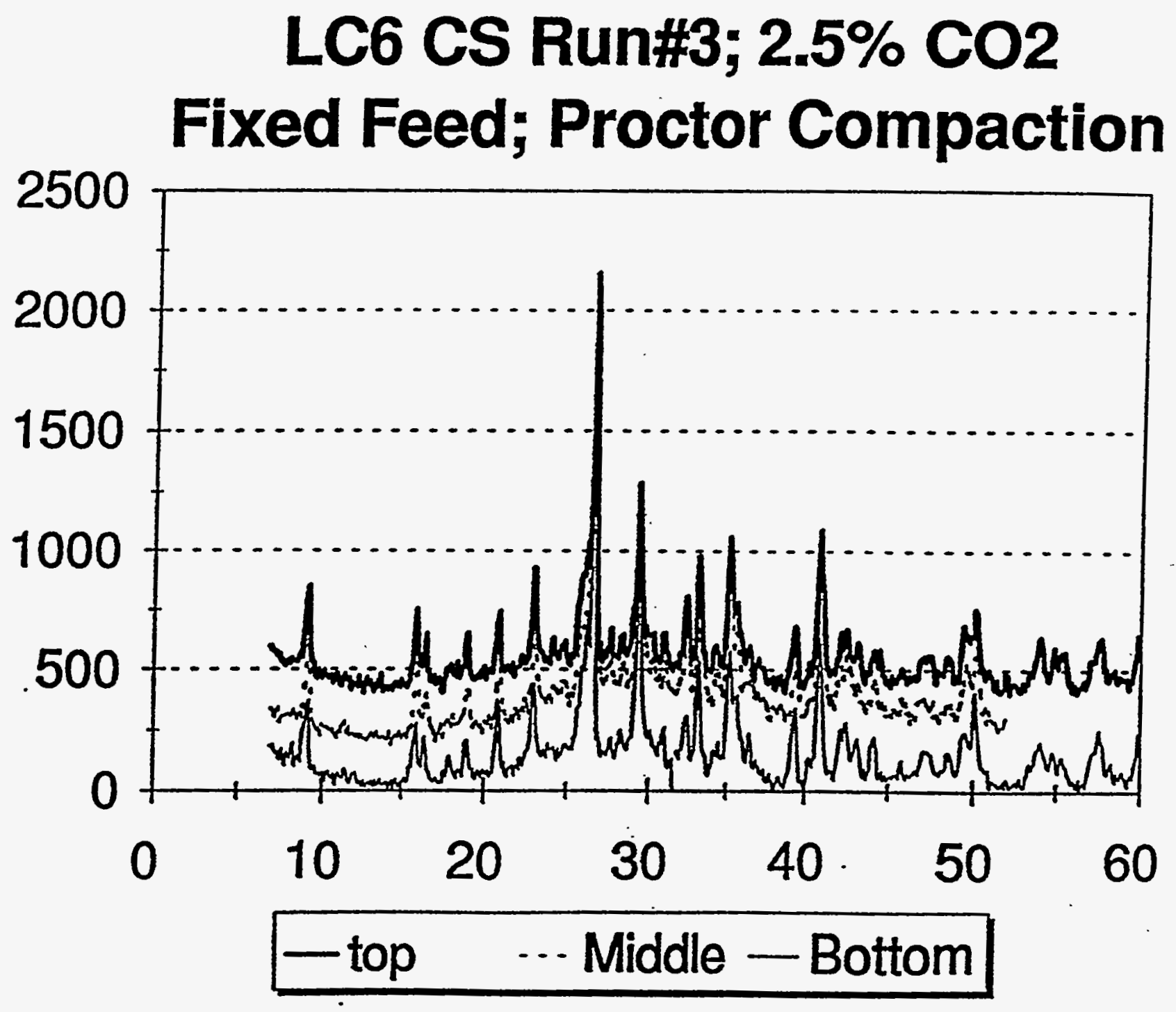

Figure 2-18. XRD analysis of Coolside Run $\# 3$ after $2.5 \% \mathrm{CO}_{2}$ was added to a fixed feed through the proctor compacted Column LC6. The ettringite peak is present at the top, middle and bottom samples ( 9.22 theta) at very similar intensities. 


\section{LC8; CS Run\#1; 2.5\% CO2 Fixed feed; Loose Compaction}

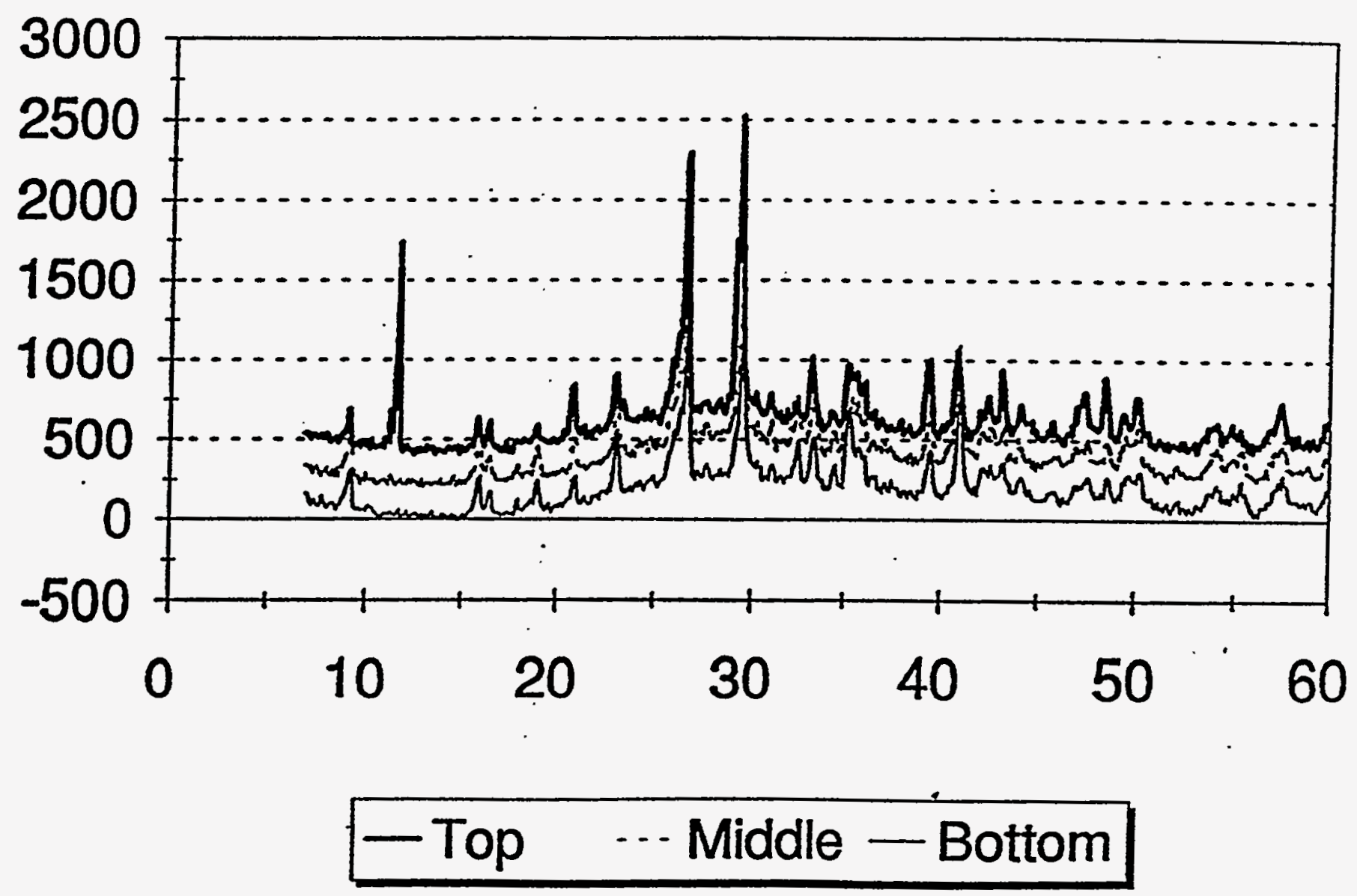

Figure 2-20. XRD analysis of Coolside Coolside Run \#1 after $2.5 \% \mathrm{CO}_{2}$ was added to a fixed feed through the loose compacted Column LC8. The ettringite peak is present at the top, middle and bottom samples (9.2 2-theta). The gypsum peak is only present at the top layer (11.4 2-theta). 
phases monitored over an extended time period. Although these conditions do not reproduce those in the field lysimeters, this experiment does provide information on mineral stability relations in a closed system, and assists in predicting reactions that would occur under other conditions.

In this study, Coolside Run \#2 material was mixed with water to a moisture content of $31 \%$, and placed into small ( 1 in. by 1.5 in.) PVC cylinders that were open at each end. Approximately $15 \mathrm{~g}$ of wet material was used per cylinder. Each sample was placed in a polypropylene jar, $50 \mathrm{ml}$ of distilled/de-ionized water added (sufficient to cover the sample), and a plastic lid (screw type) was secured tightly to the jar to seal its contents from the atmosphere. The equilibrium temperature of the solutions was $20^{\circ} \mathrm{C}$.

At specific intervals, over a period of 300 days, a sample was removed from the water bath and the FGD material extracted from the PVC cylinder. In many of the jars, a small amount of calcium carbonate was observed on the water surface, probably from uptake of $\mathrm{CO}_{2}$; these deposits were not included in the XRD analysis. The sample was ground thoroughly with a mortar and pestle, and approximately 2 grams acquired for XRD analysis, which was conducted using $\mathrm{Cu}-\mathrm{K}$ radiation, from $7-40^{\circ}$ 2theta, at an increment of $0.1^{\circ}$, and the spectra compared to that from the dry, parent material.

The solutions were decanted through a Whatman No. 4 paper filter, and the $\mathrm{pH}$ measured at $20^{\circ} \mathrm{C}$.

Figure 2-21 shows XRD spectra for the samples. The dry material comprised mainly hannebachite $\left(\mathrm{CaSO}_{3} 0.5 \mathrm{H}_{2} \mathrm{O}\right)$, portlandite $\left(\mathrm{Ca}(\mathrm{OH})_{2}\right)$, calcium carbonate, quartz, mullite, and hematite. Over the course of 300 days, calcium carbonate (Figure 2-22), hannebachite (Figure 2-23), and quartz (Figure 2-25) abundance changed a negligible amount, as did mullite and hematite (Figure 2-21). The calcium carbonate abundance was controlled in the closed system of the sealed containers. Ettringite formation (Figure 2-24) commenced within the first day of the study and increased to an equilibrium amount after 

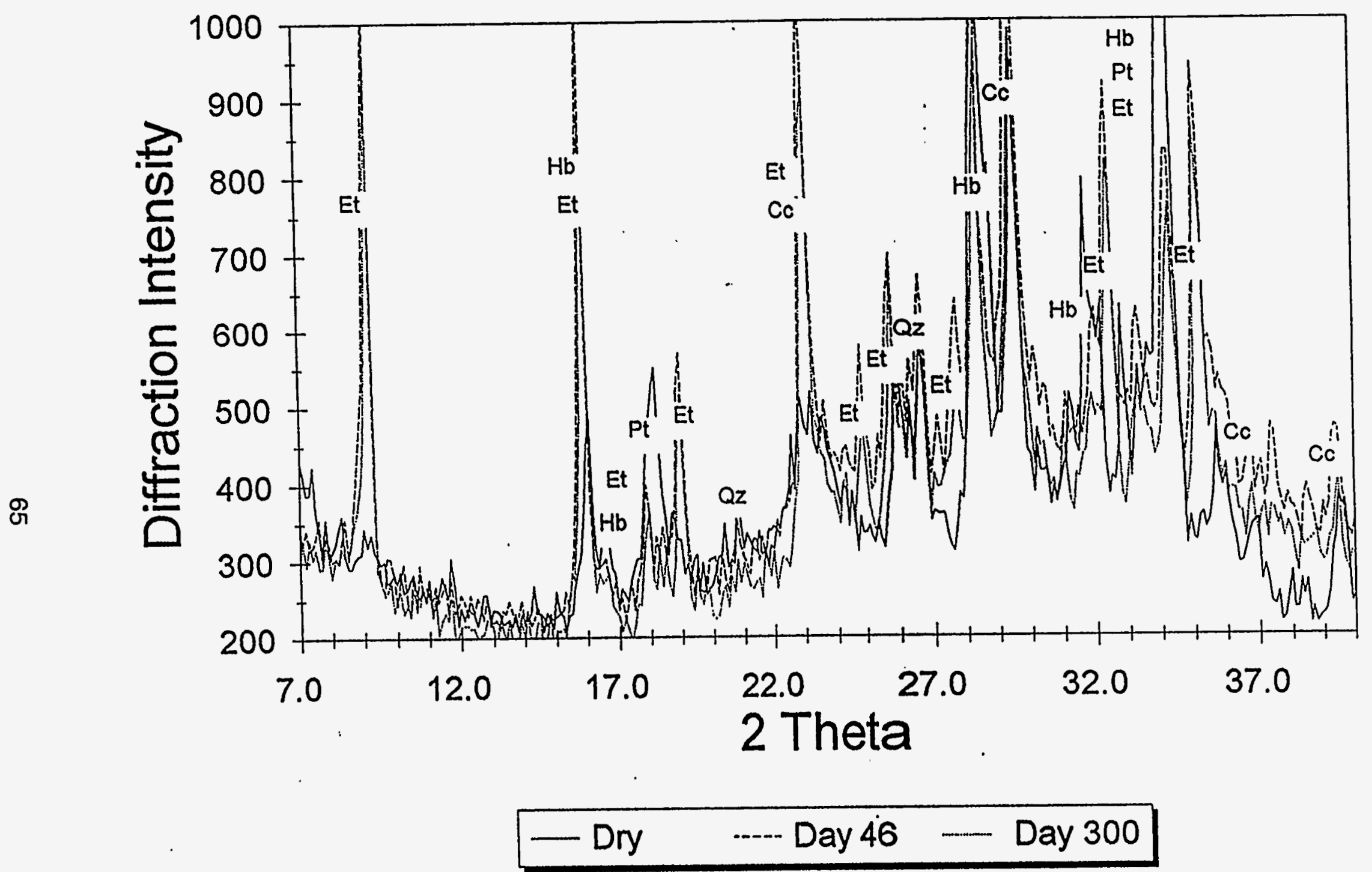

Figure 2-21. XRD spectra for Coolside material from Run \#2 used in the kinetic study. Illustrated are XRD results for the dry material compared with material aged for 46 days and for 300 days. ( $E t=$ ettringite; $\mathrm{Hb}=$ hannebachite; $\mathrm{Pt}=$ portandite; $\mathrm{Qt}=$ quartz; $\mathrm{Cc}$ $=$ calcium carbonate). Ettringite was not present in the dry material, but formed significant amounts of crystals in the aged materials. 


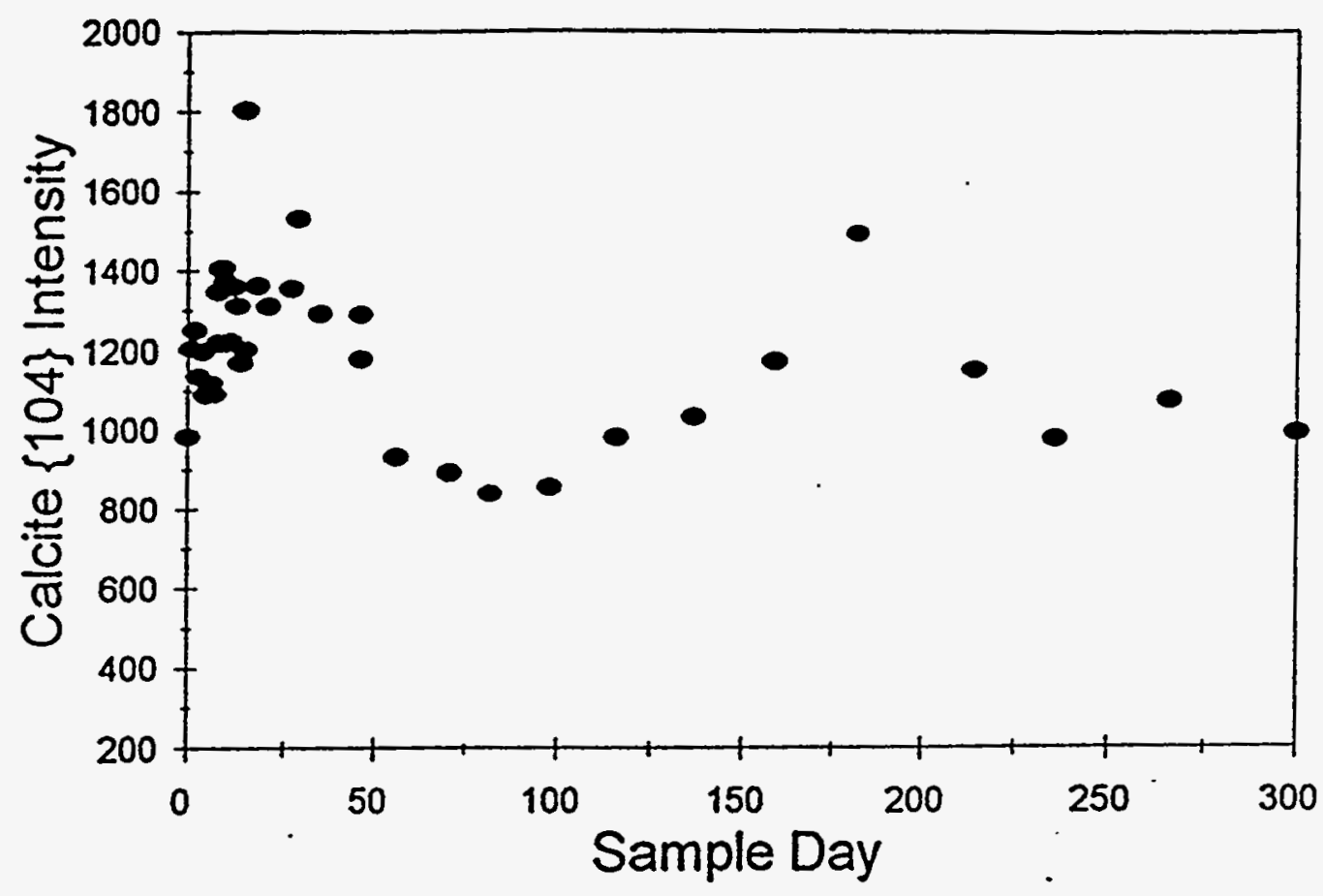

Figure 2-22. Illustration of the relative amounts of calcium carbonate in the kinetic study as a function of sample age.

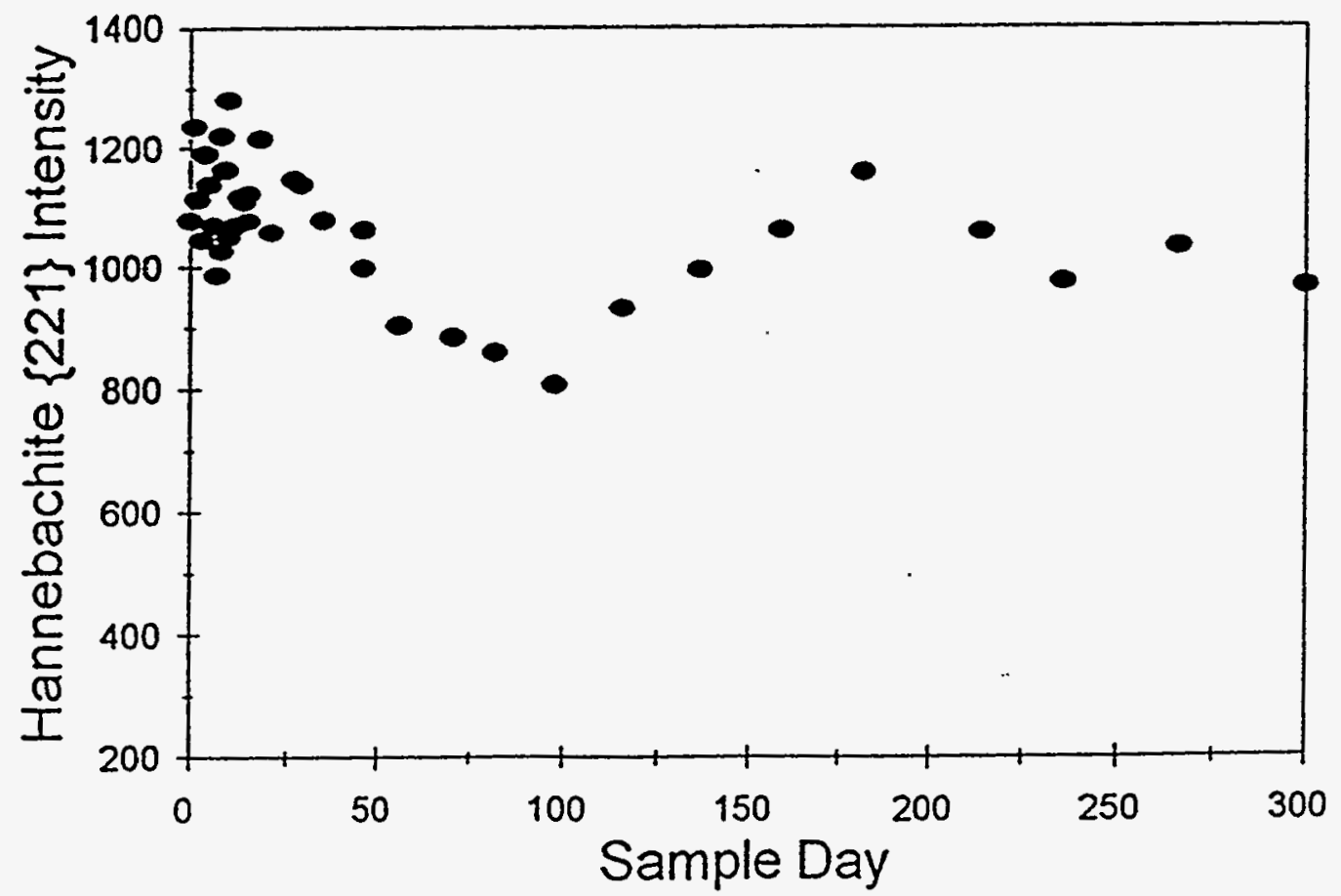

Figure 2-23. Illustration of the relative amounts of hannebachite in the kinetic study as a function of sample age. 


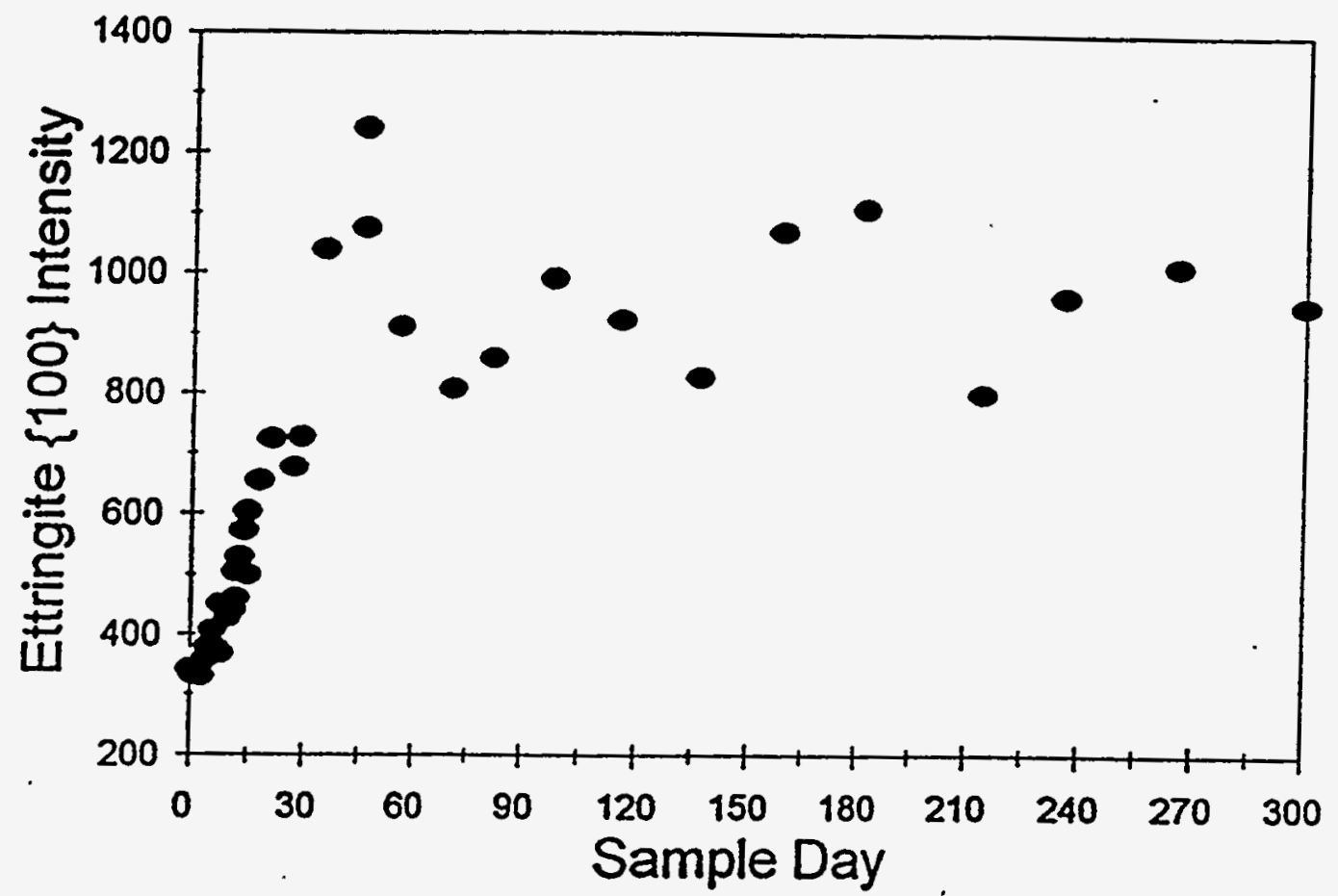

Figure 2-24. Illustration of the relative amounts of ettringite in the kinetic study as a function of sample age.

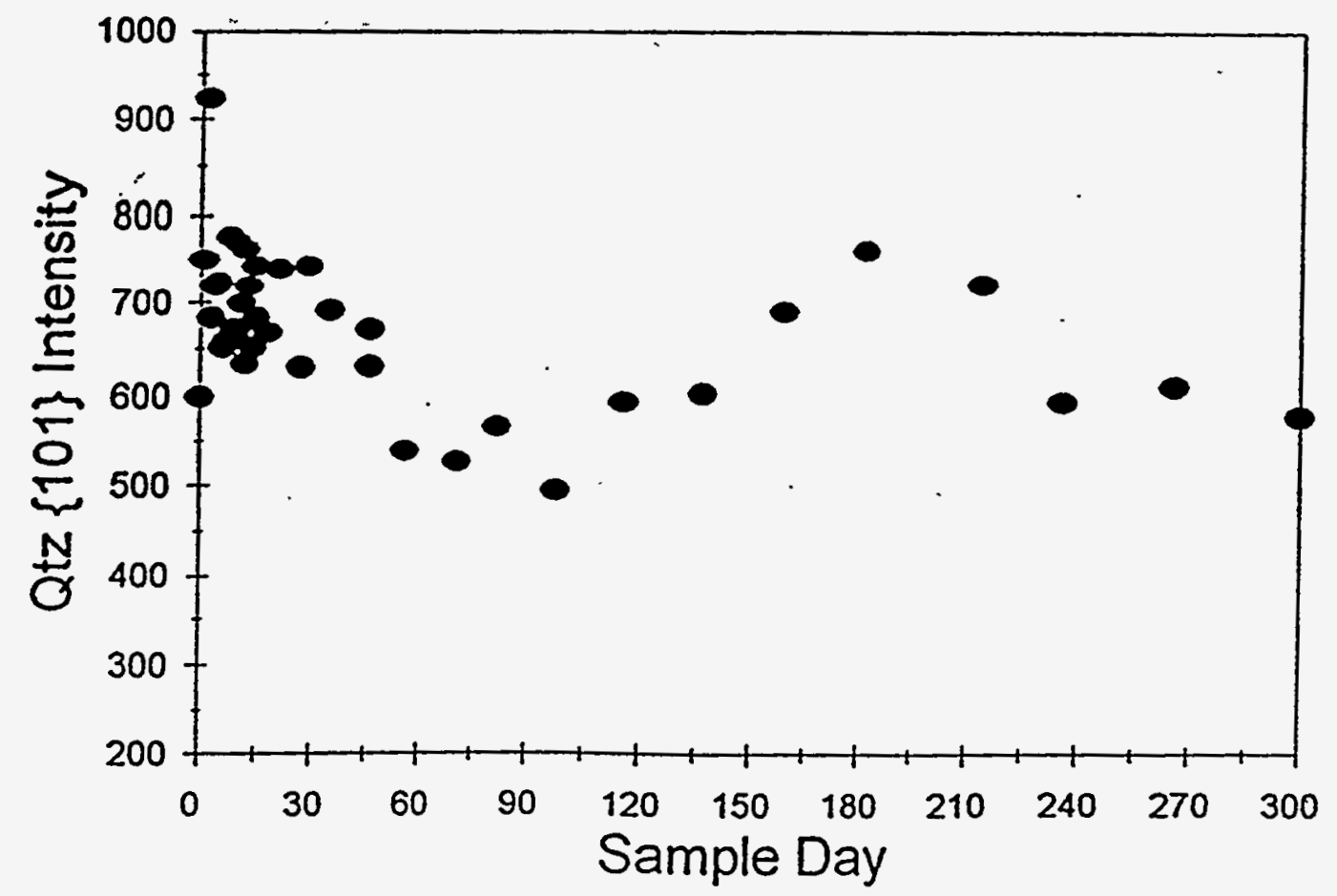

Figure 2-25. Illustration of the relative amounts of quartz in the kinetic study as a function of sample age. 
approximately 45 days; from 45 days to 300 days, the diffraction intensity fluctuated about a mean value. All of the mineral diffractions exhibited considerable variation in intensity over the course of the study, due to instrument variation and sample heterogeneity. However, a sufficient number of samples were analyzed to elucidate general trends.

Portlandite abundance decreased with aging to a minimum at approximately 45 days, as is shown in Figure 2-26. Because of overlap of the $\{101\}$ peak with that from hannebachite, the minimum intensity of about 700-750 counts mainly represents the constant abundance of hannebachite. Gypsum formed within the first day of the experiment and increased in abundance up to approximately day 10 (with considerable variation in reflection intensity) whereupon it exhibited a marked decrease and was more-or-less absent by day 15 (Figure . 2-27). Ettringite formation is the likely cause of the gypsum and portlandite decrease, which is discussed below.

The solution pH remained constant at approximately 12.5 until day 45-55, upon which it monotonically declined to about 8.5 (Figure 2-28), a value approaching that of bicarbonate buffer in equilibrium with atmospheric $\mathrm{CO}_{2}$. This suggests the occurrence of a small leak in the lid-container seal, although the system was nominally closed to the atmosphere. In fact, the surfaces of the solutions contained small amounts of calcite, although the deposits did not increase consistently over the course of the experiment, and there was negligible evaporation of the solution). However, the $\mathrm{pH}$ in the solution was possibly higher than the measured value because ettringites are reportedly unstable at a $\mathrm{pH}<11$, and the constant intensity of the ettringite XRD reflections in the solid material over time suggest that the mineral was not dissolving.

The introduction of atmospheric carbon dioxide into the system could cause portlandite dissolution via the following reactions in which portlandite is a buffer:

$$
\begin{aligned}
& \mathrm{CO}_{2}+\mathrm{OH}-=\mathrm{HCO}_{3-}^{-} \\
& \mathrm{HCO}_{3-}^{-}=\mathrm{H}^{+}+\mathrm{CO}_{3}^{2-}
\end{aligned}
$$




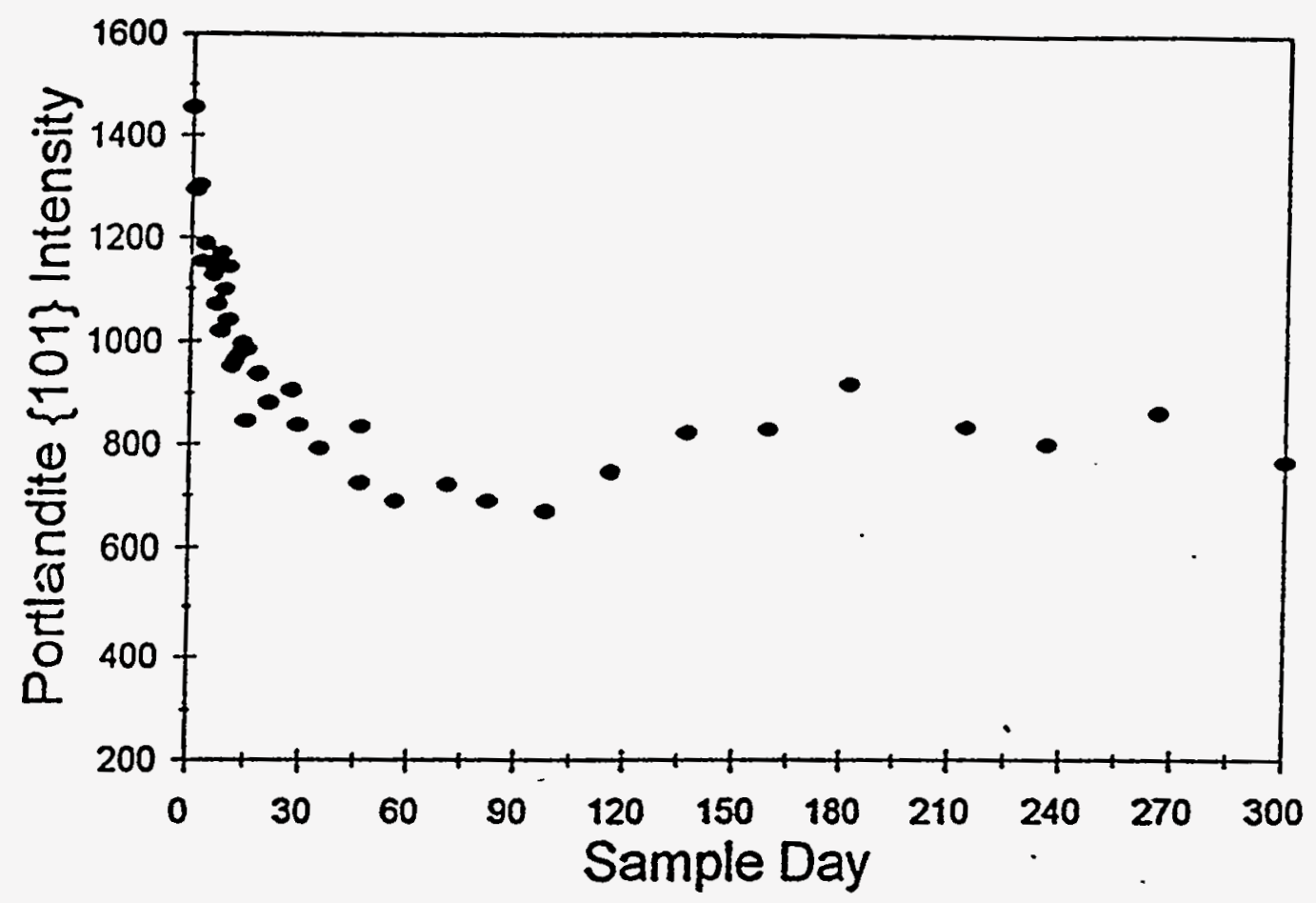

Figure 2-26. Illustration of the relative amounts of portlandite in the kinetic study as a function of sample age.

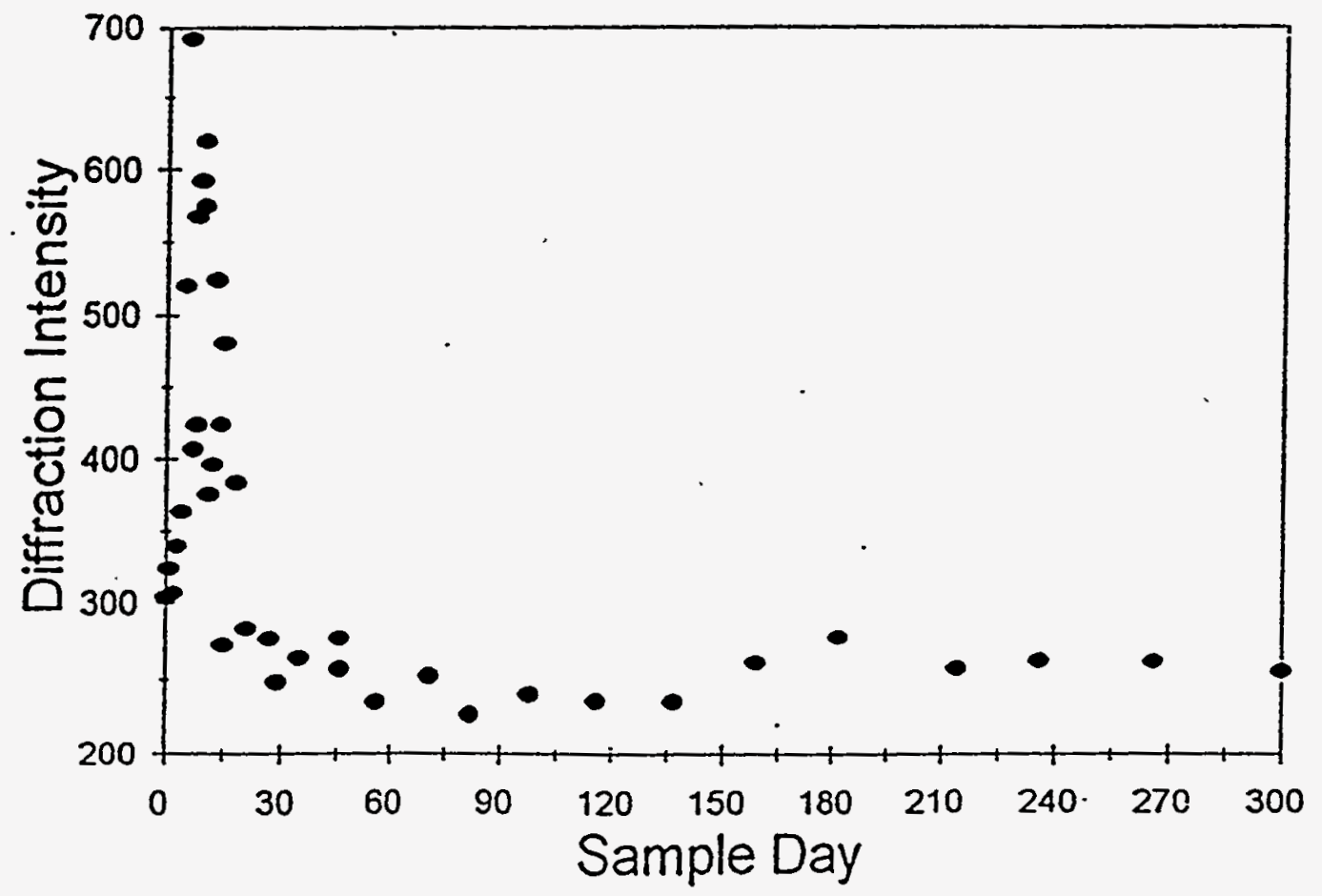

Figure 2-27. Illustration of the relative amounts of gypsum in the kinetic study as a function of sample age. 


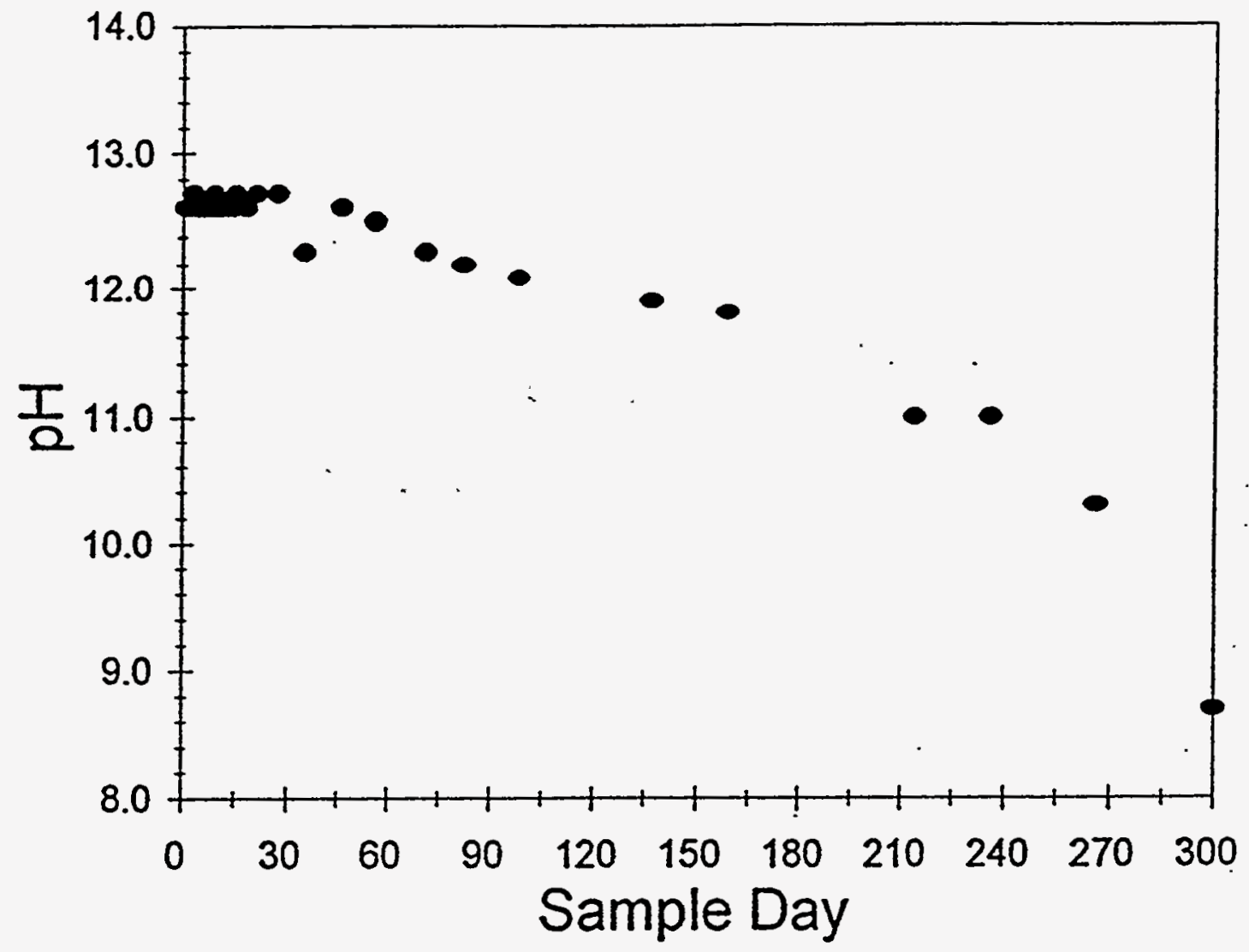

Figure 2-28. Monitoring of the solution $\mathrm{pH}$ in the Coolside samples throughout the kinetic experiments. 


$$
\begin{aligned}
& \mathrm{Ca}(\mathrm{OH})_{2}=\mathrm{CaOH}^{+}+\mathrm{OH}- \\
& \mathrm{CaOH}^{+}=\mathrm{Ca}^{2+}+\mathrm{OH}^{-}
\end{aligned}
$$

The solution $\mathrm{pH}$ would eventually decrease when all of the portlandite was depleted.

The formation of ettringite in the solid can cause dissolution of portlandite, for several reasons. Crystallization of 1 mole of ettringite, theoretically, consumes 6 moles of calcium and 3 moles of sulfate, whereas dissolution of hannebachite produces 1 mole each of calcium and sulfite ion. Assuming hannebachite to be the major source of sulfite, after gypsum-derived sulfate is depleted, then ettringite formation would cause the $\left[\mathrm{Ca}^{2+}\right] /\left[\mathrm{SO}_{3}{ }^{2-}\right]$ ratio to decrease in the solution. In order to maintain equilibrium of hannebachite with the solution, which is suggested by the XRD data, then the activity of calcium in solution needs to increase such that $\left[\mathrm{Ca}^{2+}\right]\left[\mathrm{SO}_{3}{ }^{2-}\right]=10^{-7.0}$ (the $\mathrm{KSP}$ of hannebachite). In this system, since portlandite is the most soluble calcium-bearing mineral ( $\left.\mathrm{K}_{\mathrm{SP}}=10^{-5.3}\right)$, it dissolves. Furthermore, as the glass component dissolves, aluminum is released and forms $\mathrm{Al}(\mathrm{OH})_{4}$ at the high $\mathrm{pH}$ of this solution. Ettringite is commonly observed crystallizing on the glass fly ash spheres, incorporating aluminum from the glass, and hydroxyl ions into its lattice, thereby causing portlandite to dissolve.

The formation of ettringite is, therefore, likely to be the primary cause of the portlandite dissolution, mainly in the first $\mathbf{4 5}$ days, with ancillary effects from calcite formation. It is predicted that if, under these conditions, the experiment had continued over a prolonged period, portlandite would be depleted, the $\mathrm{pH}$ would decrease to approximately 8.0 , and ettringite would dissolve incongruently (precipitating a non-crystalline, aluminum-rich gel). If the system were kept completely sealed from the atmosphere, then portlandite and ettringite, in addition to the other crystalline phases, would coexist indefinitely within the material.

\section{Series IV: Pelletization of FBC Material}


The main objective of this part of the Coolside project was to investigate the importance of different mineral components and their relative abundances in the pellet precursor materials (FBC ash versus spray dryer ash) to understand the contributions of mineralogical transformations upon hydration and aging on the overall pellet stability.

The FBC ash (Wormser) that was utilized in the preparation of pellets was $\mathrm{X}$-rayed after receiving the raw mixture. The $X R D$ results indicate the presence of portlandite, slow reacting anhydrite, and quartz, as well as amorphous $\mathrm{SiO}_{2}$, small amounts of gypsum (possibly formed from a reactive anhydrite within the raw mixture through contact with surface moisture), and small amounts of mullite.

The spray dryer ash has very similar mineral constituents compared to the FBC ash described above, however, the anhydrite fraction present consists predominantly of reactive anhydrite which readily reacts with moisture to form gypsum.

The data obtained during the entire reporting period supports the conjecture that detrimental swell associated with continued pellet aging upon exposure to humidified air or upon exposure to water is principally a function of calcium sulfate hydration reactions. The main difference between the FBC pellets and the spray dryer pellets produced by CONSOL, are summarized hereof:

Anhydrite crystals in the FBC pellets, upon exposure to moisture, undergo a dissolutionreprecipitation reaction that results in the nucleation and growth of gypsum crystals. The reaction is extremely delayed (slow reaction kinetics) since the anhydrite crystals were produced under high temperature conditions such as occurring in a fluidized bed reactor (the expression "dead burned" anhydrite may be used to describe the degree of crystallinity of the anhydrite crystals formed at elevated temperatures in an FBC unit). The mineralogical transformation is delayed because of the dense crystal structure of the anhydrite grains causing early formed cementitious bonds to rupture, and, overall, leads to poor pellet strength. The performance of the FBC pellets under weathering conditions 
(reported in laboratory and field testing by Dr. Milton Wu at Consol) was unsatisfactory and, based on this study, was attributed to the slow mineralogical transformation from anhydrite to gypsum which takes place long after initial solidification of the material. The transformation, which is associated with a 60 percent volume increase, causes detrimental crack and fracture formation in the pelletized FBC materials. In contrast, spray dryer materials are lacking "dead burned" anhydrite crystals. Therefore, the anhydrite to gypsum transformation in the spray dryer pellets occurs prior to the solidification of the material and, hence, avoids the detrimental swell.

The mineralogic analyses of the two different kinds of ash, $\mathrm{FBC}$ ash versus spray dryer ash, used in the pelletization study illustrate the differences in the mineralogical makeup of the pellet precursor materials. Chemical characterization of the $\mathrm{FBC}$ pellets indicates that the main mineral components are anhydrite $\left(\mathrm{CaSO}_{4}\right)$, portlandite $\left(\mathrm{Ca}(\mathrm{OH})_{2}\right)$, free lime $(\mathrm{CaO})$, minor calcium carbonate $\left(\mathrm{CaCO}_{3}\right)$, minor mullite $\left(\mathrm{Al}_{6} \mathrm{Si}_{2} \mathrm{O}_{13}\right)$ and, of course, quartz. During early curing the anhydrite did not hydrate to spontaneously form gypsum but rather resulted in the early hydration products portlandite and ettringite as evidenced by XRD and SEM investigations of the materials. Although ettringite needles form early bonds between mineral grains, subsequent hydration of anhydrite leads to extremely large gypsum crystals that form in voids and fractures, causing the complete disrupture of mineral bonds after 160 days curing period.

In the spry dryer material, the main mineral components after hydration are: hannebachite, ettringite, and calcite, with only minor amounts of gypsum. Mullite, rutile and quartz are present in both the starting and hydrated spray dryer pellets. The XRD peaks for the spray dryer material are typically indicative of the presence of a substantial amount of glassy phase. The glass diffraction "ramp" is centered approximately at $29-302$ theta and is relatively broad, indicative of a high $\mathrm{CaO}$ component within the glassy phase. Figure 4 compares the XRD results obtained for the spray dryer ash (SDA-F) with the pelletized material (SDA-P). It is evident from the XRD data that hannebachite $\left(\mathrm{CaSO}_{3} 0.5 \mathrm{H}_{2} \mathrm{O}\right)$ is the most important mineral participating in the "spray dryer ash" transformation reactions. 
The aged spray dryer pellets have abundant hannebachite present as well as ettringite needles, both of which contribute to mineral bonding and early strength gain of the materials. In contrast, gypsum crystals which comprise the bulk of newly formed minerals in the FBC pellets, are only a minor component in the spray dryer pellets, suggesting, that gypsum crystals easily reprecipitate in the $\mathrm{FBC}$ material from the $\mathrm{Ca}$ and $\mathrm{SO}_{4}$ supersaturated pore waters, while in the spray dryer materials oxidation of $\mathrm{SO}_{3}$ to $\mathrm{SO}_{4}$ is the rate limiting step in the reaction forming gypsum. Based on the results of this study it appears that ettringite crystals, which also require the sulfate ion, are more readily formed in an $\mathrm{SO}_{4}$ starved environment compared to gypsum crystals. One of the prerequisites for the ettringite crystals to form is the presence of aluminum ions, which, in the pore solutions of the spray dryer pellets are most likely supplied by the mullite phase available in the starting material.

In summary the present results suggest that the spray dryer ash constitutes a better precursor material for pelletization work than FBC ash. The results are based on XRD and SEM observations that indicate a lesser effect of mineral transformations and swell causing mineral growth in the spray dryer pellets compared to that of the FBC ash pellets. 


\section{CHAPTER 3: THE PHYSICAL AND GEOTECHNICAL CHARACTERISTICS OF THE COOLSIDE MATERIALS}

The Characteristics of the Coolside Materials as Emplaced in the Field

\section{Geotechnical Characteristics of the Materials As Emplaced}

Density and Moisture Content. Coolside material was placed in Lysimeter 1 with no compactive effort. The material was mixed to a target moisture content of $37 \%$ before placement. No target density was established because the material was placed loosely. The average dry density and moisture content of the 16 loosely placed lifts were $706 \mathrm{~kg} / \mathrm{m}^{3} .\left(44.1 \mathrm{lbs} / \mathrm{ft}^{3}\right)$ and $37.5 \%$, respectively. Average lift thickness was approximately $13.5 \mathrm{~cm}$ (5.3 in). Values of dry density and optimum moisture obtained for each lift are shown in Figures 3-1 and 3-2, respectively.

Coolside materials placed in the second lysimeter ( $L-2)$ were compacted to target dry density and moisture content values of $794 \mathrm{~kg} / \mathrm{m}^{3}\left(49.6 \mathrm{lbs} / \mathrm{ft}^{3}\right)$ and $41.2 \%$, respectively. These values were obtained from moisture-density tests designed to simulate the compactive efforts of a D9 bulldozer. This test was performed by applying a static pressure of $117.3 \mathrm{kPa}$ ( 17 psi--the stress exerted by the track of a D9 bulldozer) to the Coolside by-product, which was placed in one lift in a standard 10.16$\mathrm{cm}$ (4-inch) diameter Proctor mold. The material was then compacted at various moisture contents. The pressure was applied until the maximum load was reached. It was then held for 1 minute and released. The moisture-density curve was similar to those established in standard moisture-density tests. Field compaction was performed with a hand-held plate compactor. The dry density and moisture content obtained for 17 lifts averaged $788 \mathrm{~kg} / \mathrm{m}^{3}\left(49.2 \mathrm{lbs} / \mathrm{ft}^{3}\right)$ and $38.9 \%$, respectively. The average lift thickness was about $12.4 \mathrm{~cm}$ (4.9 in). Values of dry density and moisture content obtained for each lift of Lysimeter 2 are shown in Figures 3-3 and 3-4, respectivley. 


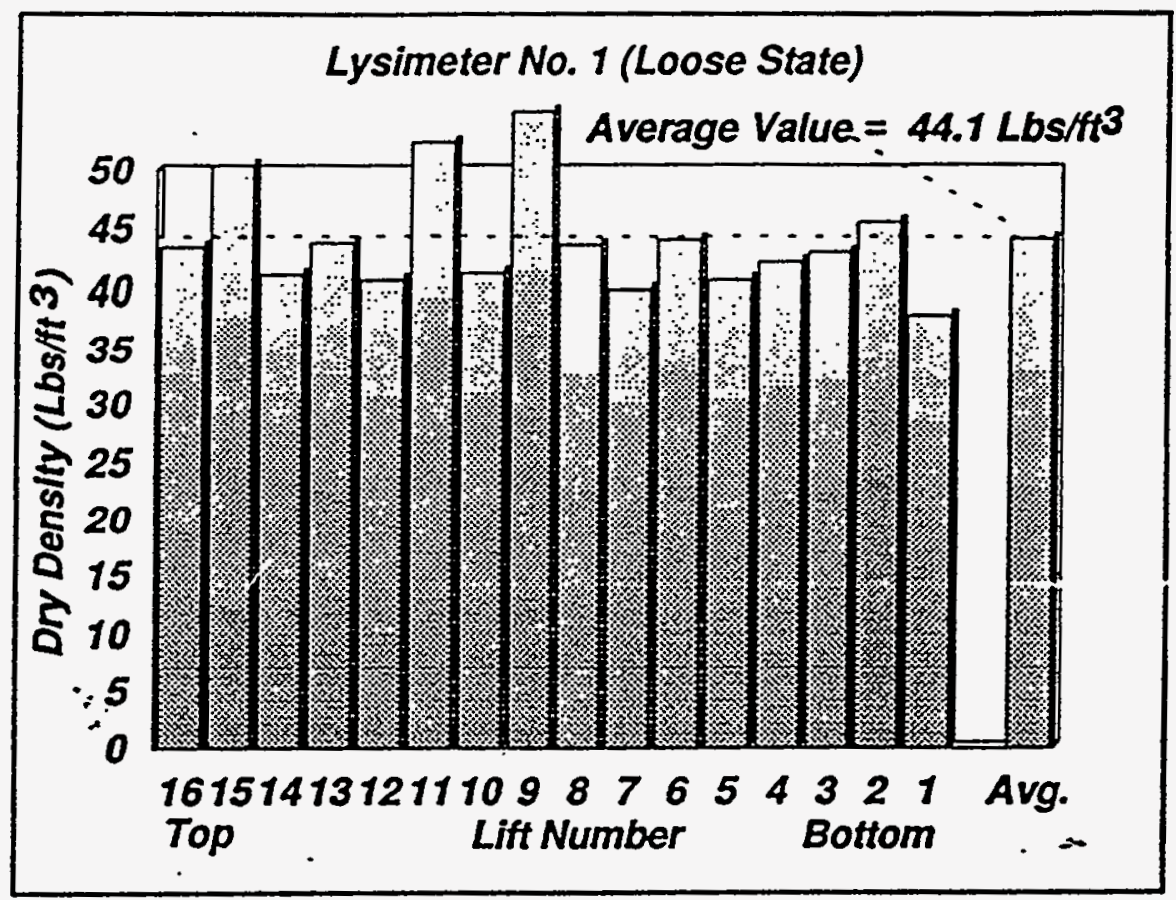

Figure 3-1. Dry densities measurements of each lift in Lysimeter 1

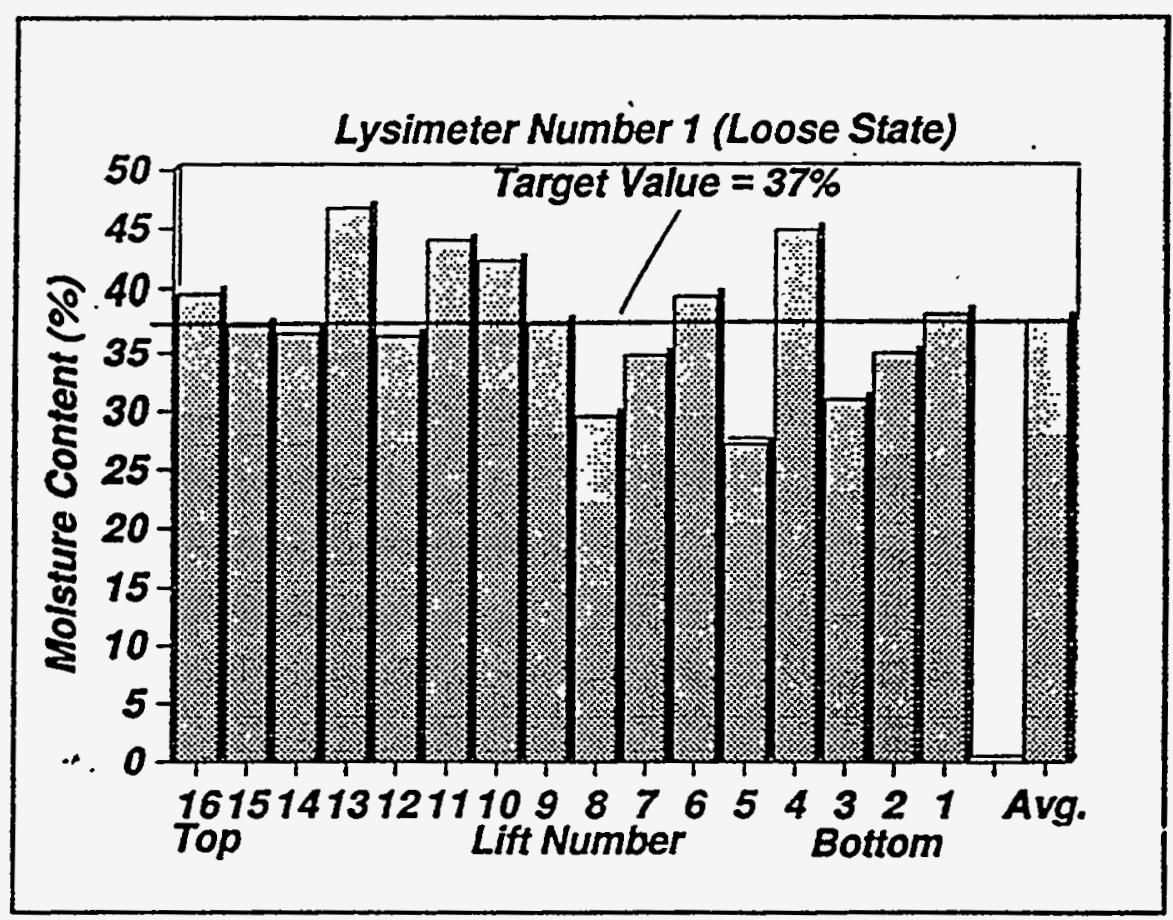

Figure 3-2. Moisture contents of each lift of Lysimeter 1 


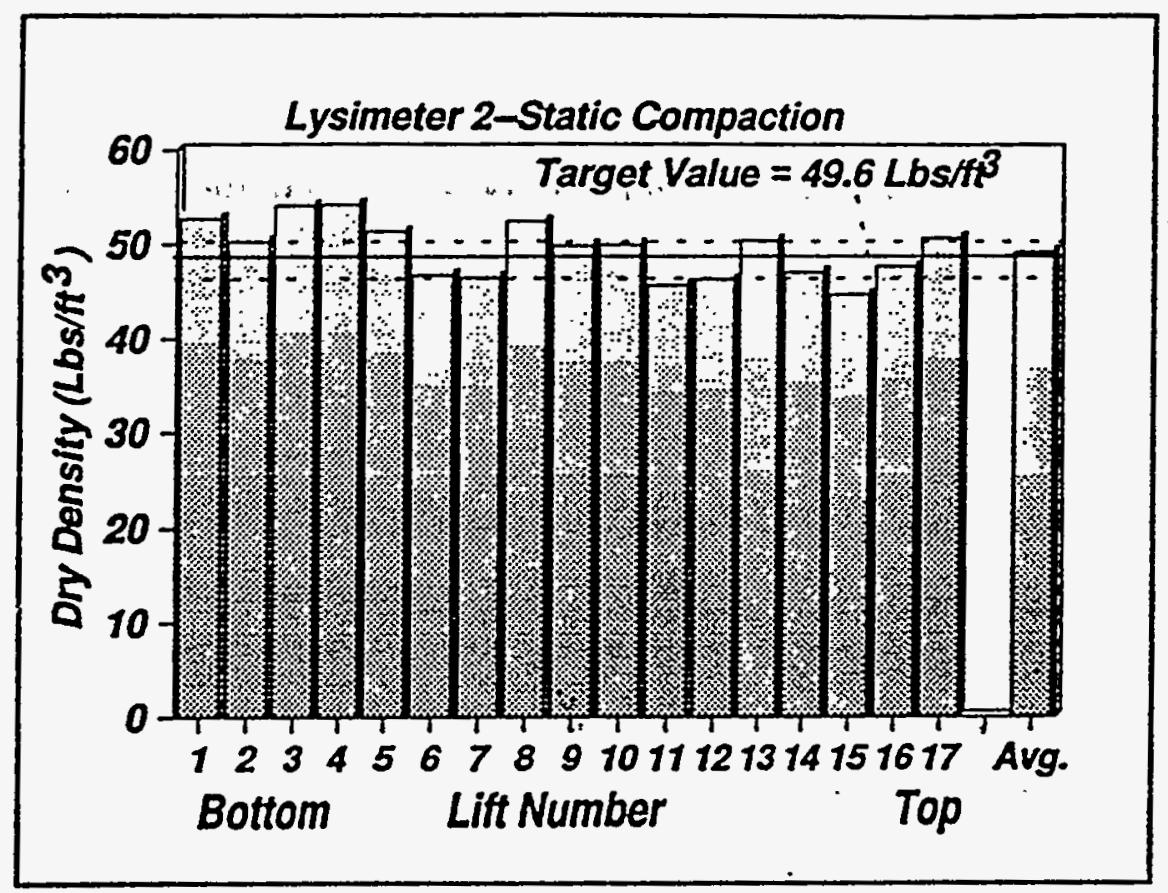

Figure 3-3. Dry density measurements of each lift of Lysimeter 2

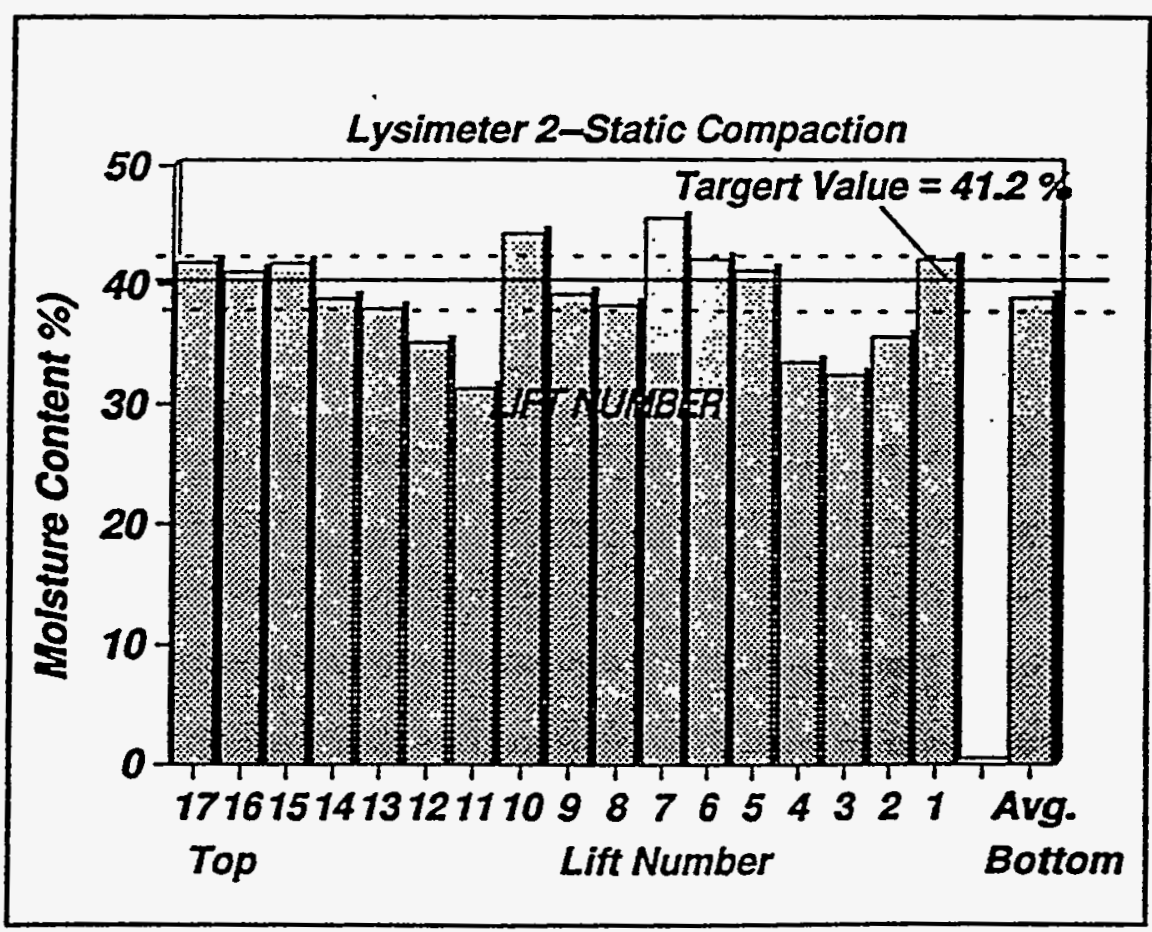

Figure 3-4. Moisture content of each lift of Lysimeter 2 
The third lysimeter ( $L-3$ ) was filled with Coolside material compacted near $95 \%$ of standard maximum dry density and optimum moisture content. Target values for density and moisture were $1065 \mathrm{~kg} / \mathrm{m}^{3}$ (66.5 lbs/ $/ \mathrm{ft}^{3}--95$ percent of standard compaction) and $37.0 \%$, respectively. Maximum dry density and optimum moisture content obtained from standard moisture-density tests were $1121 \mathrm{~kg} / \mathrm{m}^{3}\left(70.0 \mathrm{lbs} / \mathrm{ft}^{3}\right)$ and 36.5 $\%$, respectively. Dry densities and moisture contents obtained for each lift of Lysimeter 3 are compared in Figures 3-5 and 3-6, respectively. The material was compacted with hand-held gasoline-powered compactors. Vibratory plate and static (jumping jack) compactors were used. Target density was easily obtained with the static compactor; therefore, it was used more frequently. Average values of dry density and moisture content obtained for 23 lifts of compacted Coolside material were $1060 \mathrm{~kg} / \mathrm{m}^{3}(66.2$ $\mathrm{lbs} / \mathrm{ft}^{3}$ ) and $37.0 \%$, respectively. Dry density and moisture content measurements of each lift are shown in Figures 3-5 and 3-6, respectively. Moisture content exceeded optimum moisture content by more than $2 \%$ in a few lifts. Liquefaction occurred in these few lifts because of the excess moisture and the silt-sized particles.

A commercial fly ash was placed in the fourth lysimeter ( $L-4)$. Fly ash was used as a control material for leachate monitoring. The fly ash was purchased and transported to the field site in seventy pound bags. Approximately $803 \mathrm{~kg}$ (1750 lbs--25 bags) of material was used for each lift. The material was lightly compacted with a hand compactor near $90 \%$ of standard maximum dry density, $2 \%$ below optimum moisture content. Maximum dry density and a moisture content that was about optimum moisture content for the fly ash were $1374 \mathrm{~kg} / \mathrm{m}^{3}\left(85.8 \mathrm{lbs} / \mathrm{ft}^{3}\right)$ and $22 \%$. Target density and moisture content were $1236 \mathrm{~kg} / \mathrm{m}^{3}\left(77.2 \mathrm{lbs} / \mathrm{ft}^{3}-\right.$-(90\% of standard density) and $20 \%$ percent moisture, as shown in Figure 3-7. The average density and moisture content values obtained for nineteen lifts were $76.4 \mathrm{lbs} / \mathrm{ft}^{3}$ and $20.5 \%$, respectively. Dry density and moisture content of each lift are shown in Figures 3-8 and 3-9, respectively. Target and actual values of dry density and moisture content obtained for each 


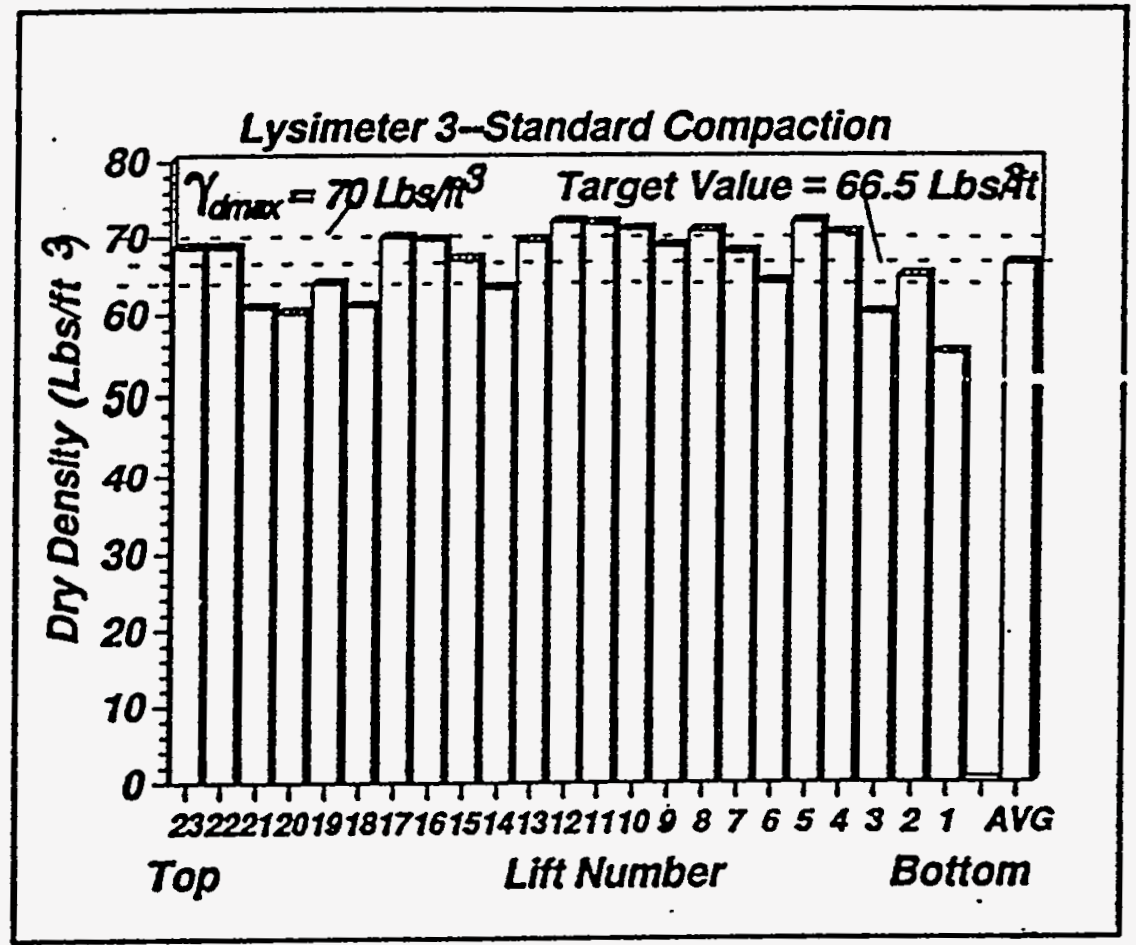

Figure 3-5. Dry density measurements of each lift of Lysimeter 3

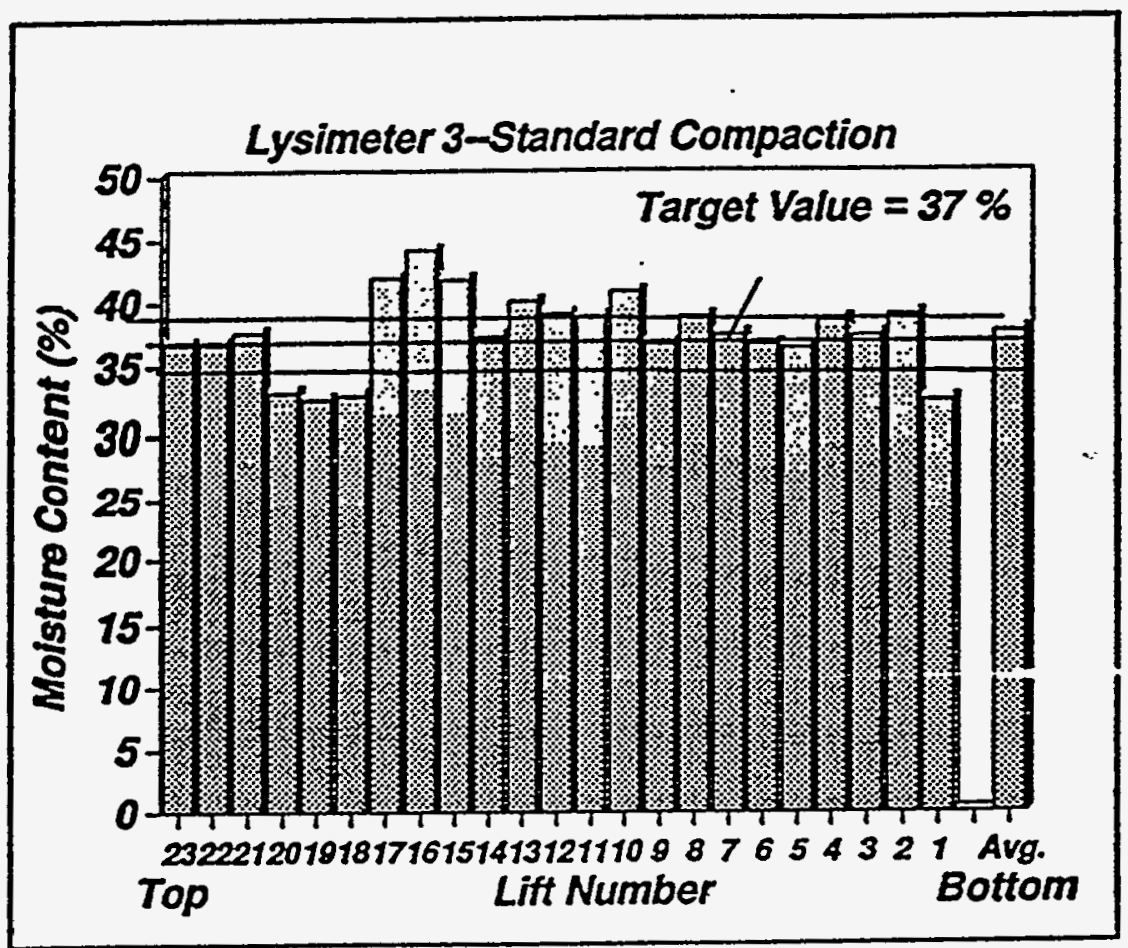

Figure 3-6. Moisture content of each lift of Lysimeter 3 


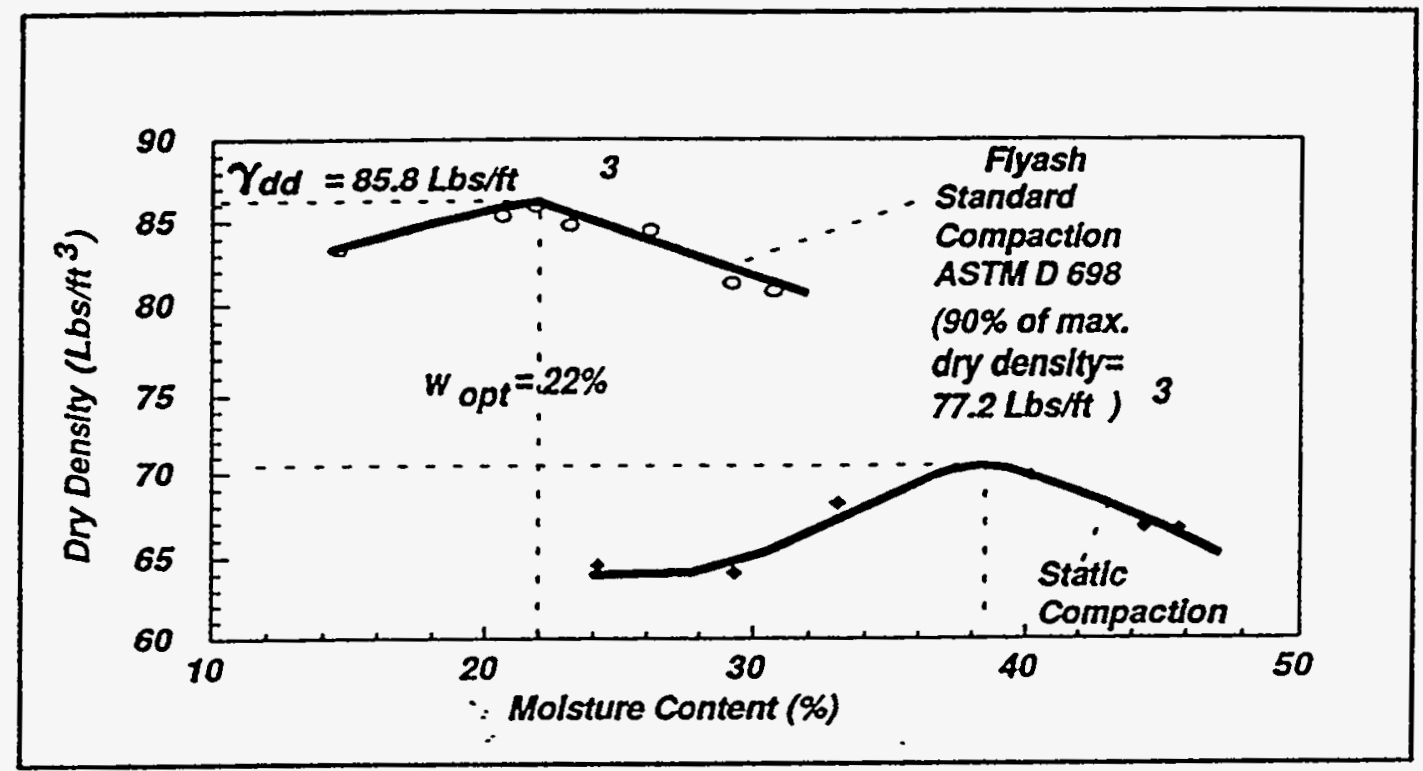

Figure 3-7. Dry density-moisture content relationships for the fly.ash used in Lysimeter 4

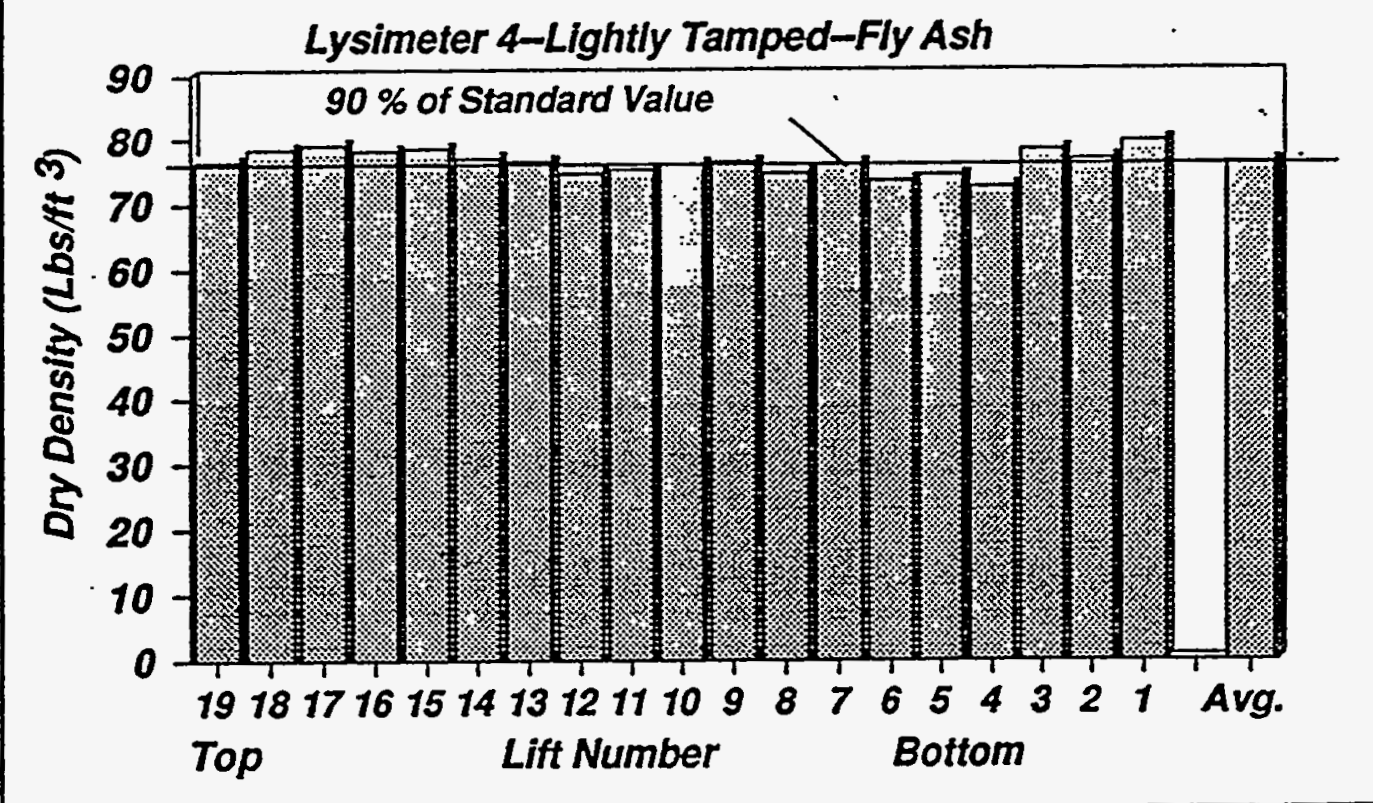

Figure 3-8. Dry density measurements of each lift of Lysimeter 4 


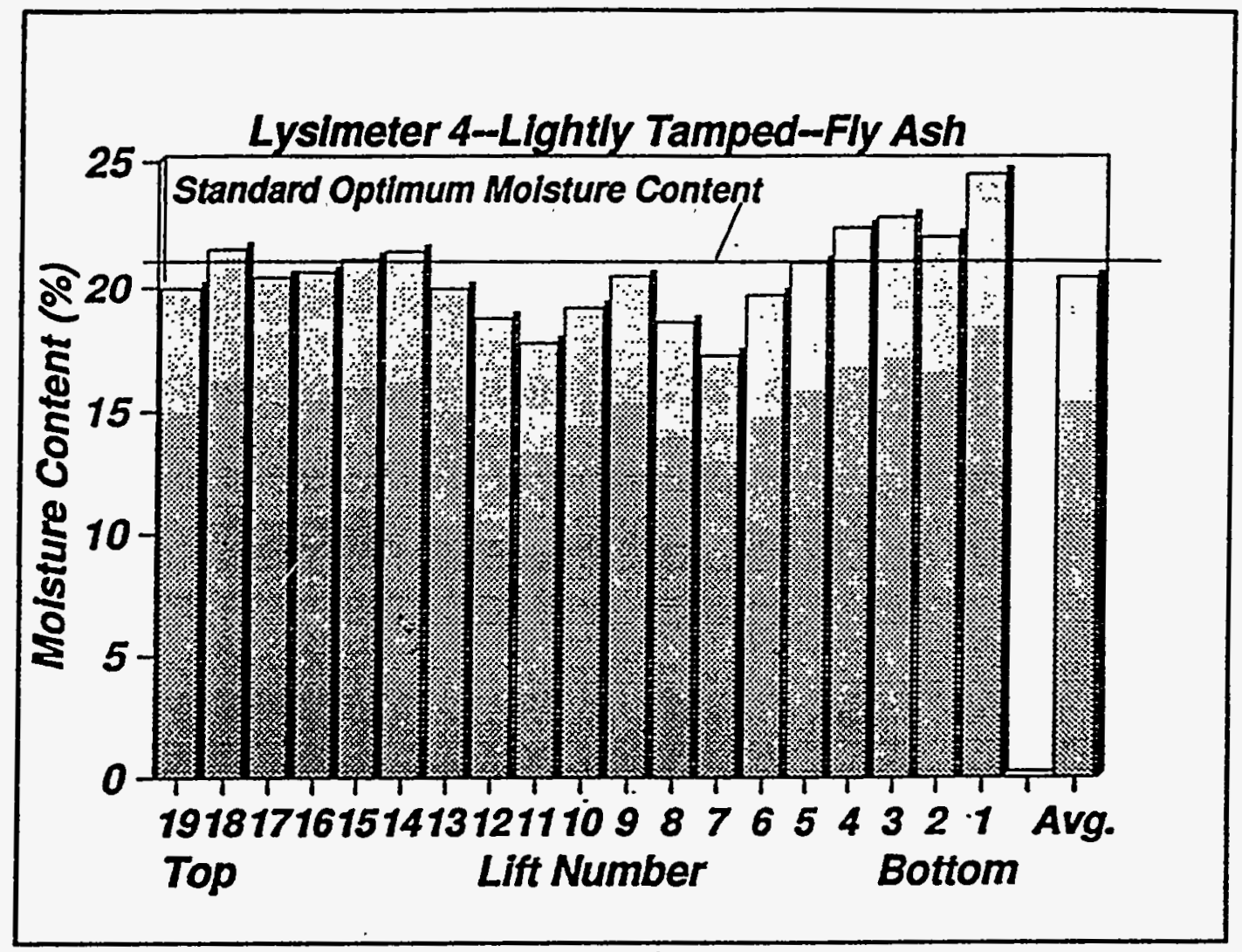

Figure 3-9. Moisture contents of each lift of Lysimeter 4

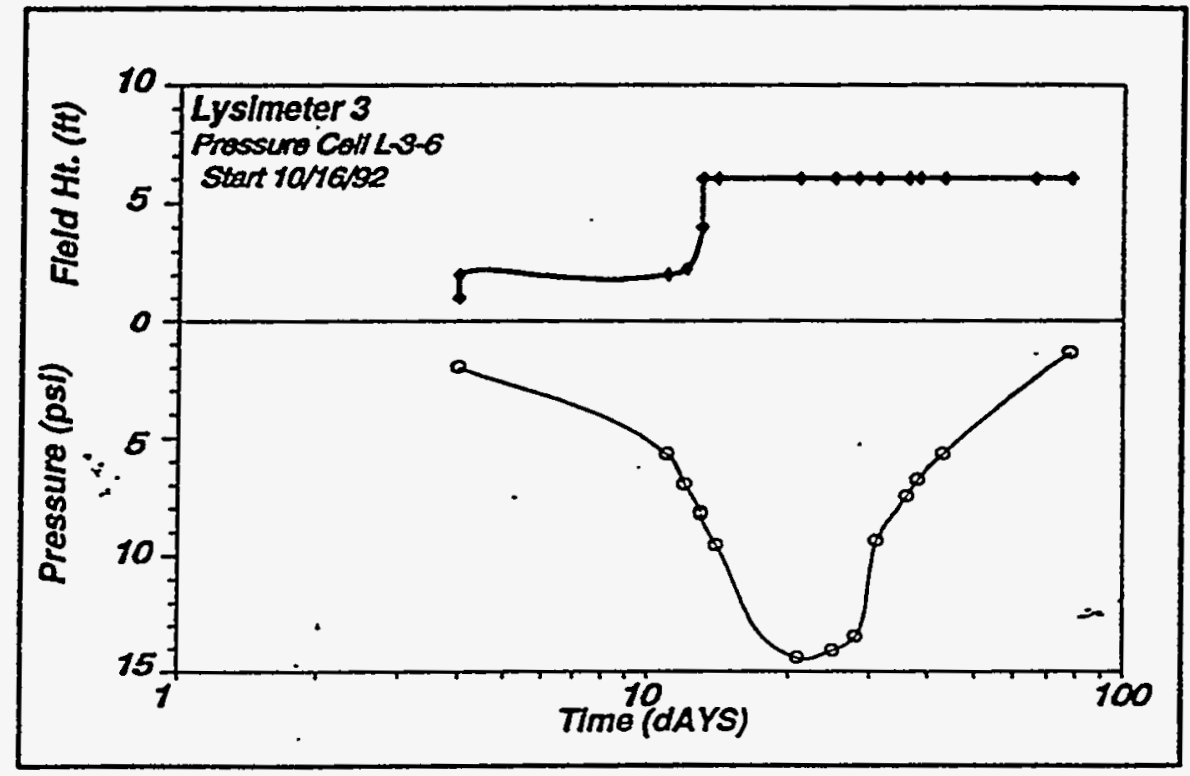

Figure 3-10. Observed side pressures in Lysimeter 3 during loading and chemical reactions 
lysimeter are compared in Table 3-1.

Table 3-1. Summary of Dry Density and Moisture Content Measurements.

\begin{tabular}{|l|l|l|l|l|l|}
\hline Lysimeter & $\begin{array}{l}\text { Average } \\
\text { Dry } \\
\text { Density } \\
\left(\text { lbs/ } / \mathrm{ft}^{3}\right)\end{array}$ & $\begin{array}{l}\text { Target Dry } \\
\text { Density } \\
\left(\mathrm{lbs} / \mathrm{ft}^{3}\right)\end{array}$ & $\begin{array}{l}\text { Average } \\
\text { Moisture } \\
\text { Content } \\
(\%)\end{array}$ & $\begin{array}{l}\text { Target } \\
\text { Moisture } \\
\text { Content } \\
(\%)\end{array}$ & $\begin{array}{l}\text { Number of } \\
\text { Lifts }\end{array}$ \\
\hline 1 & 44.1 & $\mathrm{~N} / \mathrm{A}$ & 37.5 & 37.0 & 16 \\
\hline 2 & 49.2 & 49.6 & 38.9 & 41.2 & 17 \\
\hline 3 & 66.2 & 66.5 & 37.0 & 37.0 & 23 \\
\hline 4 & 76.4 & 77.2 & 20.5 & 20.0 & 19 \\
\hline
\end{tabular}

Pressure, Temperature, and Swell Measurements. Pressure at a depth of 6 feet in Lysimeter 3 increased slightly as fill was placed. A maximum pressure of $103.4 \mathrm{kPa}$ (15 psi) was reached a few days after filling operations were completed. Variation of the pressure on the side wall, during loading and shortly after completion of loading, in Lysimeter 3 with increasing time is shown in Figure 3-10. The pressure eventually returned near to zero. Pressure at 4 feet increased to about $103.4 \mathrm{kPa}$ ( $15 \mathrm{psi}$ ) and returned near to zero (Figure 3-11). A pressure increase in the lysimeter containing fly ash was less than $13.78 \mathrm{kPa}(2 \mathrm{psi}$ ), as shown in Figure $3-12$. In the three lysimeters containing Coolside materials, the moisture accumulated near the top of the materials, initially, and gradually moved downward in the materials with increasing time. Moisture from precipitation in Lysimeter 4 initially increased at the bottom of the fly ash and gradually increased upward to the top of the material. 


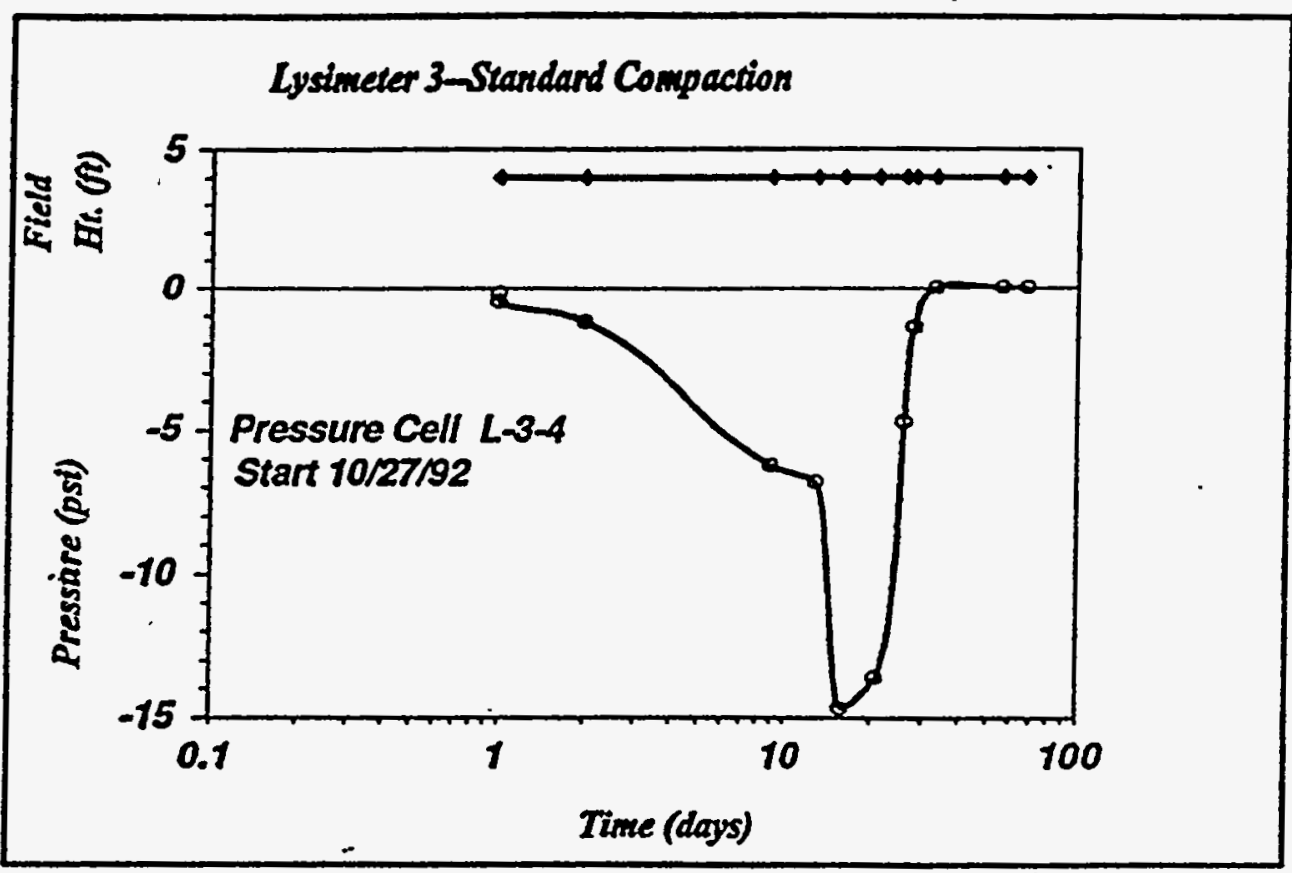

Figure 3-11. Observed side-wall pressure at a four foot depth in Lysimeter 3

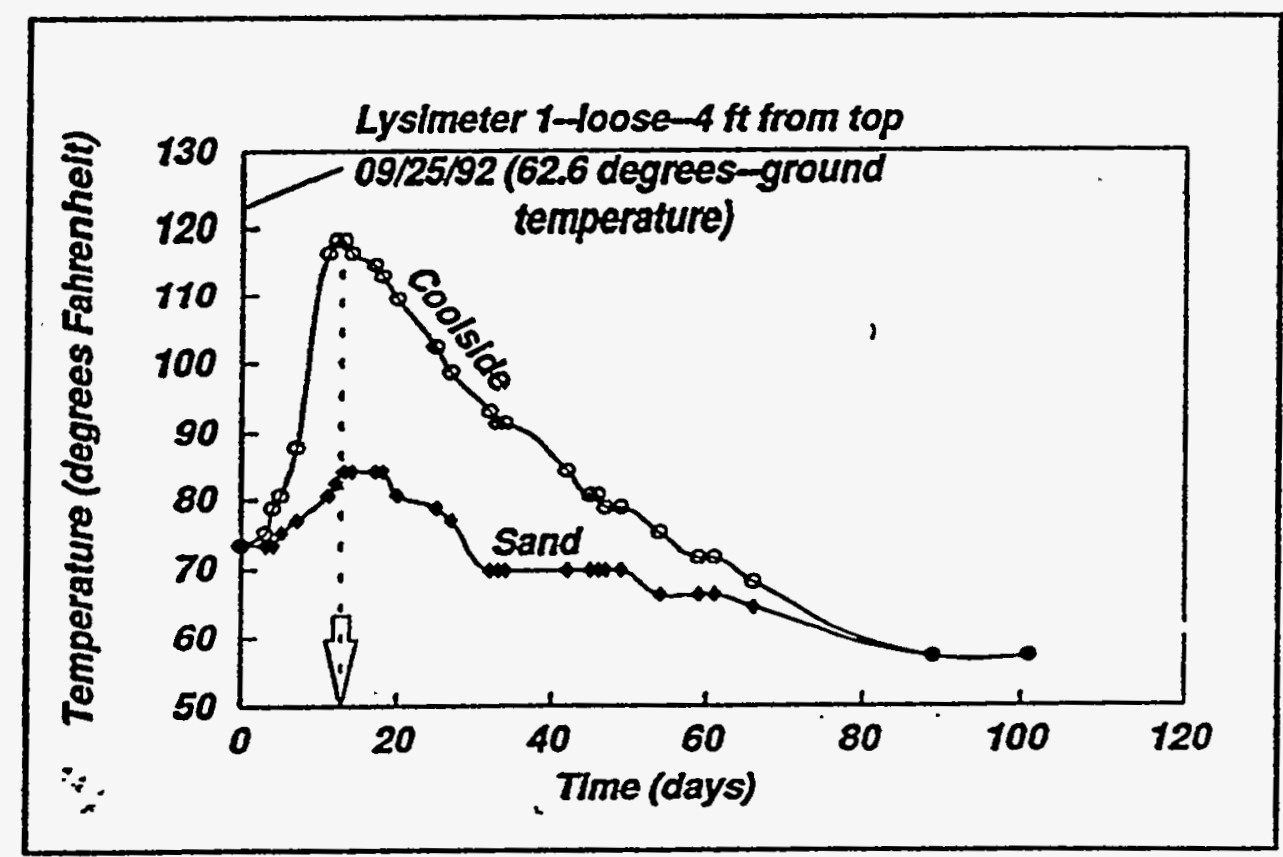

Figure 3-12. Temperatures observed in Lysimeter 1 during the filling operation 
Lysimeters containing Coolside materials increased in temperature, as shown in Figure 3-12, and reached a maximum value of $48.9^{\circ} \mathrm{C}$ (120 degrees Fahrenheit) about two weeks after placement of the Coolside residue. Subsequently, the temperature decreased. Thermocouples installed in the lysimeter containing fly ash showed no increase in temperature (see Figure 3-13). Temperatures at the interface between the fly ash and bottom of the soil layer and the interface between the bottom of the fly ash and sand during the first two months after filling are shown in Figure 3-14. At both interfaces, the temperatures decrease and appear to reach some constant values. During the 3.5-year study period, no significant swell (or consolidation) occurred in the Coolside or fly ash materials.

Collection and Testing of Specimens. Immediately after the Coolside ash had been compacted in Lysimeter 3 , thin-walled tube samples were collected. The Coolside ash in this lysimeter had been compacted to $95 \%$ of standard density. Unconfined compressive strength tests were performed on these specimens. The specimens were allowed to age for various times before testing. The strengths of the field specimens are compared in Figure 3-15 to laboratory strengths of specimens remolded near $95 \%$ of standard maximum dry density. Strength tests on lysimeter samples were performed after permeability tests were conducted. Unconfined strength of the lysimeter specimens ranged from $8.27 \mathrm{kPa}$ (1.2 psi) at a 1-day aging time to approximately $1295.51 \mathrm{kPa}$ (188 psi) at a 7-day aging time. As seen in Figure 3-15, the unconfined compressive strengths of the field lysimeter samples correlated well with unconfined compressive strengths of the laboratory specimens.

Permeability tests were performed on selected samples obtained from Lysimeters 3 and 4. The coefficients of permeability ( $k$ ) for the lysimeter samples are shown as a function of void ratio (the volume of voids divided by the volume of solids) and compared to permeability coefficients of remolded laboratory samples in Figure 3-16. The coefficient of permeability of the Coolside materials increases as the void ratio 


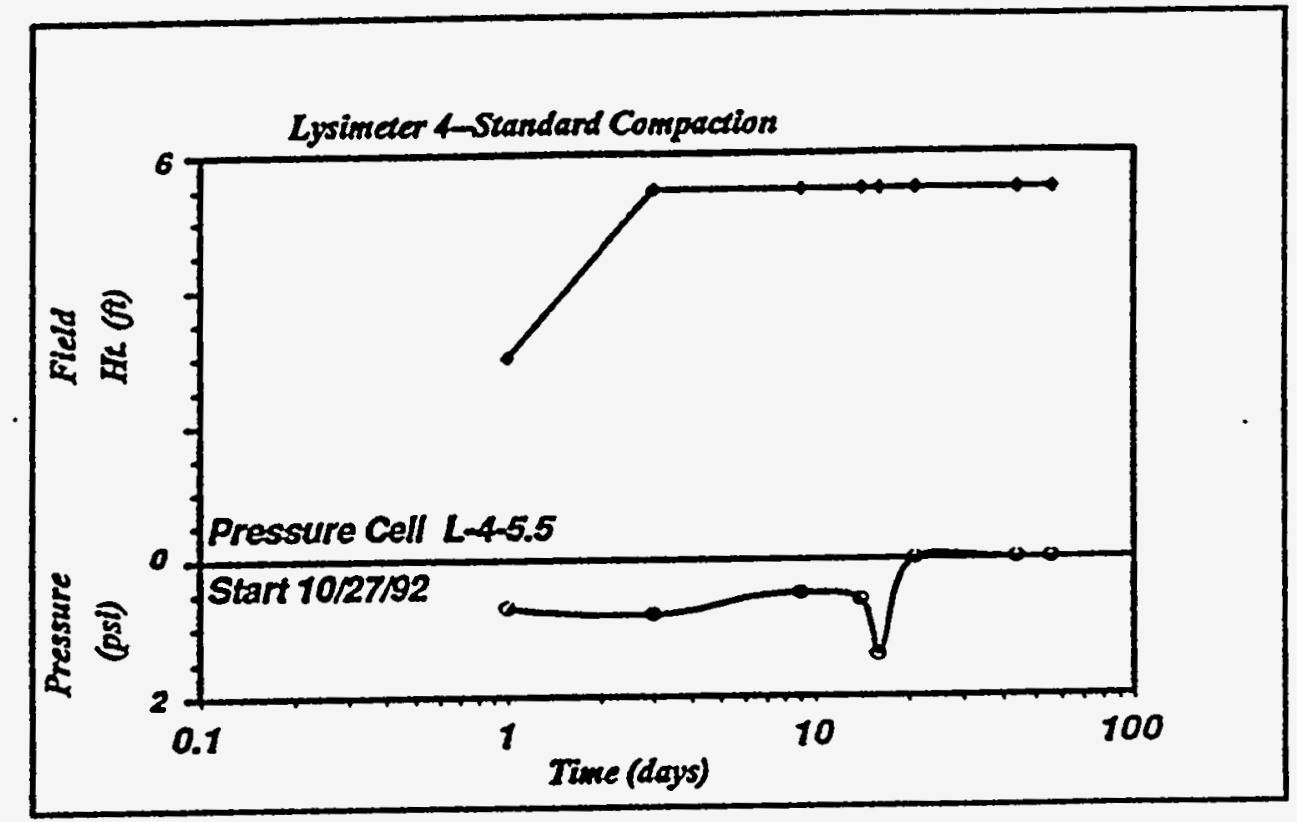

Figure 3-13. Observed side-wall pressure in Lysimeter 4

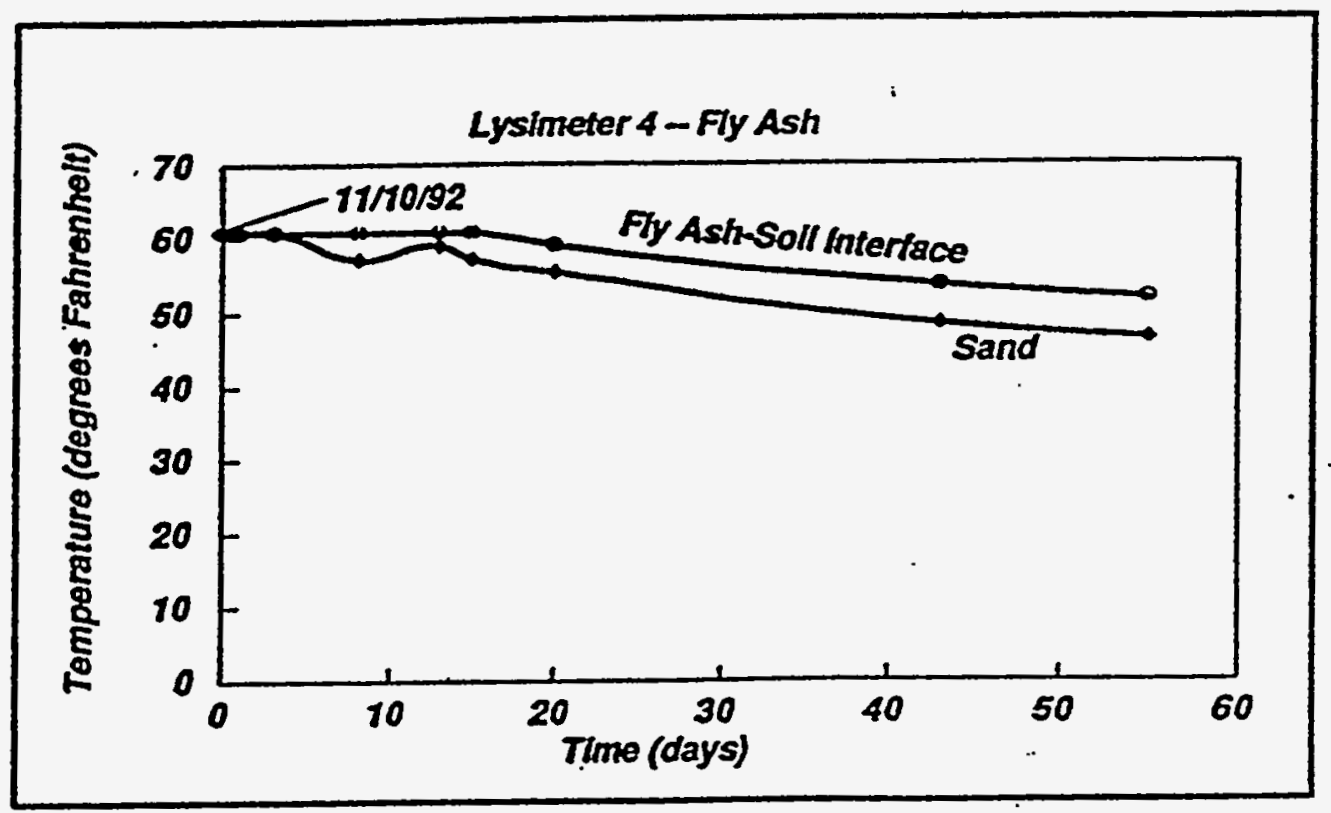

Figure 3-14. Observed temperatures at fly ash-soil and fly ash-sand interfaces 


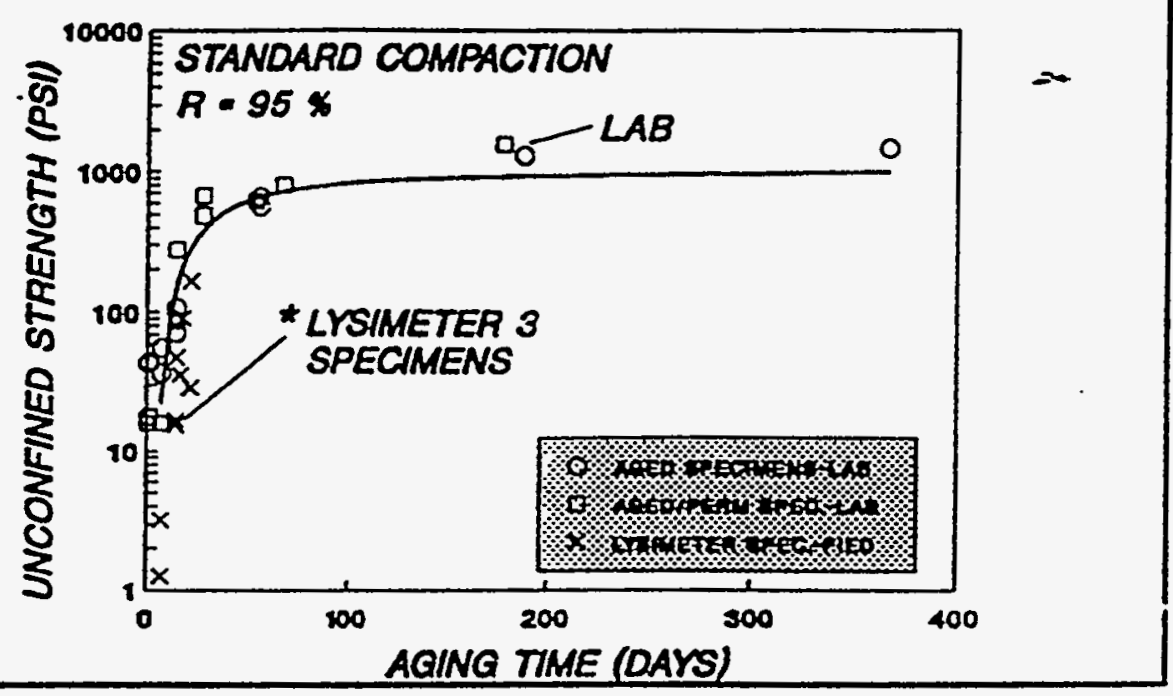

Figure 3-15. Unconfined.compressive strength of laboratory. and lysimeter samples

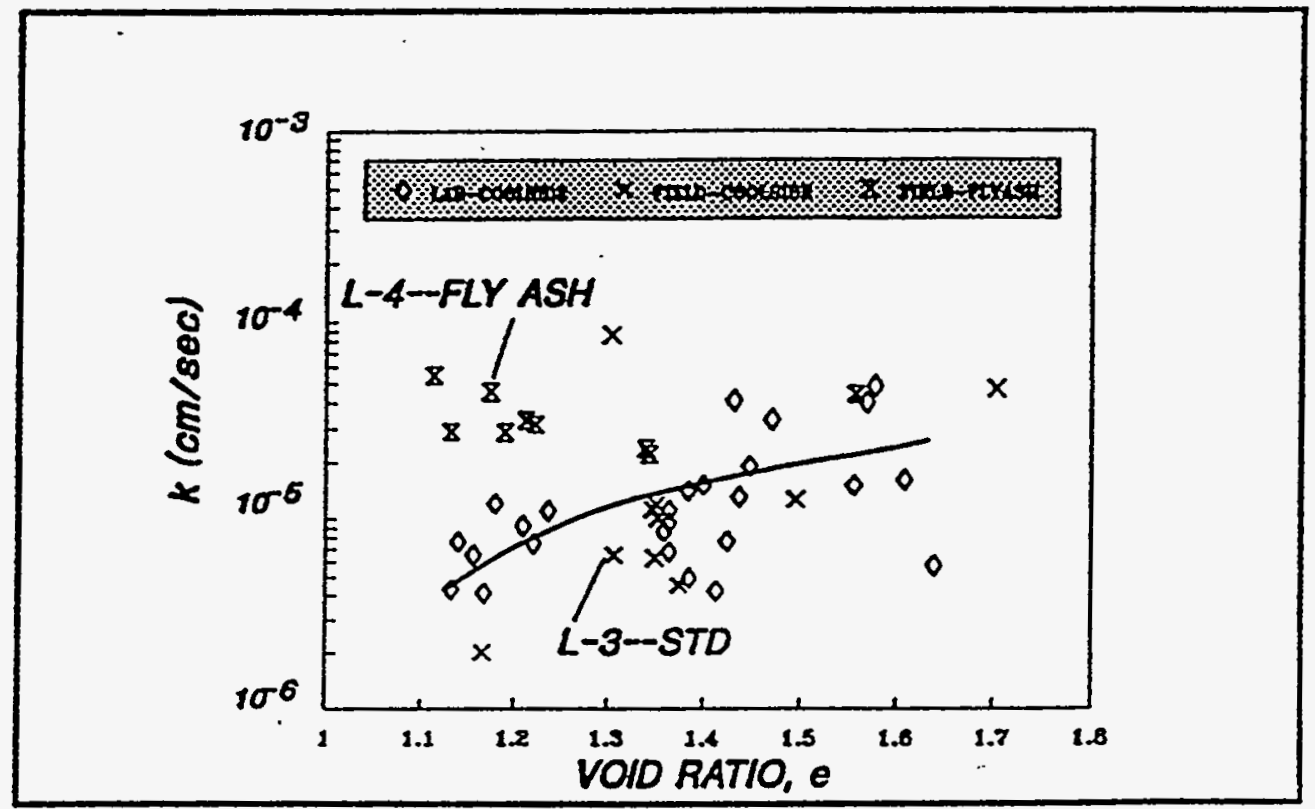

Figure 3-16. Permeability coefficient as a function of void ratio 
increases. The tests were performed in a pressurized cell using a back saturation technique and falling head, rising tailwater method.

\section{Field and Laboratory Tests on Specimens Collected after 3.5 Years}

About 3.5 years after the Coolside ash had been placed, standard penetration tests (SPT) were performed continuously in Lysimeters 1 (loose state) and 2 (simulated

static compaction of $117.2 \mathrm{kPa}$, or $17 \mathrm{psi})$. Thin-walled tube samples $(7.1-\mathrm{cm}$, or 2.8 in diameter) were obtained at $0.61-\mathrm{m}(2-\mathrm{ft})$ intervals in those lysimeters. Samples obtained from SPT tests were submitted to the University of Kentucky Center for Applied Energy Research (CAER) for a calibration of the neutron moisture gauges and mineralogical characterization. In Lysimeter 3 (the Coolside material had been compacted to $95 \%$ of maximum dry density and optimum moisture content), standard penetration tests were attempted. However, the tests had to be ended when the blows per $0.15-\mathrm{m}(0.5-\mathrm{ft})$ interval exceeded 50 at the 0.91 to $1.07-\mathrm{m}(3.0$ to $3.5 \mathrm{ft}$ ) interval. Subsequently, core samples were obtained. Portions of the core sample were submitted to the CAER for mineralogical characterization. Results of standard penetration tests are shown in Table 3-2. 
Table 3-2. Results of standard penetration tests

\begin{tabular}{|c|c|c|c|c|}
\hline $\begin{array}{l}\text { Lysimeter } \\
\text { Number }\end{array}$ & $\begin{array}{l}\text { Depth } \\
\text { (feet) }\end{array}$ & $\begin{array}{l}\text { *Blows per } 0.5 \\
\text { foot }\end{array}$ & $\begin{array}{l}\text { Types of } \\
\text { Material }\end{array}$ & $\begin{array}{l}\text { Standard } \\
\text { Penetration } \\
\text { Resistance } \\
\text { (blows per foot) }\end{array}$ \\
\hline 1 & $\begin{array}{l}0.0-2.0 \\
2.0-4.0 \\
4.0-6.0 \\
6.0-8.0 \\
8.0-10.0\end{array}$ & $\begin{array}{l}0 / 2 / 3 \\
5 / 10 / 11 / 11 \\
10 / 8 / 11 / 14 \\
7 / 6 / 6 / 16 \\
7 / 5 / 3 / 3\end{array}$ & $\begin{array}{l}\text { Soil } \\
\text { Coolside } \\
\text { Coolside } \\
\text { Coolside } \\
\text { Sand }\end{array}$ & $\begin{array}{l}2 \\
11 \\
12 \\
11 \\
3\end{array}$ \\
\hline 2 & $\begin{array}{l}0.0-2.0 \\
2.0-4.0 \\
4.0-6.0 \\
6.0-8.0 \\
8.0-10.0\end{array}$ & $\begin{array}{l}0 / 1 / 1 / 5 \\
16 / 19 / 16 / 17 / \\
12 / 17 / 19 / 16 \\
10 / 15 / 15 / 11 / \\
8 / 7 / 4 / 4\end{array}$ & $\begin{array}{l}\text { Soil } \\
\text { Coolside. } \\
\text { Coolside } \\
\text { Coolside } \\
\text { Sand }\end{array}$ & $\begin{array}{l}3 \\
16 \\
18 \\
13 \\
4\end{array}$ \\
\hline 3 & $\begin{array}{l}0.0-2.0 \\
2.0-4.0 \\
\# 0.33(4 \text { in.) Foot } \\
\text { penetration }\end{array}$ & $\begin{array}{l}0 / 0 / 1 / 2 \\
25 / 38 / 50 \#\end{array}$ & $\begin{array}{l}\text { Soil } \\
\text { Coolside }\end{array}$ & $\begin{array}{l}1 \\
\text { Refusal }\end{array}$ \\
\hline
\end{tabular}

*One blow is a 140-pound weight free falling 30 inches.

\# Test terminated-50 blows for less than 0.5 foot penetration is considered refusal during SPT testing.

In Lysimeter 4 (fly ash), attempts were made to obtain thin-walled tube samples. These efforts were unsuccessful because there was not enough cohesion between the fly ash and the sampling tube to retain the sample in the tube. Due to the soft nature of the fly ash, Dutch cone penetration tests were performed to obtain strength parameters.

Efforts to perform cone penetration in Lysimeters 1,2, and 3 were unsuccessful because the Coolside material was too hard. Results of field and laboratory tests performed on the in situ material and field specimens are summarized in Tables 3-3, 34, and 3-5, respectively, for Lysimeters 1,2, and 3. Dutch cone results for Lysimeter 4 are summarized in Table 3-6. 
Table 3-3. Field and laboratory results of tests performed on Coolside material in Lysimeter 1.

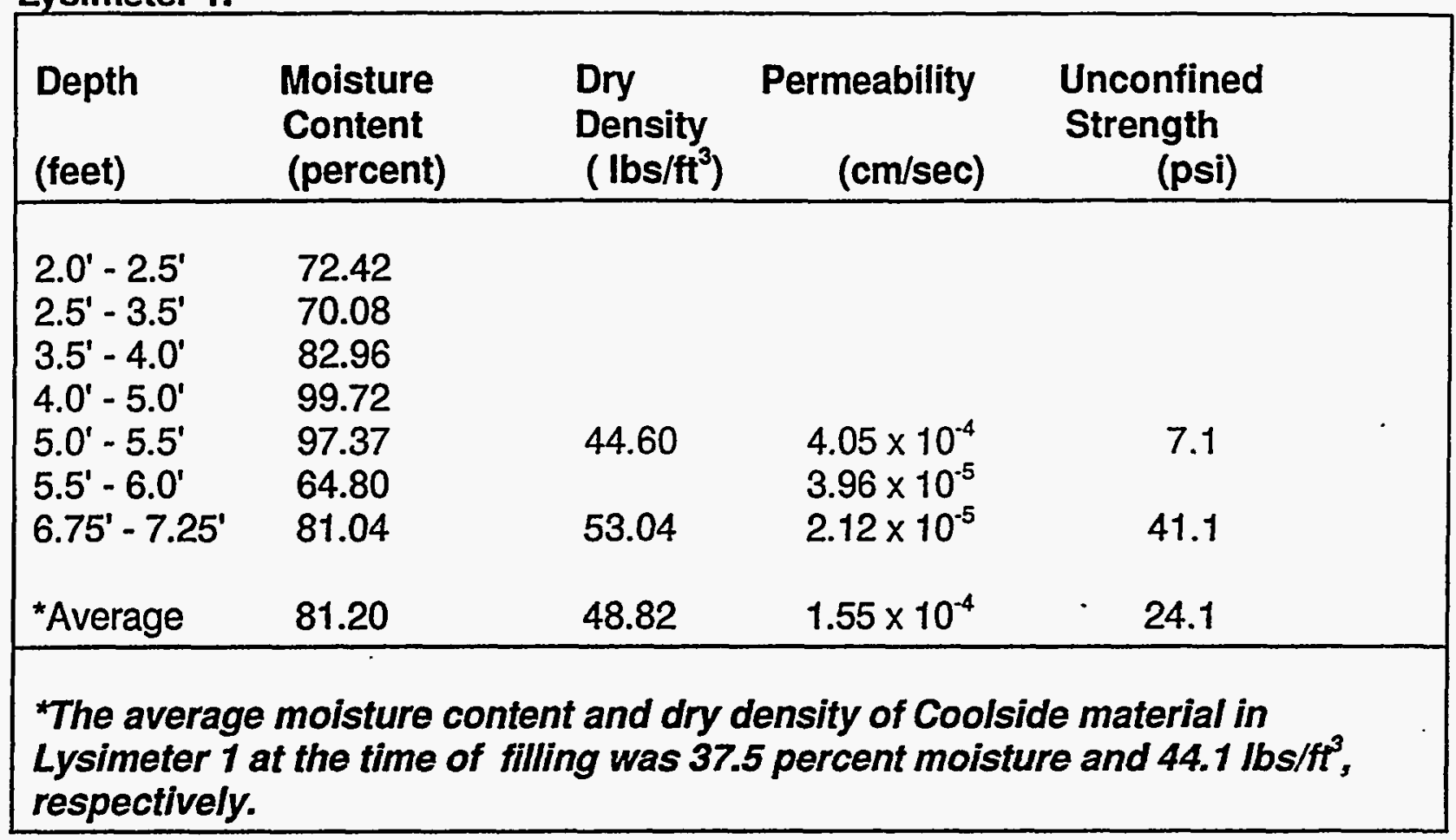

Unconfined strengths of specimens obtained from Lysimeters 1, 2, and 3 are compared in Figure 3-17. While the unconfined strength of the material in Lysimeter 1 only averaged about $542.6 \mathrm{kPa}\left(24 \mathrm{lbs} / \mathrm{in}^{2}\right)$, the strength of specimens from Lysimeter 2 averaged about $1116.3 \mathrm{kPa}\left(162 \mathrm{lbs} / \mathrm{in}^{2}\right)$, or almost seven times larger than strengths of Lysimeter 1 specimens. 


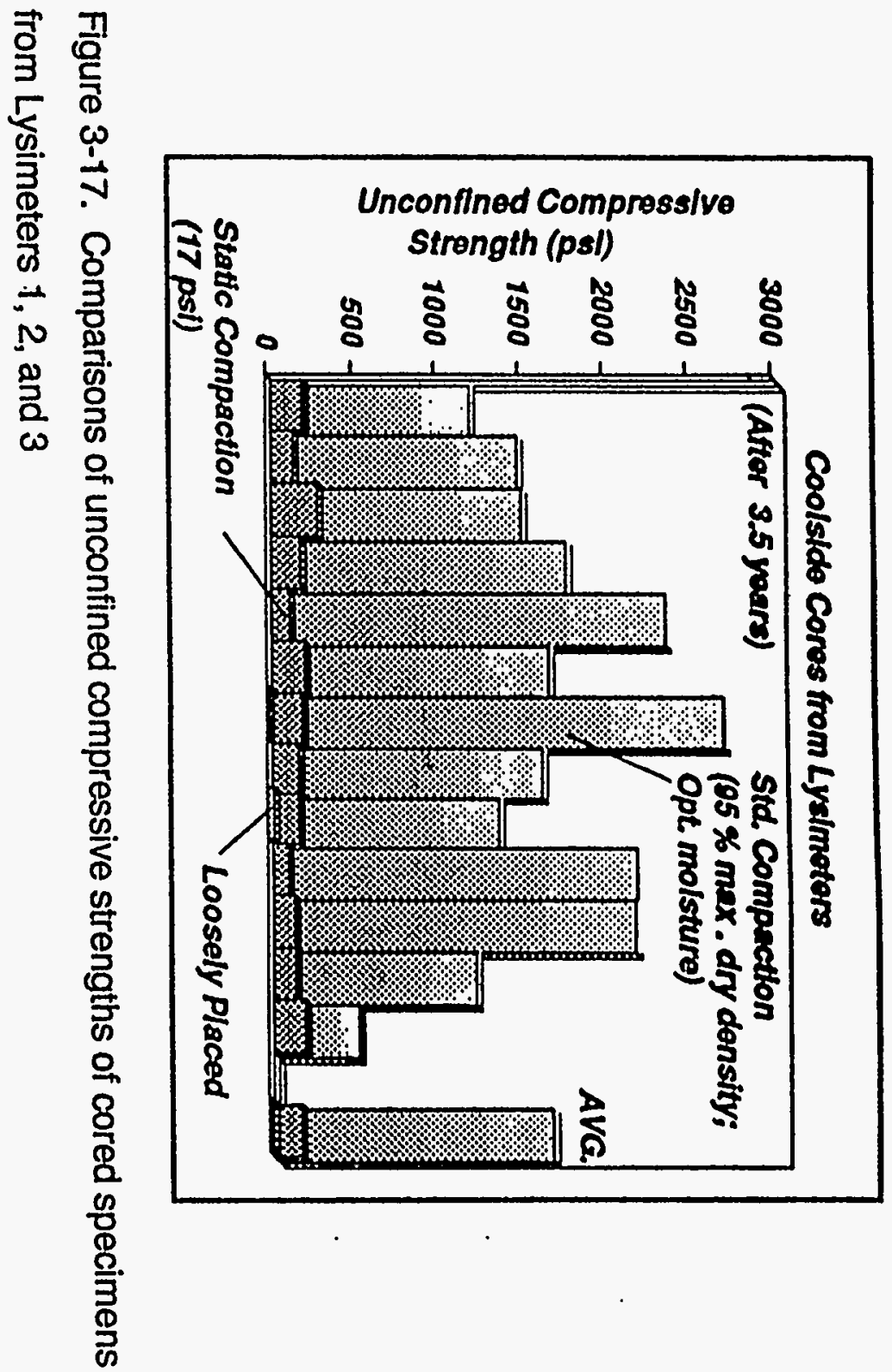


Table 3-4. Field and laboratory results of tests performed on Coolside material of Lysimeter 2.

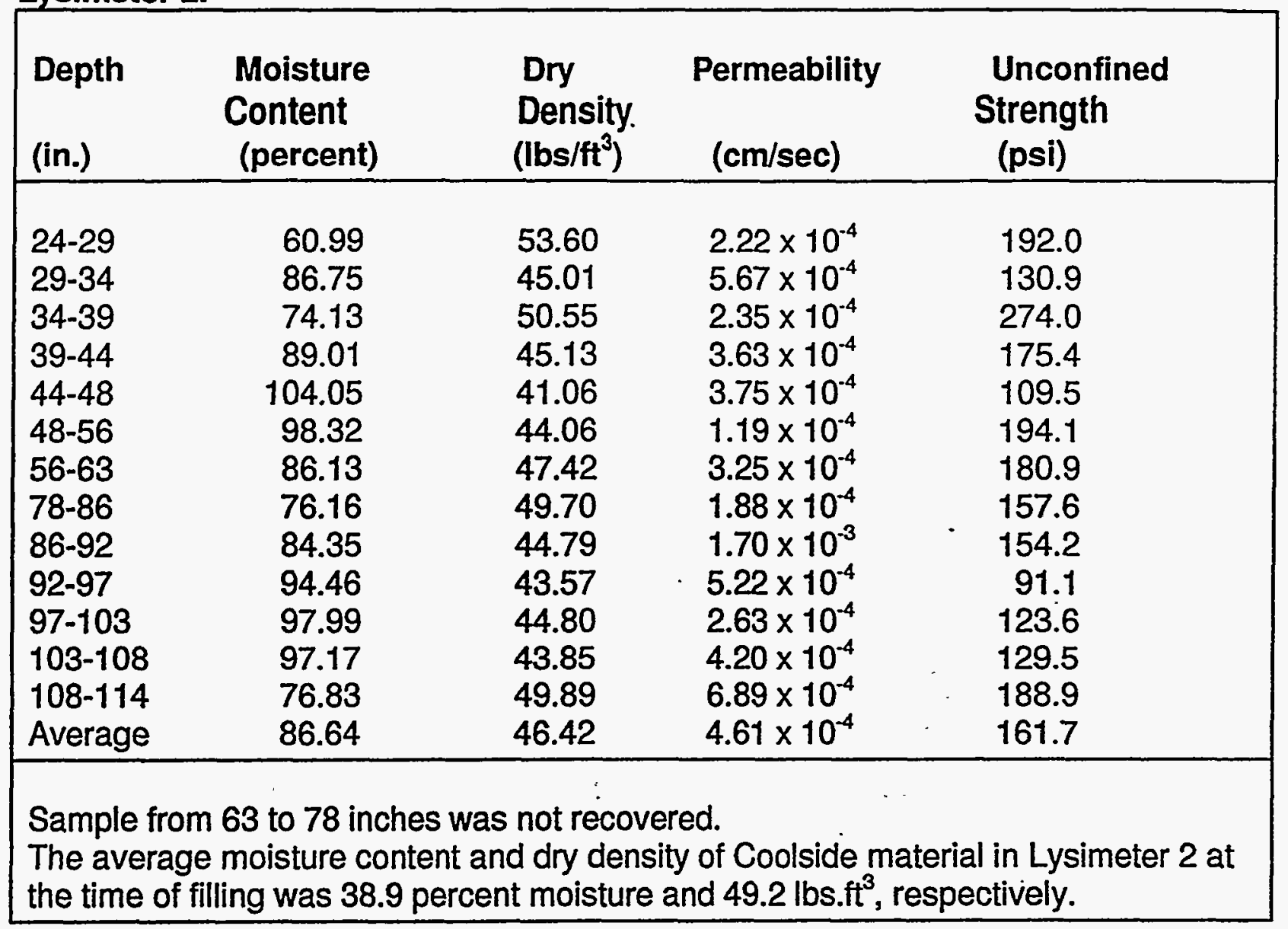


Table 3-5. Field and laboratory results of tests performed on Coolside material of Lysimeter 3.

\begin{tabular}{|c|c|c|c|c|}
\hline $\begin{array}{l}\text { Depth } \\
\text { (in.) }\end{array}$ & $\begin{array}{l}\text { Moisture } \\
\text { Content } \\
\text { (percent) }\end{array}$ & $\begin{array}{l}\text { Dry } \\
\text { Density } \\
\text { (lbs/ft') }\end{array}$ & $\begin{array}{l}\text { Permeability } \\
\text { (cm/sec)....... }\end{array}$ & $\begin{array}{l}\text { Unconfined } \\
\text { Strength } \\
\text {...(psi) }\end{array}$ \\
\hline $24-29$ & 48.90 & 69.51 & $9.16 \times 10^{-8}$ & 1153.6 \\
\hline $47-53$ & 46.18 & 68.85 & $7.91 \times 10^{-6}$ & 1436.0 \\
\hline $55-59$ & 64.46 & 60.34 & $4.76 \times 10^{-6}$ & 1457.6 \\
\hline $59-63$ & 47.34 & 68.09 & $1.56 \times 10^{-7}$ & 1728.4 \\
\hline $63-69$ & 42.05 & 73.44 & $8.23 \times 10^{-8}$ & 2319.6 \\
\hline $69-73$ & 43.20 & 72.61 & $1.98 \times 10^{-7}$ & 1619.4 \\
\hline $84-88$ & 41.24 & 74.47 & $7.86 \times 10^{-9}$ & 2654.8 \\
\hline $88-90$ & 56.25 & 65.69 & $7.66 \times 10^{-7}$ & 1578.9 \\
\hline $91-94$ & 52.48 & 67.41 & $2.18 \times 10^{-6}$ & 1319.5 \\
\hline $94-97$ & 49.15 & 69.21 & $4.34 \times 10^{-7}$ & 2138.4 \\
\hline $97-101$ & 39.63 & 74.54 & $7.39 \times 10^{-9}$ & 2130.0 \\
\hline $101-105$ & 59.16 & 62.97 & $4.89 \times 10^{-6}$ & 1179.0 \\
\hline $109-113$ & 75.96 & 54.66 & $1.17 \times 10^{-5}$ & 467.0 \\
\hline${ }^{*}$ Average & 51.23 & 67.83 & $2.20 \times 10^{-6}$ & 1629.4 \\
\hline \multicolumn{5}{|c|}{$\begin{array}{l}\text { The average moisture content and dry density of Coolside material in } \\
\text { Lysimeter } 3 \text { at the time of filling was } 37.0 \text { percent moisture and } 66.2 \text { lbs. } \mathrm{ft}^{3} \text {, } \\
\text { respectively. }\end{array}$} \\
\hline
\end{tabular}


Table 3-6. Results obtained from Dutch Cone Penetration Testing of the Fly Ash in Lysimeter 4.

\begin{tabular}{|cccc|}
\hline $\begin{array}{c}\text { Depth } \\
(\mathrm{m})\end{array}$ & $\begin{array}{c}\text { Cone } \\
\left(\mathrm{Kg} / \mathrm{cm}^{2}\right)\end{array}$ & $\begin{array}{c}\text { Cone }+ \text { Sleeve } \\
\left(\mathrm{Kg} / \mathrm{cm}^{2}\right)\end{array}$ & \\
$0.0-0.1$ & 4.5 & 5.0 & \\
$0.1-0.2$ & 4.5 & 5.0 & SOIL \\
$0.2-0.3$ & 3.0 & 8.0 & \\
$0.3-0.4$ & 4.0 & 6.0 & \\
$0.4-0.5$ & 2.5 & & \\
6.0 & & & \\
$0.5-0.6$ & 7.0 & 12.0 & \\
$0.6-0.7$ & 8.5 & 13.0 & \\
$0.7-0.8$ & 7.0 & 11.0 & \\
$0.8-0.9$ & 5.5 & 7.0 & \\
$0.9-1.0$ & 6.0 & 9.0 & \\
$1.0-1.1$ & 8.0 & 12.0 & \\
$1.1-1.2$ & 6.0 & 9.0 & \\
$1.2-1.3$ & 4.0 & 8.0 & \\
$1.3-1.4$ & 4.0 & 9.5 & \\
$1.4-1.5$ & 3.5 & 5.0 & \\
$1.5-1.6$ & 4.0 & 5.5 & \\
$1.6-1.7$ & 5.5 & 6.5 & \\
$1.7-1.8$ & 2.5 & 4.0 & \\
$1.8-1.9$ & 1.0 & 3.0 & \\
$1.9-2.0$ & 0.5 & 1.5 & \\
$2.0-2.1$ & 0.5 & 1.0 & \\
$2.1-2.2$ & 0.5 & 1.0 & \\
$2.2-2.3$ & 1.0 & 1.5 & \\
$2.3-2.4$ & 0.5 & 0.5 & \\
$2.4-2.5$ & 2.0 & & \\
3.0 & & & \\
$2.5-2.6$ & 4.0 & 18.0 & \\
$2.6-2.7$ & 13.0 & & \\
\hline
\end{tabular}

However, the average strength of the specimens from Lysimeter 3 was $11,225.5 \mathrm{kPa}$ (1629 lbs/in ${ }^{2}$ ), or a strength that was about ten times the average strength obtained for the specimens from Lysimeter 2. Hence, compacting the Coolside to $95 \%$ of maximum 
dry density (ASTM D 698) produced strengths that were much larger than strengths that would be produced from a tracked dozer. The strengths of the Lysimeter 3 specimens are similar to those that would be obtained from a low-strength concretes.

Unconfined strengths obtained for laboratory specimens compacted to $95 \%$ of maximum dry density and optimum moisture content obtained from standard compaction (ASTM D 698) are compared in Figure 3-18 to the unconfined strengths of the field specimens from Lysimeter 3. Although the unconfined strengths of the specimens from lysimeter ranged from $3,218.1 \mathrm{kPa}$ to $18,294 \mathrm{kPa}$ (467 to 2654.8 $\left.\mathrm{lbs} / \mathrm{in}^{2}\right)$, the average value was about $11,225 \mathrm{kPa}\left(1629 \mathrm{lbs} / \mathrm{in}^{2}\right)$. This strength was very similar to laboratory specimens remolded in the laboratory to the same conditions.

Coefficients of permeability of Coolside specimens from Lysimeters 1, 2, and 3 are shown in Figure 3-19 as a function of dry density. As the dry density increases, the coefficient of permeability decreases. A good correlation exists between the permeability coefficient and dry density. As the compactive effort approaches that used in standard compaction, the coefficient of permeability approaches a value of $1 \times 10^{-7}$ $\mathrm{cm} / \mathrm{sec}$.

Coefficients of permeability of the field specimens and laboratory specimens are compared and shown as a function of dry density in Figure 3-20. In both instances, the coefficients of both types of specimens decrease as the dry density increases. The coefficients of permeability of the field and laboratory specimens are very similar. As shown in Figure 3-21, the coefficient of permeability of the cored field specimens increases as the void ratio increases. The coefficient approaches a value of $1 \times 10^{-7}$ $\mathrm{cm} / \mathrm{sec}$ when the void ratio approaches a value of approximately 0.8 . 


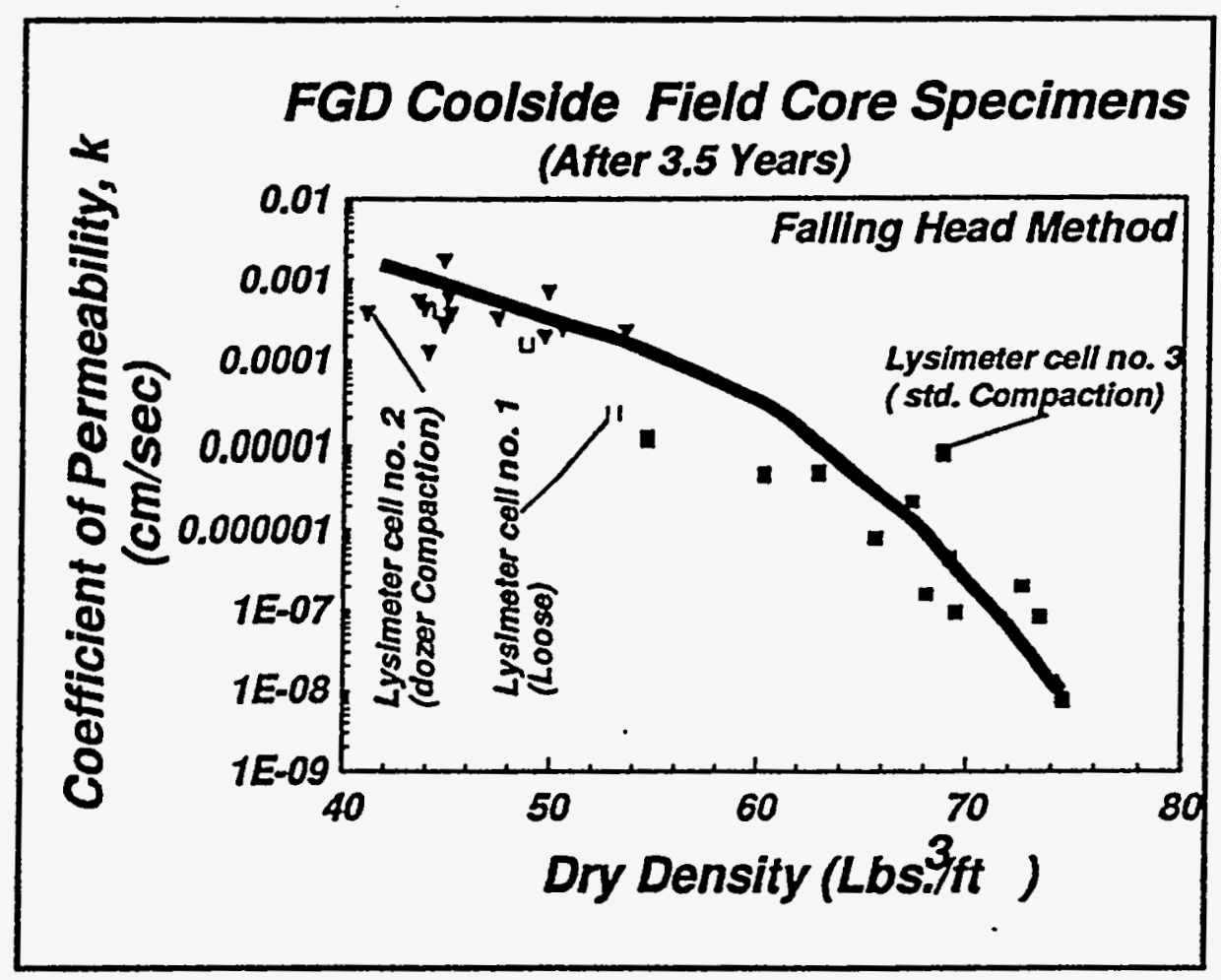

Figure 3-19. Comparisons of the coefficients of permeability of cored specimens of Lysimeters 1, 2, and 3

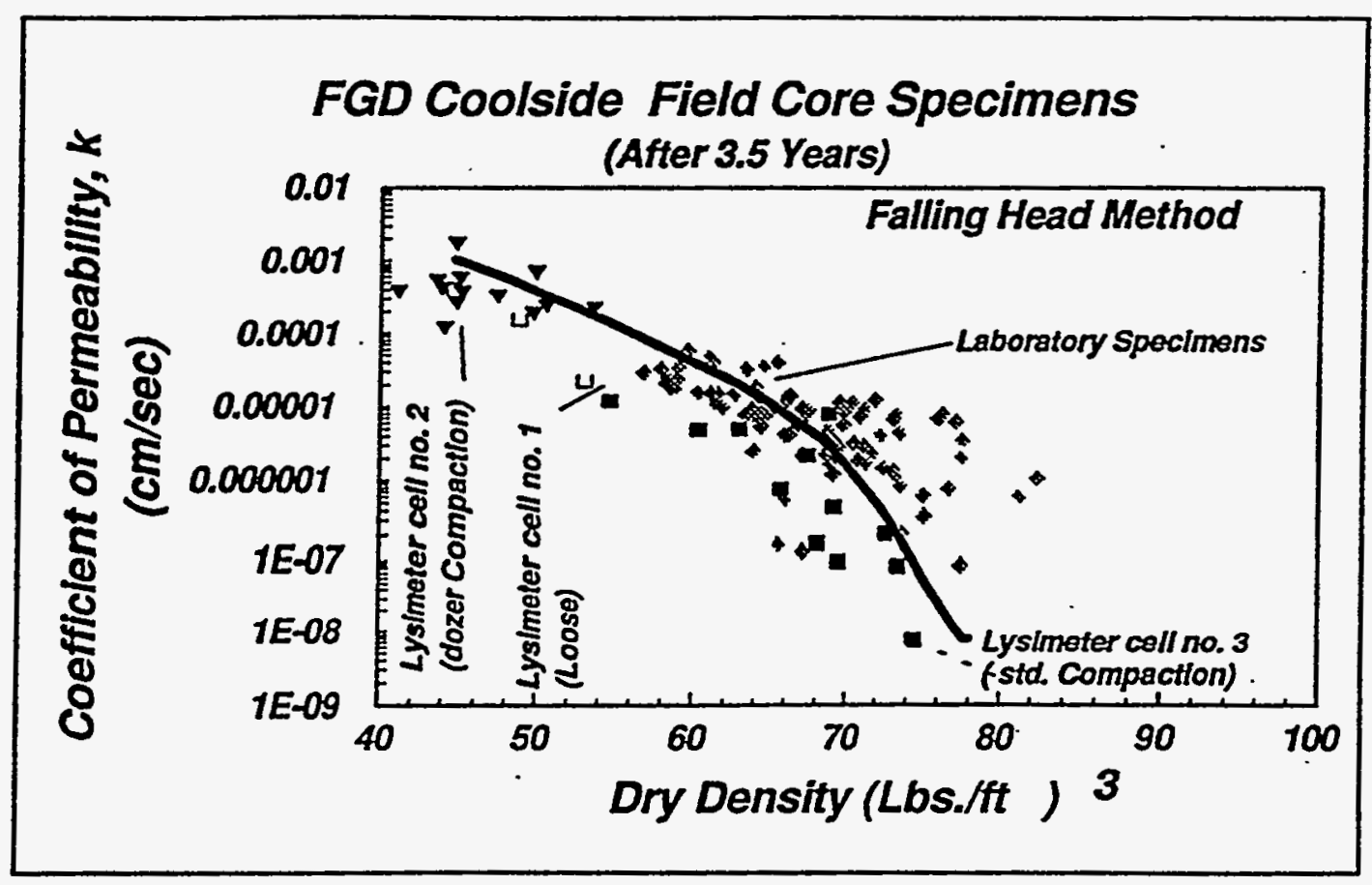

Figure 3-20. Comparison of the relationship between the coefficients of permeability of core specimens from Lysimeters 1,2, and 3 and laboratory specimens and dry density 


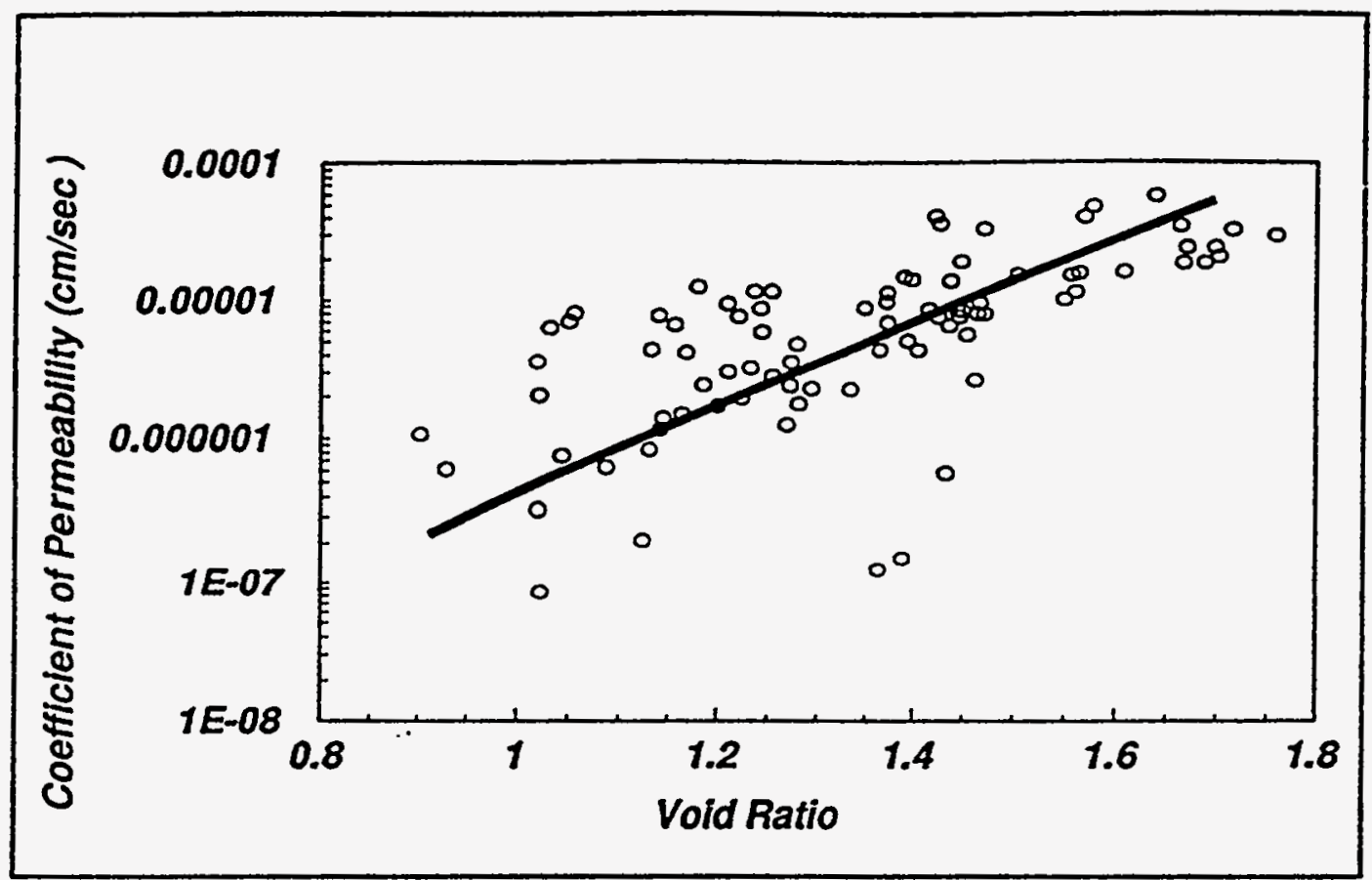

Figure 3-21. Coefficients of permeability of cored specimens from Lysimeters 1,2, and 3 as a function of void ratio

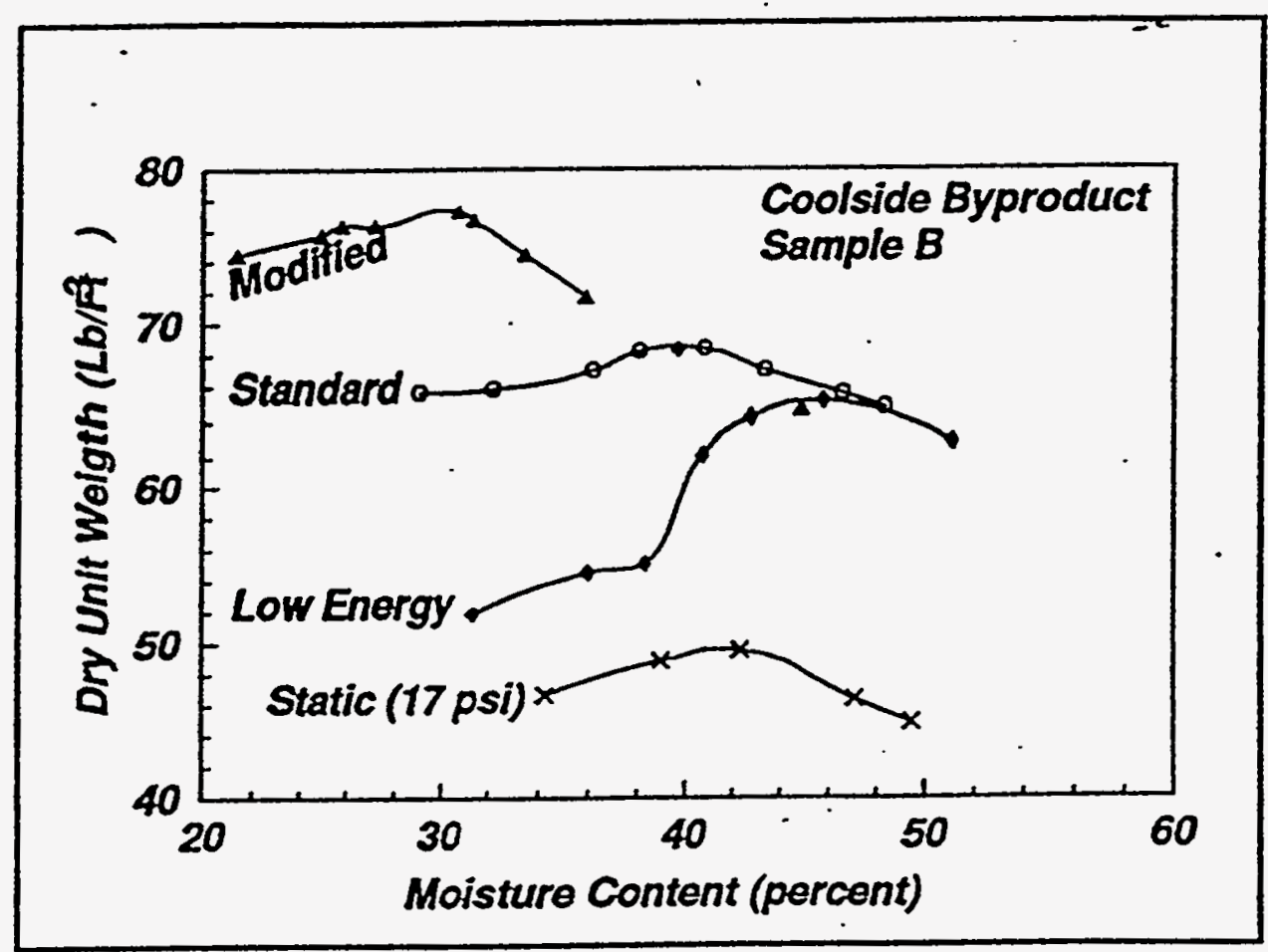

Figure 3-22. Moisture-density relations for different compactive energies 


\section{Geotechnical Laboratory Test Program}

\section{Index Properties and Classification}

Geotechnical index and classification tests were performed in accordance with ASTM procedures. These tests were performed to characterize and classify the Coolside material. ASTM test designations are summarized in Table 3-7. The index tests consisted of sample preparation, moisture content, moisture-density relationships, liquid and plastic limits, particle size analysis, specific gravity, and classification. The Coolside material was also classified according to the Association of American State Highway Transportation Officials (AASHTO).

Index properties and classifications of the Coolside byproduct are shown in Table 3-8. Samples identified as CS-1040, CS-1050, CS-388, and CS-397 were obtained from runs 1 and 3 at the Ohio Edison coal-fired power plant at Lorain, Ohio. A sample identified as CS-2 was obtained from CONSOL's Coolside pilot plant at their research facility in Library, Pennsylvania. The different samples had essentially the same properties. All of the specimens were nonplastic (NP). That is, none of the materials, when initially wetted, exhibited cohesion. All the materials passed the U.S. 200 sieve

and 95 percent of the materials were finer (by weight) than the U.S. sieve number 200. Specific gravity of the materials ranged from 2.51 to 2.55 . Based on the Unified Classification System, the Coolside materials were classified as ML (silt). According to the ASSHTO Classification System, the materials were classified as A-4.

\section{Moisture-Density Relations}

Laboratory compaction tests were performed at different compaction energies to examine the relationships between dry density and compaction effort, and optimum. moisture content and compaction energy. These tests were performed at modified 


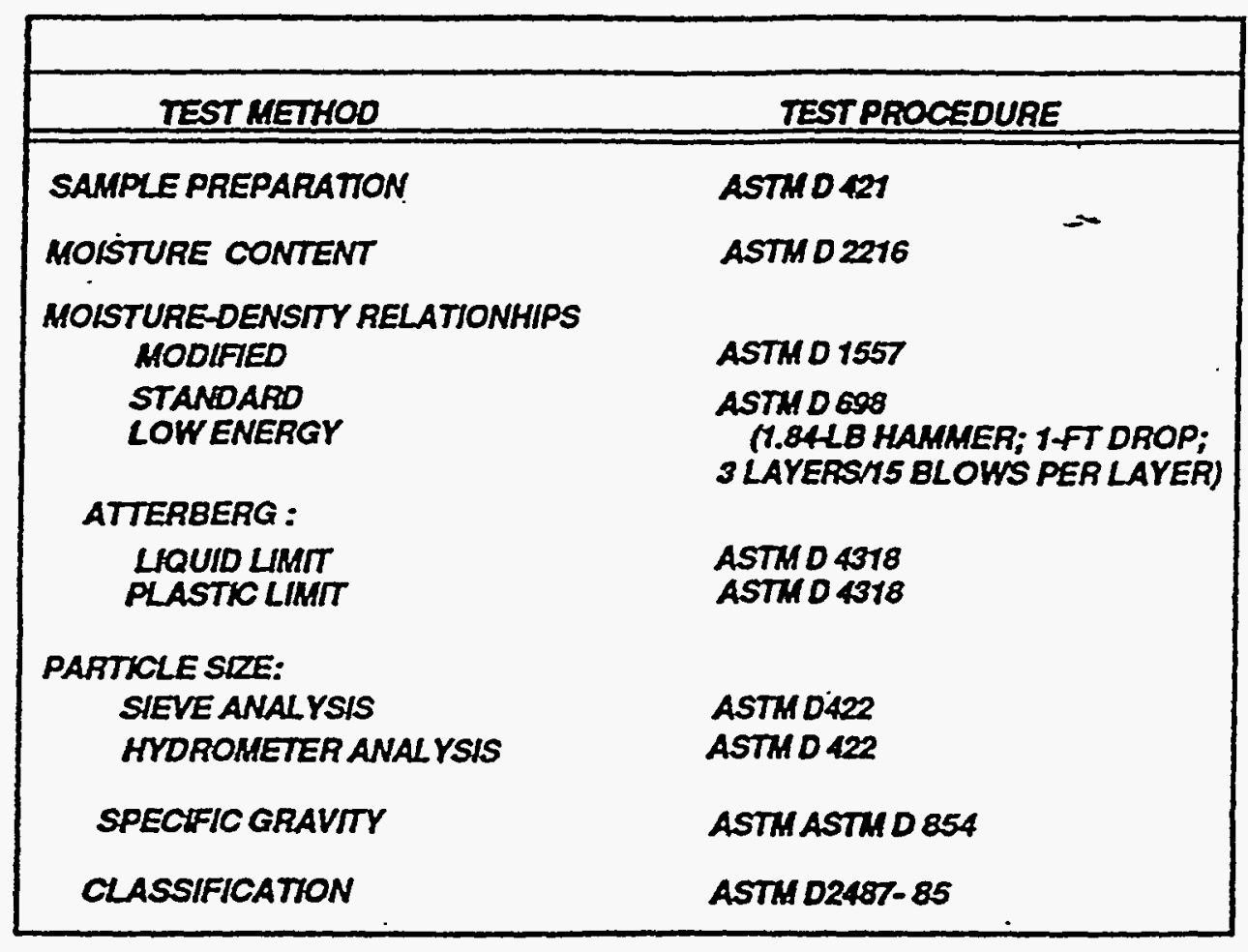

Table 3-7. Index properties test procedures

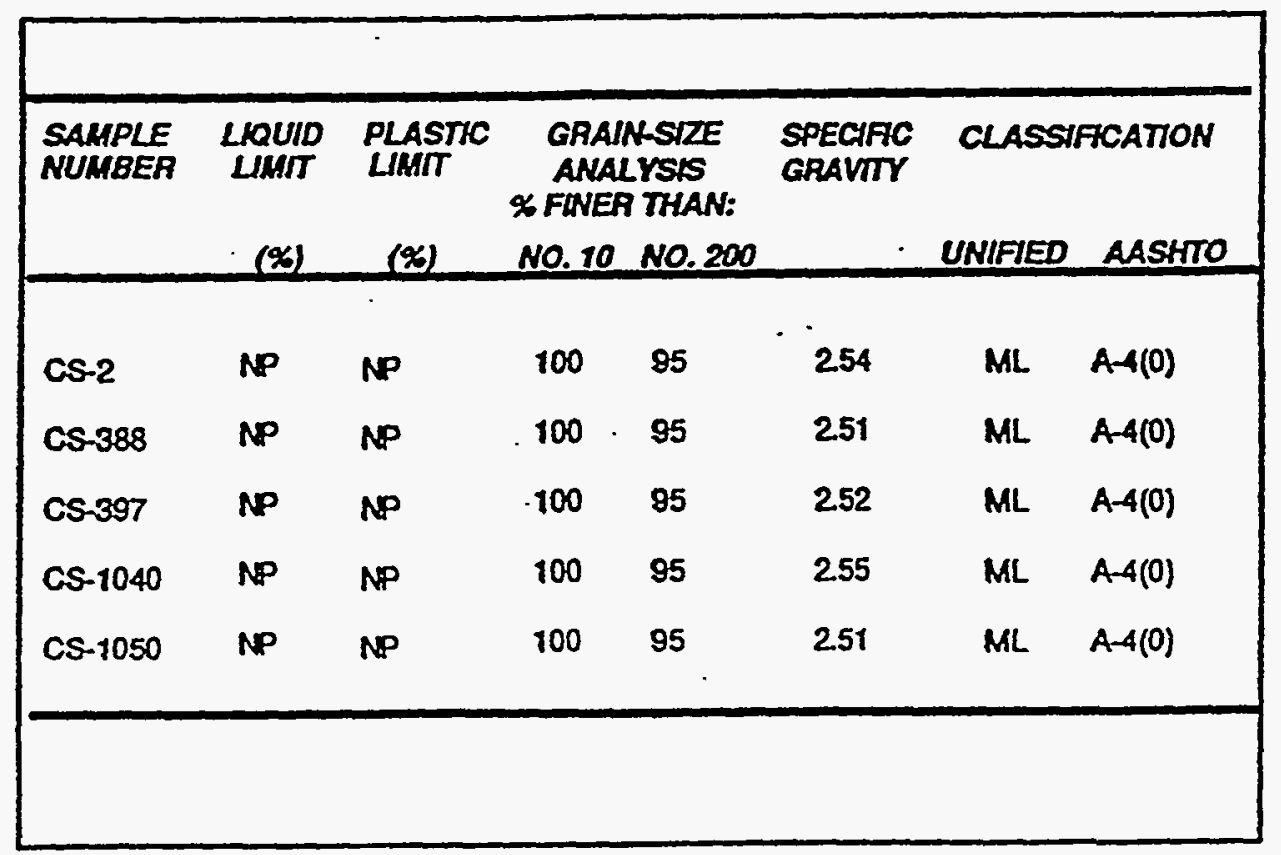

Table 3-8. Index properties and geotechnical classification of the Coolside byproduct 
compaction (ASTM D 1557), standard compaction (ASTM D 698), and a low energy compaction method (Hopkins, 1988). Compaction energies were $326,754,71,885$, and $14,450 \mathrm{~m}-\mathrm{kg} / \mathrm{m}^{3}$ (or $56,246,12,374$, and $2,025 \mathrm{ft}-1 \mathrm{~b} / \mathrm{ft}^{3}$ ). Besides the three dynamic compaction energies, compaction tests were performed using static compaction. These tests were performed to simulate the compaction action of the tracks of a Caterpillar7D9L track-type tractor (Caterpillar Performance Handbook, 1981). The static compaction tests were performed at a stress of $117 \mathrm{kPa}$ (17 psi)--the contact stress of the tracks of this equipment. Specimens were molded statically in a testing machine using this stress.

Typical compaction test results obtained for the Coolside byproduct, identified as CS-3, are illustrated in Figure 3-22. Sample CS-3 was a composite sample from the Run number 3 series of the Edgewater plant in Lorain, Ohio. Identical results --not shown-were obtained for a Coolside byproduct identified as sample CS-1. As the compaction energy increases, the maximum dry density increases and the optimum moisture content decreases. The maximum dry density ranges from $7.79 \mathrm{kN} / \mathrm{m}^{3}\left(49.5 \mathrm{lbs} / \mathrm{ft}^{3}\right)$ at static compaction to $12.10 \mathrm{kN} / \mathrm{m}^{3}\left(76.5 \mathrm{lbs} / \mathrm{ft}^{3}\right)$ at modified compaction. The dry density of the Coolside material in a loose state is $5.72 \mathrm{kN} / \mathrm{m}^{3}\left(36.5 \mathrm{lbs} / \mathrm{ft}^{3}\right)$. Consequently, the maximum dry density obtained from the static compaction is only about $36 \%$ greater than the loose state dry density.

Variation of the maximum dry density of sample CS-3 with compaction energy is illustrated in Figure 3-23. The maximum dry density $\left(10.80 \mathrm{kN} / \mathrm{m}^{3}\right)$ of the Coolside material is about 1.9 times the dry density of the material in a loose state. However, the maximum dry density at modified compaction is only about 1.12 times the maximum dry density obtained from standard compaction. Therefore, for a fourfold increase in compaction energy above the standard compaction energy, the dry density increases only about $12 \%$. Consequently, efforts to compact the Coolside material to a dry density greater than the maximum dry density obtained from standard compaction 


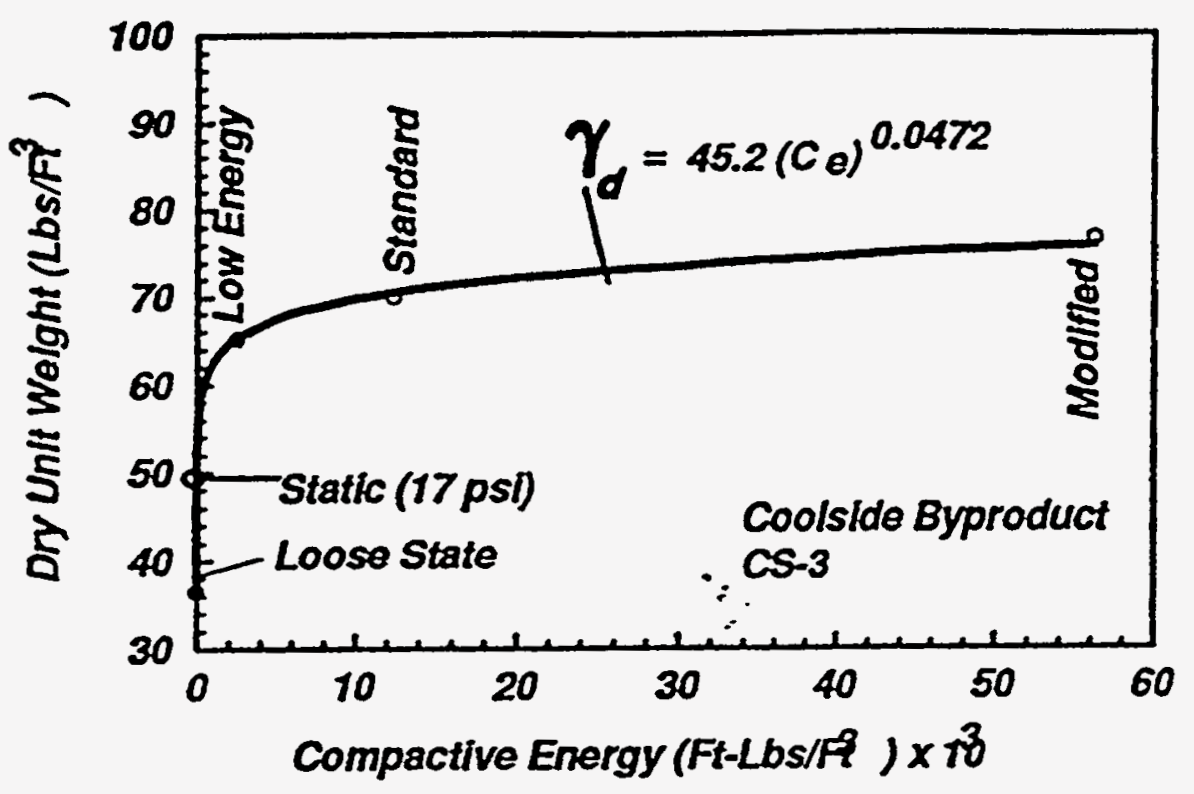

Figure 3-23. Variation of the maximum dry density with compactive energy

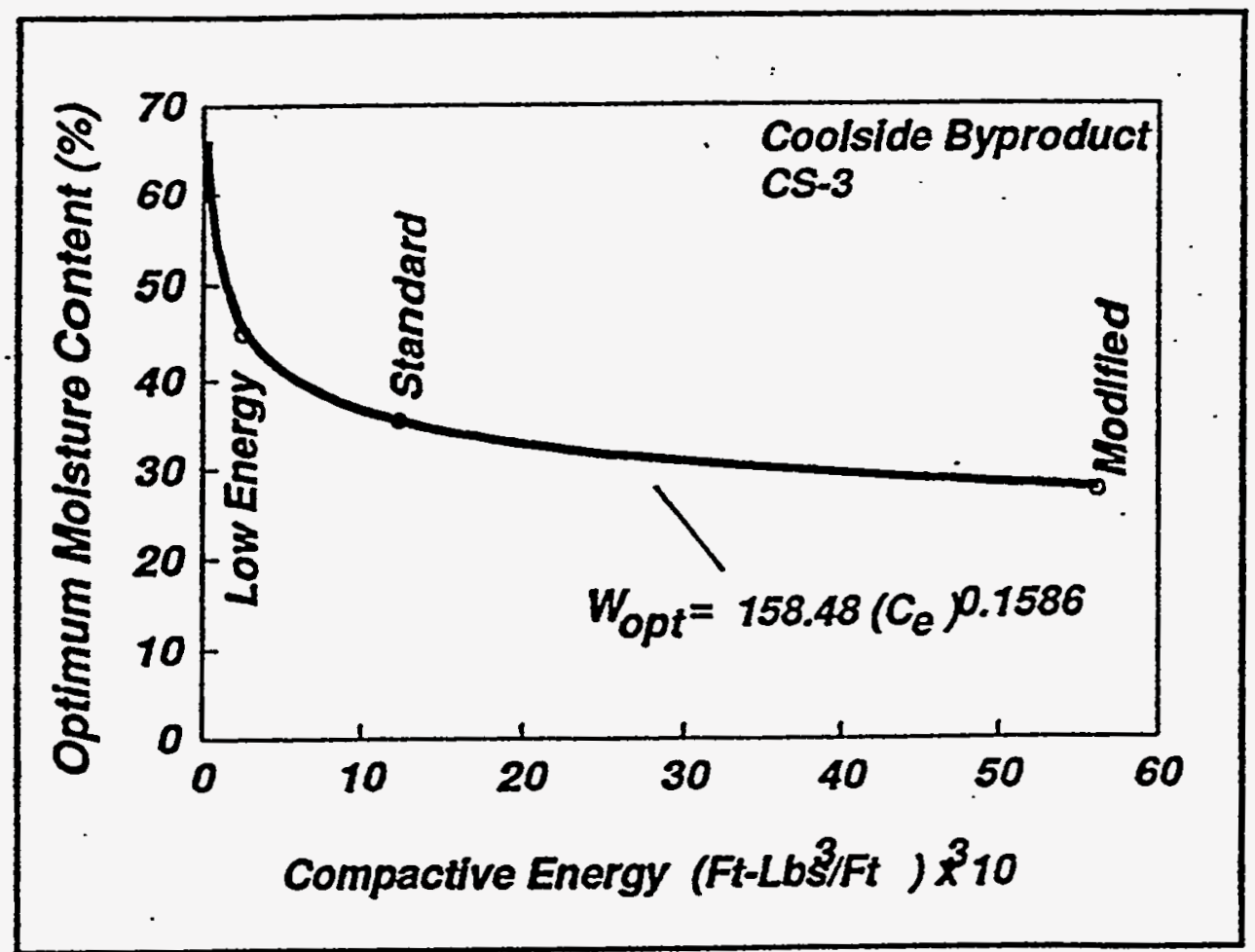

Figure 3-24. Variation of optimum moisture content with compactive energy 
would probably be uneconomical. However, the compaction energy selected would depend on the specific site variables, such as land cost, cost of compaction equipment and operation, haul distances, and availability of land for disposal. Economic evaluation of those factors would be required at a given site to select the most economical compaction energy.

Variation of optimum moisture content and compaction energy for sample CS-3 is illustrated in Figure 3-24. At standard compaction, the optimum moisture content is about $36.5 \%$. Compaction of the Coolside material could pose a problem when the water content is greater than the optimum moisture content. Because the Coolside byproduct contains a large silt content, the material may liquefy under compaction stresses when the moisture content is greater than optimum moisture content.

\section{Recommendations}

Based on the results of index tests, 95 to $100 \%$ of maximum dry density obtained from standard compaction tests may be achieved using sheep foot rollers. Compacted lift thickness should be about $15.24 \mathrm{~cm}$ ( 6 in) and 4 to 6 passes of the compaction rollers may be required to achieve a specified compactive state. Foot contact stress should range between 1380 and $2760 \mathrm{kPa}$ (200 to $400 \mathrm{psi}$ ). A self-propelled Sheep foot roller with a blade may be desirable so that the material could be spread and compacted simultaneously. Alternatively, rubber tire rollers may be used. Here, the lift thickness of $25.4 \mathrm{~cm}$ (10 in) is indicated. About 4 to 6 passes of the rollers are required. Large size tires with inflation pressures ranging from 276 to $345 \mathrm{kPa}$ are desirable to avoid. shearing and rutting failures. Field trials at a given site should be performed to ensure the proper selection of compaction equipment and to decide desired field density states. If standard compaction is selected as the compactive state, the moisture content should generally not exceed the optimum moisture. Otherwise, the silty material may liquefy under the stress imposed by construction and compaction 
equipment. A short curing period of the compacted material will improve the bearing capacity of the material since the material gains strength with increasing time. However, the total strength gain depends partly on the energy used to compact the material.

\section{Structural Properties Tests}

Remolding Procedure. In the design of untreated and chemically treated subgrades, geotechnical laboratory tests, such as triaxial tests and permeability tests, should be performed on specimens of the materials that closely simulate the compactive state of the materials as they may exist in an engineered subgrade or landfill. The laboratory compaction test (Hopkins et al, 1988 and Hopkins and Beckham, 1993) should produce specimens for physical properties tests that duplicate the anticipated, or specified, field dry density and moisture content. For example, if field compaction specifications require that a subgrade be compacted to 95 percent of maximum dry density and 2 percent of optimum moisture content (ASTM D 698), then the laboratory compaction procedure should produce specimens that duplicate, as practically as possible, those target values of dry density and moisture content.

Moreover, since the maximum dry density and optimum moisture content of a soil may change as a chemical admixture is added, then it is essential that the remolding procedure account for such a change. Otherwise, the physical properties as measured from laboratory tests may differ significantly from those that may exist in the completed engineered subgrade. Additionally, when chemical admixtures are used, a method is needed to decide the most economical amount, or percentage, of a chemical admixture to add to the soil subgrade to obtain some desired strength. The procedures developed during this study for compacting specimens that conform to specified values of dry density and optimum content and for determining the optimum percentage of a chemical admixture are described below. The procedure is applicable to a variety of 
fine-grained materials, such as soils, byproducts, and mixtures of byproducts and soils and chemical admixtures and soils.

Equations. Equations used in the proposed procedure to compact specimens to selected, or target, values of dry density and moisture content are described below. When byproducts, or other chemical admixtures, are added to soils, the matrix of the mixture consists of air, water, soil particles, and admixture particles. To determine the weights of soil solids $\left(W_{s}\right)$, water $\left(W_{w}\right)$, and a chemical admixture $\left(W_{p}\right)$, that must be mixed to form a specimen of a known volume $(V)$, dry density $\left(\gamma_{d}\right)$, and moisture content $\left(w_{1}\right)$, the phase diagram of Figure 3-9, may be used in formulating the necessary equations.

By definition, the total density $((t))$, is

$$
\gamma_{1}=W / N=\left(W_{w}+W_{s}+W_{p}\right) / N \text {. }
$$

The target moisture content, $w_{1}$, is, by definition

$$
W_{1}=W_{w} /\left(W_{s}+W_{p}\right)
$$

By definition, the dry weight of an admixture is expressed as a percentage, $P$, of the dry weight of soil particles, or

$$
W_{p}=P W_{s}
$$

Solving for $W_{w}$ in Equation 2 and inserting this quantity, and the quantity given by Equation 3, into Equation 1, then the dry weight of soil, $W_{s}$ is

$$
W_{s}=\gamma_{1} V /\left(1+W_{1}\right)(1+P)
$$

The air-dried weight, $W_{a d}$ is related to the oven-dried weight, $W_{s}$ (or weight of soil solids) by the expression:

$$
W_{a d}=W_{s}\left(1+W_{h s}\right) \text {, }
$$


where $w_{h s}$ is the (hygroscopic) moisture content existing in the soil at the time of mixing. The total density is related to the dry density by the expression,

$$
\gamma_{i}=\gamma_{d}\left(1+w_{i}\right) \text {. }
$$

Substituting Equations 4 and 6 into Equation 5, then

$$
W_{\text {ad }}={ }_{d} V\left(1+W_{h s}\right) /(1+P)
$$

Equation 7 gives the weight of air-dried soil that must be added to form the specimen of a known volume, $V$, dry density, ( $d$, and moisture content, $w_{1}$. The air-dried weight, $W_{\text {pad, }}$ of chemical admixture may be computed from the following expression.

$$
W_{\text {pad }}=W_{p}\left(1+W_{h p}\right)=P W_{s}\left(1+W_{h p}\right) \text {, }
$$

where $W_{h p}$ is the hygroscopic moisture content of the air-dried (or moisture content at the time of mixing) admixture. Equation 8 gives the weight of an air-dried admixture that must be added to the mixture.

The amount of water $\left(W_{\text {wadd }}\right)$, which must be added to the mixture depends on the amounts of moisture (or hygroscopic moisture content) existing in the soil and admixture at the time of mixing. If the materials have been air-dried, then the materials may contain only hygroscopic moisture. Hygroscopic moisture content ( $\left.W_{\mathrm{hs}}\right)$, of the soil, and $w_{\text {hp }}$ of the admixture must be determined from laboratory tests. The total amount of water required to mix the materials and form the specimen of a known volume and selected dry density may be determined from Equations 1 and 2, or,

$$
W_{w}=W_{1} W_{s}(1+P) .
$$

From the phase diagram, Figure $3-25$, the amount of water existing in the soil before mixing (by definition) is, 

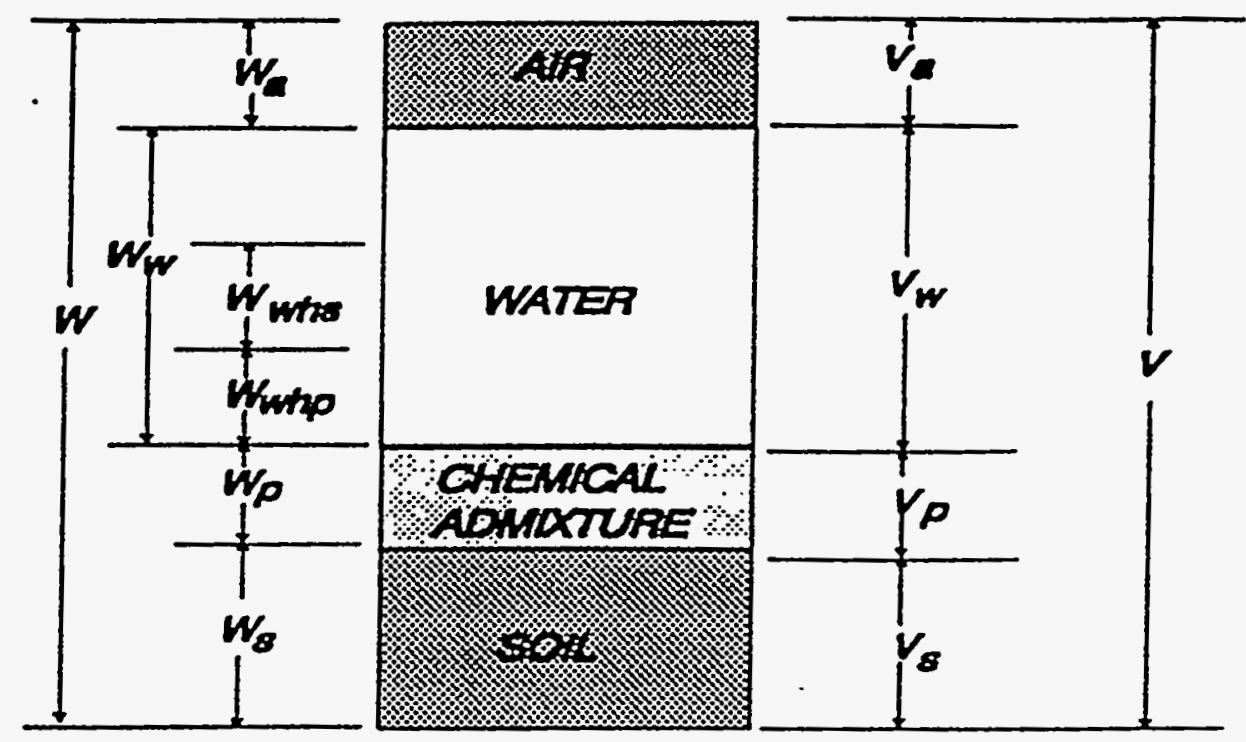

Figure 3-25. Phase diagram

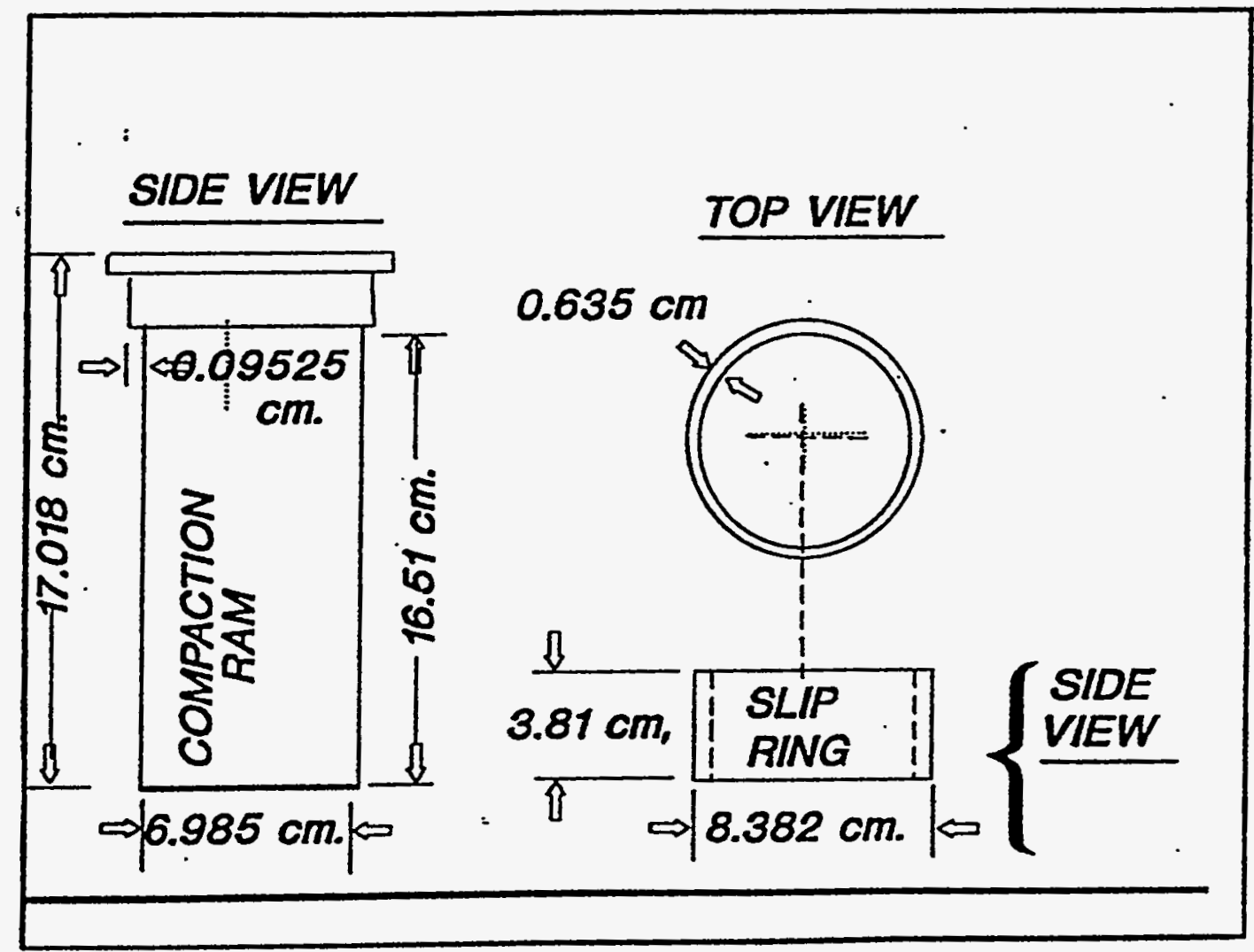

Figure 3-26. Schematic of the ram and slip rings used to compact specimens 


$$
W_{\text {whs }}=W_{h s} W_{s} \text {, }
$$

and in the admixture,

$$
W_{\text {whp }}=W_{h p} W_{p}=w_{h p} P W_{s} \text {. }
$$

The amount of water to be added at the time of mixing (Figure 3-25) is

$$
W_{\text {wadd }}=W_{w}-W_{\text {whs }}-W_{\text {whp }} \text {. }
$$

Substituting the expressions given by Equations 8, 9, and 10 into Equation 11, then

$$
W_{\text {wadd }}=W_{s}\left[P\left(W_{t}-W_{h p}\right)+W_{1}-W_{h s}\right] \text {. }
$$

A spreadsheet computer program was developed to compute the weights of air-dried soil, water, and admixture needed to compact a specimen of a known volume and selected values of dry density and moisture content.

Compaction Equipment and Method. Equipment required to compact a specimen includes some type of apparatus, or other means, for mixing the specimen, an electronic scale with a resolution of 0.01 grams, a split-type mold, and speciallydesigned ram and slip rings. Although the split-type mold for compacting the specimens may be designed for any selected dimensions, a type of mold that is convenient for forming specimens for triaxial or permeability testing measures $20.32 \mathrm{~cm}$ ( 8 in height and $7.11 \mathrm{~cm}(2.8 \mathrm{in})$ in diameter. Specimens are compacted to a height of $15.24 \mathrm{~cm}$ ( 6 inches). The inside diameter of this mold is the same as the diameter $(7.11 \mathrm{~cm})$ of a commonly-used, thin-walled, field sampling tube. Another suitable size of a split-type mold for laboratory compaction purposes measures $15.24 \mathrm{~cm}$ in height and $5.08 \mathrm{~cm}$ ( 2 inches) in diameter. Specimens are compacted to a height of 11.43 (4.5 inches). By using a split-type mold, the specimen may be removed from the mold conveniently, the need to extrude the compacted specimen from the mold is avoided, and sample disturbance after compaction is reduced. 
The function of the ram and rings, which slip over the ram, is to control the height of each layer of the compacted specimen. In the compaction standard, ASTM D 698, the specimen height is $11.6434 \mathrm{~cm}$ ( 4.584 inches); the specimen is compacted in three layers and each layer is $3.879 \mathrm{~cm}$ (1.527 inches) in height. In the proposed compaction procedure, each layer of the specimen is compacted to approximately the same height, or $3.81 \mathrm{~cm}$ (1.5 inches). For example, specimens measuring $15.24 \mathrm{~cm}$ in height are compacted in four layers but each layer is $3.81 \mathrm{~cm}$ in height. A schematic of the ram and slip rings used to compact specimens measuring $15.24 \mathrm{~cm}$ in height and $7.11 \mathrm{~cm}$ in diameter is illustrated in Figure 3-26. Views of the split mold, rings, and ram are shown in Figure 3-27.

Procedure. The purpose of the compaction procedure is to produce a specimen that has a dry density and a moisture content that are near prescribed, or target, values of dry density and moisture content. For example, if field specifications dictate that a given material must be compacted to 95 percent (or another choice) of maximum dry density obtained from a standard laboratory test, such as ASTM D698, then the target values for remolding the laboratory specimen would be selected according to the field specifications. After developing the specified moisture-dry density curve, target values of dry density and moisture content are selected. When only one material is involved $(P=0)$, Equations 45 and 41 are used to calculate the weight of air-dried material and the volume of water that must be used to remold a specimen of a known (or selected) volume. However, the material does not necessarily have to be air-dried. The only requirement here is that the existing moisture content, $w_{\mathrm{hs}}$, in the material at the time of sampling, must be equal to or less than the selected, target moisture content. After a small sample is obtained to find the existing moisture content of the material, the material may immediately be placed and sealed in a zip-lock plastic bag to prevent any further loss of moisture. The material remains sealed until the time of mixing. Sealing the material in a plastic bag is not necessary when the material is air dried. To mix the sample, the material is placed in a mixing bowl and the amount of water, as determined 
from Equation 51, is added to the material. When the specimen to be formed is 15.24 $\mathrm{cm}$ in height and $7.11 \mathrm{~cm}$ in diameter, the mixed material is divided into four parts of equal weight and stored in zip-lock bags. It is imperative that care is exercised in this portion of the procedure to avoid the loss of material when the material is weighed and transferred to the plastic bags. Normally, the material remains sealed in the plastic bags for about 24 hours before remolding to allow an even distribution of moisture.

After the mellowing period, the specimen is compacted as illustrated in Figure 3-28. The contents of the first bag are placed in the split mold and the ram is hammered down until the collar of the ram rests against the top of the mold. When the collar touches the top of the mold, the first compacted layer is exactly $3: 81 \mathrm{~cm}$ (1.5 inches) in height. The top of the first layer is scarified and the second bag of material is added to the mold. The first slip ring is slipped over the ram and the second layer is compacted. When the bottom of the first ring touches the top of the mold, the second layer is exactly $3.81 \mathrm{~cm}$. The procedure is repeated for the third and fourth layers, as shown in Figure 3-29, respectively. When the last layer is compacted, the specimen is exactly $15.24 \mathrm{~cm}$ in height. During the compaction procedure, the number of blows does not have to be counted because the exact amounts of materials and water are used to form the specimen of a selected dry density, water content, and known volume.

When admixtures, such as a byproduct (Coolside, FBC, etc.) or hydrated lime, are added to soil, chemical reactions may occur. Maximum dry density and optimum moisture content derived from a given type of compaction test depend on the percent of an admixture used in the mix. If this percent of an admixture is known (or assumed), then the maximum dry density and optimum moisture content may be determined using the known percentage. However, if the objective of the testing program is to determine the optimum percent of an admixture, then the testing procedure must be altered because the maximum dry density and optimum moisture content vary with increasing percentage of an admixture. These variations are illustrated, for example, in Figures 3- 


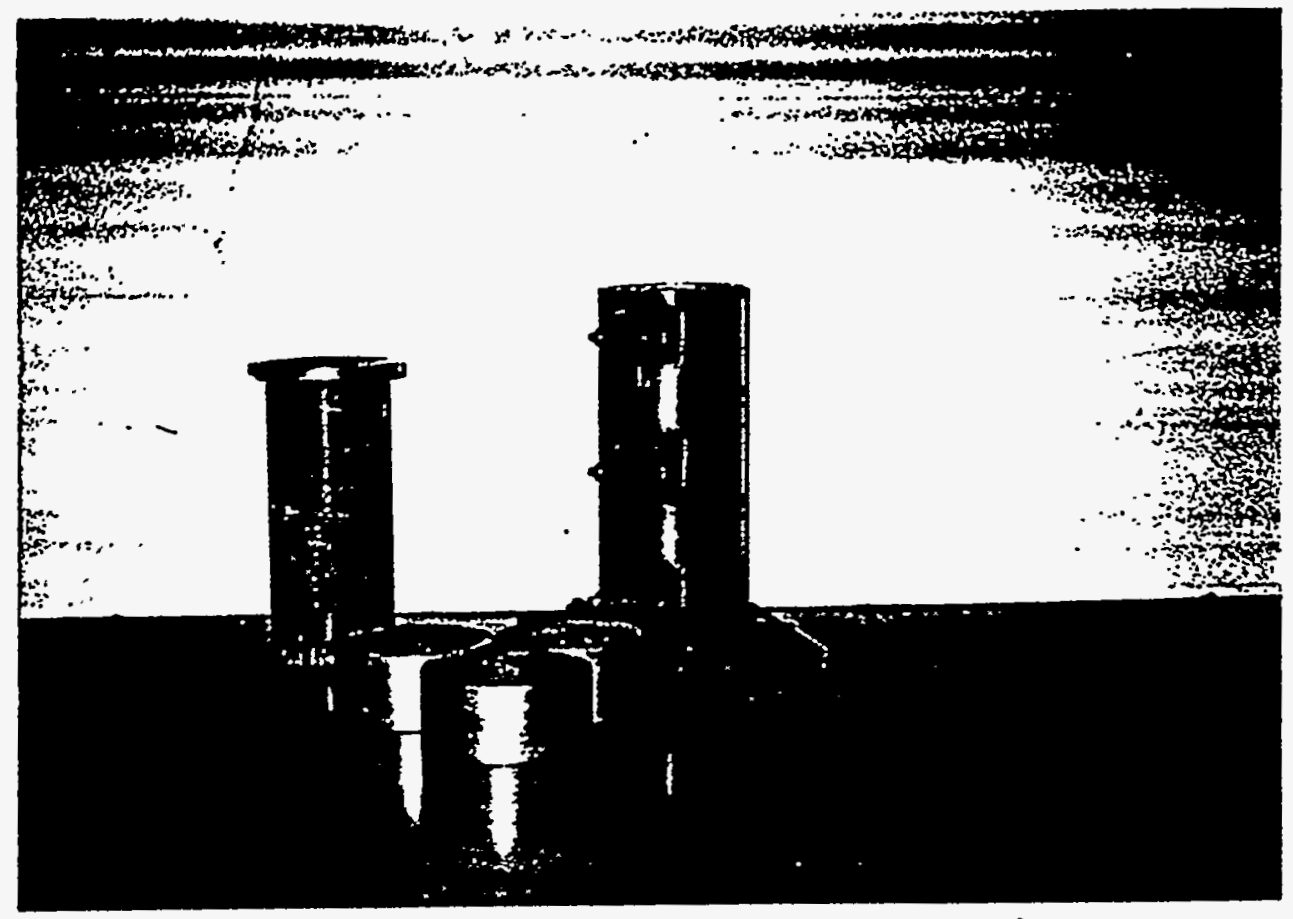

Figure 3-27. View of the split mold, rings, and ram

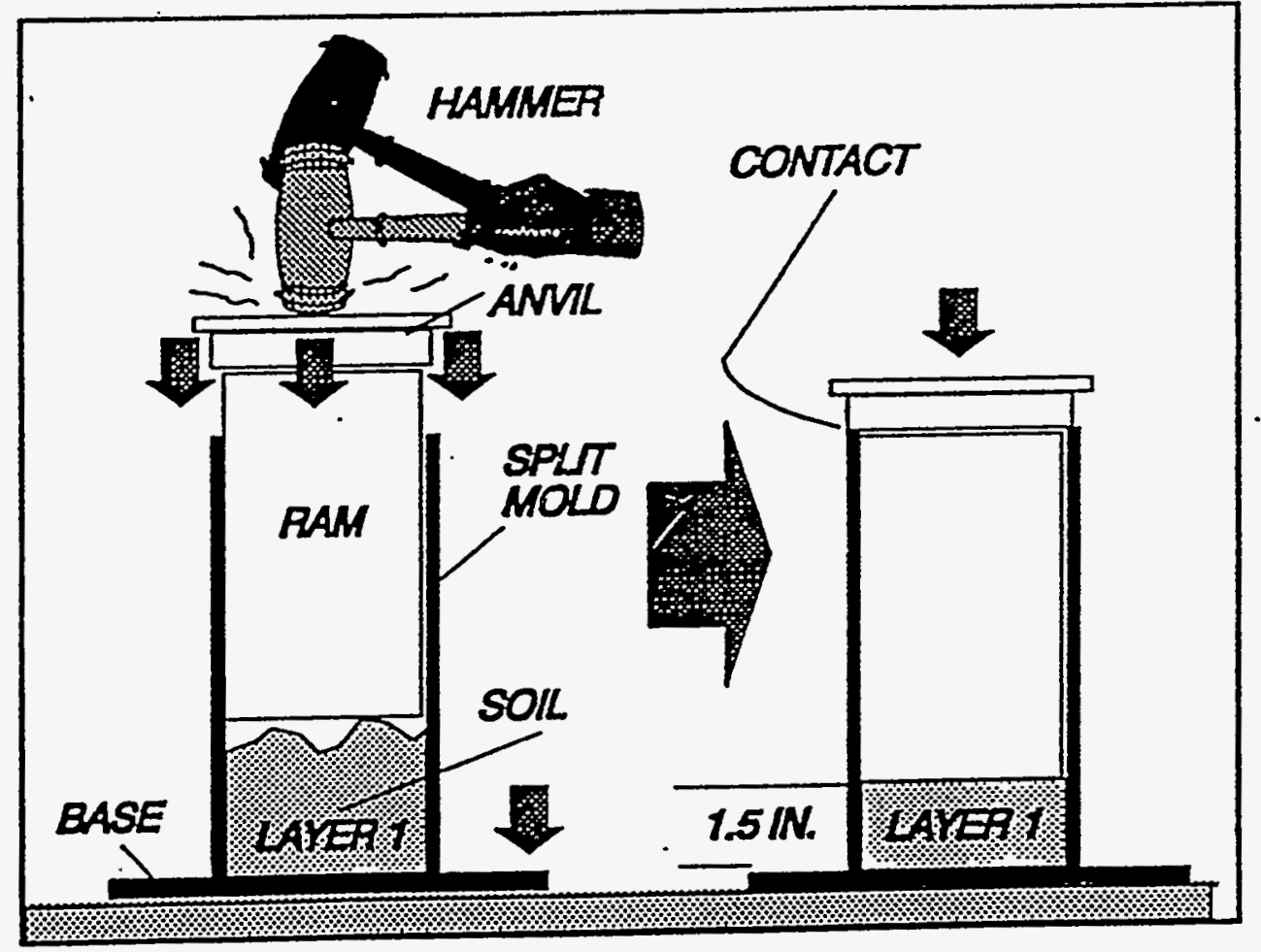

Figure 3-28. Compaction of a specimen 


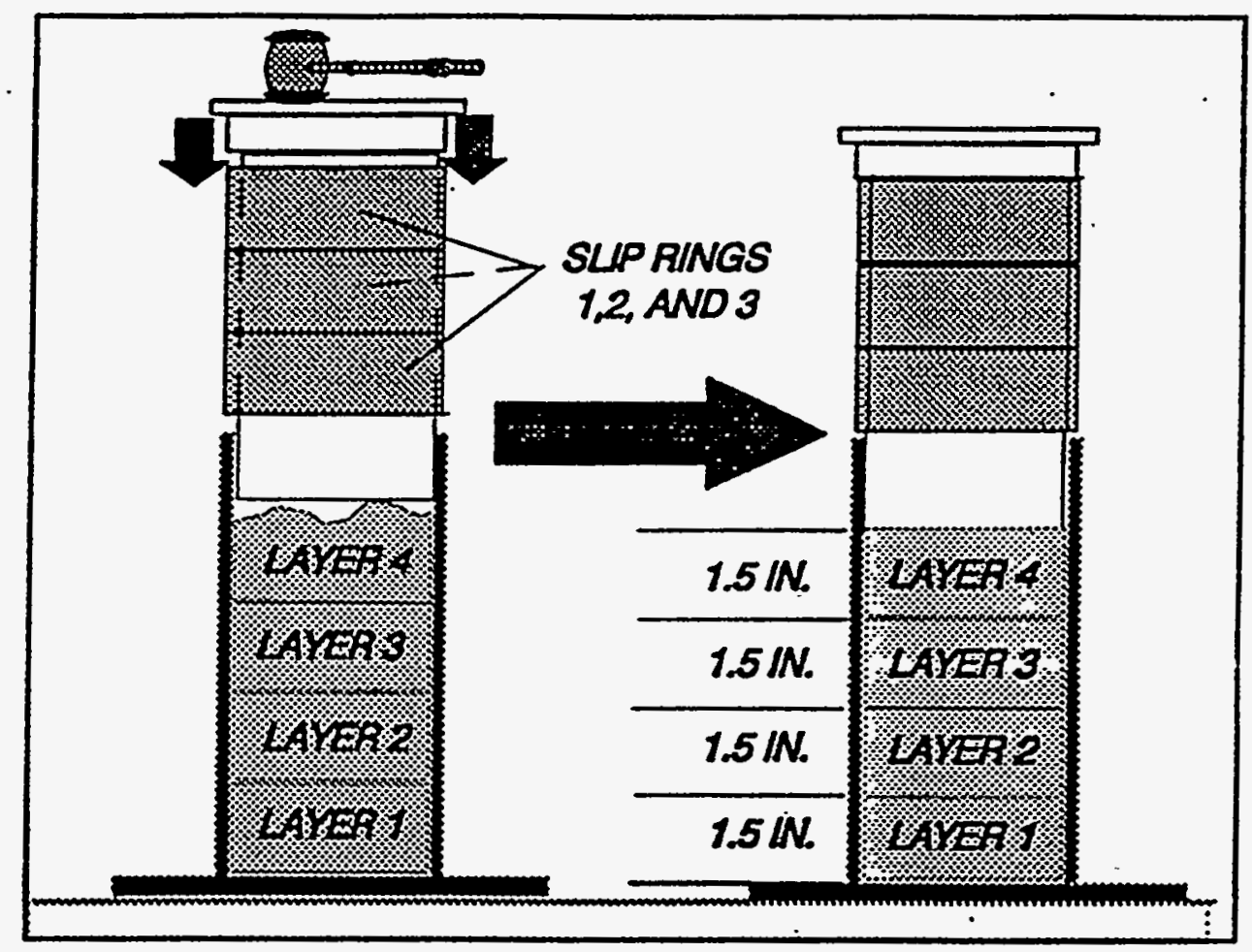

Figure 3-29. Compaction procedure is repeated for four layers

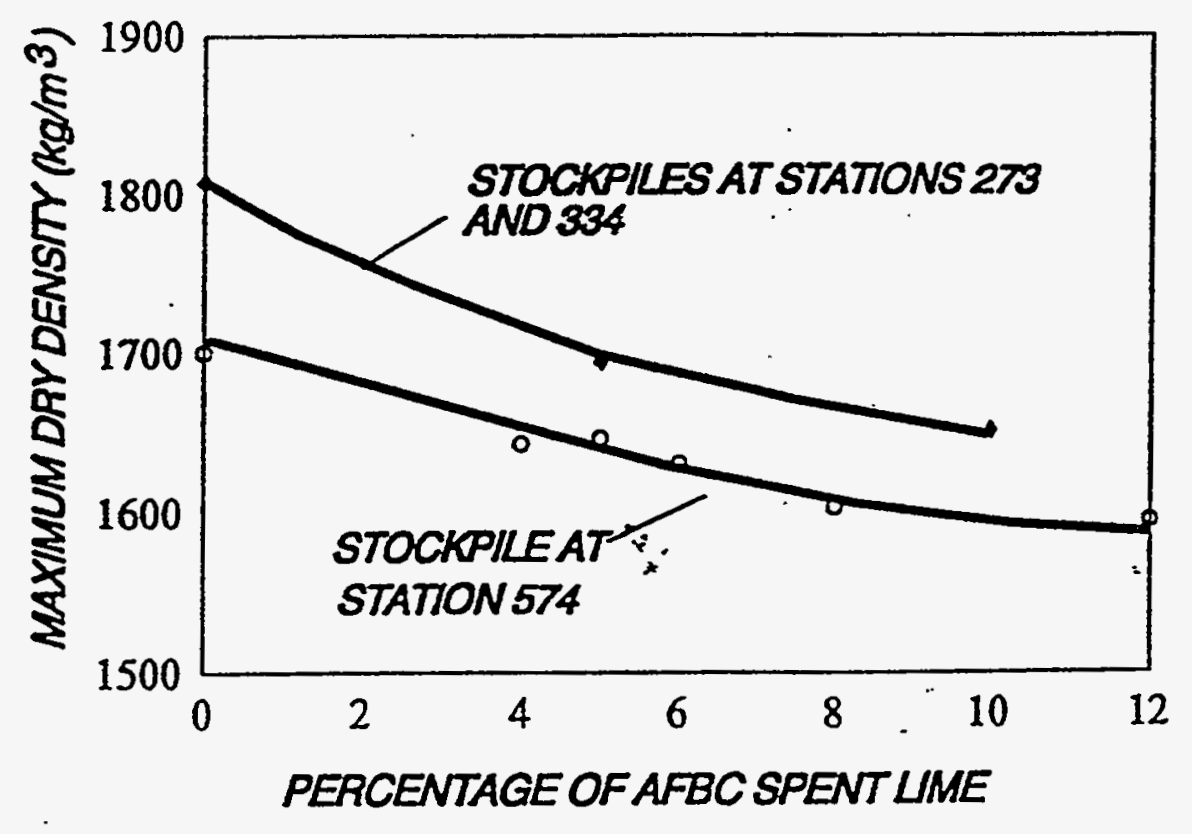

Figure 3-30. Variation of the maximum dry density with the percentage of AFBC spent lime 


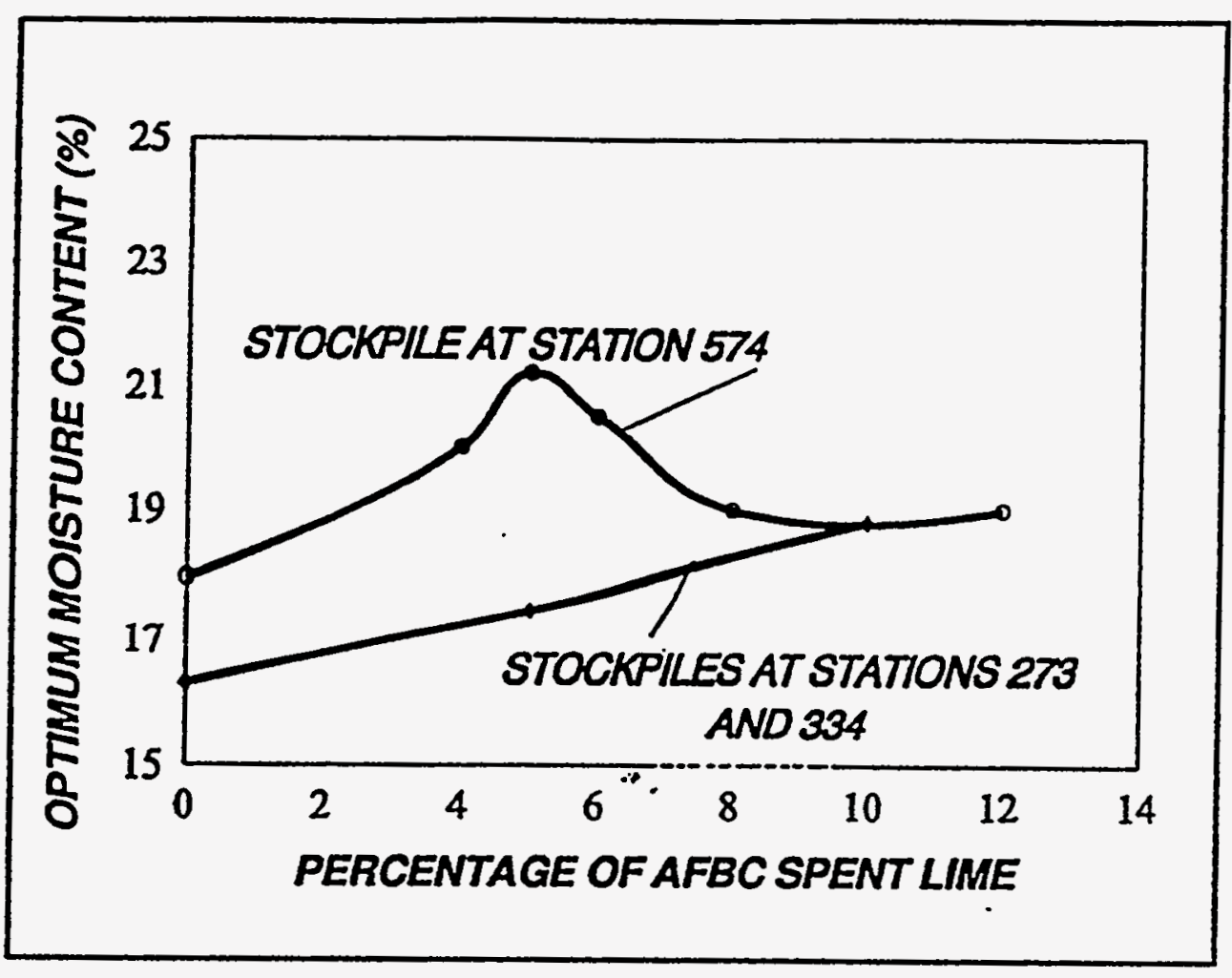

Figure 3-31. Variation of optimum moisture content with the percentage of AFBC spent lime

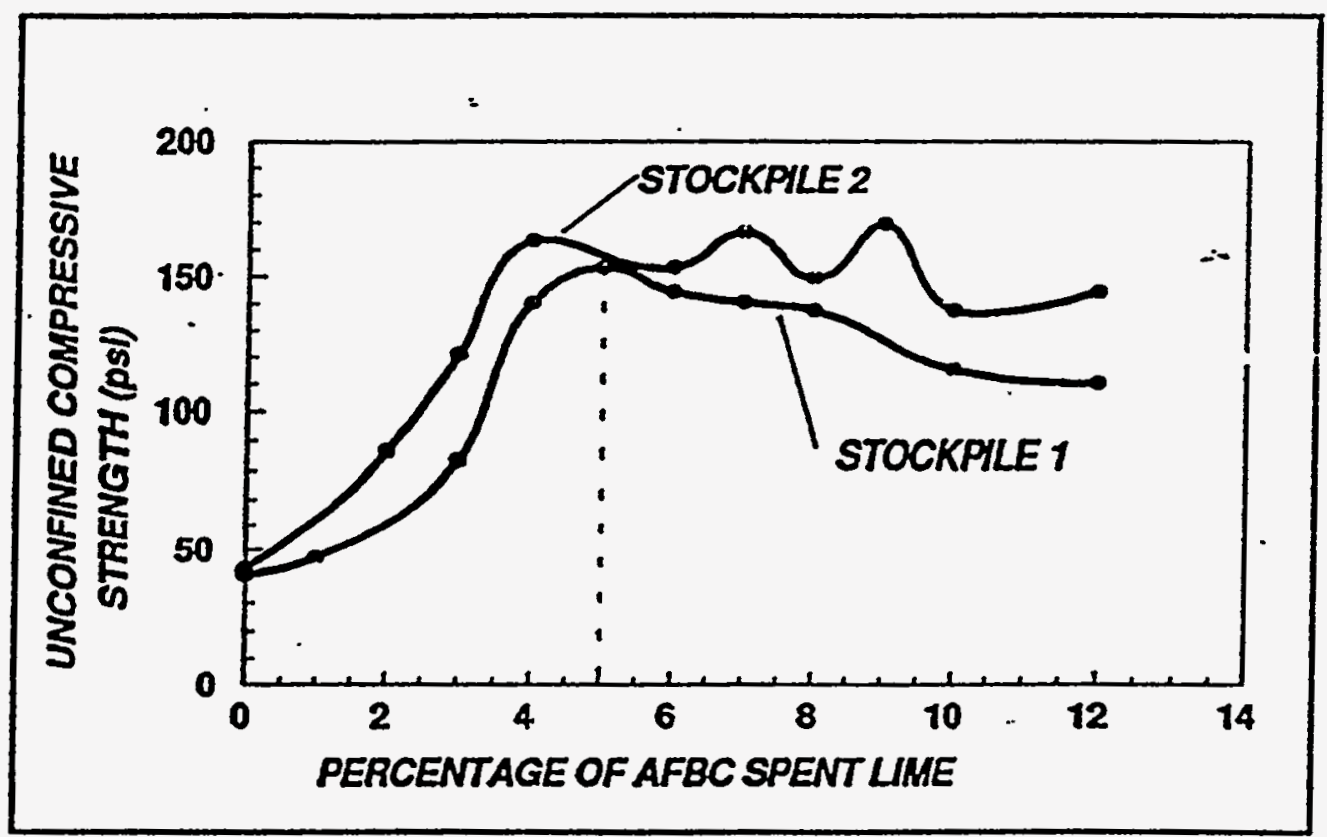

Figure 3-32. Results of unconfined compressive strength tests performed on different remolded specimens of AFBC byproduct-soil mixtures 
30 and 3-31. This example involves mixtures of an AFBC byproduct (or admixtures) and a clayey soil. As the percent of AFBC byproduct increases, the maximum dry density decreases and the optimum moisture content increases. Therefore, to account for these variations, three to five compaction tests may need to be performed on the soil-admixture mixtures using different, selected percentages.

The percent of admixture is ranged from a low percent to a high percent. Since the objective of this testing program was to determine the maximum strength (unconfined compressive strength) and corresponding optimum percent of an AFBC byproduct, several compaction tests were performed on mixtures containing different percentages. Unconfined compressive tests were performed on specimens remolded to selected percentages of the AFBC byproduct. For a selected percent of an AFBC byproduct, the maximum dry density $\left(\gamma_{d}\right)$ and optimum moisture contents were selected from the curves shown in Figures 3-30 and 3-31. Equations 7 through 13 are used to calculate the amounts of admixture, soil, and water that must be mixed to form a specimen of a known dry density, moisture content, and volume. Figure 3-32 shows the results of unconfined compressive tests performed on different remolded specimens of AFBC byproduct-soil mixtures. As these data show, the maximum strength occurred at 5 percent.

Statistical Analysis. To determine the reliability of the remolding procedure, actual dry densities and moisture contents obtained from the proposed compaction procedure were compared statistically to target values of dry densities and moisture contents. Seventy cases, which represented a variety of fine-grained soils, and different values of relative compaction, were analyzed. Deviation of the average dry density, or the difference between the actual dry density of the compacted specimen and target dry density, was $\pm 0.011 \mathrm{kN} / \mathrm{m}^{3}\left(0.07 \mathrm{lb} / \mathrm{ft}^{3}\right)$. Standard deviation was $\pm 0.121 \mathrm{kN} / \mathrm{m}^{3}(0.77$ $\left.\mathrm{lb} / \mathrm{ft}^{3}\right)$. Deviation of the average moisture content, or the difference between the actual moisture content of the compacted specimen and the target moisture content, was 
-0.31 percent. Standard deviation was \pm 0.88 percent.

\section{Triaxial Strength Properties}

The short-and long-term stabilities of geotechnical facilities constructed with, or on, the Coolside byproduct will depend on the shear strength of the Coolside material at different times of aging, since the material has pozzolanic properties. Since construction equipment must operate on the material during construction or during land filling of the material, the initial bearing, or shear strength, is important to insure mobility and efficient operation of construction traffic. A knowledge of the long-term shear strength of the material is vital to maintaining stable structures throughout their useful lives. Results of triaxial and bearing tests are described below. The triaxial testing program consisted of performing, unconsolidated-undrained (UU) triaxial compression tests and unconfined triaxial compression tests (UC). All specimens were remolded to $95 \%$ of standard maximum dry density and optimum moisture content (ASTM D 698). The specimens were aged in sealed containers at room temperature (about $21^{\circ} \mathrm{C}$, or $70^{\circ} \mathrm{F}$ ).

Unconsolidated-Undrained (UU) Triaxial Compression Tests. A minimum of three UUtests (ASTM D 2850) was performed at different cell pressures at a selected curing period. The tests were performed on unsaturated, aged specimens of the Coolside byproduct to observe the effects of aging, or curing, on the total stress parameters, the angle of internal friction, $\phi_{u}$, and cohesion, $c_{u}$. Generally, confining pressures of 138 , 276 , and $414 \mathrm{kPa}(20,40$, and $60 \mathrm{psi})$ were used for a given series at a selected aging time. Since the strength changes with aging time--as observed in the first initial testing of the remolded Coolside material--each specimen of a test series $(38,276$, and 414 $\mathrm{kPa}$ ) was tested precisely at the end of the selected aging time. Otherwise, data fits to determine the strength parameters would not have been valid. Variations of the 
strength parameters, $\phi_{u}$ and $c_{u}$, with aging time are shown in Figures 3-33 and 3-34. The strength parameter, $c_{u}$, increased from about $62 \mathrm{kPa}$ (one-day aging time) to 466 $\mathrm{kPa}$ (21-day aging time). The strength parameter, $\phi_{u}$, increased from about $29.3^{\circ}(1-$ day aging time) to $32.9^{\circ}$ (21-day aging time).

Variations of the $\phi$ - and c-values at each selected curing time are shown in Figures 333 and 3-34. In curing and testing the triaxial specimens, each specimen was tested exactly at the end of the selected curing period. Otherwise, the tests of a set would not be valid, since each specimen continued to gain strength as it cured. As shown in Figure 3-33, the internal angle of friction, $\phi$, increased slightly as the curing period increased. The $\phi$-value increases from about 29 degrees at one day of curing to about 33 degrees when the compacted specimens were cured for 21 days. The relationship between the cohesive component, $\mathrm{c}$, and aging time is shown in Figure 3-34. The ccomponent increases from about $60 \mathrm{kPa}$ at one day of curing to about $465 \mathrm{kPa}$ at 21 days of curing. Apparently, the cohesive component continues to increase with curing time after 21 days. Specimens could not be tested for longer curing periods because the strengths exceeded the capacity of the triaxial equipment.

Unconfined Compression Tests. Unconfined compression tests (ASTM D 2850) were performed on remolded specimens, which were compacted to different compactive states, to examine the effects of aging on strength. Specimens were compacted to 90 , 95 , and 100 percent of maximum dry density obtained from a low-energy compaction method, standard compaction, and modified compaction. In each case, the specimens were compacted to optimum moisture content obtained from each compaction method, respectively. Moreover, the unconfined compression tests were performed on specimens that been aged to different curing periods. Each specimen, after remolding, was wrapped in Saran ${ }^{\circledR}$ wrap and stored in PVC cylinders that measured about 6.5 inches $(16.5 \mathrm{~cm})$ in height and about 3 inches $(7.62 \mathrm{~cm})$ in diameter. Rubber (Shelbytube) caps were placed on each end of the cylinder and taped. This insured that the 


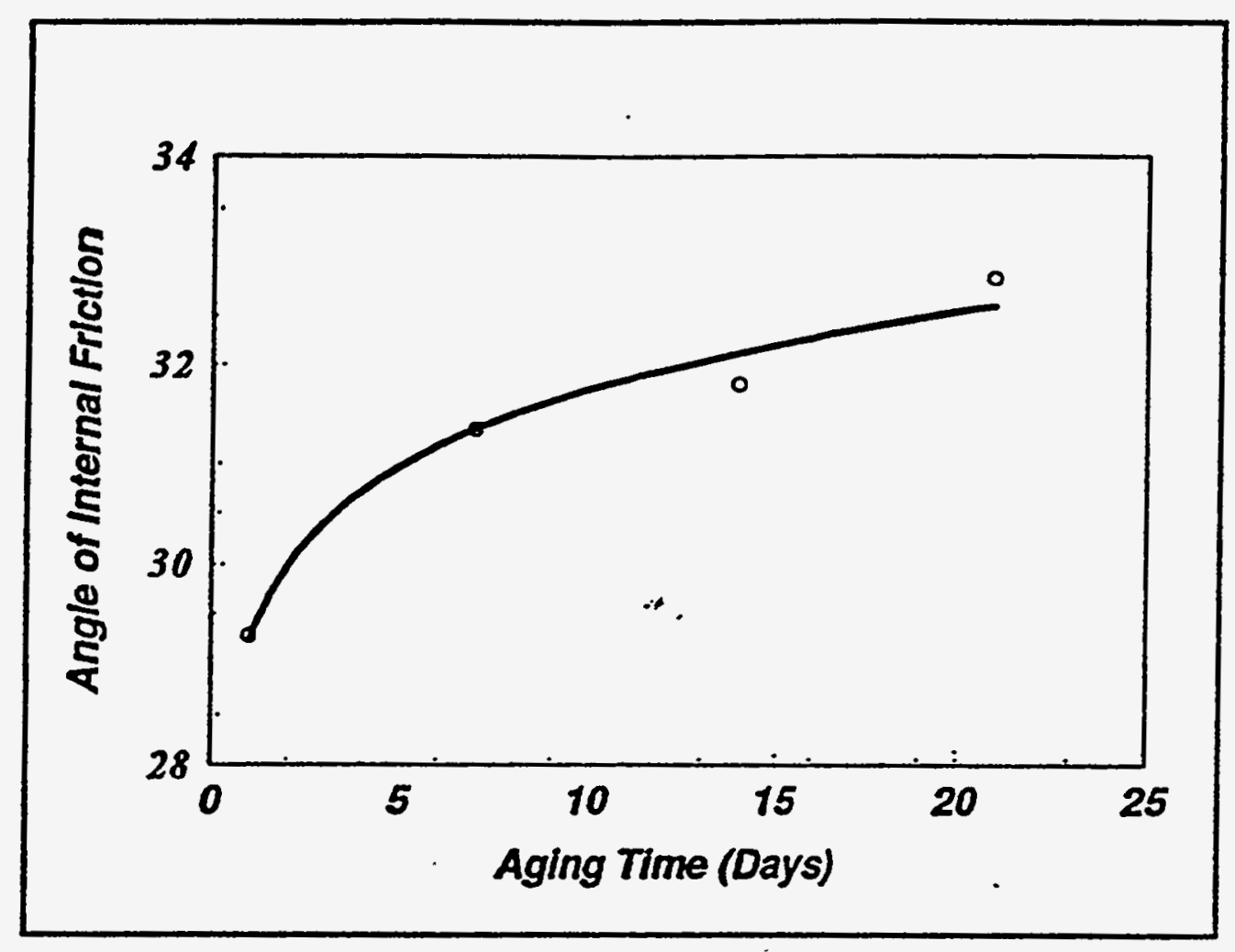

Figure 3-33. Variation of the angle of internal friction, $\phi$, with aging time

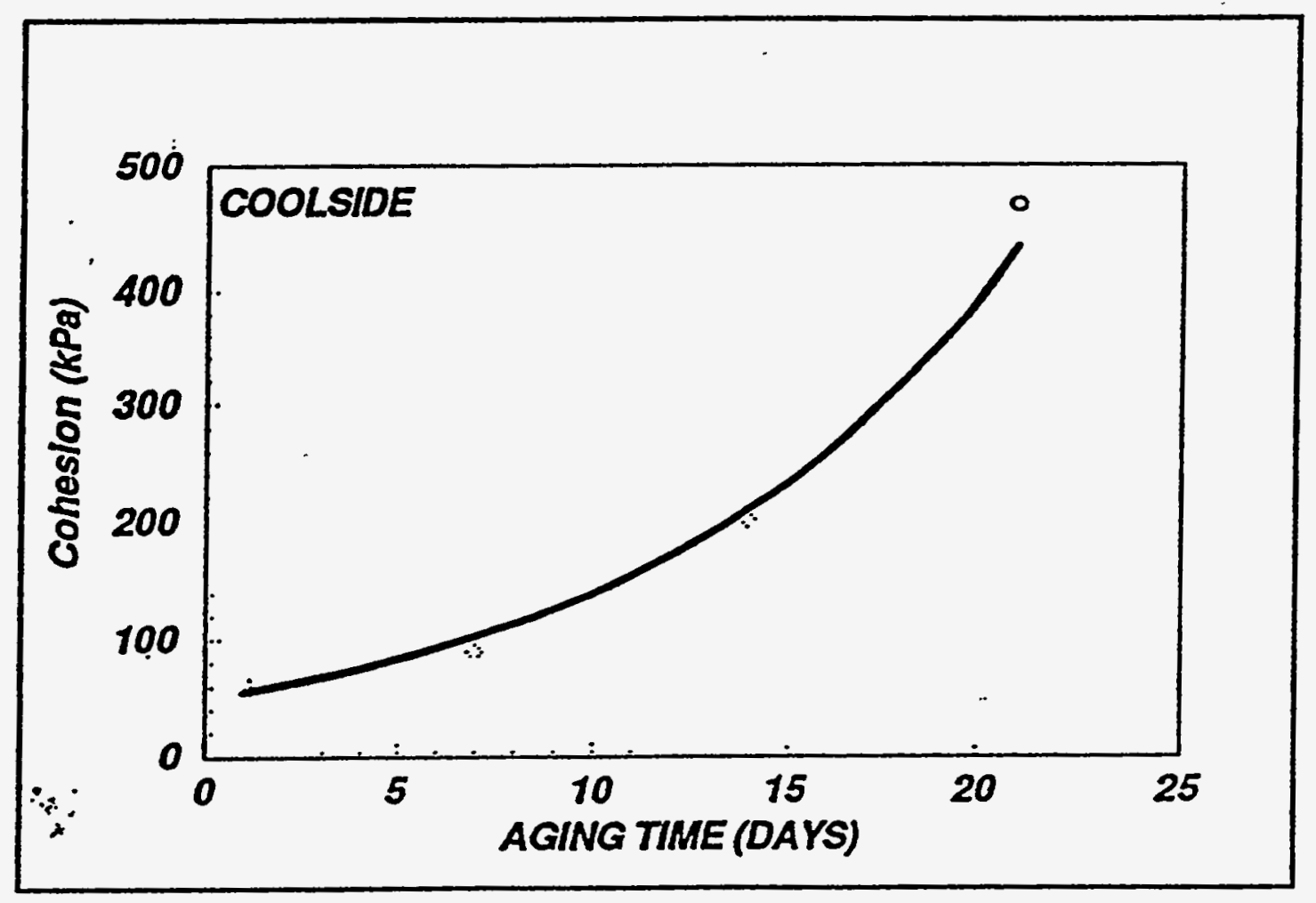

Figure 3-34. Variation of cohesion, $c_{u}$, with aging time

115 
specimens did not lose moisture doing storage. The specimens were cured, or aged, to different periods. Some specimens were aged for 3.5 years. The specimens were aged in sealed containers at room temperature (about $21^{\circ} \mathrm{C}$ ). Two series of tests were performed. The first series consisted of performing the unconfined compressive tests at different aging times in an unsaturated state. The second series consisted of performing tests on specimens saturated during permeability testing.

Results of the unconfined compression tests are shown as a function of curing time in Figures 3-35, 3-36, and 3-37. Unconfined strengths of the specimens compacted to $90 \%$ of maximum dry density obtained from low energy, standard, and modified compaction methods increased rapidly in the first 90 days. The low energy specimens obtained a strength of about $540 \mathrm{psi}(3721 \mathrm{kPa})$ in the first 90 days while the specimens compacted to standard compaction increased to $660 \mathrm{psi}(4548 \mathrm{kPa})$. The specimens that were compacted using modified energy reached a strength of $1150 \mathrm{psi}$ $(7925 \mathrm{kPa})$ in the first 90 -day period. In each of the three cases, the strengths decreased after peaking at 540,1850 , and 1850 days, respectively (to values of about 1028,542 , and $1374 \mathrm{psi}(7084,3735$, and $9468 \mathrm{kPa})$, respectively). As shown in Figure 3-30, the peak unconfined strengths of specimens compacted to $100 \%$ of maximum dry density obtained from low energy, standard, and modified were 1922 , 2488 , and $2610 \mathrm{psi}(13,245,17,145$, and $17,985 \mathrm{kPa})$, respectively. However, the strengths of the standard and modified specimens decreased with increasing curing time after the peak strengths had been reached at curing times of 365 and 730 days, respectively.

\section{California Bearing Ratio}

Both short-term and long-term California Bearing Ratio (CBR) tests were performed on compacted specimens of the Coolside material. Results of the short-term tests are shown in Figure 3-38. All specimens were compacted to $95 \%$ of maximum dry density 


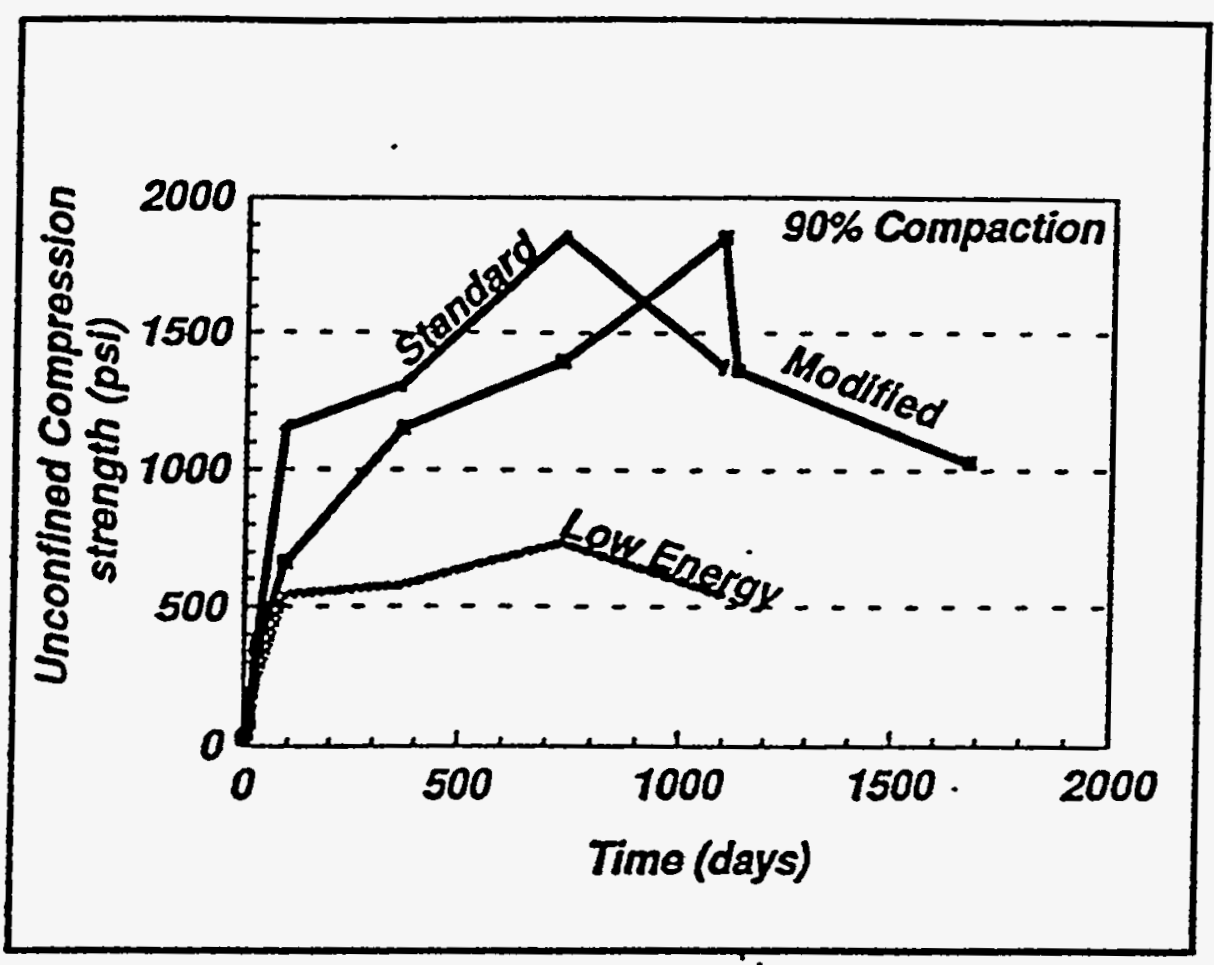

Figure 3-35. Unconfined compressive strength as a function of curing time for Coolside specimens compacted to $90 \%$ of low energy, standard, and modified compaction

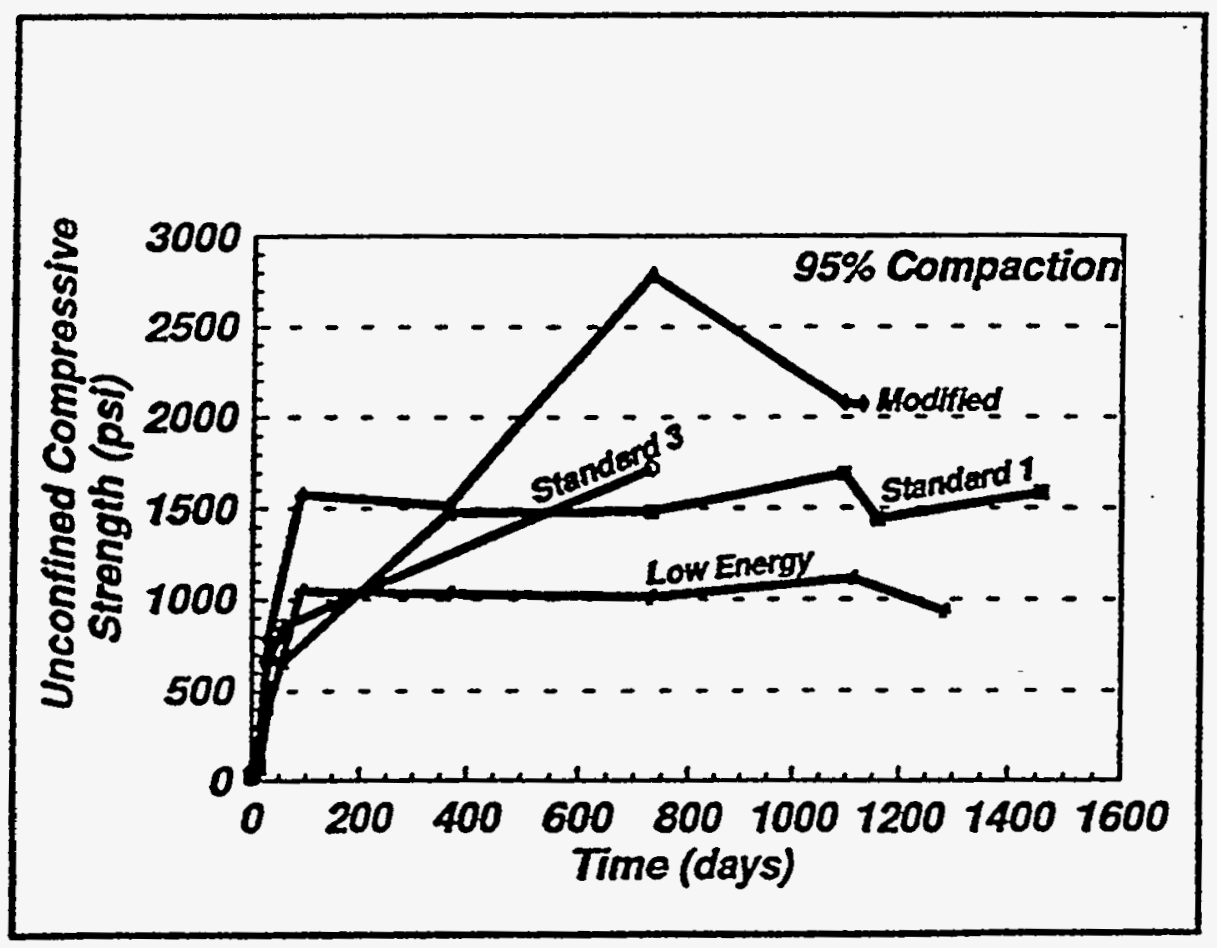

Figure 3-36. Unconfined compressive strength as a function of time for Coolside specimens compacted to $95 \%$ of low energy, standard, and modified compaction 


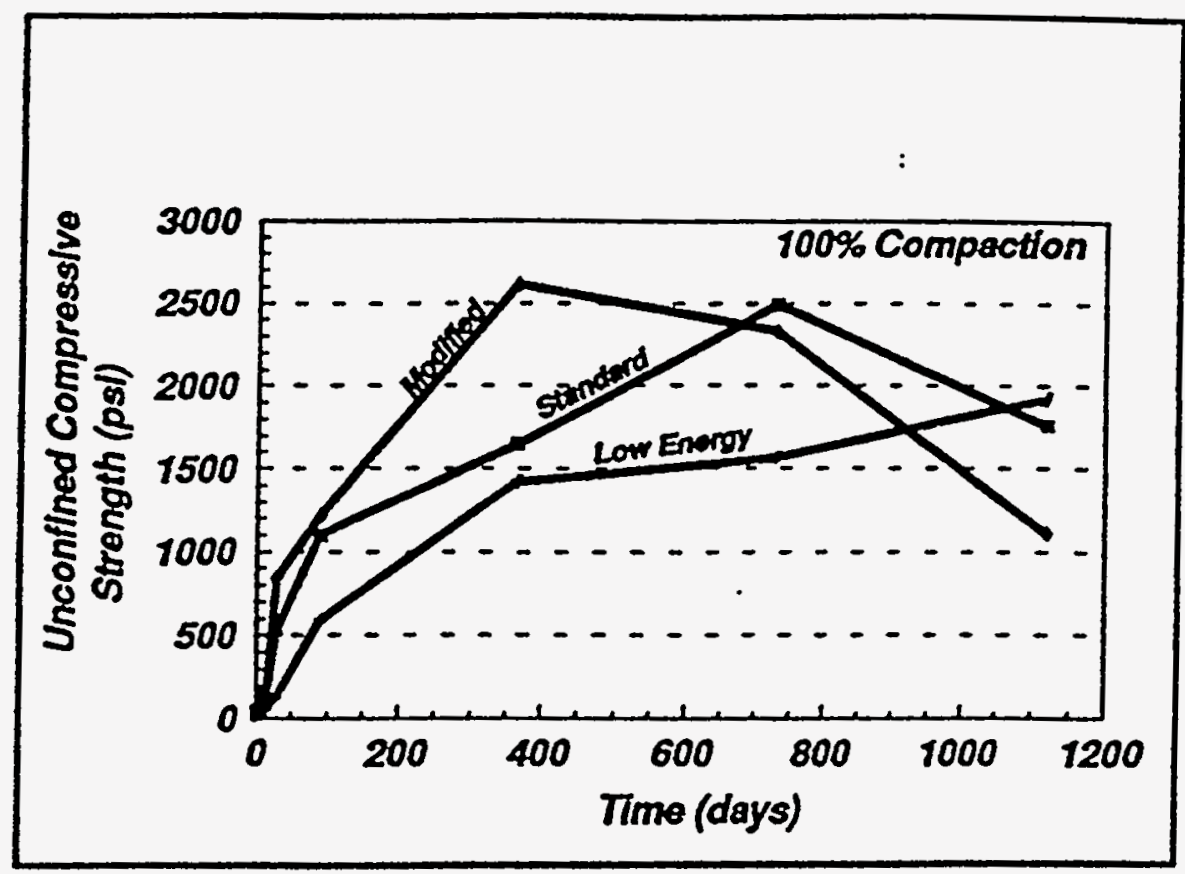

Figure 3-37. Unconfined compressive strengths as a function of time for specimens compacted to maximum dry density obtained from low energy, standard, and modified compaction

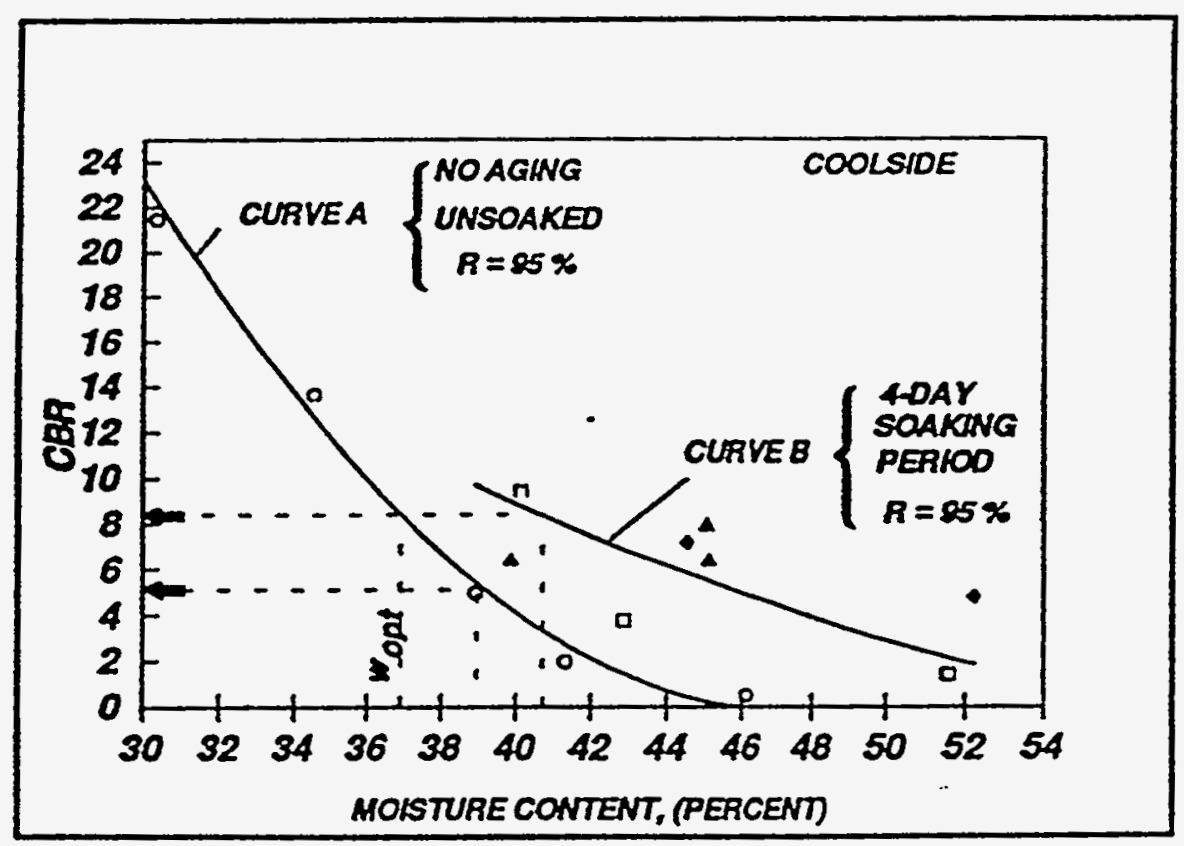

Figure 3-38. California Bearing Ratio (CBR) test values as a function of molding moisture content 
obtained from ASTM D 698. The purpose of those tests was to characterize the bearing strengths of the Coolside material during construction handling. The relationship between $\mathrm{CBR}$ and compaction moisture content is represented by curve $A$ in Figure 3-38. Here, none of the specimens were aged or soaked before the CBR test was performed. The tests were performed immediately after compaction. The intent of these tests was to simulate the field bearing strengths of the Coolside material during construction. As the moisture content of the specimens increases, the CBR value decreases. When the moisture content increases above the optimum moisture content $\left(w_{\text {opl }}\right)$, the CBR strengths decrease rapidly to small values. At two percent above optimum moisture content, the CBR value is only about 5 .

Curve B in Figure 3-38 represents the relationship between CBR and moisture content of specimens cured and soaked in water for 4 days. The tests were performed after the specimens had been soaked for four days. Although the moisture contents of the soaked specimens were greater than the moisture contents of specimens represented by Curve $A$, the CBR strengths were comparable to those values represented by Curve B. For example, at a moisture content of $41 \%$, the CBR of the 4-day soaked specimens (Curve B) was 8. At a CBR of 8 , the moisture content of the unsoaked specimen (Curve $A$ ) is about $37 \%$. Consequently, during the soaking period, the compacted Coolside material gained strength. Apparently, pozzolanic reactions occurred during the soaking, or curing period.

Long-term CBR strength of the Coolside ash was also examined. Specimens of the ash were remolded in CBR molds (Figure 3-39) to $95 \%$ of maximum dry density and . optimum moisture content. Results obtained from the CBR tests are shown in Figure 340. In these tests, the CBR specimens of the Coolside ash were allowed to swell until secondary swell was reached, or essentially most of the swelling had occurred before the CBR test penetration was performed. Time to complete swell ranged from about 2.6 to almost 6 months. The soaked values of CBR always exceeded 100--exceedingly 


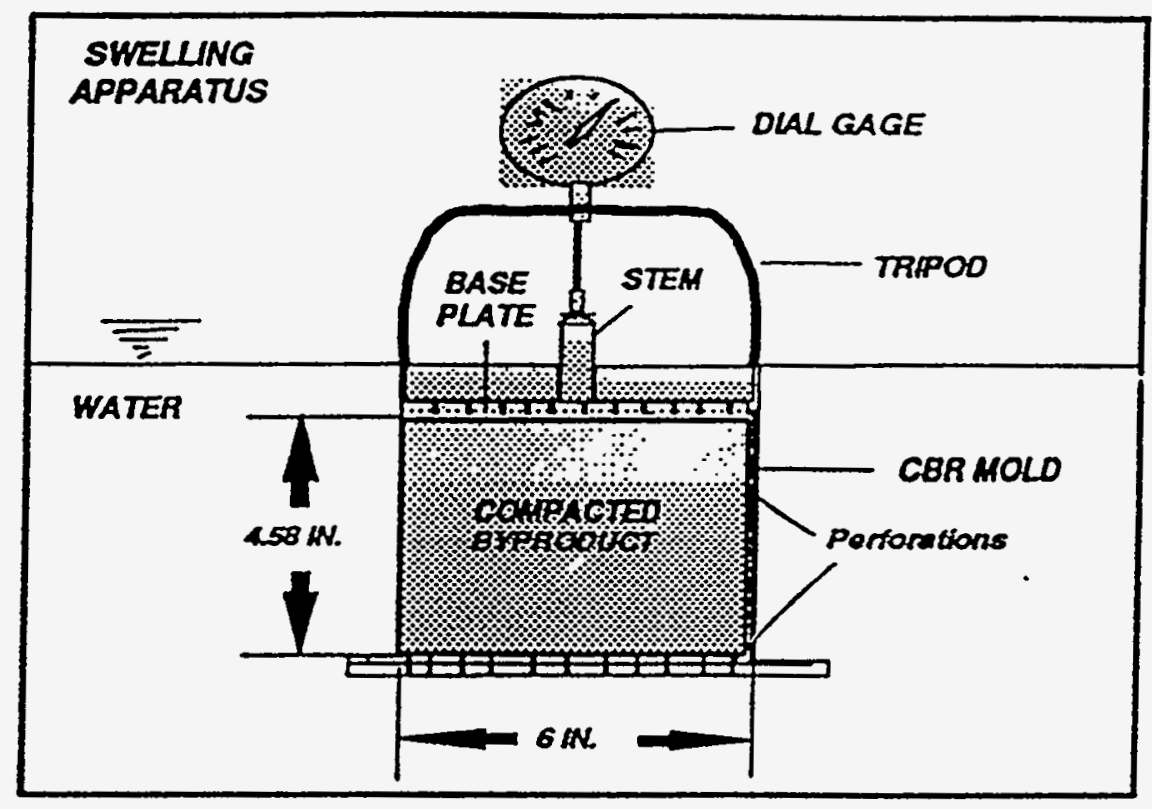

Figure 3-39. CBR mold

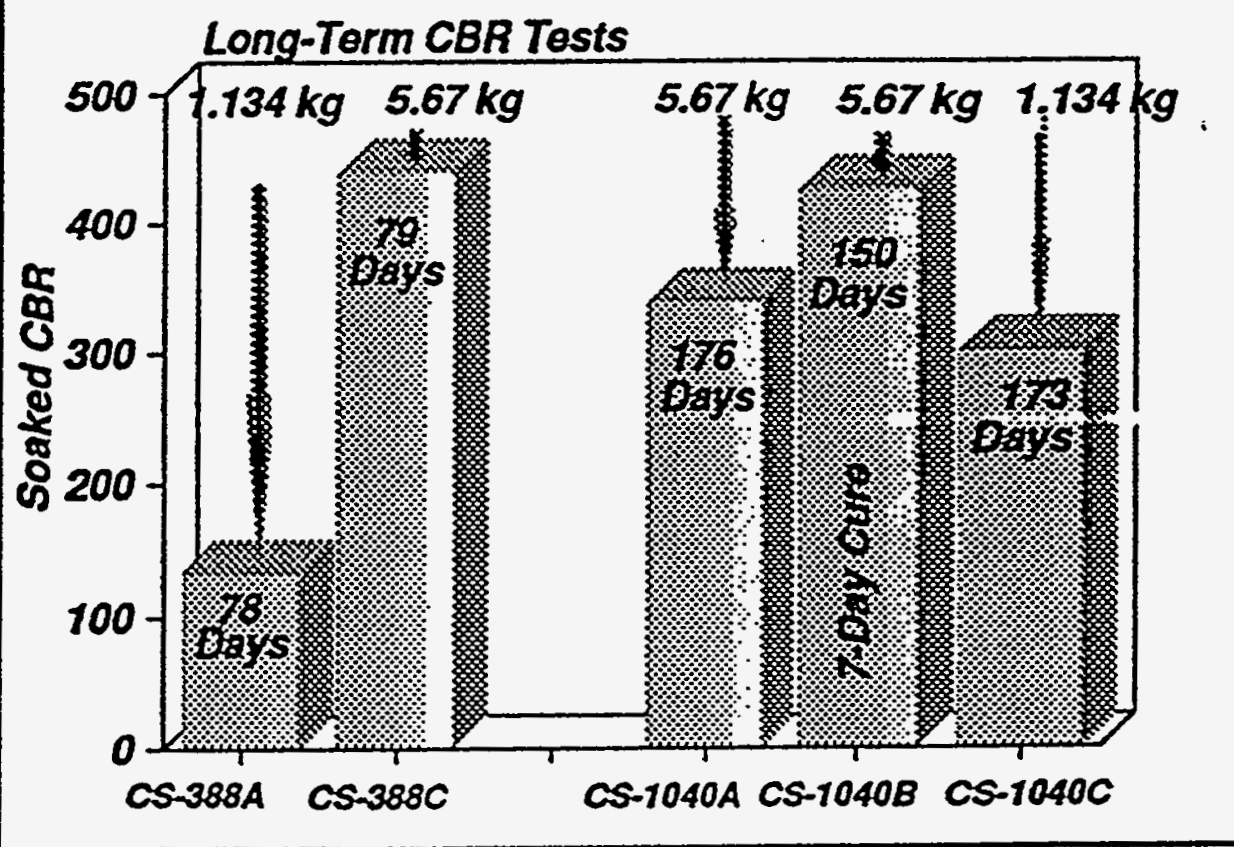

Figure 3-40. Long-term, soaked CBR values of the Coolside ash 
large values.

\section{Permeability}

Permeability, or hydraulic conductivity, is the ability of a material to transmit fluid. Hydraulic conductivity depends on the unit weight, pore spacing, and degree of saturation of the material. The coefficient of permeability, $k$, is an important parameter used in engineering applications to decide the rate and quantity of flow through a material. Permeability tests were performed on specimens remolded to different densities and different aging times. The specimens were remolded to 90, 95, and $100 \%$ of maximum dry densities obtained from low-energy, standard, and modified compaction. Optimum moisture contents obtained from the respective compaction methods were used in remolding the specimens. The tests were performed using a back saturation technique and falling head method in triaxial cells. Equations for calculating the coefficient of permeability have been described by Daniel (1989).

The coefficient of permeability, $k$, as a function of aging, or curing time, is shown in Figures $3-41,3-42$, and 3-43. Analysis shows that aging does not significantly affect the coefficient of permeability. The values decreased only slightly as the curing time increased. The coefficient of permeability ranges from about $4 \times 10^{-5} \mathrm{~cm} / \mathrm{sec}$ to $3 \times 10^{-6}$ $\mathrm{cm} / \mathrm{sec}$. The permeability of the compacted specimens of the Coolside by-product may be described as low, or very low. However, the material in a compacted state is permeable, since the coefficient of permeability is not smaller than $10^{-7} \mathrm{~cm} / \mathrm{sec}$.

Variation of the coefficients of permeability with dry density and void ratio (volume of voids divided by the volume of solids) is shown in Figures 3-44 and 3-45. As the dry density increases, the coefficient of permeability decreases and approaches $1 \times 10^{-7}$ $\mathrm{cm} / \mathrm{sec}^{2}$ as the dry density approaches a value of the maximum dry density obtained from modified compaction. The coefficient of permeability increases as the void ratio 


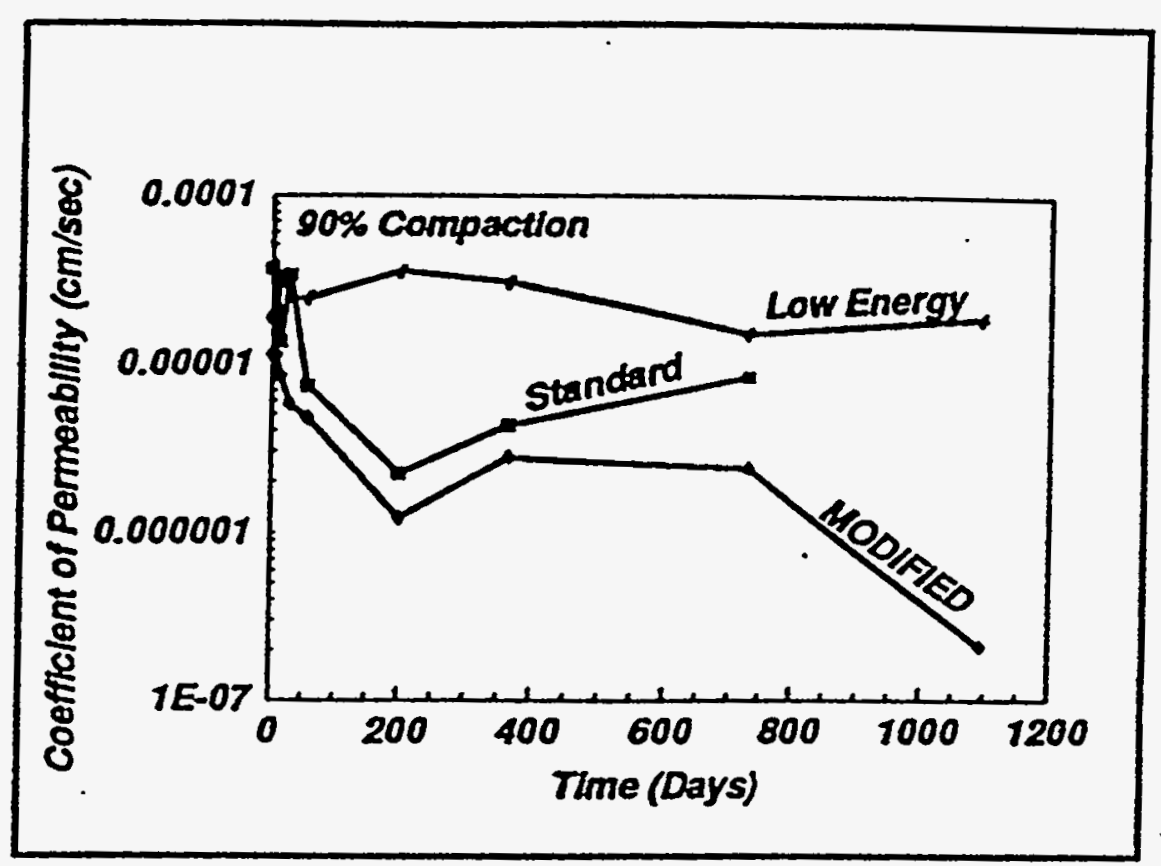

Figure 3-41. Coefficient of permeability as a function of time for specimens remolded to $90 \%$ of maximum dry density

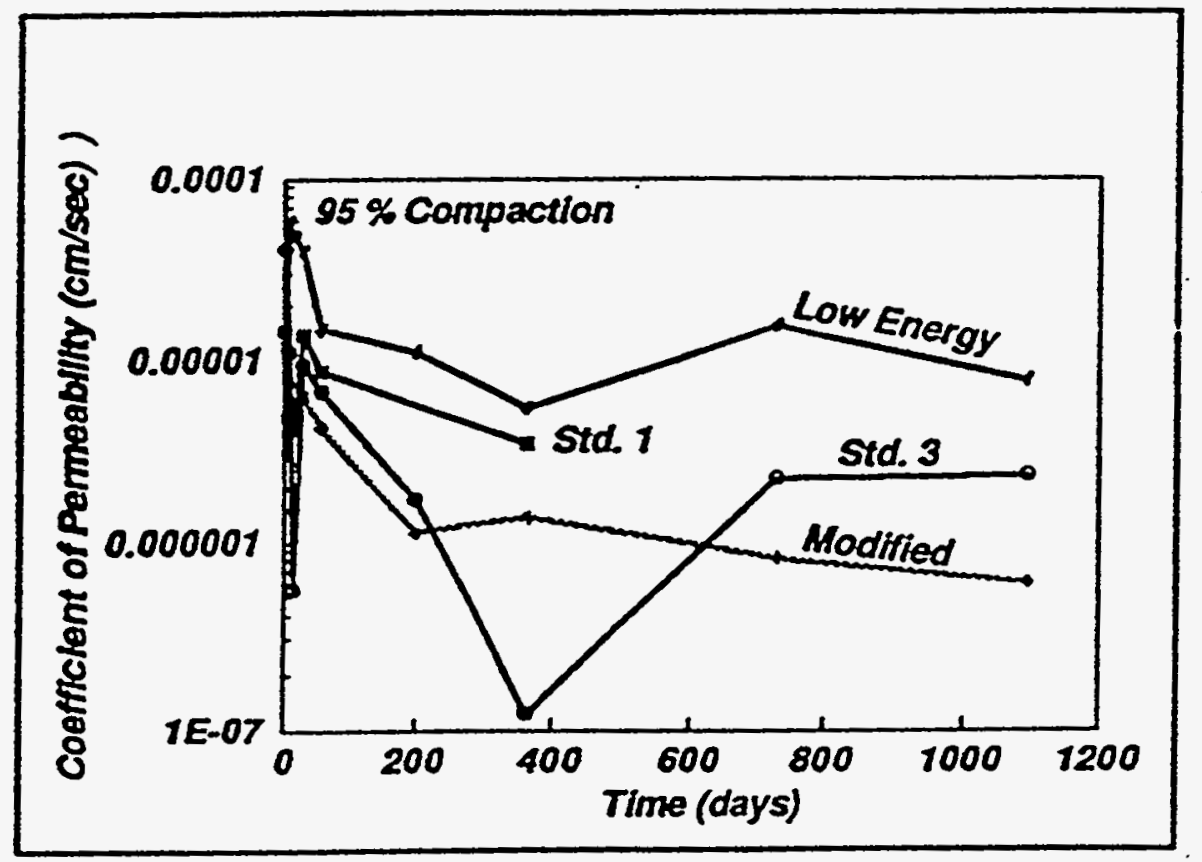

Figure 3-42. Coefficient of permeability as a function of time for specimens compacted to $95 \%$ of maximum dry density 


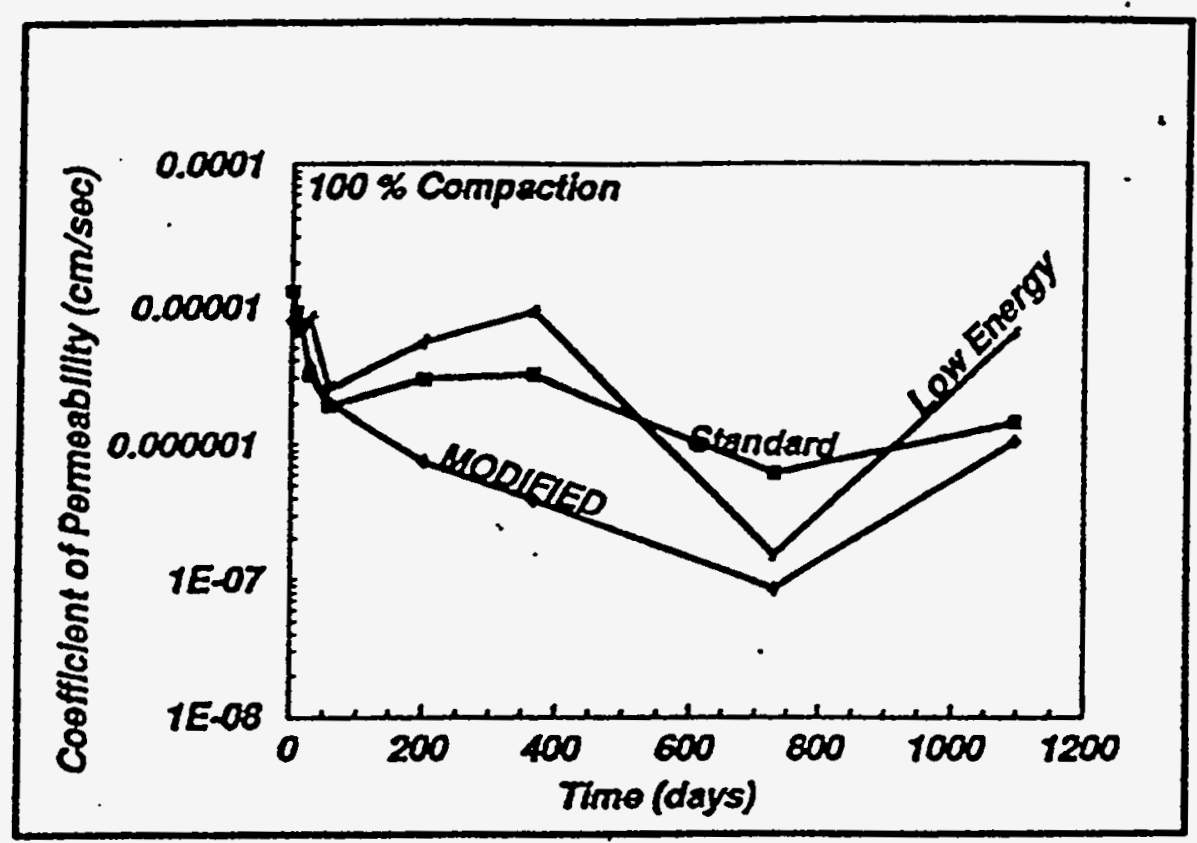

Figure 3-43. Coefficient of permeability as a function of time for specimens remolded to $100 \%$ of maximum dry density

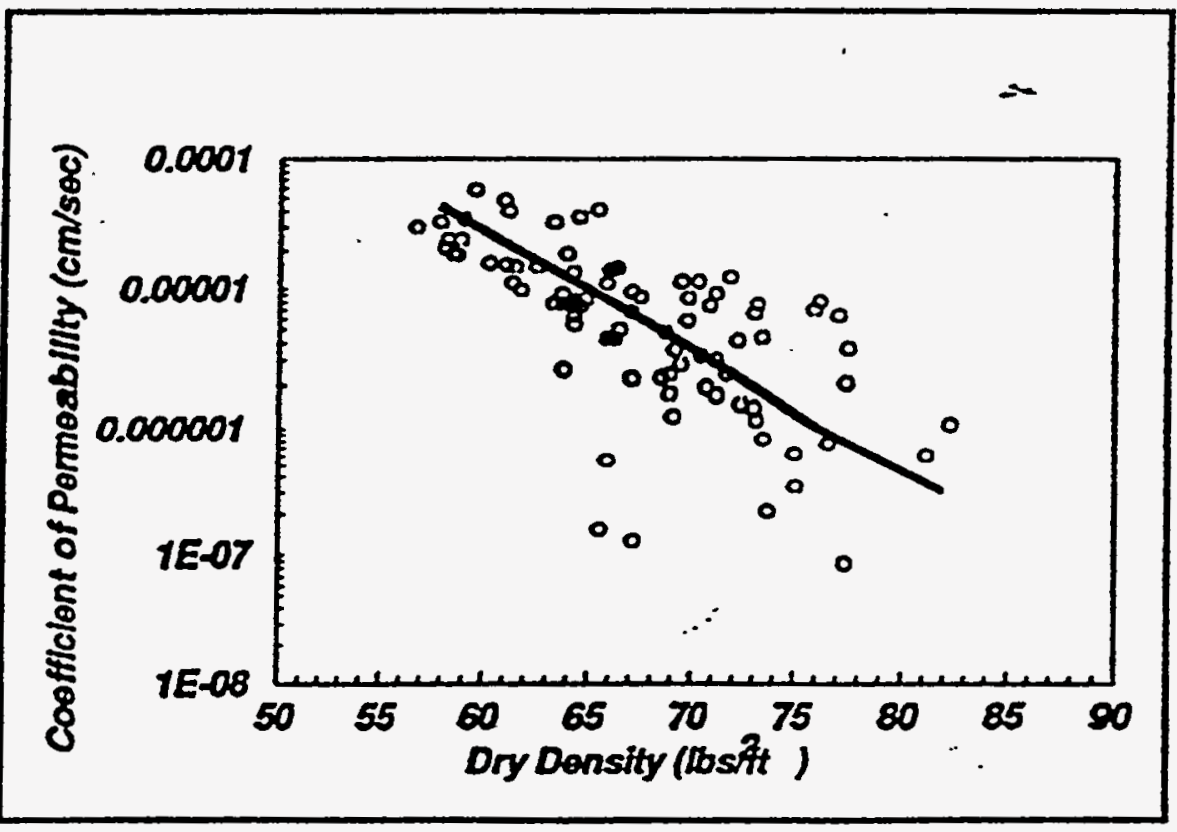

Figure 3-44. Coefficient of permeability as a function of dry density 


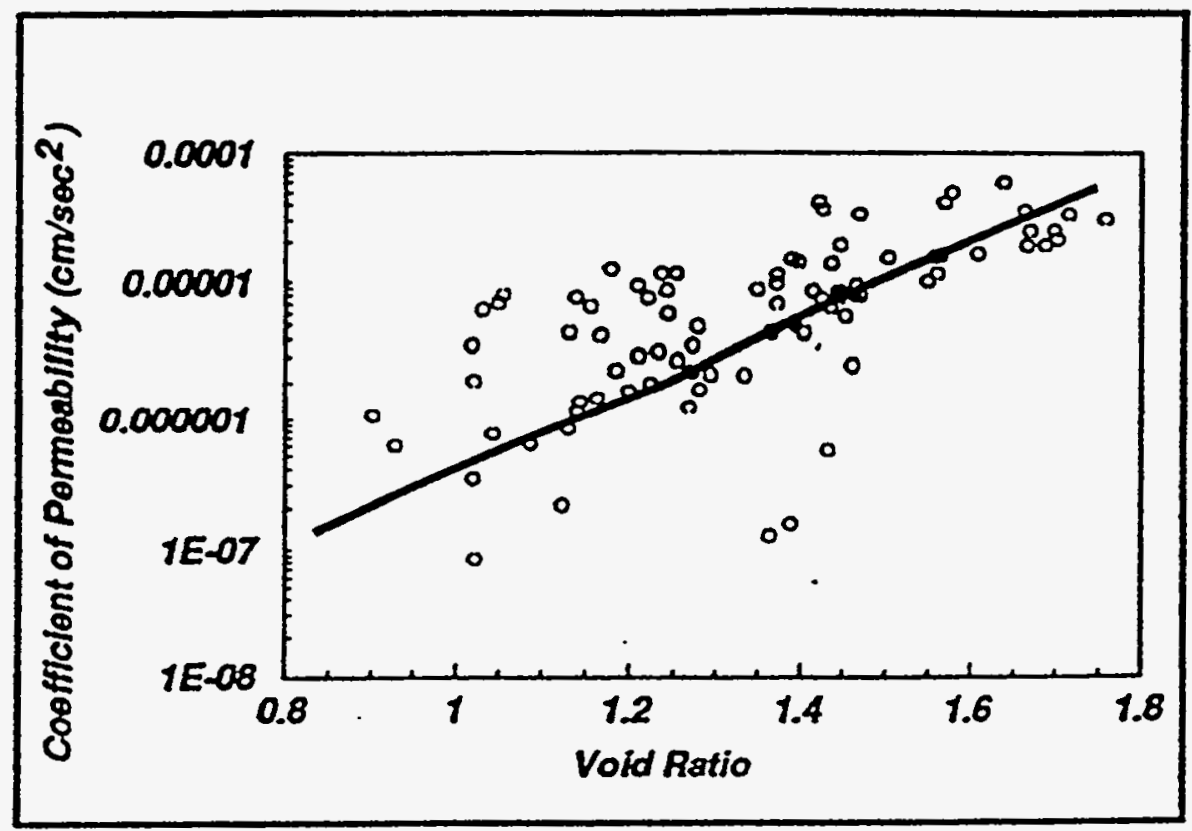

Figure 3-45. Variation of the coefficient of permeability and void ratio

COOLSIDE SAMPLES

CS-1040

CS-1050
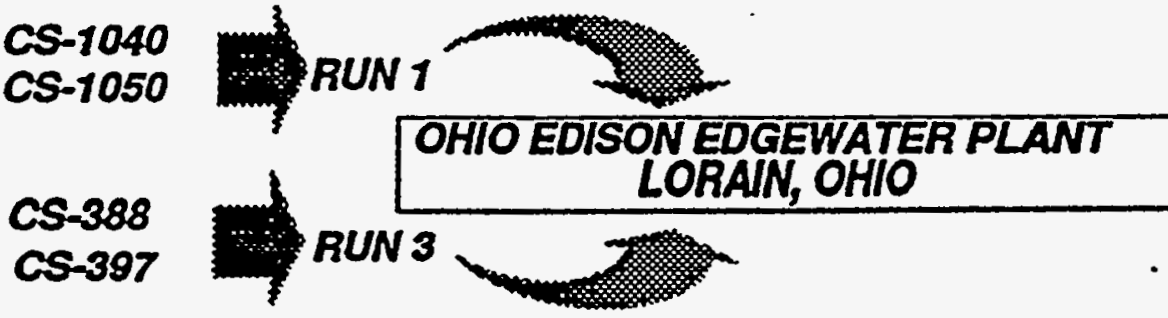

cs-2

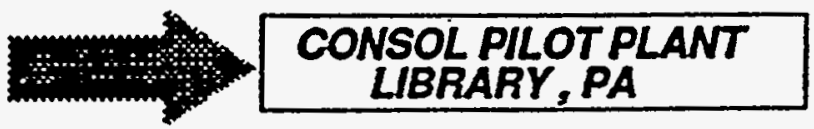

FBC SAMPLES FLUIDIZED BED COMBUSTER - PA

Figure 3-46. Sources of Coolside materials and an FBC material 
increases.

\section{Swelling Characteristics Of the Coolside Ash}

As described above, the Coolside and other FGD byproducts could be used in a number of highway applications. However, to qualify as viable materials in the highway industry, swelling of these materials must be predictable. Typically, many soils used to construct subgrades of pavements swell some zero to 20 percent. Pavements on clayey subgrades, which exhibit swelling magnitudes of $5 \%$, or greater, generally have performed poorly. When the swelling exceeds about $10 \%$, pavements have performed extremely poorly. When the swelling percentage becomes larger than about four or five, the bearing strength decreases. If swelling of the Coolside and FGD byproducts is severe, then the physical and geochemical mechanisms that may cause swelling must be understood and means to control swelling must be devised. The intent here was to examine and evaluate the swelling characteristics of these materials.

Sources Of Materials For Swelling Tests And Index Properties. Long-term swelling characteristics of dry flue gas desulfurization (FGD) byproducts obtained from a successful demonstration of Coolside technology and a Coolside pilot plant operation were examined and compared to the swelling properties of two typical clayey soils, an FBC ash, and an atmospheric fluidized bed combustion (AFBC) spent lime-clay mixture.

Coolside byproducts were obtained from the Ohio Edison Edgewater Station at Lorain, Ohio and the CONSOL Coolside pilot plant in Pennsylvania. Three series (numbers 1 , 2, and 3) of Coolside byproduct samples (Figure 3-46) were collected from the ESP hopper (Figure 3-47) of the Edgewater Station in February of 1988 during the Coolside demonstration program. Samples identified herein as CS-388 and CS-1050 were two bulk samples obtained from Edgewater sample series run numbers 1 and 3 , 


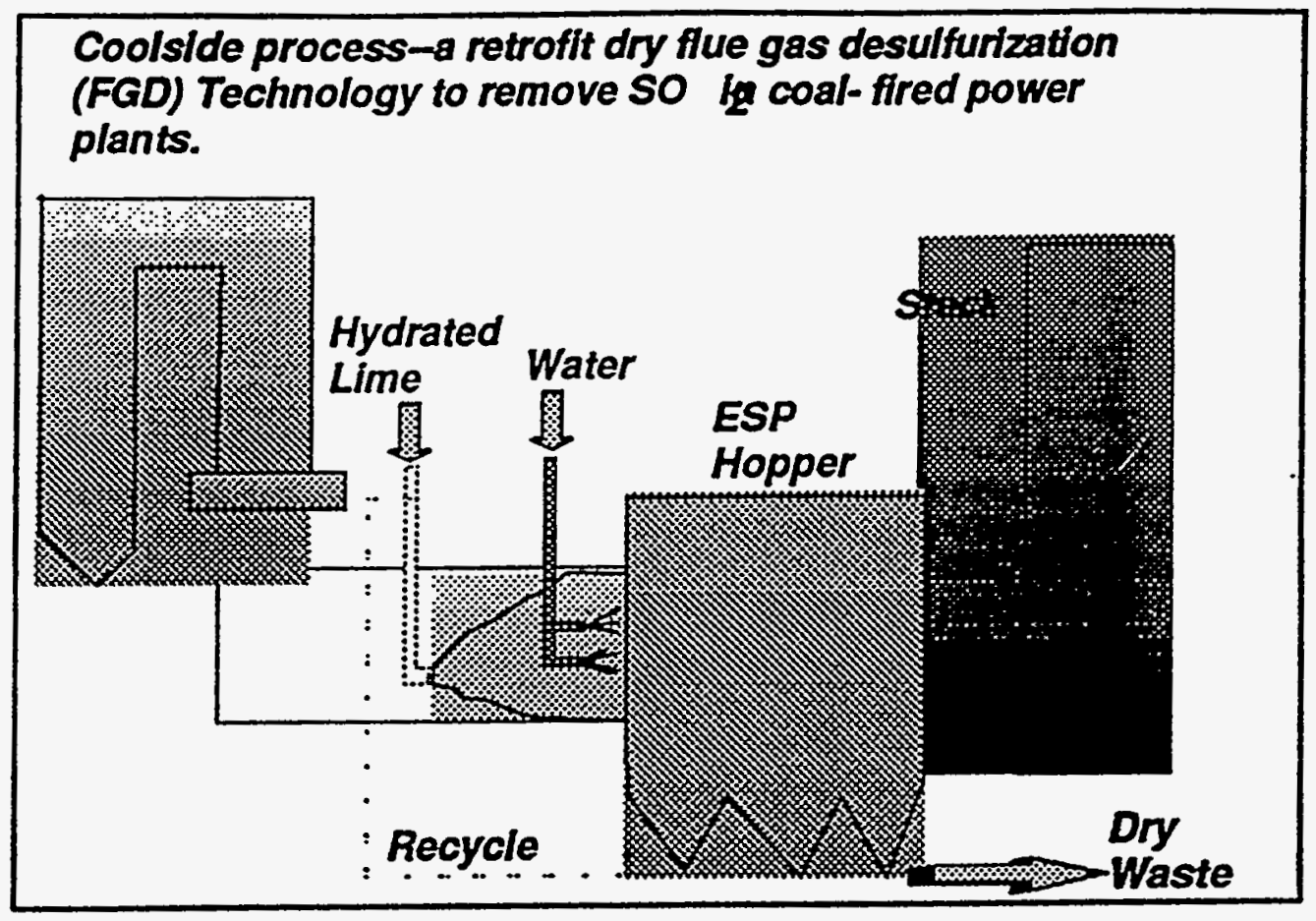

Figure 3-47. Coolside process

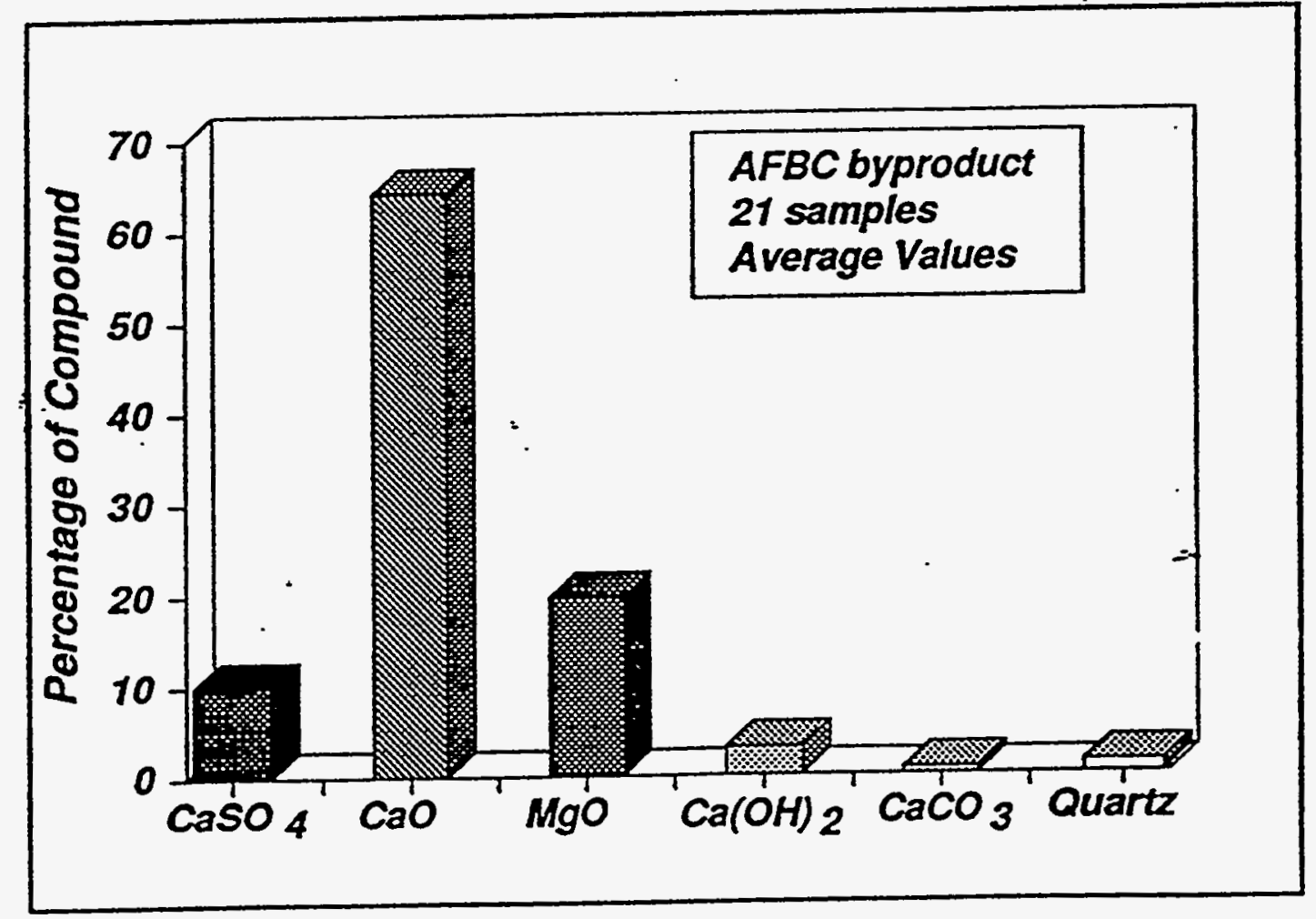

Figure 3-48. Chemical analysis of an AFBC ash 


\begin{tabular}{|l|l|l|cc|cc|}
\hline $\begin{array}{l}\text { Sample } \\
\text { Number }\end{array}$ & $\begin{array}{l}\text { Liquid } \\
\text { Limit }\end{array}$ & $\begin{array}{l}\text { Plasticity } \\
\text { Index } \\
(\%)\end{array}$ & $\begin{array}{c}\text { Grain-Size } \\
\text { Percent Finer than: } \\
\text { No. 10 No. 200 } \\
(\%)\end{array}$ & \multicolumn{2}{|c|}{ Classification } \\
\hline Coolside & NP & NP & 100 & 95 & ML & A-4(0) \\
\hline FBC ash & NP & NP & 100 & 51 & SM & A-4(0) \\
\hline Silty Clay & 36 & 11 & 90 & 70 & ML & A-6(8) \\
\hline Plastic Clay & 71 & 37 & $99: 93$ & ML & A-7-5(44) \\
\hline $\begin{array}{l}\text { AFBC(7\%)- } \\
\text { Soil Mixture }\end{array}$ & 49 & 14 & 91 & 49 & ML & A-7-5(5) \\
\hline
\end{tabular}

Table 3-9. Index properties 
respectively. Samples identified as CS-1040 and CS-1050 were two samples from Edgewater sample series number 1 . Samples identified as CS-1 were a composite sample from run number 1 (also, identified as Sample A). Samples identified as CS-3 were a composite sample from run number 3 .

Samples identified as CS-2 were obtained from the CONSOL Coolside pilot plant operating in the recycling mode. These samples were produced at conditions that simulate operation of the Coolside process installed on a unit burning medium-sulfur (2.5\%) Ohio coal. The sulfur content of CS-2 was about 8 percent. Edgewater Coolside samples contained about 2 percent of sulfur. Samples of an FBC ash were obtained from a silo of a coal-fired circulating fluidized bed combuster in a coal-fired co-generating plant in Pennsylvania on February 18, 1992. The FBC byproduct was hydrated by CONSOL. However, nonhydrated samples of the FBC ash were also received for testing. The AFBC spent lime (nonhydrated) was obtained from an oil refinery in 1987 and is a byproduct of an AFBC process used in cracking crude oil; Two typical soils were obtained in Kentucky. Index properties of the materials are compared in Table $3-9$. This AFBC material contains some $10 \%$ of calcium sulfate and about $65 \%$ of calcium oxide, as shown in Figure $3-48$.

Swell Testing Procedures. Swelling characteristics of the Coolside byproducts and the other materials selected for comparative purposes were determined by submerging compacted specimens in water in confined CBR molds and oedometers (consolidometers). Two series of tests were performed in oedometers, which commonly are used to perform consolidation tests. The third series of tests was performed in CBR molds. A schematic of the arrangement of specimens in CBR molds was illustrated previously in Figure 3-39. Diameter and height of the CBR-size specimens were 6 in $(15.24 \mathrm{~cm})$ and 4.58 in $(11.63 \mathrm{~cm})$. Consolidometer specimens measured 2.5 in $(6.35 \mathrm{~cm})$ in diameter and 1 in $(2.54 \mathrm{~cm})$ in height. All specimens were compacted to about 95 percent of maximum dry density and optimum moisture content 
was obtained from standard compaction (ASTM D 698) following a procedure described above and elsewhere (Hopkins and Beckham, 1993). This compactive state was used because field specifications of many agencies require those compaction criteria.

Swelling periods for tests conducted in the oedometer apparatus usually ranged from 18 to 21 days. Swelling periods of tests performed in the CBR molds ranged from 64 to 79 days. In the first series of oedometer tests, the Coolside material was compacted immediately after mixing. After compaction, the tests were started by submerging the specimens (and mold) in water. In the second series of tests, the Coolside material was compacted and the specimens were allowed to age, or cure, for seven days in a sealed container at room temperature (about $21^{\circ} \mathrm{C}$ ). In the third series, the swelling tests were started after the CBR specimens had aged 0,7 , and 14 days. Laboratory bearing ratio tests were performed on some of the larger specimens as a means of evaluating bearing strength properties after swell.

Test Results and Analysis. Swelling of FGD byproducts due to the absorption of water (and chemical reactions) may affect the structural integrity of the disposed material at the disposal site and the potential uses of the byproduct in structural applications, such a base material for pavements or chemical admixture for pavements subgrade. For example, swelling of a compacted byproduct, used as a base course, may do little structural damage to the pavement if it occurs very rapidly and before placement of the pavement, although the magnitude of swelling may be very large. Although the time rate of swelling may be very slow, taking many years to complete, little damage may occur if the magnitude is small. Bearing strength is directly related to the magnitude of swell, as illustrated in Figure 3-49. As the total magnitude of swell increases, the bearing decreases.

Based on previous work by Hopkins $(1994,1995)$, the minimum CBR strength of subgrades to sustain tire stresses of $552 \mathrm{kPa}(80 \mathrm{psi}$ ) should be 6.5 (for a factor of 


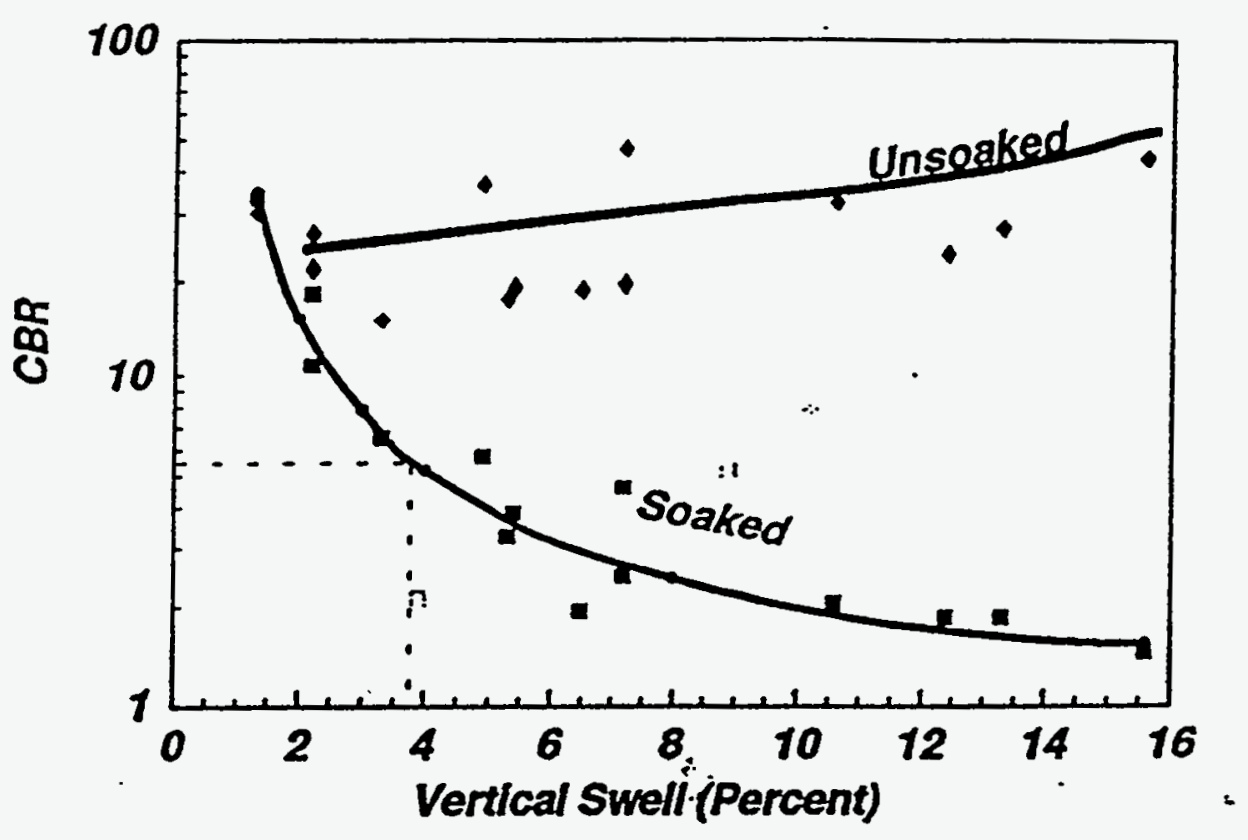

Figure 3-49. Decreases in CBR strengths as the total swell of compacted soils increases

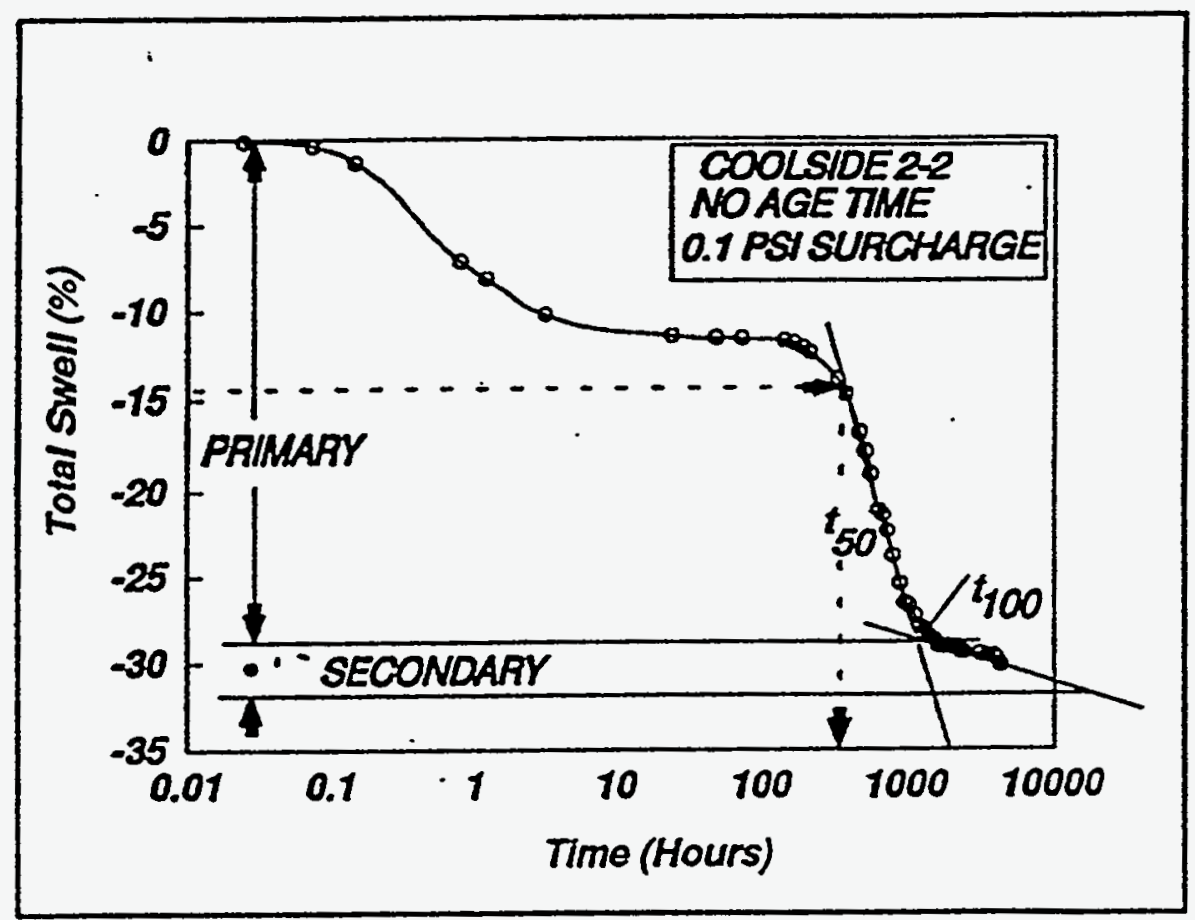

Figure 3-50. Vertical swell of Coolside specimen number 2 
safety equal to one). As shown in Figure 3-49, the magnitude of swelling should be below about 4 percent for the CBR strength to be above 6.5. A typical swelling-time curve of a compacted specimen of the Coolside material (CS-2) is shown in Figure 350. Typical swelling curves obtained for Edgewater runs 1 and 3 (CS-388 and CS1040) are shown in Figure 3-51.

Sweiling may be divided into two parts: primary and secondary swelling. Usually, primary swelling represents a large portion of the total magnitude of swelling. Secondary swelling is linear when the vertical swelling is plotted as a function of the logarithm of time. All specimens of the Coolside material behaved as shown in Figure 3-50. Hence, coefficients of primary swelling and secondary swelling may be computed using principles proposed by Terzaghi (1947) and fitting methods proposed by Casagrande (1936)--logarithmic-of-time-- and Taylor (1945)--square-root-of-time-since swelling due to the absorption of water is consolidation in reverse. The swelling rate increases with an increasing coefficient of swell at any time. These principles may be used to forecast the time rate of swelling for a given field problem. The magnitude of primary swelling (of thin layers) may be estimated by the vertical strain observed from a laboratory test. Values of coefficients of primary and secondary coefficients are summarized in Table 3-10. These values were determined from a computer program developed by McNulty, et al (1978).

In the first series of oedometer tests, where no aging was permitted and no surcharge pressures were used, the total swell of the four specimens ranged from 10 to $23 \%$. Primary swell ranged from 8 to $16 \%$. Secondary swell ranged from $0.206 .5 \%$. In comparison, total values of swell of two typical, compacted clays were 4.6 and $12.5 \%$. Two typical swelling curves of the Coolside material are compared in Figure 3-52 to swelling curves of two typical clay soils and an atmospheric fluidized bed combustion material--AFBC-- (from an oil refinery). Primary values of swell were 4.1 and $11.6 \%$, respectively, as shown at the bottom of Table 3-10. 


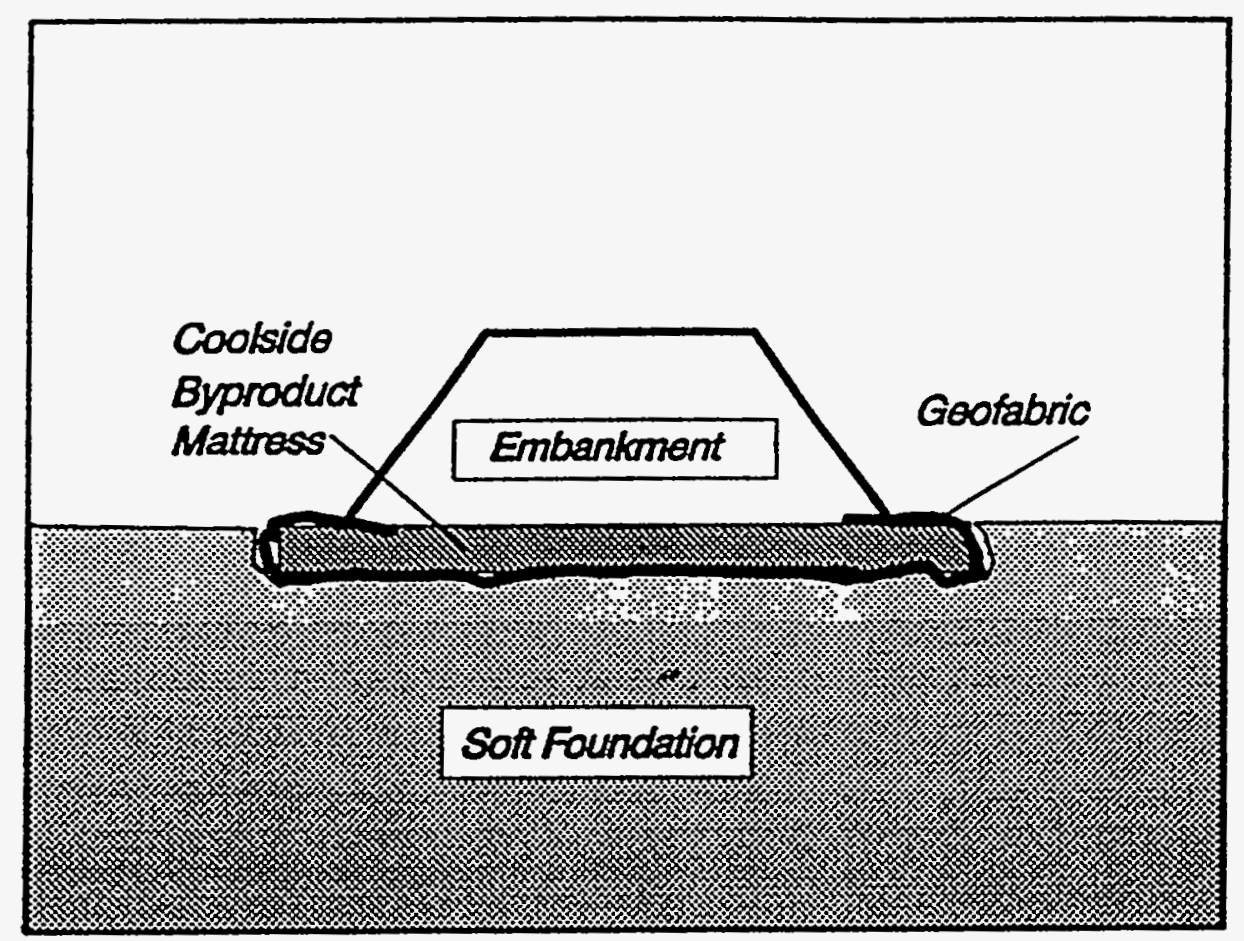

Figure 3-51. Use of the Coolside material to construct a mattress reinforced with geofabric to support an embankment on a soft foundation

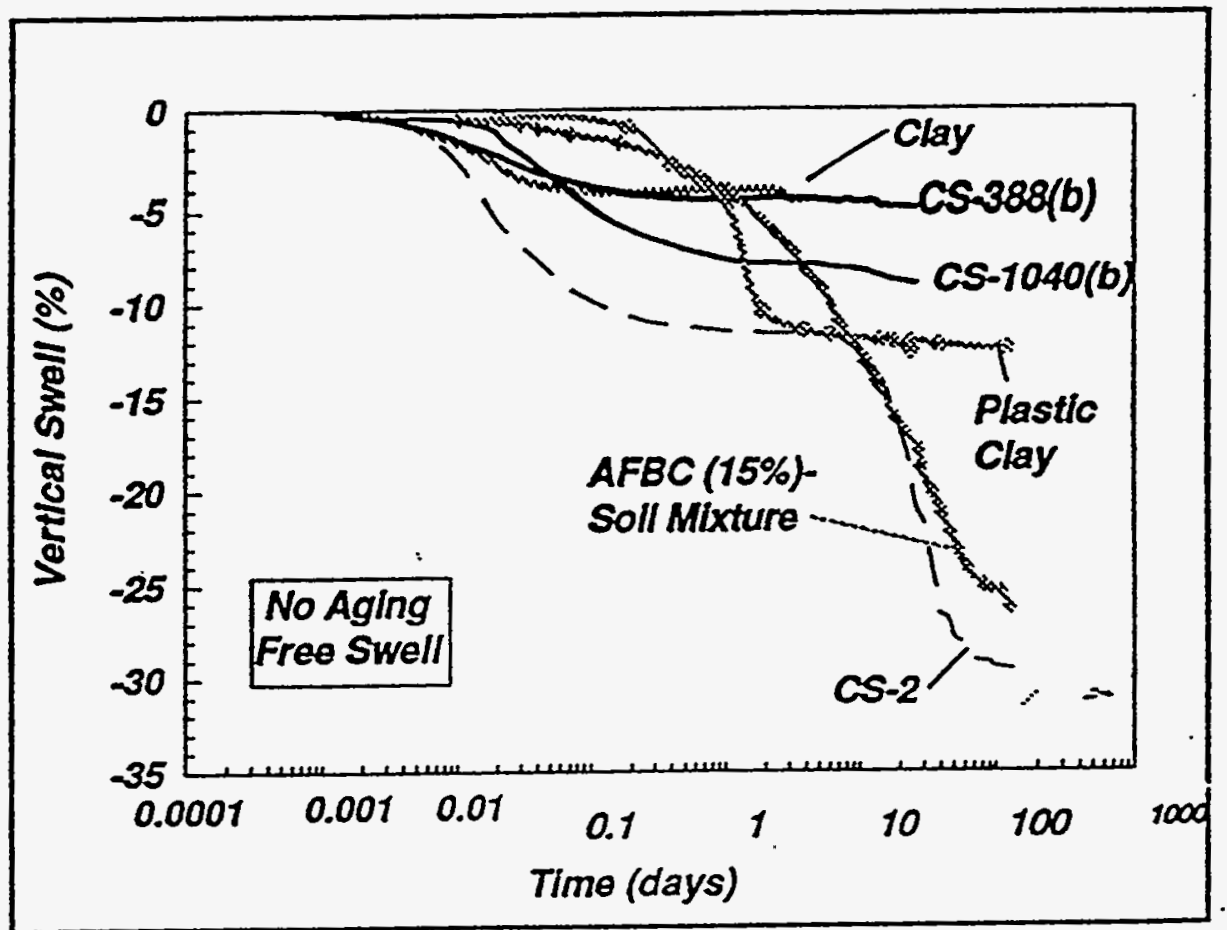

Figure 3-52. Comparisons of the swelling curves of AFBC- and FGD-type byproducts and two natural clays 


\begin{tabular}{|c|c|c|c|c|c|c|c|c|}
\hline $\begin{array}{c}\text { Specimen } \\
\text { Number Curing } \\
\text { Period } \\
\text { (days) }\end{array}$ & $\begin{array}{l}\text { Square-I } \\
t_{90} \\
\text { (Min.) }\end{array}$ & $\begin{array}{l}\text { Fitting Me } \\
\text { Root-of-Time } \\
\text { Coefficient- } \\
\text { Of Primary } \\
\text { Swell } \\
(\mathrm{cm} 2 / \mathrm{day})\end{array}$ & $\begin{array}{l}\text { ethods } \\
\text { Logarit } \\
t_{\text {so }} \\
\text { (Min) }\end{array}$ & $\begin{array}{l}\text { Coefficient } \\
\text { of Primary } \\
\text { Swell } \\
\text { (cm } 2 / \text { day) }\end{array}$ & $\begin{array}{l}\text { Coefficient } \\
\text { of } \\
\text { Secondary } \\
\text { Swell } \\
\text { Cds }\end{array}$ & $\begin{array}{l}\text { Primary } \\
\text { Swell } \\
(\%)\end{array}$ & $t_{100}$ & Total Swell \\
\hline CS- $388^{4}$ & 18.5 & 1022 & 4.3 & $\cdot 102.2$ & 0.0 & 6.0 & 25.1 & 22.5 \\
\hline $\operatorname{Cs}-397^{\circ}$ & 38.05 & 1.8 & 14.7 & 31.1 & 0.0009 & 15.4 & 56.2 & 15.6 \\
\hline CS-1040 0 & 177.8 & 11.1 & 13.0 & 35.2 & 0.0040 & 14.5 & 316.2 & 15.1 \\
\hline CS.1050 0 & 28.7 & 38.7 & 5.6 & 81.7 & 0.00 & 8.0 & 46.4 & 9.9 \\
\hline $\operatorname{CS}-388^{\circ}$ & 13,200 & 0.15 & 3,600 & 0.13 & 0.0 & 4.9 & 18,000 & 4.9 \\
\hline CS-397 & 7,200 & 0.27 & 2,700 & 0.17 & 0.0 & 2.1 & 10,020 & 2.1 \\
\hline $\operatorname{CS}-1040^{\circ}$ & 7,800 & 0.25 & 3,300 & 0.14 & 0.0011 & 0.9 & 10,998 & 1.0 \\
\hline $\operatorname{CS}-1050^{2} \quad 7$ & 6,600 & 0.30 & 4,200 & 0.11 & 0.0021 & 2.1 & 15,840 & 2.2 \\
\hline CS-388A & 9045 & 8.0 & 21 & 458.0 & 0.0041 & 4.1 & 132.8 & 5.1 \\
\hline CS-1040B $\quad 0$ & 1,050 & 39.4 & 300 & 32.1 & 0.0092 & 7.4 & 1,286 & 9.0 \\
\hline CS-388C 0 & 180 & 2462 & 39 & 229.5 & 0.0010 & 1.3 & 170.0 & 1.6 \\
\hline$C S-1040 D^{b} \quad 0$ & 300 & 137.5 & 45 & 213.7 & 0.0007 & 1.4 & 180.0 & 1.6 \\
\hline CS-1040E 7 & 17,400 & 2.4 & 4,200 & 2.3 & 0.0 & 0.2 & 24,000 & 0.2 \\
\hline CS-388F ${ }^{b} \quad 14$ & 15,000 & $11.1^{\circ}$ & 3,900 & 9.8 & 0.0 & 0.1 & 18,000 & 0.1 \\
\hline AFBCSSOil $^{8} 0$ & 67,500 & 0.5 & 17,160 & 0.4 & 0.0776 & 24.2 & 120,000 & 26.2 \\
\hline Sitty Clay " & 2,700 & 0.2 & 90 & 0.3 & 0.0025 & 4.1 & 3,600 & 4.6 \\
\hline Plastic Clay ${ }^{b} \quad$ & 2,800 & 14.4 & 390 & 24.6 & 0.065 & 11.6 & 4,500 & 12.5 \\
\hline
\end{tabular}

Notes: (a), Specimen mold = oedometer; (b). Specimen mold = CBR; the third series of tests were performed on CBR-size specimens. Four (specimen numbers 388A, 1040B, $388 \mathrm{C}$, and 1040D) of the six specimens were not aged prior to soaking.

Table 3-10. Swelling characteristics of compacted specimens of Coolside byproduct 
Magnitudes of primary swelling are shown in Figure 3-53. Numbers $(0,7$, and 14) shown at the bottom of each bar refer to the aging time (in days) of each specimen in sealed containers before submerging in water. Numbers at the top of each bar represent a small surcharge pressure $(0,0.69 \mathrm{kPa}[0.1 \mathrm{psi}]$, and $3.44 \mathrm{kPa}[0.5 \mathrm{psi}])$ applied to each specimen. A pressure of $3.44 \mathrm{kPa}$ is about equivalent to the pressure exerted by a 15.24-cm (6-in) layer of pavement. When the aging and the applied surcharge pressure were zero, the magnitude of primary swell of specimens CS-388, 397,1040 , and 1050 ranged from 8 to 16 percent.

The amount of primary swelling decreased as the surcharge pressure was increased. For example, when the surcharge pressure of CS-388 and CS-1040 was increased from zero to $3.44 \mathrm{kPa}(0.5 \mathrm{psi})$, the amount of swelling decreased from about $15 \%$ to $2 \%$. Swelling of CS-2 was reduced from $29 \%$ to $15 \%$ when the surcharge was increased from $0.69 \mathrm{kPa}$ to $3.44 \mathrm{kPa}$ (0.1 to $0.5 \mathrm{psi}$ ). A similar trend was observed for the other specimens. Additionally, the amount of swelling decreased when the specimens were allowed to age prior to soaking. For instance, the amount of swelling of CS-388 decreased from about $16 \%$ at no aging time to about $5 \%$ at a 7 -day aging period. Primary swelling of the two soils were about 5 to $12 \%$, respectively. Primary swelling of the AFBC-soil mixture was about $25 \%$. Primary swelling of the Coolside specimens that had been aged for seven days prior to soaking and surcharged to 3.44 $\mathrm{kPa}(0.5 \mathrm{psi})$ was less than $5 \%$--a value equal to or less than values of primary swelling observed for the two typical soils. The higher sulfur content of CS-2 may have played an important role in producing a swelling magnitude that was greater than those observed for the other Coolside samples.

Coefficients of primary swelling are compared in Figure 3-54. This coefficient is calculated by the equation shown in Figure 3-55. Specimen aging generally had a significant effect on the observed values. For example, coefficients of primary swelling 


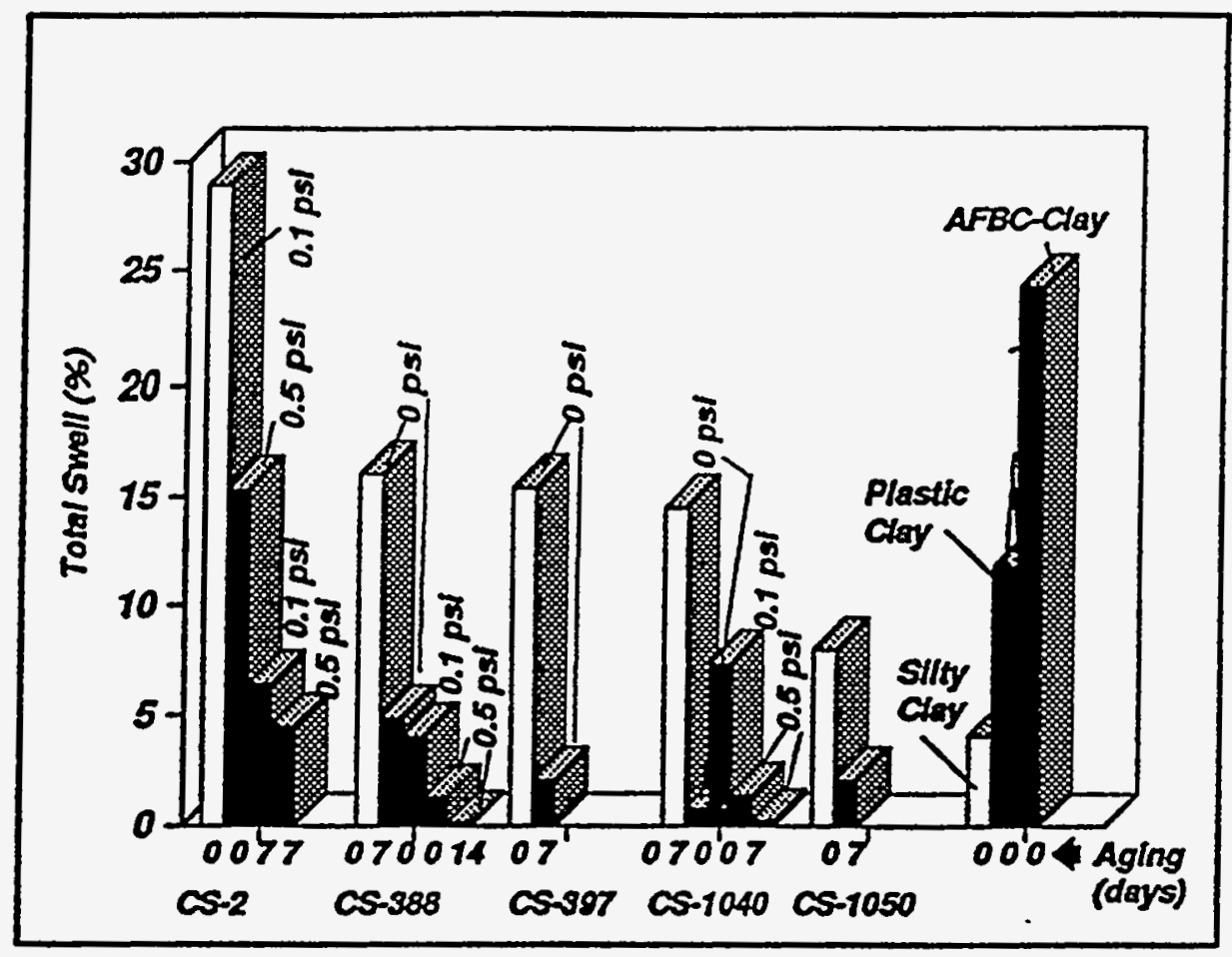

Figure 3-53. Comparison of total magnitudes of swelling

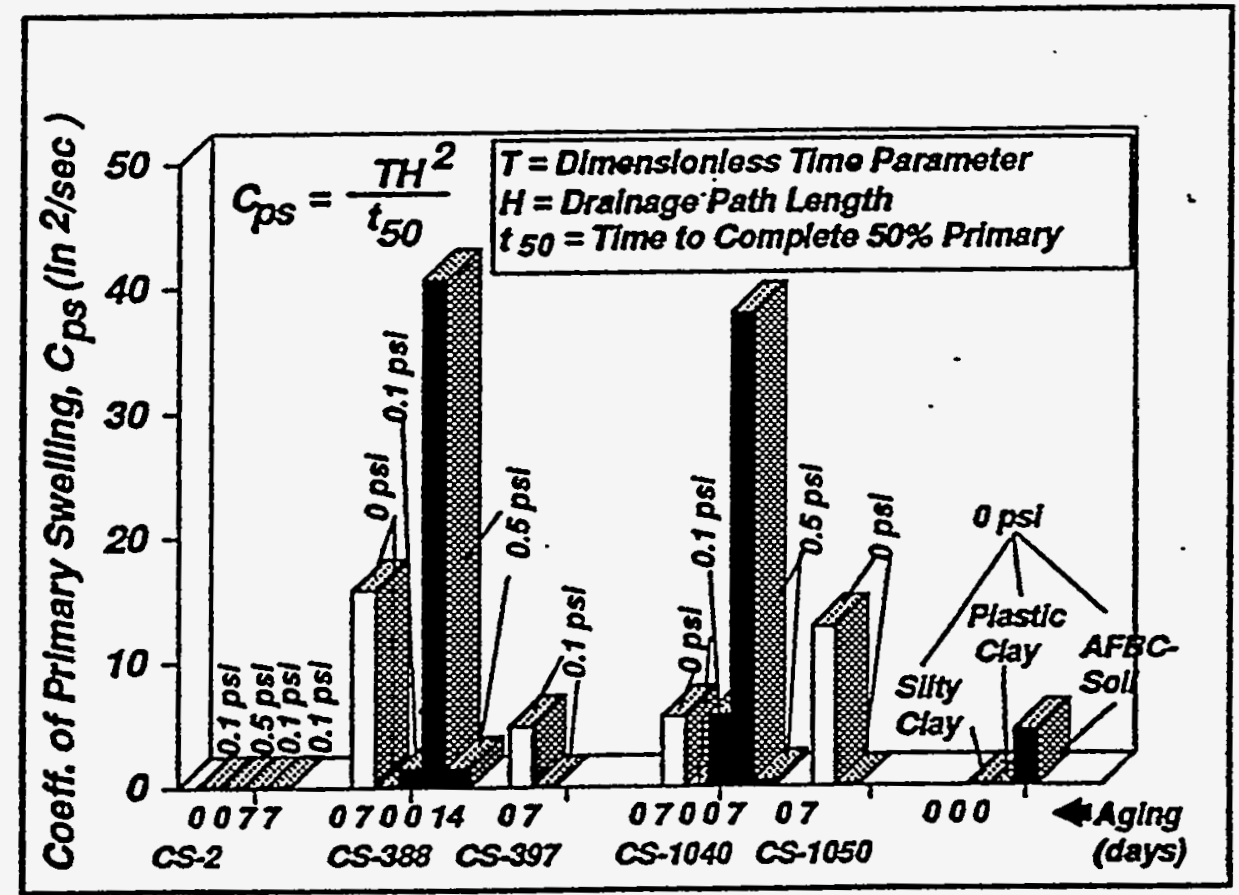

Figure 3-54. Primary swelling coefficients 


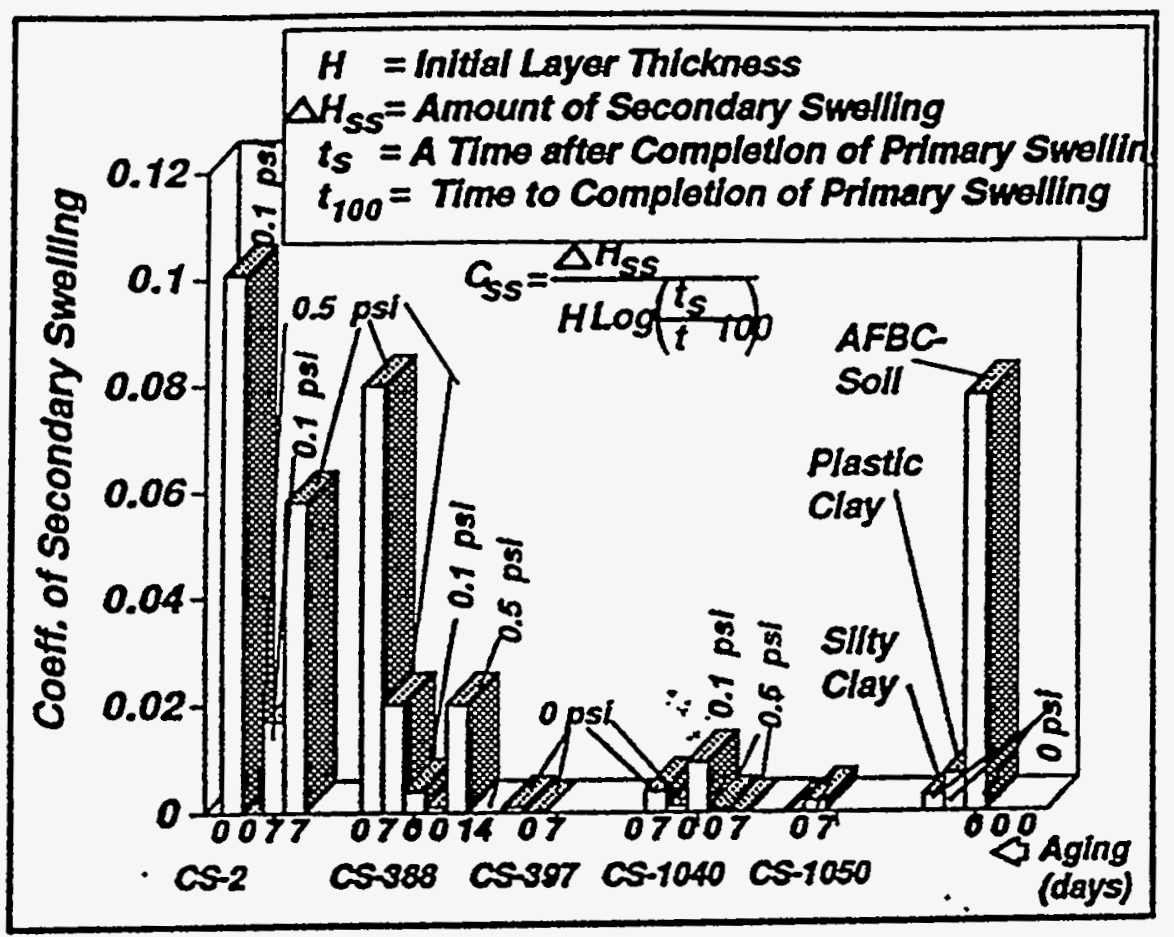

Figure 3-55. Secondary swelling coefficients

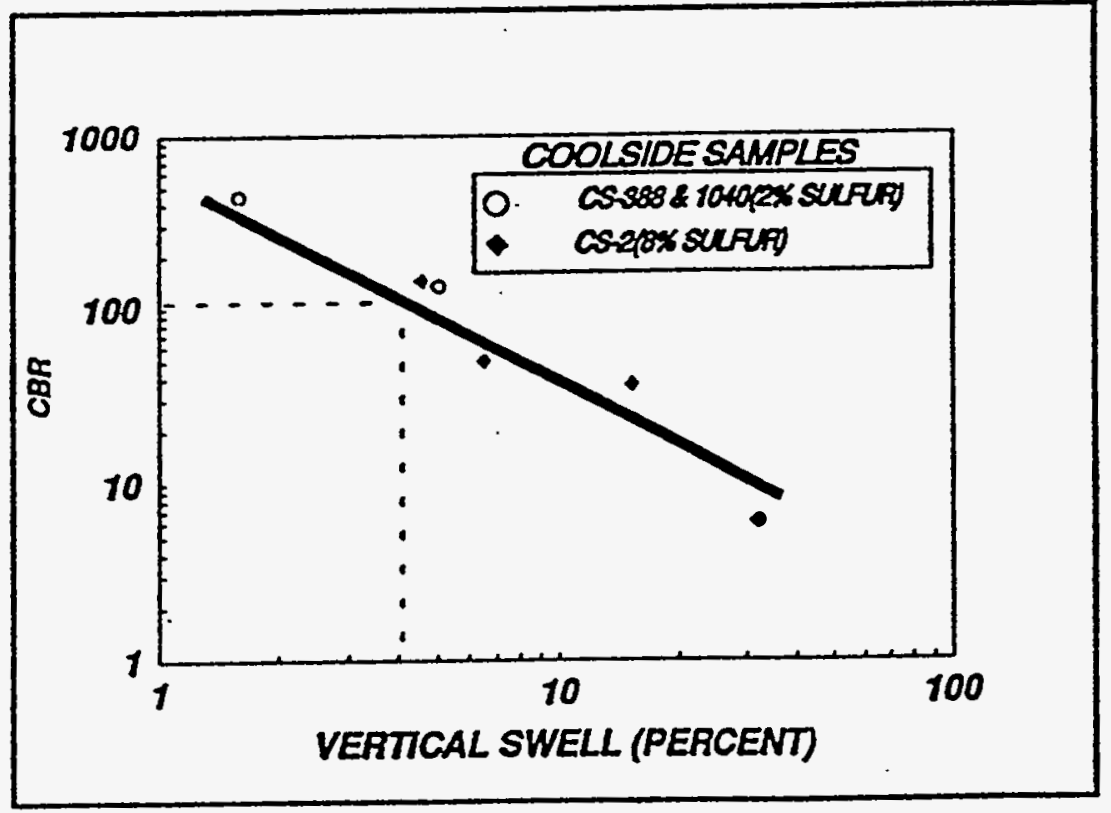

Figure 3-56. Variation of CBR strength and total magnitude of swell of compacted Coolside specimens 
of specimens, CS-388, CS-397, CS-1040, and CS-1050 at zero aging time ranged from 14.2 to $40.1 \mathrm{~cm}^{2}$ per day (5.6 to $15.8 \mathrm{in}^{2}$ per day). Coefficients of the same Coolside samples that had been aged ranged from only 0.04 to $3.9 \mathrm{~cm}^{2}$ per day $(0.017$ to 1.52 $\mathrm{in}^{2}$ per day). Primary coefficients of those specimens increased when the surcharge pressure was increased from zero to $3.44 \mathrm{kPa}(0.5 \mathrm{psi})$. Coefficients of primary swelling of the AFBC spent lime-soil mixture, the silty clay, and the plastic clay were $0.18,0.13,11.0 \mathrm{~cm}^{2}$ per day $\left(0.07,0.053\right.$, and $4.35 \mathrm{in}^{2}$ per day), respectively. Changing the aging period, or increasing the surcharge pressure did not appear to cause significant changes in the coefficient of primary swell of CS-2. Values ranged from 0.13 to $0.20 \mathrm{~cm}^{2}$ per day ( 0.05 to $0.08 \mathrm{in}^{2}$ per day).

Generally, coefficients of secondary swelling of CS-2 specimens (Figure 3-55) were slightly larger than the secondary coefficients of CS-388, CS-397, CS-1040 and CS1050. The equation shown in Figure 3-55 was used to calculate the secondary coefficients. Coefficients of secondary swelling of all Coolside specimens ranged from zero to 0.1 . Secondary coefficients of CS-2, CS-388, and CS-397 that had not been aged appeared slightly larger than coefficients of specimens that had been aged for seven to 14 days. However, secondary coefficients of specimens surcharged $0.69 \mathrm{kPa}$ ( $0.1 \mathrm{psi}$ ) were generally slightly smaller than those of specimens that were not surcharged.

CBR strengths of Coolside byproducts after swelling ranged from 133 to 430 -exceptionally high values. However, as shown in Figure 3-56, the CBR strengths decrease rapidly as the magnitude of swell increases. Whereas CBR values of natural soils compacted to values of about $95 \%$ of maximum dry density and optimum moisture content are only about 6 at a magnitude of swell of about 3.5\%, the CBR values of Coolside specimens, compacted to the same conditions, are greater than 100. In fact, at a swell magnitude of about $30 \%$, the CBR value of the Coolside material is about 7 . This implies that the Coolside material could go through an expansion period and still 
retain considerable strength.

Prehydrating FGD byproducts before use has been suggested for eliminating, or minimizing, the large magnitudes of swelling observed in these materials. To check this common assumption, two series of swelling tests were performed using an FGD byproduct in two different states. In contrast to slaking the byproduct, which involves combining water with the calcium oxide of the byproduct so that a slurry is formed, the byproduct was hydrated. In the hydration process, $99 \%$ of the water is chemically combined as hydroxide to produce a dry, finely powdered, hydrated lime. In hydration, less water is used than when the slaking process is involved. The byproduct selected for testing was an FBC ash obtained from a power plant in Pennsylvania. Some material was prehydrated by CONSOL, Inc. Additionally, FBC ash specimens were. received that had not been hydrated.

Swell tests were performed on both the hydrated and nonhydrated material. These tests were performed on specimens compacted to $95 \%$ of standard compaction and optimum moisture content. Values of maximum dry density and optimum moisture content used to remold the swell specimens were obtained from compaction tests performed on both the hydrated samples and the nonhydrated samples (Figure 3-57). Swelling (expressed as a percentage), as a function of the logarithm-of-time for the hydrated and nonhydrated FBC ash specimens is shown in Figures 3-58 and 3-59. The swelling tests were conducted for different specimens aged to two different conditions and different surcharge pressures. Two specimens were not aged while two specimens were aged for 7 days before submerging the specimens in water. Surcharge pressures of $0.69 \mathrm{kPa}(0.1 \mathrm{psi})-$-which corresponds to a weight of about $1.08 \mathrm{~kg}(2.4 \mathrm{lbs})--$ and $3.45 \mathrm{kPa}(0.5 \mathrm{psi})-$ - which corresponds to a weight of $5.8 \mathrm{~kg}$ (12.8 lbs)--were used. In both cases, the nonhydrated and prehydrated specimens are still in a primary swelling state. The nonhydrated specimens have been in primary swelling for some 1292 days--3.5 years--and have not reached a secondary swelling 


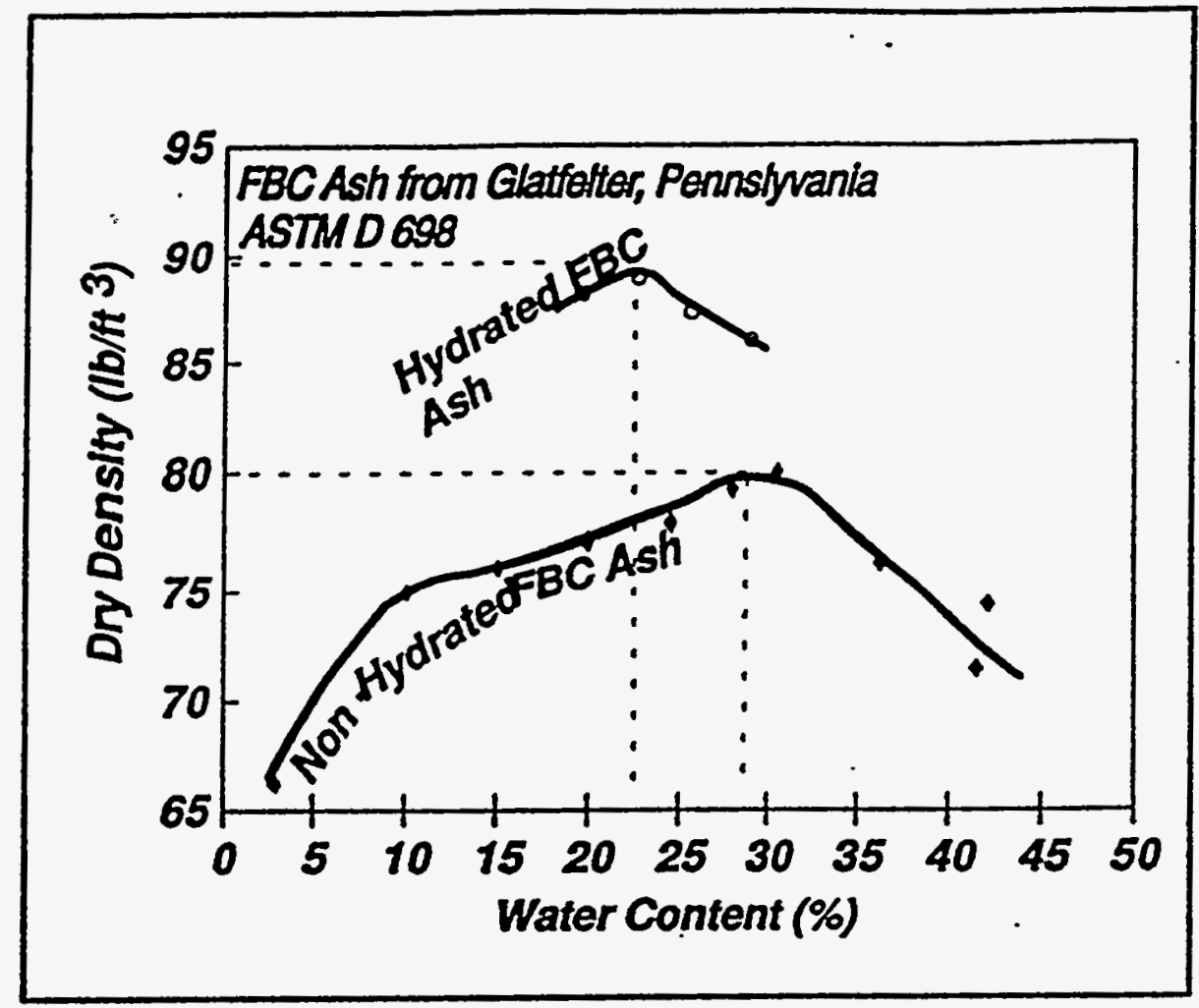

Figure 3-57. Dry density-moisture content relationships of an FBC ash from Pennsylvania

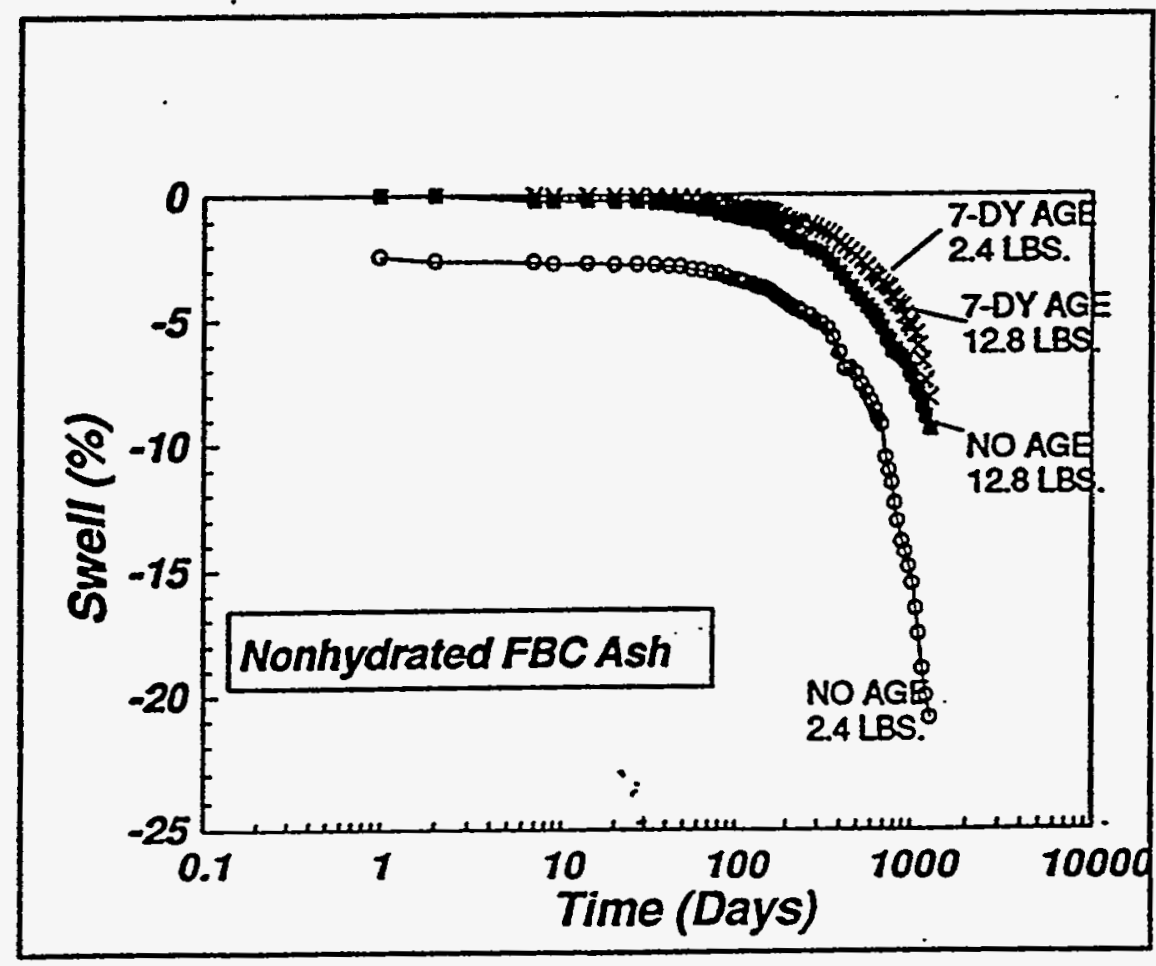

Figure 3-58. Swell curves for the nonhydrated FBC ash from Pennsylvania 


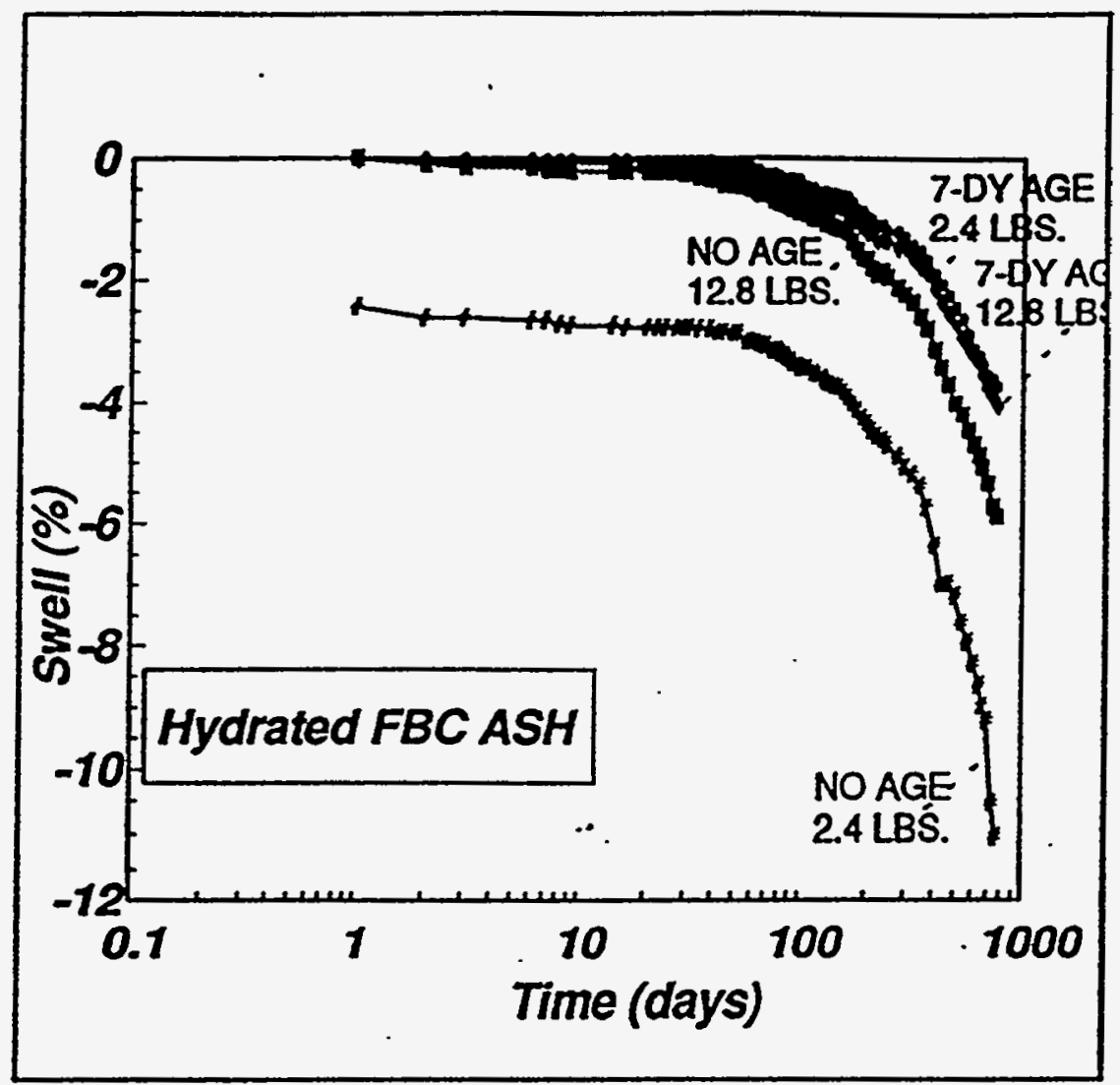

Figure 3-59. Swell curves for an FBC (prehydrated) ash from Pennsylvania

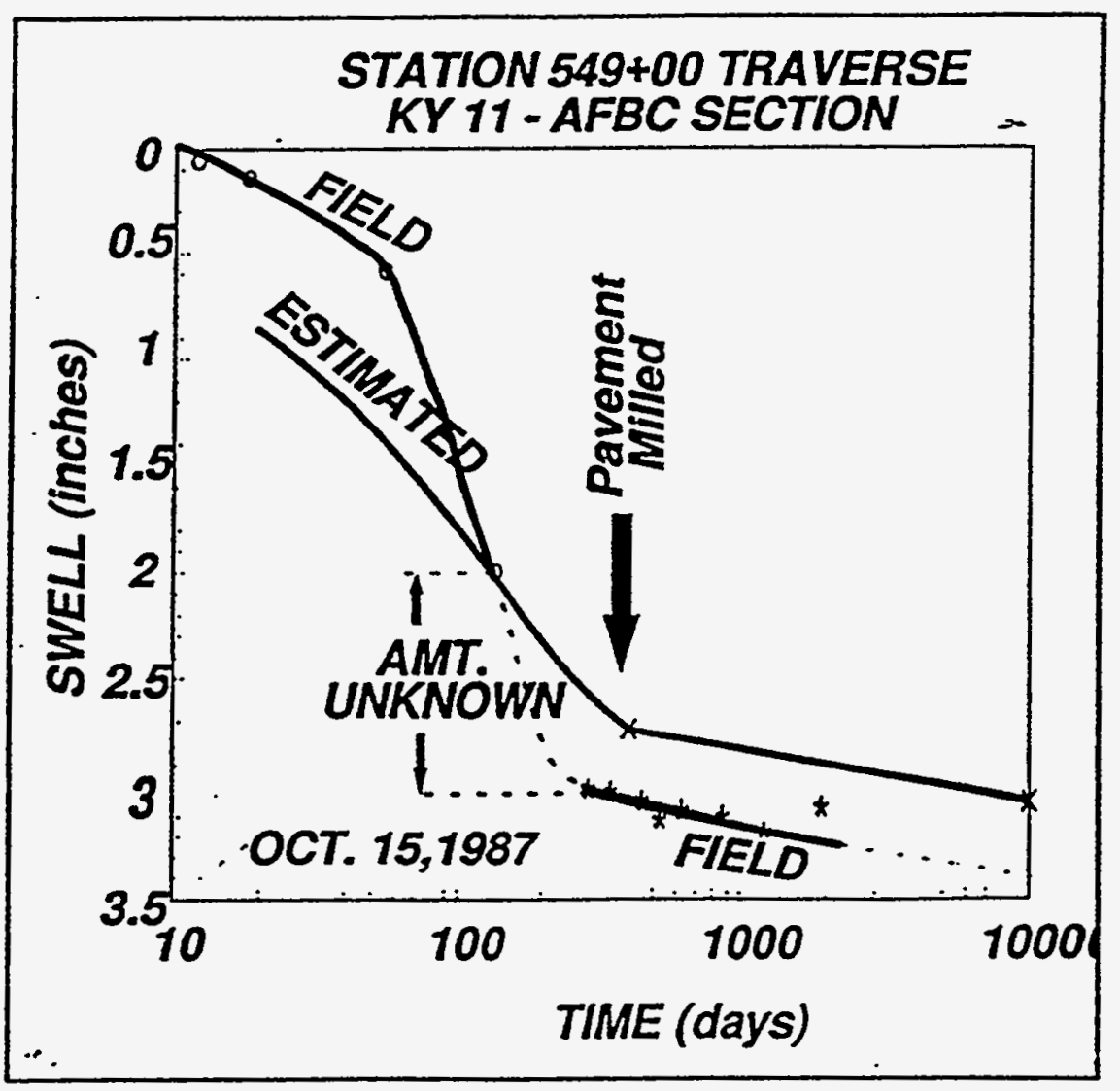

Figure 3-60. Comparison of predicted and observed swelling of the AFBC-clayey subgrades of the KY 11 Route 
condition. Similarly, the preydrated specimens, as shown in Figure 3-59, have been swelling for some 766 days, or 2.1 years, and have not reached the secondary swelling state. As shown in Figures 3-58 and 3-59, the magnitudes of swelling for aged specimens were much more than specimens that had not been aged. Moreover, the magnitudes of swelling of specimens with larger surcharge pressures are less than specimens with smaller surcharge pressures. The swelling of the prehydrated specimens ranged from about 4 to $11 \%$ after some 2.1 years (Figure 3-59). The swelling amounts for the nonhydrated specimens range from about 8 to $21 \%$ after some 3.5 years. For the same time as the period of the nonhydrated specimens (2.1 years), the swelling magnitudes of the prehydrated specimens range from about 3.8 to $11 \%$. So, only small differences in the magnitudes of swelling of prehydrated and nonhydrated specimens are suggested by the results of these tests. However, observations must continue to confirm this finding. Swelling trends of the FBC byproduct are similar to those observed for the Coolside specimens. However, the Coolside specimens reached the secondary swelling state much faster.

\section{CASE STUDY}

FGD by-products have the potential to be used to stabilize clayey subgrades of highway pavements. For example, a by-product obtained from an AFBC process of an oil refinery was mixed with a clayey subgrade of a highway route in Kentucky (Hopkins, et al., 1987; 1993). Combined length of the two highway subgrade sections was about $4.0 \mathrm{~km}$ ( 2.5 miles). The pavement section consisted of $21.6 \mathrm{~cm}$ ( 8.5 inches) of asphaltic concrete, $12.7 \mathrm{~cm}$ (5 inches) of crushed limestone aggregate, and a $30.5-\mathrm{cm}$ (12-inch) compacted mixture of the AFBC spent lime (7 percent) and clayey soil (93 percent). The AFBC-soil subgrades were allowed to cure for seven days. Approximately two months after placement of the asphaltic base courses and a rainy period, noticeable "humps", running perpendicular to the centerline, formed on the pavement surface. 
Many points were immediately established on the surface of the sections and optical surveys were performed periodically. Placement of the asphaltic surface course was delayed for several months. Magnitudes of pavement heaving as large as $8.9 \mathrm{~cm}$ (3.5 in) were measured during a 5-month period. Additional laboratory swelling tests were performed. Based on laboratory coefficients of primary and secondary swell, the magnitude and time rate of swelling were estimated. Time required to complete 95 percent of primary swell was estimated to be about 1.1 years after completion of the subgrades. About 10 months after observed differential heaving, the pavement was milled to remove "humps" and the surface course was placed. Survey points were reestablished. These points have been monitored for about 5 years. Observed swelling at one location is compared to the estimated swelling in Figure 3-60. Five years after placement of the surface course, only about $0.5 \mathrm{~cm}$ (0.2 in) of swelling has occurred. Based on the projected linear relationship of swelling and the logarithm of time, an additional $0.5 \mathrm{~cm}$ ( $0.2 \mathrm{in}$ ) will occur over the next 22 years.

In situ CBR tests performed often on top of the AFBC spent lime over a 6-year period show that the bearing strength decreased slightly after construction. However, CBR strengths during this period generally exceed 10 and range as high as 40 . Pavement rutting, 5 years after placement of the surface pavement, is nominal and generally does not exceed about $0.33 \mathrm{~cm}(0.13 \mathrm{in})$. If the $30.5-\mathrm{cm}(12-\mathrm{in})$ layer had been constructed of Coolside material of a nature similar to CS-2, the magnitude of swelling, based on the results in Figure 3-60 could, potentially, range from $8.9 \mathrm{~cm}$ (3.5 in)--no surcharge pressure--to about $1.5 \mathrm{~cm}$ (0.6 in)--with some surcharge pressure. Considering that a six-inch layer of a pavement provides a surcharge loading of about $0.5 \mathrm{psi}$, the magnitude of swell is estimated to be about $4.8 \mathrm{~cm}(1.9 \mathrm{in})$. However, based on the coefficient of primary swelling $0.13 \mathrm{~cm}^{2} /$ day $\left(0.05 \mathrm{in}^{2} /\right.$ day), a time of about 2.1 years would be required to complete 95 percent of primary swelling. Moreover, based on a coefficient of secondary swelling, approximately $2.0 \mathrm{~cm}(0.8 \mathrm{in})$ of secondary swelling 
would occur between the approximate end of primary swelling and 27.4 years after construction. Total estimated swell would be about $6.9 \mathrm{~cm}(2.7 \mathrm{in})$. This amount of swelling would probably adversely affect the structural integrity of the pavement. However, field trials and more study would be required to determine the exact swelling behavior of the Coolside byproduct.

If a $30.5-\mathrm{cm}$ (12-in) layer of plastic clay was used as the subgrade, then the magnitude of primary swelling is estimated to be about $3.6 \mathrm{~cm}$ (1.4 in). Time required to complete 95 percent of primary swelling is estimated to be about 1.6 years. Estimated secondary swelling that would occur between the end of primary swelling and 27.4 years is 0.25 $\mathrm{cm}$ (0.1 in). Total swelling is $4.3 \mathrm{~cm}(1.7 \mathrm{in})$. If the silty clay is used to construct the $30: 5-\mathrm{cm}$ (12-in) subgrade layer, then estimated primary swelling is about $1.3 \mathrm{~cm}(0.5 \mathrm{in})$ and about 1.3 years would be required for this amount of swelling to occur. Secondary swelling is estimated to be $0.13 \mathrm{~cm}(0.05 \mathrm{in})$. Total swelling is less than $1.5 \mathrm{~cm}(0.6 \mathrm{in})$. Hence, swelling of the two typical clayey soils ranges from 1.5 to $4.3 \mathrm{~cm}(0.6$ to $1.7 \mathrm{in})$, while estimated swelling of the CS-2 is $6.9 \mathrm{~cm}$ (2.7 in).

Although by-products produced from FGD technology, such as the Coolside and FBC by-products, have potential applications in the highway industry, the physical and geochemical processes that lead to swelling of these materials need to be fully understood. Means of controlling the swelling of these materials need to be devised. The physical swelling characteristics of materials from the Coolside technology and an FBC byproduct were examined briefly. Results of swelling tests performed on compacted specimens indicated that primary swelling decreased as the surcharge pressure was increased. Aging the materials before inundating them also appeared to reduce the amount of primary swelling. Coolside byproducts that contained less sulfur, or were aged, swelled less. 


\section{The Utilization of Coolside and Other FGD Materials in Highway Embankments}

Byproducts produced from flue gas desulfurization (FGD) technology, such as the Coolside technology, for the removal of $\mathrm{SO}_{2}$ in coal-fired power plants have potential applications in the highway construction industry. As shown previously, the shear and bearing strengths of compacted specimens of byproducts obtained from the Coolside (and fluidized bed combustion --FBC) processes are substantially greater than the strengths of compacted specimens of natural soils. For example, because of the pozzolanic reactions, unconfined compressive strengths of Coolside specimens compacted to $95 \%$ of the maximum dry density and optimum moisture content of standard compaction approach values of $6981 \mathrm{kPa}$ ( $1000 \mathrm{psi}$ ) to $17,228 \mathrm{kPa}$ (2500 psi). Unconfined compressive strengths of naturally occurring soils compacted to the same conditions are only about $69 \mathrm{kPa}$ (10 psi) to $414 \mathrm{kPa}$ (60 psi). These strengths approach those of some concretes. The strengths of the compacted Coolside specimens are some 120 to 700 times greater than compacted specimens of soils.

Because of the presence of substantial quantities of calcium oxide $(\mathrm{CaO})$, in the Coolside, as well as other FGD byproducts, the materials could be used in a number of potential applications. For instance, as shown in Figure 3-61, the materials could be used as a chemical admixture to construct base courses of highway pavements. Moreover, byproducts containing high percentages of $\mathrm{CaO}$ or $\mathrm{Ca}(\mathrm{OH})_{2}$ could be used to mix, stabilize, and improve the bearing strengths of clayey subgrades (Figure 3-62).

Another application of byproducts similar to the Coolside material could be in the construction of embankments with very steep slopes. Normally, clayey, or silty clays, used to construct highway embankments are constructed on a slope of about two horizontal to one vertical, or about 26.5 degrees. Because of the large strengths of the Coolside material, when compacted, embankments constructed with the Coolside material could be constructed at much steeper angles. By constructing embankments 


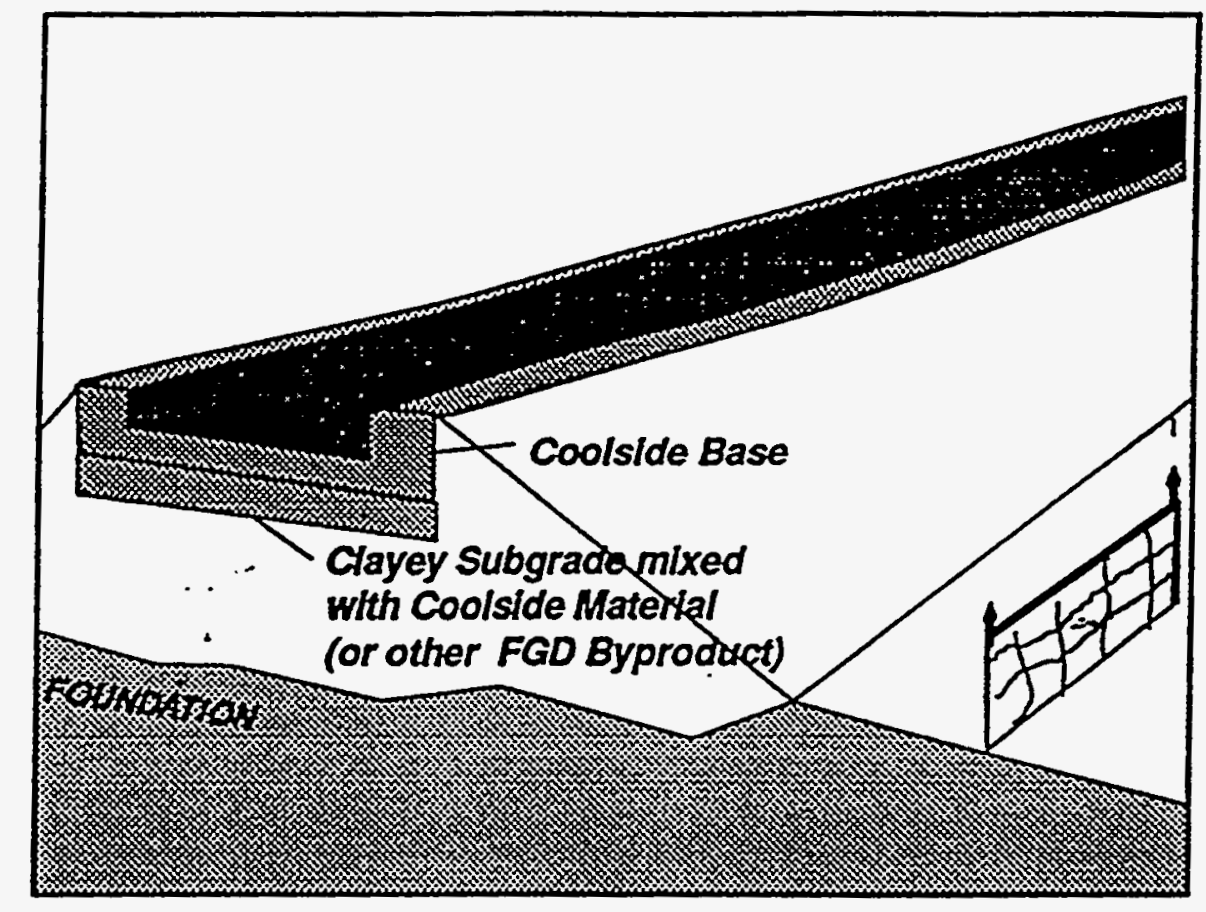

Figure 3-61. Two potential-applications of the Coolside byproduct and other FGD byproducts

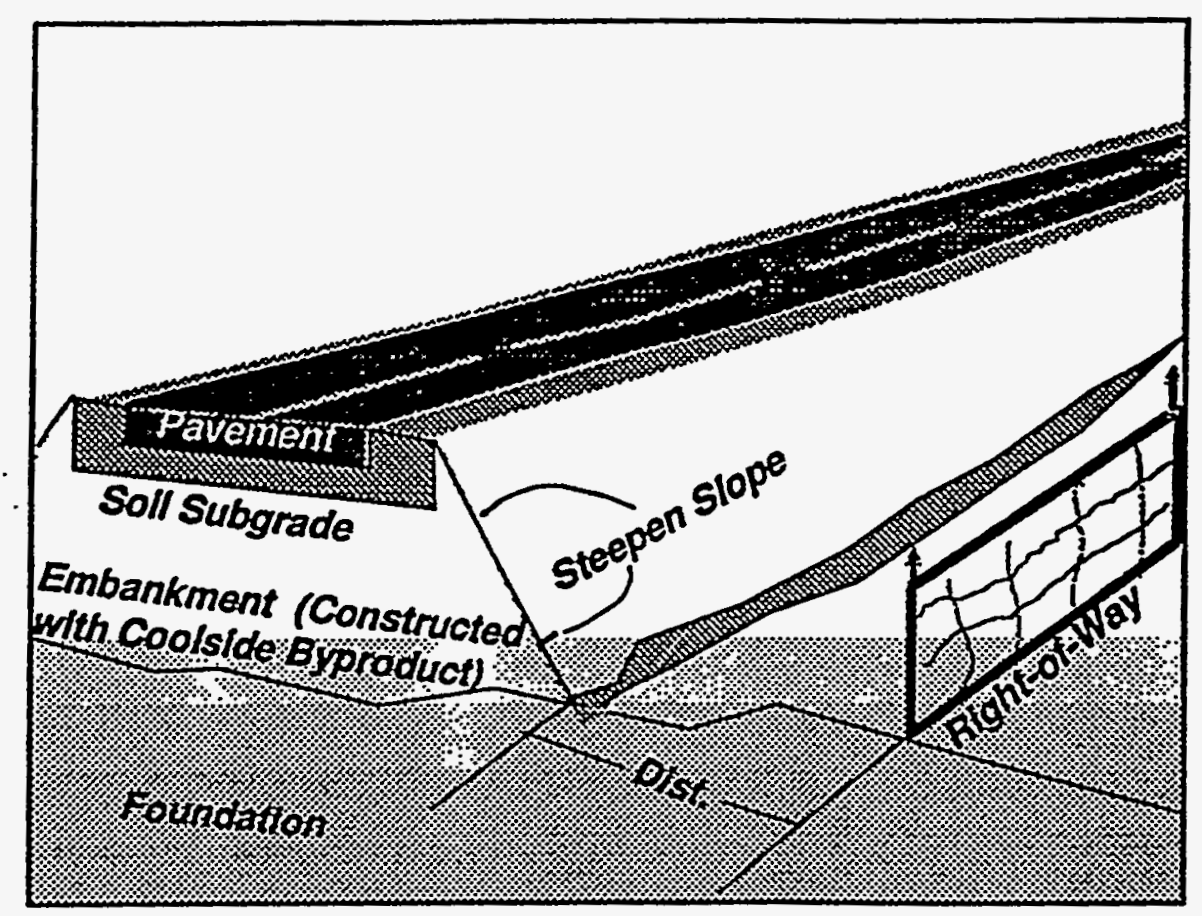

Figure 3-62. Use of Coolside byproduct to construct embankments with a steepen slope 


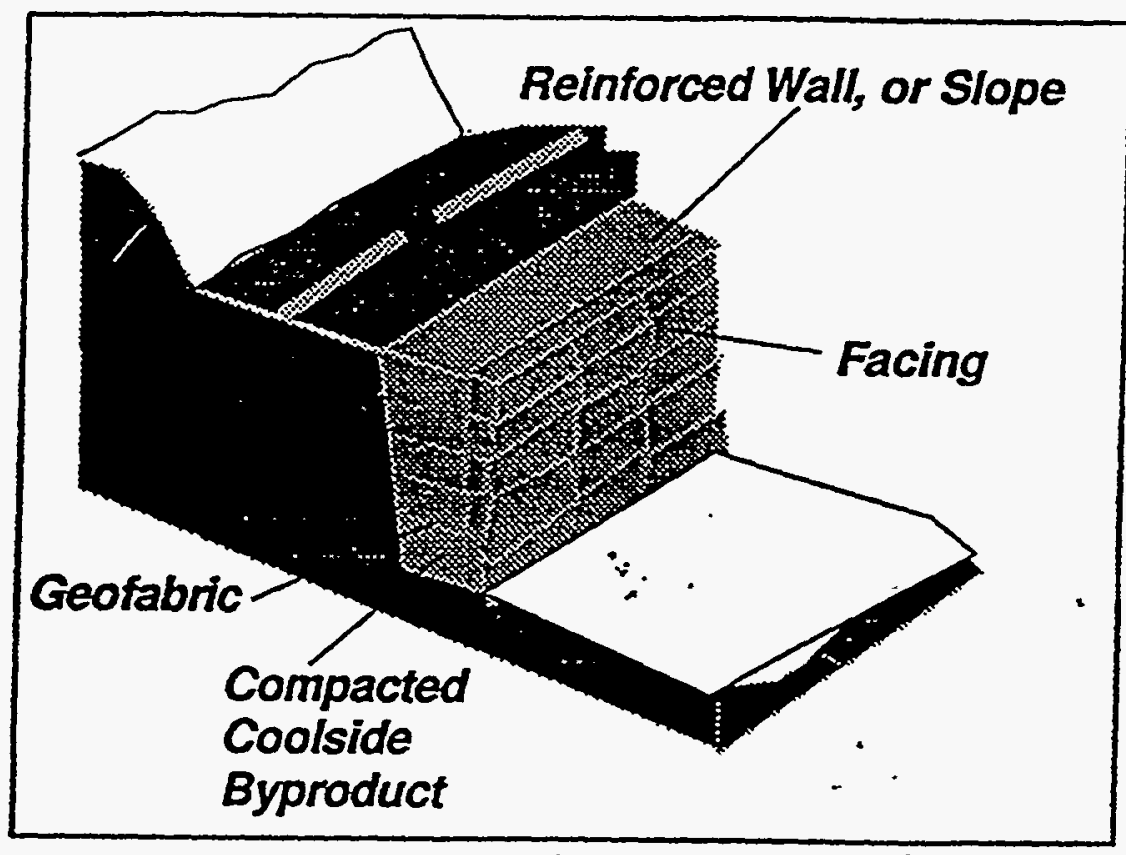

Figure 3-63. Reinforced wall, or slope, constructed with Coolside byproduct 
with steep angles, less land is required to construct a highway and a large savings could be realized, especially in urban areas where land may cost several thousands of dollars per acre. In certain instances, the Coolside material could be used with geofabrics to construct vertical walls, as illustrated in Figure 3-63. Because of the large strength of the material, when compacted to standard conditions, the Coolside material could be potentially used to support an embankment on a soft foundation. Geofabric could be used to reinforced the Coolside material, as shown in Figure 3-51.

\section{References}

AASHTO; (1995). Standard Specifications for Transportation Materials and Methods of Sampling and Testing, Part II, Seventeenth Edition, Published by the American Association of State Highway and Transportation Officials, Washington, D.C.

ASTM; (1996). Annual Book of Standards, Soil and Rock (1): D 420-D4914, Section 4 (Construction), American Society for Testing and Materials, West Conshohocken, PA.

Beckham, T. L. and Hopkins, T. C.; (September 1993). "Techniques Used in the Placement of Coolside By-Product in Field Lysimeters," Proceedings, Tenth Annual International Pittsburgh Coal Conference, Pittsburgh, Pennsylvania.

Casagrande, A., and Fadum, R. E.; (1940). Notes on Soil Testing for Engineering Purposes, Harvard University Graduate School Engineering Publication 8.

Caterpillar Tractor Co.; (October1981). Caterpillar Performance Handbook, 1981 Revised Edition, U.S.A.

Daniel, D. E.; (December 1989). "A Note on Falling Headwater and Rising Tail Water Permeability Tests," Geotechnical Testing Journal GTJODJ, Volume 12, Number 4. 
Graham, U.M., Wu, M.M., Robl, T.L., and Hopkins, T.C.; (Sept. 1993); “Mineralogical Transformation of Ettringite in Concrete Derived From Dry FGD Byproducts," Proceedings, Tenth Annual International Pittsburgh Coal Conference, Pittsburgh, Pennsylvania.

Hopkins, T.C.; (January 1980), "Shear Strength of Compacted Shales," University of Kentucky Transportation Center, Research Report 88-1, College of Engineering, Lexington, $K Y$.

Hopkins, T.C., Hunsucker, D., and Sharpe, G.W.; (October 1988) "Highway Field Trials of Chemically Stabilized Soil Subgrades," Proceedings, ORVSS XIX, Ohio River Valley Soils Seminar, Lexington, Kentucky.

Hopkins, T.C., "Bearing Capacity Analysis of Pavements," Research Report KTC91-8, University of Kentucky Transportation Center, College of Engineering, Lexington, Kentucky, June 1991.

Hopkins, T.C., and Beckham, T. L.; (September 1993) "Proposed Procedure for Compacting Laboratory Specimens for Physical Properties Testing," Proceedings, Tenth Annual International Pittsburgh Coal Conference, Pittsburgh, Pennsylvania.

Hopkins, T.C.; Hunsucker, D. Q.; and Beckham, T. L.; (October 1993). "Residue Byproduct from an Atmorpheric Bed Combustion Process Used in Highway Subgrade Modification," Syposium Proceedings, Recovery and Effective Reuse of Discarded Materials and By-Products for Construction of Highway Facilities, Sponsored by the Federal Highway Administration and the Environmental Protection Agency.

Hopkins, T.C.; Wu, M.M.; Winschel, R.A.; and Robl, T.L., (January 1993), "The Ohio Coal Development Office Coolside Waste Management Demonstration Project," Proceedings: Tenth International Ash Use Symposium, Vol.2: Ash Use R\&D and Clean Coal By-Products, American Coal Ash Association, Orlando, Florida. 
Hopkins, T.C.; Beckham, T. AL.; and Hunsucker, D. Q.; (June 1994). "Modification of Highway Soll Subgrades," Research Report KTC-94-11, University of Kentucky Transportation Center, College of Engineering, Lexington, Kentucky.

Hopkins, T.C.; (July 1994a), "Minimum Bearing Strength of Soil Subgrades Required to Construct Flexible Pavements," Proceedings, The 4th International Conference on the Bearing Capacity of Roads and Airfields, Vol.1, Minneapolis, Minnesota.

Hopkins, T.C.; (July 1994b), "Case Studies of Flexible Pavement Failures During Construction," Proceedings, The 4th International Conference on the Bearing Capacity of Roads and Airfields, Vol.1, Minneapolis, Minnesota.

McNulty, E. G.; Hopkins, T. C.; and Gorman, C. T.; (1978). "Analysis of TimeDependent Consolidation Data," Preprint 3280, ASCE Spring Convention and Exhibit.

Terzaghi, K.; (1943), "Theoretical Soil Mechanics," John Wiley and Sons, Inc., New York.

Taylor, D. W.; (1948). Fundamentals of Soil Mechanics, John Wiley \& Sons, New York, New York. 


\section{CHAPTER 4. LABORATORY-LYSIMETER STUDIES}

\section{Summary}

Twenty-two laboratory lysimeters were packed with dry flue-gas desulfurization wastes generated during tests of the Coolside duct-injection Technology. Included in the test matrix were FGD materials from Ohio Edison's 1990 demonstration runs conducted at its Edgewater power plant near Loraine, $\mathrm{OH}$ as well as materials derived from runs conducted in CONSOL's Coolside pilot plant in Library, PA. In an effort to characterize the leaching behavior of these materials, water was systematically added to the laboratory lysimeters with the resulting leachate being collected and analyzed on a weekly basis. The primary objective of the study was to generate predictive information on leaching behavior following disposal of Coolside wastes. In addition to the primary objective, the test matrix was designed to examine the impact of various experimental conditions on leaching behavior including 1) lysimeter packing density, 2) use of a constant versus a rain simulation method of water addition, 3 ) variation in the extent of prehydration of the wastes prior to loading, and 4) exposure to elevated levels of $\mathrm{CO}_{2}$ in the lysimeter headspace. Following 12 months of leaching, cores were removed from the top, middle, and bottom of each lysimeter for XRD and SEM analyses.

TCLP extractions conducted independently of the leaching study indicated the maximum concentration of extractable hazardous elements to be well below the limits specified by the Federal Resource Conservation and Recovery Act (RCRA) for non-hazardous wastes and often below the analytical detection limits. Leaching results were congruent with the TCLP extractions in that the maximum concentration of hazardous elements in the leachate waters were also well below these same limits. 
The major impact of variations in packing density as well as the level of prehydration was on flow rate (permeability) through the columns which in turn had a significant impact on leaching kinetics. Variation of $\mathrm{CO}_{2}$ concentrations in the headspace above each column altered the mineralogy of the packed beds and in turn the leachate chemistry. The largest effects of exposure to $\mathrm{CO}_{2}$ were a decrease in leachate $\mathrm{pH}$, elimination of ettringite, and formation of calcite and gypsum. When the leachate data were examined in terms of total water flow through the column, only minor differences were noted for those columns in which water was added at a fixed weekly volume versus those in which water was added in a manner to simulate regional rainfall.

Due to the massive amount of data generated over a 12-month interval from 22 lysimeters, only those data that are deemed significant or relevant are discussed in this report.

\section{Background}

Various scenarios for the use or disposal of the solid wastes generated by the Coolside or similar dry-FGD technologies have been contemplated including landfilling, use as a subbase for road construction, as soil additive, or light weight aggregate following pelletization. However, none of these strategies may be realized until it has been demonstrated that the leachates generated from such materials can meet RCRA standards for non-hazardous wastes and that the long-term leaching characteristics are clearly not detrimental to the environment. As discussed elsewhere in this report, the concentrations of hazardous elements extracted from the Coolside wastes are well below RCRA limits as determined by TCLP. However, while useful for determining compliance with RCRA, TCLP does not simulate landfill conditions, nor does it provide information on long term leaching characteristics and/or mineralogic transitions that may occur over prolonged periods. In addition, while actual field studies are the most realistic simulator of landfill conditions, capital costs coupled with sample-size and manpower requirements make it cost prohibitive 
to conduct an extensive matrix study of the impact of various packing/eaching parameters or conditions. Accordingly, the laboratory-lysimeter study was devised with the objective of complimenting a larger-scale concurrent field-lysimeter study by confirming the field results while providing basic information on the impact of leaching parameters unavailable from the larger field study due to cost considerations. To meet these objectives, a total of six Coolside-based samples were loaded to 22 laboratory lysimeters in an effort to monitor long-term leaching behavior and changes in mineralogy.

The lysimeter variables investigated in the study include the solid-waste packing density, the concentration of $\mathrm{CO}_{2}$ in the column headspace, constant versus rain simulation methods of water addition, and sample prehydration. Due to space limitations, only those results which are deemed significant and/or relevant are discussed. These discussions focus on the impact of packing density, $\mathrm{CO}_{2}$, and prehydration and for the most part are limited to one sample generated in the Coolside pilot plant (PP run \#2) and one sample from the larger scale demonstration-plant runs (Demo run \#3).

Experience gained from a preliminary round of lysimeter tests was used to select the conditions and methods employed for a final series of laboratory lysimeters. One of the more significant departures from field conditions is that nearly all the laboratory columns were packed at a relatively low density (loose/static packing) of $49 \mathrm{lb} / \mathrm{ft}^{3}$. Three of the columns were packed at a more moderate density (proctor-65 lb/ $/ \mathrm{ft}^{3}$ ) and none of the columns were packed at the high density level (modified-69 lb/tit) included in the preliminary series. Low density packing was emphasized due to problems encountered with low porosity and consequent low flow rates in columns packed at higher density, often precluding the collection of sufficient leachate for analysis. A second significant modification is that, with the exception of the rain-simulation columns, the majority of the columns received a fixed amount of water each week ( 46.5 or $93.0 \mathrm{~mL}$ ) as opposed to maintaining fully saturated conditions with a standing column of water over the packed 
beds. In this approach, the water added to each column made a single pass through the packed bed then collected in a reservoir at the bottom where it was drained weekly. Two implications of this approach were that water samples were not available for analysis until breakthrough occurred several weeks into the study and this setup provides for the wetting and partial drying of the waste materials during the study, both of which are more similar to prevailing conditions in the field lysimeters. In addition, in an effort to evaluate the impact of high levels of dissolved $\mathrm{CO}_{2}$ in groundwaters, several of the laboratory columns were blanketed with enriched $\mathrm{CO}_{2}$ atmospheres (2.5 and 5.0 vol\%). The packing and leaching procedures along with the test matrix are detailed in the following sections.

\section{Experimental Design}

\section{Samples}

A total of 6 waste samples were placed into 22 laboratory lysimeters. Table 4-1 shows the sample identification, lysimeter number, sample weight, the weight of water added to the sample prior to packing (prehydration water), packing height, and dry-sample density for each of the lysimeters. The samples identified as Coolside Run \#1 and \#3 (LC \#1-8) represent FGD wastes from the Edgewater plant demonstration runs 1 and 3 , respectively. These materials were comprised of Class $\mathrm{F}$ fly ash mixed with $\mathrm{CaSO}_{4}, \mathrm{CaSO}_{3}, \mathrm{Ca}(\mathrm{OH})_{2}$, and $\mathrm{NaOH}$. The materials loaded to the lysimeters represented blends of material taken from several of the barrels of waste collected during each run. These sample blends had been retained in sealed 5-gallon plastic buckets from the time they were blended until they were loaded to the lysimeters. The samples identified as PP \#1-4 (LC9-16, 25-26, and 3336) represent FGD wastes from the four Coolside pilot-plant tests. These samples were taken from 5-gallon plastic buckets that had remained sealed since early stages of the project. 


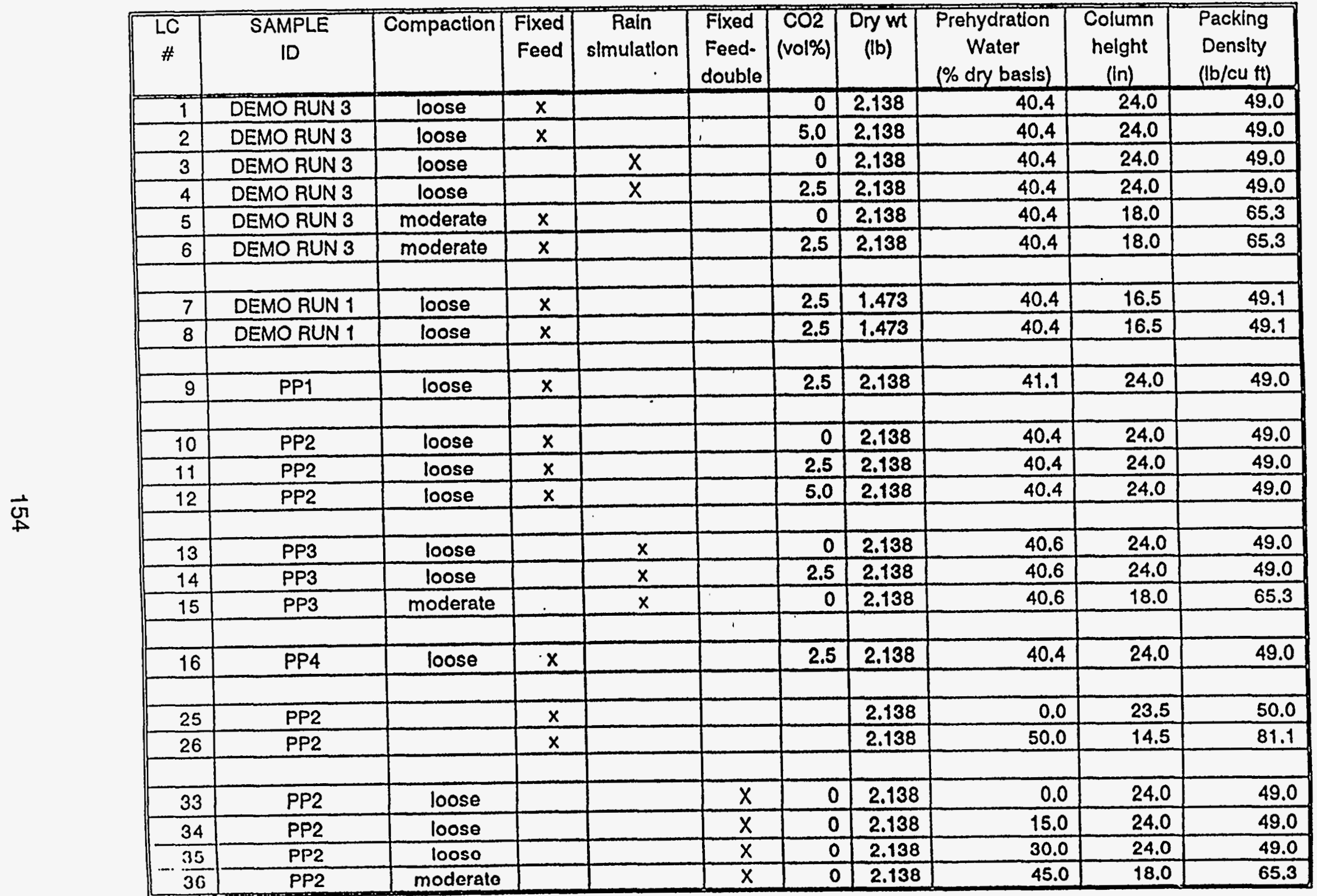

Table 4-1. Test matrix. 


\section{Laboratory-Lysimeter Description}

A schematic of the laboratory leaching columns fabricated for this study is shown in Figure 4-1. They were constructed from clear-acrylic cylinders, $39^{\prime \prime}(1 \mathrm{~m})$ in length, 2 " $(5 \mathrm{~cm})$ inner diameter, and $\sim 1 / 4^{\prime \prime}(0.6 \mathrm{~cm})$ wall thickness. A plexiglass frit, overlain with one inch of glass wool followed by one inch of Ottawa sand, was sealed in the bottom of each column within a PVC sleeve. The sleeve was in turn connected to a PVC reducing connector and plastic valve that was maintained in the closed position except during leachate collection.

There was an approximate $100 \mathrm{~mL}$ void volume between the bottom of the waste bed and the cut-off valve, sufficient for leachate waters passing through the column to collect beneath the waste bed until drained and weighed. This single-pass flow, as opposed to fully-saturated-conditions, was selected since it is believed to more closely simulate conditions encountered within a landfill.

\section{Lysimeter Packing Procedures}

Packing the laboratory lysimeters entailed weighing approximately $2.1 \mathrm{lb}(\sim 1-\mathrm{kg})$ of dry sample to a 2-L stainless-steel mixing bowl to which was added a targeted amount of distilled water (prehydration water). The sample and water were then blended with a Kitchen-Aide Classic $^{\mathrm{TM}}$ mixer at low speed for approximately five minutes (or until the prehydrated sample had a homogeneous granular appearance). The bowl with prehydrated sample was then placed onto a top-loading balance from where the targeted weight of sample was transferred to a preassembled lysimeter. Each lysimeter was then held vertically and tamped until the targeted column height was attained.

For samples that were loaded to more than one lysimeter, a series of mixtures were prepared as described above with each mixture being split equally among the lysimeters 
Rubber stopper $\longrightarrow$

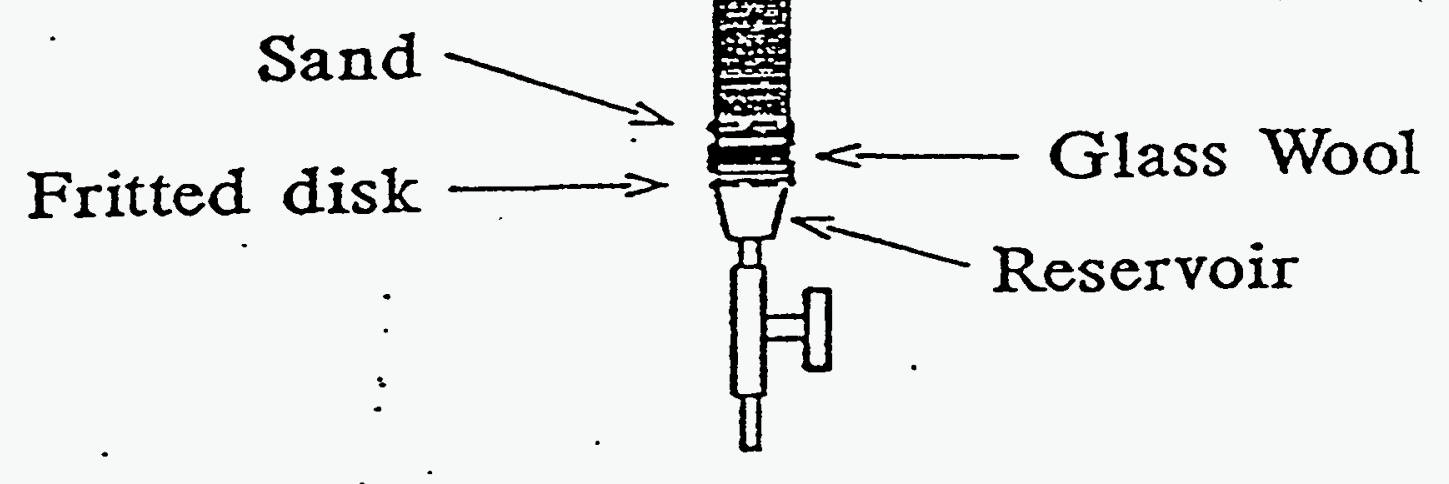

$\longleftarrow$ Solid Waste
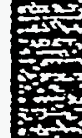

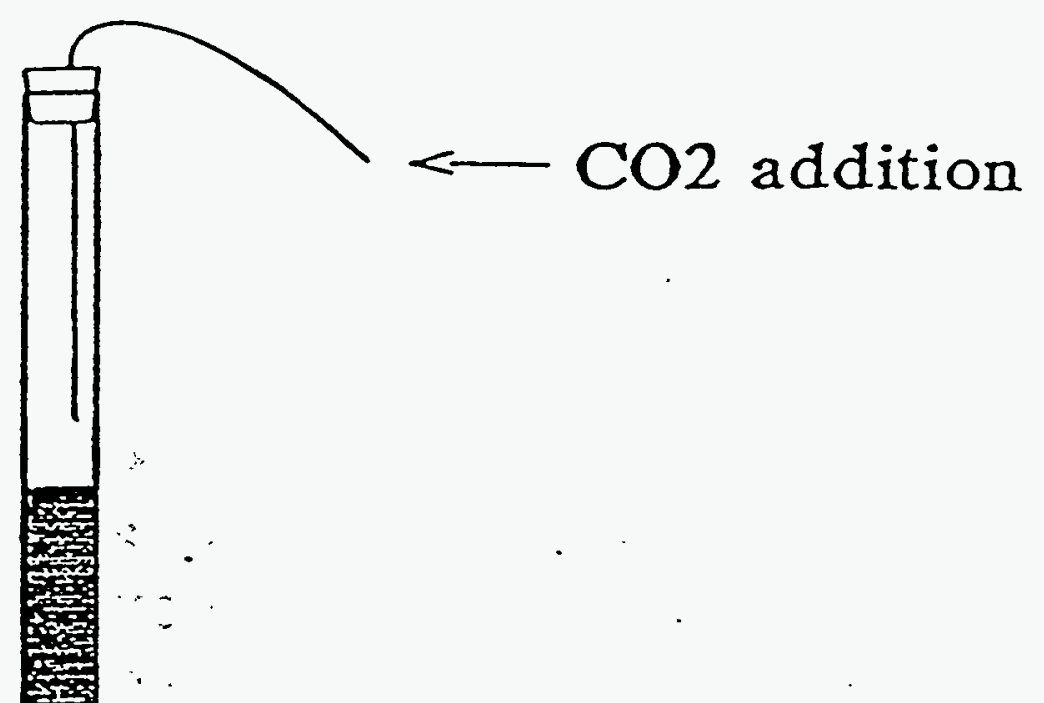


being loaded with that particular sample. Since each mixture contained just enough sample to load one lysimeter, the number of such mixtures prepared corresponded to the number of lysimeters being loaded with that sample. This approach was used to ensure sample homogeneity between different lysimeters containing the same sample.

The exceptions to the packing procedure described above were for lysimeters \#7-8 (Demo Run \#1), and lysimeters 25, 26, 33, and 36 (PP \#2). For \#7 and 8, there was an insufficient amount of sample to pack two columns to a height of $24^{\text {" }}$ at loose density. Instead, the available sample was split between two columns and was intended to serve as a measure of method reproducibility (duplicate conditions). However, as discussed in more detail later, flow problems in LC8 (no flow) prevented the gathering of comparative data for these two columns. The packing of LC25, LC26, LC33, and LC36 varied from the normal procedure because the degree of prehydration was chosen as a test variable for these columns. For LC25 and LC33, the targeted amount of dry sample was loaded to the column without prior water addition (prehydration). For LC26 and LC36, an excess of prehydration water was blended with the sample before loading. The 'mudlike' consistency of these latter high-water-content samples precluded adjusting packing height, i.e. dry density.

All columns were capped with rubber stoppers. With the exception of the rain-simulated columns and those columns exposed to $\mathrm{CO}_{2}$, these were solid stoppers which served to suppress evaporation. The rain-simulated columns were capped with two-hole stoppers in order to permit some evaporation while restricting the exchange of room air with the headspace atmosphere. Those samples exposed to elevated levels of $\mathrm{CO}_{2}$ were also capped with two-hole stoppers. For these columns, $\mathrm{CO}_{2}$-containing gases were delivered through one of the holes and permitted to exit through the other. This approach also permitted evaporation. The use of rubber plugs, as well as the manner in which $\mathrm{CO}_{2}$ is added, is described more fully in the next section. 


\section{Test Matrix}

The conditions to which each lysimeter was subjected are shown in Table 4-1. The same weight of material was targeted for each column with the exception of LC7 and LC8 (Demo run \#1). As previously discussed, the limited amount of sample was split evenly between these two columns then the packing height was adjusted to attain the targeted packing density (Table 4-1).

The column headings in Table 4-1 are defined as follows:

Compaction. Refers to the packing density of the study samples and is expressed on a drysample basis. All columns were packed at either a low-energy compaction of $49 \mathrm{lb} / \mathrm{tt}^{3}$ (static) or at $65 \mathrm{lb} / \mathrm{ft}^{3}$ (proctor). These are the packing densities previously determined to correspond to a low-energy static compaction or to that which would result from the pressure applied by the tracks of a Caterpillar D9L track-type tractor using samples from demonstration runs 1 and $3 .^{1}$

Fixed Feed and Rain Simulation. Weekly additions of distilled water were made to each column in one of three manners. For the rain-simulated columns, water additions matched the cumulative weekly rainfall measured at a weather station located approximately 15 miles from the field lysimeters (Farmers-Stanton station). The starting date for rainfall measurements was the week of November 19,1991. This interval was selected since 1) the 12 month cumulative rainfall measured from this date (46.94 in) matches within one inch the average annual rainfall for this site (46.1) and 2) the field lysimeters were uncovered and exposed to the elements during November of 1992; thus, seasonal wet and dry periods were in rough correspondence for the laboratory and field lysimeters. Weather- 
station measurements and water additions to the rain-simulation columns are shown in Table 4-2.

The annual rainfall (46.94) measured from November 19, 1991 through November 18, 1992 at the Farmers-Stanton Station equates to the addition of $46.5 \mathrm{~mL}$ of water/week to a $2^{\prime \prime}$ diameter column. Thus, $46.5 \mathrm{~mL}$ of distilled water were added to each of the fixed-feed columns as designated under the fixed water feed heading of Table 4-1. By virtue of this approach, both the rain-simulated and fixed-feed columns received equivalent amounts of water over the 12-month study interval. Water was added in a similar matter to those lysimeters as indicated in the fixed feed-double heading (LC33-36), only the volume of added water was doubled to $93.0 \mathrm{~m} /$ week. These latter columns were included in the study six weeks after water feed had been initiated to the other lysimeters. The objective for these columns was to expand the examination of prehydration, particularly with respect to the impact on flow rates. They were not intended for a full 12-month study since other columns (LC25-26), in which prehydration water was also a test variable, were already in place for that purpose. Thus, a doubling of the feed rate served to accelerate this phase of the study, permit the study to be completed on schedule, and provide additional information on the impact of prehydration.

The rain-simulated and fixed-feed columns also differ in that water added to the rainsimulated columns was permitted to evaporate and exit the column through a two-hole rubber stopper used to cap these columns. Thus, it is anticipated that some drying of the upper portions of the packed solids will occur during the study, particularly during dry periods (low or no water additions during dryer periods). This was also the case for the fixed-feed and rain-simulation columns which were continuously purged with a blend of $\mathrm{N}_{2}$ and $\mathrm{CO}_{2}$ as described in the next paragraph. The remaining fixed feed columns were capped with solid rubber stoppers for the duration of the study to suppress evaporation. 


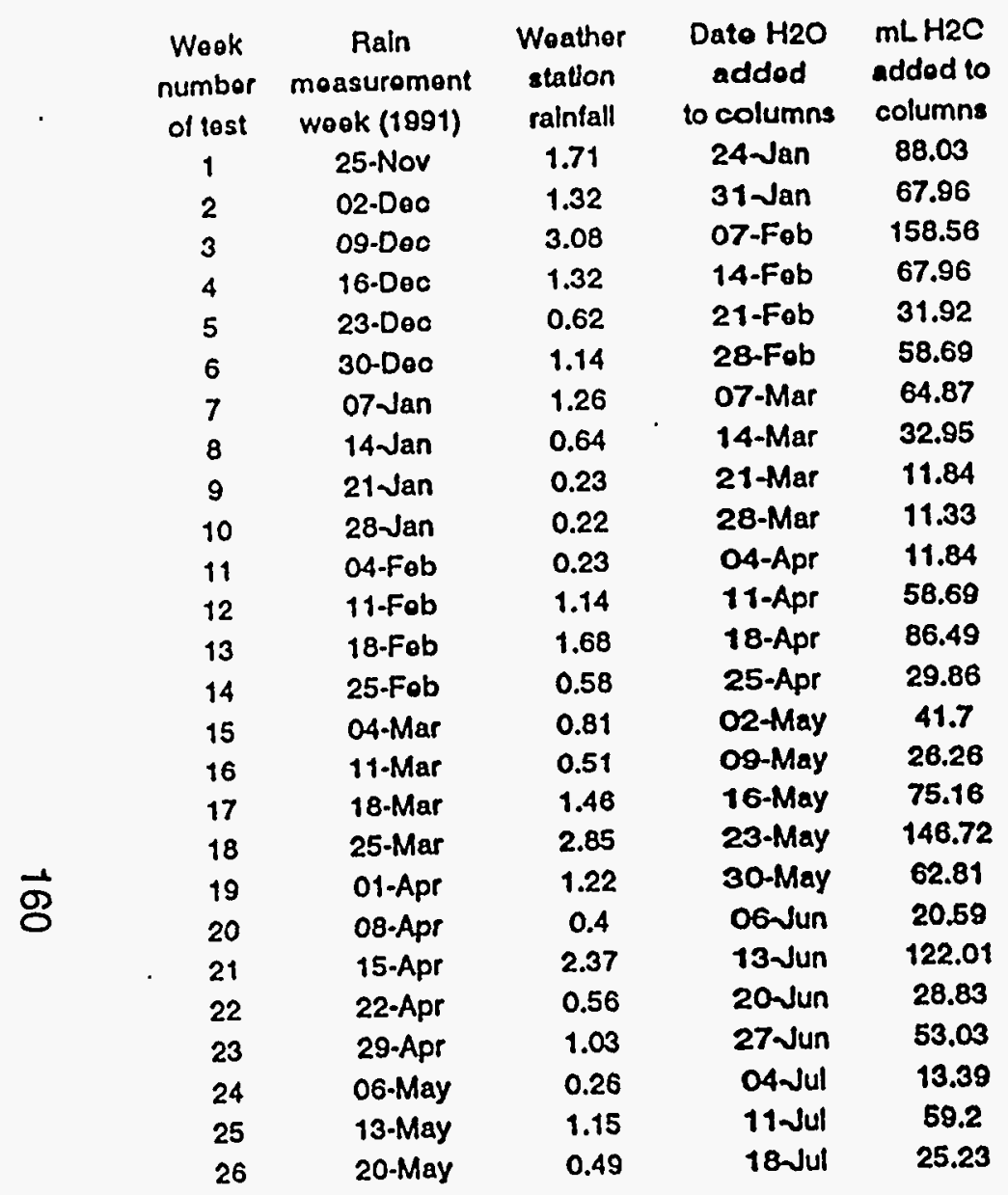

\begin{tabular}{|c|c|c|c|c|}
\hline $\begin{array}{l}\text { Wook } \\
\text { numbor } \\
\text { of tost }\end{array}$ & $\begin{array}{l}\text { Rain } \\
\text { moasuromont } \\
\text { wook (1991) }\end{array}$ & $\begin{array}{l}\text { Weathor } \\
\text { station } \\
\text { ralnfall }\end{array}$ & $\begin{array}{l}\text { Dato H2O } \\
\text { added } \\
\text { to columns }\end{array}$ & $\begin{array}{l}\mathrm{mL} \mathrm{H} 2 \mathrm{C} \\
\text { addod to } \\
\text { columns }\end{array}$ \\
\hline 27 & 27-May & 0.04 & 25 Jut & 2.08 \\
\hline 28 & 03Jun & 1.25 & 01-Aug & 64.35 \\
\hline 29 & 10-Jun & 0 & OB-Aug & 0 \\
\hline 30 & 17Jun & 0.18 & 15-Aug & 8.27 \\
\hline 31 & 24Jun & 0.87 & 22-Aug & 44.79 \\
\hline 32 & 01 Jul & 1.03 & 29-Aug & 53.03 \\
\hline 33 & OBDJul & 0.5 & 05-Sep & 25.74 \\
\hline 34 & 15 Jul & 2.44 & 12-Sep & 125.5 \\
\hline 35 & 22Jul & 1.97 & 10-Sop & 101.5 \\
\hline 36 & 29-Jul & 0.51 & 26-Sep & 26.26 \\
\hline 37 & 05.Aug & 0.01 & $03-0 \mathrm{ct}$ & 0.51 \\
\hline 38 & 12-Aug & 2.16 & $10-0$ at & 111.2 \\
\hline 39 & 19-Aug & 0.48 & $17-0 \mathrm{ct}$ & 24.71 \\
\hline 40 & 26-Aug & 0.39 & 24-Oct & 20.08 \\
\hline 41 & 02-Sep & 0.03 & $31-O c t$ & 1.54 \\
\hline 42 & 09-Sop & 1.06 & 07-Nov & 54.57 \\
\hline 43 & 16-Sep & 1.25 & 14-Nov & 64.35 \\
\hline 44 & 23-Sop & 0.17 & 21-Nov & 8.75 \\
\hline 45 & 30-Sop & 2.38 & 28-Nov & 122.53 \\
\hline 46 & $07-0 c t$ & 0.68 & 05-Doc & 35.01 \\
\hline 47 & $14-$ Oct & 0.01 & 12-Doc & 0.51 \\
\hline 48 & 21-Oct & 0.21 & 19-Dec & 10.81 \\
\hline 49 & 28-Oct & 0.77 & 26-Doc & 39.64 \\
\hline 50 & 04-Nov & 0.01 & 02Jan & 0.51 \\
\hline 51 & 11-Nov & 0.01 & 09-Jan & 0.51 \\
\hline 52 & 18-Nov & 0.25 & 16 Jan & 12.87 \\
\hline
\end{tabular}

$\begin{array}{cccc} & \text { total } & 46.94 & 2416.5 \\ 1 & \text { average } & 0.90 & 46.47\end{array}$

Table 4-2. Weekly rainfall measurements from the Farmers-Stanton weather station and equivalent water additions to the rain-simulation lysimeters. 
$\% \mathrm{CO}_{2} . \mathrm{CO}_{2}$ concentrations in the lysimeter headspace were maintained at either $\leq 0.03$ (atmospheric abundance), 2.5 , or 5.6 vol\% (Table 4-1). The $\mathrm{CO}_{2}$-containing gas streams were continuously routed in parallel to the appropriate columns at a rate of 10$15 \mathrm{~cm}^{3} / \mathrm{min} /$ column for the duration of the study. These gases were dispensed from compressed gas cylinders via 1/8-i.d. x 1/4"-o.d. tygon tubes through a two-hole rubber stopper used to cap the $\mathrm{CO}_{2}$-purged columns. The ends of the delivery tubes were positioned approximately $12^{\prime \prime}$ down into the void space above each packed bed. The 1/4" exit holes of each of the rubber stoppers were constricted with $1 / 8^{\prime \prime} \times 1 / 16^{\prime \prime}-0 . d$. plastic reducing unions which served to suppress exchange of void-space gases with room air as well as to provide a more uniform distribution of the $\mathrm{CO}_{2}$-containing gas stream among the columns. This parameter was selected for study since groundwaters typically contain $\mathrm{CO}_{2}$ levels well in excess of atmospheric abundance and it is believed that the carbonate anions present in such waters may have a significant impact on the leachate $\mathrm{pH}$, leaching kinetics, and waste mineralogy. ${ }^{2}$

Prehvdration Water. After encountering problems with heating, swell, and loss of porosity in an initial series of tests, a decision was made to prehydrate the Coolside samples prior to loading, with the exception of LC25 and LC33 which were loaded dry. The values shown for prehydration water are expressed as a percentage of the dry sample weight and indicate the amount of distilled water blended with the sample prior to loading. Except for $L C 25,26$, and $33-36$ in which prehydration was a test variable, a moisture content of $42 \%$ was targeted (proctor moisture) since this level provided a granular consistency that was conducive to water flow. Addition of excess water to LC26 and LC36 resulted in a 'mudlike' consistency and precluded adjusting packing density. Immediately after loading, the drypacked column could only be tamped to a height of $26-27^{\text {n }}$ without risk of breaking the column. However, these columns settled slowly for several days to a final height of 23.5 and 24" for LC25 and LC33, respectively, following the first water addition. 
Dry Weight. The weight of dry sample loaded to each column.

\section{Flow Problems}

As previously mentioned, LC7 and LC8 were packed as duplicate columns and were included as a means to evaluate reproducibility. However, while LC7 flowed freely, LC8 did not. Since this rendered the data from LC8 meaningless, these data will not be presented or discussed in this report. Data on total ion elution from LC7 is presented in Table 4-3 to serve as a comparison to other Coolside samples. LC8 was dissected at the conclusion of the study and the problem was diagnosed as an insufficient amount of glass wool in the bottom of the column. This permitted the overlying sand to block the drain holes in the fritted disk thereby preventing flow through the column.

In addition to the flow problems encountered with LC8, problems with low flow rates were also encountered in LC15, 16, 25, 26, 33, and 36 . These low flow rates were apparently due to the level of prehydration selected for LC25, 26, 33, and 36 as discussed in the results section. However, for LC14-16, the reasons for the unacceptably low flow rates are unknown. Elemental release from these latter columns appeared to be dictated by total flow rendering comparisons between these lysimeters and the free-flowing lysimeters invalid. For this reason, discussion of leaching data for LC15-16 is also omitted. In addition, discussion of the data from LC13 and LC14, which were coupled with LC15 in the matrix study, will be limited.

\section{Leachate Analysis}

Each weekly collection of leachate waters was analyzed for $\mathrm{pH}$, alkalinity, conductivity, anions $\left(\mathrm{SO}_{4}, \mathrm{SO}_{3}, \mathrm{Cl}, \mathrm{NO}_{3}, \mathrm{Br}\right.$ ), and cations/metals ( $\mathrm{Ag}, \mathrm{Al}, \mathrm{As}, \mathrm{B}, \mathrm{Ba}, \mathrm{Be}, \mathrm{Ca}, \mathrm{Cd}, \mathrm{Co}, \mathrm{Cr}$, $\mathrm{Cu}, \mathrm{Fe}, \mathrm{K}, \mathrm{Mg}, \mathrm{Mn}, \mathrm{Mo}, \mathrm{Na}, \mathrm{Ni}, \mathrm{P}, \mathrm{Pb}, \mathrm{Se}, \mathrm{Si}, \mathrm{Ti}, \mathrm{V}, \mathrm{Zn}$ ). Splits of each leachate were 


\begin{tabular}{|c|c|c|c|c|c|c|c|c|c|c|c|c|c|c|c|c|c|}
\hline & LC1 & LC2 & LC3 & LC4 & LC5 & L.C6 & LC7 & LC9 & $L C 10$ & $\frac{\text { Lysim }}{\text { LC11 }}$ & LC12 & LC13 & LC14 & $\underline{L} \mathrm{~L} 25$ & $\angle C 33$ & LC34 & LC35 \\
\hline & Dem3 & Dem3 & Dom3 & Dem3 & Dem3 & Dem3 & Dem1 & PP1 & PP2 & PP2 & PP2 & PP3 & PP3 & PP2 & PP2 & PP2 & PP2 \\
\hline Water Feed & Fxod & Flxed & Rain & Raln & Fixid & Fixed & Fxod & Fixod & Fxod & Fined & Fixed & Roin & Rain & Fixed & Double & Double & Double \\
\hline $\mathrm{CO} 2$ & 0 & 5 & 0 & 2.5 & 0 & 2.5 & 2.5 & 2.5 & 0 & 2.5 & 5 & 0 & 2.5 & 0 & 0 & 0 & 0 \\
\hline Compaction & Loose & Loose & Loose & Loose & Proctor & Proctor & Loose & Loose & Loose & Loose & Looes & 10001 & 1000 & Loose & 1000 & Loose & Loose \\
\hline Water added-mL & 2442 & 2442 & 2435 & 2435 & 2442 & 2412 & 2347.7 & 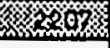 & 2442 & 2442 & 2142 & 2337 & 2334 & 2207 & s. & 1228 & 1228 \\
\hline Recovery ( $\%$ ) & 89.5 & 77.8 & 81.0 & 89.5 & 87.8 & 02.4 & 85.0 & 84.8 & 92.2 & 94.7 & 87.8 & 68.3 & 51,8 & 81.8 & 27.8 & 82.7 & 81.0 \\
\hline $\mathrm{Ag}(\mathrm{mg})$ & 0.00 & 0.00 & 0.00 & 0.00 & 0.00 & 0.00 & 0 & 0.00 & 0.00 & 0.00 & 0.00 & 0.00 & 0.00 & 0.00 & 0.006 & 0.00 & 0.00 \\
\hline$A l(m g)$ & 8.84 & 8.95 & 8.84 & 1.43 & 36.66 & 24.65 & 5.38 & 7.47 & 25.32 & 18.56 & 8.10 & 0.33 & 0.22 & 24.30 & 5.30 & 86.7 & 64.8 \\
\hline As (mg) & 2.14 & 1.68 & 2.17 & 2.85 & 4.18 & 4.38 & 2.22 & 0.063 & 0.55 & 0.60 & 0.61 & 0.0053 & 0.12 & 1.18 & 1.11 & 1.55 & 2.40 \\
\hline $8(\mathrm{mg})$ & 7.57 & 90.3 & 5.40 & 29.0 & 0.48 & 7.13 & 5.00 & 0.42 & 0.08 & 1.37 & 11.38 & 0.37 & 0.28 & 2.24 & 2.10 & 4.98 & 4.57 \\
\hline$B a(m g)$ & 0.058 & 0.022 & 0.041 & 0.021 & 0.018 & 0.012 & 0.021 & 0.058 & 0.023 & 0.185 & 0.308 & 0.080 & 0.040 & 0.040 & 0.023 & 0.032 & 0.027 \\
\hline $\mathrm{Ca}(\mathrm{mg})$ & 142.7 & 858.8 & 115.3 & 94.5 & 30.7 & 33.0 & 73.2 & 39.0 & 68.0 & 166.0 & 609.5 & 134.0 & 82.3 & 79.6 & 10.4 & 88.0 & 81.7 \\
\hline Cd (mg) & 0.000 & 0.000 & 0.000 & 0.000 & 0.00 & 0.00 & 0.00 & 0.000 & 0.000 & 0.000 & 0.000 & 0.000 & 0.000 & 0.005 & 0.000 & 0.0004 & 0.0010 \\
\hline $\mathrm{Cr}(\mathrm{mg})$ & 0,0003 & 0.000 & 0.0011 & 0.000 & 0.000 & 0.0007 & 0.0000 & 0.0015 & 0.004 & 0.0061 & 0.000 & 0.000 & 0.0680 & 0.0273 & 0.0004 & 0.0005 & 0.0000 \\
\hline$K(\mathrm{mg})$ & 3302 & 1834 & 2841 & 3315 & 2520 & 2830 & 2053 & 2843 & 1111 & 1345 & 1203 & 1243 & 830 & 1850 & 1120 & 1978 & 1813 \\
\hline $\mathrm{Mg}$ (mg) & 0.063 & 1.87 & 0.070 & 0.071 & 0.045 & 0.058 & 0.067 & 0.081 & 0.064 & 0,062 & 1.73 & 0.110 & 0.087 & 0.031 & 0.018 & 0.10 & 0.12 \\
\hline $\mathrm{Na}(\mathrm{mg})$ & 10632 & 6404 & 8617 & 11181 & 8269 & 8762 & 5846 & 16684 & 0608 & 12107 & 12702 & 624 & 374 & 21072 & 12035 & 17565 & 16837 \\
\hline $\mathrm{NI}(\mathrm{mg})$ & 0.00 & 0.00 & 0.00 & 0.00 & 0.00 & 0.00 & 0.00 & 0.00 & 0.00 & 0.00 & 0.00 & 0.00 & 0.00 & 0.014 & 0.000 & 0.001 & 0.0010 \\
\hline $\mathrm{Pb}(\mathrm{mg})$ & 0.011 & 0.001 & 0.013 & 0.000 & 0.001 & 0.000 & 0.000 & 0.000 & 0.000 & 0.000 & 0.000 & 0.000 & 0.0060 & 0.000 & 0.000 & 0.000 & 0.000 \\
\hline So $(m g)$ & 1.23 & 1.14 & 1.17 & 1.40 & 1.02 & 1.25 & 1.01 & 0.14 & 0.12 & 0.23 & 0.15 & 0.005 & 0.00 & 0.13 & 0,12 & 0.25 & 0.21 \\
\hline $\mathrm{Cl}(\mathrm{mg})$ & 10801 & 1286 & 8078 & 10568 & 9320 & 10516 & 1041 & 688 & 391 & 628 & 935 & 136 & 74 & 772 & 738 & 1509 & 1449 \\
\hline $\operatorname{Br}(m g)$ & & & & & 1031 & 1078.2 & 756.9 & 1159 & 1073 & 1345.0 & & & & 2502 & 1041 & 5437 & 5474 \\
\hline $\mathrm{SO} 4(\mathrm{mg})$ & 11747 & 10413 & 10032 & 10631 & 3454 & 4790 & 6399 & 30421 & 10231 & 22200 & 21063 & 2755 & 1864 & 42689 & 33100 & 34538 & 32644 \\
\hline Alkallnity & 2079 & 1048 & 2054 & 2078 & 5311 & 4050 & 2031 & 17078 & 1903 & 1118 & 3680 & 100 & & 3778 & & 7108 & 8076 \\
\hline AvopH & 11.73 & 10.76 & 11.90 & 11.76 & 12.22 & 12.16 & 11.05 & 12.01 & 12.14 & 11.02 & 10.07 & 8.07 & 0.42 & 11.02 & 11.61 & 11.03 & 11.82 \\
\hline Avo Conduetlutty & 21.77 & 10.07 & 21.04 & 22.64 & 20.80 & 21.70 & 14.48 & 18,88 & 17.40 & 20.30 & 21.34 & 4.10 & 3,81 & 34.87 & 00.31 & 16.51 & 17.38 \\
\hline
\end{tabular}

Table 4-3. Total eluted weights for selected ions calculated as the product of the 
treated with acid immediately after collection to stabilize the samples prior to metals (cation) analysis. All analyses were conducted as soon as practical following leachate collection, particularly for $\mathrm{pH}$ and anions (usually same day).

Anions were determined by EPA Method 300.0, The Determination of Inorganic Anions in Water by lon Chromatography. Conductivity and $\mathrm{pH}$ were determined using classical electrometric techniques. An Orion Microprocessor lonanalyzer/901 was used to measure pH and a YSI Model 32 conductance meter to measure conductivity. EPA Method 1620, Metals by Inductively Coupled Plasma Atomic Emission Spectroscopy, was followed for major and trace elements with a Spectrametrics ICP-AES coupled with a CETAC U-5000 ultrasonic nebulizer.

At the end of the 12-month study period, $1 / 2^{\prime \prime}$ cores were removed one inch from the top, one inch from the bottom, and from the middle of each packed bed. XRD spectra were obtained on each core sample with a Philips APD 3500 X-ray diffractometer, using Cu-K radiation $(=1.5418 \mathrm{~nm})$, scan speed of two counts $/$ second, and an increment of $0.1^{\circ}$ over a 2q range from 7 to $60^{\circ}$.

\section{Results}

The presentation of the laboratory-lysimeter results that follow is divided into sections. In the first section, total ion elution from the majority of the lysimeters, including information on RCRA elements, is shown. This is followed by discussion of the lysimeter results in subsets. That is, LC1-6 are treated separately as they were designed to examine the impact of the method of water addition, packing density, and an enriched $\mathrm{CO}_{2}$ atmosphere on materials from Demonstration Run \#3. Likewise, LC10-12 examines the impact of $\mathrm{CO}_{2}$ enriched atmospheres on material from PP2 and LC33-36 will be used to demonstrate the impact of differences in prehydration on leaching behavior, also on material from PP2. 


\section{Total Ion Elution}

Table 4-3 shows the total weight of selected ions that was eluted from each laboratory lysimeter over the course of the study. These values were obtained by multiplying the weight of leachate water collected each week by the coinciding ion concentrations then summing over the full study interval. The total leachate weights listed in Table 4-3 represent a 52 week interval with the exception of LC7 (50 wks), LC9 (47 wks), LC25 (47 wks), and LC33-35 (45 wks). Since not all columns exhibited free flow, Table 4-3 also shows the weight of water added to each column on an absolute basis as well as the amount of water collected, expressed as a percentage of the weight of added water. As discussed in the experimental section, low flow rates were a particular problem in lysimeters LC8 (660 mL recovered), LC15 (173 mL), LC16 (185 mL), LC25 (1364 mL), LC26 (2 mL), LC33 (438 mL), and LC36 (0 mL). For LC 25, 26, 33, and 36, the low flow rates were due to the level of prehydration water added to the samples prior to packing. The reason for flow problems in LC8 was found to be blockage of the drain holes in the fritted disk underlying the sample bed and the same problem is suspected for LC15 and LC16. Since the low flow rates in these latter columns precluded valid comparisons with the other columns that exhibited good flow characteristics, leaching data for several of these lysimeters are omitted from Table 4-3.

Scrutiny of the data of Table 4-3 shows the leachates from LC9 (PP1) exhibited the highest average $\mathrm{pH}$ and total alkalinity values. Leachates from LC13-14 (PP3) exhibited the lowest $\mathrm{pH}$, alkalinity, and conductivity. LC1-7 (demonstration runs 1 and 3) exhibited the greatest release of Se, As, and $\mathrm{Cl}$. The remaining lysimeters (PP2) were unremarkable with the exception of relatively high $\mathrm{SO}_{4}$ release. Unfortunately, $\mathrm{LC} 16$ which was packed with the remaining Coolside sample (PP4), did not flow. Due to the voluminous amount of data in 
Table 4-3, additional discussion of the leaching results is deferred to subsequent sections which examine the column data in a less cumbersome form.

\section{TCLP Extraction Data}

Results from TCLP extractions for the six Coolside samples are shown in Table 4-4. Also shown are the RCRA hazardous element limits which are used in defining hazardous versus non-hazardous wastes for disposal purposes. Materials from Demonstration runs 1 and 3 were blended prior to conducting this analysis. As can be seen, all of the RCRA elements were either below detection limits or well below limits specified by RCRA. However, pH values for both pilot plant \#3 and \#4 exceeded the RCRA limit of 12.5 in this analysis. This is somewhat surprising in that it was pilot plant tests 1 and 2 to which a sodium promoter was added and the presence of elevated $\mathrm{Na}$ would be expected to lead to greater release of $\mathrm{NaOH}$ which in turn yields $\mathrm{pH}$ levels higher than those from $\mathrm{Ca}(\mathrm{OH})_{2}$.

The top half of Table 4-5 shows the maximum concentrations of the RCRA elements along with maximum pH values that were measured in the column leachates over the course of the study.* $\mathrm{Ag}$ and $\mathrm{Cd}$ were below detection limits in all leachates while $\mathrm{Pb}$ was below detection limits in all of the pilot plant samples and at trace levels in the remainder. For the lysimeters packed with pilot plant materials, only arsenic in LC33 exceeded RCRA limits às defined for TCLP extraction. In contrast, all of the lysimeters packed with demonstration run materials exceeded the TCLP-defined limits for Se and all but two (LC1 and LC7) exceeded the limit for As. Though detected, none of the remaining hazardous elements exceeded RCRA limits in any of the lysimeters.

*Note that ion concentrations and $\mathrm{pH}$ values for the leachate waters are not required to meet RCRA limits. The data is presented in this format for comparative purposes only. 


\begin{tabular}{|c|c|c|c|c|c|c|}
\hline & \multirow{2}{*}{$\begin{array}{l}\text { RCRA } \\
\text { Limit } \\
\text { (ppom) }\end{array}$} & \multicolumn{5}{|c|}{ Concentration (ppm) } \\
\hline & & $\begin{array}{c}\text { Demonstretion Runs } \\
\# 1+\# 3\end{array}$ & $\begin{array}{c}\text { Pllot Plant } \\
\text { Run } \# 1\end{array}$ & $\begin{array}{c}\text { Pilot Plant } \\
\text { Run H2 } \\
\end{array}$ & $\begin{array}{c}\text { PIlot Plant } \\
\text { Run } \# 3\end{array}$ & $\begin{array}{l}\text { Pllot Plant } \\
\text { Run } \# 4\end{array}$ \\
\hline $\mathrm{Ag}$ & 5.0 & $<0.01$ & $<0.01$ & $<0.01$ & $<0.01$ & $<0.01$ \\
\hline As & 5.0 & 0.70 & $<0.03$ & $<0.03$ & $<0.03$ & $<0.03$ \\
\hline $\mathrm{cd}$ & 1.0 & $<0.001$ & $<0.001$ & $<0.001$ & $<0.001$ & $<0.001$ \\
\hline $\mathrm{Cr}$ & 5.0 & 0.002 & 0.018 & 0.023 & 0.033 & 0.019 \\
\hline $\mathrm{Hg}$ & 0.2 & $<0.0005$ & $<0.0005$ & $<0.0005$ & $<0.0005$ & $<0.0005$ \\
\hline $\mathrm{Ba}$ & 100.0 & 0.095 & 0.114 & 0.151 & 0.236 & 0.235 \\
\hline $\mathrm{Pb}$ & 5.0 & $<0.02$ & $<0.02$ & $<0.02$ & $<0.02$ & $<0.02$ \\
\hline Se & 1.0 & 0.12 & $<0.03$ & $<0.03$ & $<0.03$ & $<0.03$ \\
\hline $\mathrm{PH}$ & 12.5 & 12.07 & 12.44 & 12.45 & 12.59 & 12.58 \\
\hline
\end{tabular}

Table 4-4. TCLP extraction data. 


\begin{tabular}{|c|c|c|c|c|c|c|c|c|c|c|c|c|c|c|c|}
\hline & \multirow{3}{*}{$\begin{array}{l}\text { RCRA } \\
\text { Limit } \\
\text { (ppm) }\end{array}$} & \multicolumn{9}{|c|}{ Maximum Measured Concentrations (ppm) } & \multirow[b]{2}{*}{ PP2 } & \multirow[b]{2}{*}{ PP2 } & \multirow[b]{2}{*}{ PP3 } & \multirow[b]{2}{*}{ PP3 } & \multirow[b]{2}{*}{ PP2 } \\
\hline & & Dem3 & Dem3 & Dem3 & Dem3 & Dem3 & Dem3 & Dem1 & PP1 & PP2 & & & & & \\
\hline & & LCI & LC2 & L.C3 & LC4 & LC5 & LC6 & LC7 & LCQ & LC10 & LC11 & LC12 & LC13 & $\mathrm{LC14}$ & $\lfloor C 33$ \\
\hline Ag & 5.0 & $<.01$ & $<0.01$ & $<0.01$ & $<0.01$ & $<0.01$ & $<0.01$ & $<0.01$ & $<0.01$ & $<0.01$ & $<0.01$ & $<0.01$ & $<0.01$ & $<0.01$ & $<0.01$ \\
\hline As & 5.0 & 2.9 & 5.75 & 6.5 & 5.2 & 8.75 & 5.0 & 2.55 & 0.50 & 4.1 & 2.8 & 3.0 & 0.35 & 4.1 & 6.6 \\
\hline $\mathrm{cd}$ & 1.0 & $<0.001$ & $<0.001$ & $<0.01$ & $<0.01$ & $<0.01$ & $<0.09$ & $<0.01$ & $<0.01$ & $<0.01$ & $<0.01$ & $<0.01$ & 0.01 & $<0.01$ & $<0: 01$ \\
\hline $\mathrm{Cr}$ & 5.0 & .018 & $<0.001$ & 0.017 & $<0.001$ & 0.01 & $<0.001$ & $<0.001$ & 0.055 & 0.037 & 0.038 & $<0.001$ & $<0.001$ & 0.007 & 0.075 \\
\hline $\mathrm{Ba}$ & 100.0 & .165 & 0.12 & 0.121 & 0.042 & 0.091 & 0.065 & 0.083 & 0.145 & 0.079 & 1.10 & 1.75 & 0.08 & 0.064 & 0.16 \\
\hline $\mathrm{Pb}$ & 5.0 & 0.13 & 0.14 & 0.15 & $<0.02$ & 0.95 & $<0.02$ & $<0.02$ & $<0.02$ & $<0.02$ & $<0.02$ & $<0.02$ & $<0.02$ & $<0.02$ & $<0.02$ \\
\hline Se & 1.0 & 3.5 & 2.80 & 3.2 & 2.7 & 3.6 & 3.2 & 4.0 & 0.50 & 0.58 & 0.80 & 0.45 & 0.40 & $<0.03$ & 0.9 \\
\hline $\mathrm{pH}$ & 12.5 & 11.96 & 12.19 & 12.15 & 11.99 & 12.81 & 12.49 & 12.08 & 13.02 & 12.53 & 12.77 & 12.73 & 10.43 & 10.7 & 12.15 \\
\hline
\end{tabular}

semples in excess of RCRA (Imit (\# of reeks)

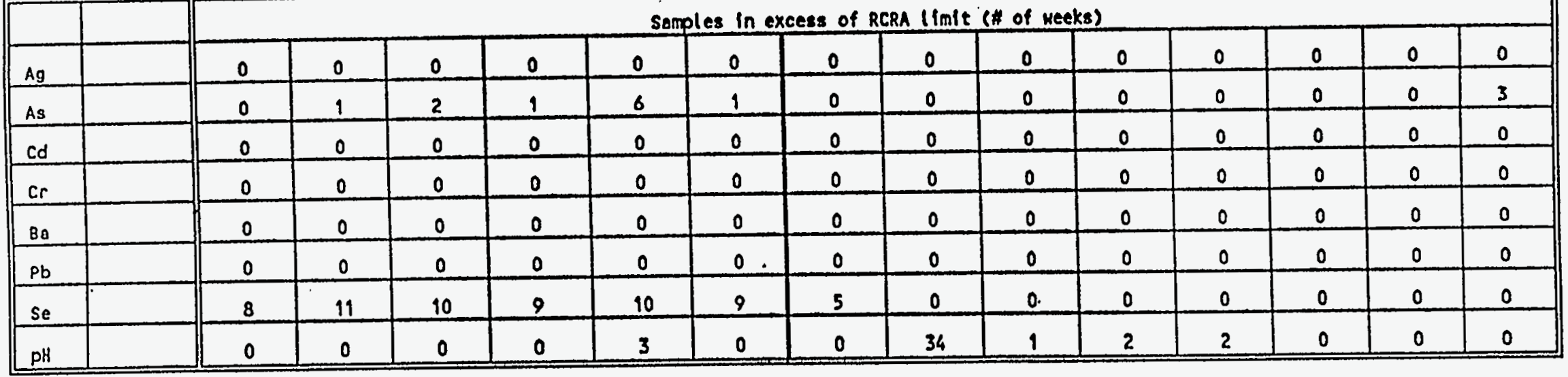

Table 4-5. Maximum concentrations of RCRA elements and pH measured in the leachate waters from the lysimeters packed with Coolside wastes. 
The $\mathrm{pH}$ limit of 12.5 was exceeded in one or more of the leachate collections in LC5 and in LC9-LC12, particularly in LC9 (PP1) which remained above 12.5 for most of the study. These high $\mathrm{pH}$ values from LC9 likely reflects the use of sodium promoter in this run with the sodium eluting as $\mathrm{NaOH}$ which tends to increase $\mathrm{pH}$ above the 12.5 limit. Sodium promoter was also used in pilot plant \#2 tests but the use of sorbent recycle in this run may have effectively had a dilution effect on the $\mathrm{Na}$. Despite $\mathrm{pH}$ values being in excess of 12.5 by TCLP for the pilot plant 3 sample, such was not the case for the leachate from the lysimeters packed with this sample (LC13-LC15).

The bottom portion of Table 4-5 shows the number of collected leachate samples in which the RCRA limits were exceeded. For the most part, those lysimeters that exceeded RCRA limits did so in only 1-3 samples during the study, again with the exception of $\mathrm{pH}$ in LC 9 (34 samples), As in LC5 (6 samples), and Se in all the lysimeters packed with demonstration run materials (LC1-LC7). It is interesting to note that the only difference in LC1 and LC5 was that LC5 was packed at a higher density yet LC5 was the only lysimeter packed with demonstration run materials that exceeded the $12.5 \mathrm{pH}$ limit, doing so on three different occasions. This compares to zero samples above the $\mathrm{pH}$ limit from the other six lysimeters.

Demonstration Run \#3.The test matrix for the six columns packed with wastes from demonstration run \#3 (LC 1-6) was devised to examine the impact of packing density, the method of water addition, and variation of gaseous $\mathrm{CO}_{2}$ concentrations in the column headspace (Table 4-1). In this study, the fixed-feed (LC1-2) and rain-simulation columns (LC3-4) exhibited free flow from about week 5 onward, i.e., collection rates essentially tracked water addition rates (Figure 4-2). LC5 and LC6, packed at higher densities, did not exhibit free flow until about week 18 at which time a large 'slug' of water passed from each column, followed by free flow for the remainder of the study. Despite differences in packing density and the manner of water addition, $\mathrm{pH}$ values were relatively constant and similar for 


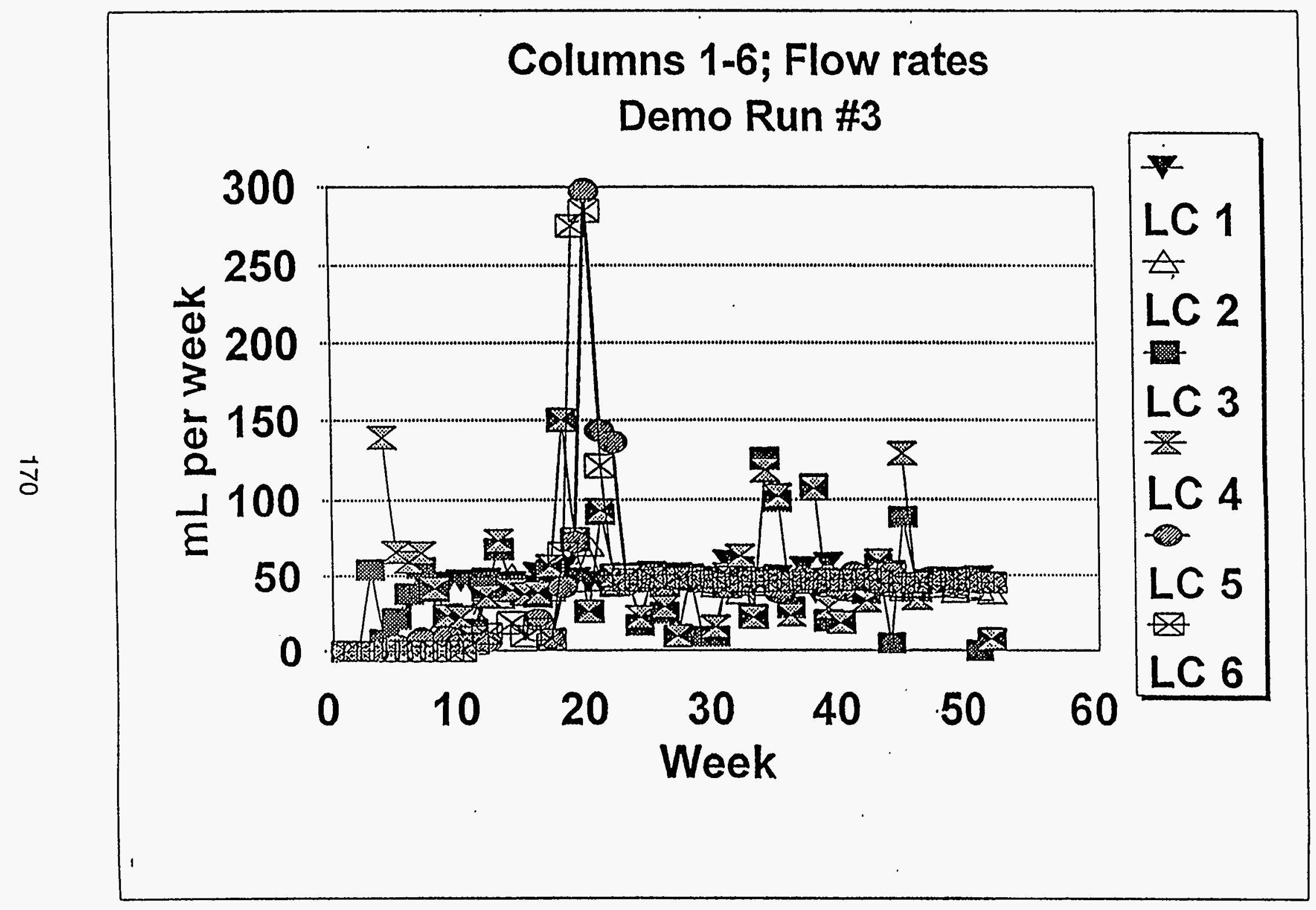

Figure 4-2. Water collections from LC1-LC6. 
LC1, LC3, and LC5 which were blanketed with room air (Figure 4-3). In contrast, pH values for $\mathrm{LC} 2\left(5.0 \% \mathrm{CO}_{2}\right)$ declined midway through the study to the $9-11$ range, indicative of $\mathrm{CO}_{2}$ uptake and ensuing carbonate buffering. For LC4 (rain simulation/2.5\% $\mathrm{CO}_{2}$ ), $\mathrm{pH}$ remained around 12.0 until near the end of the study at which time $\mathrm{pH}$ values also dropped to the 911 range for the latter three measurements (six weeks of collections). The $\mathrm{pH}$ for LC6 (higher density/2.5\% $\mathrm{CO}_{2}$ ) was initially at approximately 11.5 then increased to near 12.0 and remained at that level for the remainder of the study. Thus, LC2 which was blanketed with the highest level of $\mathrm{CO}_{2}$ exhibited the largest $\mathrm{pH}$ drop followed by $\mathrm{LC} 4$ blanketed with $2.5 \% \mathrm{CO}_{2}$ and loosely compacted. LC6, packed at a greater density, was relatively unaffected by the presence of elevated levels of $\mathrm{CO}_{2}$ in the column headspace despite exhibiting total flows similar to those of LC2 and LC4.

Elemental release patterns for LC1-6 can roughly be divided into three patterns. The first, represented by the plots of Figure 4-4, appears to include highly soluble ions that were rapidly depleted as a function of flow rate. Thus, the rain-simulated columns (LC3 and LC4), to which relatively large volumes of water were added early in the study, were the first to be depleted. The columns packed at higher density, LC5 and LC6, which were the last to flow, were the last to be depleted. LC1 and LC2 were intermediate in both flow rate and time to depletion of the highly soluble elements, relative to LC1, LC2, LC5, and LC6. In addition to the ions shown in Figure 4-4, Se and Mo also followed this pattern with maximum Mo values in the $100 \mathrm{ppm}$ range and Se maximizing around $3 \mathrm{ppm}$. Since with the exception of hydroxyl ions, $\mathrm{Na}$ and $\mathrm{Cl}$ are by far the most prevalent ions in the leachate waters, conductivity followed trends similar to those shown in Figure 4-4 as well.

The second type of leaching pattern for LC-LC6, illustrated by the plots of $\mathrm{Ca}, \mathrm{Mg}$, and $\mathrm{B}$ in Figure 4-5, appears to correlate with leachate $\mathrm{pH}$. For all three plots, ion concentrations varied inversely with $\mathrm{pH}$. Even the 'hump' in $\mathrm{Ca}$ ion concentration around week 10 in LC1 and LC3 corresponds to a dip in $\mathrm{pH}$ that occurred at that time. At high $\mathrm{pH}, \mathrm{Ca}$ and $\mathrm{Mg}$ 


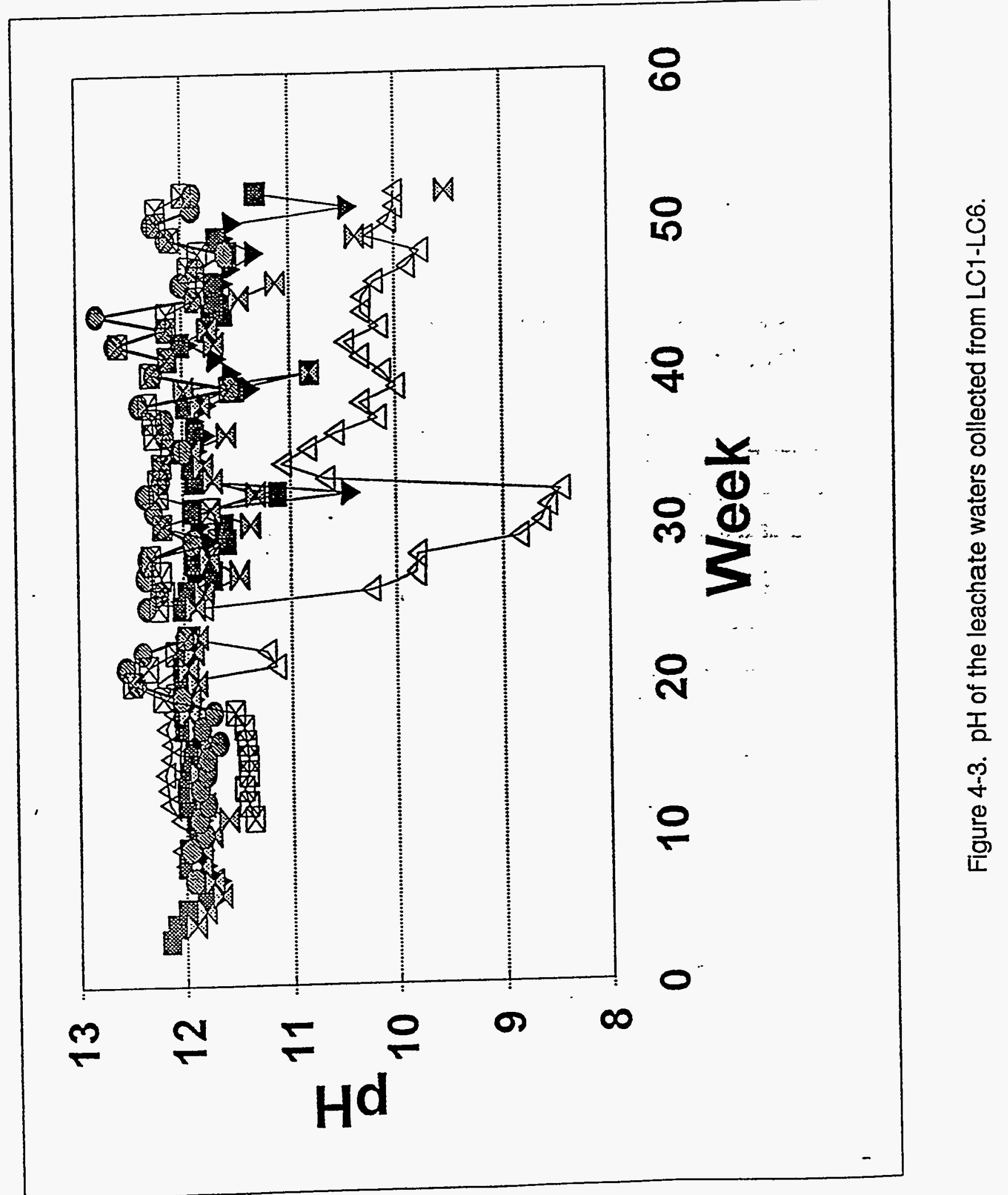




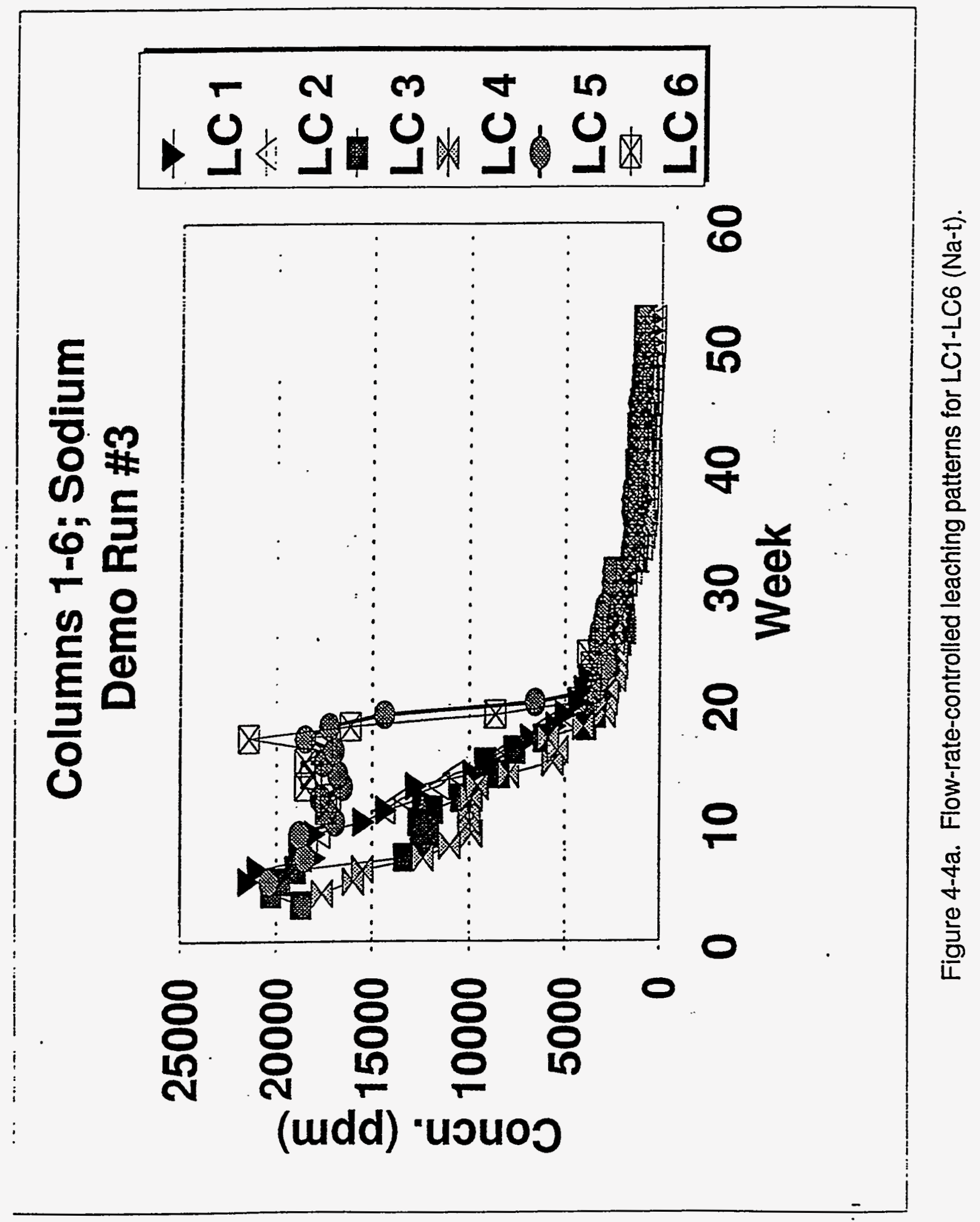




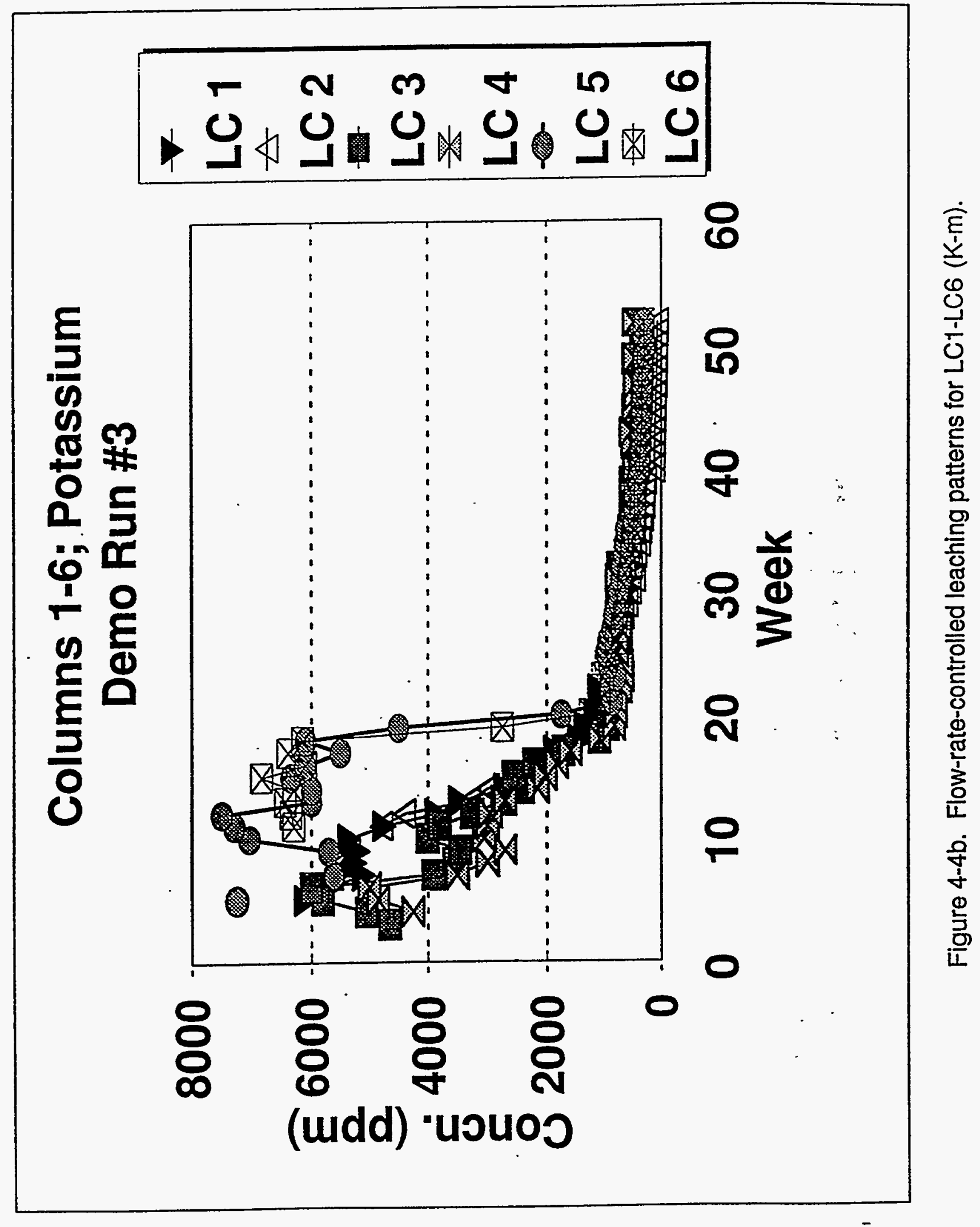




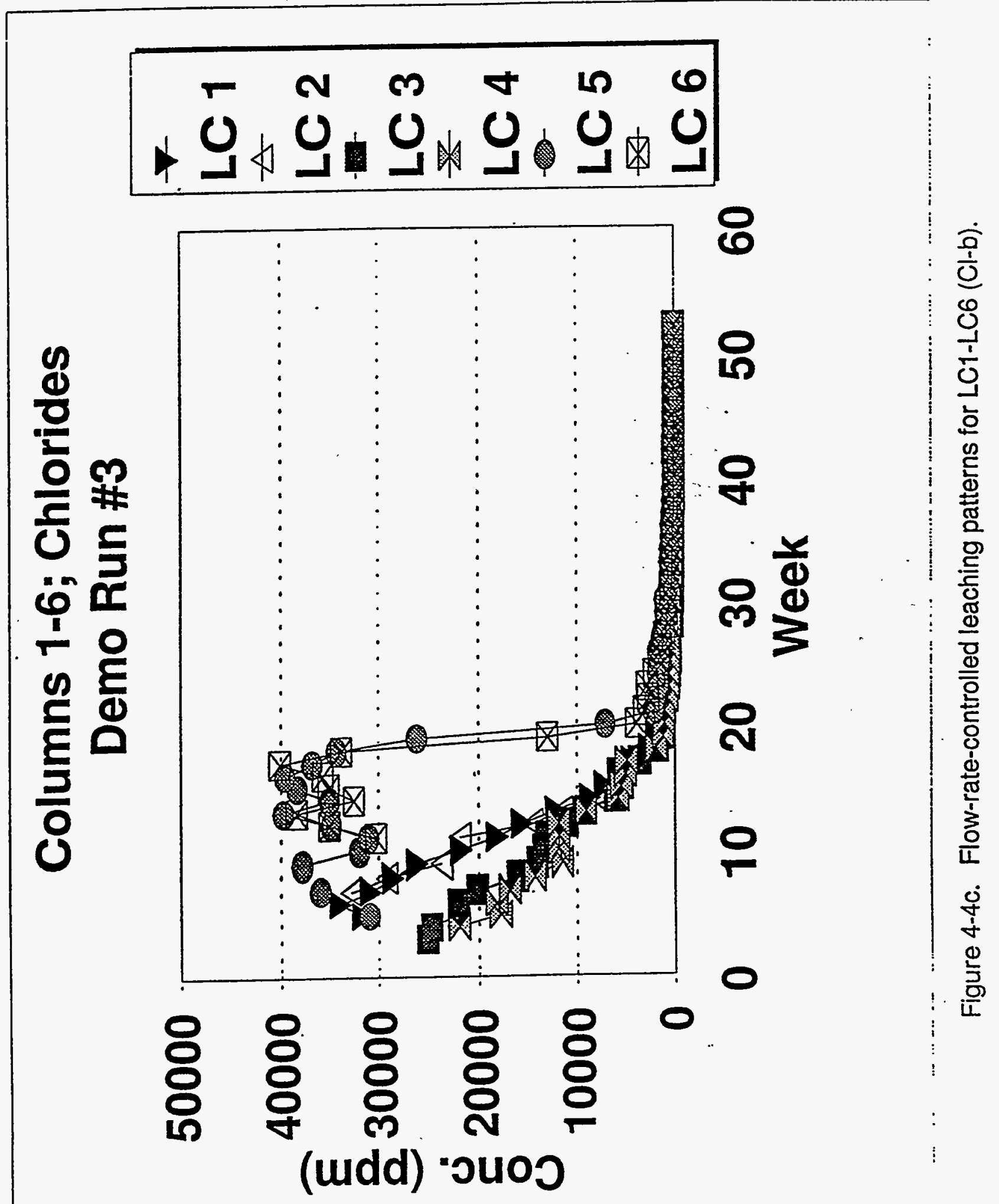




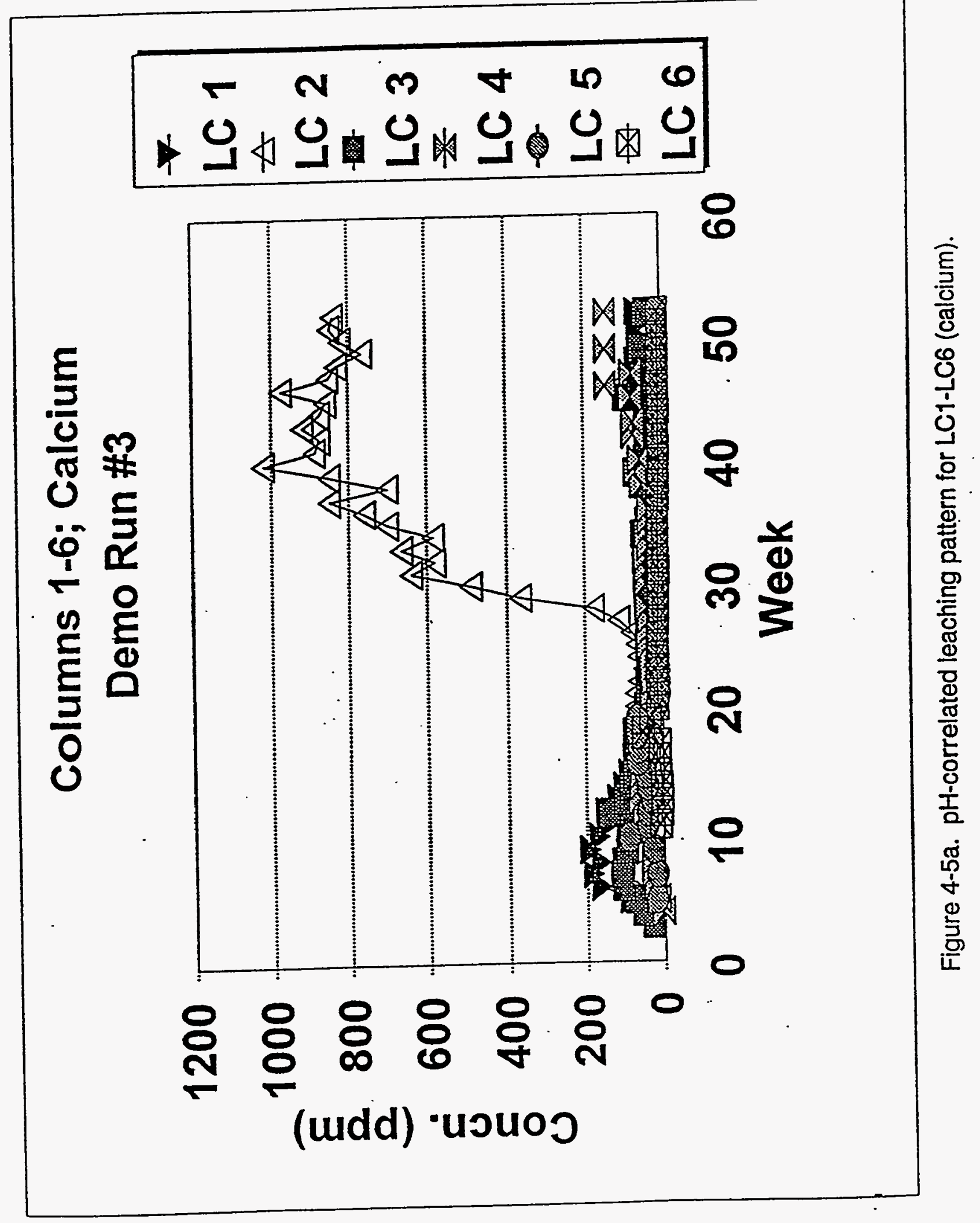




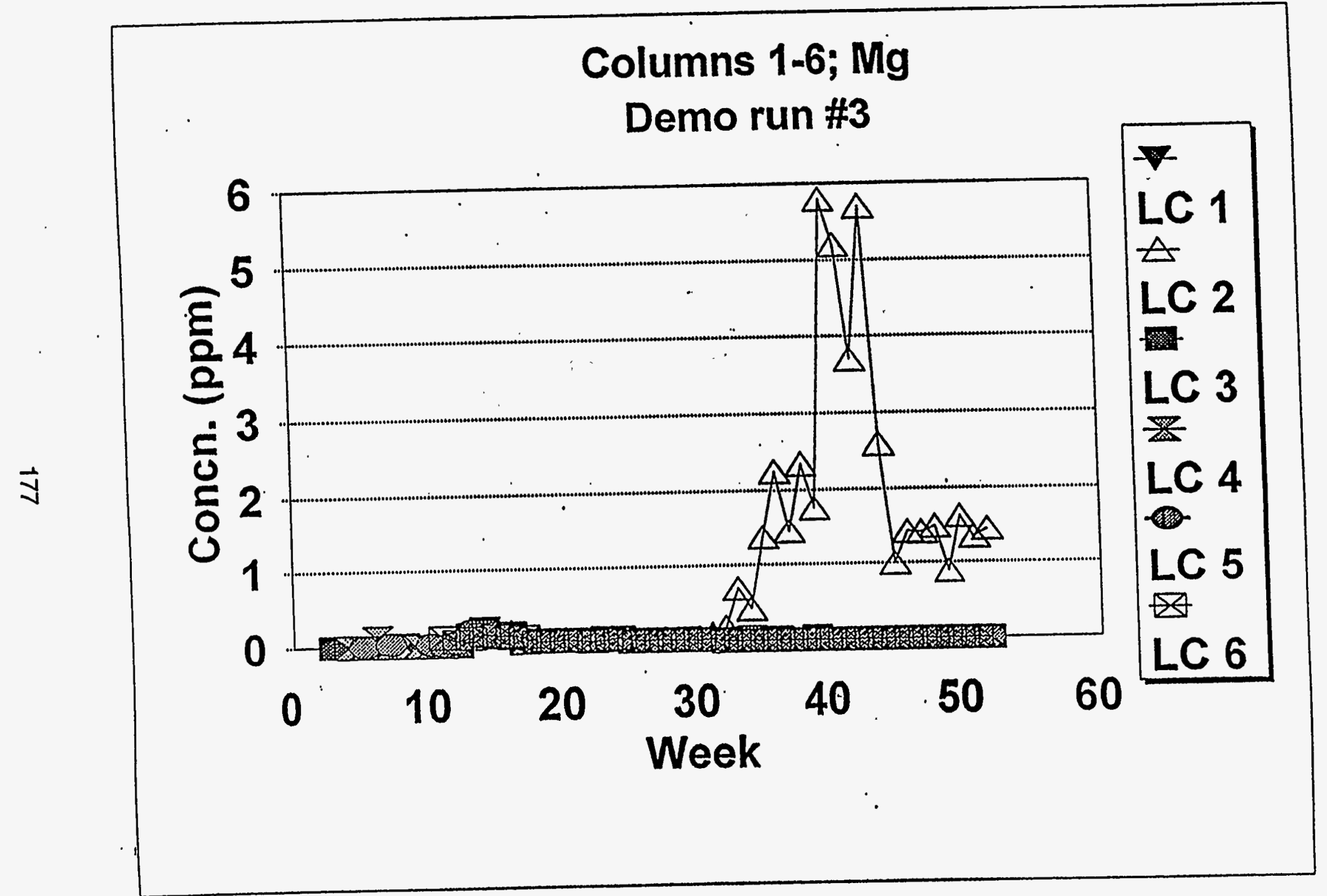

Figure 4-5b. pH-correlated leaching pattern for LC1-LC6 (Mg). 


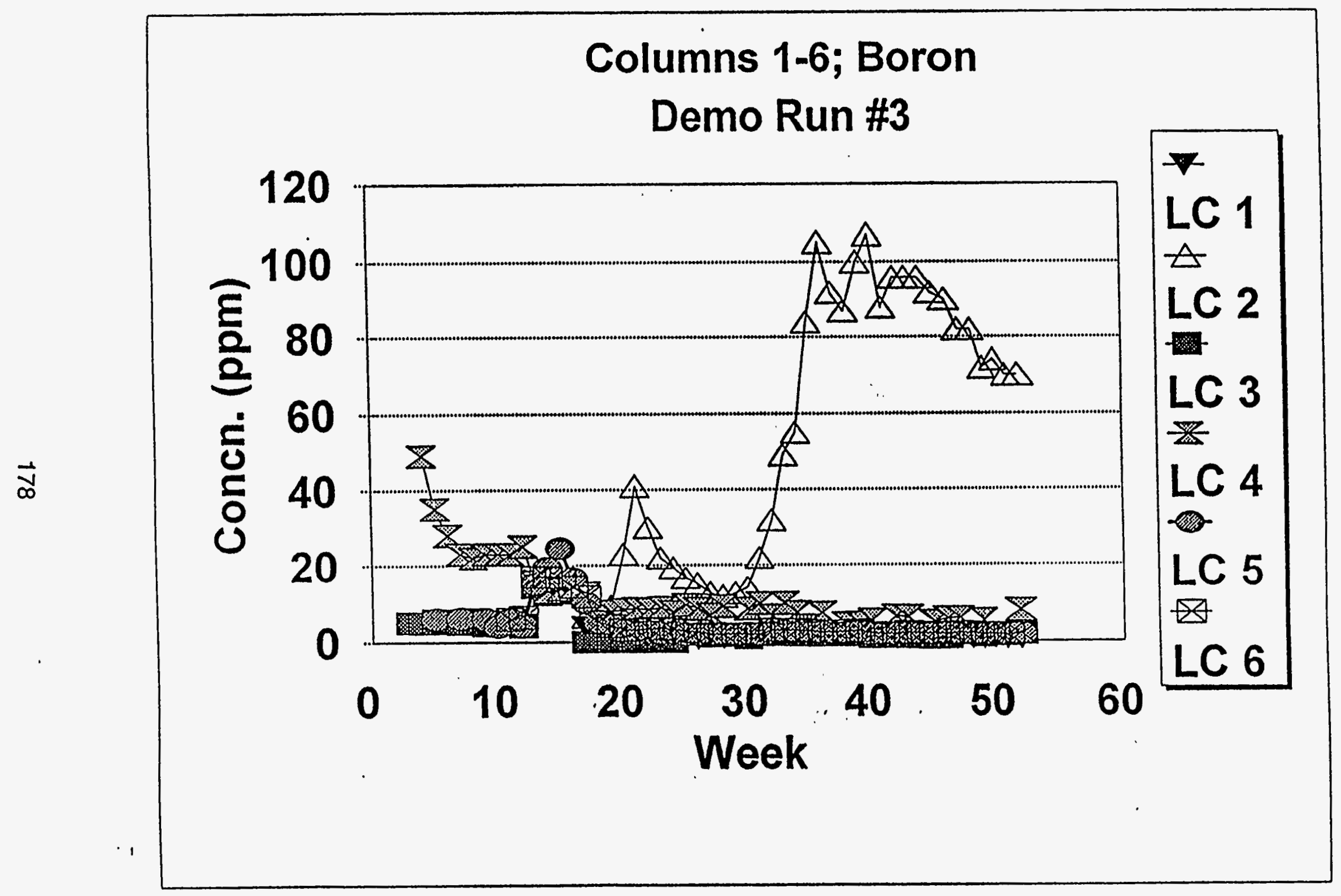

Figure 4-5c. pH-correlated leaching pattern for LC1-LC6 (Boron). 
remain in the solid phase largely as portlandite $\left[\mathrm{Ca}(\mathrm{OH})_{2}\right]$ and brucite $\left[\mathrm{Mg}(\mathrm{OH})_{2}\right]$. However, in those leachates in which $\mathrm{pH}$ declines following the uptake of $\mathrm{CO}_{2}$ and subsequent reaction with hydroxyl $(\mathrm{OH})$ to form carbonates $\left(\mathrm{HCO}_{3}{ }^{-}\right)$, the release of $\mathrm{Ca}$ and $\mathrm{Mg}$ to the leachate increased substantially. This effect is most obvious in the leachate from LC2 (Figure 4-5) which was blanketed with the highest concentration of $\mathrm{CO}_{2}(5 \%)$. Unlike $\mathrm{Ca}$ which continued to increase in concentration once the $\mathrm{pH}$ declined in $\mathrm{LC} 2, \mathrm{Mg}$ concentrations rose sharply before dropping to $1-2 \mathrm{ppm}$ around week 45 . This is believed to reflect the depletion of $\mathrm{Mg}$ ions from the packed bed. Boron follows a similar pattern with the exception of relatively high levels of $B$ released from LC4 in the initial 3-4 leachate collections. In contrast to its behavior in LC2, boron from LC4 passed through a minimum before increasing inversely with $\mathrm{pH}$. Since $\mathrm{pH}$ was initially near 12 in the leachate from $\mathrm{LC} 4$, the reason for the early $\mathrm{B}$ release from $\mathrm{LC} 4$ is not understood. It is notable that the concentrations of $\mathrm{Ca}, \mathrm{Mg}$, and $\mathrm{B}$ in the leachate from $\mathrm{LC} 6$ never exhibited an increase with time as in LC2 and LC4. As previously discussed, LC6 was packed a higher density and the leachate $\mathrm{pH}$ did not exhibit a decline with time as did LC2 and LC4 which were also blanketed with elevated levels of $\mathrm{CO}_{2}$.

The third type of leaching pattern is represented by $\mathrm{Al}, \mathrm{SO}_{4}$, and $\mathrm{V}$ in Figure 4-6 (As and $\mathrm{Br}$ also followed this pattern). This pattern is similar to that exhibited by $\mathrm{Cl}, \mathrm{Na}$, and $\mathrm{K}$ in .Figure 4-4 (flow controlled) only with a more prolonged rate of release. The primary factor(s) that dictate the slower release is unknown but is believed to be related to either the slow kinetics of dissolution-transformation of the minerals that retain these ions in the solid phase, the formation of oxyanions, or perhaps a combination of the two. For example, in LC2 which was blanketed with $5 \% \mathrm{CO}_{2}, \mathrm{XRD}$ analysis (discussed in a later section) indicated early formation of ettringite in the hydrated starting materials. However, by the end of the study, ettringite was completely depleted from the upper and middle cores, apparently having been replaced by calcite in the top and gypsum in the middle. Ettringite had also disappeared from the top of both LC2 and LC4 (blanketed with enhanced levels of 


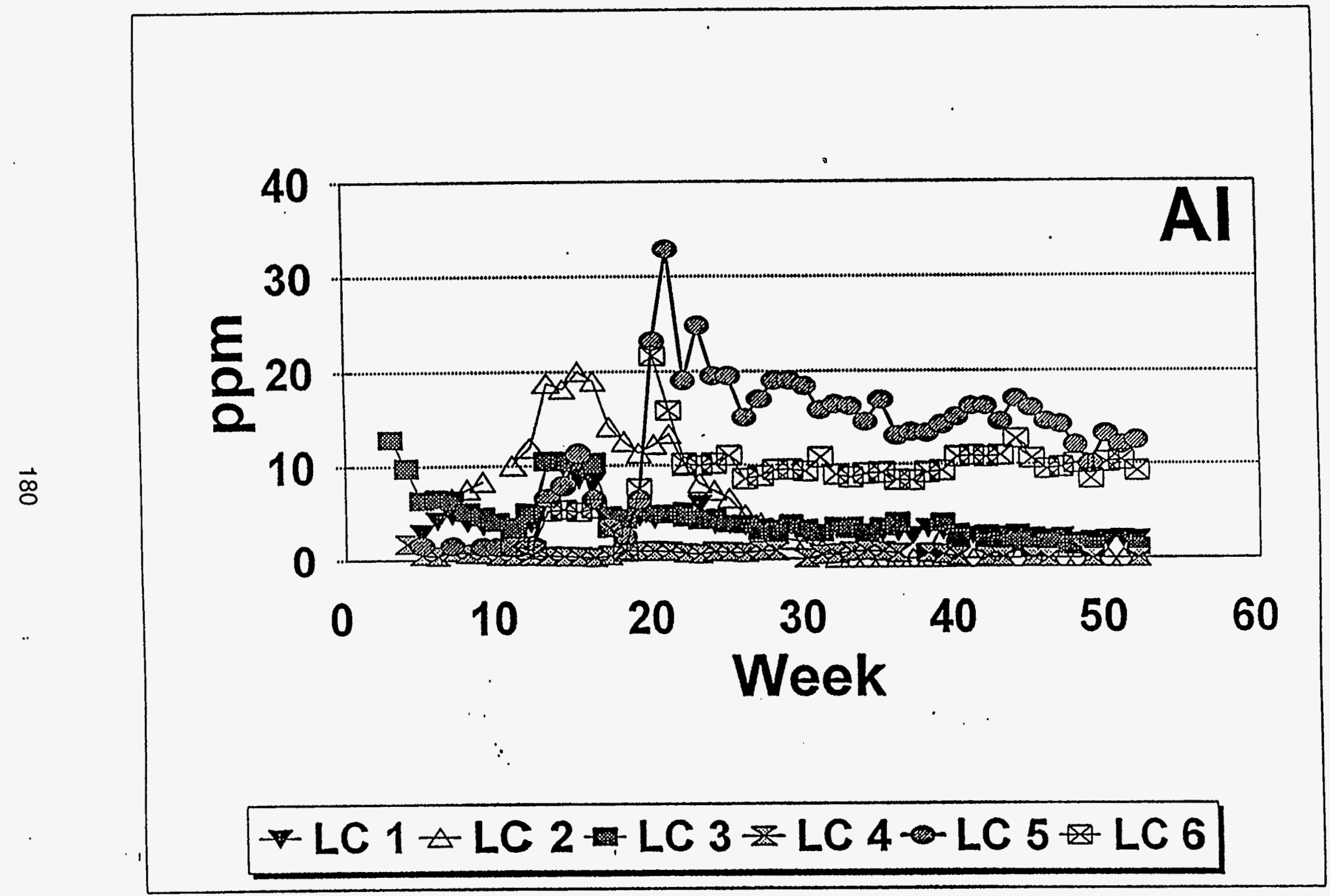

Figure 4-6a. Examples of ions exhibiting more prolonged release patterns from LC1LC6 (Al-t). 


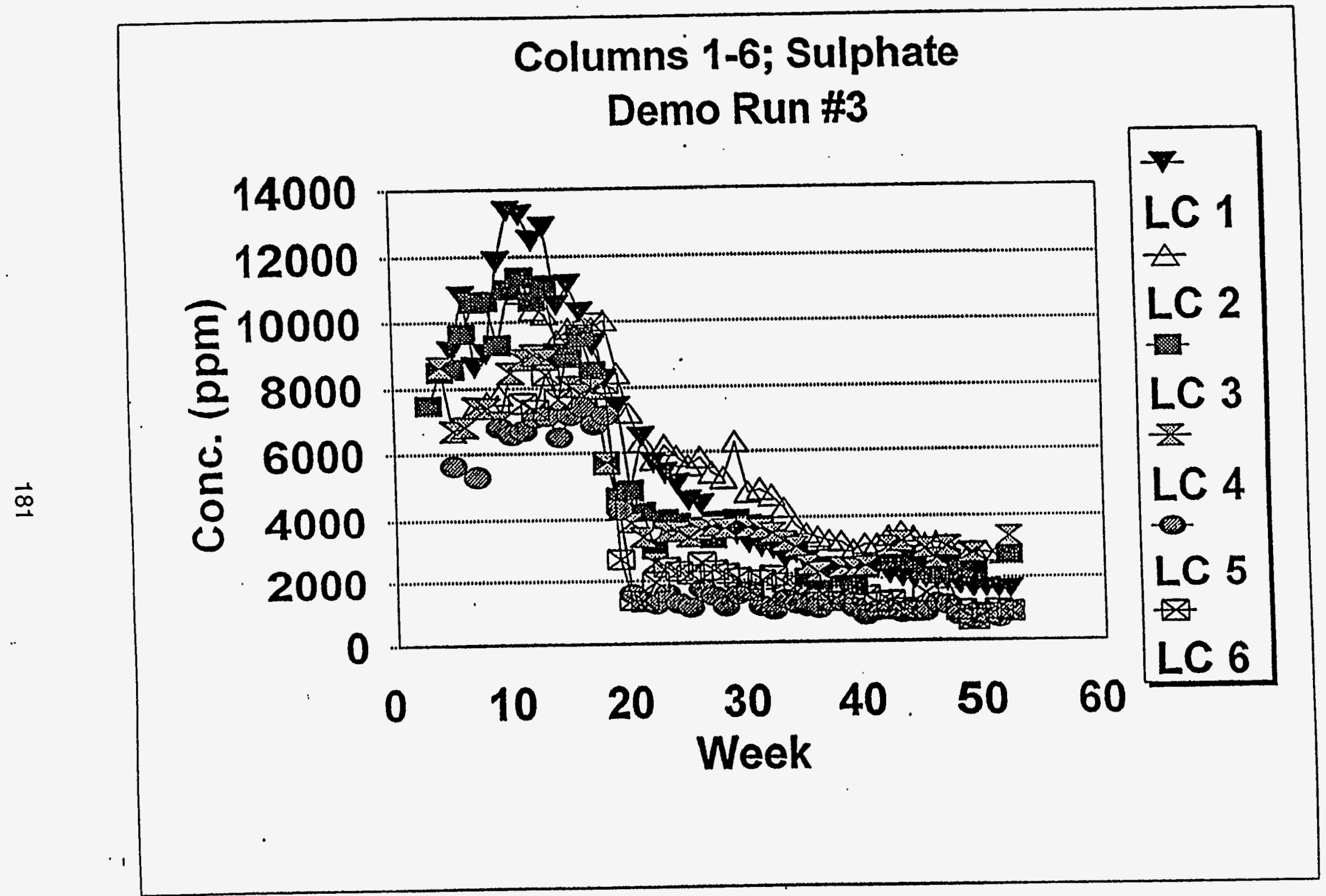

Figure 4-6b. Examples of ions exhibiting more prolonged release patterns from LC1LC6 $\left(\mathrm{SO}_{4}-\mathrm{m}\right)$. 


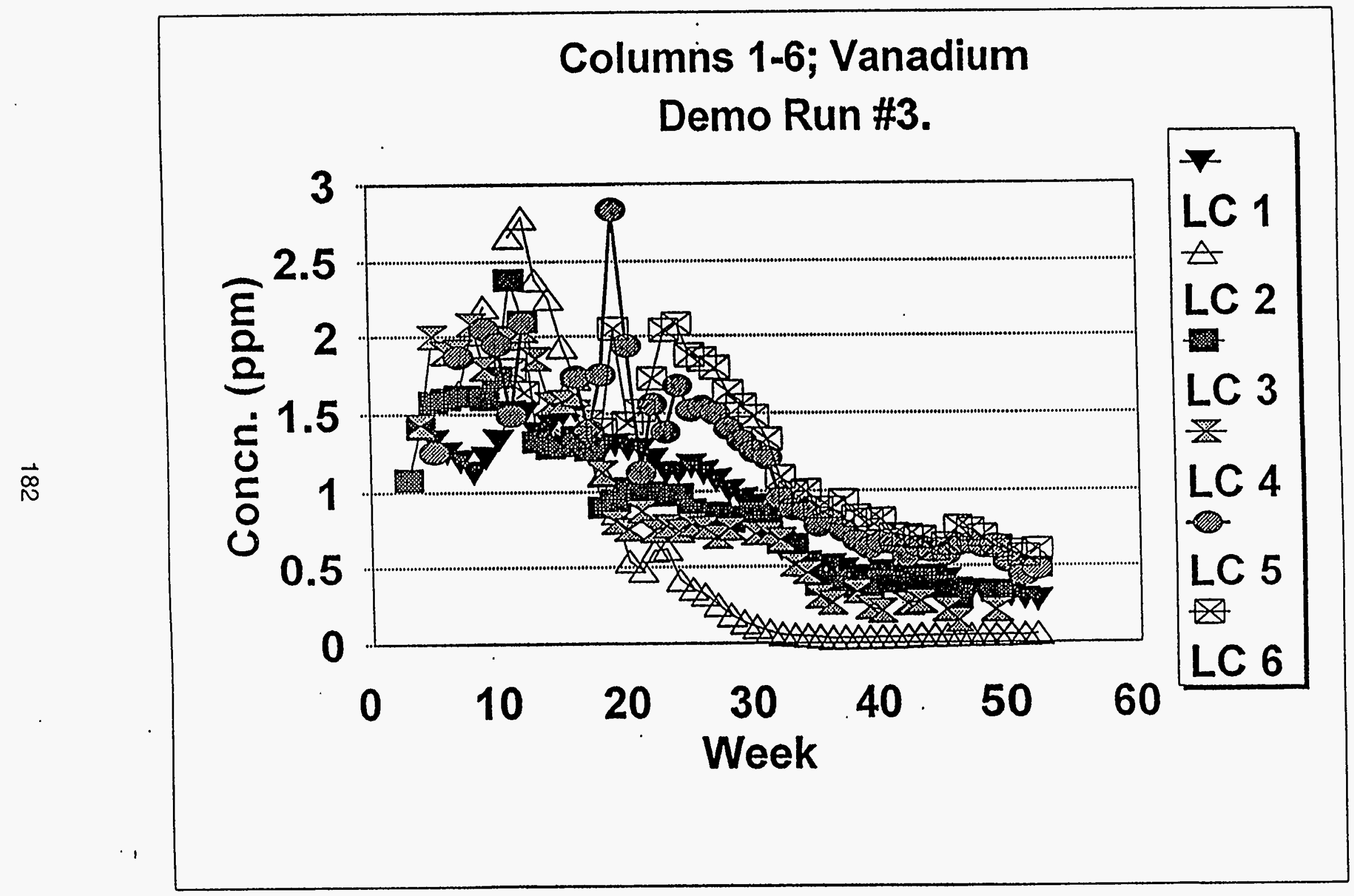

Figure 4-6c. Examples of ions exhibiting more prolonged release patterns from LC1: LC6 (V-b). 
$\mathrm{CO}_{2}$ ) but remained throughout the three columns blanketed with room air and LC6 (dense packing/2.5\% $\mathrm{CO}_{2}$ ).

Formation of calcite was observed in the upper portions of LC2 and 4 but not in LC6. Thus, the early formation of ettringite would tend to initially retain Al. This ion may be released at a later time as the ettringite is depleted whereas the $\mathrm{Ca}$ initially present in ettringite is incorporated into calcite or gypsum. The net effect would be prolonged release of Al. Similar arguments can be made for $\mathrm{SO}_{4}$ which is also present in ettringite. Though a portion of the $\mathrm{SO}_{4}$ would be incorporated to gypsum, $\mathrm{SO}_{4}$ release would be greater in those portions of the column where $\mathrm{CO}_{2}$ is available and calcite formation is favored. It is speculated that the delayed release of $V$ may be due to slow kinetics of formation of oxyanions of vanadium.

$\mathrm{CO}_{2}$ Effects. All three columns exhibited essentially free flow from about week 5 onward with the exception of LC10. This latter lysimeter flowed erratically until about week $18 \mathrm{a}$ which time a large slug of water passed through the column followed by free flow for the remainder of the study (Figure 4-7). Essentially the same cumulative volumes of water passed through each of these columns during the study.

In the initial leachate collections, pH levels were in the 12.5-12.6 range for all three lysimeters (Figure 4-8), indicative of $\mathrm{pH}$ control by portlandite. However, with time, $\mathrm{pH}$ values exhibited clear declines that varied inversely with the concentration of $\mathrm{CO}_{2}$ in the lysimeter headspace. By the end of the study, pH levels for $\mathrm{LC10}\left(0 \% \mathrm{CO}_{2}\right)$ were approximately 12.0 , approximately 11.4 for $\mathrm{LC11}\left(2.5 \% \mathrm{CO}_{2}\right)$, and in the 9.5-10.0 range for $\mathrm{LC1} 12\left(5.0 \% \mathrm{CO}_{2}\right)$. As discussed previously, such behavior is consistent with carbonate buffering following absorption of $\mathrm{CO}_{2}\left(\mathrm{CO}_{2}+\mathrm{OH}-\rightarrow \mathrm{HCO}_{3}{ }^{-}\right)$. 


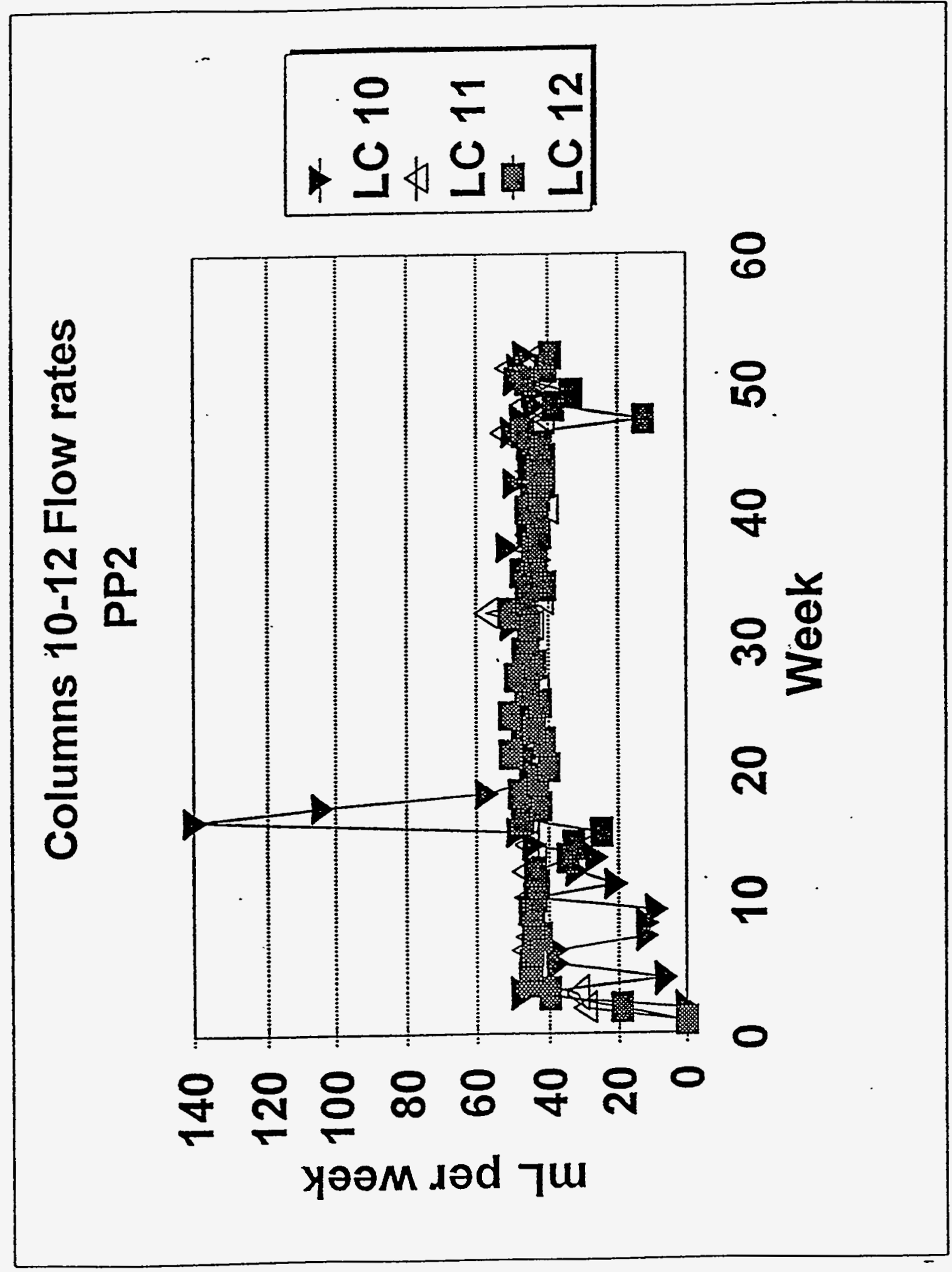




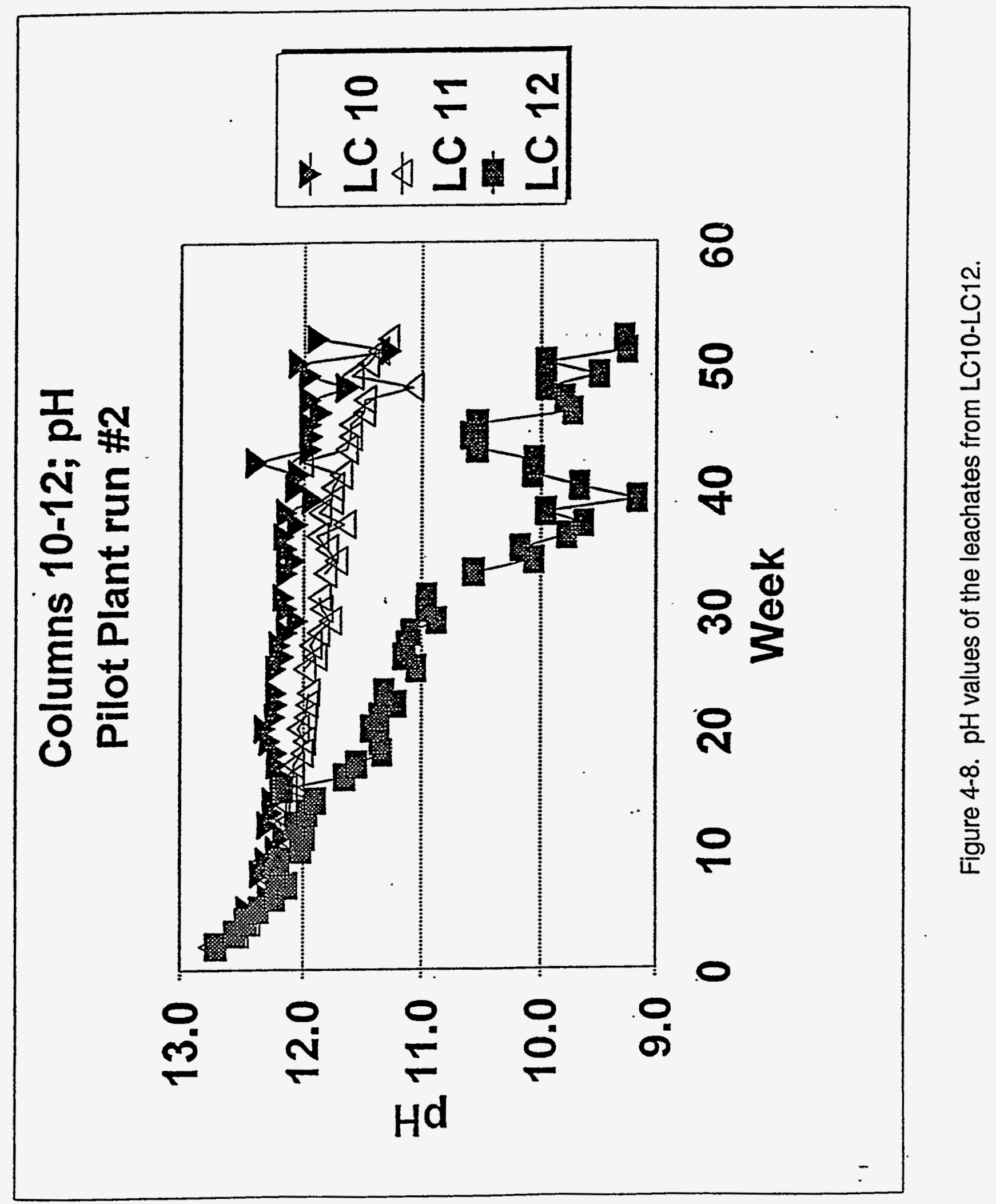


Many of the ions measured in the leachate waters from LC10-LC12 either eluted too early in the study or their concentrations were too low to demonstrate clear trends as a function of $\mathrm{CO}_{2}$ concentration ( $\mathrm{As}, \mathrm{Zn}, \mathrm{Ba}, \mathrm{Mn}, \mathrm{Se}, \mathrm{Cl}, \mathrm{Mo}$ ). The release rate for several other ions, such as $\mathrm{K}$ and $\mathrm{Na}$, appear to have been unaffected by the uptake of $\mathrm{CO}_{2}$ and exhibited release patterns similar to those of the highly soluble ions shown in Figure 4-4. These ions rapidly eluted from the columns until depleted. However, the leachate concentrations for $\mathrm{Ca}, \mathrm{Mg}, \mathrm{B}, \mathrm{Al}, \mathrm{V}$, and $\mathrm{SO}_{4}$ did exhibit variations which appear to correlate with the concentration of $\mathrm{CO}_{2}$ in the lysimeter headspace.

The relative concentrations of $\mathrm{Ca}, \mathrm{Mg}$, and $\mathrm{B}$ in the leachate waters increased with decreasing $\mathrm{pH}$ as illustrated in Figure 4-9. This trend is particularly apparent for the leachates from $\mathrm{LC1} 2$ which was blanketed with a higher concentration $(5 \%)$ of $\mathrm{CO}_{2}$. This inverse relation between $\mathrm{pH}$ and ion concentration is similar to that previously discussed in the $\mathrm{CO}_{2}$-enriched columns in the LC1-LC6 series with the same arguments being applicable to the behavior of this set of lysimeters.

In contrast to the inversely related plots of Figure 4-9, $\mathrm{Al}$ and $\mathrm{V}$ concentrations in the leachate waters directly correlated with higher $\mathrm{pH}$ (lower $\mathrm{CO}_{2}$ headspace concentrations) as illustrated by the plots in Figure 4-10. The behavior exhibited by $\mathrm{Al}$ in this plot is consistent with release from $\mathrm{Al}(\mathrm{OH})_{3}$ (gibbsite) as a function of $\mathrm{pH}^{3}$ The more prolonged release of $V$ from LC10 is again believed to be related to the slow formation of soluble oxyanions. ${ }^{4}$ On a relative basis, $\mathrm{SO}_{4}$ concentrations increased from $\mathrm{LC10}$ to $\mathrm{LC1} 2$ (Figure 4-11). As will be discussed in more detail in a later section, XRD of cores taken from these columns showed ettringite from top to bottom of LC10, some formation of gypsum and calcite in the top and middle cores of LC11, and complete destruction of ettringite and formation of gypsum and calcite in all three cores from LC12. The release of $\mathrm{SO}_{4}$ is believed to be retarded by incorporation of $\mathrm{SO}_{4}$ into ettringite in $\mathrm{LC10}$ and the partial 


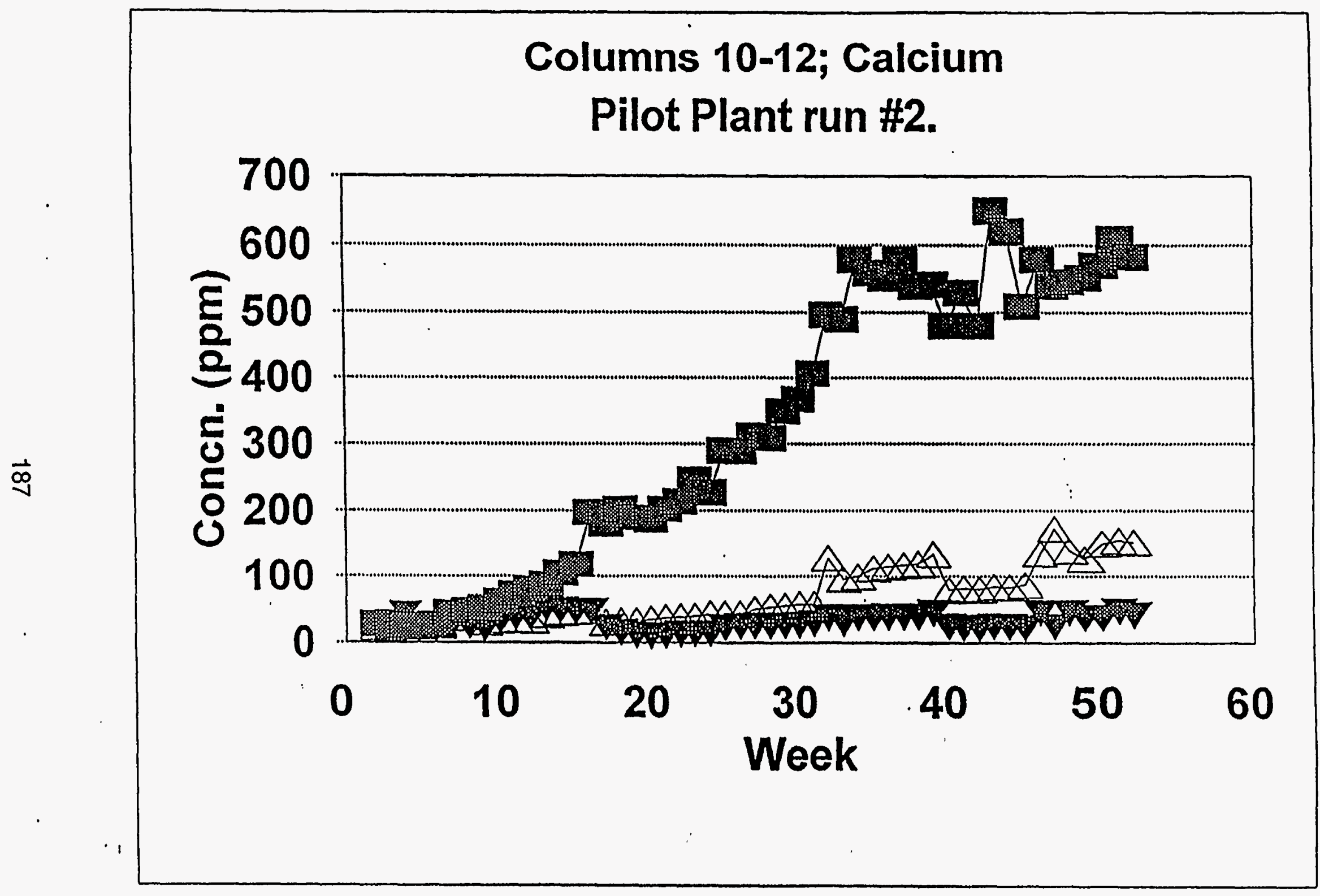

Figure 4-9a. The impact of an enriched $\mathrm{CO}_{2}$ atmosphere on the release of $\mathrm{Ca}(\mathrm{t})$ from LC10-LC12. 


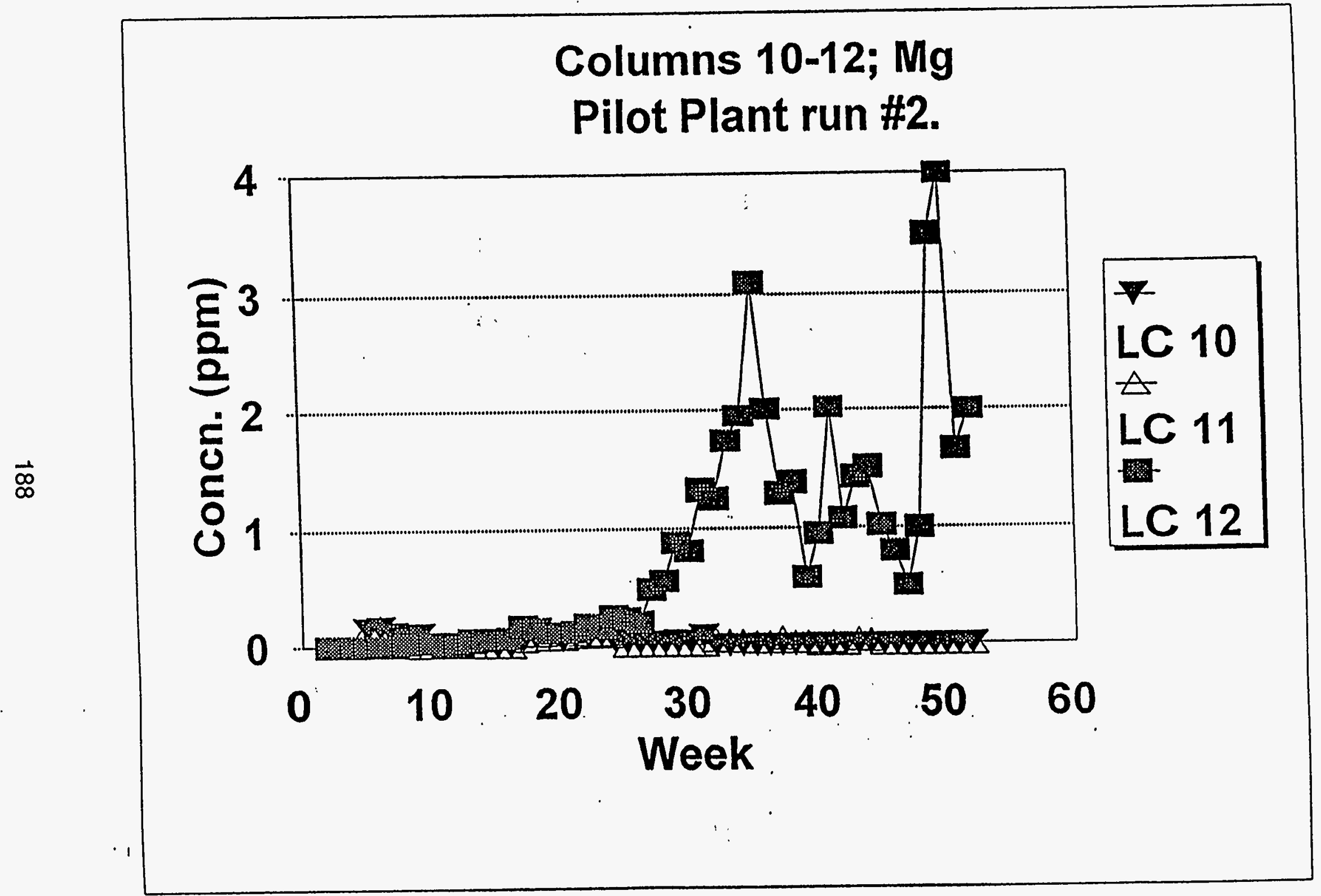

Figure 4-9b. The impact of an enriched $\mathrm{CO}_{2}$ atmosphere on the release of $\mathrm{Mg}(\mathrm{m})$ from LC10-LC12. 


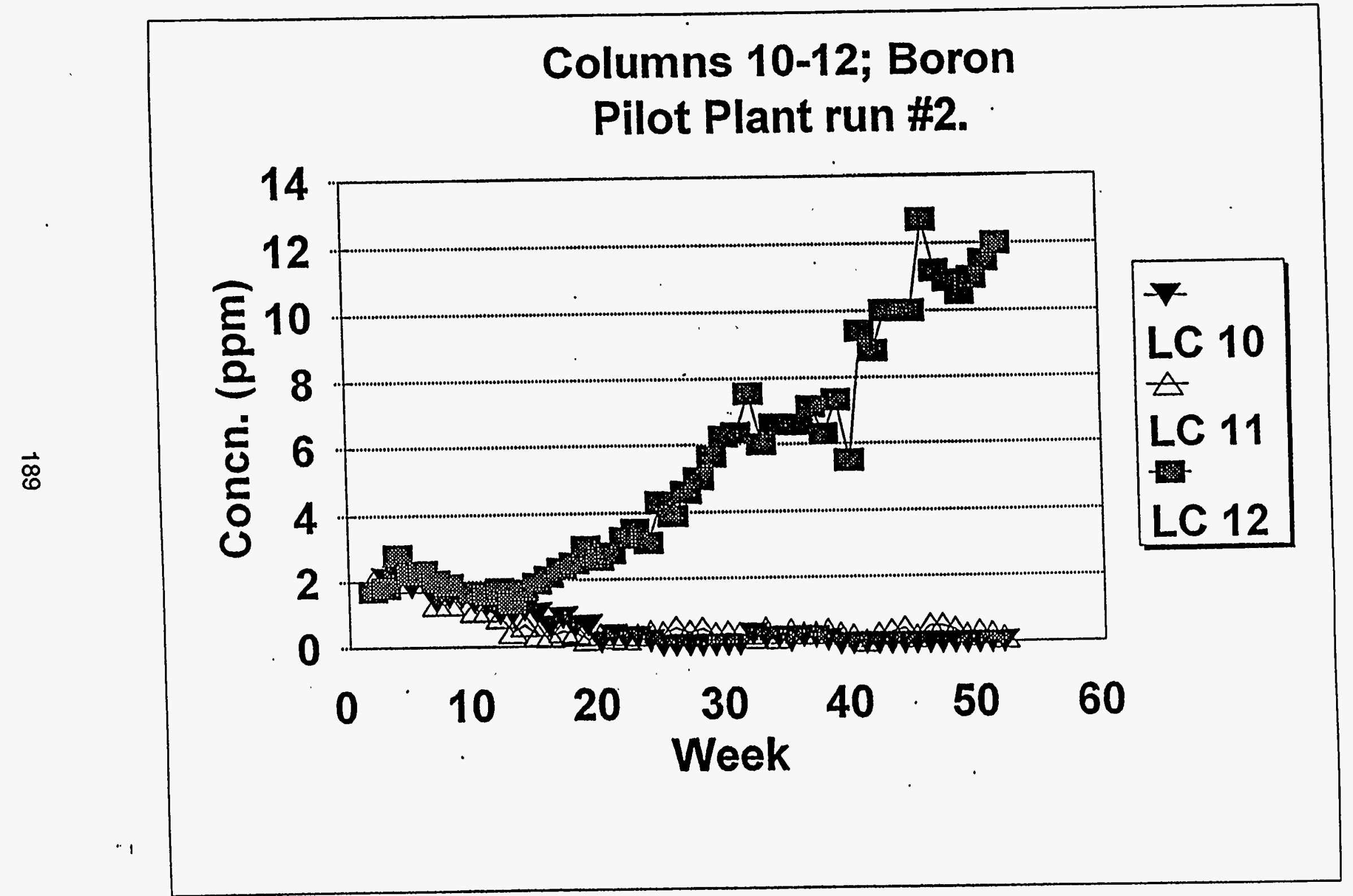

Figure 4-9c. The impact of an enriched $\mathrm{CO}_{2}$ atmosphere on the release of $B(b)$ from LC10-LC12. 

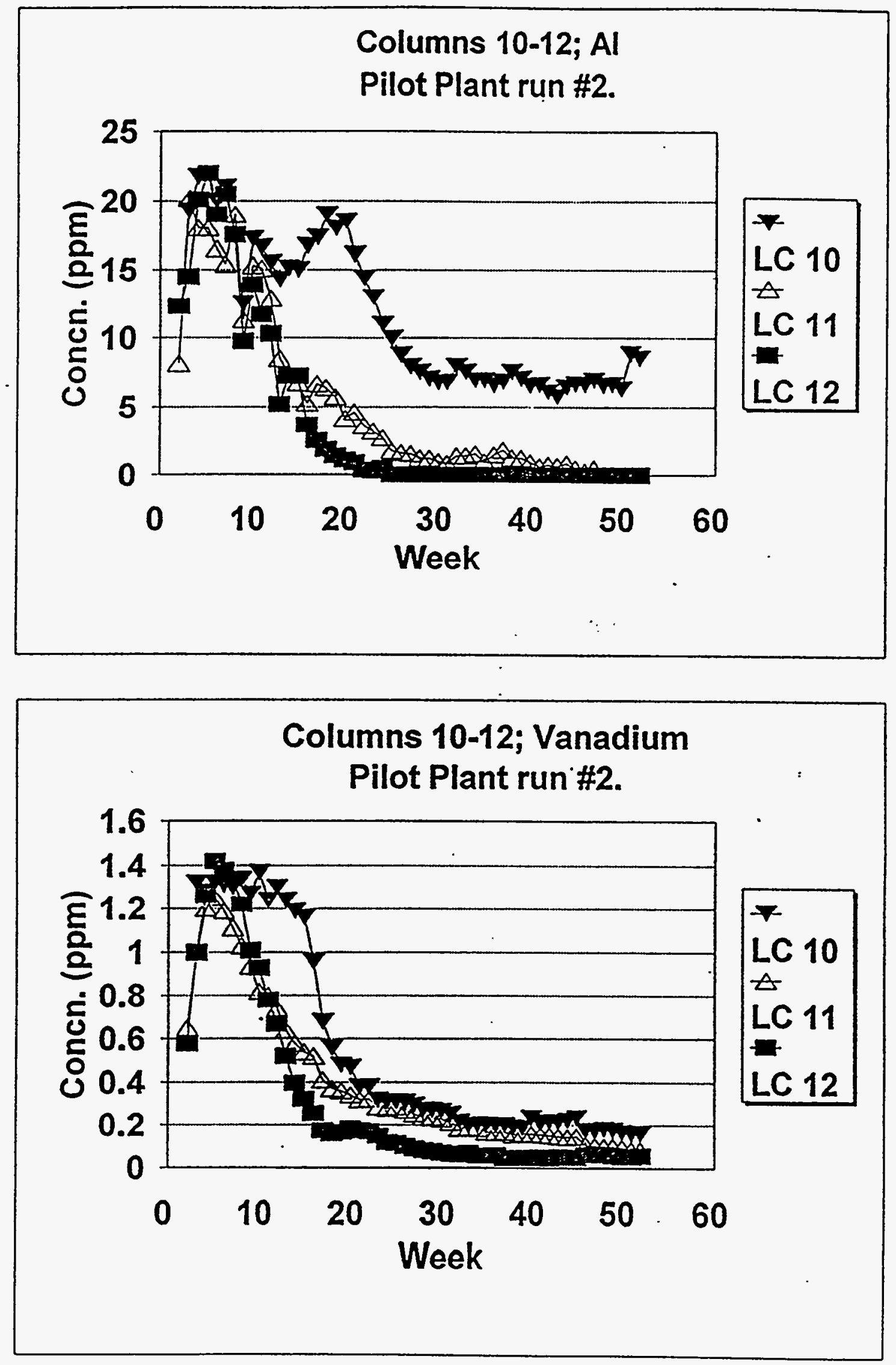

Figure 4-10. The impact of enriched $\mathrm{CO}_{2}$ atmosphere on the release of $\mathrm{Al}(\mathrm{I})$ and $\mathrm{V}(\mathrm{r})$ in LC10-12. 


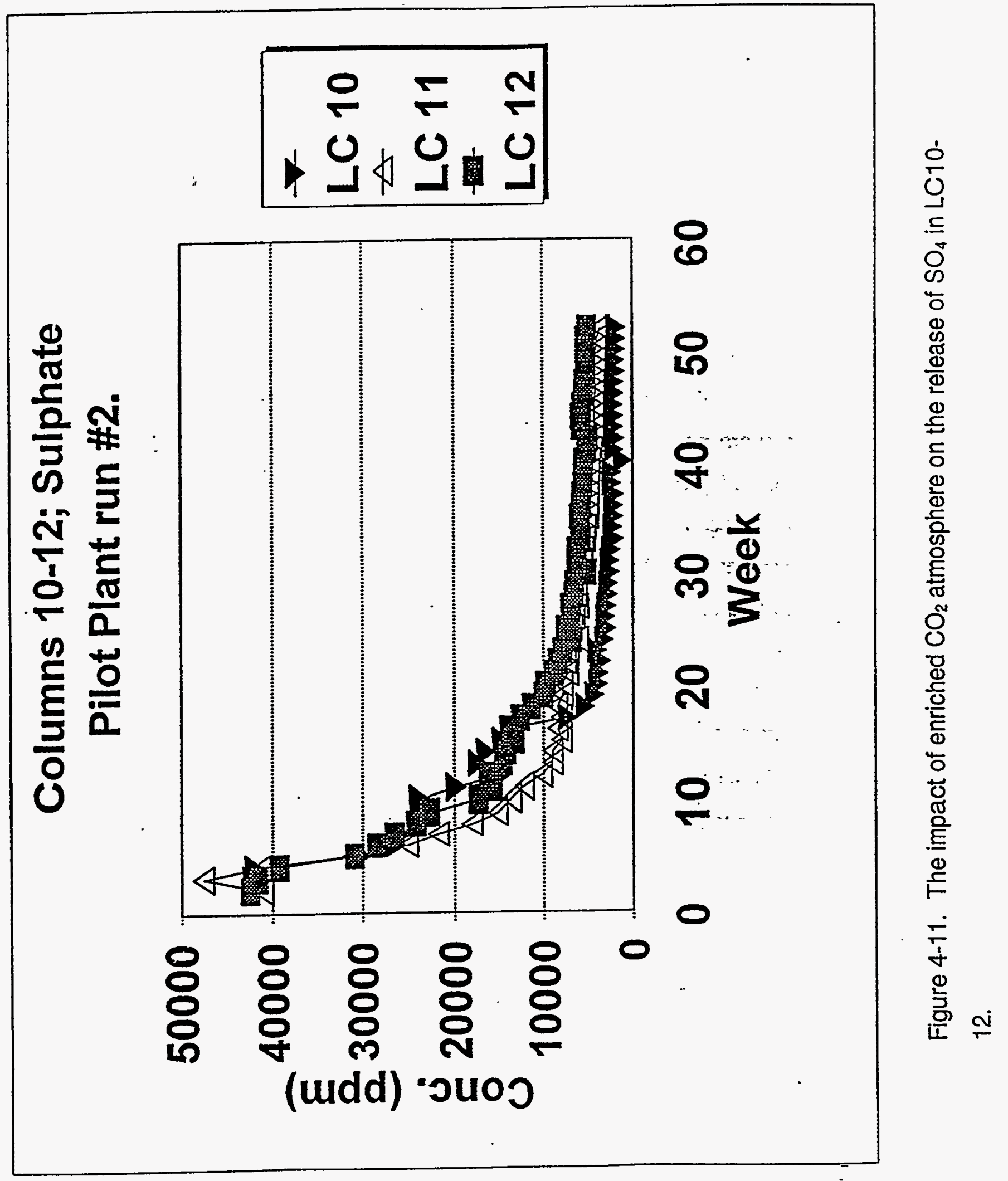


destruction of the same with concurrent sulfate release and gypsum formation in LC11 and LC12.

Prehydration Effects. LC33-LC36 were devised to examine the effect of sample prehydration on leaching behavior. The most notable effect was reflected in differences in the rate of water flow through these columns (Figure 4-12). None of the columns flowed until about week 12 to 14 at which time LC34 and LC35 (15 and 30\% prehydration, respectively) exhibited breakthrough followed by free flow around week 15 . LC33 (0\% prehydration water) began to flow slowly at week 15 then slowly increased to $\sim 20 \%$ of the feed rate around week 30 . LC36 (45\% prehydration) never flowed. LC25 and LC26 which were included in the original set of columns and were prehydrated at $0 \%$ and $40 \%$, respectively, showed similar trends, i.e., LC25 flowed slowly while LC26 did not flow. Thus, the level of prehydration water added to the FGD materials prior to packing appears to have significantly affected the bed permeability. This has implications with respect to disposal in that excess hydration of FGD wastes prior to disposal may produce a material that is effectively impermeable to or at least substantially reduces the percolation of groundwater through the bed.

The leachate chemistry was very similar for LC34 and LC35, the two columns that exhibited similar flow rates during the study. $\mathrm{pH}$ values for the leachates from these columns was initially around 12.5 before declining to 11.5-12.0 near the end of the study (Figure 4-13). LC33 which began to flow later and continued to flow more slowly exhibited a relatively low $\mathrm{pH}$ of 9.5-11.5 in the initial collections before increasing to the 12.0 range.

Elemental release from LC34 and LC35 was also similar with the exception of $\mathrm{Cl}^{-}$which was more concentrated and shifted about plus 10 weeks for LC35 relative to LC34. Leachate concentrations for LC33, which exhibited slower flow rates, were substantially delayed relative to both LC34 and LC35. Examples of these patterns are shown in the plots of 


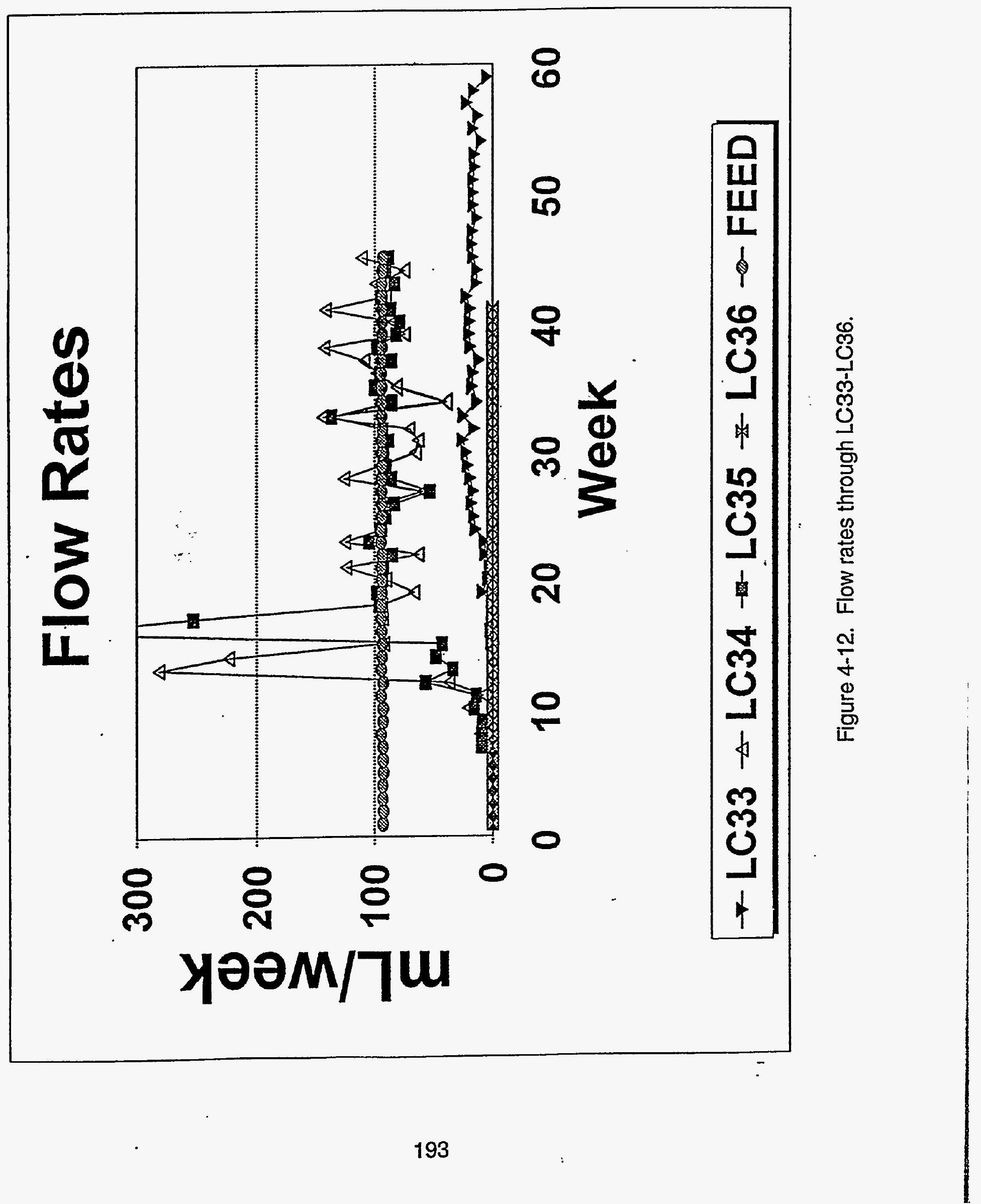




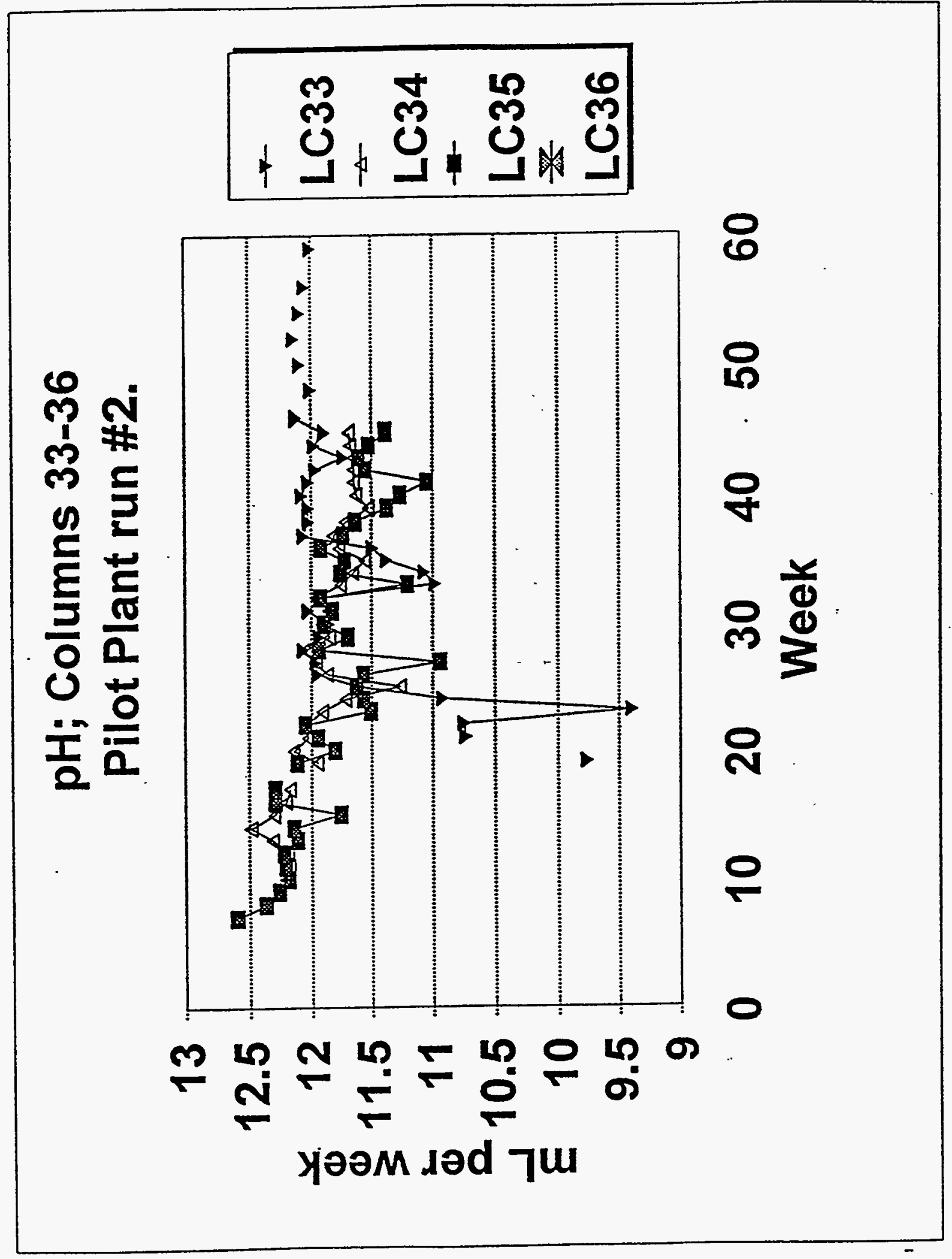

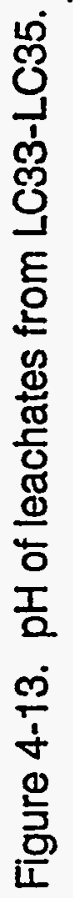



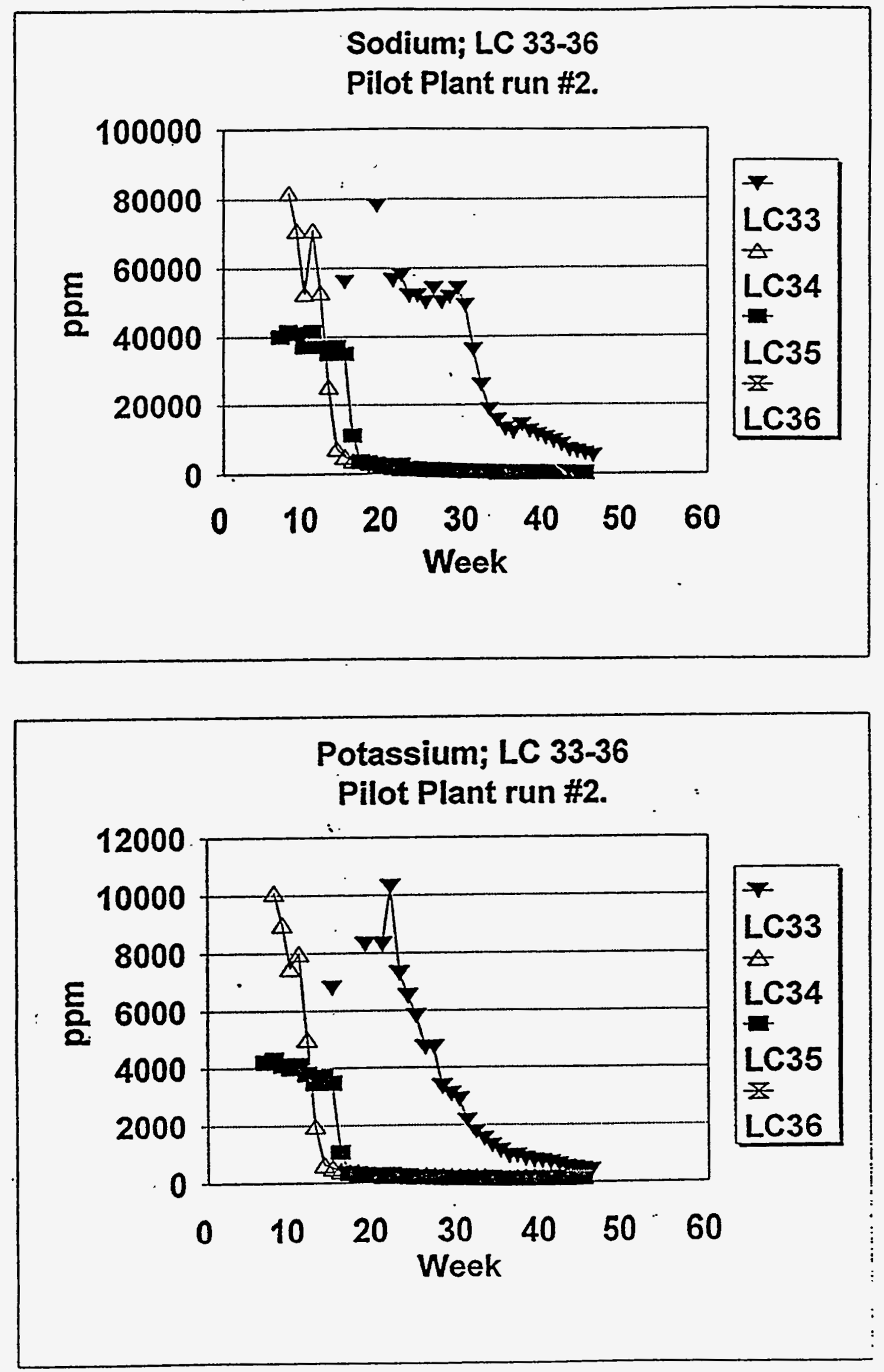

Figure 4-14a, b. Leaching behavior for selected ions collected from LC33=LC35 (sodium, potassium). 

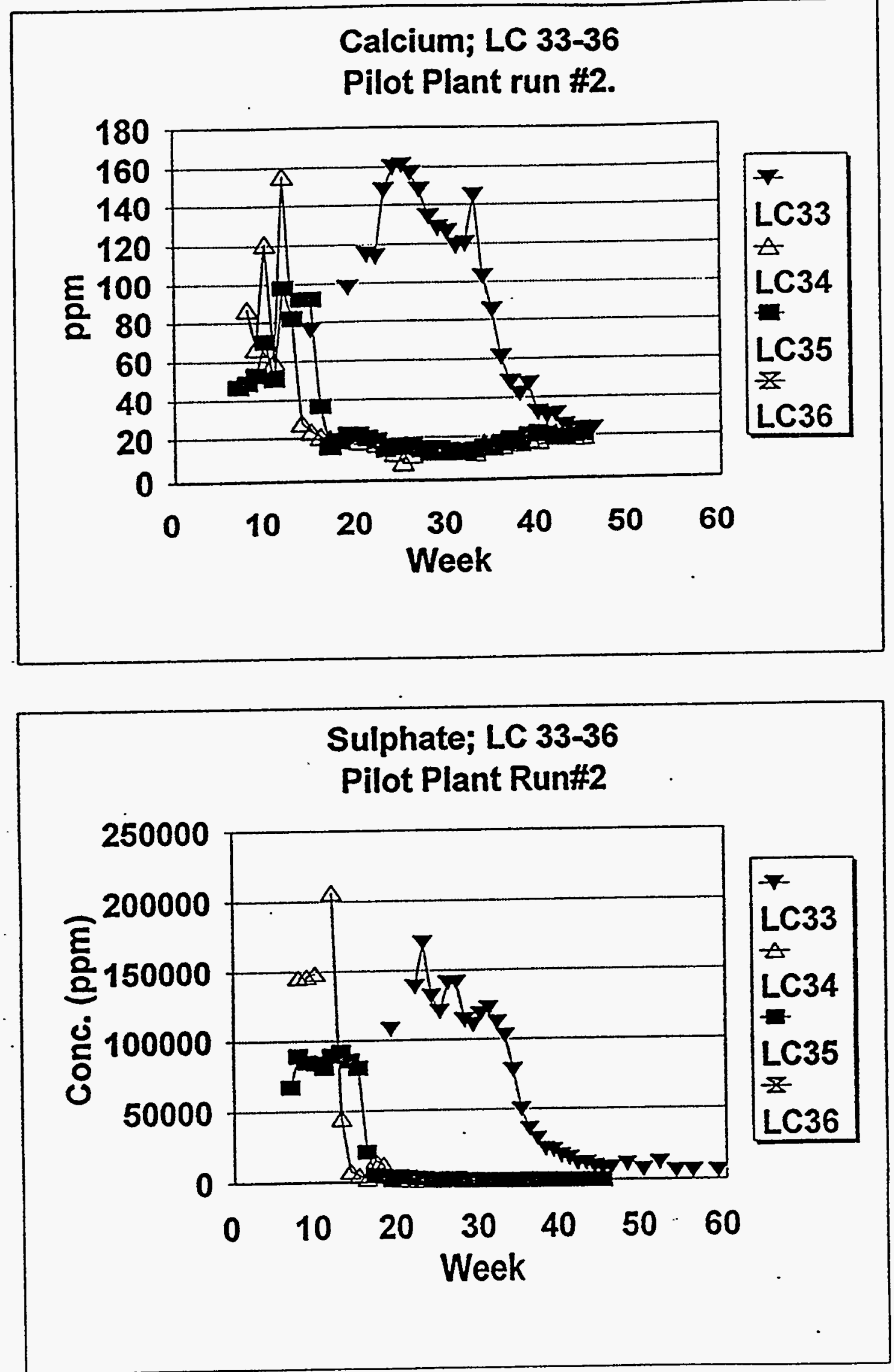

Figure 4-14c, d. Leaching behavior for selected ions collected from LC33-LC35 (calcium, sulphate). 
Figure 4-14. When ion concentrations were examined as a function of total flow, release patterns for all three lysimeters were similar. That is, release appeared to be governed by the volume of water passing through the columns and not by differences in mineralogy. Thus, although the rate of release is affected by the level of prehydration, release as a function of the volume of water flow through the columns does not appear to be affected. The XRD spectra for post-leaching samples from these columns are presented in a later section.

\section{Summary}

The results indicate that packing density, the presence of a $\mathrm{CO}_{2}$ atmosphere, and extent of prehydration all measurably impact leaching behavior. Packing density and particularly the level of prehydration significantly impacted flow rates which in turn affected leaching kinetics. Packing density also appeared to have some influence on leachate chemistry and $\mathrm{CO}_{2}$ uptake. Provision of an enriched $\mathrm{CO}_{2}$ atmosphere in the column headspace was found to lower leachate $\mathrm{pH}$ which in turn affects both mineralogy and release patterns for several of the monitored ions including $\mathrm{Al}, \mathrm{B}, \mathrm{Ca}, \mathrm{Mg}, \mathrm{SO}_{4}, \mathrm{~V}$, and possibly $\mathrm{As}$ and $\mathrm{Br}$. With the exception of Se from the demonstration run $\# 1$, none of the ions being monitored were released at concentrations that would be of concern environmentally. Even this material met RCRA standards for non-hazardous waste.

1. Coolside Waste Management Research Annual Techn. Progress Report for Period October 1991 through September 1992. Prepared for the USDoE under Contract No. DE-AC2191MC28162 by the University of Kentucky-Center for Applied Energy Research and Kentucky Transportation Center; October, 1992; 61 p.

2. Coolside Waste Management Research Annual Techn. Progress Report for Period October 1992 through September 1993. Prepared for the USDoE under Contract No. DE-AC2191MC28162 by the University of Kentucky-Center for Applied Energy Research and Kentucky Transportation Center; October, 1993. 
3. R Garavaglia and P. Caramuscio, Coal Fly-Ash Leaching Behavior and Solubility Controlling Solids, in J.J.J.M. Goumans, H.A. van del Sloot, Th. G. Aalbers (eds.), Proc. Intnl. Conf. on Env. Implications of Construction materials and Techn. Developments, Maastricht, the Netherlands, June 1-3 1994, Elsevier, Amsterdam, 1994, pp.87-102.

4. Lafferty, C.J.; Robl, T.L.; Schram, W.H.; Yewell, M.E.; "Elemental Release Characteristics of Dry Flue Gas Desulfurization By-Products" 10th Annual Intnl. Pittsburgh Coal Conf. S.-H. Chiang, Ed.; Univ. of Pittsburgh School of Engineering.-Center for Energy Res.; 1993; 858863. 


\section{CHAPTER 5. CHEMISTRY OF THE FIELD LEACHATES}

\section{Sample Collection and Analytical Methodology}

\section{Analytical Protocol}

Protocols for analysis were established in the project's infancy. Analytical method 1620 was the protocol followed by the CAER analytical staff in the determination of metals concentration. Method 1620 was developed by the Industrial Technology Division (ITD) within the United States Environmental Protection Agency (USEPA) Office of Water Regulations and Standards (WORS) to provide improved precision and accuracy of analysis of pollutants in aqueous and solid matrices. Below is the routine method of analysis for metals concentration:
a) Calibration of Instrument
b) Initial Calibration Verification (ICV)
c) Continuing Calibration Verification (CCV)
d) Interference Check Sample A (ICS A)(interference- Ca, Al, Mg 500 ppm- Fe 200 ppm)
e) Interference Check Sample AB (ICS AB)(analytes mixed with the interference) control limit $\pm 20 \%$
f) Reagent Blank (RB) (must follow steps as samples)
g) Quality Control (QC) (Spex QC-19, QC-7 Quality Control Standards)

Prior to analysis of the leachate samples, the initial calibration, check samples, and quality control samples were analyzed and accepted. After every ten samples, a quality control standard was analyzed to secure accuracy of the instrument.

Following the collection of leachate samples from the field lysimeters the following EPA methods of analysis were implemented by the CAER staff on a routine basis. Method 310.1 (Titrimetric, pH 4.5) was the EPA method of analysis for the determination of alkalinity. Method 300.0 was the EPA test method used for the determination of inorganic anions in water by Ion Chromatography. SW-846 Method 9050A was used for the determination of specific conductance. SW-846 Method 9040A was used for the determination of $\mathrm{pH}$. EPA Method 160.2 was used for total residual 
non-filterable solids (TDS).

\section{Sample Collection Methodology}

The precise chain of events of field and laboratory analytical methods and procedures were as follows. Collection occurred every Monday and Thursday in the beginning of the project (on rare occasions, circumstances dictated alternate days). The early samples were known to have high concentrations of metals, and it was determined that at the onset of leaching samples were to be collected twice a week until the metals trends showed an equilibrium or depletion in concentration.

Upon arrival at the field site the scientific field crew would interpret and record the rainfall data of the preceding week. Moisture readings (counts) were taken with a Troxler Neutron Probe Model 4300 Depth Moisture Gauge within the four lysimeters. The Neutron Probe, containing both a neutron source and a neutron detector encased within a probe, was placed into a $2^{\prime \prime} \mathrm{OD}$ stainless steel tube positioned in the center of the lysimeter in a vertical position. These tubes were sealed at the bottom and fitted with a cap at the top to prevent moisture from entering the tube. Measurements were taken at .5 feet, at the sand layer, at $2.5,4.5$, and 6.5 feet within the Coolside material, and at 8.5 feet, a measurement at the soil layer.

Multiple K-type stainless steel sheathed $1 / 8^{\prime \prime}$ OD thermocouples were placed in specified areas within the lysimeters positioned near the collection tubes. Temperatures were recorded at the soil, Coolside material and sand in the bottom of the lysimeters using an Omega HH11 Portable Digital Thermometer.

It has been proven in previous column leachate studies that $\mathrm{CO}_{2}$ concentrations can affect the chemistry and mineralogy of the material and leachate, resulting in the decision to measure the $\mathrm{CO}_{2}$ in the soil and Coolside layers. Four borosilicate glass tubes (suction lysimeters) were placed in each of the four lysimeters in April, 1994 at 
depths of 8, 18, and 30 inches. A 42 inch suction lysimeter was added in April of 1995. The tubes were filled with glass beads, and a rubber septum was placed on the top of the tubes. Measurements occurred by placing a Kitagawa $\mathrm{CO}_{2}$ Precision Gas Detector Tube through the septum and into the tube. A Kitagawa model 8014-400A hand operated pump was used to pull a vacuum. With the vacuum established, the gas would flow through the Kitagawa detection tube resulting in the recorded measurement of $\mathrm{CO}_{2}$ concentration.

After an event (precipitation), the volume (L) was determined from each collection port from each lysimeter. The volume $(L)$ in the center collection cell was measured to establish a complete percolation profile. The samples were collected in 25 liter carboys and transported to the laboratory. $500 \mathrm{mls}$ of sample were collected from each carboy for analysis (if more than one carboy was collected from a single port, the sample was mixed homogeneously). The unused portion of the samples was then treated with nitric acid to neutralize the $\mathrm{pH}$, and it was then diluted with tap water and discarded. The $500 \mathrm{ml}$ homogeneous sample was analyzed for $\mathrm{pH}$ using the Orion Research Microprocessor lonalyzer 1901 following SW-846 Method 9040A. A small portion of the sample was then decanted to measure conductivity using the YSI Model 32 Conductance Meter following SW-846 Method 9050A.

$200 \mathrm{mls}$ of the sample were then filtered through a $.45 \mu \mathrm{m}$ membrane filter; acidified with nitric acid, and analyzed for metals following Method 1620. The aqueous sample was analyzed by a Plasma-Therm ICP 2500 for the determination of trace metals. It was introduced to the ICP via the CETAC U-5000 Ultrasonic Nebulizer. The ultrasonic nebulization system contains a sample inlet, a piezoelectric transducer, an aerosol chamber, a gas inlet, a heating chamber, a condenser, and an interface to the plasma torch. Detection limits obtained with an ultrasonic nebulizer are generally 5 to 25 times better than those achieved with a pneumatic nebulizer. Major elements were obtained by utilizing a Spectrometric DCP Optical Emission Spectrometer. 
The determination of mercury was accomplished by using a Perkin Elmer 3100 Atomic Absorption Spectrometer along with the Perkin Elmer FIAS 200 following the SW-846 Method 7470 cold vapor generation of $\mathrm{Hg}$.

The unacidfied portion of the sample was used for the determination of alkalinity, anions, and total dissolved solids. $50 \mathrm{mls}$ of the unacidified sample was analyzed by the Fisher Computer Aided Titrimeter to generate aikalinity results following EPA Method 310.1. Another portion of the unacidified sample was used in the determination of anions using a Dionex 2120i lon Chromatograph following EPA Method 300.0. The last portion of unacidified sample was used to determine the Total Dissolved Solids following EPA Method 160.2.

Collection of field samples twice a week continued from approximately March, 1993 at the beginning of the first collected leachate sample to approximately December, 1994 when it was decided to collect once a week because the data had shown a decline in concentration nearing equilibrium of most elements. March 29,1996 was the last visit to the field site by the field operators, ending the $31 / 2$ year field study. All samples have been analyzed and data recorded.

\section{Chemistry of the Leachates Collected from the Field Lysimeters}

\section{Leachate Composition}

The samples were analyzed for $\mathrm{Ag}$ (0.01), Al (0.05), As (0.05), B, Ba, Be, Ca, Cd, Co, $\mathrm{Cr}$ (0.005), Cu (0.01), Fe (0.005), Hg, K, Mg (0.02), Mn (0.005), Mo, Na, Ni (0.01), P (0.05), $\mathrm{Pb}(0.02)$, Se (0.03), Si, Ti, V (0.005) and $\mathrm{Zn}(0.002)$. Alkalinity was determined and was titrated to both $\mathrm{pH} 8.3$ and $\mathrm{pH} 4.5$ for the samples which were highly alkaline. $\mathrm{Cl}$, sulfate, $\mathrm{pH}$, conductivity, volume, total dissolved solids, total suspended solids and temperature were also determined for all samples. Bromine $(\mathrm{Br})$ was not in thē original 
protocol, but was added later in the study and was determined for the leachates from the L3 lysimeter.

The chemistry for the leachates is summarized in Tables 5-1 to 5-3. In these tables, leachate data is presented for the lysimeter transport tubes which had the longest continuous collection record. The data are presented by year, starting on March 1.

The data for the soil $\mathrm{CO}_{2}$ is summarized in Table 5-4. The data was collected from soil gas wells as placed at $8,18,30$ and 42 inches of depth $(20,46,76$ and $107 \mathrm{~cm})$. The wells were emplaced in April of 1994. The 42-inch deep tube was added in April of 1995.

Of the elements determined, $\mathrm{Ag}, \mathrm{Be}, \mathrm{Cd}, \mathrm{Co}, \mathrm{Cu}, \mathrm{Hg}, \mathrm{Ni}, \mathrm{P}, \mathrm{Pb}$ and $\mathrm{Zn}$ were either never detected, or were found only rarely in extremely low concentrations. Similarly, while data is presented in the tables for $\mathrm{Cr}, \mathrm{Fe}, \mathrm{Mn}$ and $\mathrm{Ti}$, they were not found in high enough concentrations to compile meaningful averages and trends. Although one of the primary mineral forms bearing calcium is hannebachite $\left(\mathrm{CaSO}_{3} \cdot 1 / 2 \mathrm{H}_{2} \mathrm{O}\right)$, the primary anion from its solution is sulfate $\mathrm{SO}_{4}^{-2}$, as $\mathrm{SO}_{3}^{-2}$ is quickly oxidized in the weathering environment.

Major lons. The state of compaction of the materials was found to have a strong impact on the chemistry of the leachates with two distinct patterns emerging over the study interval. The lysimeters which were uncompacted or compacted with minimal effort (L1 and L2) initially had much higher elemental concentrations compared to $L 3$ which was compacted to optimum density. Sodium, $\mathrm{Cl}, \mathrm{K}$ and sulfate were all higher in concentration by factors of 3 to 4 and Ca by a factor of more than 10 in L1 and 2 compared to L3 (Figures 5-1 to 5-4). The elemental release pattern over time also showed marked contrast, with the less compacted lysimeters showing a rapid decline in . leachate elemental concentrations. For example, $\mathrm{Na}$ in $\mathrm{L} 1$ dropped from a high of $30,000 \mathrm{ppm}$-which is approaching the concentration of a brine--the first year, tō a 
Table 5-1 Summary of Chemistry from Feild Leachates for First Project Year (March 1, 1993-February 28, 1994)

\begin{tabular}{|c|c|c|c|c|c|c|c|c|c|c|c|c|}
\hline & $L 1-3$ & $\sqrt{ }=44$ & & $2-3$ & $\mathrm{~N}=38$ & & L3-4 & $\mathrm{N}=52$ & & L4-3 & $N=50$ & \\
\hline & Year 1 & Year 1 & Year 1 & Year 1 & Year 1 & Year 1 & Year 1 & Year 1 & Year 1 & Year 1 & Year 1 & Year 1 \\
\hline & Mean & Min & $\operatorname{Max}$ & Mean & Min & $\operatorname{Max}$ & Mean & Min & $\operatorname{Max}$ & Mean & Min & $\operatorname{Max}$ \\
\hline $\mathrm{Na}$ & 15767 & 5000 & 30000 & 15863 & 5300 & 33000 & 5480 & 3200 & 9100 & 465 & 11 & 830 \\
\hline $\mathrm{Ca}$ & 800 & 335 & 1350 & 640 & 220 & 1340 & 45 & 18 & 90 & 522 & 240 & 730 \\
\hline $\mathrm{K}$ & 5378 & 1640 & 8100 & 4764 & 1610 & 8000 & 1856 & 1000 & 3300 & 1031 & 56 & 1690 \\
\hline $\mathrm{Al}$ & ns & dl & dl & ns & dl & 0.93 & 14.26 & 6.00 & 29.00 & 0.56 & 0.05 & 1.70 \\
\hline As & 0.70 & 0.30 & 1.50 & 1.17 & 0.40 & 2.30 & 4.88 & 0.80 & 11.20 & 0.57 & 0.30 & 1.00 \\
\hline B & 1.79 & 0.70 & 3.70 & 1.37 & 0.50 & 2.60 & 0.96 & 0.20 & 2.80 & 87.16 & 37.00 & 115.00 \\
\hline $\mathrm{Ba}$ & 0.06 & 0.02 & 0.08 & 0.05 & 0.01 & 0.10 & ns & dl & 0.03 & 0.03 & 0.02 & 0.14 \\
\hline $\mathrm{Cr}$ & ns & dl & 0.04 & ns & dl & 0.05 & ns & dl & 0.18 & 0.52 & 0.23 & 0.76 \\
\hline $\mathrm{Fe}$ & ns & $\mathrm{dl}$ & 0.49 & ns & $\mathrm{dl}$ & 1.29 & ns & dl & 0.62 & 0.02 & $\mathrm{dl}$ & 0.32 \\
\hline $\mathrm{Mg}$ & 1.34 & 0.09 & 4.94 & 1.16 & 0.02 & 4.00 & ns & dl & 0.15 & 7.51 & 2.59 & 35.00 \\
\hline $\mathrm{Mn}$ & 0.01 & $\mathrm{dl}$ & 0.04 & ns & $\mathrm{dl}$ & 0.05 & 0.03 & dl & 0.62 & ns & dl & 0.02 \\
\hline Mo & 56.21 & 6.00 & 110.00 & 50.69 & 6.10 & 138.00 & 20.31 & 5.50 & 41.00 & 41.97 & 2.35 & 85.00 \\
\hline $\mathrm{Se}$ & 2.44 & 0.30 & 3.70 & 1.85 & 0.25 & 3.55 & 1.09 & 0.24 & 2.00 & 0.12 & 0.03 & 0.33 \\
\hline Si & 11.89 & 4.10 & 19.70 & 14.02 & 4.20 & 32.00 & 44.39 & 23.00 & 58.00 & 0.83 & 0.51 & 1.95 \\
\hline $\mathrm{Ti}$ & 0.08 & 0.03 & 0.12 & 0.07 & 0.02 & 0.13 & 0.01 & $\mathrm{dl}$ & 0.02 & 0.03 & 0.01 & 0.53 \\
\hline V & 0.54 & 0.28 & 0.98 & 0.85 & 0.07 & 1.80 & 1.56 & 0.92 & 3.47 & 0.41 & 0.13 & 0.64 \\
\hline Alk-8.3 & 72 & 0 & 126 & 125 & 9 & 315 & 1905 & 275 & 4710 & & & \\
\hline Alk-4.5 & 186 & 139 & 314 & 249 & 143 & 550 & 3366 & 1573 & 7735 & 540 & 150 & 1006 \\
\hline $\mathrm{Cl}$ & 23908 & 1645 & 37590 & 23940 & 2380 & 46230 & 6897 & 2446 & 13200 & 153 & 18 & 570 \\
\hline Sulphate & 17304 & 9000 & 20580 & 14311 & 2450 & 21410 & 2202 & 1214 & 3460 & 3187 & 1506 & 4238 \\
\hline $\mathrm{Br}$ & nd & nd & nd & nd & nd & nd & 1018 & 506 & 1960 & nd & nd & nd \\
\hline $\mathrm{pH}$ & 9.77 & 8.64 & 11.10 & 10.37 & 8.38 & 12.24 & 12.26 & 12.06 & 12.48 & 9.54 & 8.93 & 9.78 \\
\hline Cond. & 70.18 & 19.40 & 102.00 & 66.21 & 19.80 & 108.30 & 28.56 & 14.90 & 48.50 & 6.50 & 2.31 & 9.02 \\
\hline Dis. Solid & 65677 & 16452 & 112500 & 59165 & 15772 & 107676 & 18462 & 8472 & 31122 & 6123 & 921 & 8504 \\
\hline Sus. Solid & 43 & 5 & 136 & 35 & 5 & 84 & 27 & 5 & 427 & 8 & 5 & 48 \\
\hline
\end{tabular}

$n d=$ not determined, $n s=$ not significant, $d=$ below limits of detection 
Table 5-2 Summary of Chemistry from Feild Leachates for Second Project Year (March 1, 1994-February 28, 1995)

\begin{tabular}{|c|c|c|c|c|c|c|c|c|c|c|c|c|}
\hline & L1-3 & $N=55$ & & L2-3 & $N=42$ & & L3-4 & $N=56$ & & L4-3 & $N=38$ & \\
\hline & $\begin{array}{r}\text { Year } 2 \\
\text { Mean }\end{array}$ & $\begin{array}{r}\text { Year } 2 \\
\text { Min }\end{array}$ & $\begin{array}{r}\text { Year } 2 \\
\text { Max }\end{array}$ & $\begin{array}{r}\text { Year } 2 \\
\text { Mean }\end{array}$ & $\begin{array}{r}\text { Year } 2 \\
\text { Min }\end{array}$ & $\begin{array}{r}\text { Year } 2 \\
\text { Max }\end{array}$ & $\begin{array}{r}\text { Year } 2 \\
\text { Mean }\end{array}$ & $\begin{array}{r}\text { Year } 2 \\
\text { Min }\end{array}$ & $\begin{array}{r}\text { Year } 2 \\
\text { Max }\end{array}$ & $\begin{array}{r}\text { Year } 2 \\
\text { Mean }\end{array}$ & $\begin{array}{r}\text { Year } 2 \\
\text { Min }\end{array}$ & $\begin{array}{r}\text { Year } 2 \\
\text { Max }\end{array}$ \\
\hline $\mathrm{Na}$ & 1637 & 800 & 4800 & 2960 & 1425 & 6650 & 2302 & 1500 & 3500 & 12 & 4 & 79 \\
\hline $\mathrm{Ca}$ & 214 & 129 & 358 & 141 & 78 & 224 & 33 & 24 & 46 & 594 & 525 & 680 \\
\hline $\mathrm{K}$ & 687 & 325 & 1600 & 1019 & 625 & 2120 & 860 & 550 & 1155 & 46 & 25 & 96 \\
\hline Al & 0.43 & dl & 0.74 & 1.23 & 0.41 & 1.96 & 11.57 & 6.70 & 19.00 & ns & dl & dl \\
\hline As & 0.36 & 0.07 & 0.63 & 1.44 & 0.28 & 2.08 & 1.95 & 1.10 & 2.90 & 0.38 & 0.30 & 0.47 \\
\hline B & 0.66 & 0.05 & 1.35 & 0.91 & 0.13 & 1.40 & 0.48 & 0.23 & 0.80 & 19.85 & 10.00 & 36.00 \\
\hline $\mathrm{Ba}$ & 0.02 & 0.01 & 0.04 & 0.01 & 0.01 & 0.04 & 0.01 & 0.01 & 0.02 & 0.07 & 0.03 & 0.22 \\
\hline $\mathrm{Cr}$ & 0.02 & $\mathrm{dl}$ & 0.15 & 0.03 & $\mathrm{dl}$ & 0.62 & ns & dl & dl & 0.08 & 0.04 & 0.22 \\
\hline $\mathrm{Fe}$ & ns & dl & 0.50 & 0.23 & dl & 3.00 & ns & dl & 0.12 & ns & $\mathrm{dl}$ & 0.11 \\
\hline $\mathrm{Mg}$ & 0.05 & dl & 0.18 & 0.03 & 0.02 & 0.13 & ns & dl & 0.12 & 21.00 & 13.00 & 30.00 \\
\hline Mn & 0.01 & dl & 0.02 & 0.04 & dl & 0.52 & ns & dl & 0.02 & ns & dl & 0.02 \\
\hline Mo & 2.10 & 1.04 & 5.50 & 2.37 & 1.00 & 7.75 & 4.65 & 3.15 & 8.00 & 0.75 & 0.30 & 2.18 \\
\hline $\mathrm{Se}$ & 0.14 & 0.00 & 0.35 & 0.16 & dl & 0.29 & 0.35 & 0.18 & 0.55 & 0.03 & 0.02 & 0.10 \\
\hline Si & 20.15 & 16.50 & 24.30 & 29.75 & 16.00 & 39.00 & 41.96 & 24.30 & 451.00 & 1.16 & 0.58 & 2.85 \\
\hline $\mathrm{Ti}$ & 0.03 & 0.02 & 0.05 & 0.03 & 0.02 & 0.06 & 0.01 & 0.01 & 0.01 & 0.01 & 0.01 & 0.02 \\
\hline v & 0.57 & 0.11 & 0.90 & 1.14 & 0.23 & 1.45 & 0.86 & 0.65 & 1.20 & 0.25 & 0.22 & 0.28 \\
\hline Alk-8.3 & 125 & 91 & 158 & 269 & 178 & 348 & 712 & 540 & 890 & & & \\
\hline Alk-4.5 & 165 & 125 & 196 & 420 & 283 & 541 & 1301 & 1100 & 1528 & 88 & 60 & 150 \\
\hline $\mathrm{Cl}$ & 432 & 164 & 1660 & 740 & 62 & 2960 & 2323 & 1250 & 3170 & 21 & 0 & 66 \\
\hline Sulphate & 3987 & 1984 & 9455 & 5761 & 3890 & 8550 & 1140 & 692 & 2124 & 1667 & 1500 & 1939 \\
\hline $\mathrm{Br}$ & nd & nd & nd & nd & nd & nd & 463 & 228 & 746 & nd & nd & nd \\
\hline $\mathrm{pH}$ & 11.14 & 10.95 & 11.31 & 11.54 & 11.39 & 11.68 & 12.08 & 11.90 & 12.31 & 9.20 & 8.88 & 9.51 \\
\hline Conductiv & 8.21 & 4.47 & 16.90 & 11.86 & 7.23 & 19.70 & 12.77 & 8.74 & 16.40 & 2.27 & 1.97 & 3.00 \\
\hline Dis. Solid & 7036 & 3512 & 17528 & 9760 & 4962 & 17544 & 7854 & 5534 & 9430 & 2706 & 1368 & 3030 \\
\hline Susp Soli & 9 & 5 & 32 & 12 & 5 & 33 & 10 & 5 & 21 & 6 & 5 & 17 \\
\hline
\end{tabular}


Table 5-3 Summary of Chemistry from Feild Leachates for Third Project Year (March 1, 1995-March 29, 1996)

\begin{tabular}{|c|c|c|c|c|c|c|c|c|c|c|c|c|}
\hline & $\begin{array}{l}1-3 \\
\text { Year } 3 \\
\text { Mean }\end{array}$ & $\begin{array}{l}N=39 \\
\text { Year } 3 \\
\text { Min }\end{array}$ & $\begin{array}{l}\text { Year3 } \\
\text { Max }\end{array}$ & $\begin{array}{l}\text { L2-3 } \\
\text { Year } 3 \\
\text { Mean }\end{array}$ & $\begin{array}{l}n=26 \\
\text { Year } 3 \\
\text { Min }\end{array}$ & $\begin{array}{l}\text { Year3 } \\
\text { Max }\end{array}$ & \begin{tabular}{|l} 
L3-4 \\
Year 3 \\
Mean
\end{tabular} & $\begin{array}{l}n=35 \\
\text { Year } 3 \\
\text { Min }\end{array}$ & $\begin{array}{l}\text { Year3 } \\
\text { Max }\end{array}$ & \begin{tabular}{|l} 
L4-3 \\
Year 3 \\
Mean
\end{tabular} & $\begin{array}{l}\mathrm{N}=23 \\
\text { Year } 3 \\
\text { Min }\end{array}$ & $\begin{array}{l}\text { Year3 } \\
\text { Max }\end{array}$ \\
\hline $\mathrm{Na}$ & 604 & 495 & 774 & 876 & 352 & 1635 & 1436 & 1035 & 2180 & 9 & 7 & 17 \\
\hline $\mathrm{Ca}$ & 109 & 48 & 167 & 83 & 40 & 122 & 33 & 23 & 47 & 473 & 375 & 640 \\
\hline $\mathrm{K}$ & 241 & 180 & 330 & 530 & 368 & 715 & 594 & 410 & 780 & 26 & 21 & 32 \\
\hline Al & 0.47 & 0.20 & 0.92 & 1.11 & 0.45 & 1.74 & 12.69 & 10.50 & 16.20 & ns & $\mathrm{dl}$ & 0.22 \\
\hline As & 0.30 & 0.21 & 0.44 & 0.47 & 0.15 & 0.77 & 1.16 & 0.85 & 1.60 & 0.43 & 0.40 & 0.47 \\
\hline$B$ & 0.57 & 0.30 & 0.85 & 0.68 & 0.30 & 0.95 & 0.21 & 0.05 & 0.48 & 8.76 & 7.25 & 9.70 \\
\hline $\mathrm{Ba}$ & 0.01 & 0.01 & 0.02 & 0.02 & 0.01 & 0.03 & 0.01 & 0.01 & 0.02 & 0.03 & 0.03 & 0.05 \\
\hline $\mathrm{Cr}$ & 0.01 & 0.01 & 0.03 & ns & dl & $d l$ & ns & dl & $\mathrm{dl}$ & 0.05 & 0.03 & 0.06 \\
\hline $\mathrm{Fe}$ & ns & dl & 0.28 & ns & dl & 0.20 & ns & dl & 0.12 & ns & dl & 0.08 \\
\hline $\mathrm{Mg}$ & ns & $\mathrm{dl}$ & 0.10 & 0.06 & dl & 1.01 & ns & dl & 0.05 & 16.40 & 13.00 & 20.50 \\
\hline $\mathrm{Mn}$ & 0.01 & 0.01 & 0.10 & ns & dl & 0.01 & ns & dl & $\mathrm{dl}$ & ns & dl & 0.01 \\
\hline Mo & 0.93 & 0.52 & 2.00 & 1.36 & 0.61 & 2.90 & 3.40 & 1.75 & 5.00 & 0.45 & 0.36 & 0.69 \\
\hline $\mathrm{Se}$ & 0.06 & dl & 0.11 & 0.09 & dl & 0.18 & 0.16 & d! & 0.45 & 0.03 & dl & 0.08 \\
\hline $\mathrm{Si}$ & 25.10 & 18.70 & 34.00 & 22.03 & 13.50 & 28.00 & 28.45 & 23.00 & 34.00 & 1.23 & 0.97 & 1.84 \\
\hline $\mathrm{Ti}$ & 0.01 & 0.01 & 0.02 & 0.01 & 0.01 & 0.02 & 0.01 & 0.01 & 0.01 & 0.01 & 0.01 & 0.03 \\
\hline V & 0.84 & 0.68 & 0.96 & 0.44 & 0.19 & 0.65 & 0.58 & 0.40 & 0.70 & 0.25 & 0.23 & 0.28 \\
\hline Alk-8.3 & 129 & 0 & 262 & 144 & 66 & 258 & 563 & 288 & 920 & & & \\
\hline Alk -4.5 & 175 & 121 & 318 & 215 & 104 & 336 & 1033 & 799 & 1500 & 53 & 36 & 76 \\
\hline $\mathrm{Cl}$ & 109 & 75 & 219 & 46 & 21 & 75 & 1408 & 838 & 2213 & 24 & 3 & 40 \\
\hline Sulphate & 1506 & 1031 & 2052 & 2652 & 1671 & 5680 & 886 & 562 & 1510 & 1335 & 907 & 1591 \\
\hline $\mathrm{Br}$ & nd & nd & nd & nd & nd & nd & 319 & 0 & 523 & nd & nd & nd \\
\hline $\mathrm{pH}$ & 11.15 & 10.95 & 11.34 & 11.32 & 10.91 & 11.54 & 12.00 & 11.84 & 12.19 & 8.97 & 8.64 & 9.17 \\
\hline Conductiv & 3.64 & 2.77 & 4.97 & 5.64 & 3.47 & 8.08 & 238.38 & 6.48 & 8032.00 & 1.97 & 1.60 & 2.36 \\
\hline Dis. Solid & 2654 & 1850 & 3584 & 4276 & 2576 & 6870 & 5404 & 3872 & 7294 & 2272 & 1804 & 2700 \\
\hline Susp Soli & 5 & 5 & 10 & 5 & 5 & $\begin{array}{r}5 \\
\end{array}$ & 6 & 5 & 25 & 5 & 5 & 10 \\
\hline
\end{tabular}


Table 5-4. Carbon dioxide data for lysimeter soil gases. Dectection limit $=0.01 \%(-100 \mathrm{ppm})$. Data in percent atmosphere.

\begin{tabular}{|c|c|c|c|c|c|c|c|c|c|}
\hline & $\begin{array}{l}1994 \\
N=25\end{array}$ & Depth & MEAN & MAX & MIN & $\begin{array}{r}1995 \\
N=24\end{array}$ & MEAN & MAX & MIN \\
\hline \multirow[t]{4}{*}{ L1 } & & 8 & 0.49 & 1.90 & 0.05 & L1 & 0.40 & 1.20 & 0.05 \\
\hline & & 18 & 0.12 & 0.40 & dl & & 0.13 & 0.35 & $\mathrm{dl}$ \\
\hline & & 30 & ns & 0.20 & dl & & ns & 0.05 & dl \\
\hline & & 42 & nd & nd & nd & & ns & 0.05 & d) \\
\hline \multirow[t]{4}{*}{ L2 } & & 8 & 0.46 & 1.40 & 0.10 & L2 & 0.34 & 1.20 & 0.05 \\
\hline & & 18 & 0.07 & 0.18 & dl & & 0.16 & 0.63 & $\mathrm{dl}$ \\
\hline & - & 30 & ns & 0.15 & dl & & ns & 0.05 & dl \\
\hline & & 42 & nd & nd & nd & . & ns & 0.02 & dl \\
\hline \multirow[t]{4}{*}{ L3 } & & 8 & 0.61 & 1.80 & 0.05 & L3 & 0.42 & 0.01 & dl \\
\hline & & $18^{\circ}$ & 0.78 & 2.30 & dl & & 0.43 & 3.20 & dl \\
\hline & & 30 & ns & 0.20 & dl & & ins & 2.00 & dl \\
\hline & & 42 & nd & nd & nd & & sn & 0.02 & d! \\
\hline \multirow[t]{4}{*}{ L4 } & & 8 & 0.15 & 0.75 & d! & L4 & 0.24 & 0.80 & 0.03 \\
\hline & & 18 & 0.06 & 0.30 & dl & & 0.11 & 0.30 & 0.03 \\
\hline & & 30 & 0.16 & 0.80 & dl & & 0.25 & 0.84 & 0.05 \\
\hline & & 42 & nd & nd & nd & & 0.25 & 1.00 & 0.02 \\
\hline
\end{tabular}


$\mathrm{Na}$ in Leachates

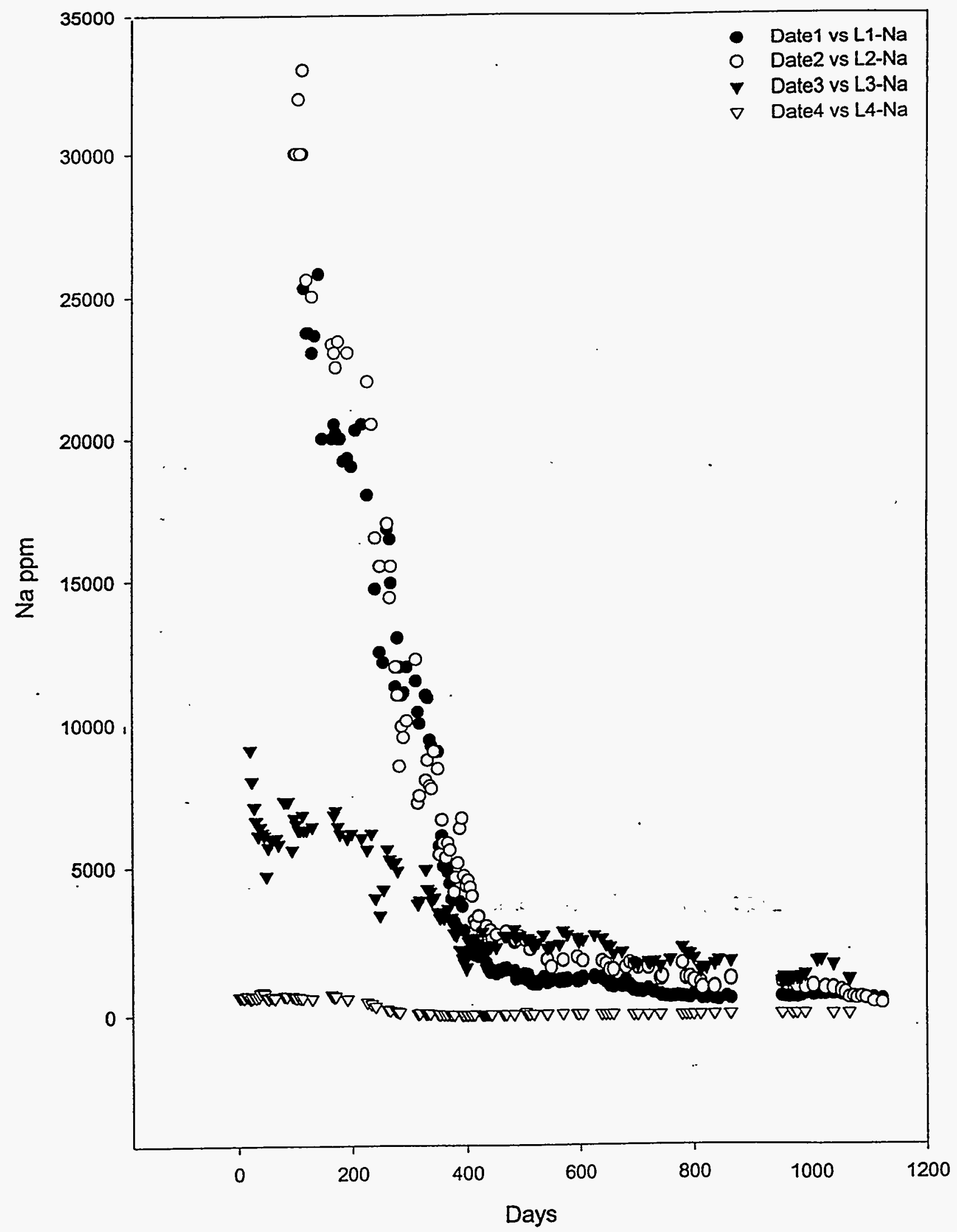

Figure 5-1. Na concentration as ppm in the leachates from the field lysimeters.

Data for $L 1$ is from transport tube $L 1-3, L 2$ from $L 2-3, L 3$ from $L 3-4$ and $L 4$ from L4-3. Day $1=$ March 1, 1993. 


\section{$\mathrm{Cl}$ in Leachates}

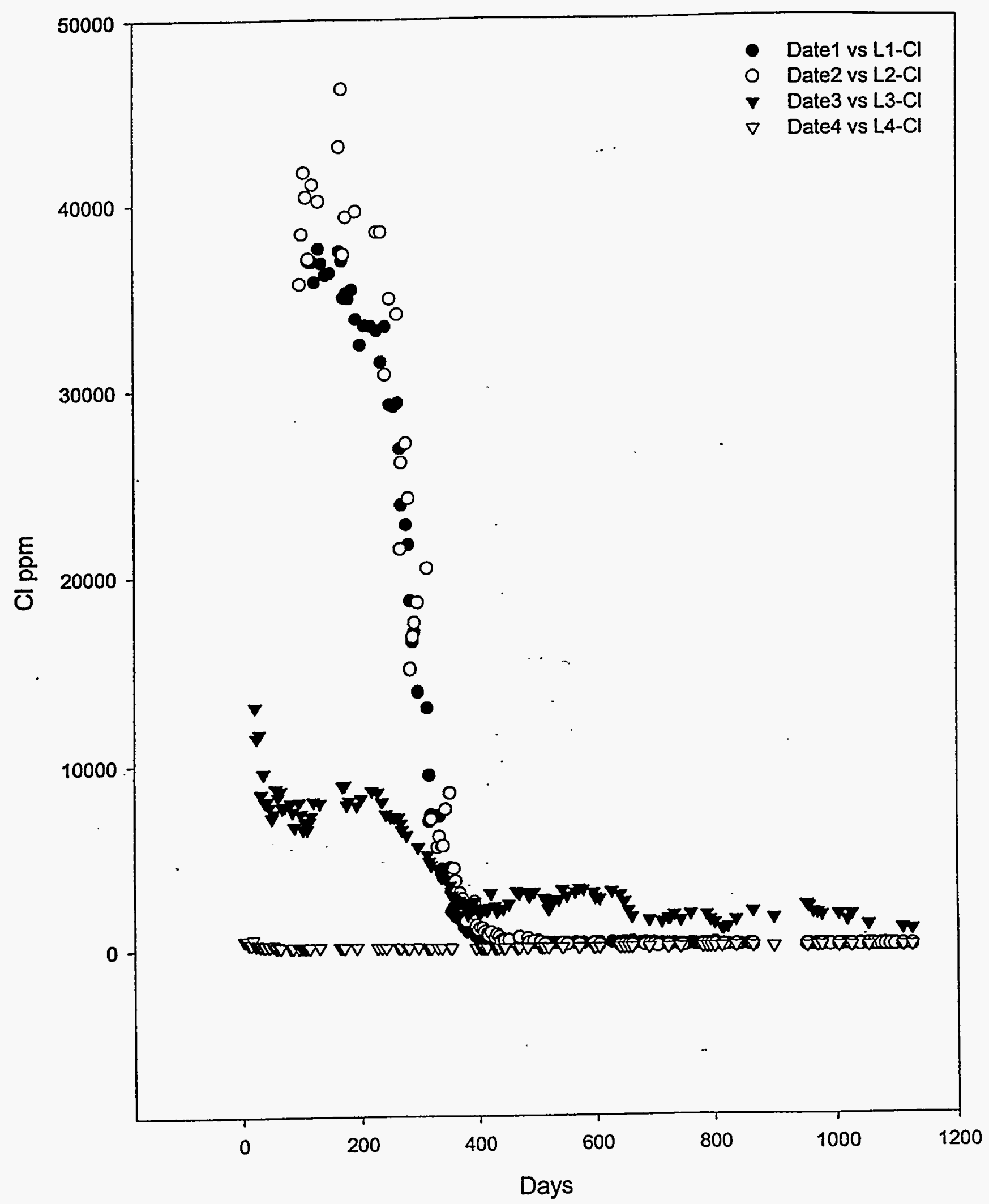

Figure 5-2. $\mathrm{Cl}$ concentration as $\mathrm{ppm}$ in the leachates from the field lysimeters.

Data as Figure 5-1. 
$\mathrm{K}$ in Leachates

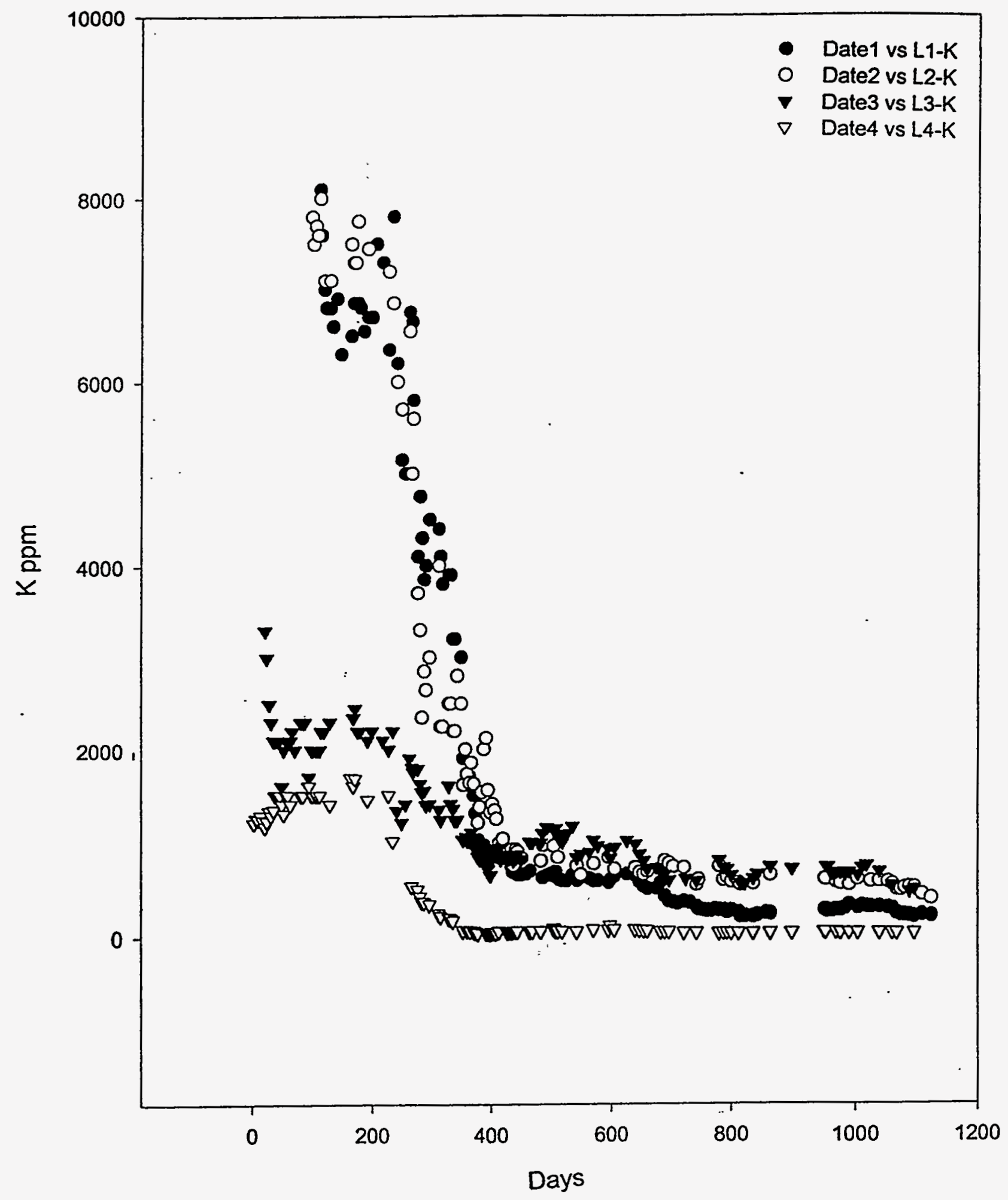

Figure 5-3. $\mathrm{K}$ concentration as ppm in the leachates from the field lysimeters.

Data as in Figure 5-1. 
maximum of $4,800 \mathrm{ppm}$ the second year, and $774 \mathrm{ppm}$ the third. Thus, the year three maximum was less than $3 \%$ of the first years. The decline in L3 was much less, with a maximum of $9,100 \mathrm{ppm}$ the first year, followed by maximums of $3,500 \mathrm{ppm}$ and 2,180 ppm the second and third year (Figure 5-4).

The control lysimeter, L4, filled with Class F fly ash, also showed these patterns, although the maximum concentrations were much less. For example, Na dropped from a maximum of $830 \mathrm{ppm}$ the first year to $79 \mathrm{ppm}$ the second and $17 \mathrm{ppm}$ the third in the L4 leachates.

Minor and Trace lons. The leaching patterns for the other elements do not necessarily show a similar pattern to that of the major elements. For example, $\mathrm{Al}$ and $\mathrm{Si}$ increased in concentration during the second year of the study in the L1 and L2 leachates (Figure $5-5,5-6)$. These trends can be related to increases in the $\mathrm{pH}$ of the leachates over time (Figure 5-7). The average pH of the L1, L2 and L3 leachates was 9.7, 10.4, and 12.3 during the first year of collection. $\mathrm{pH}$ increased as a function of material density. Most transition elements such as $\mathrm{Fe}, \mathrm{Co}$, etc. are essentially insoluble under these conditions. However, the higher $\mathrm{pH}$ resulted in the mobilization of elements which can form oxyanionic complexes such as $\mathrm{Mo}\left(\mathrm{MoO}_{4}^{-2}\right), \mathrm{Se}\left(\mathrm{SeO}_{4}^{-2}\right), \mathrm{As}\left(\mathrm{AsO}_{4}^{-3}\right)$ and $\mathrm{V}\left(\mathrm{VO}_{4}^{-3}\right)$ (e. g., Figures 5-8, 5-9). Additional equlibria with hydroxide, for example, is also expected.

In comparison to the Class $\mathrm{F}$ fly ash used for control, the concentrations of $\mathrm{Al}, \mathrm{As}, \mathrm{Mo}$, $\mathrm{Se}, \mathrm{Si}, \mathrm{Ti}$ and $\mathrm{V}$ were higher in the Coolside leachates. On the other hand the concentrations of $\mathrm{B}$ and $\mathrm{Mg}$ were significantly higher in the Class $\mathrm{F}$ leachates (Figure 510, 5-11).

Leachate elements of environmental concern include As and Se, which have an RCRA limit of 5 Pam and $1 \mathrm{ppm}$ respectively. Many of the samples from the L3 (Figure 5-12) lysimeter exceeded the $5 \mathrm{ppm}$ limit during the first year of the study with a maximum 
Sulfate in Leachates

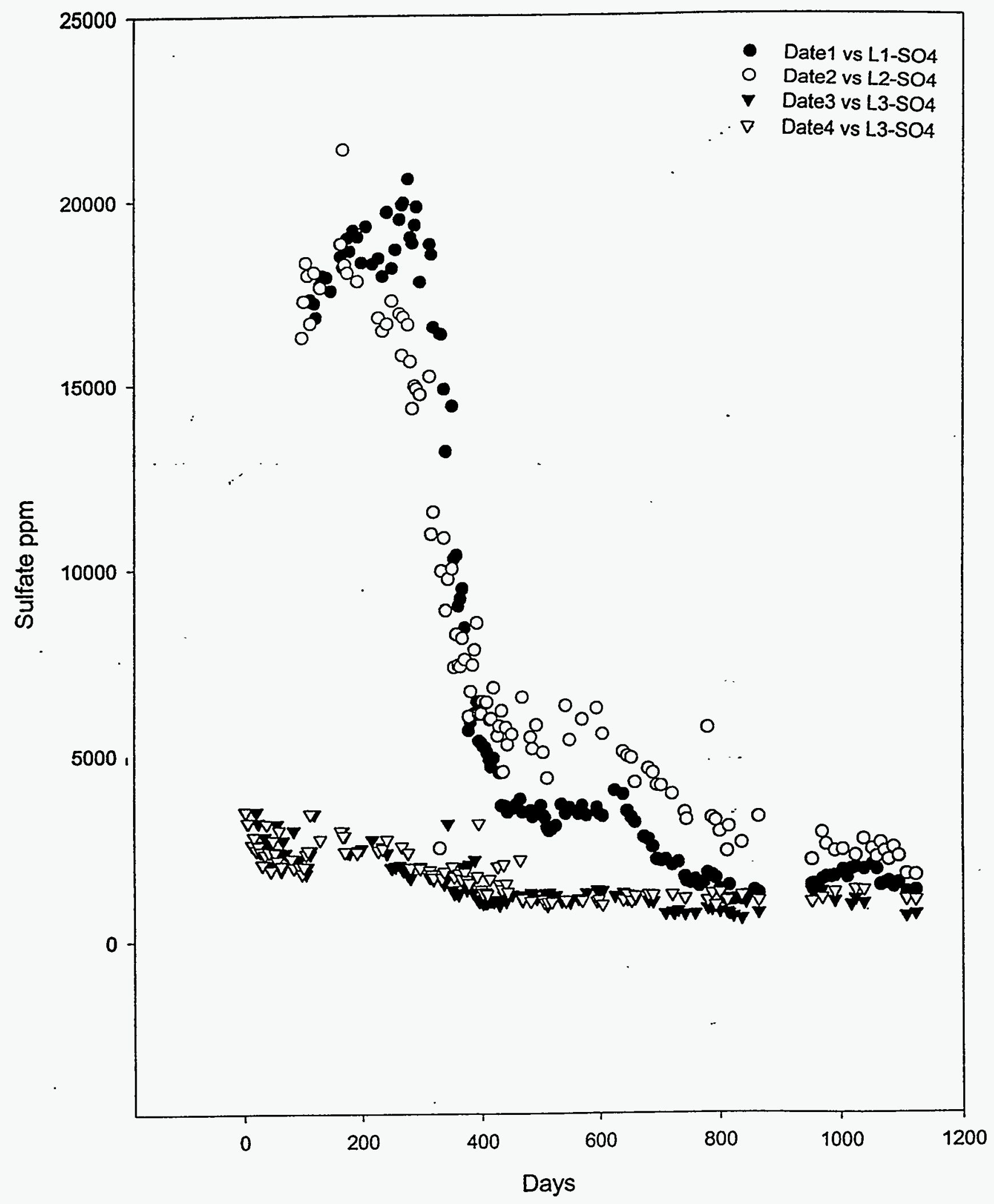

Figure 5-4. Sulfate concentration as ppm in the leachates from the field lysimeters. Data as in Figure 5-1. 


\section{Al in Leachates}

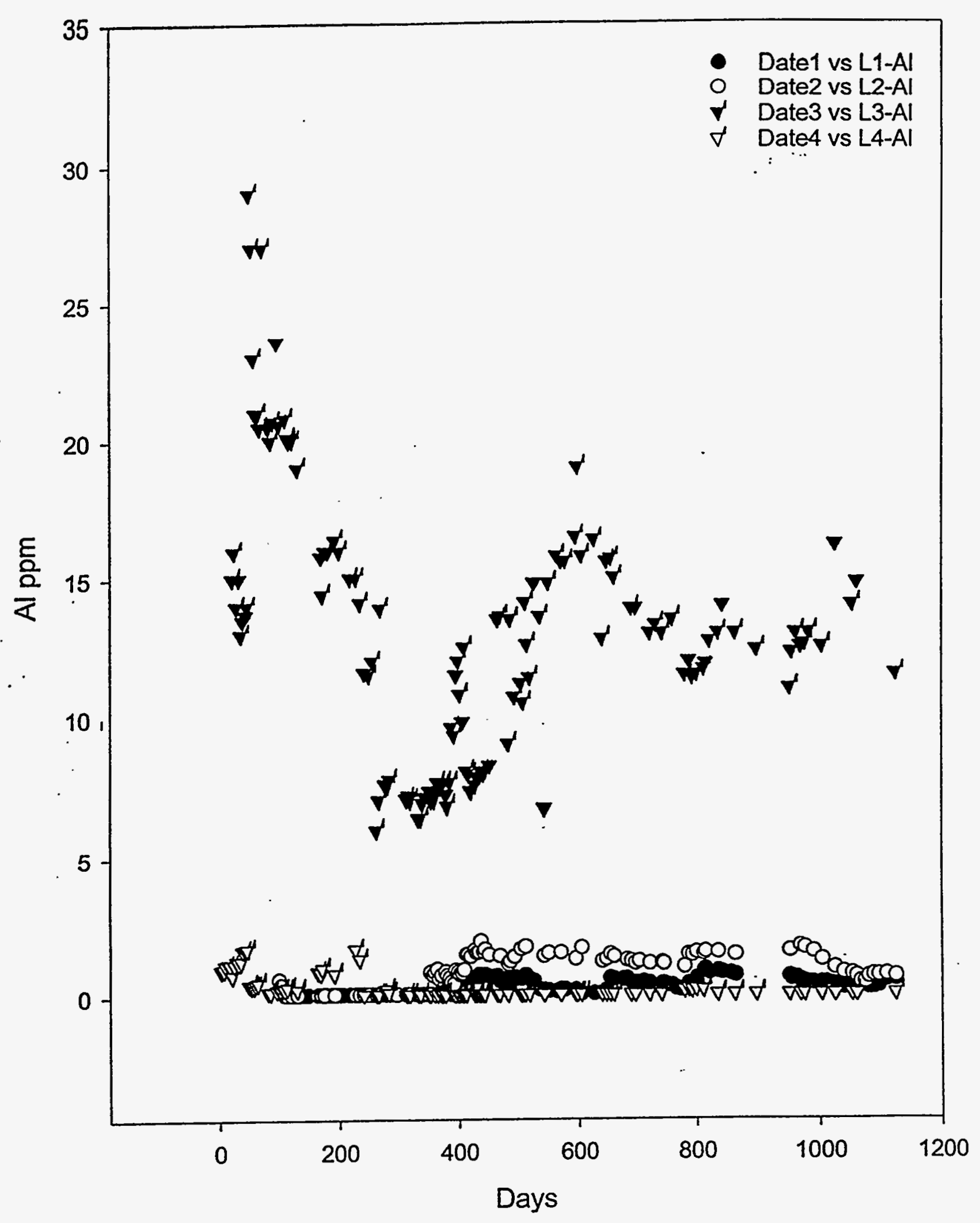

Figure 5-5. Al concentration as ppm in the leachates from the field lysimeters.

Data as in Figure 5-1. 


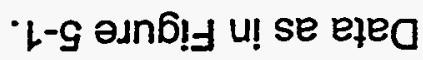

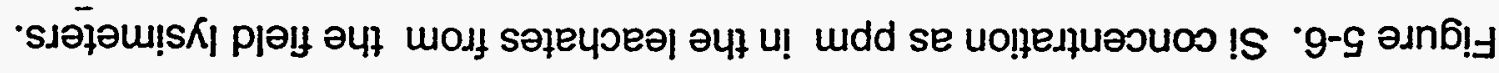
sरeo

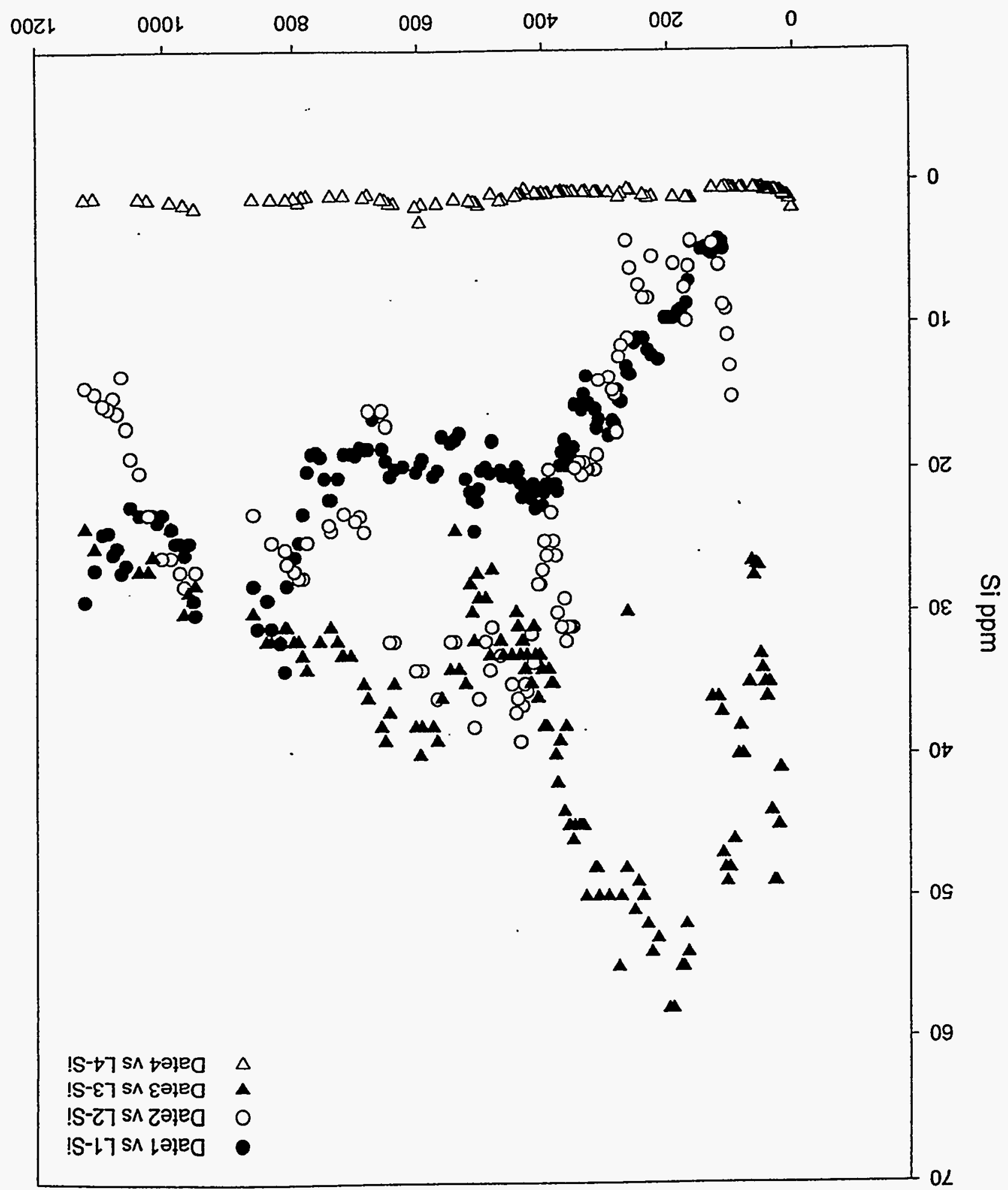


$\mathrm{pH}$ of Leachates

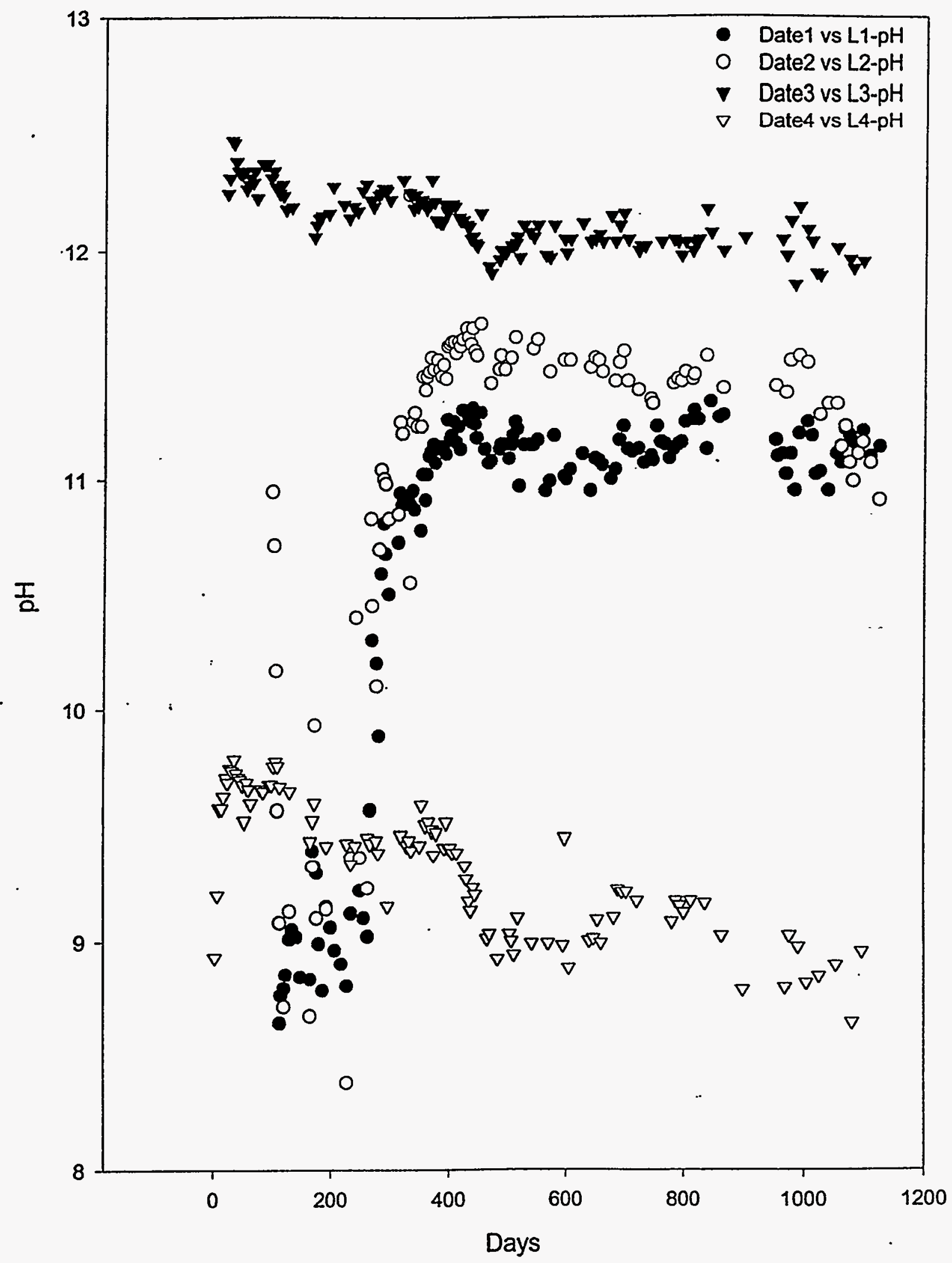

Figure 5-7. $\mathrm{pH}$ of the leachates from the field lysimeters. Data as in Figure 5-1. 
Mo in Leachates

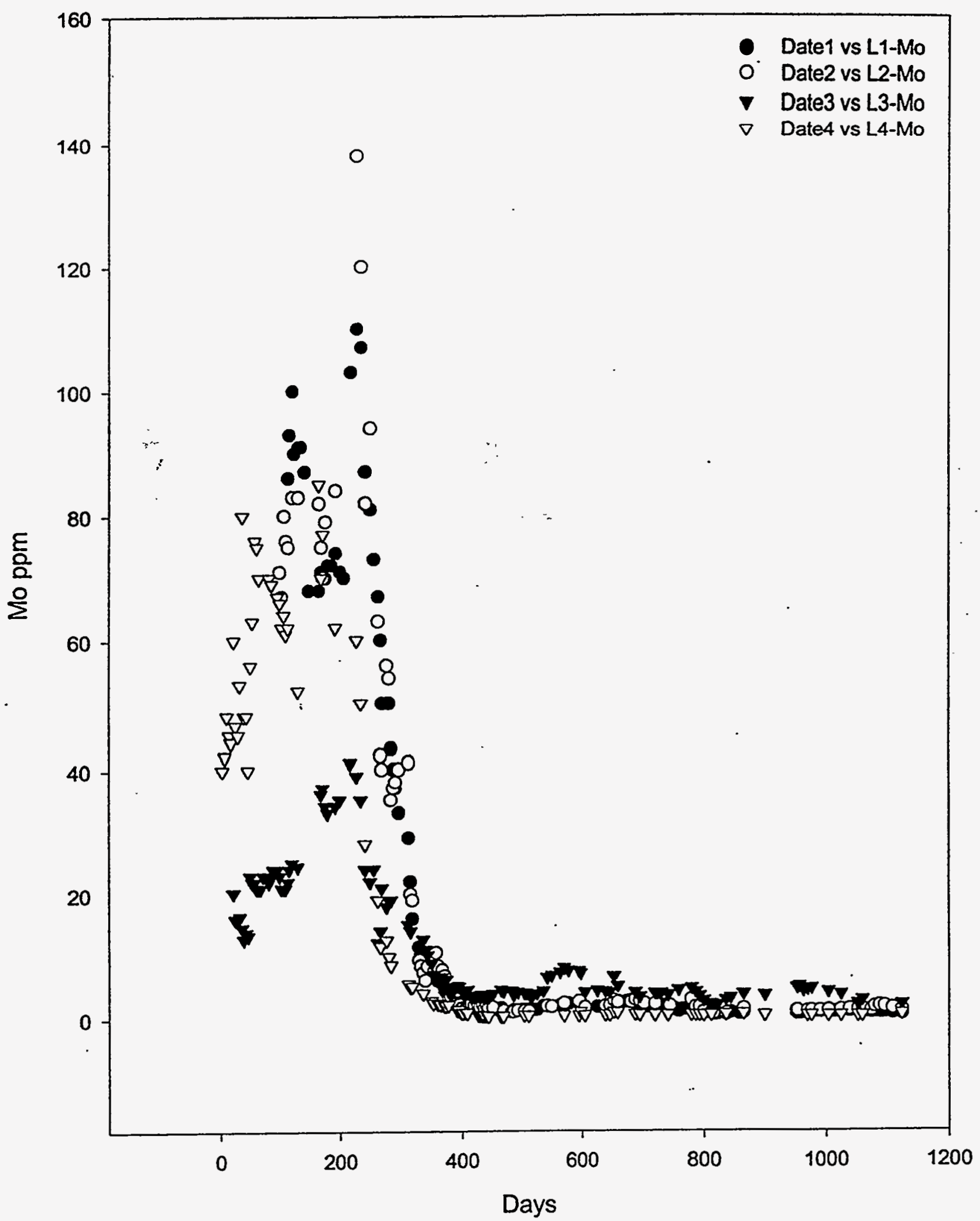

Figure 5-8. Mo concentration as ppm in the leachates from the field lysimeters. Data as in Figure 5-1. 
$V$ in Leachates

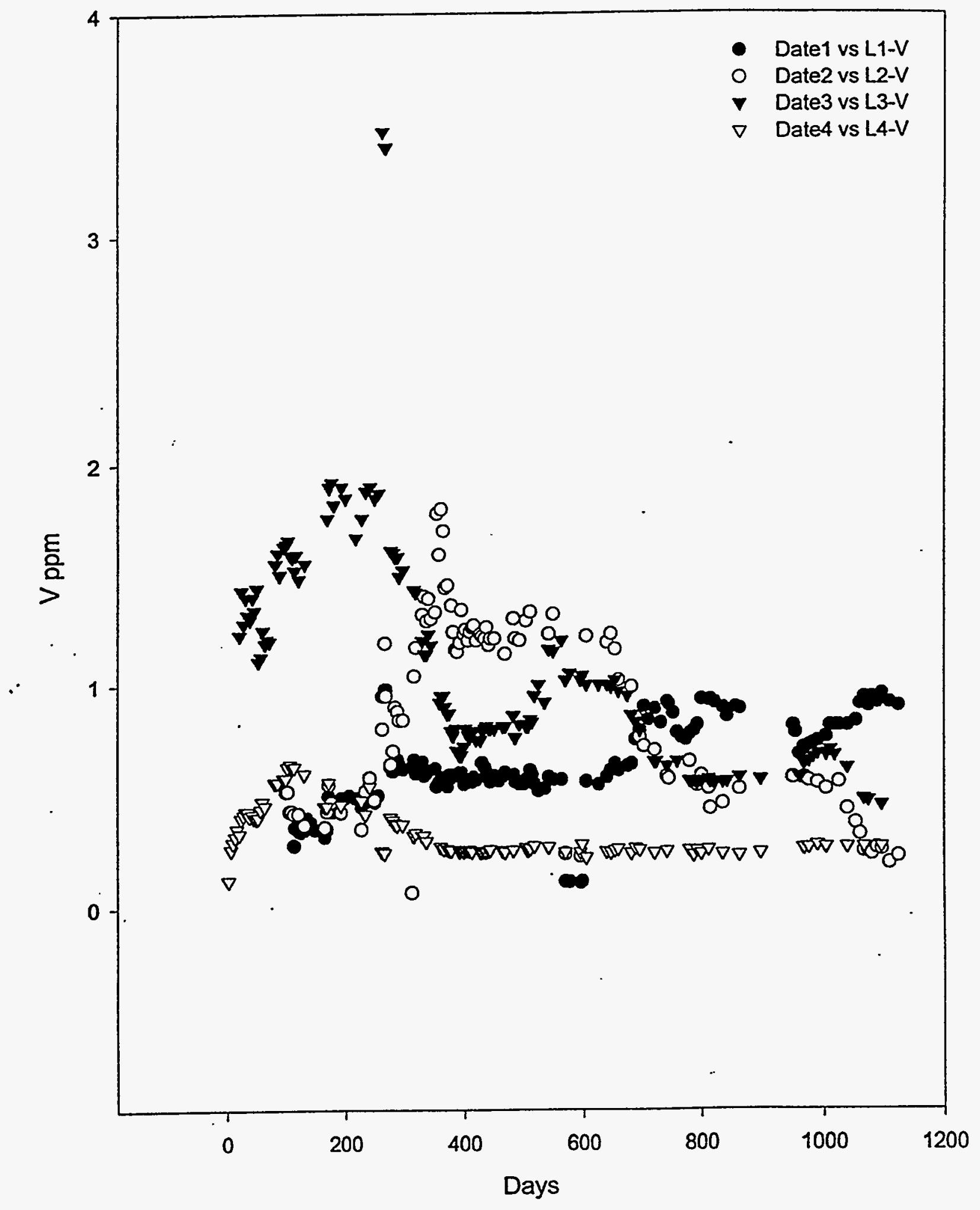

Figure 5-9. $V$ concentration as ppm in the leachates from the field lysimeters.

Data as in Figure 5-1. 


\section{$B$ in Leachates}

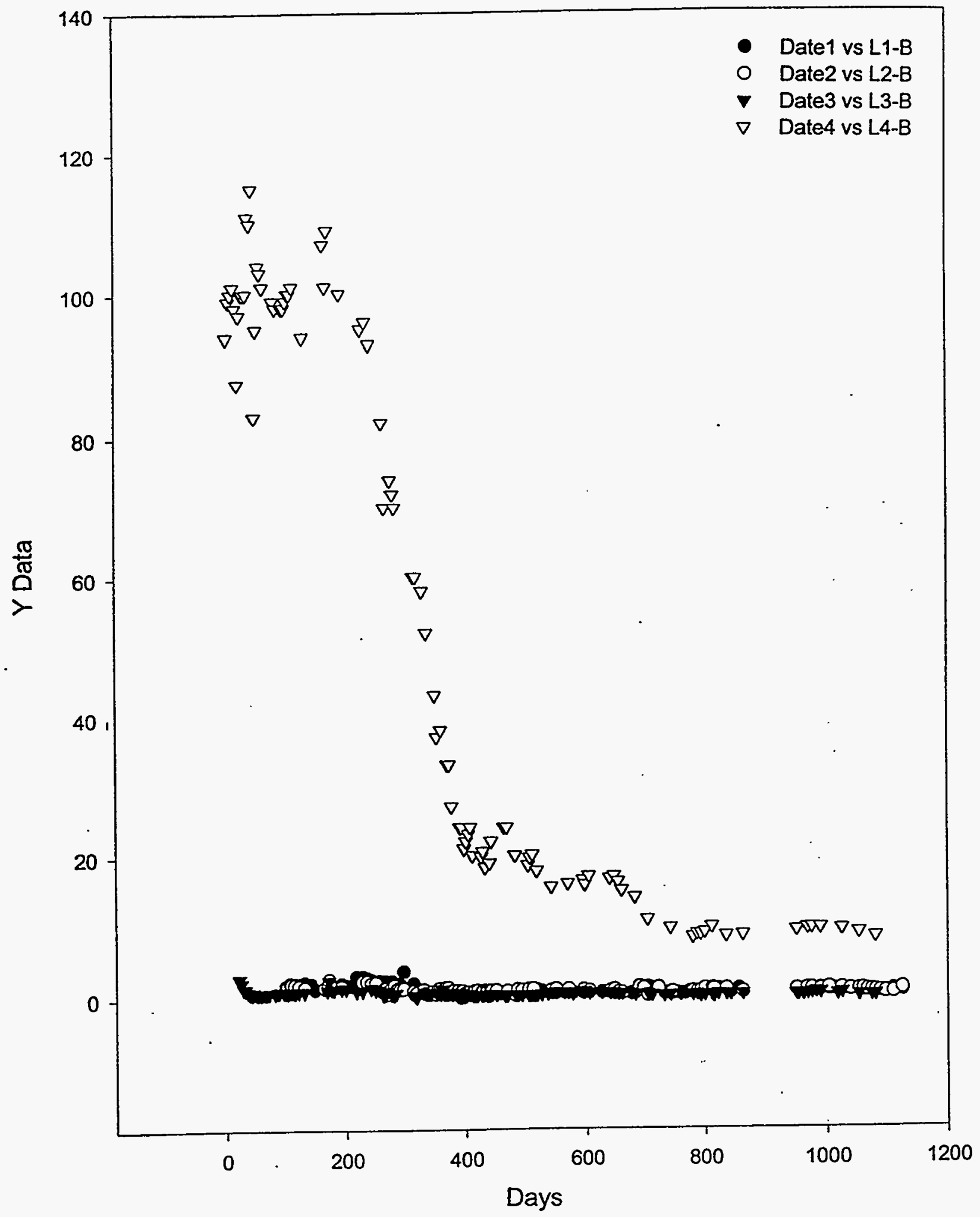

Figure 5-10. B concentration as ppm in the leachates from the field lysimeters. Data as in Figure 5-1. 


\section{$M g$ in Leachates}

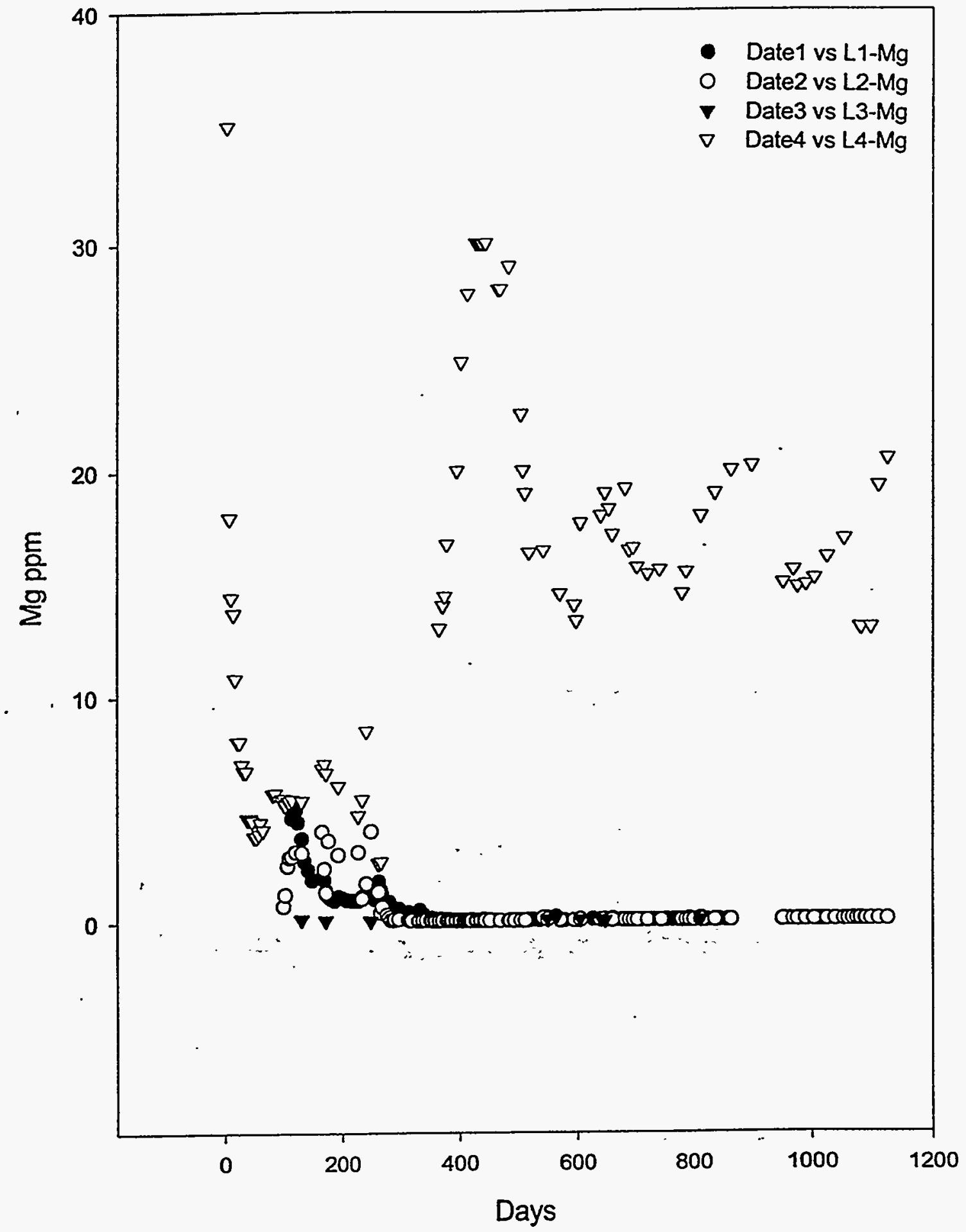

Figure 5-11. Mg concentration as ppm in the leachates from the field lysimeters.

Data as in Figure 5-1. 
As in Leachates

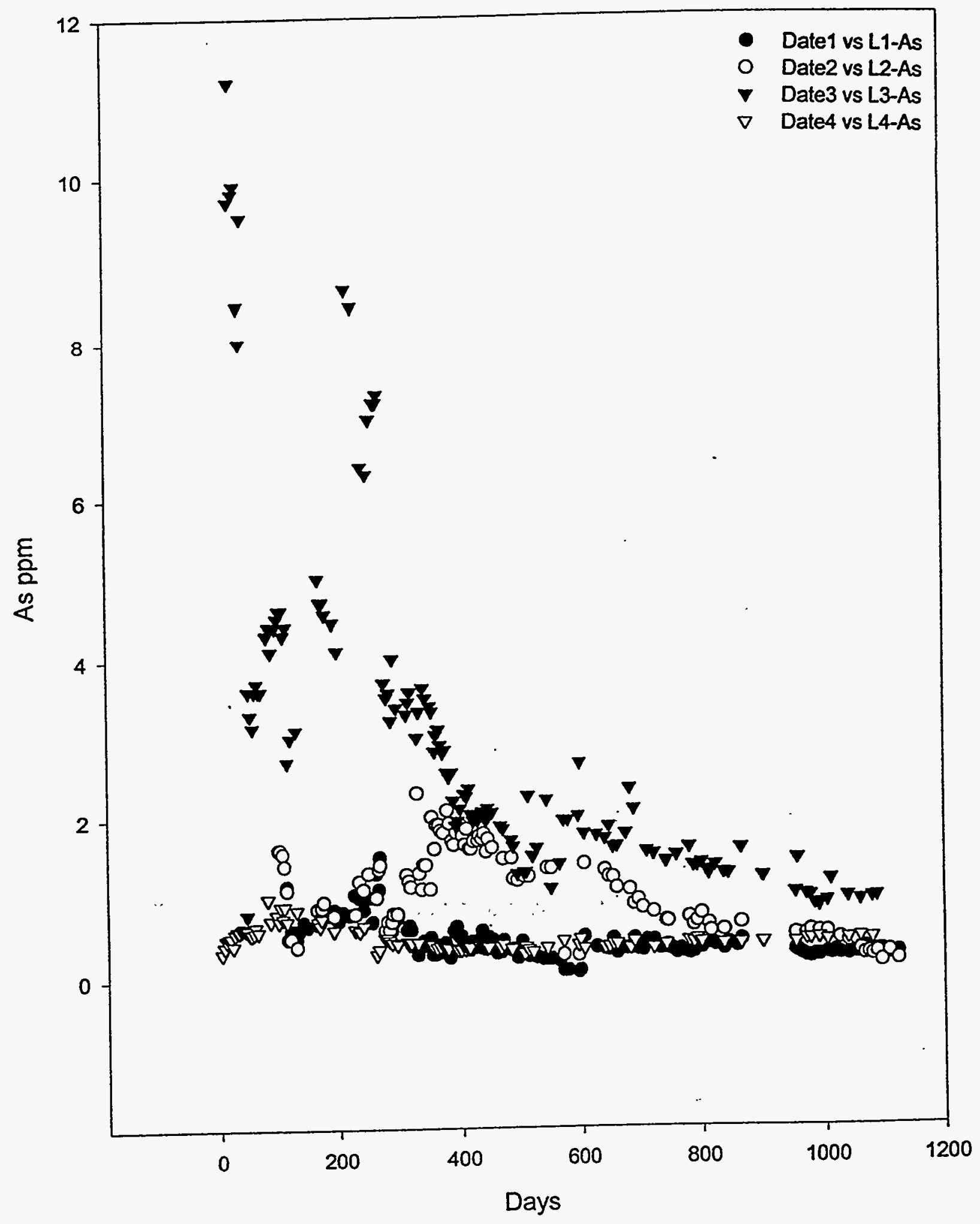

Figure 5-12. As concentration as ppm in the leachates from the field lysimeters. Data as in Figure 5-1. 
concentration of $11.2 \mathrm{ppm}$ in L3-3 and $19 \mathrm{ppm}$ in L3-5. The Se concentration for the L1-3, L2-3 and L3-4 leachates during the first year of the study (Figure 5-13) averaged $2.44 \mathrm{ppm}, 1.85 \mathrm{ppm}$ and $1.09 \mathrm{ppm}$, respectively, well above the RCRA limit. These elements did not exceed the RCRA limits in the TCLP extractions (see Chapter 4).

Carbon Dioxide. After the first few months of the study, grasses and other plants established themselves on the top of the lysimeters. The plants were entirely volunteers, no attempt was made to either seed or fertilize the soil capping the lysimeters. There was considerable variation in plant density and, therefore, rate of respiration, as the best growth was found on the lysimeters with the Coolside material, most notable L1 and L2. Lysimeter L4 had the sparsest growth. Soil gas monitoring began in April 1994 and lasted to the end of the project.

Carbon dioxide concentrations varied throughout the year as a function of time and depth of collection. In general, the highest concentrations were reached during the summer and the lowest during mid-winter (e.g. Figure 5-14). "This is a function of respiration. Plants generally conduct photosynthesis in their leaves and respire in their roots, e.g.:

1)

$$
\mathrm{CH}_{2} \mathrm{O}+\mathrm{O}_{2}=\mathrm{CO}_{2}+\mathrm{H}_{2} \mathrm{O}
$$

Also contributing to $\mathrm{CO}_{2}$ in the soil zone is bacterial respiration.

The concentration of $\mathrm{CO}_{2}$ in the soil zone typically greatly exceeds that of the atmosphere. For example, the highest reading recorded in the study, from the $18^{\text {" level }}$ of $L 2$, was $3.2 \%(32,000 \mathrm{ppm})$. Typical atmospheric concentration in the study area is $\sim 350 \mathrm{ppm}(0.035 \%)$, thus soil activity increased the level of $\mathrm{CO}_{2}$ by two orders of magnitude. The amount of $\mathrm{CO}_{2}$ in the soil is also a function of its defusivity which is related to permeability. In general, $\mathrm{CO}_{2}$ concentrations increase with soil depth. The profiles are typified by that of $L 4$. The profiles for $L 1$ through $L 3$ are not typical. In fact, 
Se in Leachates

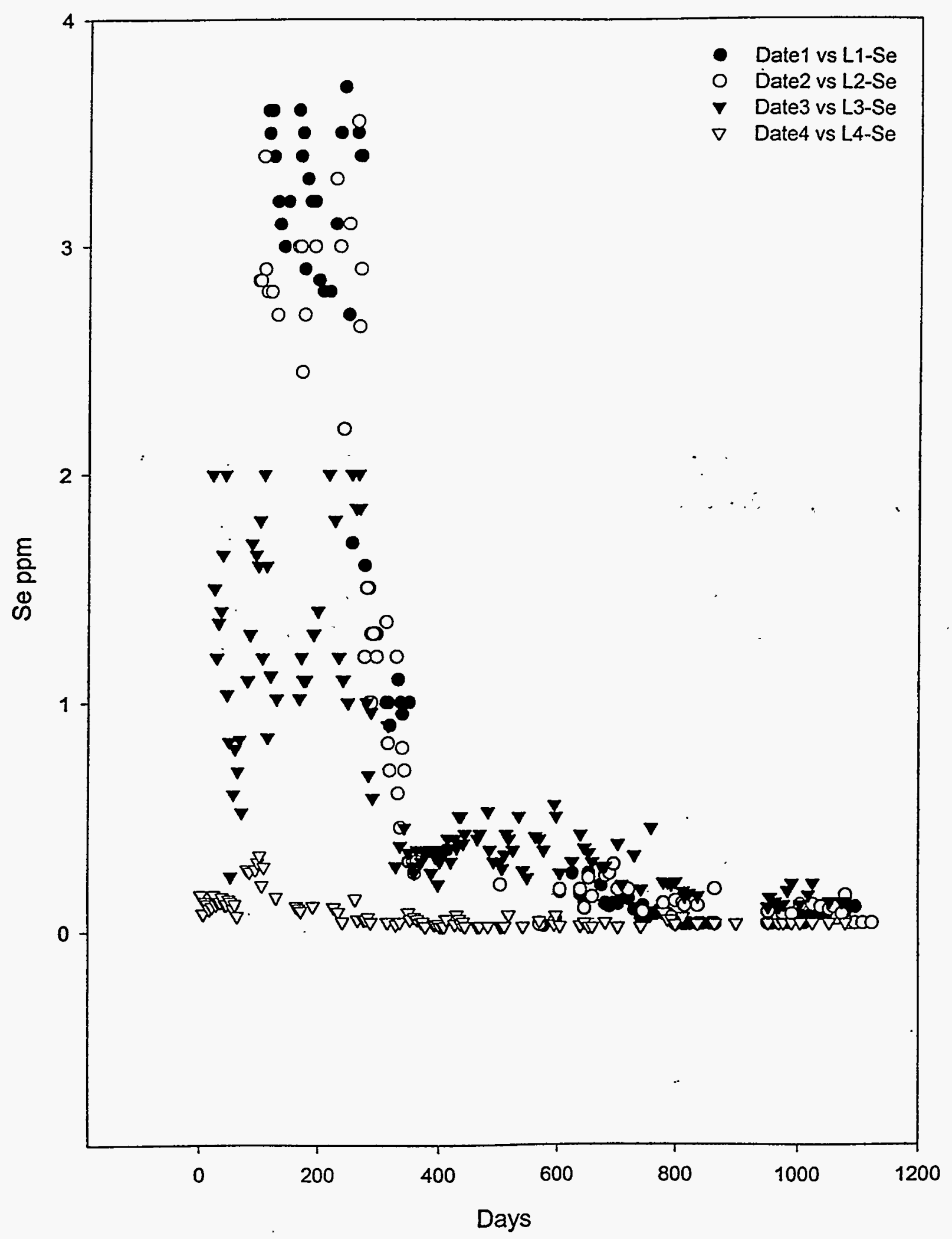

Figure 5-13. Se concentration as ppm in the leachates from the field lysimeters. Data as in Figure 5-1. 


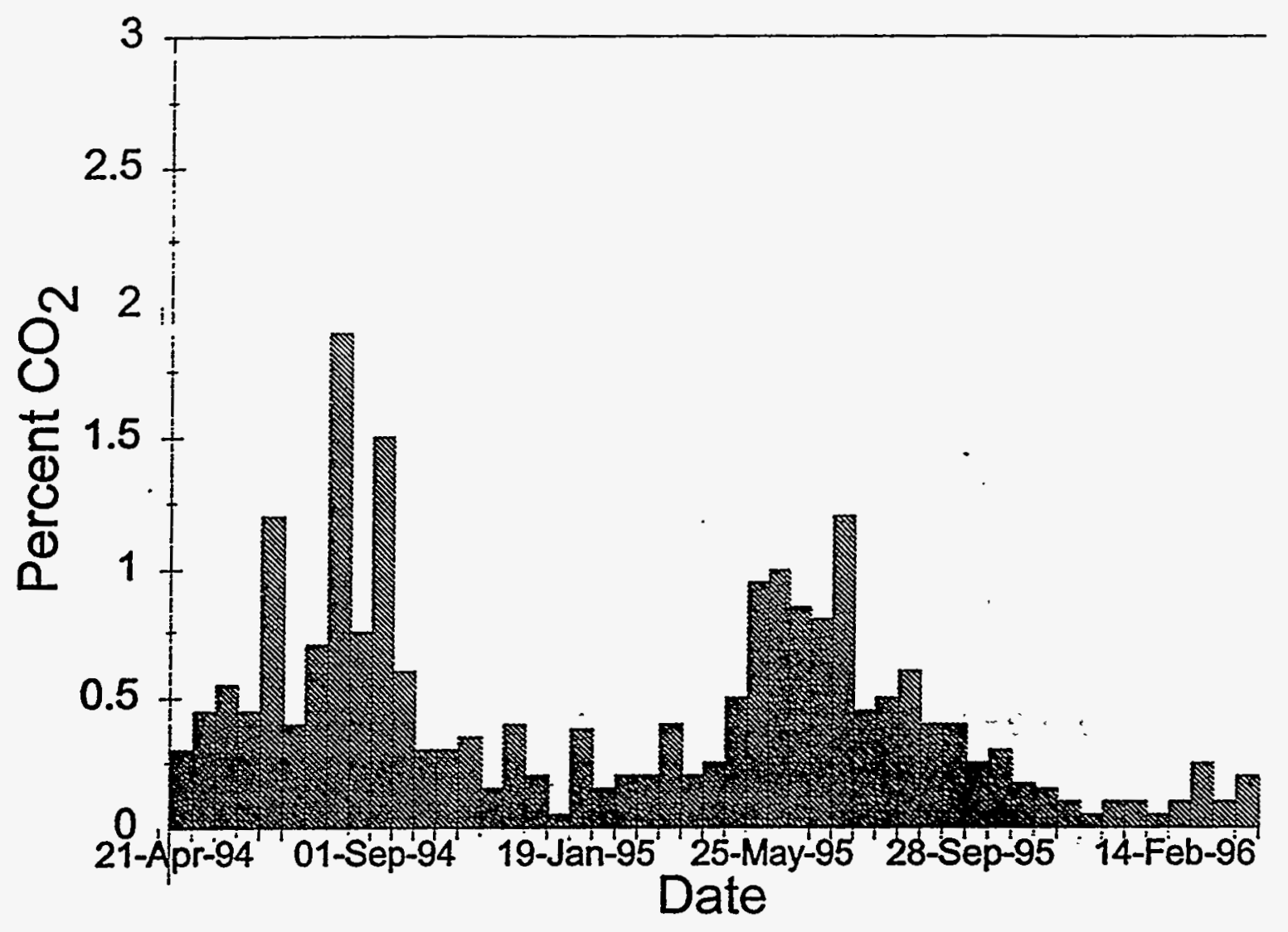

Figure 5-14. $\mathrm{CO}_{2}$ concentration as measured in the gases of the soils overlaying the L1 Lysimeter. Data is from the 8-inch soil gas well. 
the gases within the Coolside material typically were below the detection limit of the equipment employed $(\sim 100 \mathrm{ppm})$, well below atmospheric $\mathrm{CO}_{2}$. This is clear and conclusive evidence of how reactive the Coolside materials are with respect to $\mathrm{CO}_{2}$.

\section{Factors Affecting Leachate Chemistry}

Early Reactions. As discussed in other chapters of this report, the Coolside materials are highly reactive and undergo extensive physical and chemical changes during and after emplacement. The principal early changes include completion of hydration reactions, the partial solution of portlandite,

2) $\mathrm{Ca}(\mathrm{HO})_{2}=\mathrm{Ca}^{+2}+2 \mathrm{OH}^{-}$

the partial solution and oxidation of hannebachite

3) $2 \mathrm{CaSO}_{3} \cdot 1 / 2 \mathrm{H}_{2} \mathrm{O}+\mathrm{O}_{2}=2 \mathrm{Ca}^{+2}+2 \mathrm{SO}_{4}^{-2}+\mathrm{H}_{2} \mathrm{O}$

and the formation of ettringite:

4)

$$
6 \mathrm{Ca}^{+2}+2 \mathrm{Al}^{+3}+3 \mathrm{SO}_{4}^{-2}+12 \mathrm{OH}+26 \mathrm{H}_{2} \mathrm{O}=\mathrm{Ca}_{6} \mathrm{Al}_{2}\left(\mathrm{SO}_{4}\right)_{3} \mathrm{OH}_{12} \cdot 26 \mathrm{H}_{2} \mathrm{O}
$$

Ettringite is the principal cementitious mineral formed and is responsible for the high strength of the materials, particularly that of the L3 lysimeter. Ettringite is found in all of the lysimeters as well as all of the lab test columns.

Longer Term Weathering Reactions. Weathering begins upon the first infiltration of meteoric water. The most important factor in the early weather is the rapid solution of the most readily soluble components, particularly $\mathrm{Na}$ and $\mathrm{Cl}$, resulting in brine-like solutions which are very high in ionic strength. High ionic strengths enhance the solution's ability to dissolve additional ionic species. For example, an equilibrium 
Ca in Leachates

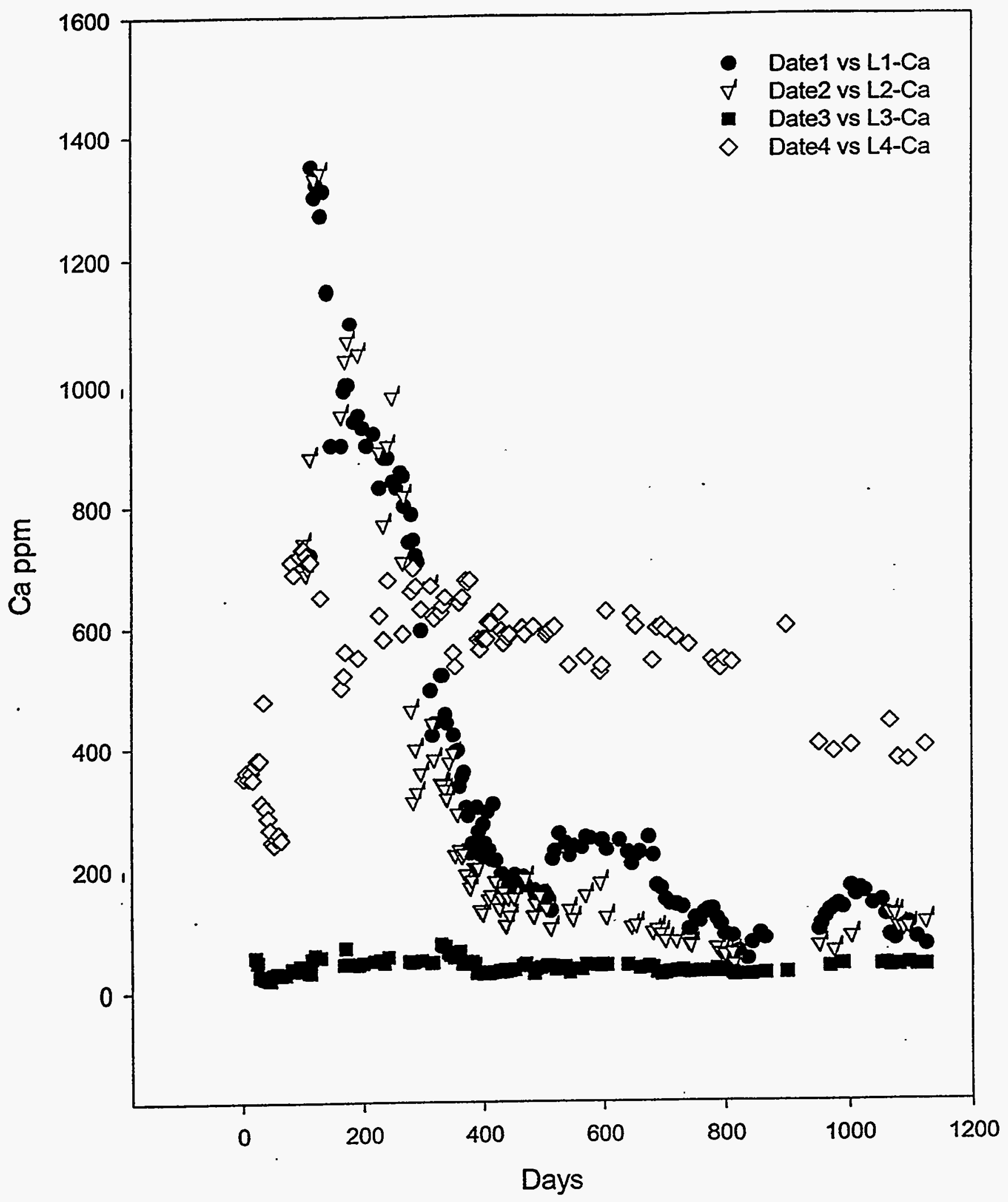

Figure 5-15. Calcium concentration as ppm in the leachates from the field lysimeter. Data as in Figure 1. 


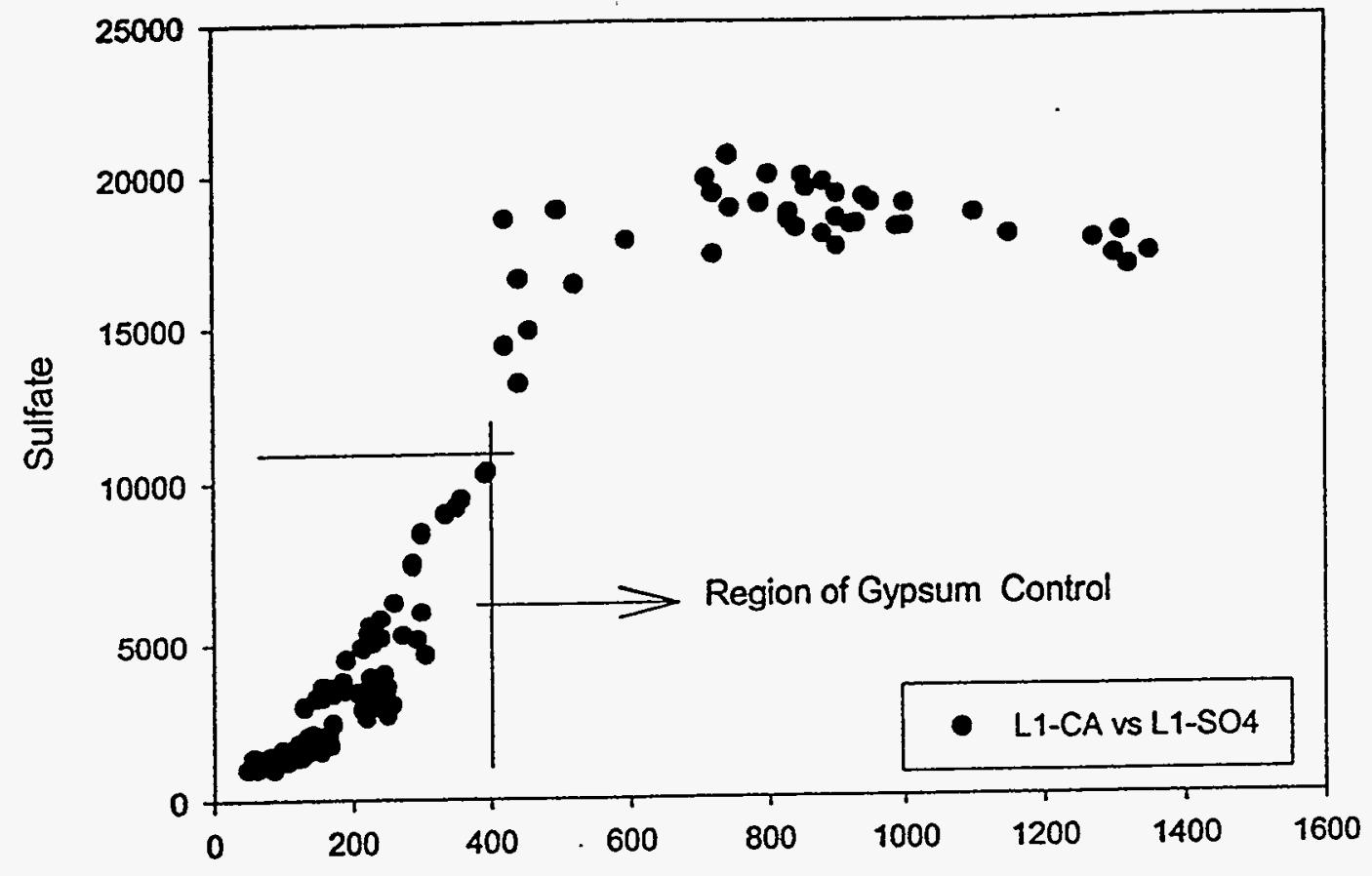

Ca ppm

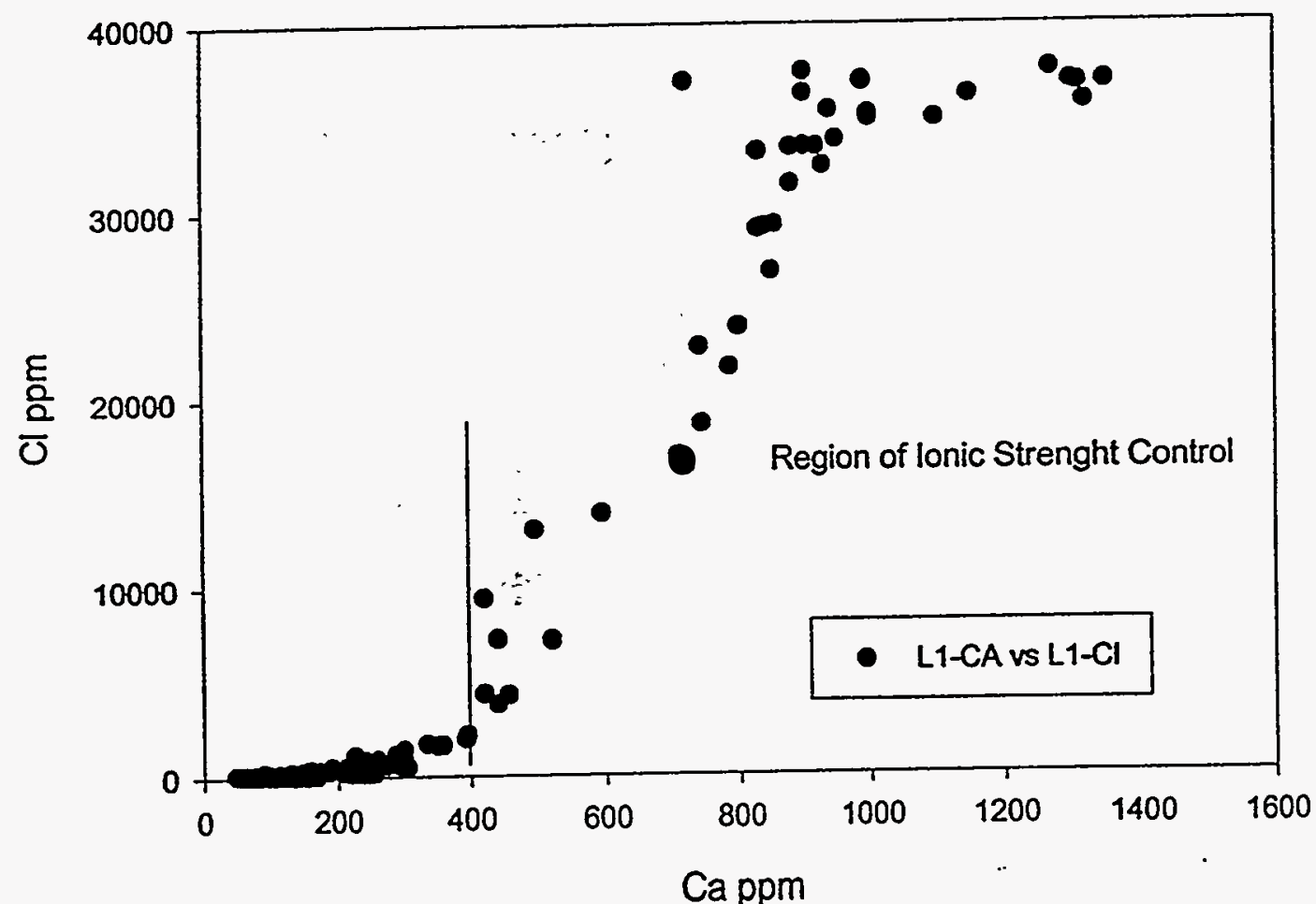

Figure 5-16. Plot of $\mathrm{Ca}$ versus Sulfate and $\mathrm{Cl}$ for $\mathrm{L} 1-3$. The plots illustrate the influence of gypsum dissolution and ionic strength on $\mathrm{Ca}$ in solution. 
solution of gypsum contains $1,480 \mathrm{ppm}$ of $\mathrm{SO}_{4}^{-2}$. One with $2,500 \mathrm{ppm}$ of dissolved $\mathrm{NaCl}$ will contain $1,800 \mathrm{ppm} \mathrm{SO}_{4}^{-2}$. This is illustrated in Figures 5-15 and 5-16 which suggests a strong influence on $\mathrm{Ca}$ concentration in the high ionic strength region. This correlation changes as ionic strength decreases to one of $\mathrm{Ca}$ and sulfate, as would be expected from the thermodynamic data.

As noted above, the absorption of $\mathrm{CO}_{2}$ is important. At pH's above $\sim 10, \mathrm{CO}_{2}$ reacts directly with hydroxide and forms a carbonate ion,

5) $\mathrm{CO}_{2}+2 \mathrm{OH}^{-}=\mathrm{HCO}_{3}^{-}=\mathrm{H}^{+} \mathrm{CO}_{3}^{-2}$

We know from the thermodynamic analysis as presented in Chapter 6 of this report that the leachates are all supersaturated with respect to calcite and undersaturated with respect to gypsum and hannebachite. Thus the materials are both dissolving and precipitating minerals simultaneously. The carbonation of the hannebachite to calcite may be summarized as follows:

6) $\mathrm{CaSO}_{3 .} 1 / 2 \mathrm{H}_{2} \mathrm{O}+1 / 2 \mathrm{O}_{2}+\mathrm{CO}_{3}^{-2}=\mathrm{CaCO}_{3}+\mathrm{SO}_{4}^{-2}+1 / 2 \mathrm{H}_{2} \mathrm{O}$

The mineralogical and thermodynamic data all indicate that in the longer term, the most stable set of minerals in the longer term are calcite quartz and ettringite. Both the mineralogical and thermodynamic data indicate that ettringite will also break down over time. However this does not appear to proceed until the sulfate is largely exhausted. Thus, in effect, the sulfate acts as a buffer to extend the stability of the ettringite.

Material Compaction and Leachate Chemistry. One of the more interesting findings of the study is the strong effect of compaction on the chemistry of the leachates. The L1 and L2 leachates have considerably higher elemental concentrations, higher sulfate and lower alkalinity than the L3 leachates. 
We believe that this is largely a function of ettringite formation. The higher compaction allowed the more extensive formation of ettringite, which tied up the available sulfate in unreactive mineral form. Thus, instead of sulfate carbonation, the reaction with portlandite is more significant, or

$$
\mathrm{Ca}\left(\mathrm{OH}_{2}\right)+\mathrm{CO}_{3}^{-2}=\mathrm{CaCO}_{3}+2 \mathrm{OH}^{-}
$$

This contributes to the significantly higher alkalinity from $\mathrm{L} 3$ and also the higher $\mathrm{pH}$. The leachates from L1 and L2 eventually increase in both $\mathrm{pH}$ and alkalinity as the amount of readily available sulfate (or sulfite) declines. The greater amount of ettringite also decreases pore volume, reduces percolation, and increases strength. This reduces the rate of weathering, resulting in the slower release of soluble salts.

\section{Summary}

The leachates from the Coolside material were found to be initially highly alkaline and contained high concentrations of dissolved ions. During the three years of the study the elemental concentration in leachates declined by factors of three to more than an order of magnitude. Initially, the overall leachate chemistry was controlled by the rapid dissolution of highly soluble salts and the formation ettringite. Later, the carbonation of calcium sulfite and calcium hydroxide played important roles.

Overall the Coolside material had significantly higher concentrations of $\mathrm{Na}, \mathrm{Cl}$, sulfate, $\mathrm{Ca}, \mathrm{K}$, alkalinity, $\mathrm{Al}, \mathrm{As}, \mathrm{Se}, \mathrm{Mo}, \mathrm{Si}, \mathrm{Ti}$ and $\mathrm{V}$ in its leachates compared to PCC Class F fly ash used as a control. The Coolside material also had lower $\mathrm{B}, \mathrm{Ba}, \mathrm{Cr}$ and $\mathrm{Mg}$ concentrations compared to the fly ash.

Compacting the material resulted in higher alkalinity and $\mathrm{pH}$ and a significantly lower rate of release for highly soluble salts. It also appeared to greatly enhance material's strength and improve its resistance to weathering. 
Elements of environmental concern which were found in concentration which exceeded RCRA limits included Se and As. Some of the leachates from the L3 lysimeter exceeded $5 \mathrm{ppm}$ As during the first year of the study. The average concentration of Se from all of the leachates from the Coolside material exceeded the limit for Se (1 ppm). 


\title{
CHAPTER 6. THERMODYNAMIC DATA BASE
}

\author{
Introduction
}

The data obtained from the lysimeters include $\mathrm{pH}$, conductivity and ionic concentrations. This solute data must be manipulated to infer chemical species concentrations and the mineral phases present in the leaching environment. For this a public domain program WATEQ4F ${ }^{1}$ was selected. This program, written in Fortran, expects a single input file and produces a single output file.

Given the large amount of data collected in lysimeter experiments and the difficulty of extracting relevant data from the volumnious output provided by this program, the original program was converted from Fortran to $C$ and tested in DOS using WATEQ4F test case one, modified to run under Windows 3.0/3.1 with menu items and input screens, using Borland $\mathrm{C}++\mathrm{V} 3.1$ and debugged with all four test cases. Batch data input via Windows clipboard, and output of user-selected data to either commadelimited files or Windows clipboard was implemented. Options were added to allow saving, reloading or editing input preferences (input species, redox options, input units, and alkalinity options), output preferences (convergence criteria, triggers for species percentage and mineral equilibrium departure for inclusion in output, whether or not to include a thermodynamics table and/or weight/molar concentration ratios in the output), and paste preferences (selection of input and individual output items for inclusion in a "cut/paste" process). The resulting program is referred to as WATEQ, to differentiate it from the $4 \mathrm{~F}$ (Fortran) version.

${ }^{1}$ WATEQ4F with Revised Thermodynamic Data Base and Test Cases for Calculating Speciation of Major, Trace, and Redox Elements in Natural Waters. USGS Open File Report 91-183. James W. Ball and D. Kirk Nordstrom 
The WATEQ program attempts to infer theoretically, given $\mathrm{pH}$ and ionic concentrations of water-borne ions, the active chemical species and approach to saturation of multiple mineral entities of the water. It measures mineral approach to equilibrium by comparing the products of the activities of the constituent ions (Ion Activity Product or IAP) with the equilibrium solubility product $\left(K_{\text {sp }}\right)$ : specifically $\log \left(\operatorname{IAP} / K_{1}\right)$, where $K$ is corrected for temperature. A value greater than zero indicates supersaturation. Individual ion activities are the product of the ion concentration and an activity coefficient which attempts to correct for the ionic strength of the solute. The concentrations are determined by an iterative mass and charge balance over the possible species present given the analytical input data of concentrations and $\mathrm{pH}$.

\section{Caveats to Computation}

There are enough sources of error inherent in these calculations that measures of approach to equilibrium must not be viewed as absolute indicators of the presence or absence of a particular mineral, or that any equilibrium has been reached. One source of error is the equilibrium solubility product, $K$, for each mineral. The range of solubilities depend on the degree of crystallinity, particle size effects, order-disorder phenonema, defect structures, range of solid solutions, interlayering and meta-stability. For instance, the Calcite $\left(\mathrm{CaCO}_{3}\right)$ group consists of Calcite plus the substitution in varying amounts of $\mathrm{Mg}, \mathrm{Mn}, \mathrm{Zn}, \mathrm{Fe}$, and $\mathrm{Cd}$ in the matrix for $\mathrm{Ca}$, depending on availability. Thus, formation of Calcite may also include Magnesite $\left(\mathrm{MgCO}_{3}\right)$, Rhodocrosite $\left(\mathrm{MnCO}_{3}\right)$, Smithsonite $\left(\mathrm{ZnCO}_{3}\right)$, Siderite $\left(\mathrm{FeCO}_{3}\right)$ and Otavite $\left(\mathrm{CdCO}_{3}\right)$ as part of the solid solution. These individually do not have equal solubilities. At primary saturation, or thermodynamic equilibrium, the aqueous phase will be undersaturated with respect to the solid components. Conversely, a high degree of supersaturation of the most soluble components will exist at stoichiometric saturation. Other examples exhibiting this behavior include Quartz, Gibbsite, Kaolinite, Sepiolite, Oxides and 
Hydroxides, Feldspars and Micas. Additionally, the solid phase initially formed may be meta-stable with respect to the thermodynamically preferred state. For example, Calcite is thermodynamically preferred over Aragonite, but Aragonite may form initially as a very fine solid phase with a disordered lattice and slowly convert to the more stable, relatively inactive, less soluble Calcite form. Silica $\left(\mathrm{SiO}_{2}\right)$ forms, Anhydrite/Gypsum and Hydroxides also exhibit this behavior.

Another source of error is the determination of the ion activity coefficient over large ranges of ionic strength. In very dilute solutions, the activity coefficient is 1 , so that the ion activity is equal to the molar concentration. As ionic strength increases, the coefficient first increases. This increase in ion activity drives the equilibrium toward formation of the neutral species, resulting in a "salting out" of the solid phase. As the ionic strength increases toward and past one, the activity coefficient may drop below one due to greater ionic association in the solute. The solid phase "sees" less ionic activity and the equilibrium is driven towards dissolution of the solid phase. For ionic strengths above about 0.5 , the activity coefficients become increasingly uncertain. The ionic strengths encountered in the Coolside leachate range from $\sim 0.01$ to 3.5 . For comparison, the effective ionic strength of a free flowing stream is $\sim 0.01$, groundwater $\sim 0.1$, seawater $\sim 1$. lonic strength also affects alkalinity. WATEQ estimates available carbonate by correcting measured alkalinity for non-carbonate alkalinity, primarily $\mathrm{OH}^{-}$. It is also known that other species, notably silicate forms, introduce errors in measured alkalinity. Despite these uncertainties in solubility constants and ion activity products, the equilibrium calculations performed are useful as an aid in interpreting observed fact.

Many mineral forms addressed by WATEQ show regions of supersaturation but do not control leachate chemistry and are unlikely to be formed in any but the most minor amounts. Albite is a Sodium feldspar. Its saturation index generally has an inverse 
relation with $\mathrm{pH}$ (Figures 6-13 through 6-15). Diopside is a Ca-Mg pyroxene which follows $\mathrm{pH}$ (Figures 6-16 through 6-18). These are the most common behaviors seen. The mineral forms which must be suspected of having control of the leachate chemistry are those whose saturation indices remain not far from zero and do not show wild variability, such as Calcite, Diaspore, Gypsum and Silica (Figures 6-22 and 6-23).

\section{Preliminary Investigations}

As an initial premise, it was assumed that, regardless of lysimeter contents and degree of compaction, $\mathrm{pH}$ will be the controlling parameter affecting leachate species and lysimeter mineralization. If so, then the approach to saturation of various minerais should vary smoothly with $\mathrm{pH}$, except for errors derived from inherent inaccuracies in test results; any departure from this assumption indicates that there are, indeed, differences in the lysimeters and their leachates. To test this, WATEQ runs were made on a composite of individual collections of various initial column and field results which gave a total $\mathrm{pH}$ range of 13.13 to 8.00 . While $\mathrm{pH}$ trends were evident, the result indicated there are lysimeter differences.

A second preliminary investigation involved selecting mineral phases approaching saturation within five orders of magnitude ( $\log [\mathrm{IAP} / \mathrm{kT}]>-5)$ ). 108 of the 322 mineral forms treated by WATEQ met this criteria. Plots of saturation behavior over time were produced. Based upon these plots, the minerals were partitioned into groups exhibiting like behavior for both initial laboratory column 25 (Coolside compressed pellets, wet/dry with average $\mathrm{pH}$ about 11.5, rising slightly) and field lysimeter 3, level 3 (Edgewater Coolside, 66\#/ft $@ 37 \% \mathrm{H}_{2} \mathrm{O}$ with average pH about 12.25, falling slightly) in order to compare saturation behavior. While the partitioning remains valid, saturation behavior is different, again indicating differences in the lysimeters and their resulting leachate. 
The third preliminary investigation compared the behavior of selected species with concentrations greater than one ppm in the same two lysimeters. The results also show differences in the lysimeters.

The results of the preliminary investigation, therefore, indicated that lysimeter contents, compaction and $\mathrm{CO}_{2}$ loading may all affect leachate chemistry.

\section{Selection of Primary Mineral Forms and lonic Species and Results}

Note: Due to the volume of data, graphical illustrations will be restricted to the field lysimeter results in most instances. As the different sample levels of the field lysimeters generally do not show major differences, only one representative level will normally be shown. In addition, as field lysimeters 1 and 2 (the least compacted) are very similar thermodynamically, only one will usually be illustrated.

The initial group of mineral forms and ionic species were further investigated to select those which may drive leachate chemistry, are driven by the chemistry and may be present in significant amounts, or are the predominant carriers of minor ions of interest. Thirty-three aqueous species and seventy-one mineral species were selected for final analysis of the lysimeters based on the following reasoning.

\section{Ionic Species}

The major components of coal combustion waste are normally oxides of $\mathrm{Mg}, \mathrm{Na}, \mathrm{Fe}, \mathrm{Ti}$, $\mathrm{Si}, \mathrm{Ca}, \mathrm{K}, \mathrm{P}$, and $\mathrm{Al}$. In addition there are significant amounts of $\mathrm{Cl}$ and $\mathrm{SO}_{3}$. There are trace amounts of other metals such as $\mathrm{Zn}, \mathrm{Cu}, \mathrm{Ni}, \mathrm{Co}, \mathrm{Cr}, \mathrm{Ba}, \mathrm{V}, \mathrm{As}, \mathrm{B}, \mathrm{V}, \mathrm{Mn}, \mathrm{Sr}, \mathrm{Zr}$ and $\mathrm{Cd}$. The Coolside sulfur control regime results in the addition of Anhydrite $\left(\mathrm{CaSO}_{4}\right)$ / Gypsum $\left(\mathrm{CaSO}_{4}{ }^{\star} 2 \mathrm{H}_{2} \mathrm{O}\right)$, Portlandite $\left(\mathrm{Ca}(\mathrm{OH})_{2}\right)$, Dolomite $\left(\mathrm{CaMg}\left(\mathrm{CO}_{3}\right)_{2}\right)$ and, in some cases, Na. Co, Cr, Mo, Ti, Zr, V, B and Se are given little or no treatment in WATEQ, 
so will not be considered further. Upon leaching, it would be expected that hydrolysis would result in the release of the metal ion and $\mathrm{OH}^{-}$into solution, resulting in a highly alkaline solute. Free trivalent ions, such as those of $\mathrm{Al}, \mathrm{Fe}$ and $\mathrm{Mn}$, are not expected to be seen in large amounts, as they tend to form under conditions of low ionic strengths and high acidity. With the exception of $\mathrm{Al}^{+3}$, they tend to form insoluble hydroxides. Therefore, $\mathrm{Al}(\mathrm{OH})_{3}{ }^{\circ}$ and $\mathrm{Al}(\mathrm{OH})_{4}{ }^{-}$are expected, as are divalent $\mathrm{Ca}, \mathrm{Mg}, \mathrm{Zn}, \mathrm{Cu}$ and $\mathrm{Ba}$, monovalent $\mathrm{Na}$ and $\mathrm{K}$ ions, and $\mathrm{OH}$. The leaching water is generally in equilibrium with a $\mathrm{CO}_{2}$ source. This source may be the atmosphere or soil. The partial pressure of $\mathrm{CO}_{2}\left(\mathrm{pCO}_{2}\right)$ in water in equilibrium with the atmosphere is approximately $10^{-3.5}$. The $\mathrm{pCO}_{2}$ in soil may be much higher due to organic respiration. It is expected that leaching will introduce significant amounts of $\mathrm{CO}_{2}$ into the solute. The steps in $\mathrm{CO}_{2}$ hydration are

$$
\begin{aligned}
& \text { 1: } \mathrm{CO}_{2}+\mathrm{H}_{2} \mathrm{O}=\mathrm{H}_{2} \mathrm{CO}_{3}^{0} \\
& \text { 2: } \mathrm{H}_{2} \mathrm{CO}_{3}^{0}+\mathrm{H}_{2} \mathrm{O}=\mathrm{HCO}_{3}^{-}+\mathrm{H}_{3} \mathrm{O}^{+} \\
& \text {3: } \mathrm{CO}_{2}+\mathrm{OH}^{-}=\mathrm{HCO}_{3}^{-}
\end{aligned}
$$

Step 2 is rapid at room temperature while steps 1 and 3 are slow. At higher $\mathrm{pH}$, however, excess hydroxide increases the relative importance of step 3 . Therefore, $\mathrm{H}_{2} \mathrm{CO}_{3}{ }^{0} \mathrm{HCO}_{3}^{-}$and $\mathrm{CO}_{3}{ }^{2-}$ are expected. Quartz $\left(\mathrm{SiO}_{2}\right)$ is slowly acted upon by alkali at room temperature, with long equilibrium time. This reaction rate increases rapidly above $\mathrm{pH} 9$ due to ionization of silicic acid $\left(\mathrm{H}_{4} \mathrm{SiO}_{4}\right.$ or $\left.\mathrm{Si}(\mathrm{OH})_{4}\right)$ and formation of monomeric or multimeric domains, so that $\mathrm{Si}(\mathrm{OH})_{4}{ }^{0}$ and $\mathrm{SiO}(\mathrm{OH})_{3}{ }^{-}$are expected. $\mathrm{SO}_{4}{ }^{2-}$ and $\mathrm{HSO}_{4}{ }^{-}$are expected to be available from the dissolution of Anhydrite/Gypsum.

Based on these arguments, the following solute species were initially selected for analysis:

$\mathrm{CO}_{2}$ total, $\mathrm{Al}(\mathrm{OH})_{4}{ }^{\circ}, \mathrm{Ca}, \mathrm{Cl}, \mathrm{CO}_{3}{ }^{2}, \mathrm{HCO}_{3}{ }^{-}, \mathrm{Na}, \mathrm{OH}^{-}, \mathrm{PO}_{4}{ }^{2-}, \mathrm{SiO}_{2}{ }^{0}, \mathrm{SO}_{4}{ }^{2-}, \mathrm{Zn}, \mathrm{Al}, \mathrm{H}, \mathrm{K}, \mathrm{Mn}$, 


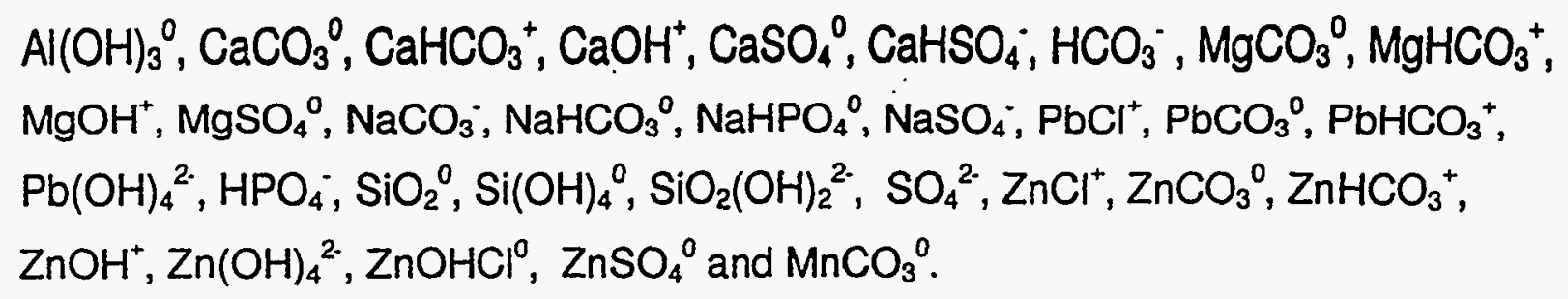

After further investigation, all $\mathrm{Pb}, \mathrm{Zn}$ and $\mathrm{PO}_{4}$ forms were dropped due to very low concentrations and lack of effect on leachate chemistry. In addition, the following items were also selected:

$\mathrm{pH},-\log \left(\mathrm{pCO}_{2}\right)$, Effective lonic Strength, $\mathrm{aH}_{2} \mathrm{O}$ (activity of water), $-\log (\mathrm{Ca}$ activity) and $\log$ (Mg activity).

\section{Mineral Forms}

Given the nature of Coolside waste, the characteristics of the oxides are of immediate importance. Of greatest interest are those present in large amounts: those of $\mathrm{Ca}, \mathrm{Al}$, $\mathrm{Fe}, \mathrm{K}$, and $\mathrm{Na}$. It is expected that hydrolysis of these oxides will result in free aqueous hydroxides and metal ions, with a resulting high $\mathrm{pH}$. On introduction of atmospheric $\mathrm{CO}_{2}$, metal carbonate precipitation will act to lower $\mathrm{pH}$ to some equilibrium value over some unknown period. The $\mathrm{pH}$ range seen in Coolside leaching roughly corresponds to that of Portlandite $\left(\mathrm{Ca}(\mathrm{OH})_{2}\right)$. in equilibrium with pure water (about 12.45) down to Calcite $\left(\mathrm{CaCO}_{3}\right)$ in equlibrium with water that is in equilibrium with atmospheric $\mathrm{CO}_{2}$ (pH about 8.4 at $\left.\log \left(\mathrm{pCO}_{2}\right)=-3.5\right)$. The dissolution of silica and Gypsum/Anhydrite add the possibility of silicate and sulfate mineral formation.

Silica. The forms of $\mathrm{SiO}_{2}$ possible are Quartz, Cristabolite, Chalcedony and Silica Gel. The higher forms, such as Tridymite would naturally convert to the lower forms over time. XRD data show the presence of Quartz. Cristabolite is formed under high temperature up to melting and persists in metastable form on cooling, with eventual conversion to Quartz. Its highly symmetric, open structure is thermodynamically slightly 
favored over Tridymite and Quartz. Chalcedony is a fibrous silica, often occurring in small residues as crusts or cavity filling. It is readily soluble in alkaline solutions. Silica Gel is a form favored on formation at STP, most soluble, and ages into poorly crystallized Cristabolite. Quartz is most easily recognized by XRD, and is the expected initial form, least soluble. Earlier inspection of initial leachate column indicated that, of the Silica members, Quartz most nearly approaches equilibrium, but usually remains below. If any other form predominated, then Quartz would be supersaturated, as discussed earlier. Its dissolution is expected to supply $\mathrm{SiO}_{2}$ to the solute. Silica formation, if any, would be expected to be of the Silica Gel dimorph. Quartz was chosen as the Silica member of interest. The field lysimeter results indicate that Quartz remains in dissolution under higher $\mathrm{pH}$ conditions, under low $\mathrm{pH}$ conditions in the fly ash lysimeter, and may initially approach equilibrium durng the low pH excursion for those lysimeters which exhibit an initial pH drop. (Figures 6-1 through 6-3)

Na. The initial Sodium mineral forms selected based on approach to equilibrium were Halite $(\mathrm{NaCl})$, Nahcolite $\left(\mathrm{NaHCO}_{3}\right)$, Trona $\left(\mathrm{Na}_{3} \mathrm{H}\left(\mathrm{CO}_{3}\right)_{2}{ }^{\star} 2 \mathrm{H}_{2} \mathrm{O}\right)$, Thermonatrite $\left(\mathrm{Na}_{2} \mathrm{CO}_{3}{ }^{*} \mathrm{H}_{2} \mathrm{O}\right)$, Natron $\left(\mathrm{Na}_{2} \mathrm{CO}_{3}{ }^{*} 10 \mathrm{H}_{2} \mathrm{O}\right)$, Mirabilite $\left(\mathrm{Na}_{2} \mathrm{SO}_{4}{ }^{*} 10 \mathrm{H}_{2} \mathrm{O}\right)$ and Thenardite $\left(\mathrm{Na}_{2} \mathrm{SO}_{4}\right)$. All of these are extremely water soluble, however, and are not expected to be formed. Na may be involved in minor silicate mineral formation mentioned later. It is expected that $\mathrm{Na}$ from oxide dissolution and $\mathrm{Na}$ addition due to Sulfur control regimes will be found as a free ion in the solute and will be involved in a very minor way in mineral formation. Its major effect on leachate chemistry is in contributing to the ionic strength of the leachate. Inspection of results for a representative group of these minerals (Figures 6-4 through 6-6) reveals uniform dissolution, except initially in the fly ash lysimeter, where Mirabilite and Analcime (a Sodium Alumino-Silicate) are indicated to possibly form, then go into dissolution.

Cl. No major cation interacts significantly with the Chloride ion. Minor cations $\mathrm{Pb}, \mathrm{Zn}$, 


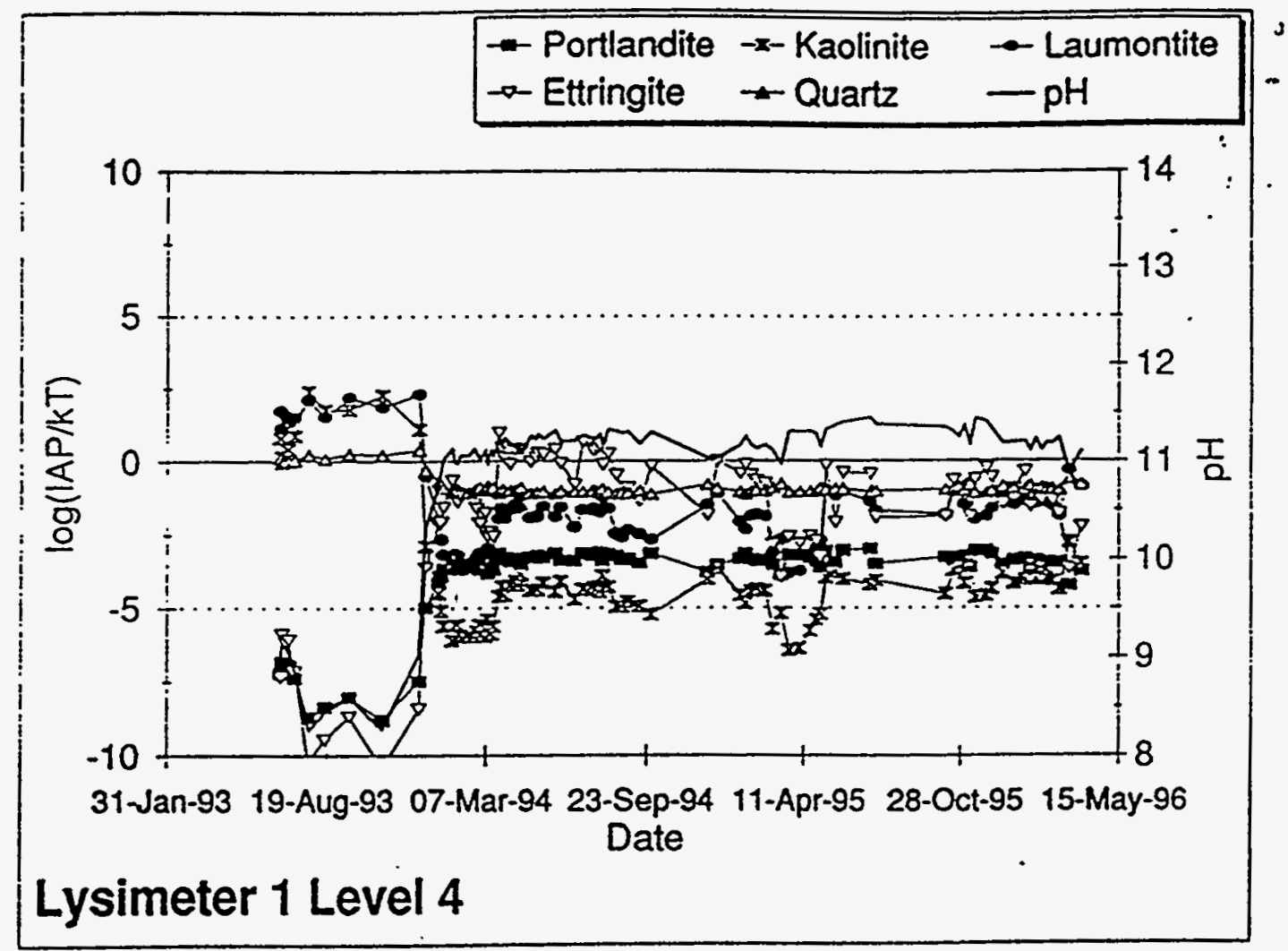

Figure 6-1. Portlandite, Kaolinite, Laumontite, Ettringite, and Quartz variations by date and $\mathrm{pH}$ in Lysimeter 1, Level 4

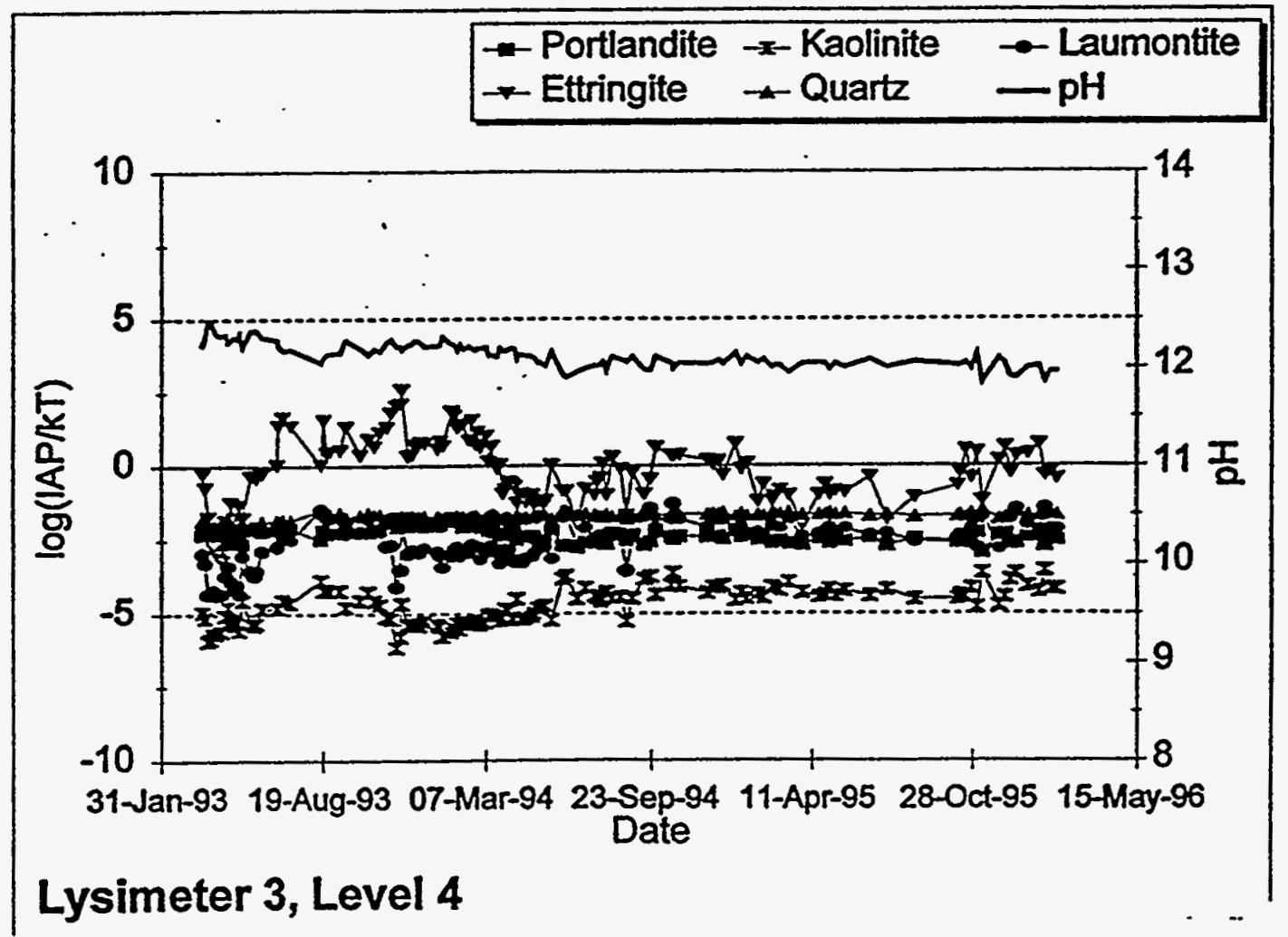

Figure 6-2. Portlandite, Kaolinite, Laumontite, Ettringite, and Quartz variations by date and $\mathrm{pH}$ in Lysimeter 3, Level 4 


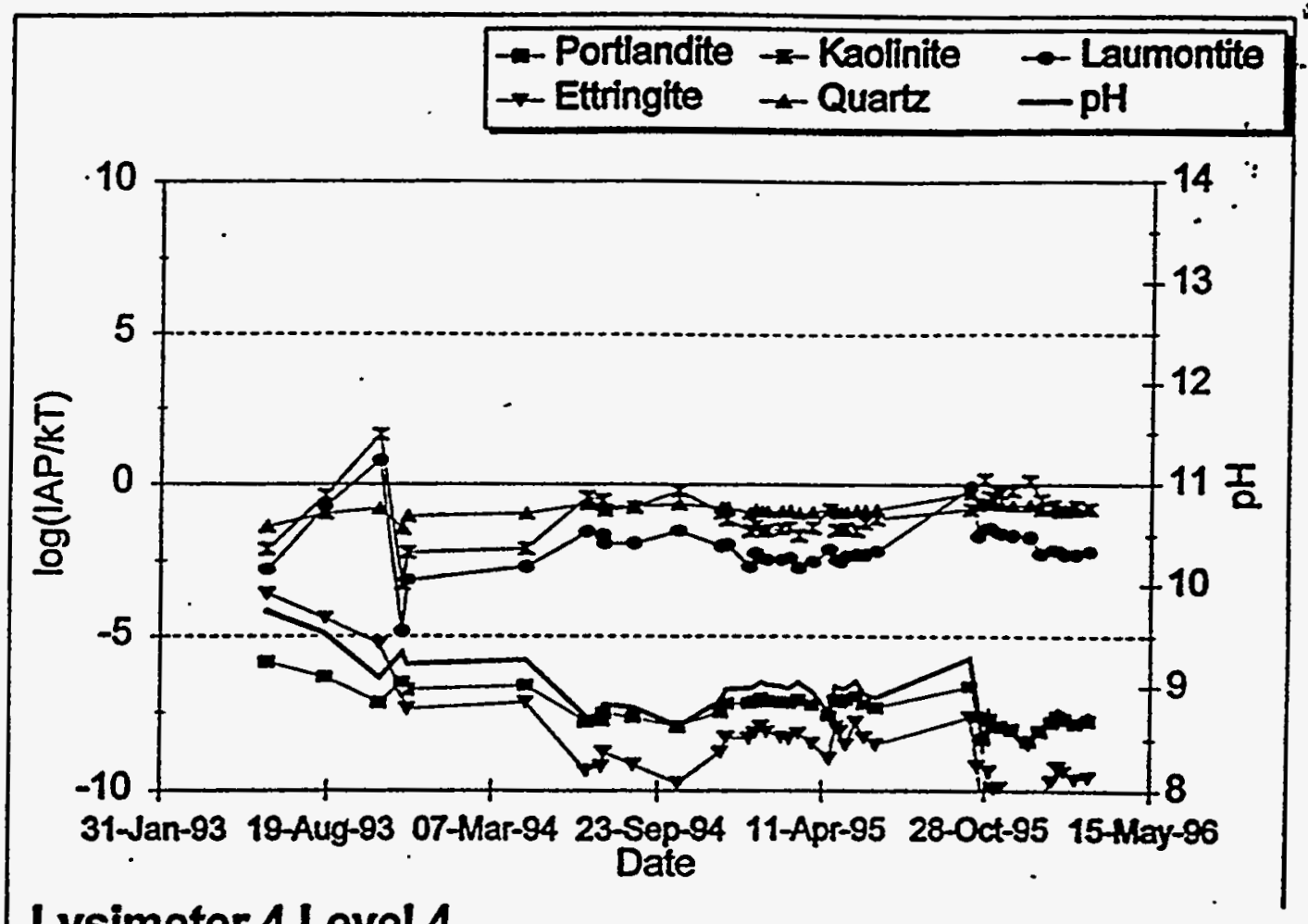

\section{Lysimeter 4 Level 4}

Figure 6-3. Portlandite, Kaolinite, Laumontite, Ettringite, and Quartz variations by date and $\mathrm{pH}$ in Lysimeter 4, Level 4

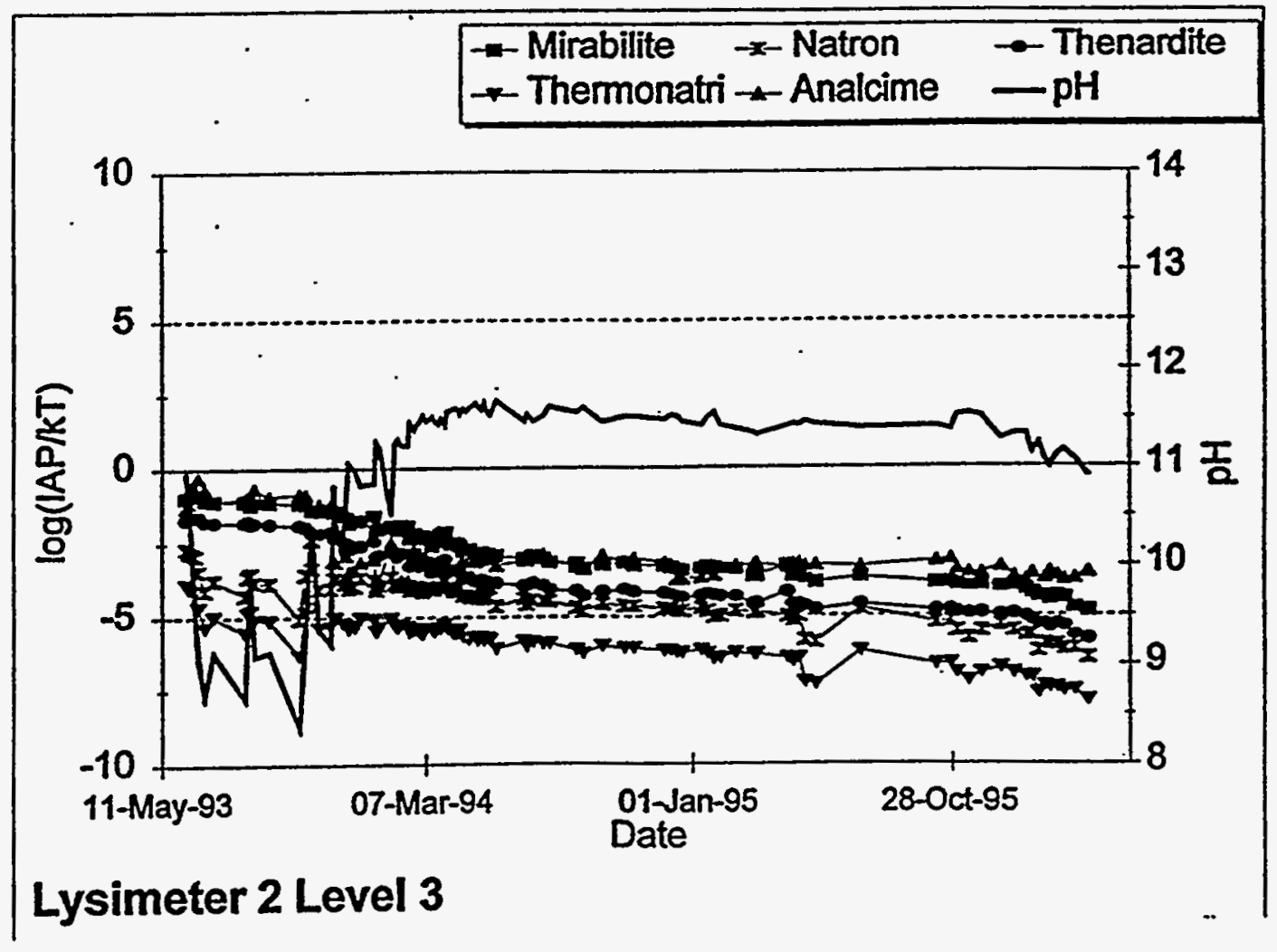

Figure 6-4. Mirabilite, Natron, Thenardite, Thermonatrite, and Analcime variations by date and $\mathrm{pH}$ in Lysimeter 2, Level 3 


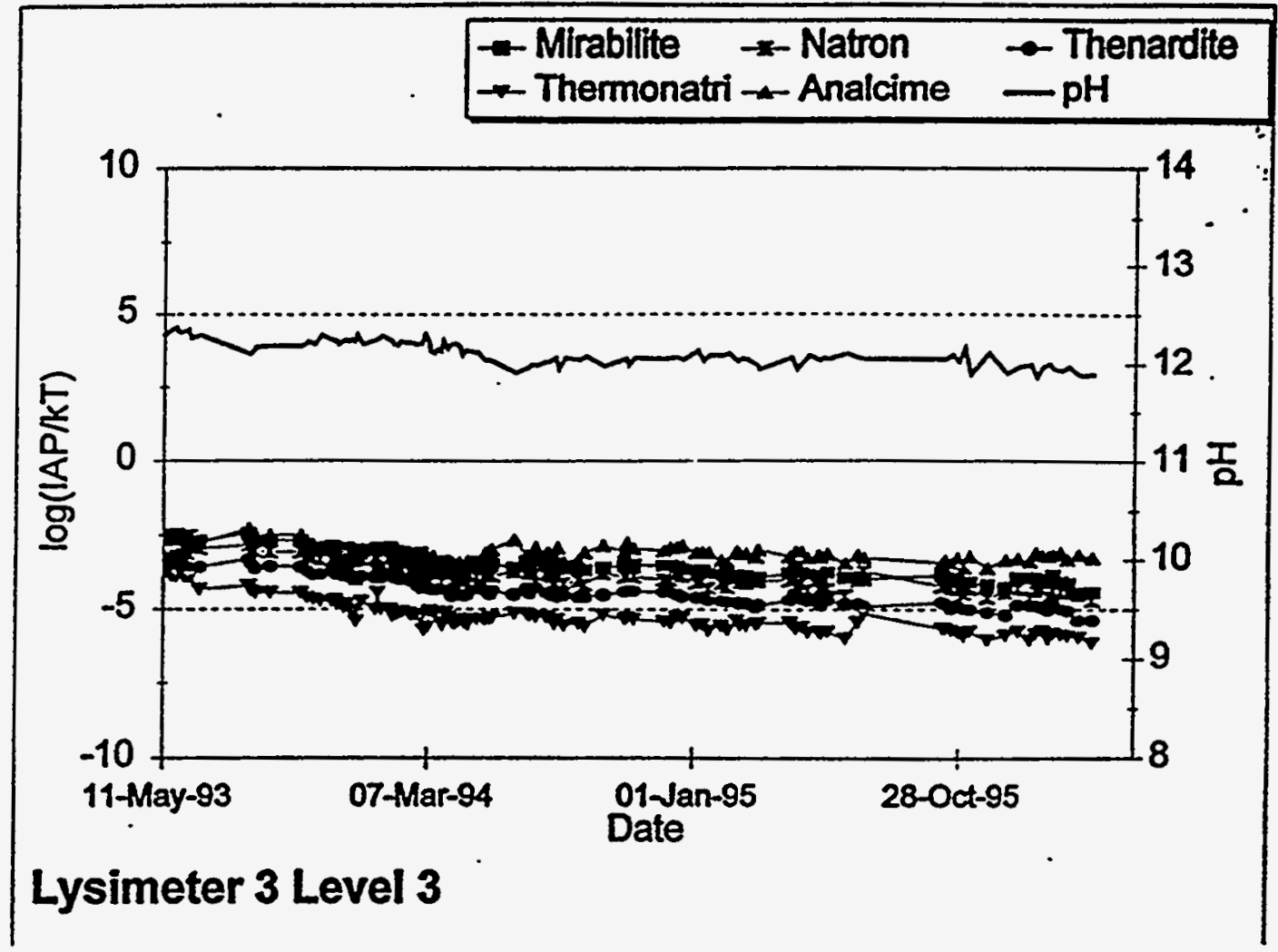

Figure 6-5. Mirabilite, Natron, Thenardite, Thermonatrite, and Analcime variations by date and $\mathrm{pH}$ in Lysimeter 3, Level 3

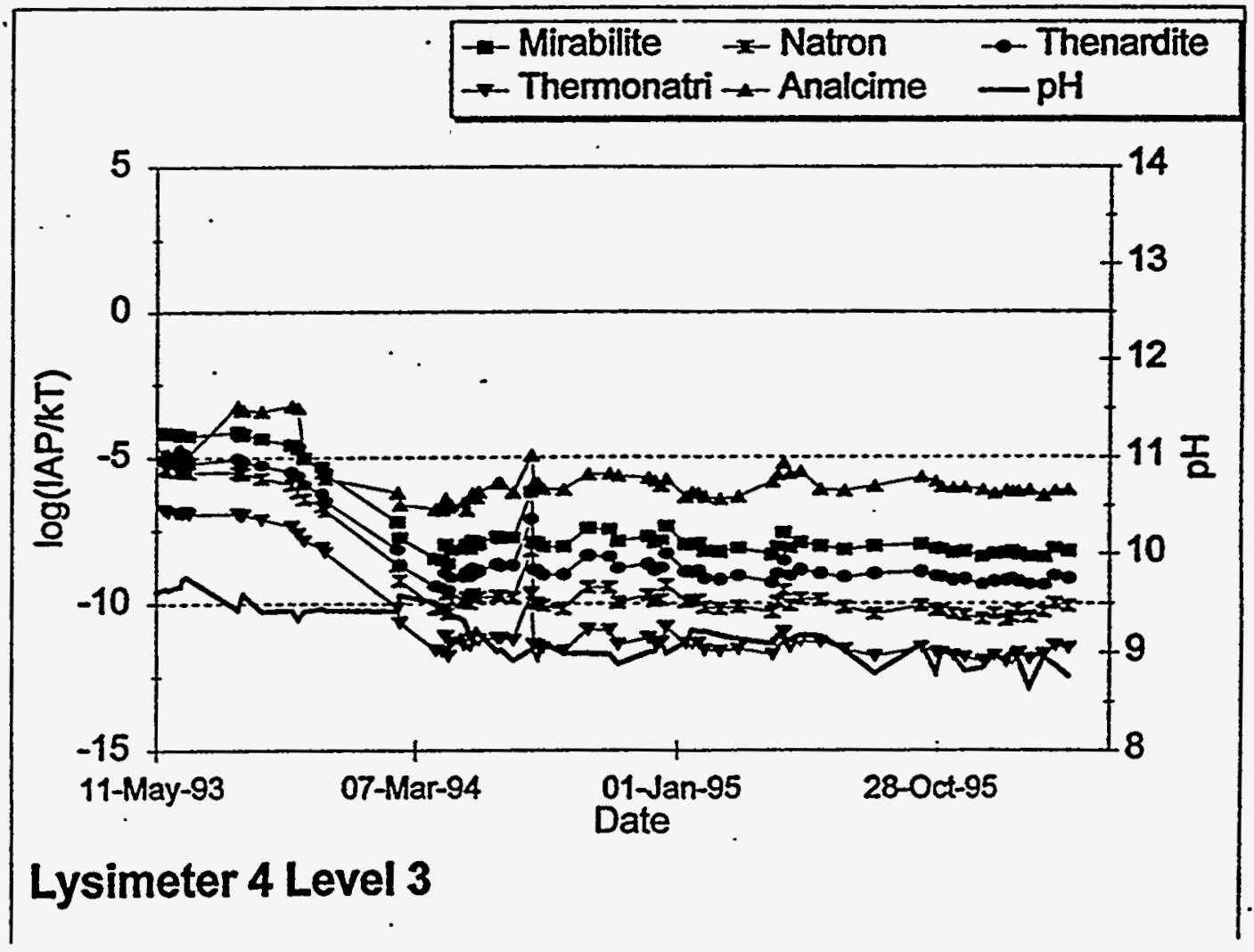

Figure 6-6. Mirabilite, Natron, Thenardite, Thermonatrite, and Analcime variations by date and $\mathrm{pH}$ in Lysimeter 4, Level 3 
Fe and Mn may form minor amounts of hydroxy-chloride minerals. Its major effect is also in contributing to the ionic strength of the leachate. Representative samples, $\mathrm{Pb}_{5}\left(\mathrm{PO}_{4}\right)_{3} \mathrm{Cl}$ and $\mathrm{Pb}_{2}(\mathrm{OH})_{3} \mathrm{Cl}$ (Figures 6-7 through 6-9), show occasional indications of supersaturation of the phosphate containing member during the low $\mathrm{pH}$ transients of the Coolside lysimeters and small supersaturation at the end of the fly ash lysimeter sampling period. Only extremely minor amounts of any $\mathrm{Cl}$ containing minerals may be formed.

Al. Free Al will be available from the dissolution of glassy alumino-silicates. It is the only trivalent ion which forms a soluble hydroxide. Initially formed is dimorphous Boehmite/Diaspore (AlOOH or $\mathrm{Al}_{2} \mathrm{O}_{3}{ }^{*} \mathrm{H}_{2} \mathrm{O}$ ), the basic oxide of Alumina. Diaspore is favored over Boehmite by $\sim 0.1 \mathrm{kcal} / \mathrm{mole}$. Further hydration in high $\mathrm{pH}$ solutions yields $\mathrm{Al}(\mathrm{OH})_{4}{ }^{-}$, the major ionic carrier of available Aluminum. Diaspore/Boehmite hydrates slowly to Gibbsite $\left(\mathrm{Al}(\mathrm{OH})_{3}\right.$ or $\left.\mathrm{Al}_{2} \mathrm{O}_{3}{ }^{*} \mathrm{H}_{2} \mathrm{O}\right)$ at room temperature. Gibbsite is also artificially formed by passing $\mathrm{CO}_{2}$ into alkali aluminate solution, which describes Coolside leachate conditions. Kaolinite $\left(\mathrm{Al}_{2} \mathrm{Si}_{2} \mathrm{O}_{5}(\mathrm{OH})_{4}\right)$, which WATEQ indicates approaches or passes saturation at lower $\mathrm{pH}$ levels, also dissolves to or forms from Gibbsite and Silicic acid. Diaspore, Gibbsite and Kaolinite were chosen as minerals of interest. For further discussion of Gibbsite and Diaspore, see the discussion of Ettringite below. Various other Aluminosilicates are discussed in the sections on micas, feldspars, pyroxenes and $\mathrm{Ca} / \mathrm{Mg}$ mineral forms.

A clay-like deposit was reported during sample augering in the field lysimeters. Inspection of Kaolinite saturation indices indicate that clay mineral formation is possible very early in the low $\mathrm{pH}$ portions of the lysimeter sample periods (Figures 6-10 through 6-12) and is not indicated at high $\mathrm{pH}$. As XRD data do not generally support the existence of detectable amounts of kaolinite, it is probable that the clay-like deposit is a diffuse alumino-silicate gel consisting of poorly consolidated mixtures of, for example, 


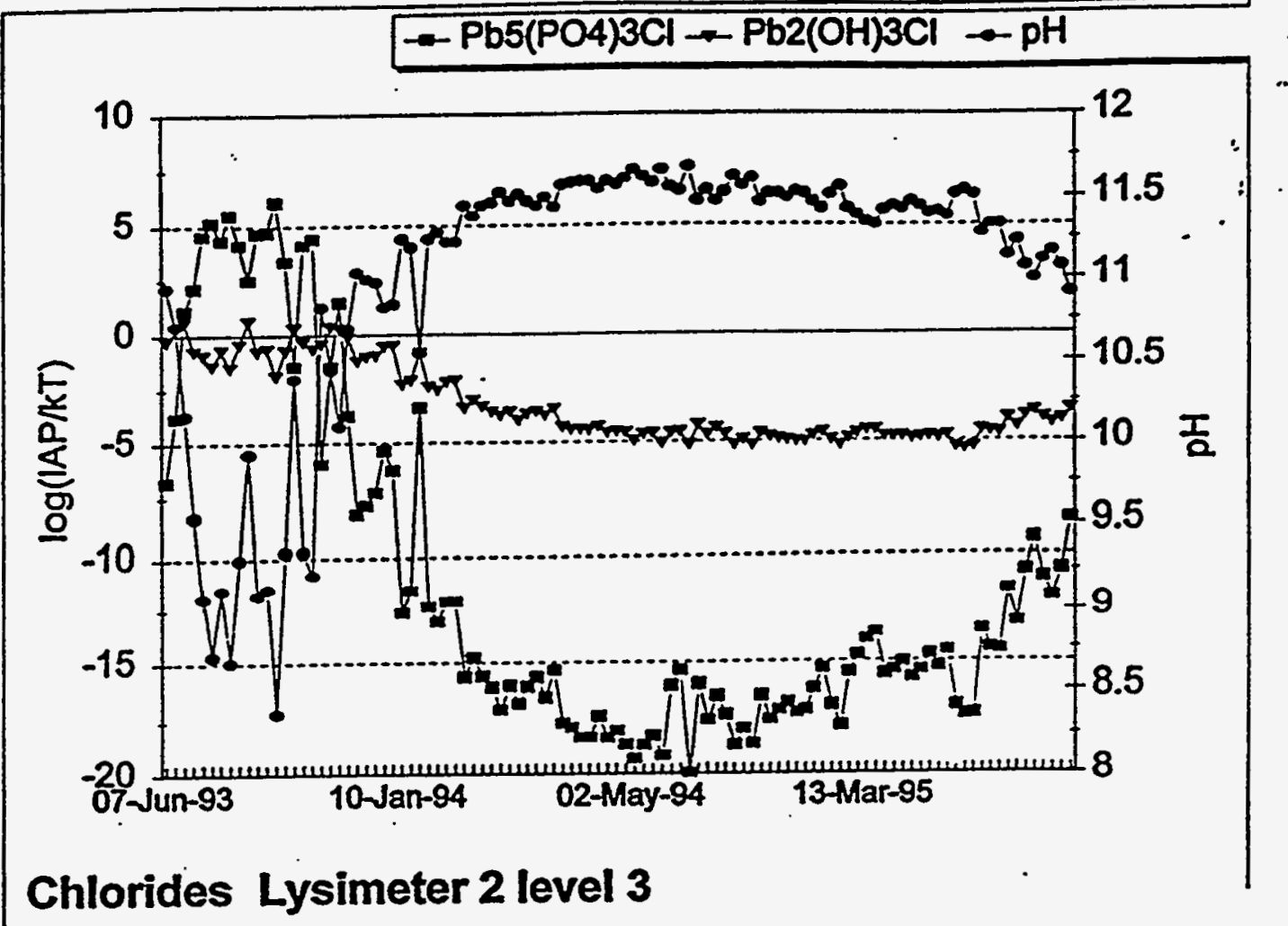

Figure 6-7. $\mathrm{Pb}_{5}\left(\mathrm{PO}_{4}\right)_{3} \mathrm{Cl}$ and $\mathrm{Pb}_{2}(\mathrm{OH})_{3} \mathrm{Cl}$ variations by date and $\mathrm{pH}$ in Lysimeter 2 , Level 3

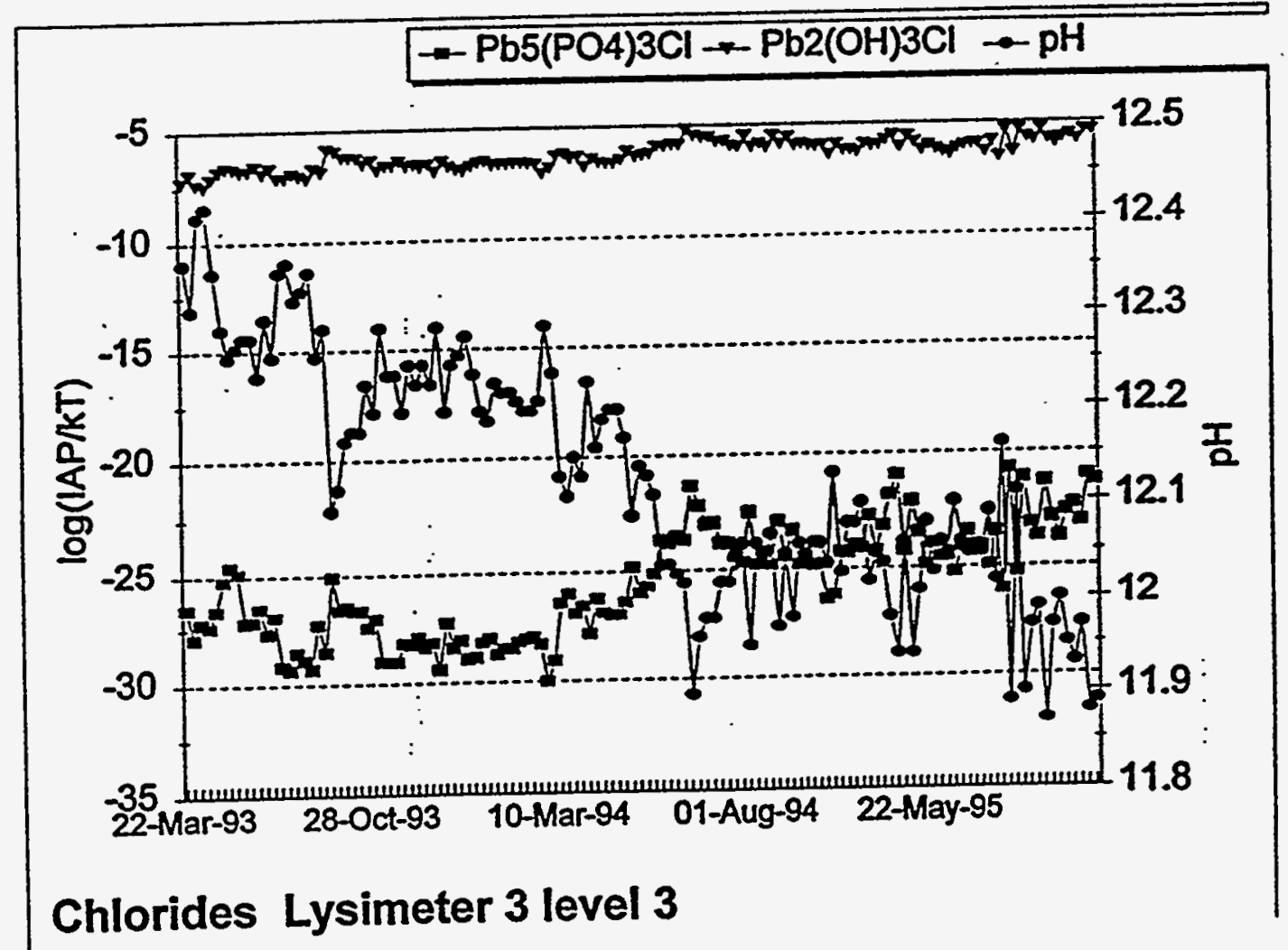

Figure 6-8. $\mathrm{Pb}_{5}\left(\mathrm{PO}_{4}\right)_{3} \mathrm{Cl}$ and $\mathrm{Pb}_{2}(\mathrm{OH})_{3} \mathrm{Cl}$ variations by date and $\mathrm{pH}$ in Lysimeter 3 , Level 3 


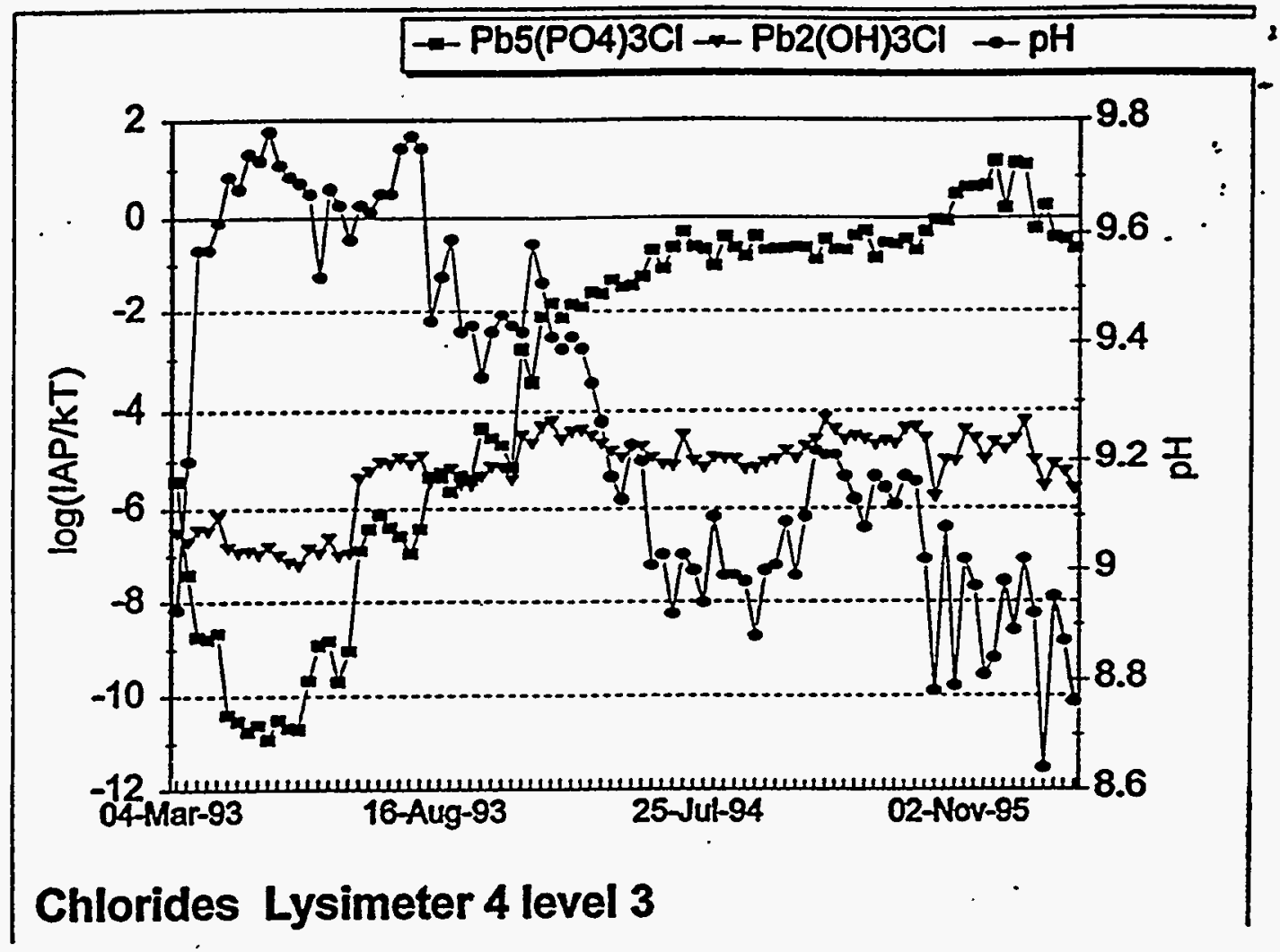

Figure 6-9. $\mathrm{Pb}_{5}\left(\mathrm{PO}_{4}\right)_{3} \mathrm{Cl}$ and $\mathrm{Pb}_{2}(\mathrm{OH})_{3} \mathrm{Cl}$ variations by date and $\mathrm{pH}$ in Lysimeter 4, Level 3

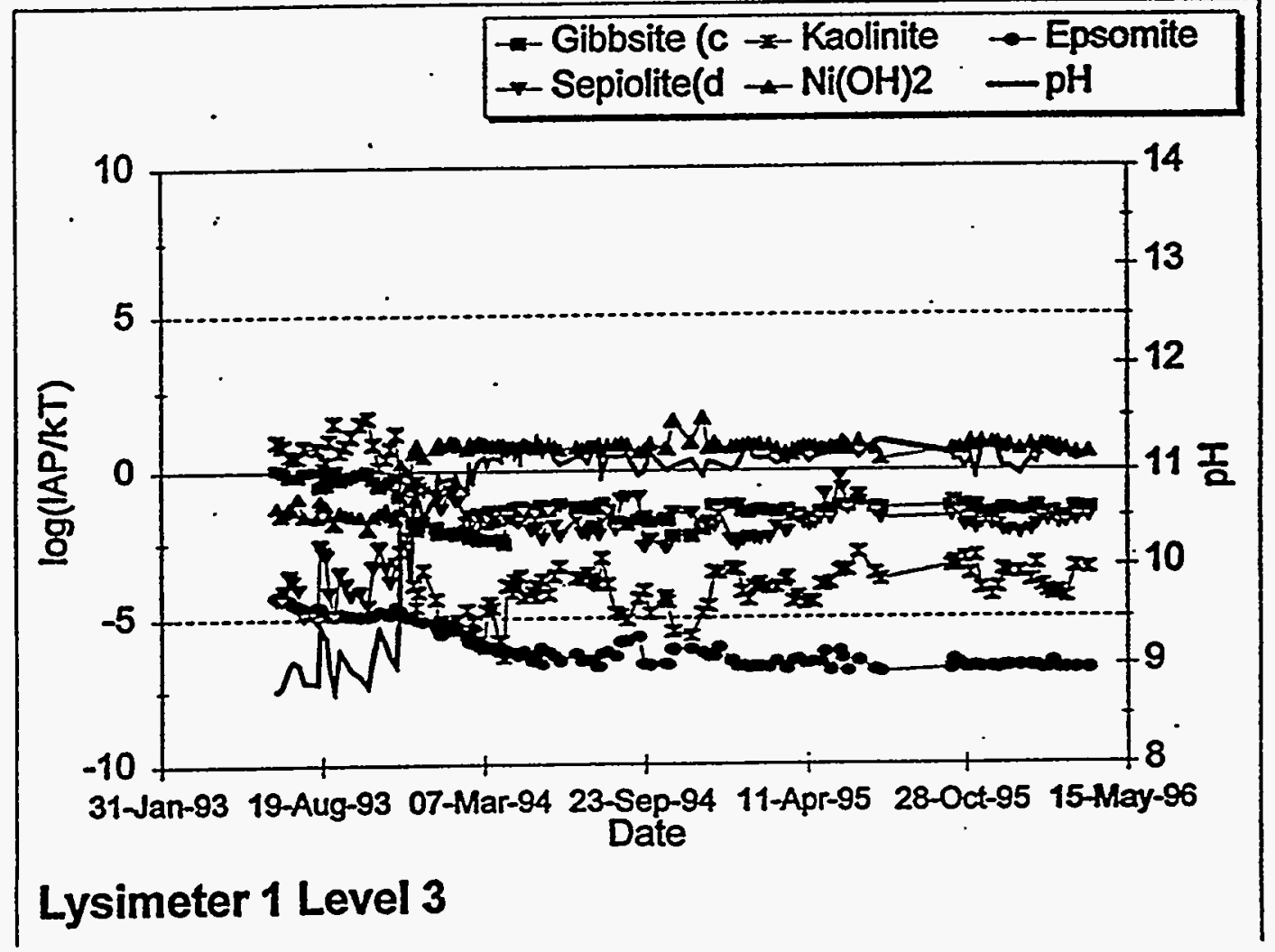

Figure 6-10. Gibbsite, Kaolinite, Epsomite, Sepiolite, and $\mathrm{Ni}(\mathrm{OH})_{2}$ variations by date and $\mathrm{pH}$ in Lysimeter 1 , Level 3 


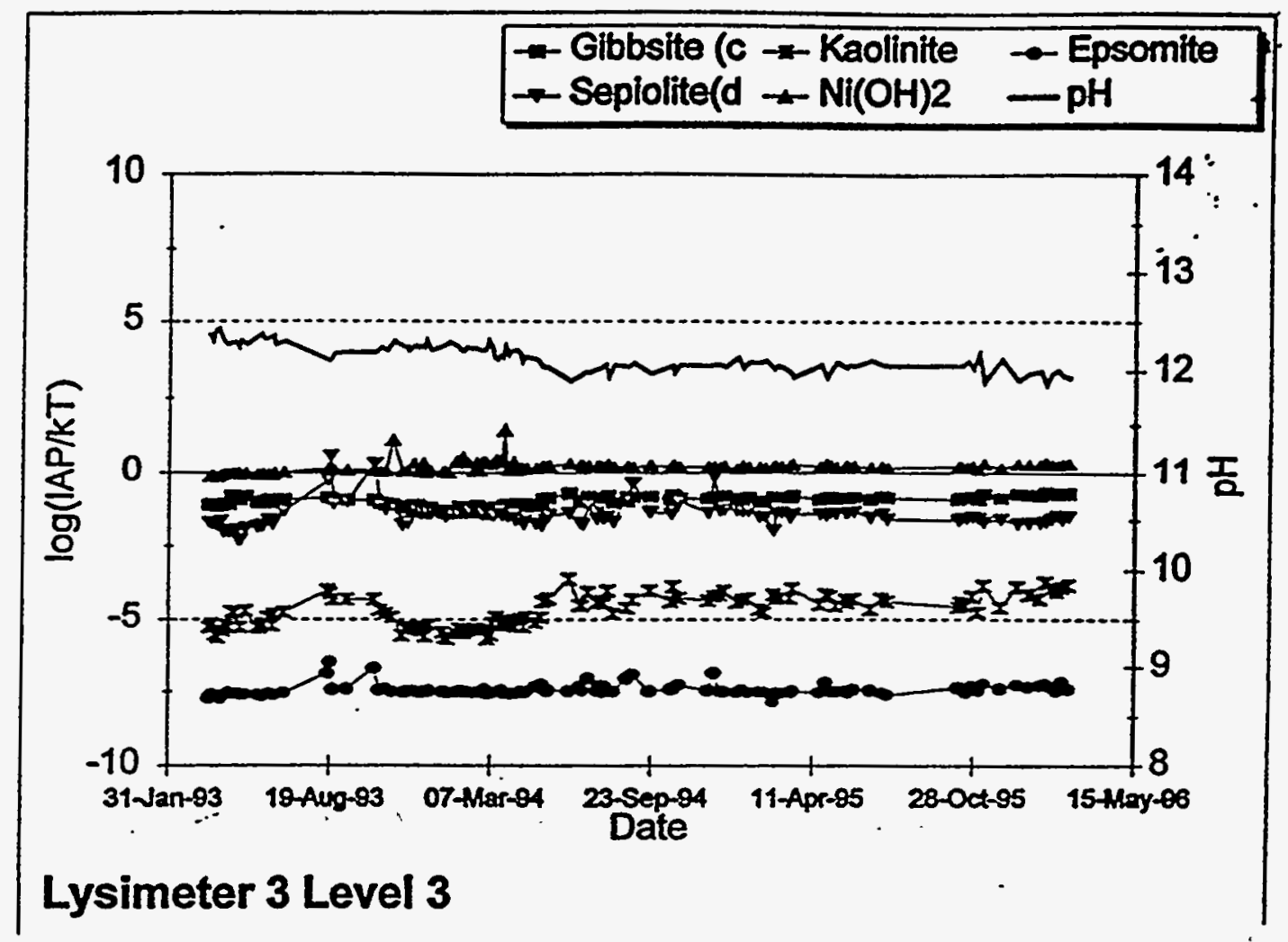

Figure 6-11. Gibbsite, Kaolinite, Epsomite, Sepiolite, and $\mathrm{Ni}(\mathrm{OH})_{2}$ variations by date and $\mathrm{pH}$ in Lysimeter 3 , Level 3

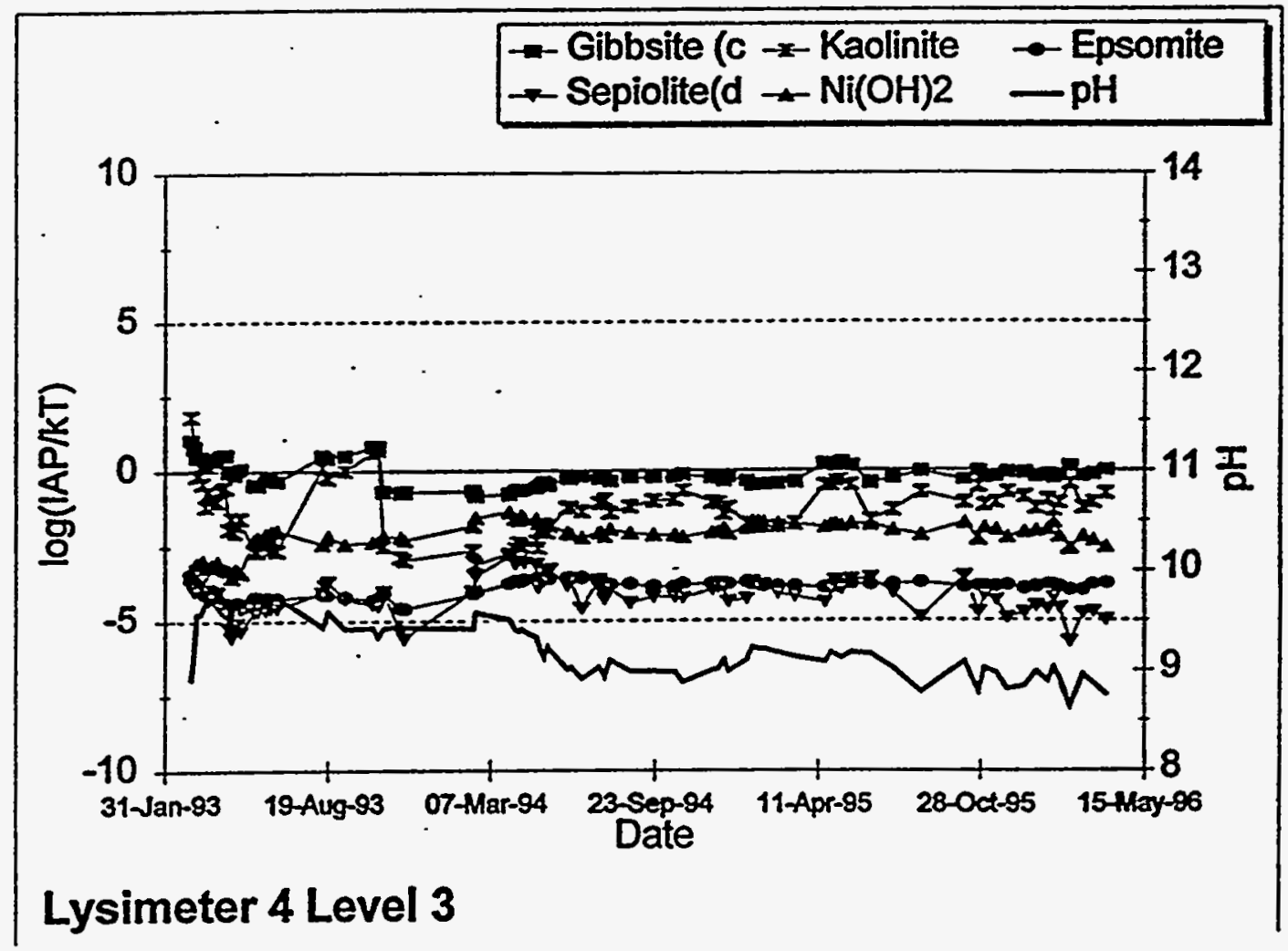

Figure 6-12. Gibbsite, Kaolinite, Epsomite, Sepiolite, and $\mathrm{Ni}(\mathrm{OH})_{2}$ variations by date and $\mathrm{pH}$ in Lysimeter 4, Level 3 
Adularia, Albite, Anorthite, Pyrophillite, Kaolnite, Wairakite and Halloysite. XRD results generally support this.

Micas. $\mathrm{K}$-mica $\left(\mathrm{KAl}_{2}\left(\mathrm{Si}_{3} \mathrm{Al}\right) \mathrm{O}_{10}(\mathrm{OH})_{2}\right)$ remains supersaturated under nearly all lysimeter conditions and shows a sometimes extreme supersaturation at lower leachate $\mathrm{pH}$ (Figures 6-13 through 6-15). Micas have never been shown to control water chemistry. Reversibility has not been shown, so there is no reason to believe that they attain equilibrium solubilities or will form. The supersaturation merely reflects the solute availability of the component ions.

Feldspars. The Feldspars Anorthite $\left(\mathrm{CaAl}_{2} \mathrm{Si}_{2} \mathrm{O}_{8}\right)$, Albite $\left(\mathrm{NaAISiȘi} \mathrm{O}_{8}\right)$ and Adularia $\left(\mathrm{KAlSiSi}_{2} \mathrm{O}_{8}\right)$ were invesitgated. These minerals form a substitutional solid solution with degree of saturation generally Adularia $>$ Albite $>$ Anorthite. They appear to be driven by leachate chemistry. The dominant members, Adularia and Albite (Figures 6-13 through 6-15), show supersaturation at low $\mathrm{pH}$ and dissolution at high $\mathrm{pH}$ in the Coolside lysimeters, and general undersaturation in the fly ash lysimeters. Formation of these minerals is expected to be slight and contributes only to the broad aluminosilicate peak detected by XRD.

Pyroxenes. Clinoenstatite $\left(\mathrm{Mg}_{2} \mathrm{Si}_{2} \mathrm{O}_{6}\right.$ or $\left.\mathrm{MgSiO}_{3}\right)$, Analcime $\left(\mathrm{NaAlSi}_{2} \mathrm{O}_{6}{ }^{*} \mathrm{H}_{2} \mathrm{O}\right)$ (hydrated Jadeite) and Diopside $\left(\mathrm{CaMgSi}_{2} \mathrm{O}_{6}\right)$ at times exhibit supersaturation. Diopside generally appears to be driven by $\mathrm{pH}$ mediated silica availability (Figures 6-16 through 6-18). Its often very high degree of supersaturation argues against its importance in leachate chemistry. Clinoenstatite, the major Magnesium Silicate, only approaches saturation at high $\mathrm{pH}$ in the most compacted lysimeter, and not at all in the others (Figures 6-13 through 6-15). This also reflects Silica availability at high pH and low Silica availability even in the presence of elevated $\mathrm{Mg}$ in the fly ash lysimeters. Analcime (Figures 6-4 through 6-6) approaches or exceeds saturation only in the early 


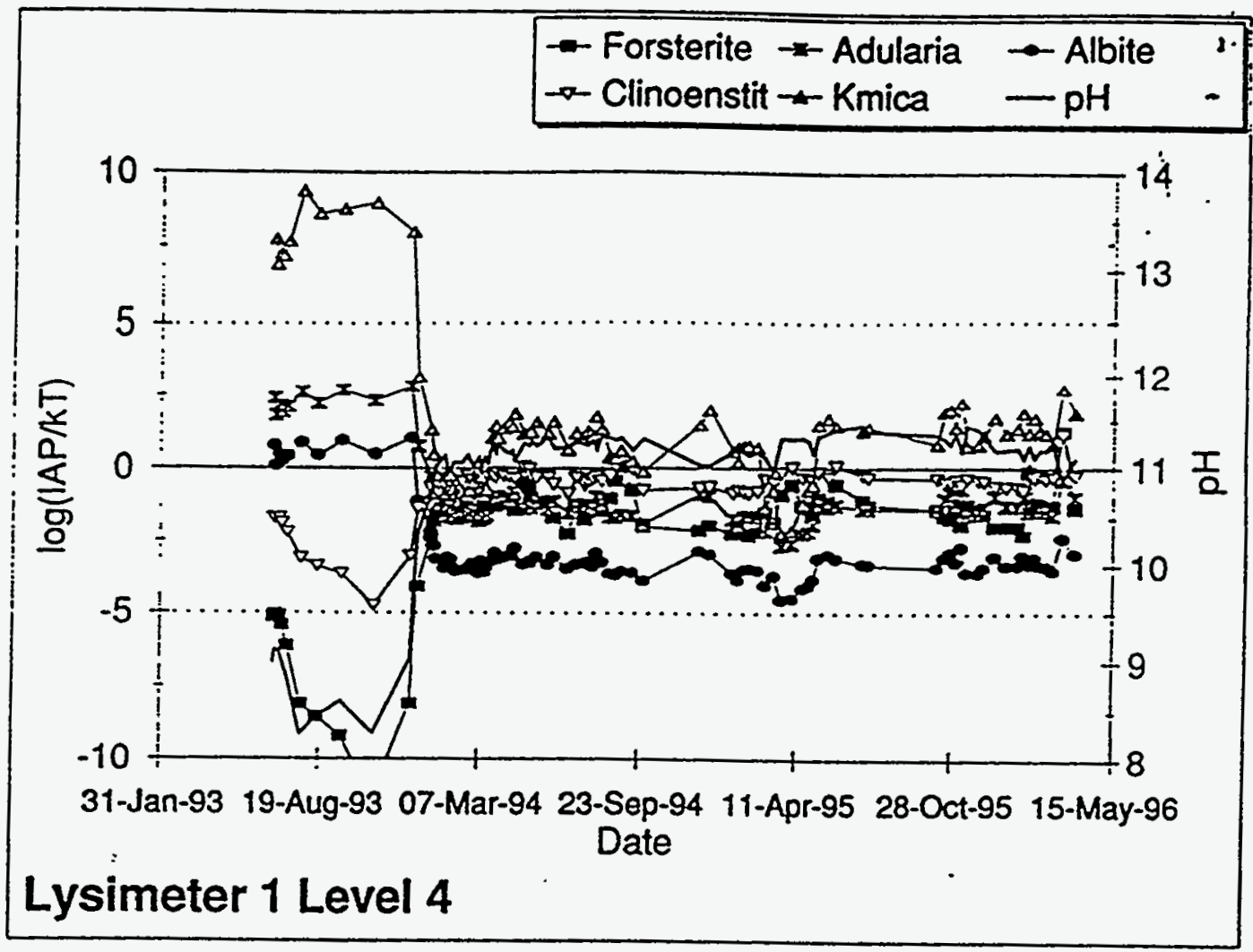

Figure 6-13. Forsterite, Adularia, Albite, Clinoenstatite, and Kmica variations by date and $\mathrm{pH}$ in Lysimeter 1, Level 4

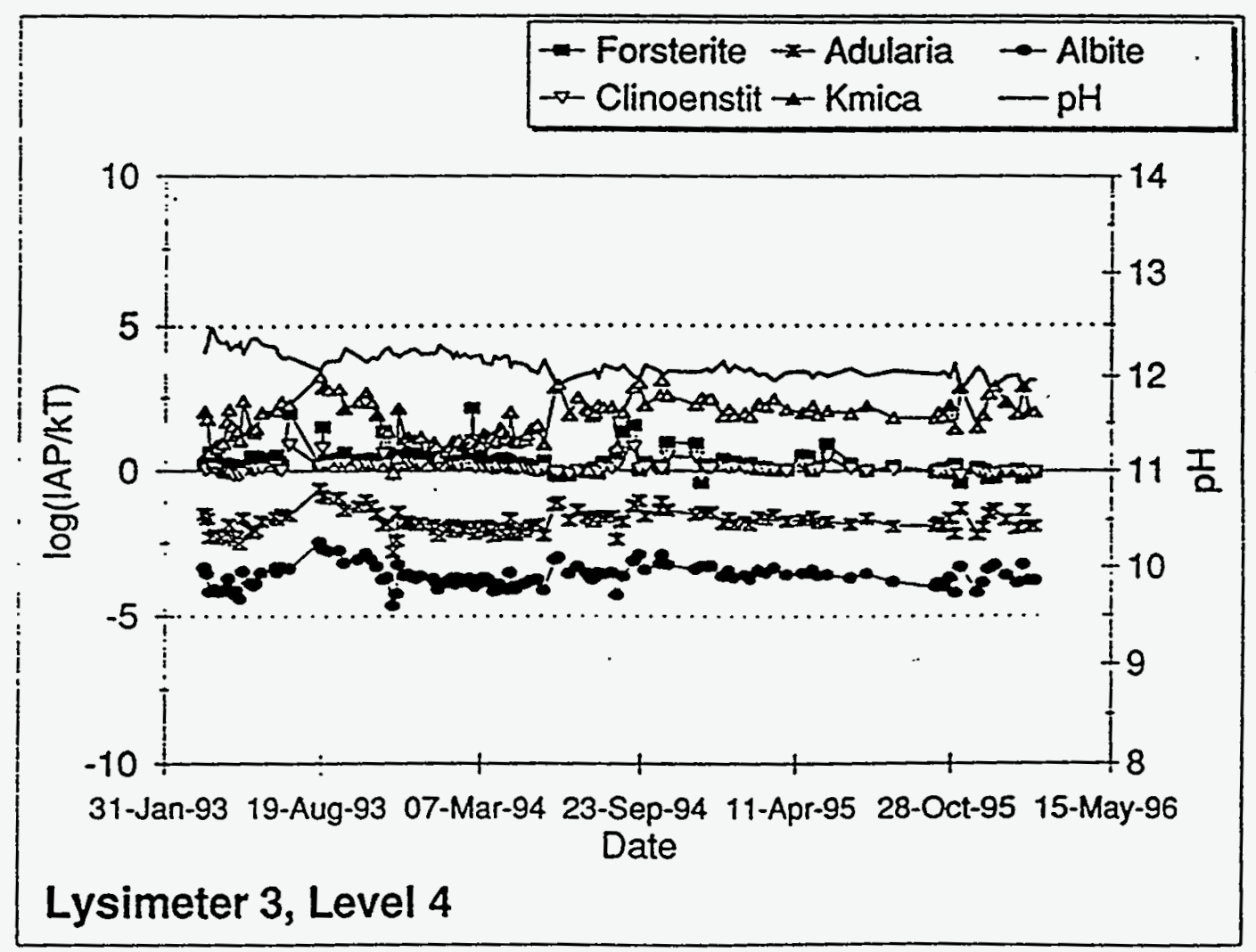

Figure 6-14. Forsterite, Adularia, Albite, Clinoenstatite, and Kmica variations by date and $\mathrm{pH}$ in Lysimeter 3 , Level 4 


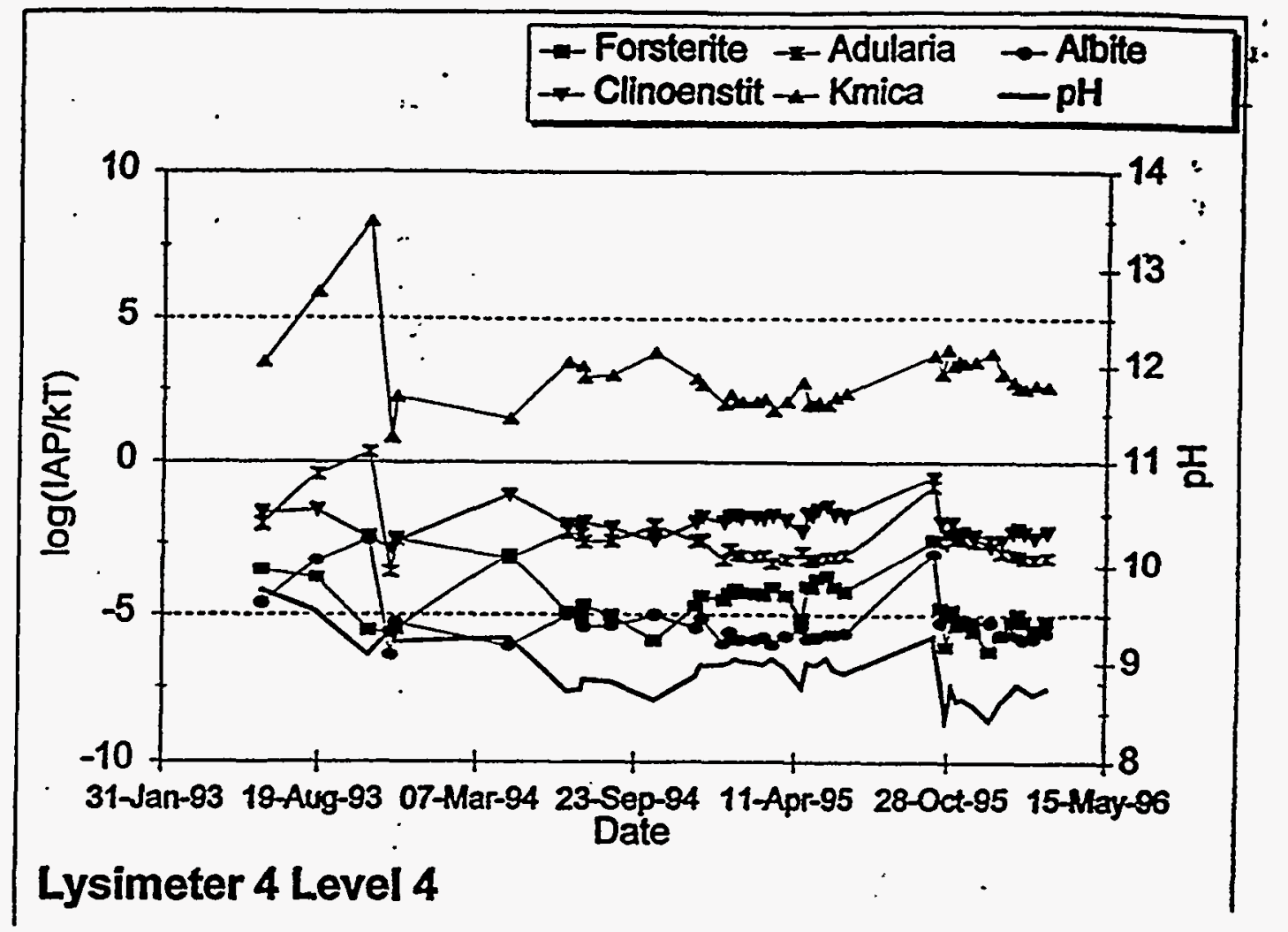

Figure 6-15. Forsterite, Adularia, Albite, Clinoenstatite, and Kmica variations by date and $\mathrm{pH}$ in Lysimeter 4, Level 4

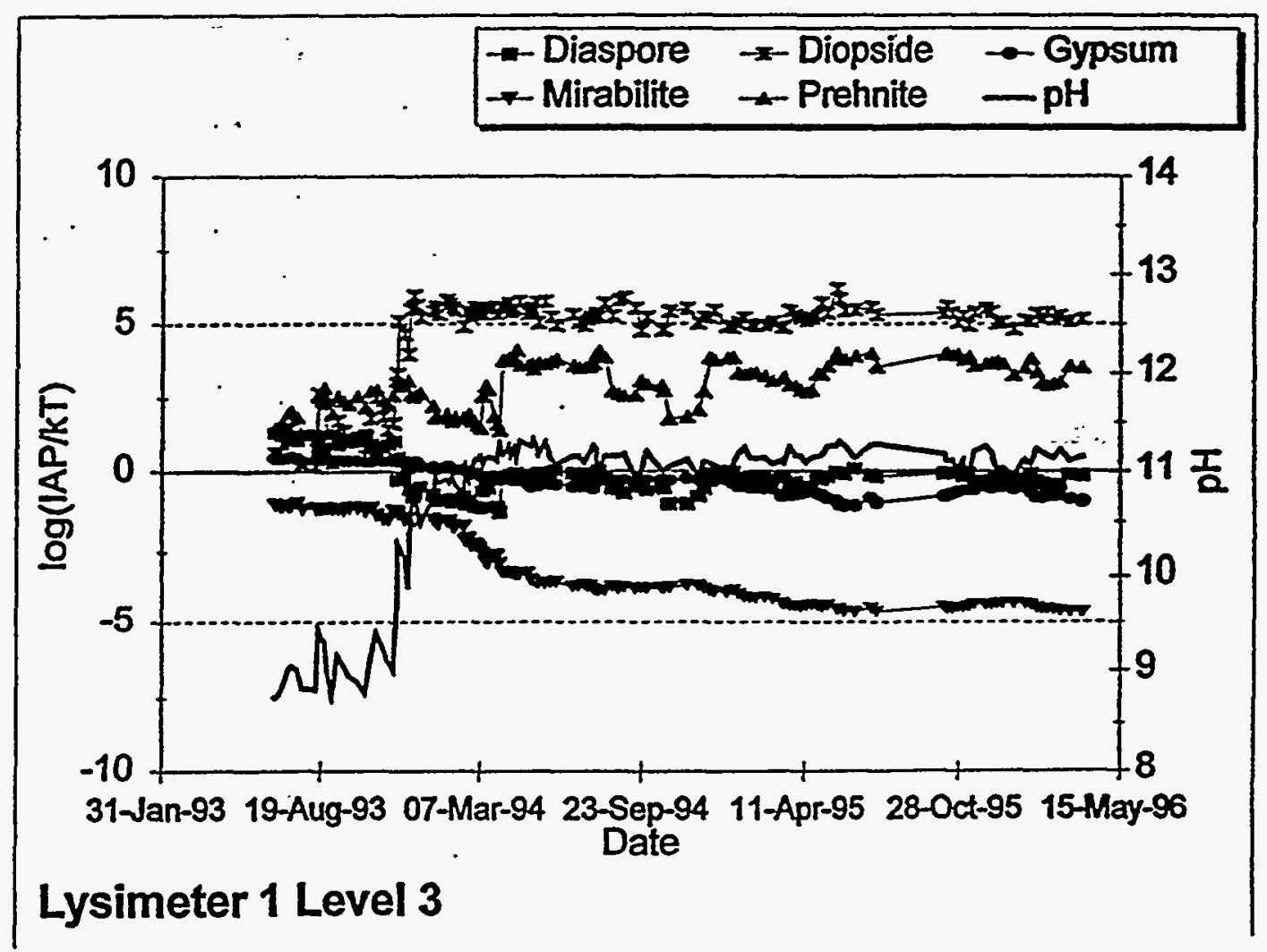

Figure 6-16. Diaspore, Diopside, Gypsum, Mirabilite, and Prehnite variations by date and $\mathrm{pH}$ in Lysimeter 1, Level 3 


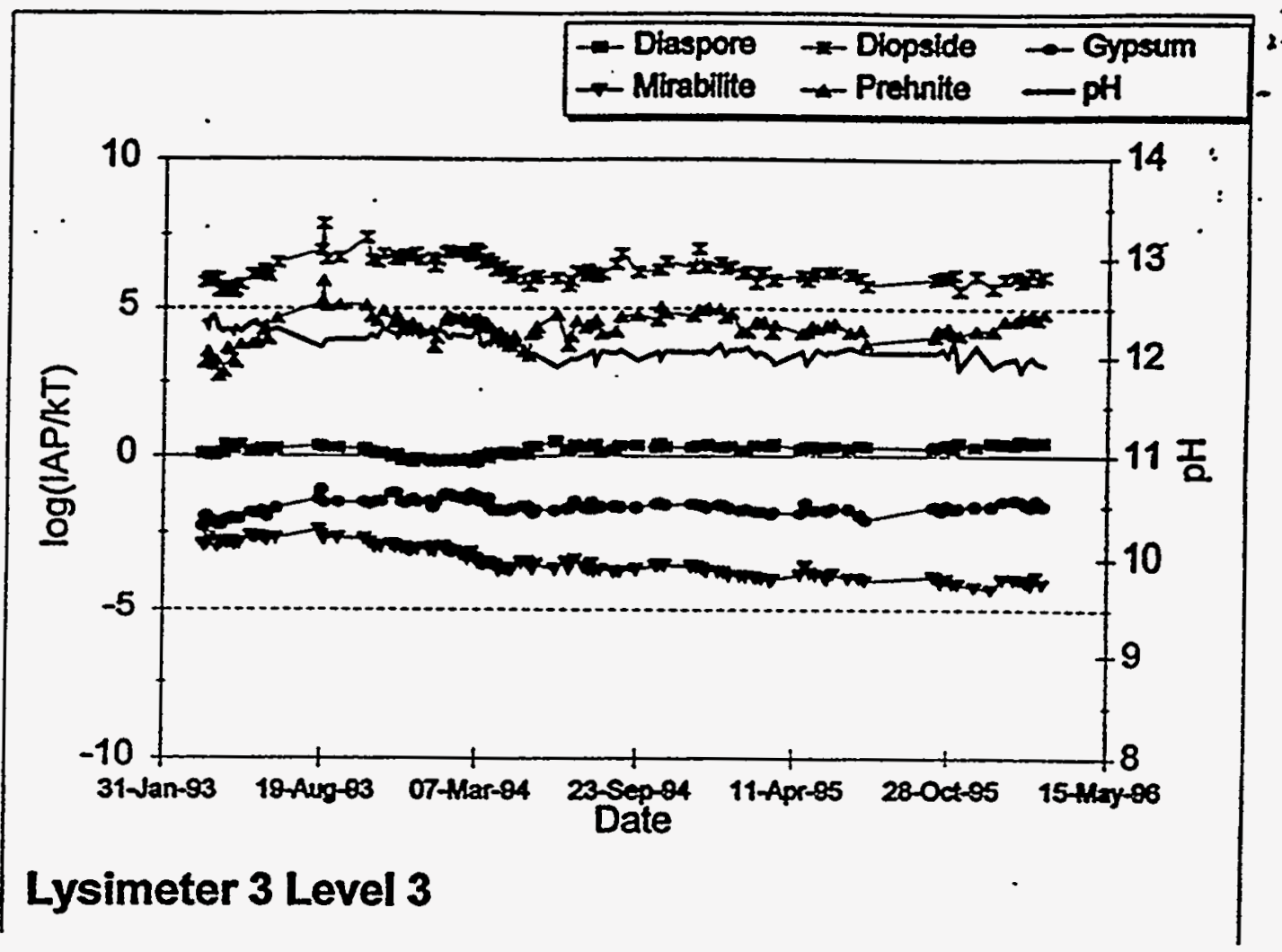

Figure 6-17. Diaspore, Diopside, Gypsum, Mirabilite, and Prehnite variations by date and $\mathrm{pH}$ in Lysimeter 3 , Level 3

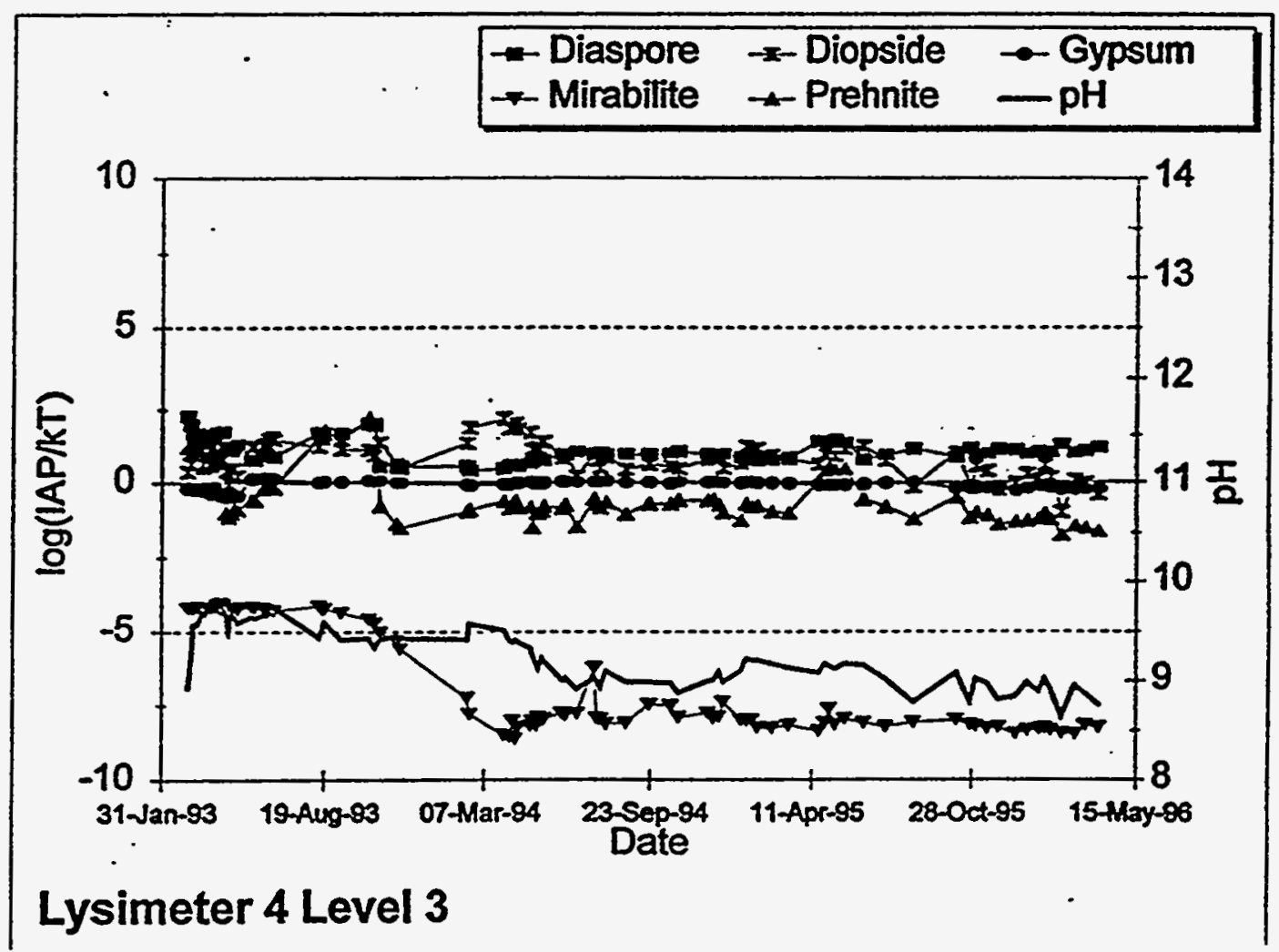

Figure 6-18. Diaspore, Diopside, Gypsum, Mirabilite, and Prehnite variations by date and $\mathrm{pH}$ in Lysimeter 4, Level 3 
stages of fly ash leaching and also appears to be driven by leachate chemistry. Pyroxenes are not expected to form in any measurable amount nor exert any control on lysimeter conditions.

SO $_{4}$. Sulfate availability is controlled by the equilibrium dissolution of Anhydrite $\left(\mathrm{CaSO}_{4}\right)$ and Gypsum $\left(\mathrm{CaSO}_{4}{ }^{*} 2 \mathrm{H}_{2} \mathrm{O}\right)$. Anhydrite crystals are not common. Anhydrite converts slowly to Gypsum with a corresponding increase in volume giving long-term swell. It is also slightly more soluble than Gypsum. WATEQ indicates that both are uniformly at or slightly under saturation, with Anhydrite slightly less saturated, as expected. There are ranges of local environmental conditions which lead to more efficient congruent dissolution of the least favored form and simultaneous precipitation of the most favored. The primary $\mathrm{SO}_{4}$ mineral in arid Coolside waste is Anhydrite. This will spontaneously hydrate to Gypsum with concommitant swell due to differing densities. Comparison of saturation indices of Gypsum and Anhydrite illustrate this. Note that the $\mathrm{pH}$ range may be fairly narrow for the most efficient conversion, with Anhydrite in dissolution and Gypsum in formation in the case of the least compacted lysimeters (Figure 6-19). Excess or insufficient hydration and the resulting $\mathrm{pH}$ (as exhibited by the pH differences in the least (Figure 6-19) and most compacted lysimeters (Figure 6-20)) may control the pace of this congruent dissolution/precipitation phenomena. When both are thermodynamically likely to precipitate $(\log (\operatorname{IAP} / k T)$ above 0$)$ or dissolve $(\log (\operatorname{IAP} / k T)$ less than 0$)$ (Figure 6-20), equilibrium formation/dissolution will still result in conversion, but at a lower rate. The fly ash lysimeter (Figure 6-21), interestingly, indicates efficient conversion during the entire sampling period. In addition, as Gypsum remains at or near saturation independently of $\mathrm{pH}$ swings, it is probable that it will exert a significant control over the fly ash lysimeter chemistry. For comparison, the saturation indices of two corresponding Calcium Carbonate forms, Calcite and Aragonite, were included in Figures 6-19 through 6-21. 


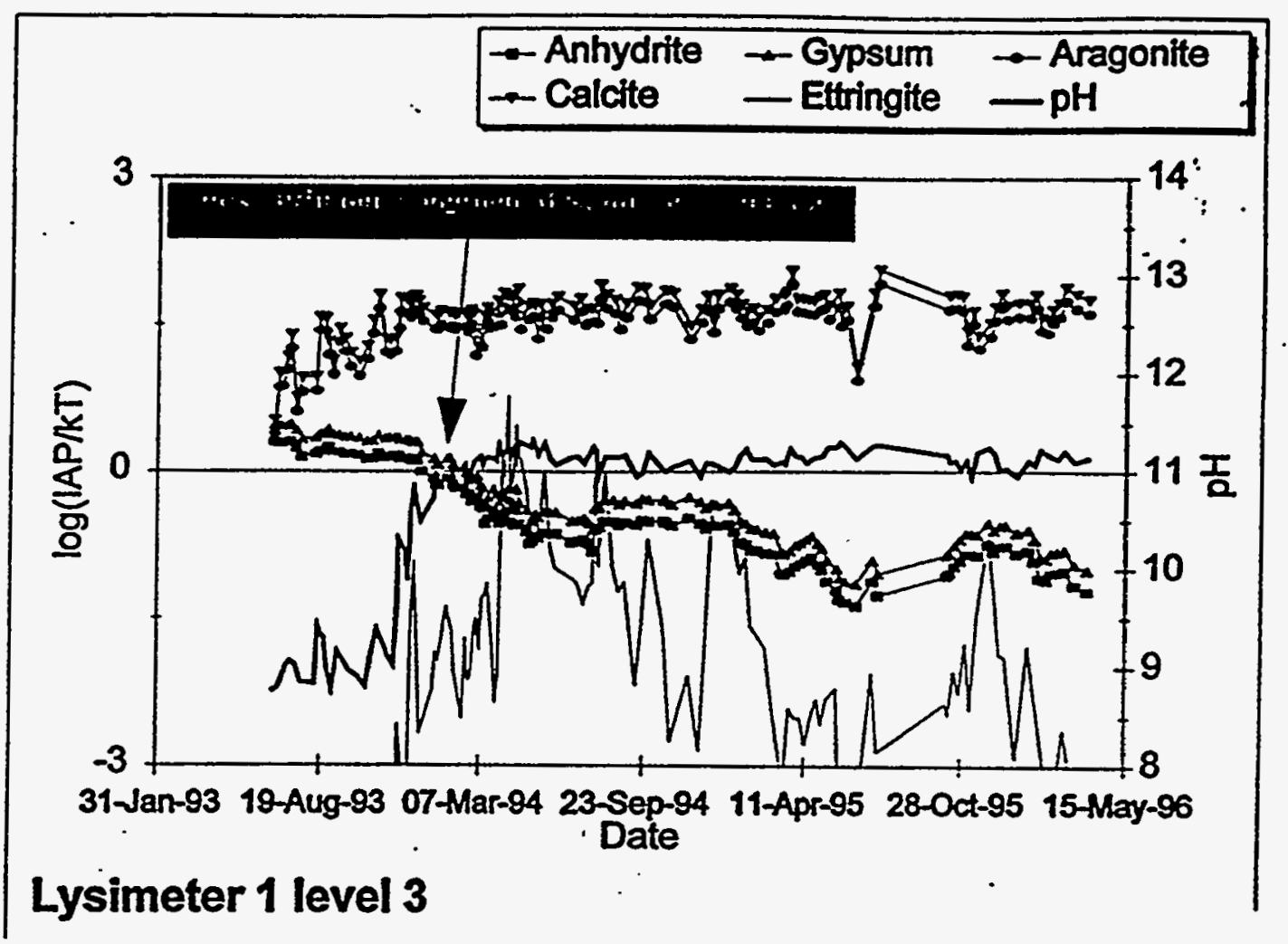

Figure 6-19. Anhydrite, Gypsum, Aragonite, Calcite, and Ettringite variations by date and $\mathrm{pH}$ in Lysimeter 1, Level 3

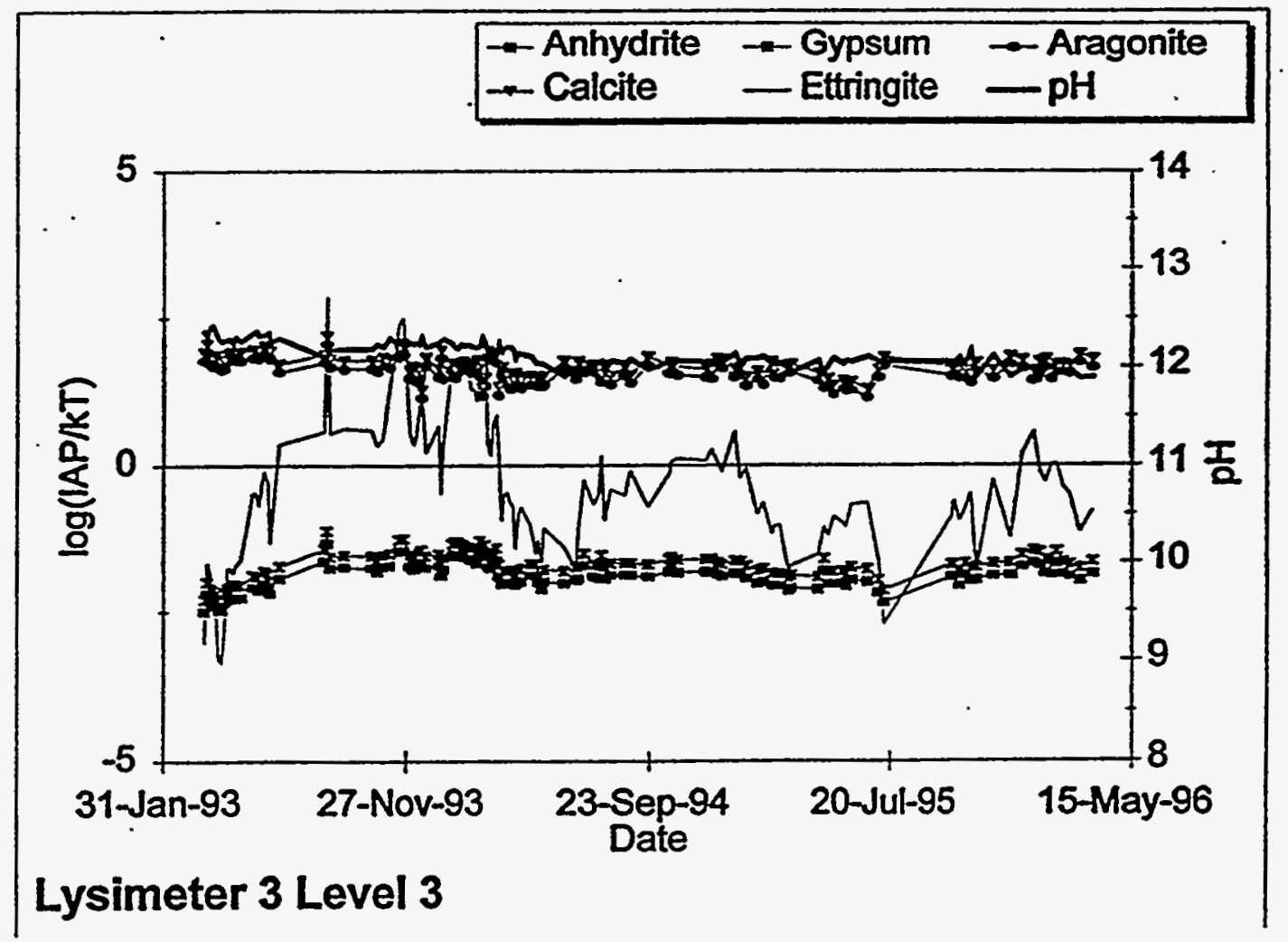

Figure 6-20. Anhydrite, Gypsum, Aragonite, Calcite, and Ettringite variations by date and $\mathrm{pH}$ in Lysimeter 3, Level 3 


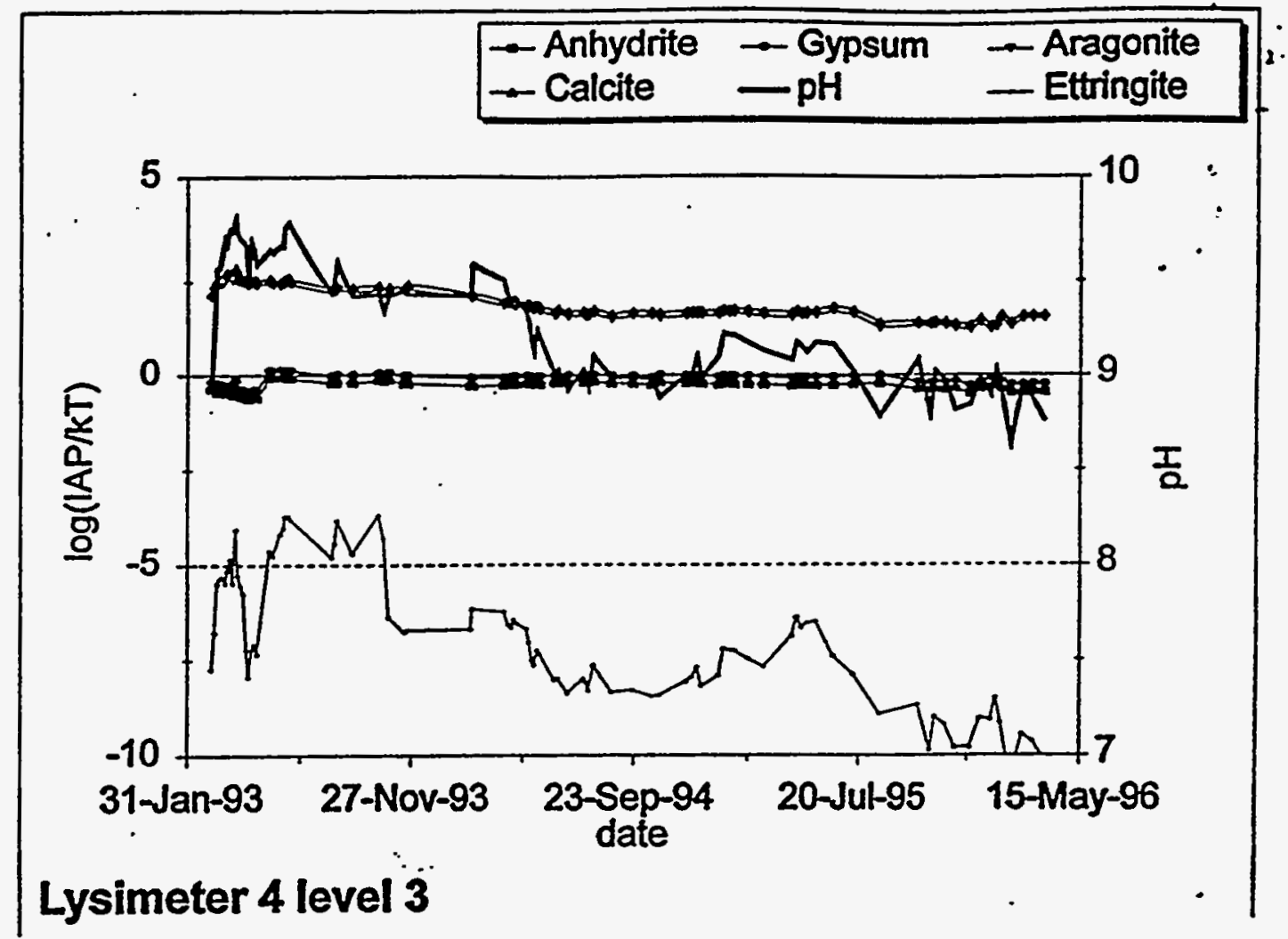

Figure 6-21. Anhydrite, Gypsum, Aragonite, Calcite, and Ettringite variations by date and $\mathrm{pH}$ in Lysimeter 4, Level 3

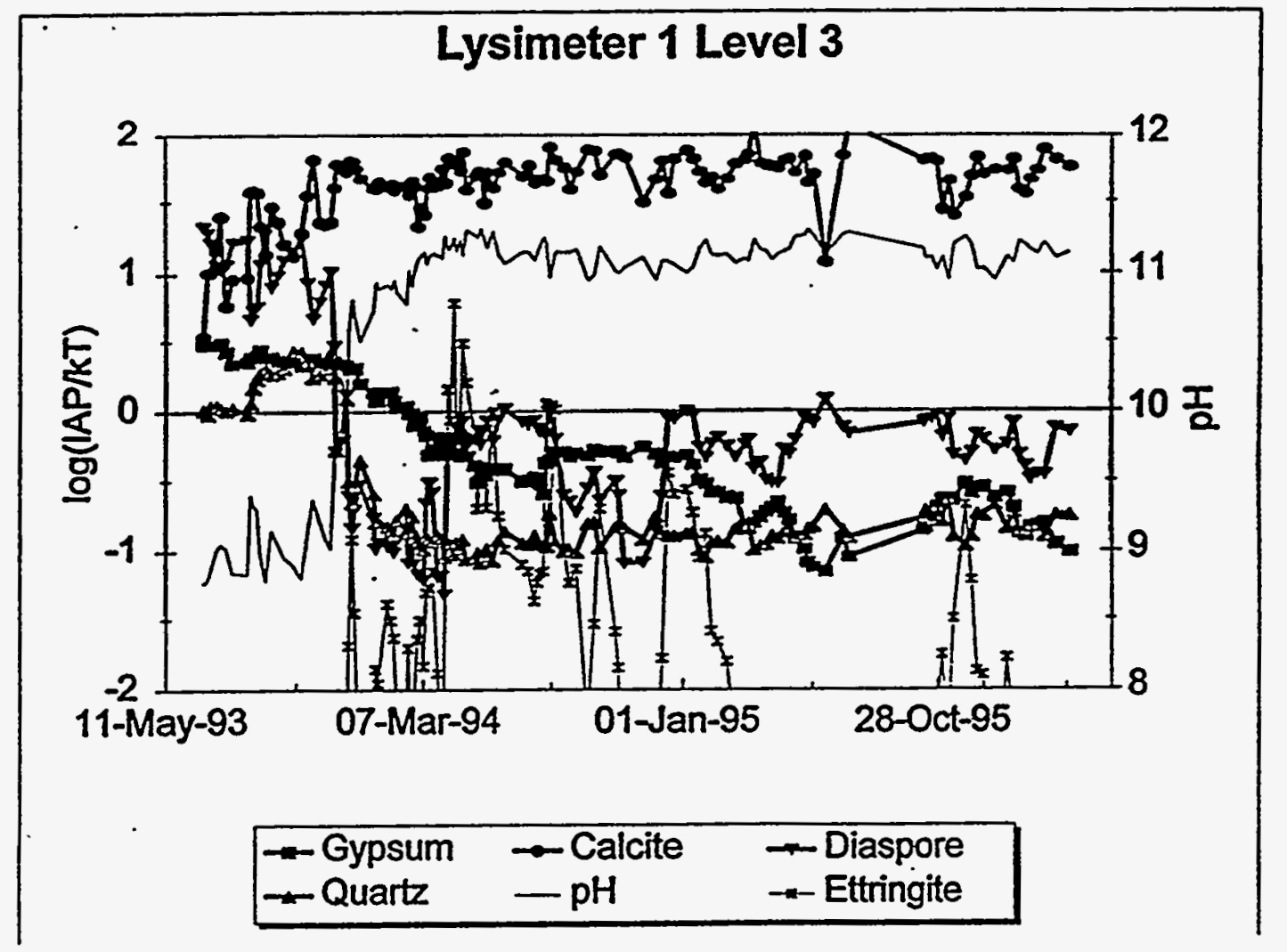

Figure 6-22. Gypsum, Calcite, Diaspore, Quartz, and Ettringite variations by date and $\mathrm{pH}$ in Lysimeter 1, Level 3 
Another source of Sulfate is the dissolution of Epsomite $\left(\mathrm{MgSO}_{4}{ }^{*} 7 \mathrm{H}_{2} \mathrm{O}\right.$ ) (Figures 6-10 through 6-12). It is extremely soluble and is expected to contribute to the very high initial Sulfate concentration in the leachate but is not expected to be present or control chemistry except initially.

Ettringite. $\left(\mathrm{Ca}_{6} \mathrm{Al}_{2}\left(\mathrm{SO}_{4}\right)_{3}(\mathrm{OH})_{12}{ }^{*} 26 \mathrm{H}_{2} \mathrm{O}\right)$ is commonly found in $\mathrm{CaO}-\mathrm{Al}_{2} \mathrm{O}_{3}-\mathrm{CaSO}_{4}-\mathrm{H}_{2} \mathrm{O}$ systems on mineral surfaces and in pore spaces. It is partly decomposed by water to an alkali. Because of its implications in swell and indications of presence in XRD results, it was added to the program calculations. While in the proper $\mathrm{pH}$ range, Ettringite formation will be controlled by the availability of Calcium, Sulfate and Aluminum and by competition with other minerals for these ions. Comparison of solubility indices of Calcite and Gypsum show no obvious correlation (Figures 6-22 and 6-23). This is expected, as the solute contains an abundance of free Sulfate and Calcium, as well as $\mathrm{OH}$. It was considered probable, therefore, that Ettringite formation is controlled by the availability of soluble Aluminum. Diaspore (AIOOH) is the basic oxide of $\mathrm{Al}_{2} \mathrm{O}_{3}$ formed by hydration. Further hydration in a high $\mathrm{pH}$ solution yields $\mathrm{Al}(\mathrm{OH})_{4}$, the major ionic carrier of available Aluminum. However, comparison of Ettringite saturation and Diaspore (Figures 6-22 and 6-23) and $\mathrm{Al}(\mathrm{OH})_{4}^{-}$(Figure 6-24) also reveals no obvious correlation. Also, comparison of Ettringite (Figure 6-25) and Diaspore (Figure 6-26) for second laboratory column lysimeters 1 through 6, which comprise a wide range of packing density and $\mathrm{CO}_{2}$ regimes, shows no correlation. It is concluded that the precipitation of Ettringite is a locally controlled phenomenom rather than a function of the bulk lysimeter/solute thermodynamics.

Other Sulfate minerals will be mentioned below.

Ca and $M q$. Carbonates and Hydroxides. The interactions between these drive much 


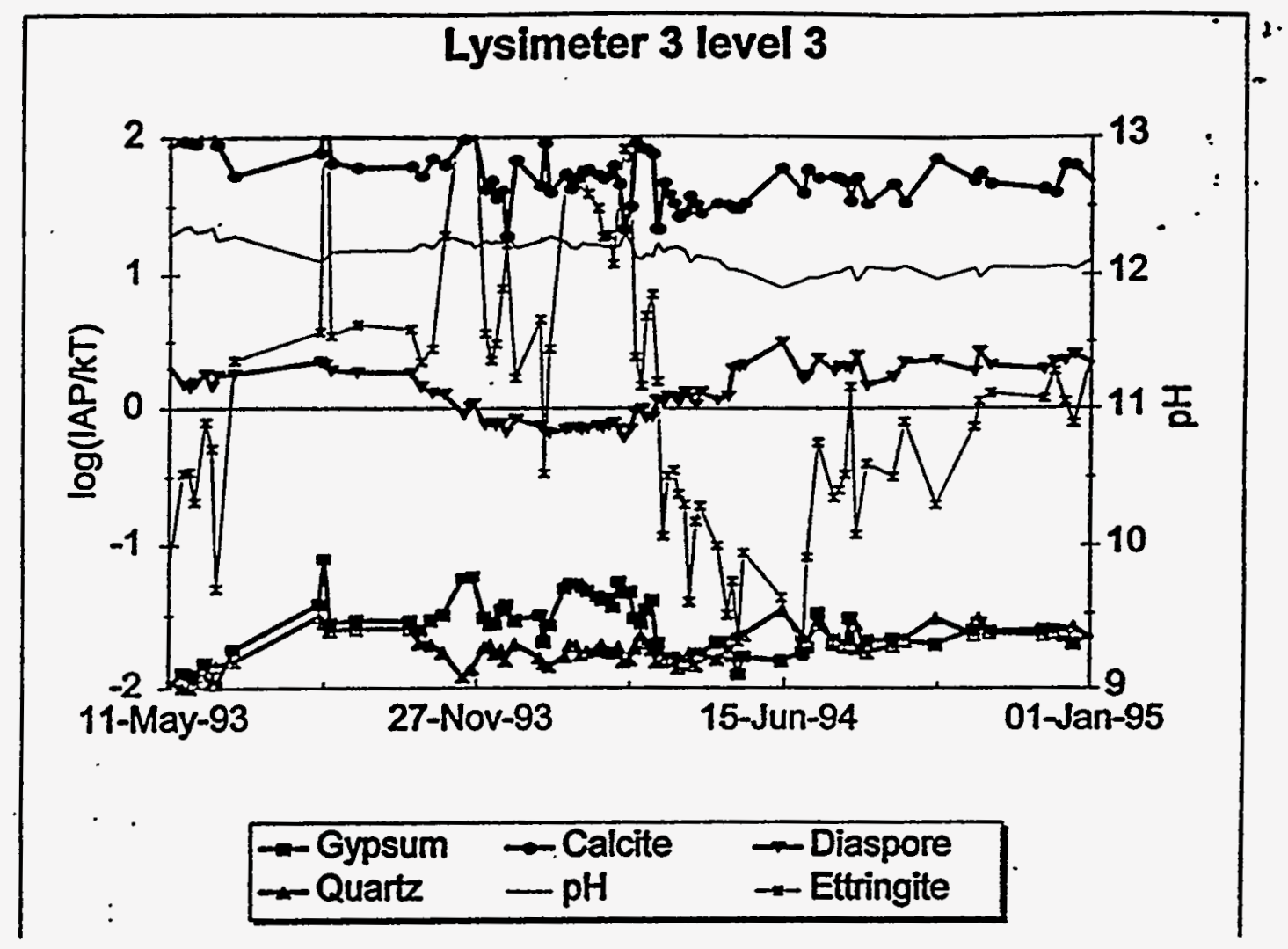

Figure 6-23. Gypsum, Calcite, Diaspore, Quartz, and Ettringite variations by date and $\mathrm{pH}$ in Lysimeter 3, Level 3

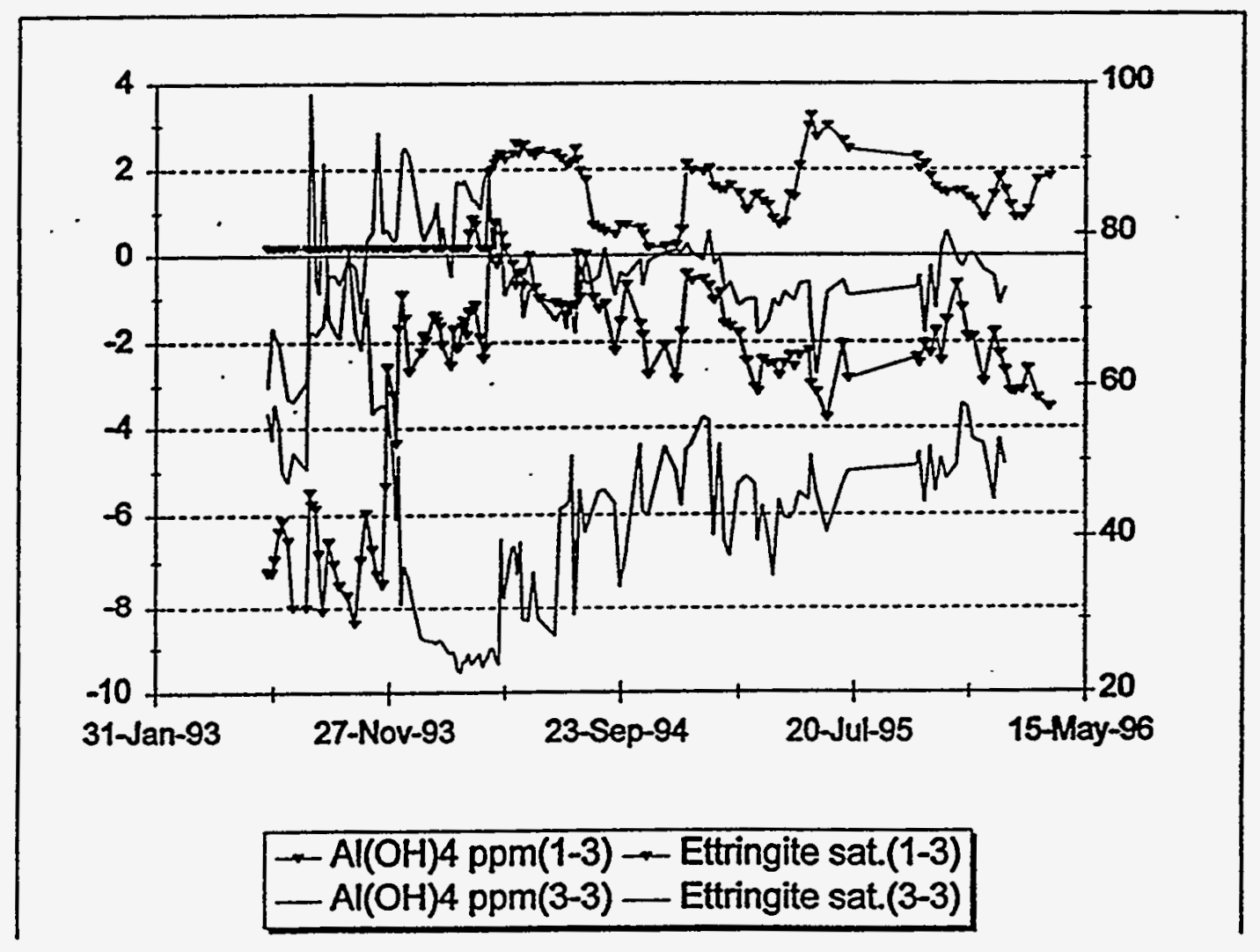

Figure 6-24. Comparison of Ettringite saturation and $\mathrm{Al}(\mathrm{OH})_{4}$ variations over time 


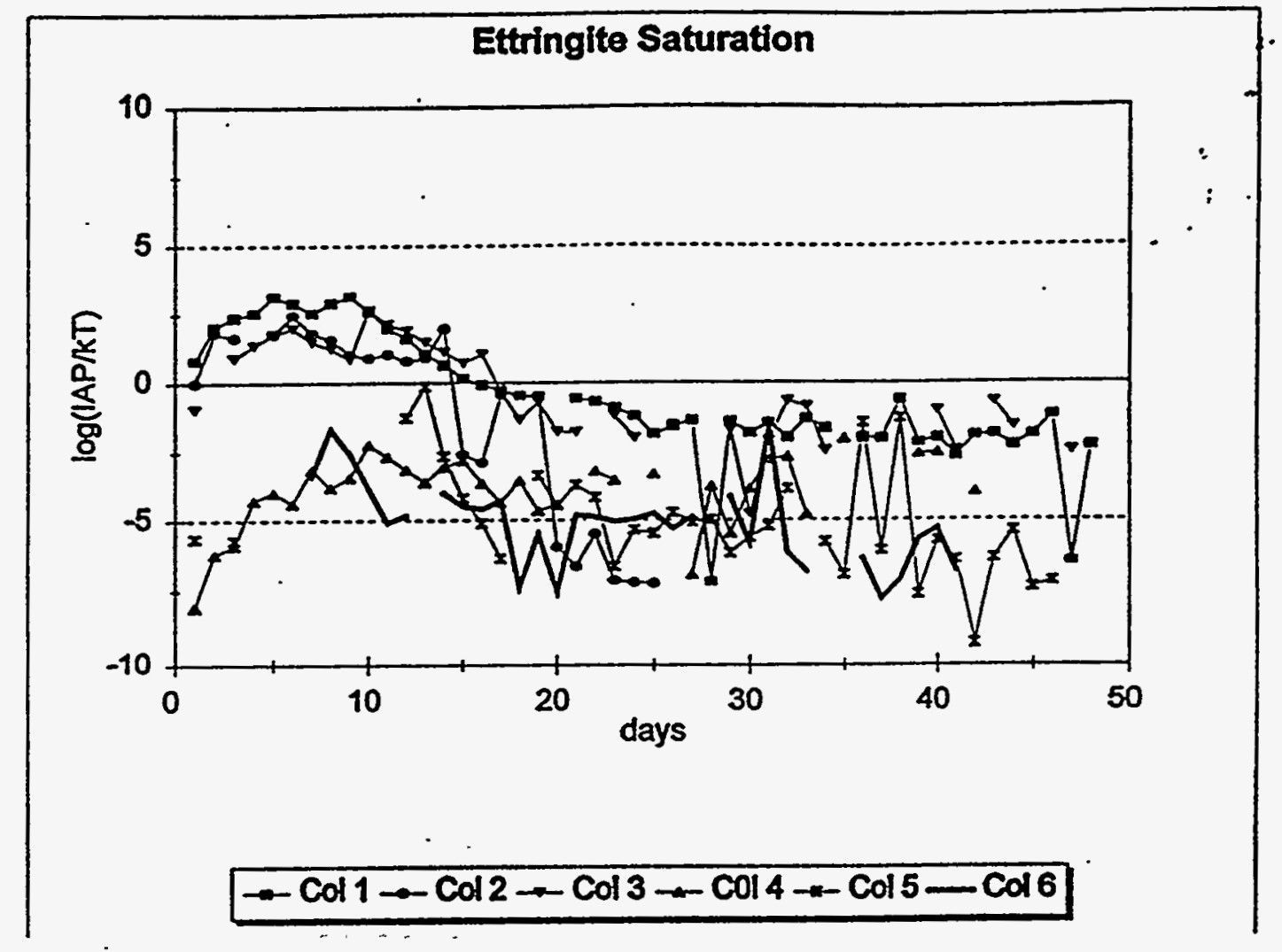

Figure 6-25. Ettringite saturation variations for second laboratory column lysimeters 1 through 6 over 50 days

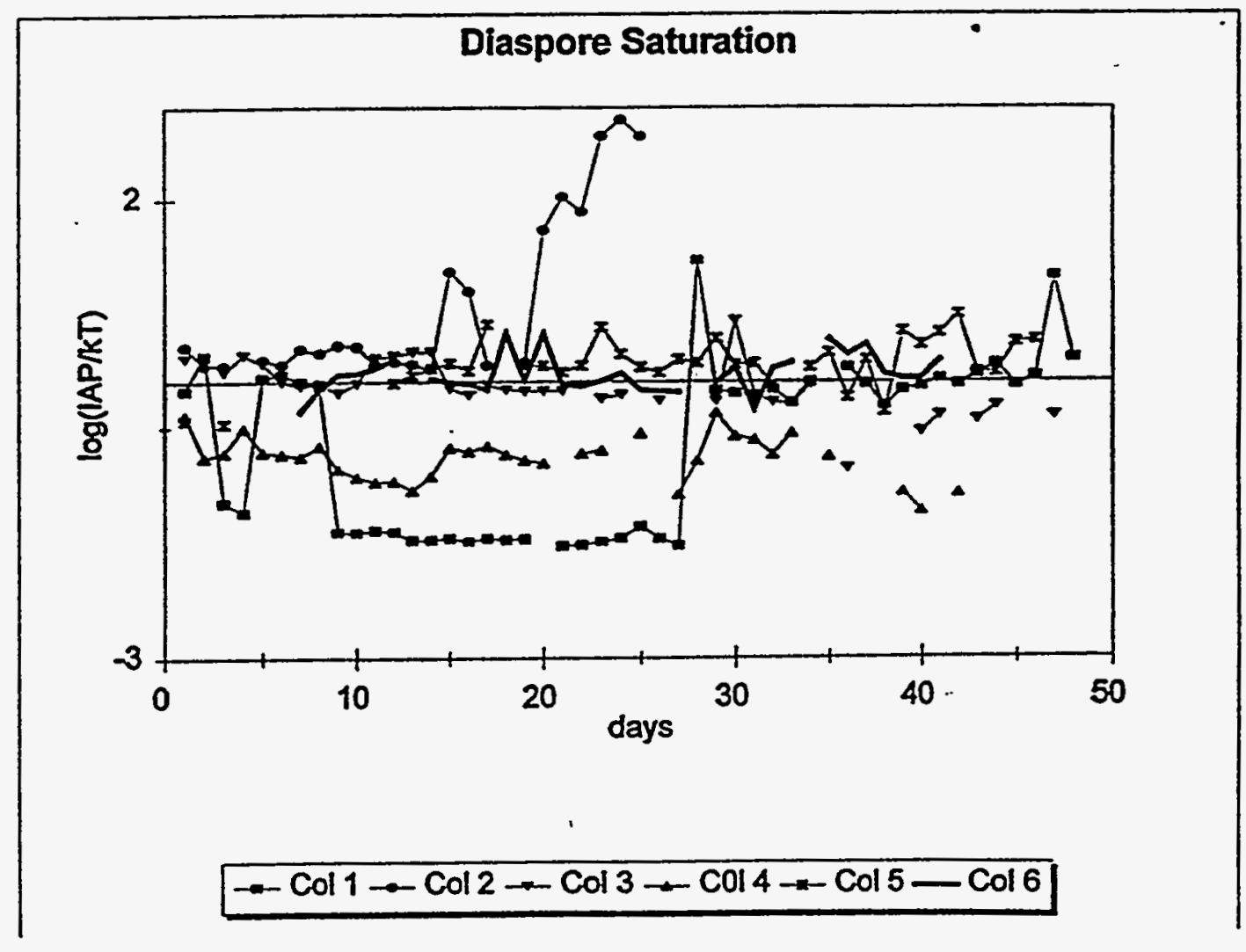

Figure 6-26. Diaspore saturation variations for second laboratory column lysimeters 1 through 6 over 50 days 
of the leachate chemistry: $\mathrm{Ca}$ because of its abundance, $\mathrm{Mg}$ because of its interchangability with $\mathrm{Ca}$, when available, and Carbonates and Hydroxides because of their implication in $\mathrm{pH}$. Possible major mineral forms which WATEQ indicates are often or always supersaturated are Calcite $\left(\mathrm{CaCO}_{3}\right)$, Aragonite $\left(\mathrm{CaCO}_{3}\right)$, Dolomite $\left(\mathrm{CaMg}\left(\mathrm{CO}_{3}\right)_{2}\right)$, Brucite $\left(\mathrm{Mg}(\mathrm{OH})_{2}\right)$, Hydromagnesite $\left(\mathrm{Mg}_{5}\left(\mathrm{CO}_{3}\right)_{4}(\mathrm{OH})_{2}{ }^{*} 4 \mathrm{H}_{2} \mathrm{O}\right)$, Magnesite $\left(\mathrm{MgCO}_{3}\right)$, dimorphic $\mathrm{MgCO}_{3}$, and Huntite $\left(\mathrm{Mg}_{3} \mathrm{Ca}\left(\mathrm{CO}_{3}\right)_{4}\right)$. Periclase $(\mathrm{MgO})$ hydrates to Brucite, which may further alter to Magnesite and Hydromagnesite. A precipitate of the hydrate Nesquehonite $\left(\mathrm{MgCO}_{3}{ }^{\star}{ }^{3} \mathrm{H}_{2} \mathrm{O}\right)$, whose slight solubility is enhanced by the presence of $\mathrm{CO}_{2}, \mathrm{NaCl}$, and $\mathrm{Na}_{2} \mathrm{SO}_{4}$, may also be formed at STP. To determine which forms are likely at leachate conditions, an equilibrium diagram which uses log[(activity $\mathrm{Ca}) /($ activity $\mathrm{Mg})]$ vs $\log \left(\mathrm{pCO}_{2}\right)$ to predict equlibrium conditions was plotted over the leachate collection periods (Figures 6-27 and 6-28). It was found that the Calcitic domain is favored for Coolside lysimeters, with brief movements into Brucite, Aragonite or Dolomitic regimes. For fly ashes, with a lower $\mathrm{Ca} / \mathrm{Mg}$ ratio, most of the collection period remains in the Dolomitic regime.

The conditions under which Dolomite $\left(\mathrm{CaMg}\left(\mathrm{CO}_{3}\right)_{2}\right)$ is precipitated in nature are not well understood. Attempts to precipitate a Dolomite phase from an oversaturated solution have not been successful. It is possible, however, that Dolomite might be formed under conditions of oversaturation and high ionic strength found in Coolside leachate but this is not confirmed. It is more likely that Dolomite is present originally due to the Coolside Sulfur control regime. Artinite $\left(\mathrm{Mg}_{2} \mathrm{CO}_{3}(\mathrm{OH})_{2}{ }^{*} 3 \mathrm{H}_{2} \mathrm{O}\right)$ is significantly favored at $\mathrm{pH}$ above about 9.5 in the Coolside lysimeters (Figures 6-29 and 6-30) and briefly favored at the beginning of fly ash lysimeter sampling (Figure 6-31). It is one of the few mineral forms which may be detectable by XRD in the Coolside samples. Forsterite $\left(\mathrm{Mg}_{2} \mathrm{SiO}_{4}\right)$ formation is indicated in minor amounts only in the most packed lysimeter (Figures 6-13 through 6-15). Sepiolite $\left(\mathrm{Mg}_{4} \mathrm{Si}_{6} \mathrm{O}_{15}(\mathrm{OH})_{2}{ }^{*} 6 \mathrm{H}_{2} \mathrm{O}\right)$ only rarely becomes supersaturated and should rarely form (Figures 6-10 through 6-12). The 


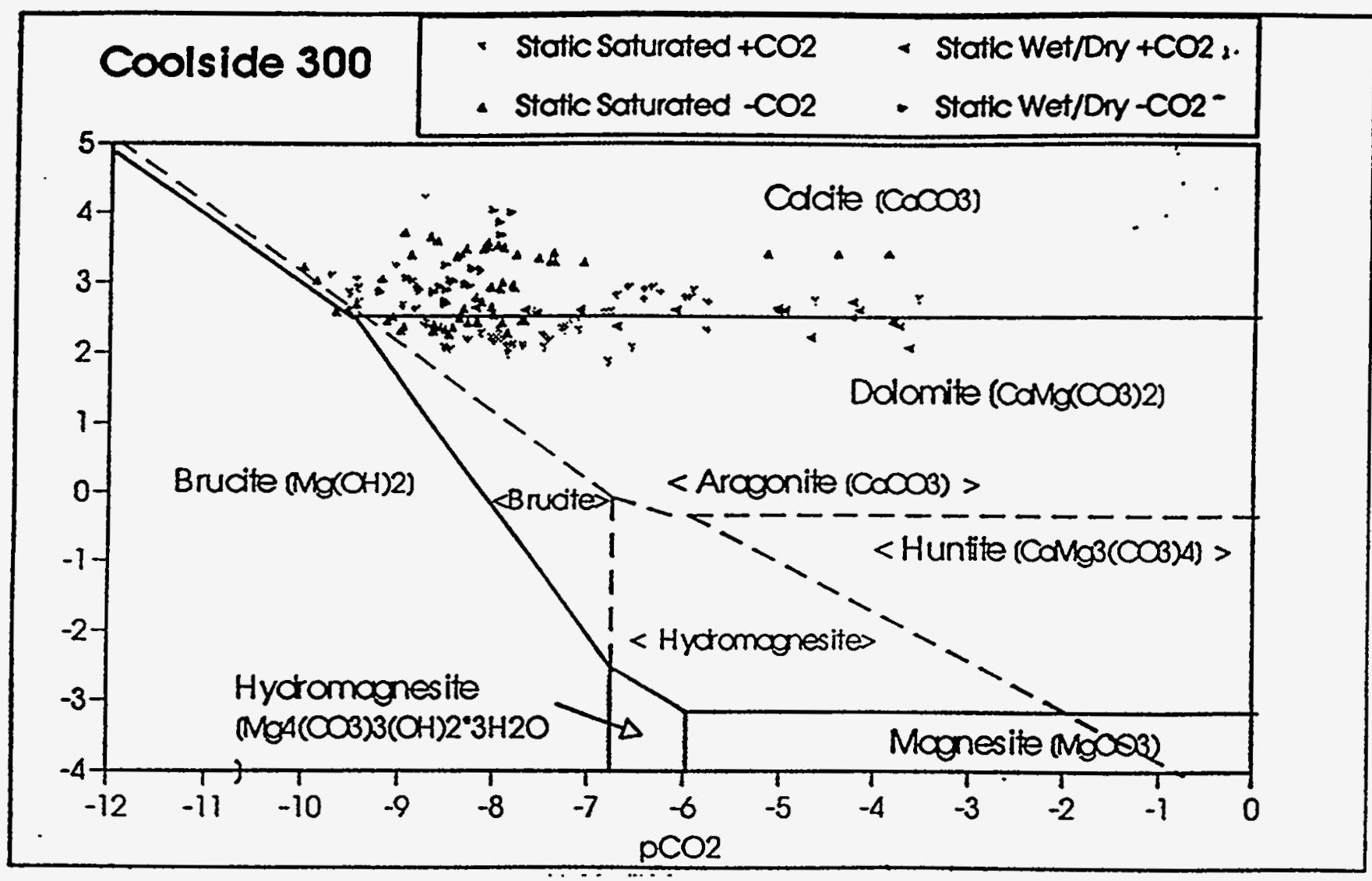

Figure 6-27. Representative Coolside equilibrium - $\log [(\mathrm{Ca}$ activity)/(Mg activity)] vs $\log \left(\mathrm{pCO}_{2}\right)$

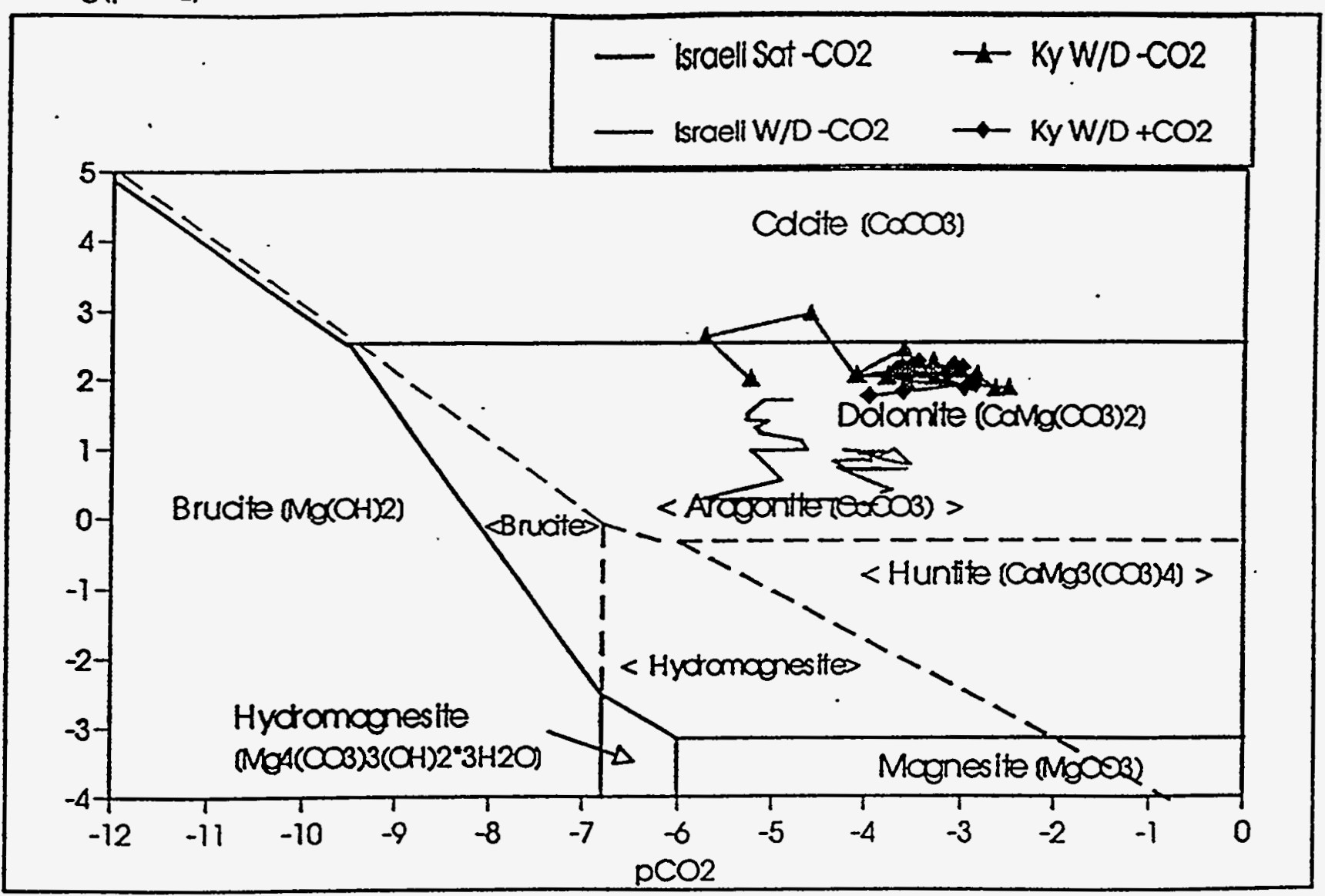

Figure 6-28. Fly ash equilibrium diagram - $\log [(\mathrm{Ca}$ activity $) /(\mathrm{Mg}$ activity $)]$ vs $\mathrm{pCO}_{2}$ 


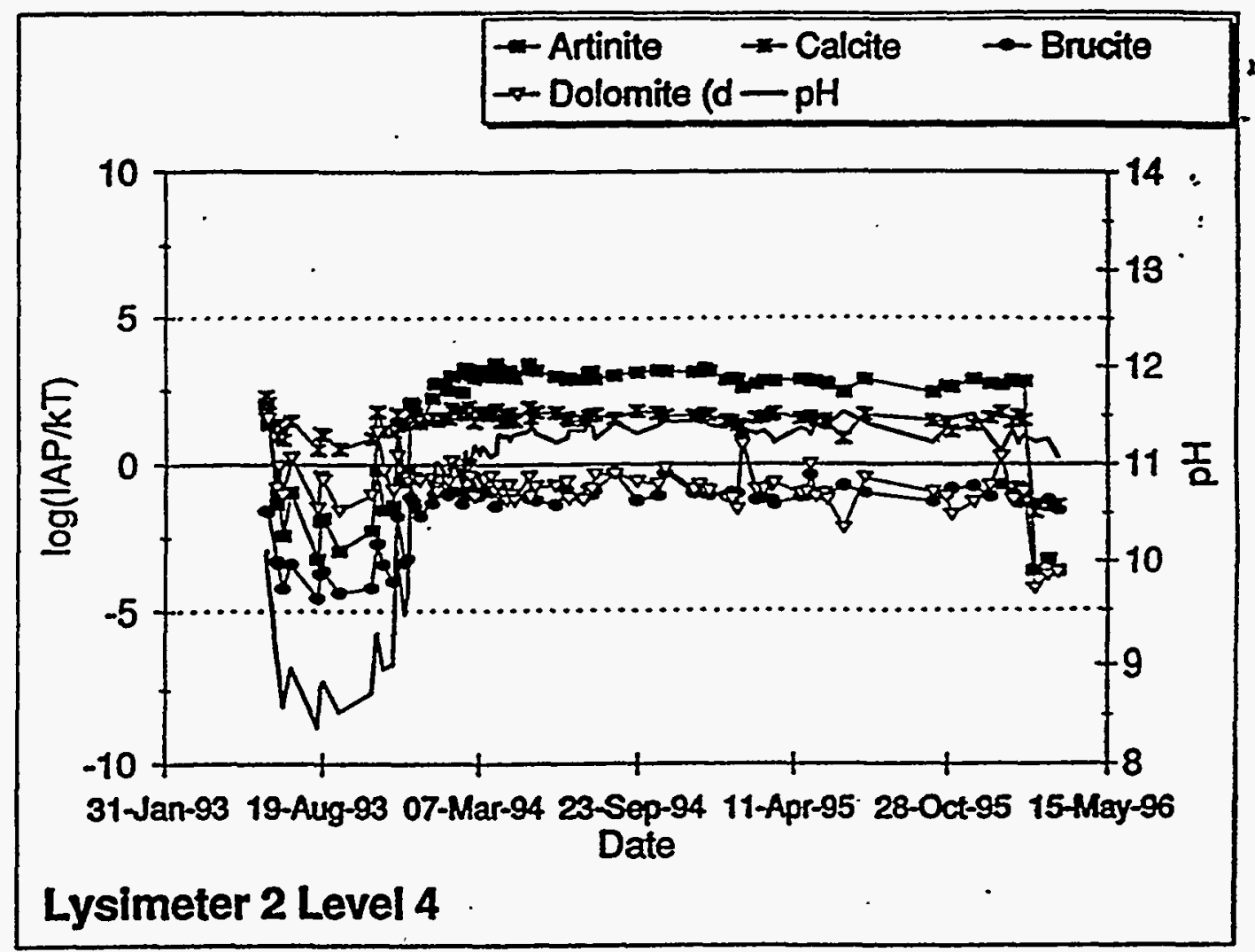

Figure 6-29. Artinite, Calcite, Brucite, and Dolomite variations by date and $\mathrm{pH}$ in Lysimeter 2, Level 4

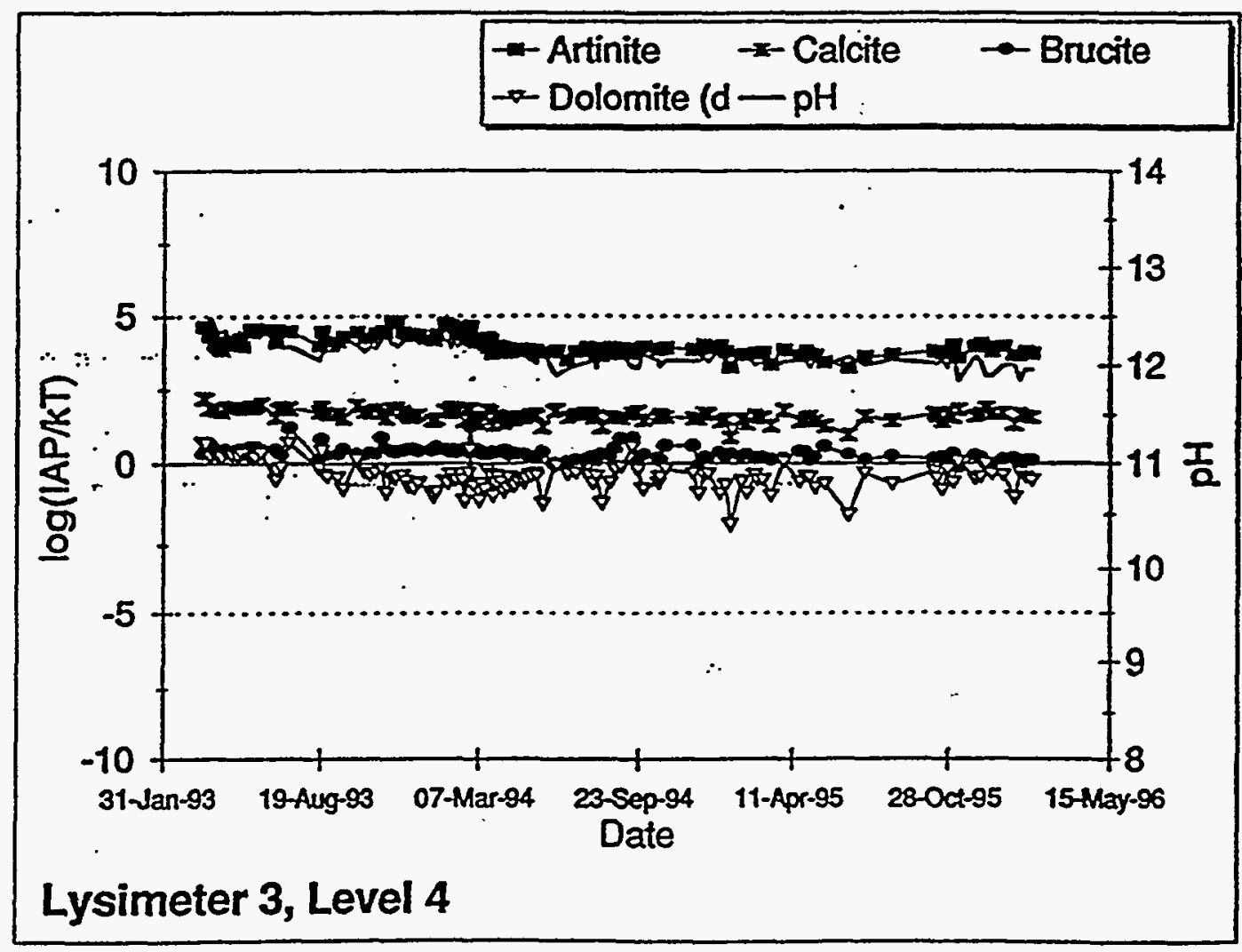

Figure 6-30. Artinite, Calcite, Brucite, and Dolomite variations by date and $\mathrm{pH}$ in Lysimeter 3, Level 4 


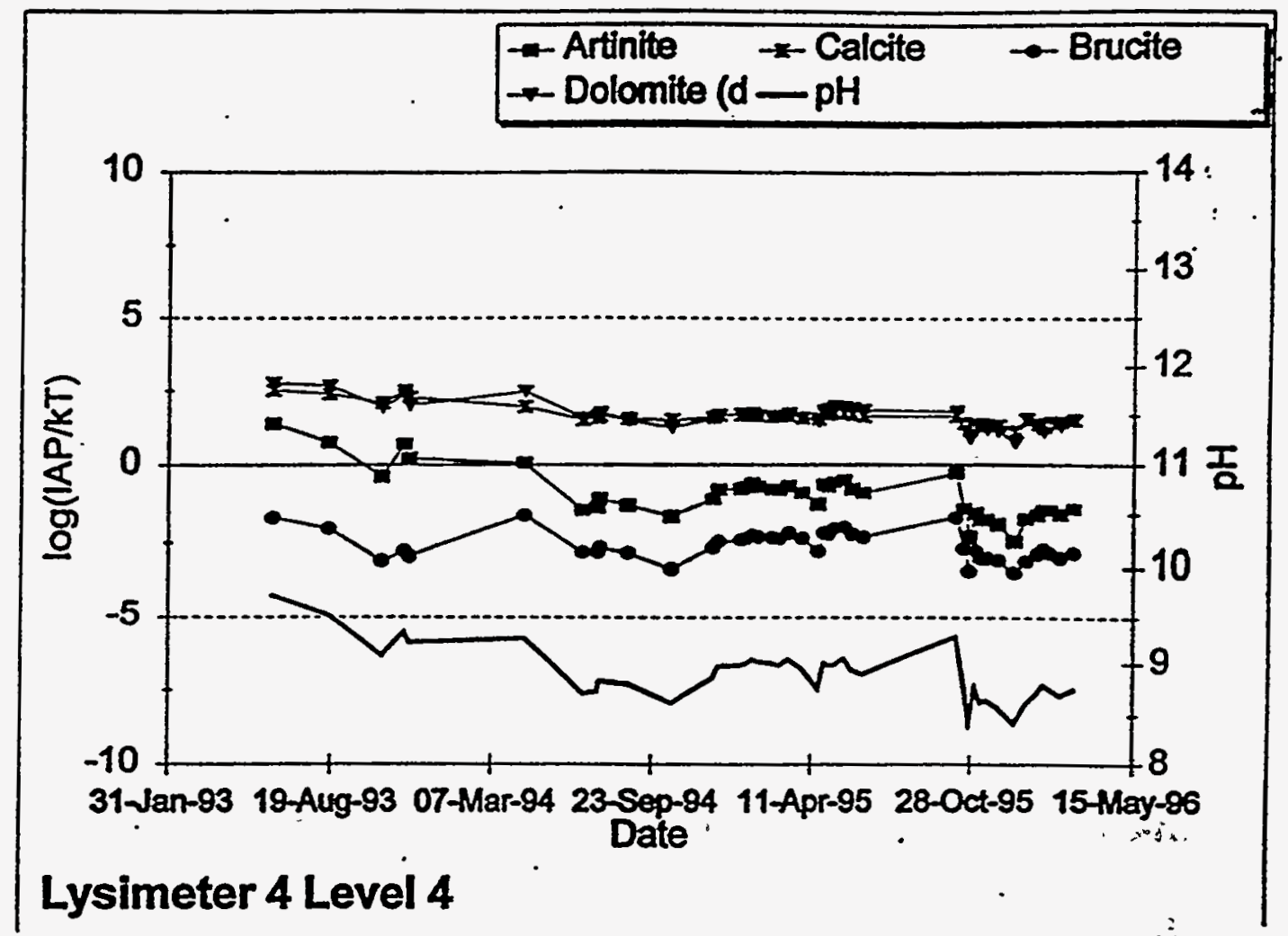

Figure 6-31. Artinite, Calcite, Brucite, and Dolomite variations by date and $\mathrm{pH}$ in Lysimeter 4, Level 4

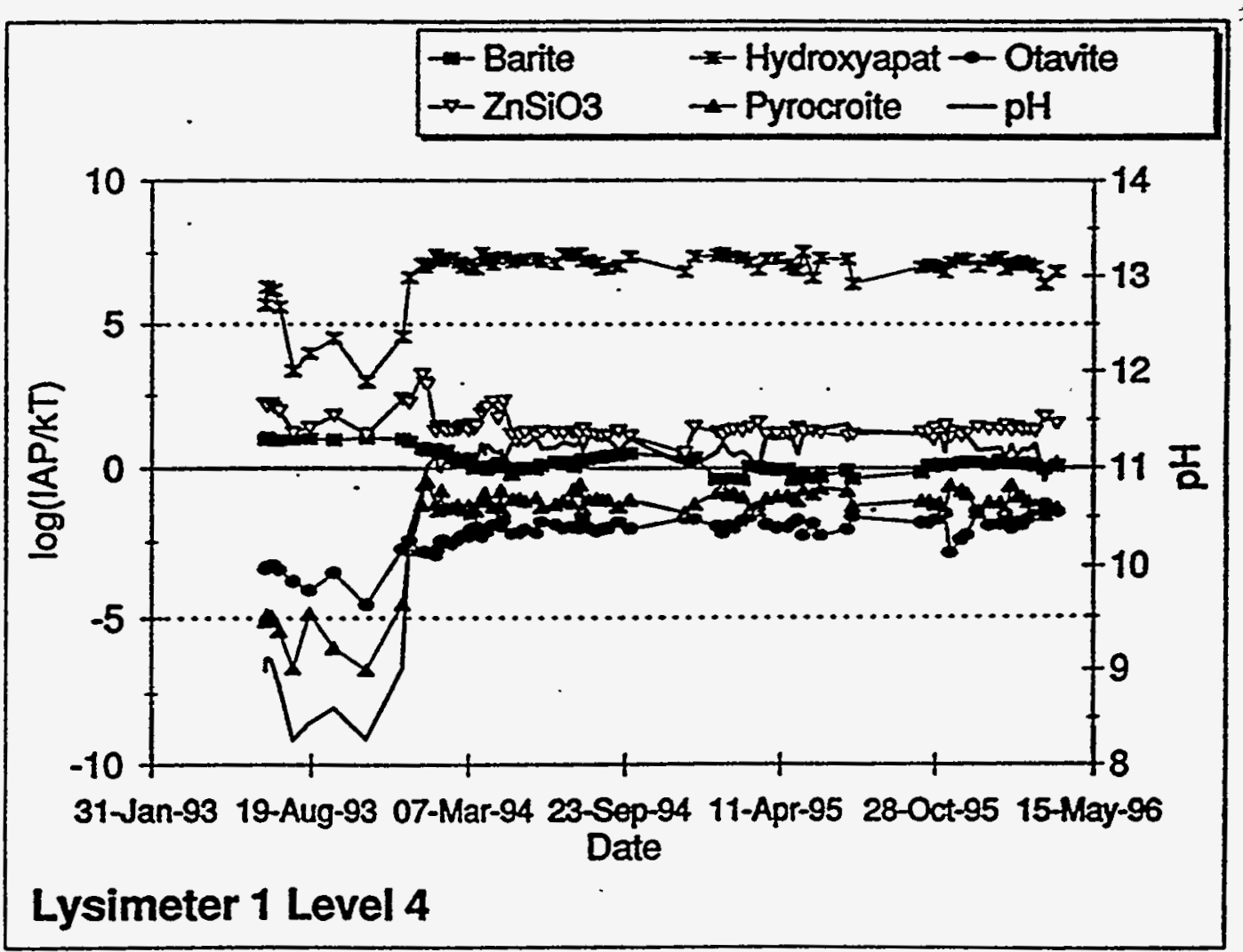

Figure 6-32. Barite, Hydroxylapatite, Otavite, $\mathrm{ZnSiO}_{3}$, and Pyrocroite variations by date and $\mathrm{pH}$ in Lysimeter 1, Level 4 
formation of Sepiolite would have been significant as its precipitation lowers $\mathrm{pH}$. Talc $\left(\mathrm{Mg}_{3} \mathrm{Si}_{4} \mathrm{O}_{10}(\mathrm{OH})_{2}\right)$, formed in high ionic strength brines at low temperature, is indicated to be supersaturated, but should not be formed, as Sepiolite tends io precipitate preferentially and is not found to form significantly. Brucite $\left(\mathrm{Mg}(\mathrm{OH})_{2}\right)$ shows a slight tendency to form only in the most packed lysimeter (Figures 6-29 through 6-31). Two other Calcium-Aluminum silicates, Prehnite $\left(\mathrm{Ca}_{2} \mathrm{Al}_{2} \mathrm{Si}_{3} \mathrm{O}_{10}(\mathrm{OH})_{2}\right)$ (Figures 6-16 through 6-18) and Laumontite $\left(\mathrm{CaAl}_{2} \mathrm{Si}_{4} \mathrm{O}_{12}{ }^{*} 4 \mathrm{H}_{2} \mathrm{O}\right)$ (Figures 6-1 through 6-3), show occasional supersaturation but neither their formation nor affect on leachate chemistry should be significant.

\section{Minor Species Carriers}

Mineral carriers of minor ions have little effect on leachate chemistry but are followed to predict which might be dominant at.points in the leachate cycle because the ions are of general interest. It is doubtful that any of these could be confirmed by XRD as the amounts would be very small. In general, the oxide, hydroxide, carbonate and silicate forms are indicated at appropriate times in the collection cycle.

Ba. Barite $\left(\mathrm{BaSO}_{4}\right)$ is relatively insoluble, but becomes more so in a salt solution. WATEQ indicates that it is sometimes slightly supersaturated at lower pH (Figures 6-32 through 6-34). Witherite $\left(\mathrm{BaCO}_{3}\right)$ is indicated to be supersaturated, but less often.

PO $_{4}$. Ca-Hydroxylapatite $\left(\mathrm{Ca}_{5}\left(\mathrm{PO}_{4}\right)_{3} \mathrm{OH}\right)$ is the major phosphate carrier. WATEQ indicates a fairly significant degree of supersaturation (Figures 6-32 through 6-34). If Fluorine were present, the Fluor-apatite would be thermodynamically preferred. It forms a solid solution with $\mathrm{Ca}>\mathrm{Pb}>\mathrm{Na}>\mathrm{K}$.

$\underline{P b}$. Litharge/Massicot ( $\mathrm{PbO}$ dimorphs) may hydrate and form minor amounts of $\mathrm{Pb}(\mathrm{OH})_{2}$ under high $\mathrm{pH}$ conditions (Figures 6-35 through 6-38). Other $\mathrm{Pb}$ minerals 


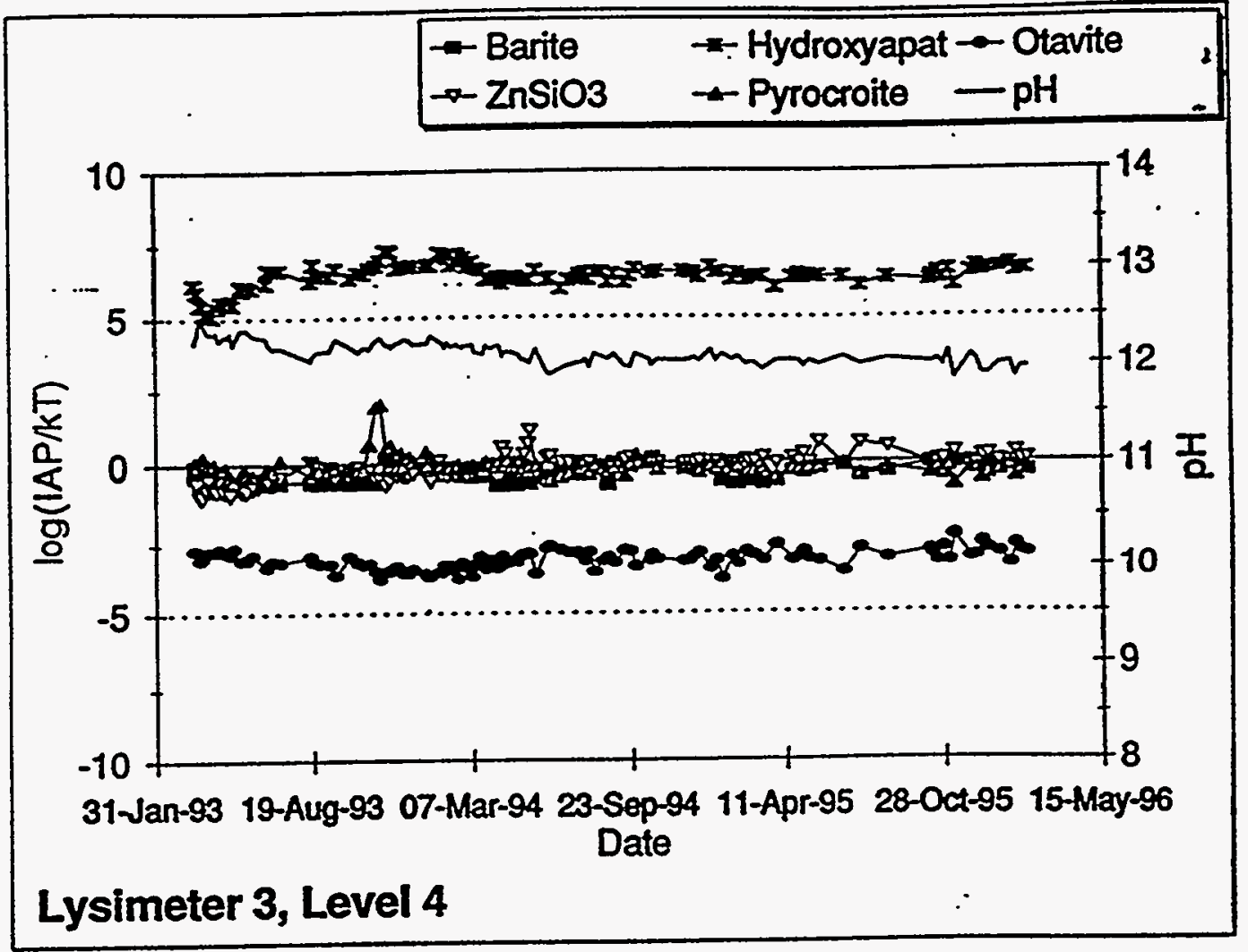

Figure 6-33. Barite, Hydroxylapatite, Otavite, $\mathrm{ZnSiO}_{3}$, and Pyrocroite variations by date and $\mathrm{pH}$ in Lysimeter 3, Level 4

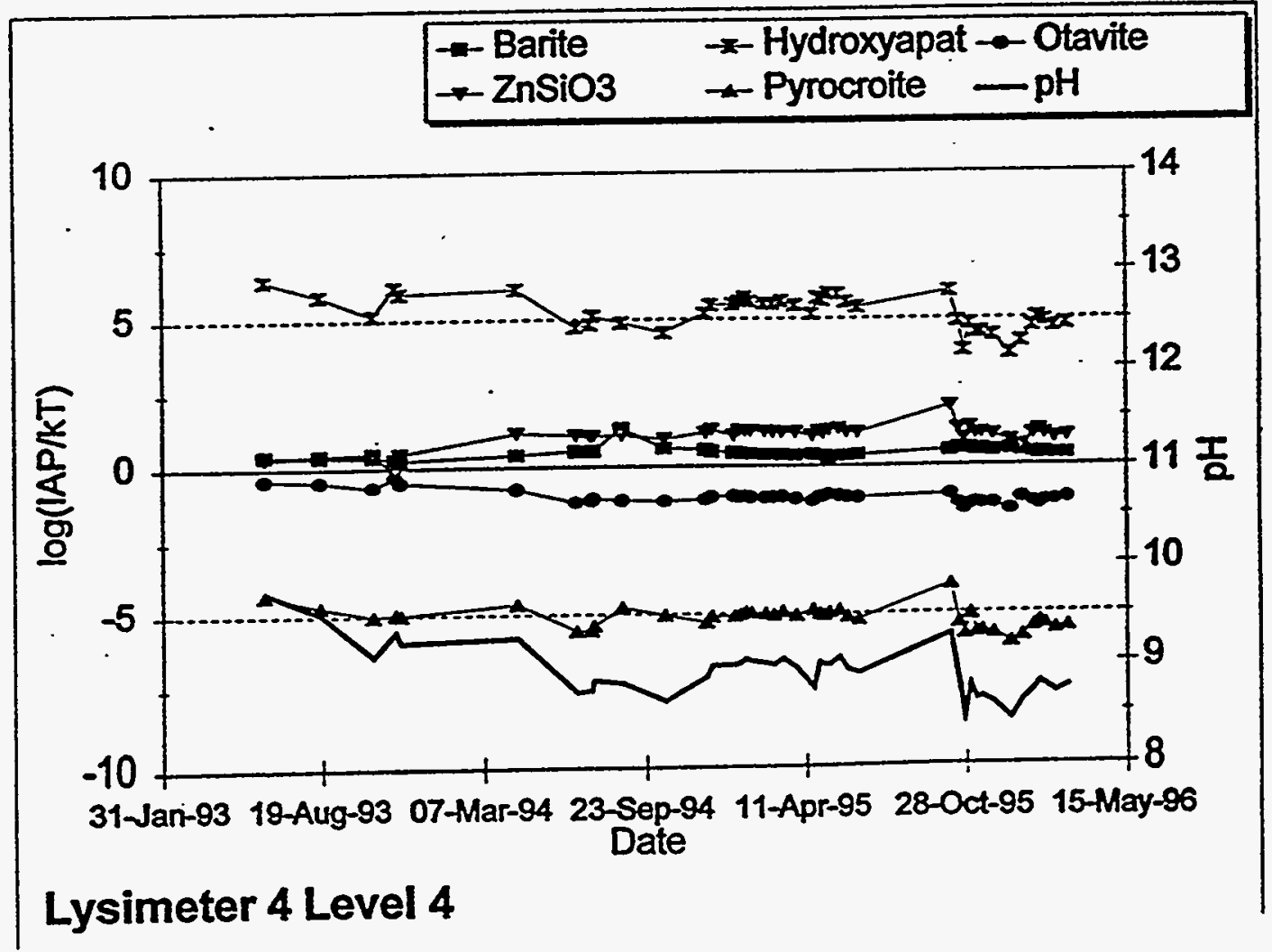

Figure 6-34. Barite, Hydroxylapatite, Otavite, $\mathrm{ZnSiO}_{3}$, and Pyrocroite variations by date and $\mathrm{pH}$ in Lysimeter 4, Level 4 


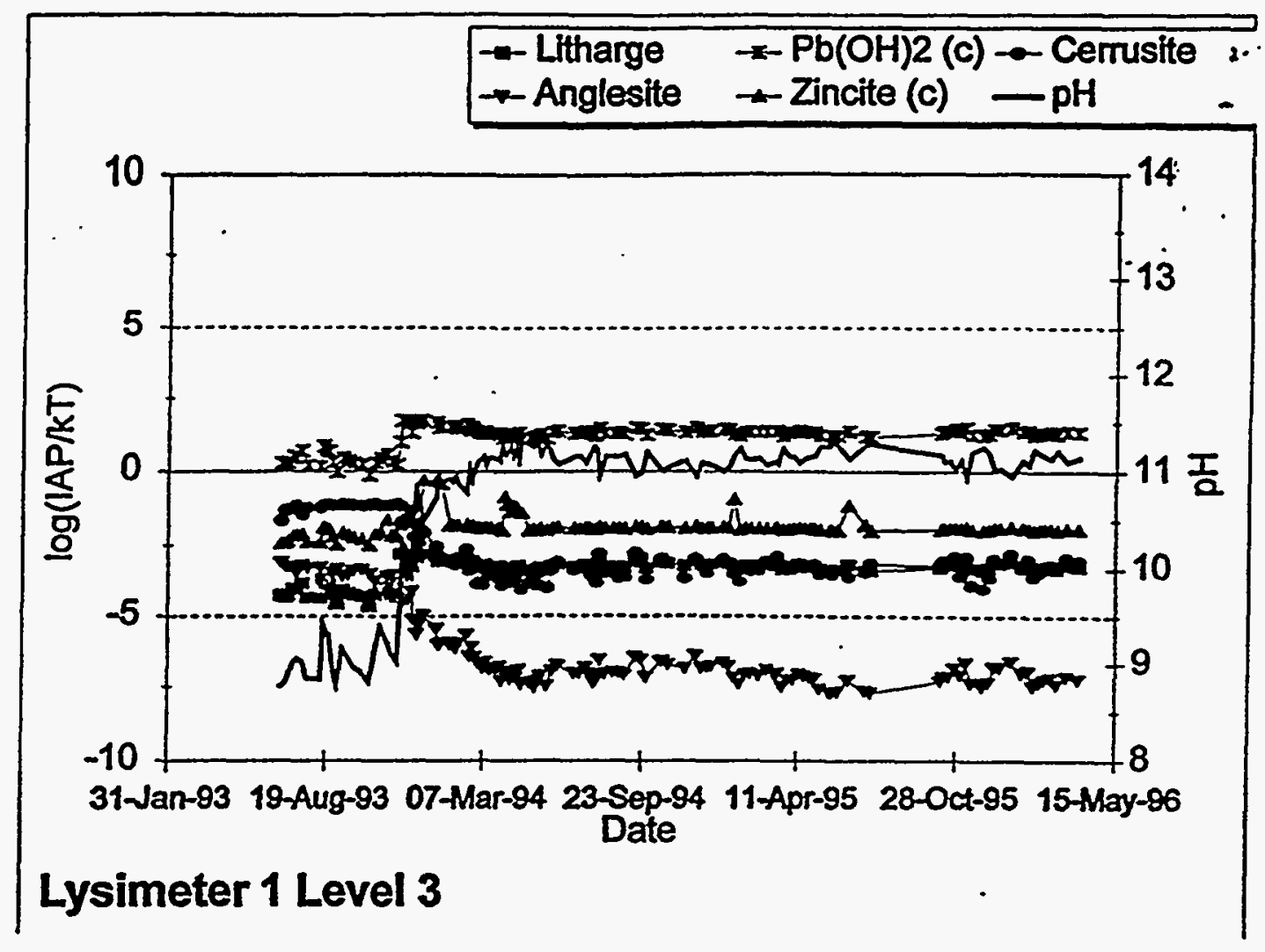

Figure 6-35. Litharge, $\mathrm{Pb}(\mathrm{OH})_{2}$, Cerrusite, Anglesite, and Zincite variations by date and $\mathrm{pH}$ in Lysimeter 1 , Level 3

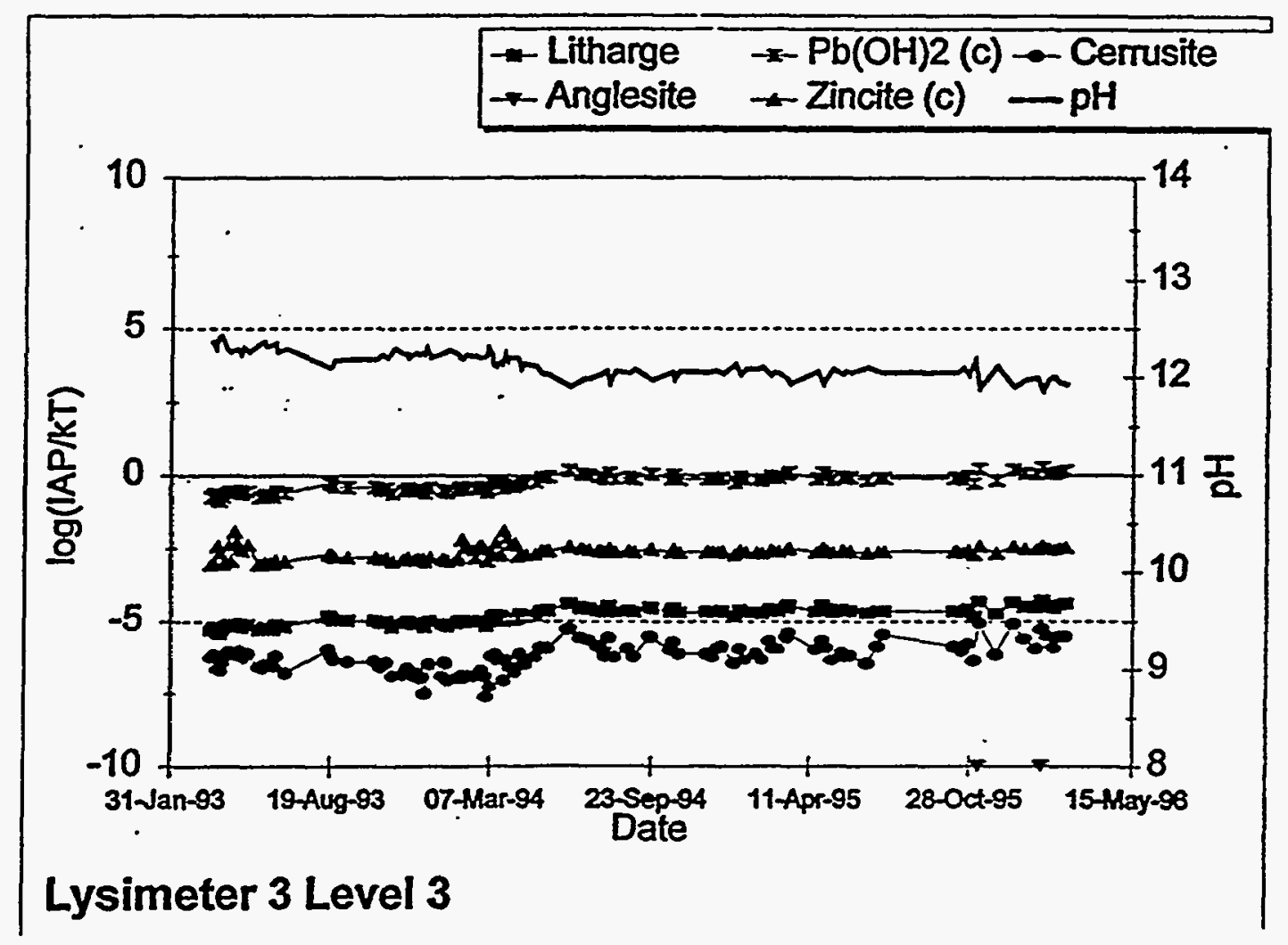

Figure 6-36. Litharge, $\mathrm{Pb}(\mathrm{OH})_{2}$, Cerrusite, Anglesite, and Zincite variations by date and $\mathrm{pH}$ in Lysimeter 3 , Level 3 


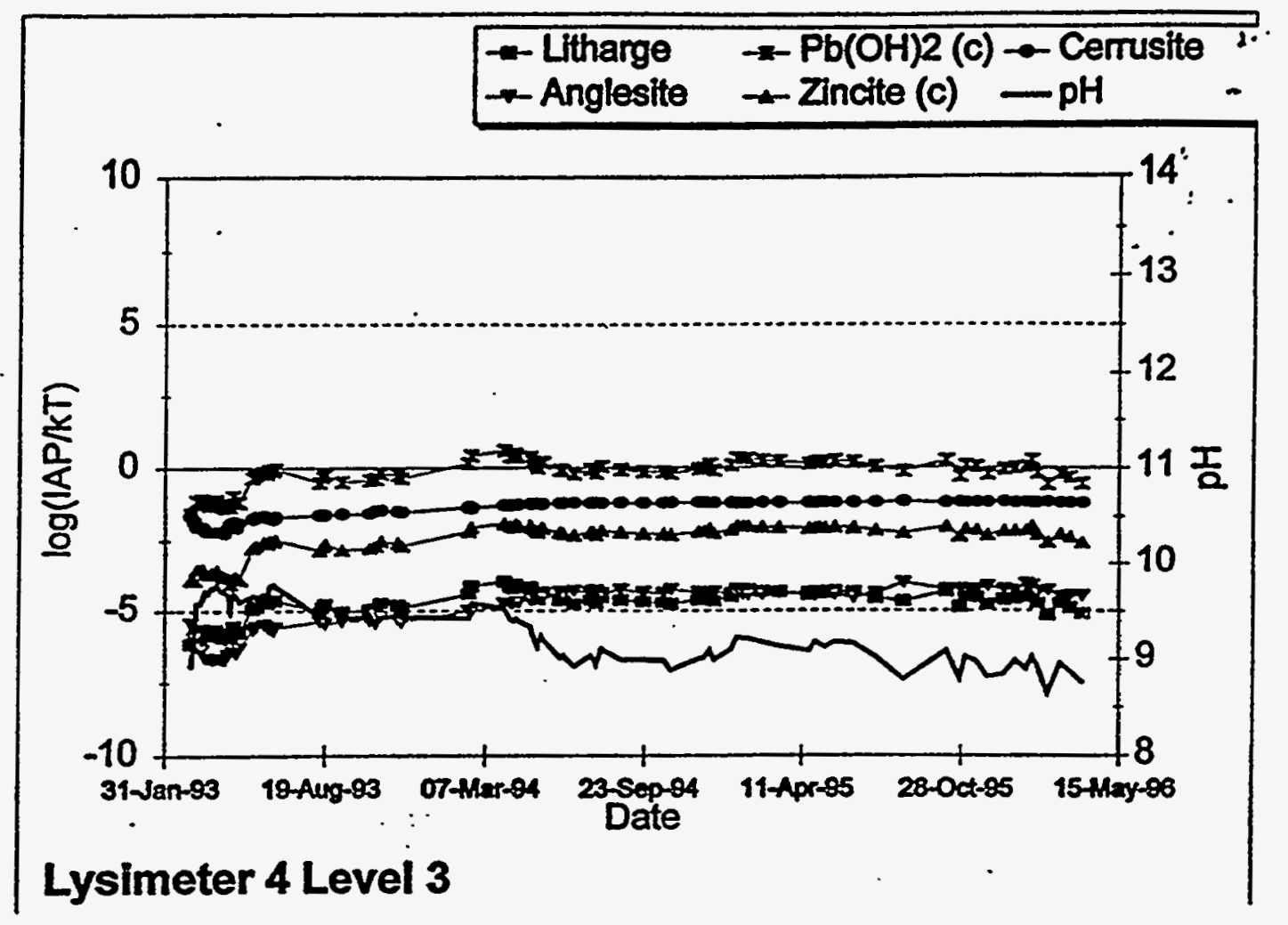

Figure 6-37. Litharge, $\mathrm{Pb}(\mathrm{OH})_{2}$, Cerrusite, Anglesite, and Zincite variations by date and $\mathrm{pH}$ in Lysimeter 4, Level 3

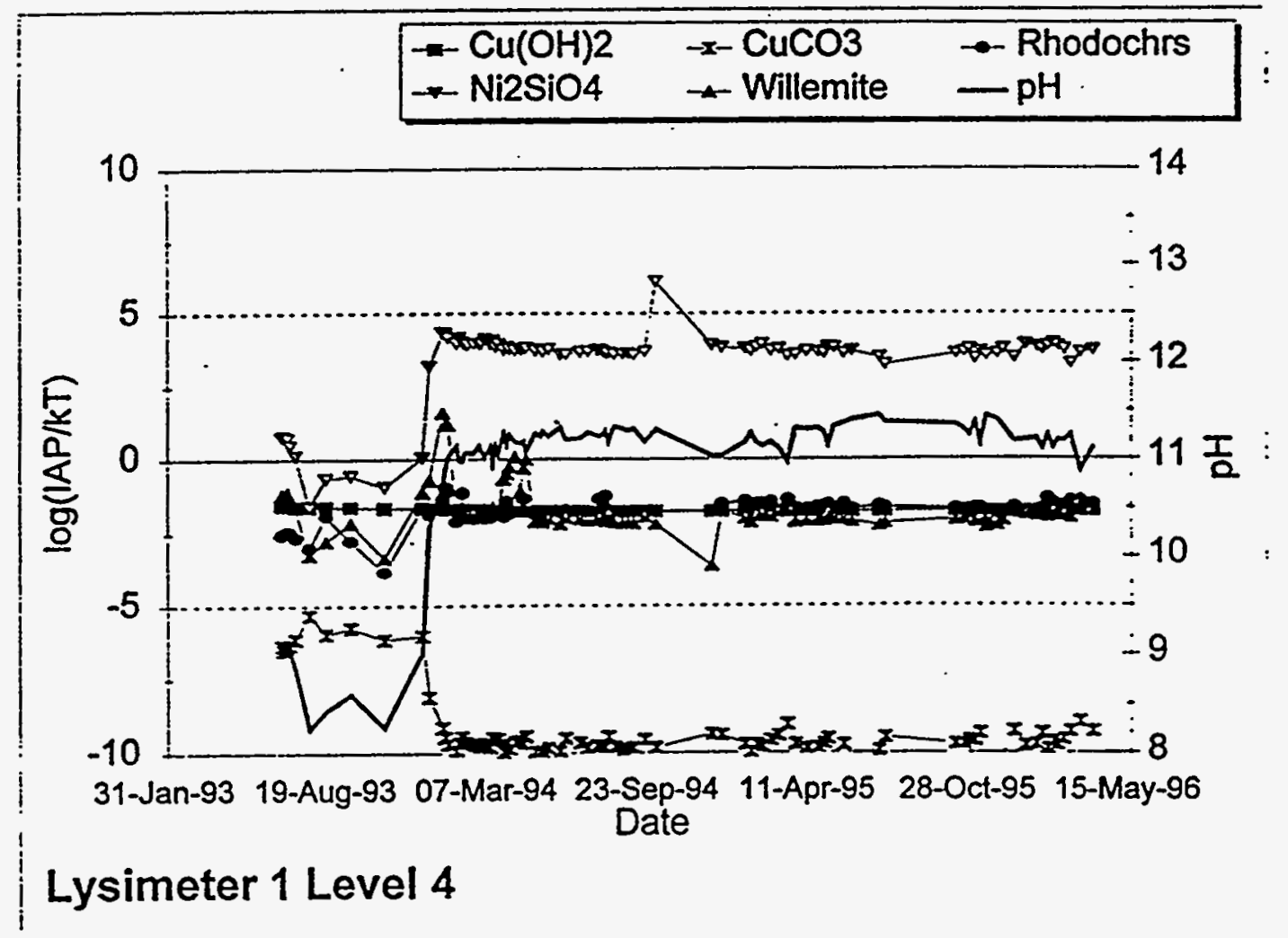

Figure 6-38. $\mathrm{Cu}(\mathrm{OH})_{2}, \mathrm{CuCO}_{3}$, Rhodocrosite, $\mathrm{Ni}_{2} \mathrm{SiO}_{4}$, and Willemite variations by date and $\mathrm{pH}$ in Lysimeter 1, Level 4 
possibly formed include Cerrusite $\left(\mathrm{PbCO}_{3}\right)$, Anglesite $\left(\mathrm{PbSO}_{4}\right)$ (Figures 6-35 through 638). Larnakite $\left(\mathrm{Pb}_{2}\left(\mathrm{SO}_{4}\right) \mathrm{O}\right)$, and Laurionite $(\mathrm{Pb}(\mathrm{OH}) \mathrm{Cl})$ are indicated as unlikely to form. $\mathrm{Pb}$ compounds, however, are not known for solubility.

Cu. Copper is probably initially available as Tenorite $(\mathrm{CuO})$ and slowly hydrated to free copper and hydroxide, as $\mathrm{Cu}(\mathrm{OH})_{2}$ remains undersaturated (Figures 6-38 through 640). Malachite $\left(\mathrm{Cu}_{2}\left(\mathrm{CO}_{3}\right)(\mathrm{OH})_{2}\right)$ (crystals rare, but slightly water- $\mathrm{CO}_{2}$ soluble), $\mathrm{CuCO}_{3}$ (Figures 6-38 through 6-41) and Atacamite $\left(\mathrm{Cu}_{2}(\mathrm{OH})_{3} \mathrm{Cl}\right)$ (another of the few $\mathrm{Cl}$ minerals; alters to malachite; formed by oxidation of other Cu minerals, especially in arid saline environments) are indicated as unlikely to form. Tsumebite $\left(\mathrm{CuPb}_{2}\left(\mathrm{PO}_{4}\right)\left(\mathrm{SO}_{4}\right)(\mathrm{OH})\right)$ is unlikely to be found even though supersaturation is indicated by WATEQ.

Cd. Monteponite (CdO), $\mathrm{Cd}(\mathrm{OH})_{2}, \mathrm{Cd}(\mathrm{OH}) \mathrm{Cl}, \mathrm{CdSiO}_{3}$ and Otavite $\left(\mathrm{CdCO}_{3}\right)$ (Figures 632 through 6-34) exhibit varying degrees of supersaturation. Otavite is supersaturated only in the fly ash system. In the Coolside leaching, the higher $\mathrm{Ca} / \mathrm{Mg}$ ratio leads to preferential Calcite formation.

$\mathrm{Zn}$. Zincite $(\mathrm{ZnO})$ may be present initially. It is indicated to remain in dissolution in all lysimeters (Figures 6-35 through 6-37). $\mathrm{Zn}(\mathrm{OH})_{2}$ and $\mathrm{ZnCO}_{3}{ }^{*} \mathrm{H}_{2} \mathrm{O}$ may form under appropriate $\mathrm{pH}$ conditions. $\mathrm{Zn}_{2}(\mathrm{OH})_{3} \mathrm{Cl}$ is unlikely. $\mathrm{ZnSiO}_{3}$ formation may take place in the least compacted lysimeters and in fly ash lysimeters (Figures 6-32 through 6-34). Willemite $\left(\mathrm{Zn}_{2} \mathrm{SiO}_{4}\right)$ is unlikely to form (Figures 6-38 through 6-40).

Mn. Pyrocroite $\left(\mathrm{Mn}(\mathrm{OH})_{2}\right)$ (Figures 6-32 through 6-34) and Rhodochrisite $\left(\mathrm{MnCO}_{3}\right)$ (Figures 6-38 through 6-40), as well as the oxide form, Pyrolusite, generally remain undersaturated, indicating that $\mathrm{Mn}$ from oxide dissolution should primarily contribute to solute ionic strength. 


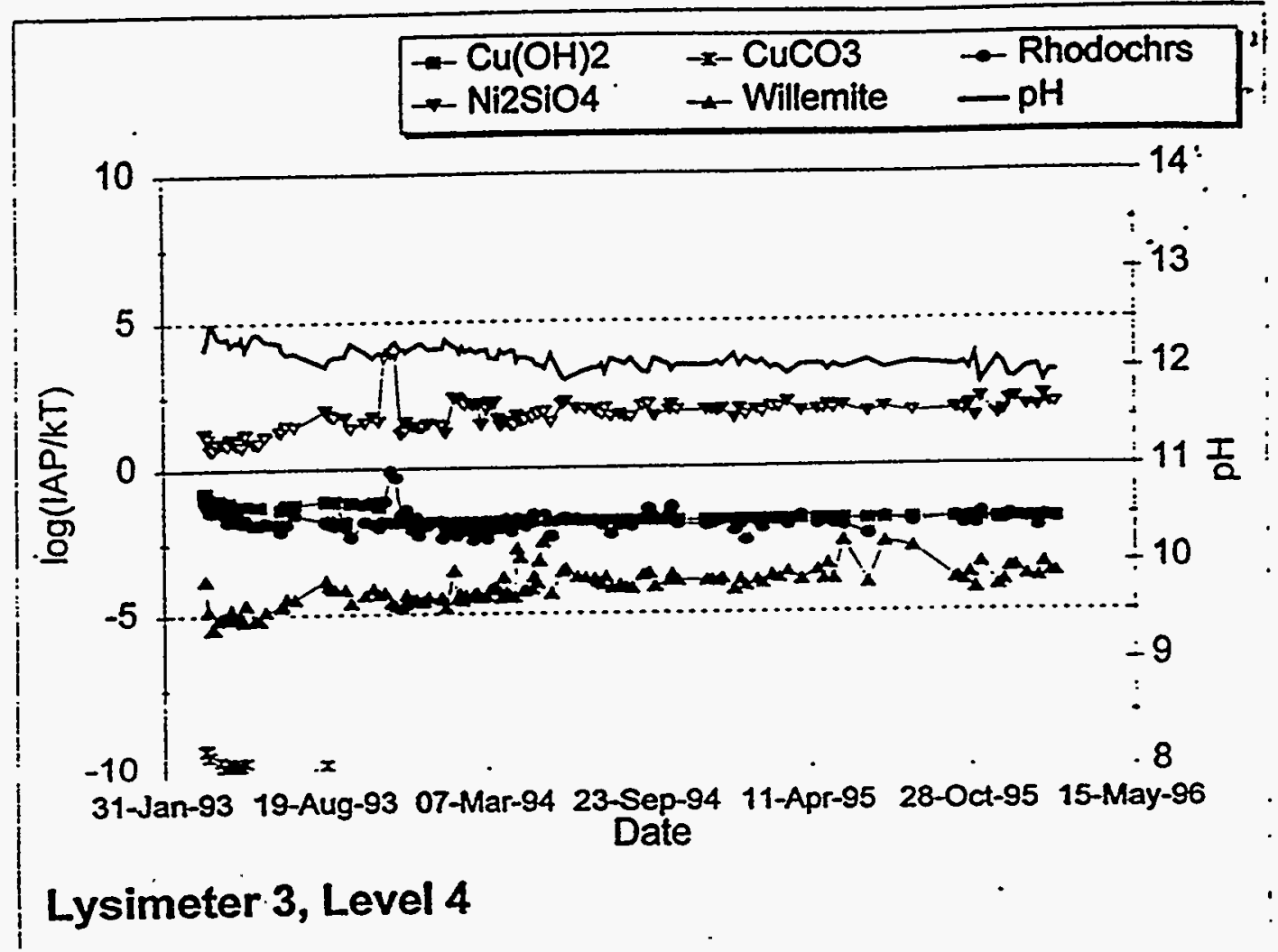

Figure 6-39. $\mathrm{Cu}(\mathrm{OH})_{2}, \mathrm{CuCO}_{3}$, Rhodocrosite, $\mathrm{Ni}_{2} \mathrm{SiO}_{4}$, and Willemite variations by date and $\mathrm{pH}$ in Lysimeter 3, Level 4

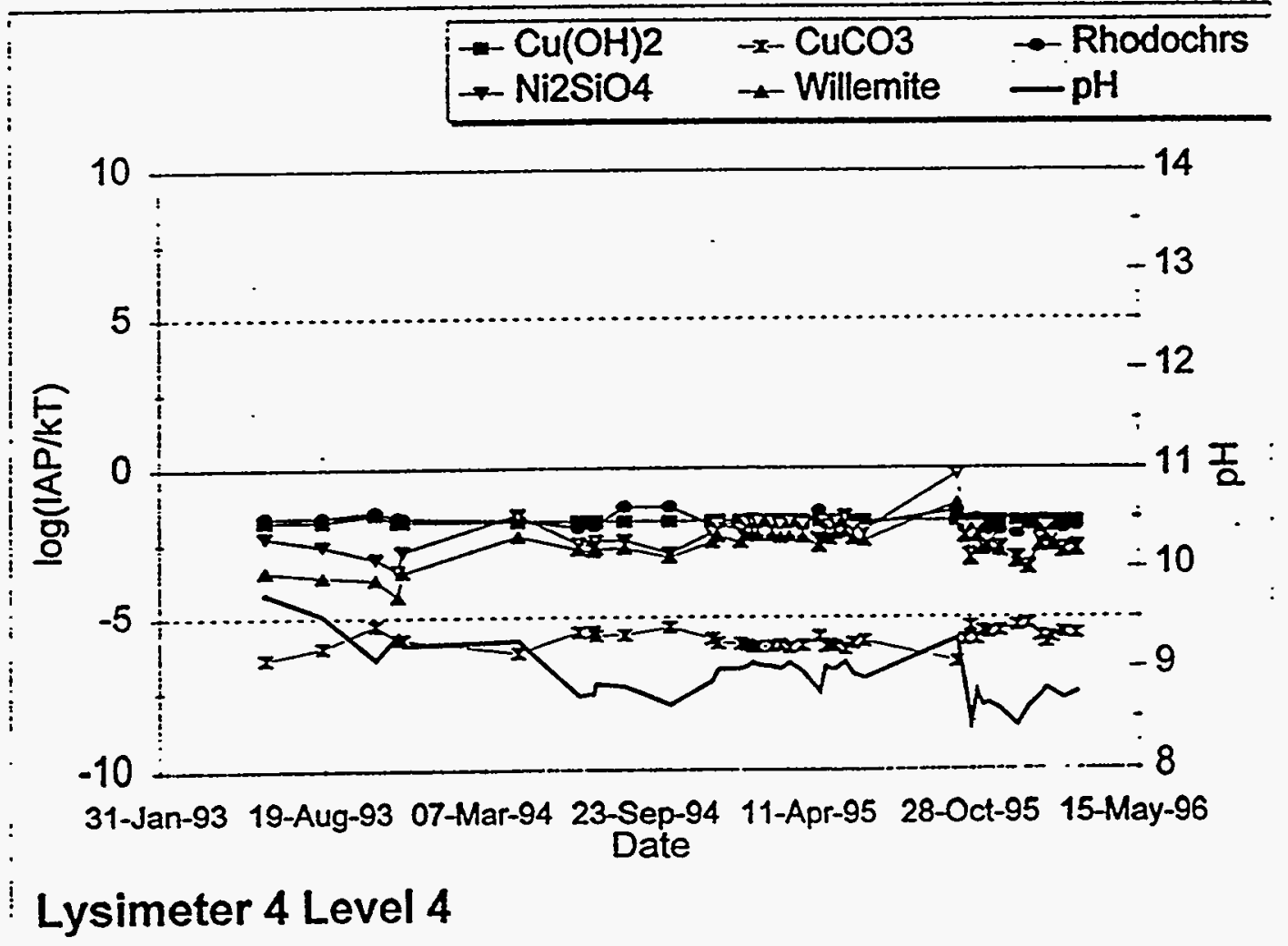

Figure 6-40. $\mathrm{Cu}(\mathrm{OH})_{2}, \mathrm{CuCO}_{3}$, Rhodocrosite, $\mathrm{Ni}_{2} \mathrm{SiO}_{4}$, and Willemite variations by date and $\mathrm{pH}$ in Lysimeter 4, Level 4 


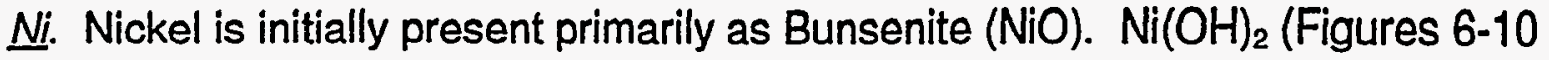
through 6-12) and $\mathrm{Ni}_{2} \mathrm{SiO}_{4}$ (Figures 6-38 through 6-40) may form under higher $\mathrm{pH}$ conditions.

Fe. There is no analytical information on relative amounts of divalent and trivalent $\mathrm{Fe}$. Goethite $(\mathrm{FeOOH})$, the trivalent analogue to Diaspore ( $\mathrm{AlOOH}$ ), does not show saturation. Also, no divalent $\mathrm{Fe}$ mineral approaches saturation, so it is probable that Hematite/Maghemite $\left(\mathrm{Fe}_{2} \mathrm{O}_{3}\right)$ and Magnetite $\left(\mathrm{FeFe}_{2} \mathrm{O}_{4}\right)$ are nearly insoluble.

\section{Investigation of Initial pH Dip, Field Lysimeters 1 and 2}

The ionic strength of the solute appears to have a great deal of influence on the $\mathrm{pH}$ - behavior. A comparison of ionic strength over time for the field lysimeters shows that the lysimeters with anomolous $\mathrm{pH}$ behavior also have the highest initial ionic strength (Figure 6-41). The three Coolside lysimeters approach the same ionic strength and about the same $\mathrm{pH}$ over time but the two least compacted exhibit the $\mathrm{pH}$ lowering during the high ionic strength period. Additional graphic illustration of the relationship of $\mathrm{pH}$ and ionic strength (Figure 6-42) shows a transition in behavior between ionic strengths of about 0.5 to 1 for the field lysimeters. The three data sets corresponding to the fly ash lysimeter, the most compacted lysimeter, and the group representing the two least compacted lysimeters can easily be seen.

lonic strength has a direct effect on the solubility of mineral species, as discussed in the section on caveats to calculation. As an example, the solubility of Calcite $\left(\mathrm{CaCO}_{3}\right)$ increases by a factor of three as ionic strength increases from 0.5 to 2.0. Another effect of high ionic strength is the reduction in activity of $\mathrm{H}_{2} \mathrm{O}$ because of polar bonding with species in solution. That portion of the water is no longer available as a solvent. 


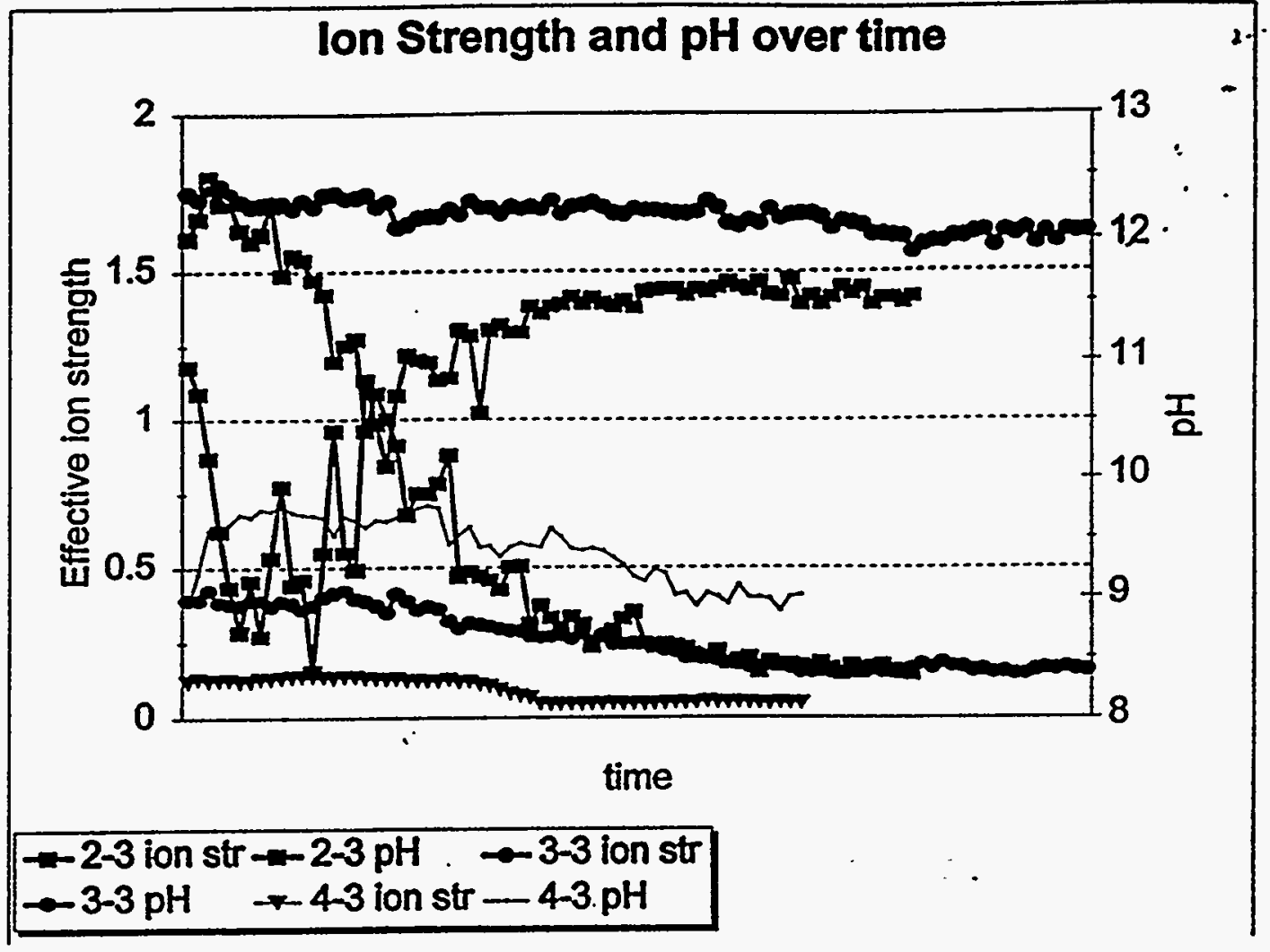

Figure 6-41. Ion strength variations and $\mathrm{pH}$ variations over time

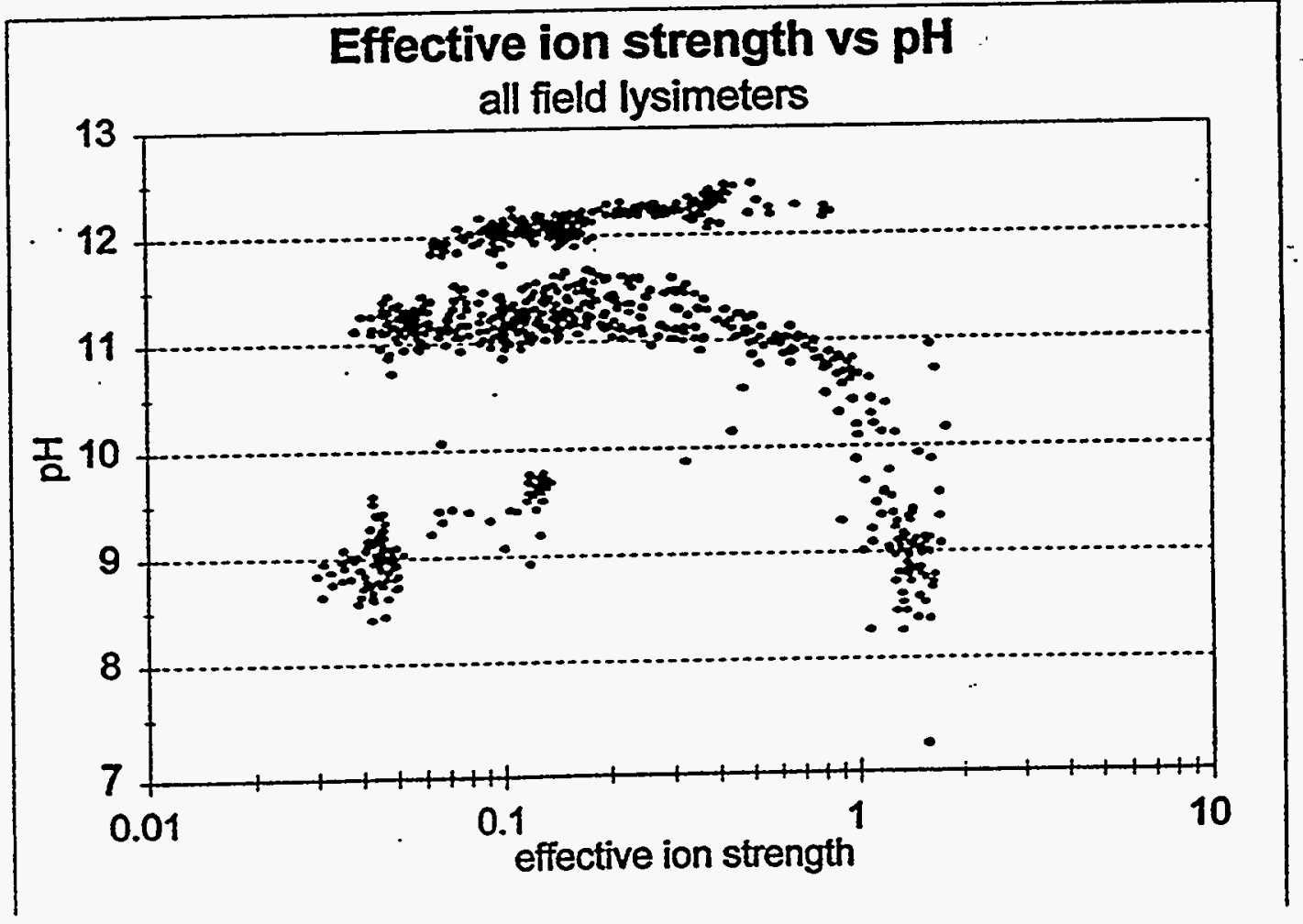

Figure 6-42. Effective ion strength vs $\mathrm{pH}$ in all field lysimeters 
The effect is evident when comparing the computed activity of $\mathrm{H}_{2} \mathrm{O}$ with observed $\mathrm{pH}$ (Figure 6-43). The initial lowering of $\mathrm{pH}$ does not appear to be related to leachate water carbonate loading (Figure 6-44, no loading for column 10 through high loading for column 12). The effect is content related. Pilot plant run 2 material (Figure 6-44) shows no dip. Coolside 3 laboratory column (Figure 6-45) shows a slight, but measurable dip. The field lysimeters (Figure 6-41) show a significant dip. The lack of observed dip in the column lysimeters could also be related to column size. It is concluded that the initial high ionic strength plays a major role in the initial lowering of $\mathrm{pH}$, similar to the observed high buffering capacity of sea water, which has an ionic strength of approximately 1.0. This capacity is enhanced by the diversity of ionic constituents available for the formation of neutral aqueous species and polar bonding, as illustrated by plots of major ion elution for the least compacted (Figure 6-46) and most compacted (Figure 6-47) field lysimeters.

It is known that Calcium and Sodium Oxide readily absorb $\mathrm{CO}_{2}$ from the atmosphere to form Carbonates. WATEQ also predicts a significant carbonate-bicarbonate buffering from both the $\mathrm{Ca}$ and $\mathrm{Na}$ forms during the low pH transient (Figures 6-48 through 6-49). It is proposed that the $\mathrm{pH}$ dip is flow related. In the least compacted lysimeters, higher flow results in increased ionic strength. The higher ionic strength simultaneously increases the solubility of the Calcium and Sodium Carbonates, resulting in significant carbonate-bicarbonate buffering, and reduces the activity of water, thereby lowering the availability for oxide hydration. This results in an initial lower $\mathrm{pH}$. As the major ions are eluted (Figure 6-46), the ionic strength decreases and oxide hydration returns $\mathrm{pH}$ to high levels. At lower flows in the most compacted lysimeter, the ionic strength is reduced, favoring oxide hydration over carbonate dissolution. 


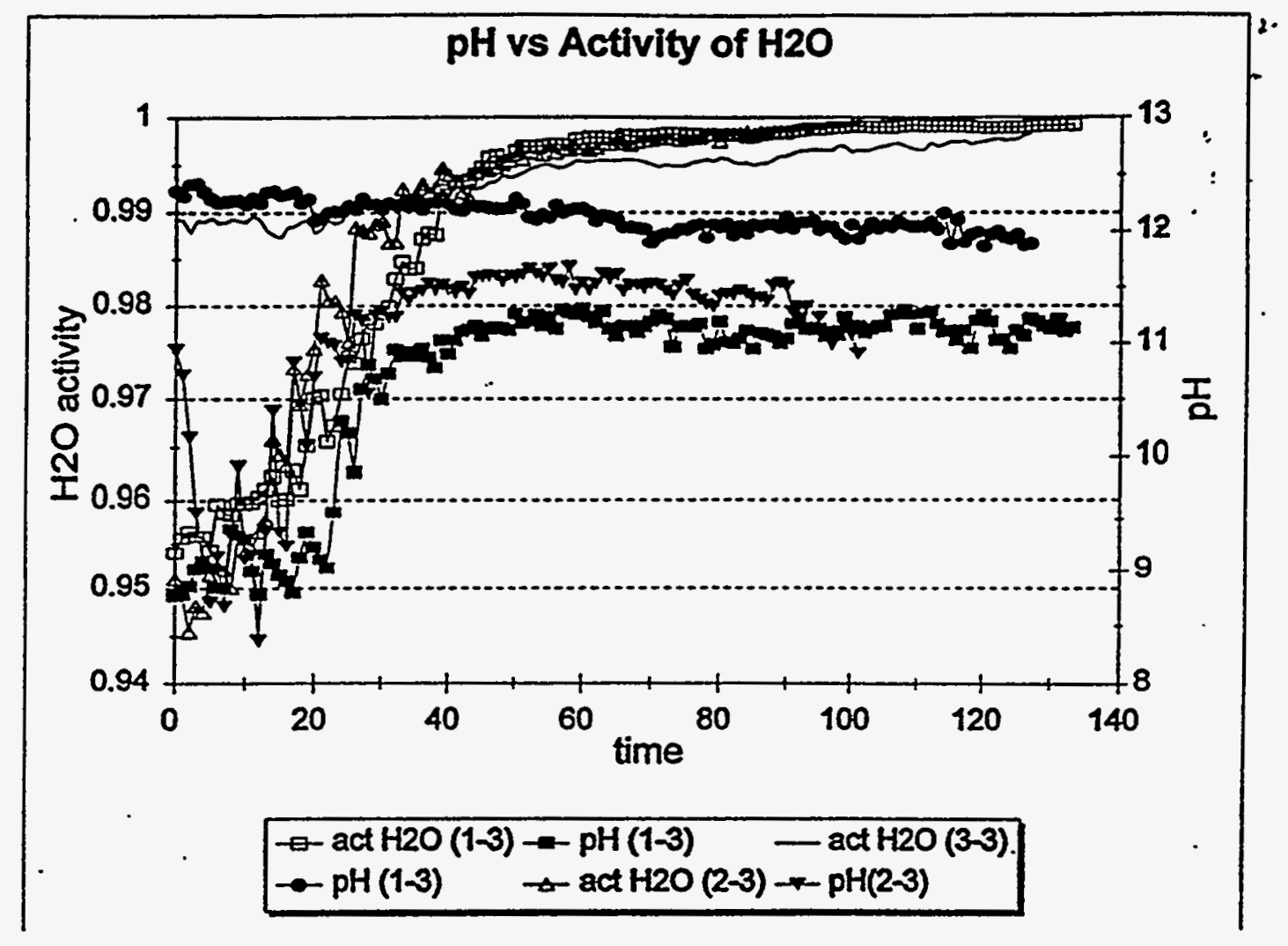

Figure 6-43. $\mathrm{pH}$ vs activity of $\mathrm{H}_{2} \mathrm{O}$ over time

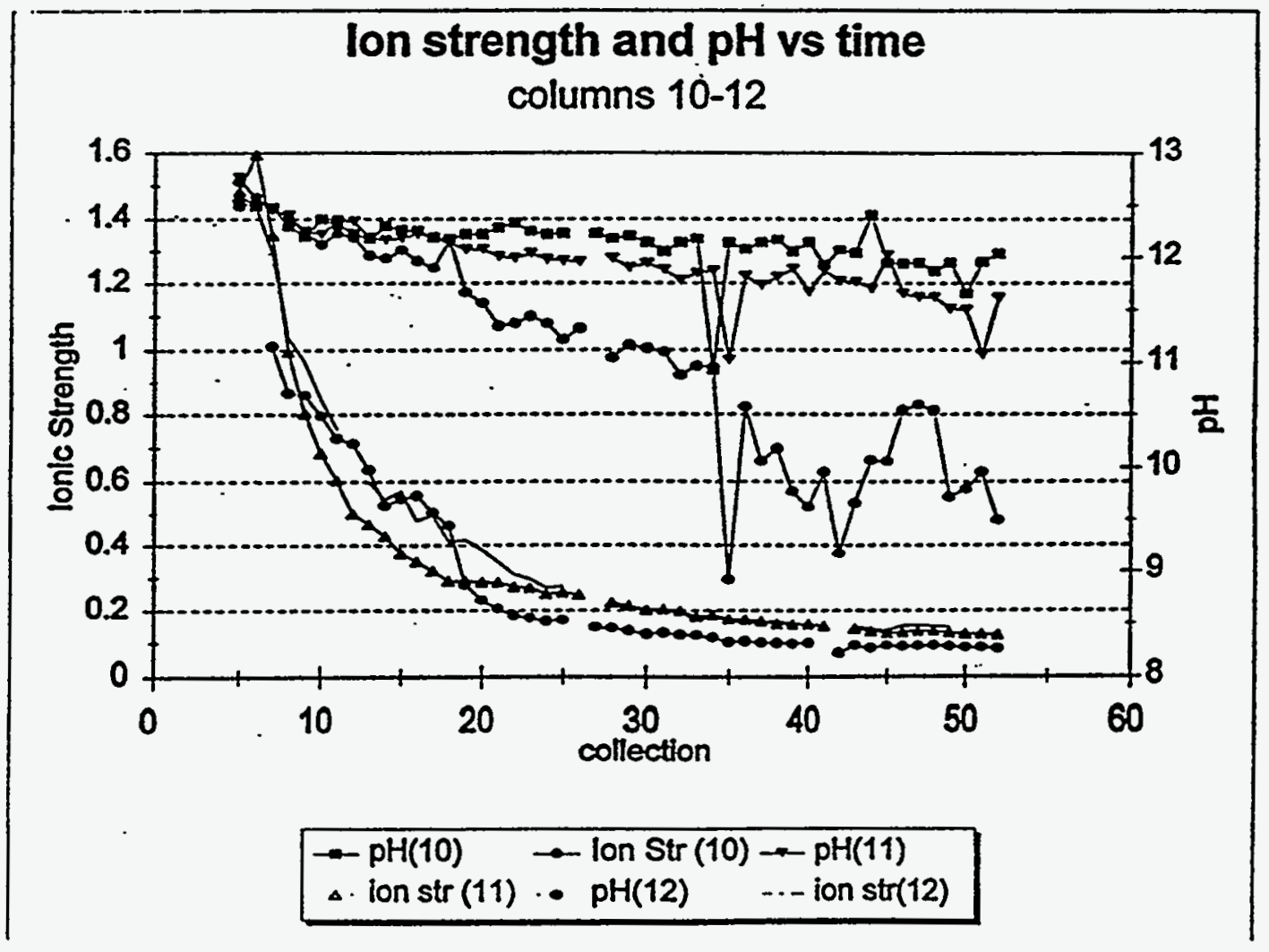

Figure 6-44. Ion strength and $\mathrm{pH}$ vs time in columns 10-12 


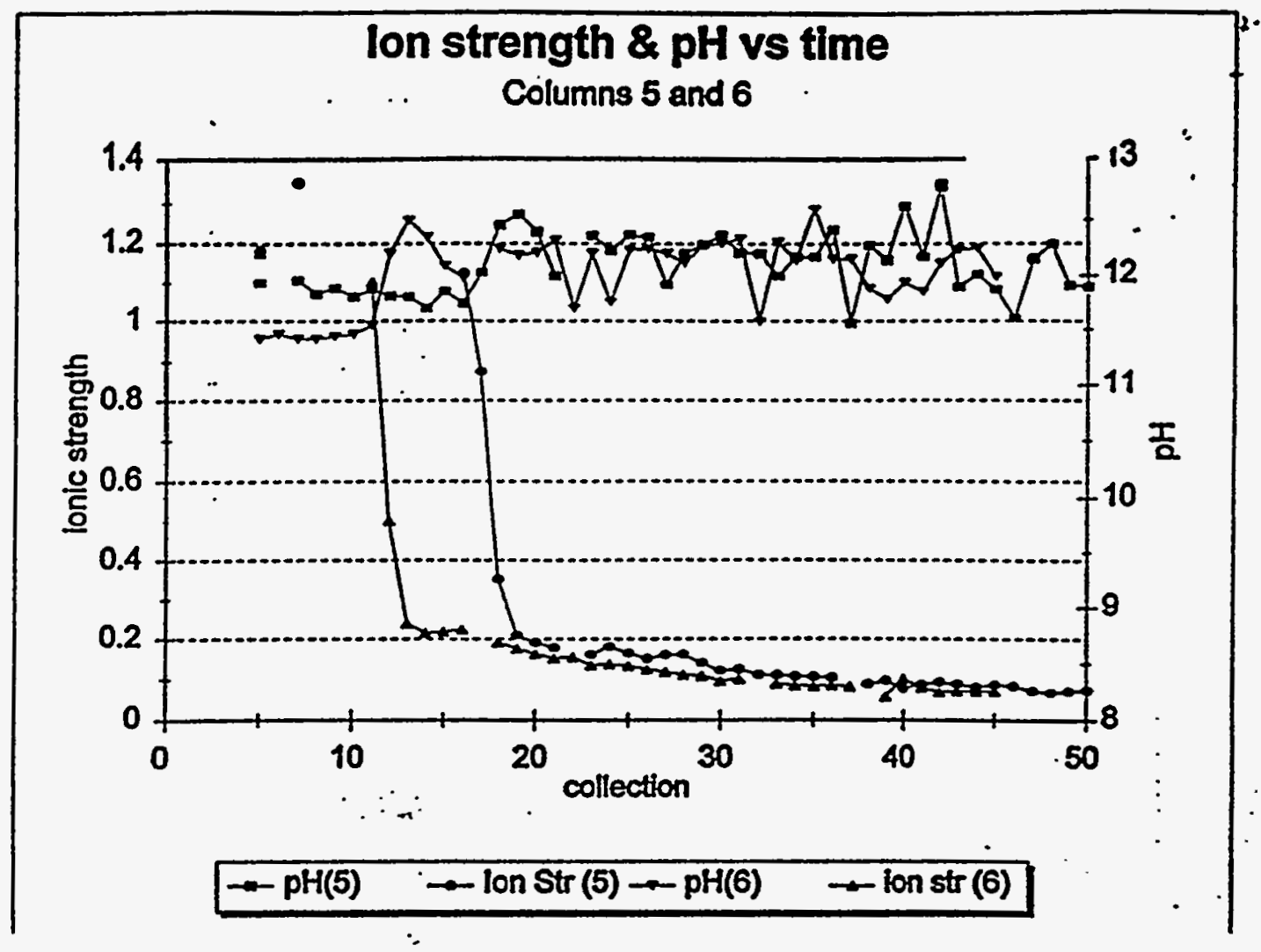

Figure 6-45. Ion strength and $\mathrm{pH}$ vs time in columns 5 and 6

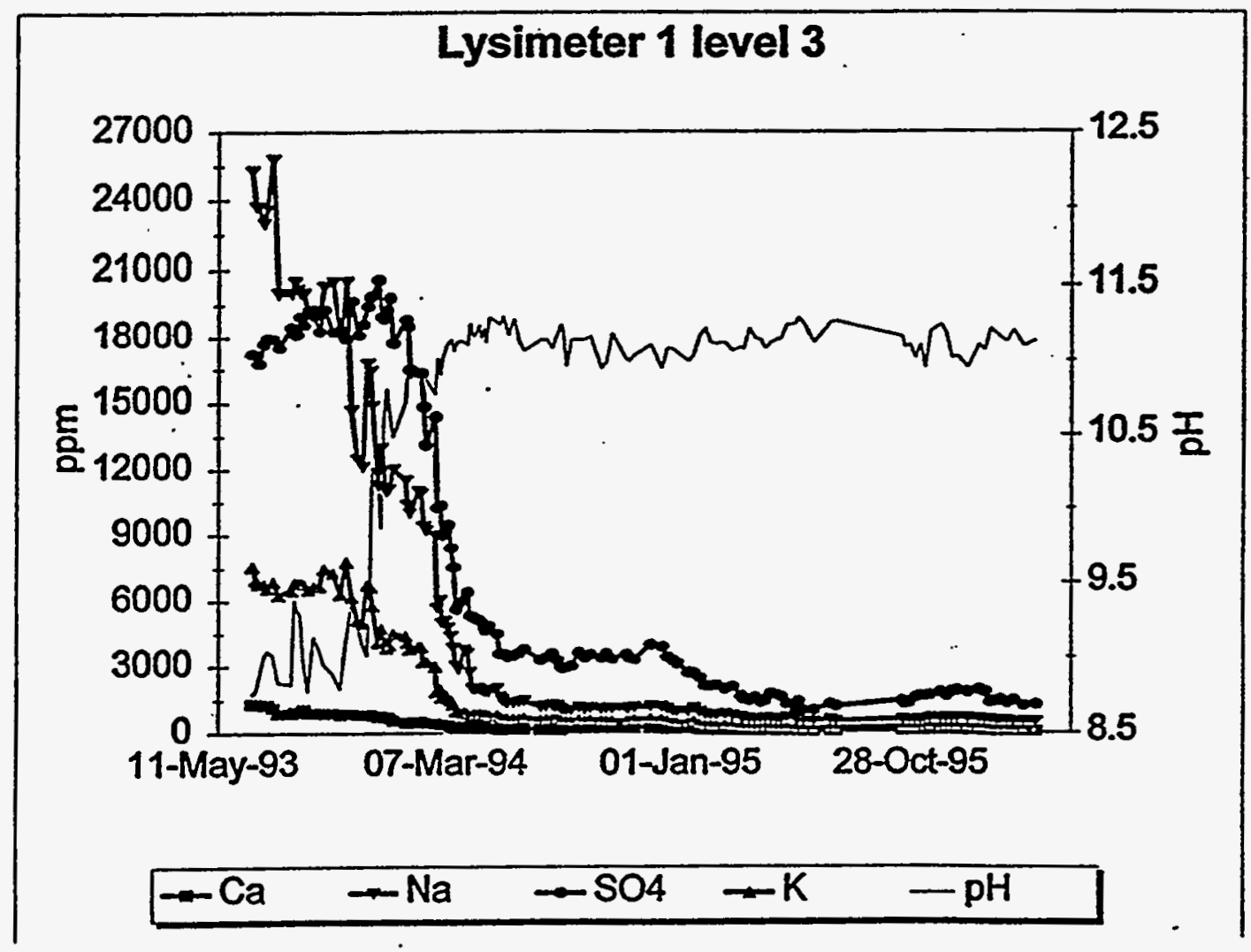

Figure 6-46. $\mathrm{Ca}, \mathrm{Na}, \mathrm{SO}_{4}$, and $\mathrm{K}$ variations by date and $\mathrm{pH}$ in Lysimeter 1 , Level 3 


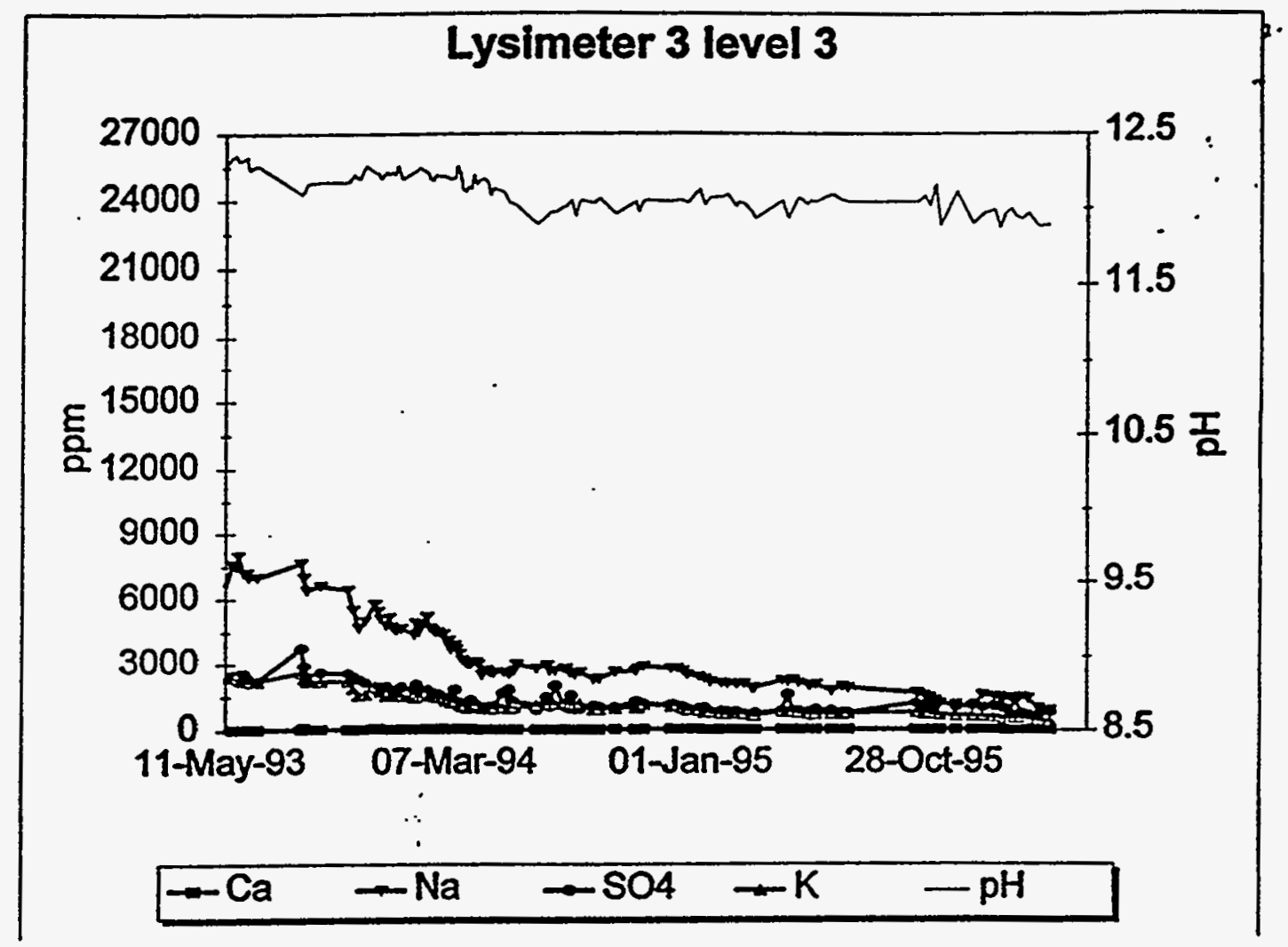

Figure 6-47. $\mathrm{Ca}, \mathrm{Na}, \mathrm{SO}_{4}$, and $\mathrm{K}$ variations by date and $\mathrm{pH}$ in Lysimeter 3, Level 3

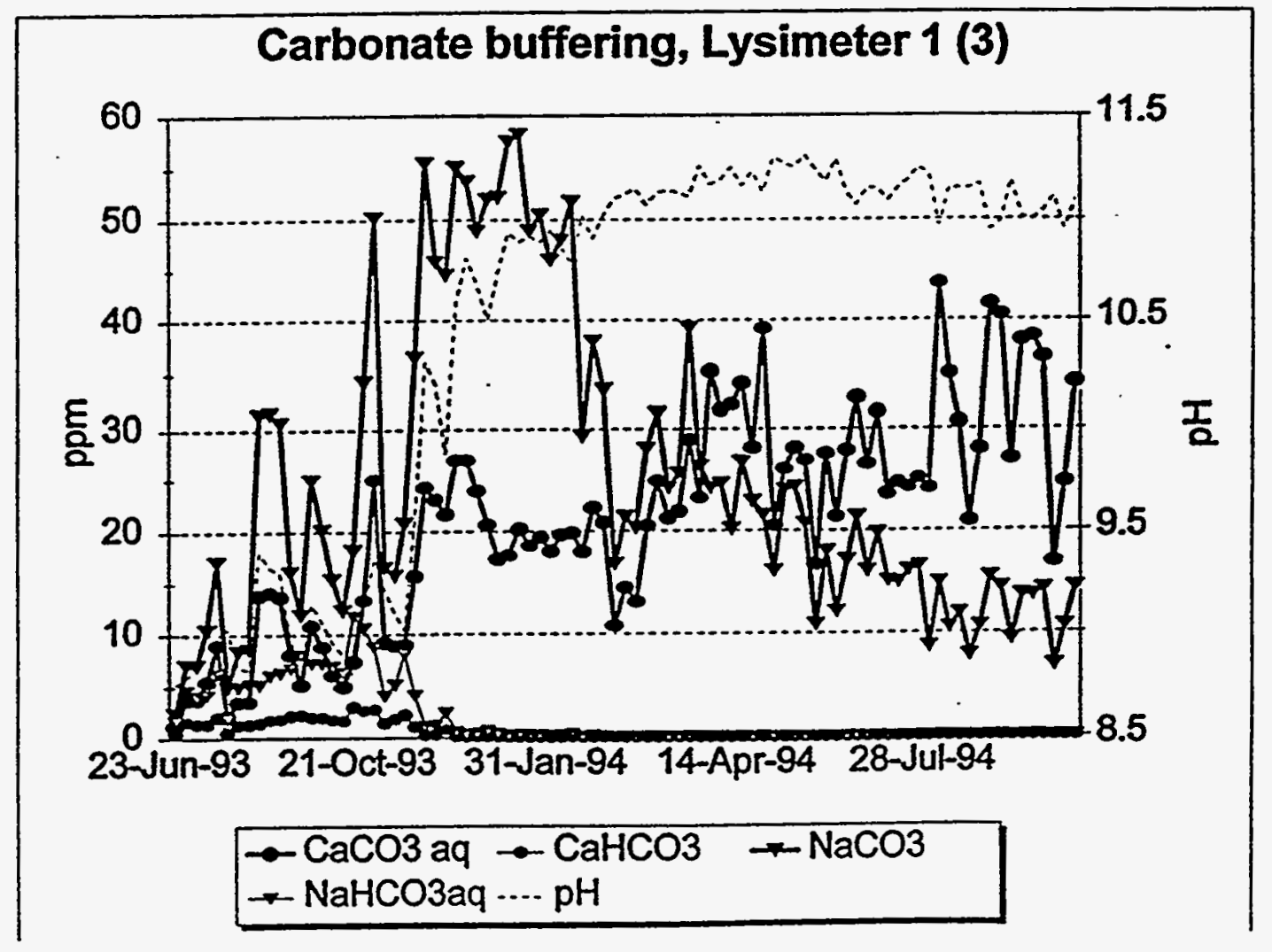

Figure 6-48. Carbonate buffering over time in Lysimeter 1 (3) 


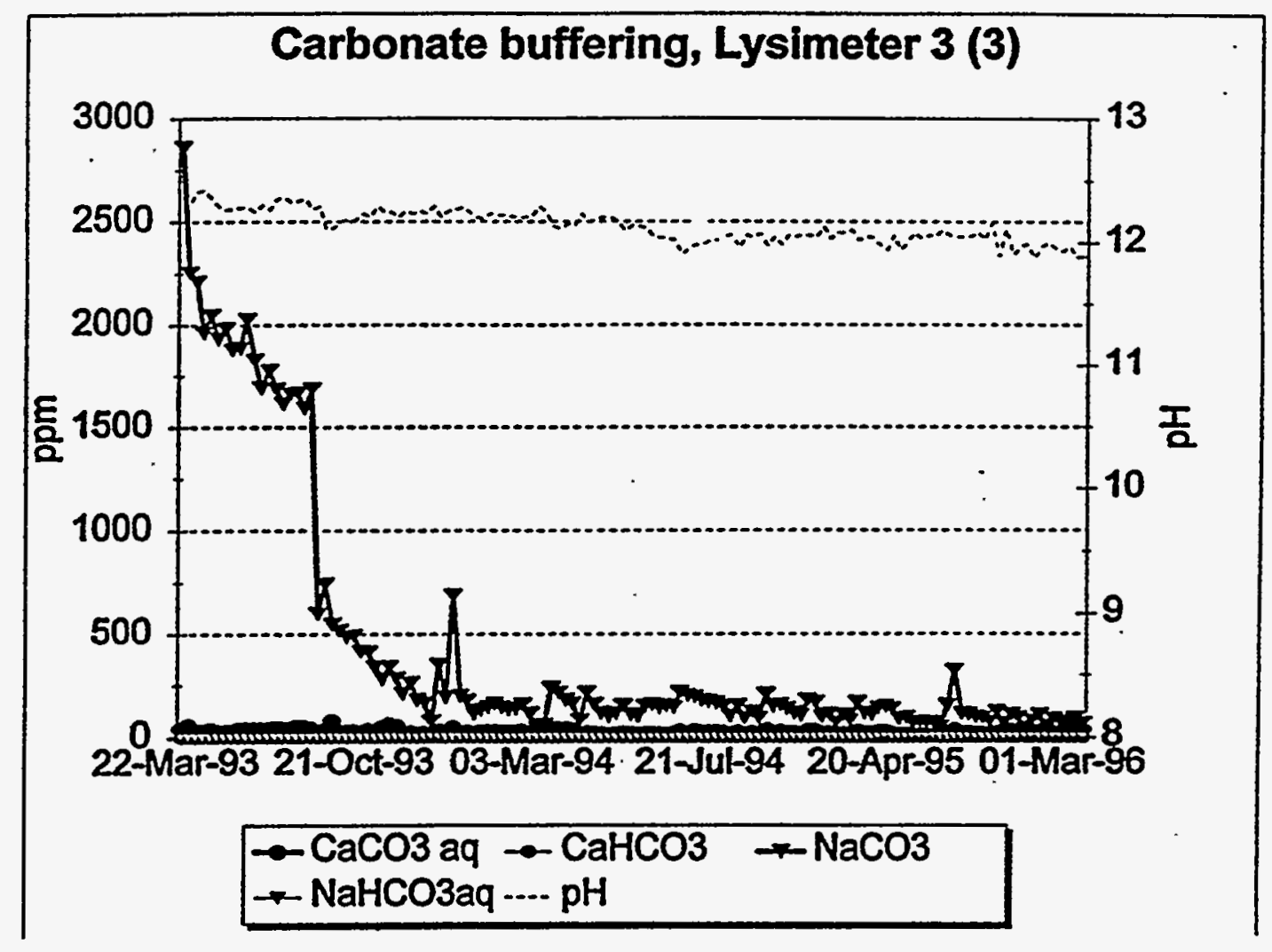

Figure 6-49. Carbonate buffering over time in Lysimeter 3 (3) 
APPENDIX 2 


\title{
COOLSIDE WASTE MANAGEMENT DEMONSTRATION OCDO GRANT AGREEMENT NO. CDO/D-902-9
}

\author{
Milestone Report - Phase 2 \\ Investigation of the Utilization Potential of Coolside, LIMB, and FBC Waste \\ as Construction Materials
}

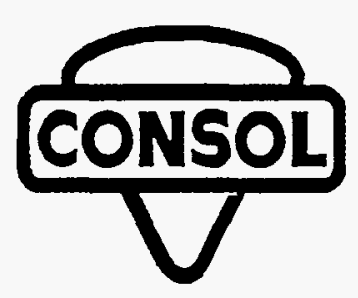

M. M. Wu 412-854-6708

R. A. Winschel 412-854-6683

CONSOL Inc. Research \& Development 4000 Brownsville Road Library, PA 15129

T. C. Hopkins

T. Beckham

University of Kentucky

Kentucky Transportation Center

113 Transportation Building

Lexington, Ky 40506-0043 


\section{TABLE OF CONTENTS}

\section{Page}

$\begin{array}{ll}\text { Summary } & 1\end{array}$

Objective 3

Results and Discussion 4

Phase 2 - Investigation of the Utilization Potential of Coolside, LIMB, and FBC Wastes as Construction Materials

Task 1 - The Utilization of Coolside, LIMB, and AFBC Wastes as Highway

Construction Materials

Task 1.1 - Material Acquisition

Task 1.2 - Evaluation of Coolside, LIMB, and AFBC Materials as Chemical Admixes for Road Base Application Admixtures of FBC Waste and Clay Soil

Swelling of FBC Waste

CBR and Swelling of Coolside Waste

Properties of Wormser FBC Waste and P.C. Fly Ash

Tasks 1.3 and 1.4 - Laboratory Testing and Field Trials of Coolside, LIMB, and AFBC Wastes as Road Construction Materials and Field Test Monitoring and Data Evaluation

Evaluation of Synthetic FBC Waste Aggregates as Construction

Materials

Preparations for Field Demonstration

Task 2 - Waste Pelletization Research with Coolside, LIMB, and FBC Wastes

Task 2.1 - Sample Acquisition

Tasks 2.2 and 2.3 - Baseline Pelletization and Improved Product Development

Coolside Waste Pellets

LIMB Waste Pellets

FBC Waste Pellets

Leaching Test of FBC Waste Aggregates

Asphalt Mix Design for FBC Waste Aggregates

Task 2.4 - Large Sample Production and Field Testing

Task 2.5 - Monitoring and Report Writing

\section{LIST OF TABLES}

\section{Table}

Index Properties of Hydrated FBC Waste and Soil Used for Subgrade

Results of CBR Tests on Clay Soil-Hydrated FBC Waste Mixtures $\quad 30$

3

Results of CBR Tests, Coolside Waste Generated in Recycle Mode

CBR Values at $2.54 \mathrm{~mm}(0.1 \mathrm{in})$ Penetration from Coolside Waste

Samples Obtained from the Edgewater Power Plant, Demo Runs 1 and 3 


\section{LIST OF TABLES}

Results of Freeze Thaw Test (AASHTO T-103) on Aggregates Made with $100 \%$ FBC Waste

Results of Particle Size Analysis (AASHTO T-27) on Aggregates Made with $100 \%$ FBC Waste

Specific Gravity and Absorption (AASHTO T-85) Test Results on Aggregates Made with $100 \%$ FBC Waste

Results of the Los Angeles Abrasion Test (AASHTO T-96) on Aggregates Made with $100 \%$ FBC Waste

Sodium Sulfate Soundness Test (AASHTO T-104) on Aggregates Made with $100 \%$ FBC Waste

Results of Sodium Sulfate Soundness Tests on Synthetic Aggregate Produced from FBC Waste Blended with $10 \%$ Fly Ash or $5 \%$ Microsilica

Results of Los Angeles Abrasion Tests on Synthetic Aggregate Produced from FBC Waste Blended with $10 \%$ Fly Ash or $5 \%$ Microsilica Analyses of Coolside Waste Samples Analyses of LIMB Waste Samples from Ohio Edison Edgewater Station Analyses of FBC Waste Samples from Glatfelter and GM

Properties of Coolside Waste Pellets and the Ohio Specifications for Road Aggregates

Properties of Coolside Waste Pellets With and Without Blended Fly Ash

Properties of LIMB Waste Pellets

Properties of LIMB Waste Pellets With and Without Blended Fly Ash

Properties of FBC Waste Pellets

Properties of Pellets Prepared with GM and Glatfelter FBC Wastes

Comparison of Properties of Fresh and Aged Pellets Made with 100\% FBC Waste with Natural Aggregates

The Effect of Aging on the Properties of Aggregates Made from 100\%

FBC Waste and the 90/10 FBC Waste and Fly Ash Blend

Properties of FBC Waste Pellets With and Without Blended Microsilica

Properties of FBC Waste Pellets Blended with $5 \%$ Processed and Waste Microsilica

The Effect of Aging on the Properties of FBC Waste Pellets Made with $5 \%$ and $10 \%$ Blended Processed Microsilica

Properties of $90 / 10$ FBC Waste/Fly Ash Pellets from the Undrained Weathering Test

Properties of 90/10 FBC Waste/Fly Ash Pellets from the Drained Weathering Test

Properties of FBC Waste Pellets with Blended Fly Ash

Properties of Pellets Made from the 65/35 Blends of FBC Waste and

Fly Ash with Additives

Degradation of 65/35 FBC Waste/Fly Ash Pellets with Asphalt Emulsion

Addition and Asphalt Coating after 54 Cycles in the Freeze/Thaw Test

Analysis of ASTM Leachate of 90/10 FBC Waste/Fly Ash Pellets and

Comparison with Relevant SDWA Standards

Comparison of Marshall Test Results in Asphalt Mix Design

Properties of Synthetic Aggregates Used in Field Trial 


\section{LIST OF TABLES}

Table

Page

36 Size Distribution of the THF-Extracted Aggregate from the Pug Mill Before and After Freeze/Thaw Treatment

37 Size Distribution of the THF-Extracted Aggregates from the Pug Mill and the Test Patch Cores

Estimated Composition of Fines in the THF-Extracted Test Patch Cores after Freeze/Thaw Treatment

\section{LIST OF FIGURES}

Fiqure

$\underline{\text { Page }}$

Maximum Dry Density vs Percent of Hydrated FBC Waste in Clay SoilFBC Waste Mixture

Optimum Moisture Content vs Percent of Hydrated FBC Waste in Clay Soil Waste Mixture

Unconfined Compressive Strength as a Function of Percent of Hydrated FBC Waste in Clay Soil-FBC Waste Mixture

Swell of Clay Soil and Soil-FBC Waste Mix During 19-Day Coal-CBR Samples

Long-Term Swell of Hydrated FBC Waste

Swell of CBR Samples from Coolside Waste Generated in Recycle Mode

Effect of Moisture Content on CBR Specimens from Coolside Demonstration Run No. 1, Barrel No. 1050

Percent Air Voids vs Asphalt Content -- Control Mix 52

Stability vs Asphalt Content -- Control Mix

Flow vs Asphalt Content -- Control Mix

Voids in Mineral Aggregate vs Asphalt Content -- Control Mix 53 Amounts of Fly Ash as a Function of Time in the Undrained Weathering Test 


\title{
COOLSIDE WASTE MANAGEMENT DEMONSTRATION \\ OCDO GRANT AGREEMENT No. CDO/D-902-9
}

\section{MILESTONE REPORT - PHASE 2 \\ INVESTIGATION OF THE UTILIZATION POTENTIAL OF COOLSIDE, LIMB, AND FBC WASTE AS CONSTRUCTION MATERIAL}

\begin{abstract}
SUMMARY
High-strength synthetic aggregates with low abrasion indices were made by pelletizing fluidizedbed combustion (FBC) wastes. The synthetic aggregates produced from FBC waste meet all American Association of State Highway and Transportation Officials (AASHTO) specifications (unit weight, soundness index, LA abrasion index and size distribution) for use as Class A aggregate for road construction. Although they met all specifications, the aggregates produced from $100 \% \mathrm{FBC}$ waste degraded when they were fully immersed in water during long-term natural weathering and laboratory freeze/thaw tests. Characterization of the degraded aggregate indicates that the slow hydration of anhydrite $\left(\mathrm{CaSO}_{4}\right)$ to gypsum $\left(\mathrm{CaSO}_{4} \cdot 2 \mathrm{H}_{2} \mathrm{O}\right)$ is the major cause for the degradation. Durability was improved by blending the FBC waste with pulverized coal (p.c.) fly ash and by coating with asphalt.
\end{abstract}

The utilization potential of pelletized FBC waste was evaluated for use as highway construction materials. The Ohio EPA determined that the use of aggregates made from FBC waste in road construction is a beneficial use. A flexible bituminous test pavement was constructed in April 1995 with 1.5 tons of synthetic aggregate made from a $65 / 35$ blend of FBC waste and p.c. fly ash to evaluate the aggregate durability under heavy traffic and natural weathering conditions. Equal quantities of crushed limestone (Class A aggregate) were blended with the synthetic aggregates to provide a larger surface area for the test and to provide an internal standard for evaluating aggregate performance. The evaluation ended in May 1996. Inspections revealed no visible degradation of the test patch or aggregate after 13 months of service. Monthly analysis of core samples showed that neither the FBC waste aggregate nor the limestone aggregate degraded between June 1995 and May 1996.

FBC waste in unconsolidated form was evaluated as a chemical admix for road base stabilization. FBC waste greatly improved the strength and bearing capacity of clay soil; however, the FBC waste-clay soil admixture swelled slowly over a long time. This swelling behavior indicates that 
special precautions would need to be taken for use of the materials in road base construction, and indicates that laboratory swelling tests should be conducted before using FBC waste, Coolside waste, or any alkaline FGD by-product in road-base construction. Coolside waste developed high load-bearing capacity, a desirable characteristic for road base use, but also swelled over time.

High-strength synthetic aggregates with low abrasion indices also were made from Coolside and LIMB wastes. Although these materials fail to meet the road aggregate specifications, they pass the ASTM specifications as lightweight aggregates. Both types of aggregates have potential for use as coarse lightweight aggregates in concrete masonry. The addition of fly ash to the waste mix during aggregate preparation further improved the aggregate properties. 


\section{OBJECTIVE}

Waste management is an important element in the application of flue gas desulfurization (FGD) processes. The Coolside and lime injection multistage burner (LIMB) sorbent injection processes were demonstrated successfully at the Ohio Edison Edgewater Station (Lorain, Ohio) as part of the DOE Clean Coal Technology (CCT) program from 1989 to 1992. Fluidized-bed combustion (FBC) is a commercial technology which combines steam generation with $\mathrm{SO}_{2}$ control. Each of these processes produce a dry solid waste that must be managed to ensure environmental compliance and economical feasibility. The objectives of this project were to specify landfill disposal criteria for waste from the Coolside process (Phase 1), and to evaluate the utilization potential for road construction of wastes generated from the Coolside, LIMB and FBC processes (Phase 2). Improved options for utilization of the wastes can reduce the cost of these FGD technologies and benefit Ohio's high-sulfur coal industry. The Phase 2 study was divided into two tasks. Task 1 was directed toward evaluating the utilization of the unconsolidated wastes (as-is and hydrated) as chemical admixes for road base construction and evaluating the utilization of the unconsolidated and consolidated wastes (as-is, hydrated, and pelletized) as construction materials. Task 2 was directed toward the development of waste pelletization technology and evaluation of the pelletized wastes as synthetic aggregates for highway construction. The objective of Phase 3 was to provide additional waste samples from the CONSOL Coolside pilot plant for use in the Phase 1 and Phase 2 studies. This report describes the results of the Phase 2 study. The results of the Phase 1 and Phase 3 studies have been reported. A final, overall report will be released. 


\section{RESULTS AND DISCUSSION}

PHASE 2 - INVESTIGATION OF THE UTILIZATION POTENTIAL OF COOLSIDE, LIMB, AND FBC WASTES AS CONSTRUCTION MATERIALS

Task 1. The_Utilization of Coolside, LIMB, and AFBC Wastes as_Highway Construction Materials

Task 1.1 Material_Acquisition

Two FBC waste samples (as-is and hydrated) were used in the study. The "as-is" sample was collected from the ash silo of a coal-fired, fluidized-bed combustor (FBC) at the P. H. Glatfelter Co. (Spring Glove, PA) on February 18, 1992. The FBC waste is the composite material from the baghouse and the bed drain of the FBC. The "hydrated" FBC waste used in the study was prepared by CONSOL R\&D by mixing the as-is waste with water in a Littleford Brothers mixer. The hydrated FBC waste contained $3.9 \%$ free moisture, and over $90 \%$ of the $\mathrm{CaO}$ was converted to $\mathrm{Ca}(\mathrm{OH})_{2}$.

Task 1.2 Exaluation of Coolside, LIMB, and AFBC Materials_as Chemical Admixes for Road Base Application

The objectives of this evaluation are to determine the potential use of FBC and Coolside wastes as a chemical admixture for pavement subgrades, to evaluate the geotechnical properties of the admixture, and to determine an optimum formulation of the admixture.

\section{Admixtures of FBC Waste and Clay Soil}

The hydrated FBC waste sample prepared by CONSOL R\&D was blended with a silty clay having marginal strength properties to determine if the soil properties could be improved. Hydrated lime (typically $5 \%-6 \%$ by dry weight) is used to improve strength and bearing capacity of clayey soil subgrades. The residual clay sample was obtained from the Franklin County landfill, which is located a few miles south of Columbus, Ohio.

Standard classification tests, including Atterberg limits (ASTM D4318), particle size (ASTM D422), and specific gravity (ASTM D854) were performed to determine engineering properties of the clay and hydrated FBC waste. Results are shown in Table 1. 
Standard moisture-density relation tests (ASTM D698) were performed on FBC waste-soil mixtures to determine changes in maximum dry density and optimum moisture content as the percent of FBC waste increases. Tests were performed on admixes containing $0,4,8,12$, and $100 \%$ of the FBC waste. Maximum dry density decreased from $1,711 \mathrm{~kg} / \mathrm{m}^{3}\left(106.8 \mathrm{lb} / \mathrm{ft}^{3}\right)$ at $0 \% \mathrm{FBC}$ waste to $1,229 \mathrm{~kg} / \mathrm{m}^{3}\left(76.7 \mathrm{lb} / \mathrm{ft}^{3}\right)$ at $100 \% \mathrm{FBC}$ waste; optimum water content increased simultaneously from 19.4 to 27.8 percent, as seen in Figures 1 and 2.

Unconfined compressive strength tests were performed to determine if the addition of hydrated FBC waste to compacted clay would result in greater unconfined compressive strengths. Samples were remolded at $95 \%$ maximum dry density, sealed in plastic containers, and aged at room temperatures for seven and fourteen days before testing. As seen in Figure 3 , the strength of the specimens aged for fourteen days increased from $145.4 \mathrm{kPa}\left(21.1 \mathrm{lb} / \mathrm{in}^{2}\right)$ at $0 \%$ of $\mathrm{FBC}$ waste (clay specimen) to $2,274 \mathrm{kPa}\left(330 \mathrm{lb} / \mathrm{in}^{2}\right)$ at $100 \% \mathrm{FBC}$ waste.

California bearing ratio tests (ASTM D1883) were performed on the clay soil sample and on an $8 \%$ hydrated FBC waste-soil mixture. California bearing ratio (CBR) is a measure of the bearing capacity of a substance, expressed in terms of the bearing capacity of a standard material. The CBR values were obtained after a long-term soaking period (19 days). Bearing ratio values are usually obtained after a soaking period of 96 hours. CBR values at $2.54 \mathrm{~mm}(0.1 \mathrm{in})$ penetration increased from 2.1 to 5.2 with the addition of $8 \%$ hydrated FBC waste. Similar increases occurred at penetration values of $5.1,7.6,10.2$, and $12.7 \mathrm{~mm}(0.2,0.3,0.4$, and $0.5 \mathrm{in}$, respectively), as seen in Table 2 . The sample modified with hydrated FBC waste swelled about $9 \%$ during the 19-day soaking period, whereas the specimen of natural clay soil swelled only about $4 \%$, as seen in Figure 4. Although the FBC waste modified clay has very high strength, a swell of $9 \%$ would be detrimental to a pavement if the swell occurred after the pavement was placed. Therefore, FBC waste should not be used as a chemical subgrade admixture unless it can be demonstrated by long-term swelling tests that the total magnitude of swelling does not exceed about $4 \%$, or that the total magnitude of swelling is completed before placement of a pavement, and that unconfined compressive strengths of the modified subgrade generally exceed about $210 \mathrm{kPa}$ (30 psi), or the value of CBR exceeds about 6.5.

\section{Swelling of FBC Waste}

Long-term swelling tests were performed on hydrated and as-is FBC wastes remolded at near $95 \%$ of standard maximum dry density and optimum moisture content. Swell data for as-is and 
hydrated FBC wastes formed in CBR molds and soaked in water are shown in Figures 5 and 6. Test results indicate that hydration, aging, and static loading (surcharge) decrease the swelling of FBC waste. In pavement applications, the total magnitude of long-term swelling should be kept below about $4 \%$ or the total swelling should be nearly completed before the placement of pavement.

\section{CBR and Swelling of Coolside Waste}

CBR tests were performed on four samples of Coolside waste from Run No. 2 (recycle mode) of the Phase 3 program. These tests were performed to determine if Coolside waste could be used as a pavement subgrade or base. The samples were compacted at near $95 \%$ of maximum dry density and optimum moisture content (ASTM D 698), then subjected to long-term swelling tests (periods ranging from 99 to 220 days). The standard test (ASTM D1883) specifies a swelling period of 96 hours. CBR tests were performed after swell had stopped or reached a secondary swelling phase. Two samples were sealed in plastic containers and aged seven days before submerging in water. As seen in Table 3, CBR values at $2.54 \mathrm{~mm}(0.1 \mathrm{in})$ penetration ranged from 6.0 to 144. All of the samples had high CBR, except for the sample with a small surcharge (or static-loading) weight and no age time. Although CBR values were high, large amounts of swell, measuring as much as $30 \%$ (see the sample with a $1.03 \mathrm{~kg}(2.27 \mathrm{lb})$ surcharge and no aging time in Figure 7) would cause pavements to heave or buckle.

A series of CBR tests was performed on Coolside waste samples obtained from demonstration Run Nos. 1 and 3 at the Edgewater Power Plant in Lorain, Ohio (see Phase 1 report for sample origin). Tests were performed on samples compacted at near 90,95 , and $100 \%$ of maximum dry density and optimum moisture content, as defined from low energy $\left(2,484 \mathrm{ft}-\mathrm{lb} / \mathrm{ft}^{3}\right.$--a procedure of the Kentucky Transportation Center), standard (12,374 $\mathrm{ft}$-lb/ft ${ }^{3}$--ASTM D698), and modified $\left(56,246 \mathrm{ft}-\mathrm{lb} / \mathrm{ft}^{3}-\mathrm{ASTM} \mathrm{D} 1557\right)$ moisture-density relation tests. The low energy and standard tests were conducted with Coolside waste samples from Run No. 1 and modified tests were conducted with Coolside waste samples from Run No. 3. A surcharge (or static loading) was placed on the compacted specimens. All samples were soaked for 96 hours and a surcharge weight of approximately $5.7 \mathrm{~kg}(12.5 \mathrm{lb}$.) was used. CBR values generally increased as the energy of compaction increased (Table 4).

Another series of CBR tests was performed on Coolside waste samples at various moisture contents to determine the effect of moisture on the value of CBR. Samples for this series of tests 
were obtained from barrel No. 1050, which was collected during the Edgewater demonstration Run No. 1 (see Phase 1 report for sample origin). Tests were performed on samples compacted at $95 \%$ of standard maximum dry density and at molding moisture contents of $4 \%$ below optimum moisture content (OMC), OMC, $4 \%$ above OMC, $6 \%$ above OMC, and $10 \%$ above OMC. CBR values increased as the molding moisture content increased from a value of $4 \%$ below OMC to a value at $O M C$ (Figure 8). However, as the molding moisture content increased above a value of OMC, the CBR values decreased. These results show that the CBR strength of the Coolside waste material is very sensitive to small changes in moisture content. Moreover, moisture contents slightly above optimum moisture could result in landfill problems from the excess moisture (the material will tend to liquefy under tire stresses).

\section{Properties of Wormser FBC Waste and P.C. Fly Ash}

Standard (ASTM D698) moisture-density relation tests were performed on samples of an FBC waste and a p.c. fly ash received from the Wormser Development Company. Classification tests are summarized in Tables 5. Maximum dry densities and optimum moisture contents of these ashes are presented in Table 6 . No further tests were conducted with these materials..

Tasks 1.3 and 1.4 Laboratory Testing and Field Trials of Coolside, LIMB and AFBC Wastes as Road Construction Materials and Field Test Monitoring and Data Evaluation

The purpose of this task was to prepare for and conduct a field trial demonstrating the use of waste in highway construction applications. The use of synthetic aggregates produced from FBC waste in bituminous concrete or as an aggregate base, and the use of unconsolidated FBC waste as a chemical subgrade stabilizer were considered for the field trial. Described below under Tasks 1.3 and 1.4 are support activities conducted to help select and conduct the field trial. The actual field trial is described under Tasks 2.4 and 2.5 .

\section{Evaluation of Synthetic FBC Waste Aggregates as Construction Materials}

A bulk sample of synthetic aggregates produced from $100 \%$ FBC waste, was evaluated. To evaluate the durability and potential uses of the aggregate, the following tests were performed: freeze thaw, particle size, specific gravity and absorption, Los Angeles abrasion, sodium sulfate soundness, triaxial shear strength, and swell. Results are shown In Tables 7 through 11.

The test results indicate that the synthetic aggregates made from $100 \% \mathrm{FBC}$ waste are coarse aggregates that meet AASHTO No. 8 size distribution. The aggregates meet the Ohio DOT 
specification for LA abrasion index, but fail the sodium sulfate soundness test. The improvement in soundness index will be discussed further in Task 2, Waste Pelletization Research.

To evaluate the shear strength of the $100 \%$ FBC waste aggregate, consolidated-undrained triaxial compression tests with pore pressure measurements and consolidated-drained triaxial compression tests were performed on the FBC waste aggregates. The drained and undrained tests were performed in triplicate to determine the effective stress parameters of the FBC waste aggregates. The effective stress parameters, the angle of internal friction ( $\left.\phi^{\prime}\right)$, and cohesion $\left(c^{\prime}\right)$, obtained from the consolidated-undrained triaxial tests with pore pressure measurements were $36^{\circ}$ and 0 , respectively. Values of $\phi^{\prime}$ and $c^{\prime}$ obtained from the consolidated-drained triaxial tests were similar, $38.7^{\circ}$ and 0 , respectively. The results obtained from the two different approaches were nearly identical. The values of ' $\phi$ ' and c' obtained for the $100 \%$ FBC waste aggregate are comparable to values of $\phi^{\prime}$ and $c^{\prime}$. for uncrushed, river bank gravel. Typically, values of $\phi^{\prime}$ and $c^{\prime}$ of river bank gravel are about $39^{\circ}$ and 0 , respectively. Values $\phi^{\prime}$ and $c^{\prime}$ of crushed limestone are generally about $43^{\circ}$ to $47^{\circ}$ and 0 , respectively. If the synthetic aggregates were crushed, the $\phi$ ' value would be greater than the value obtained for the uncrushed synthetic aggregate. Based on the values of $\phi^{\prime}$ and $c^{\prime}$, the synthetic aggregates made from FBC waste could be used as a base aggregate.

Long-term swell tests (995 days) were performed on the $100 \%$ FBC waste aggregates by placing the material in CBR molds and submerging them in water. Two tests were performed with surcharge weights of $1.12 \mathrm{~kg}(2.47 \mathrm{lb})$ and $5.74 \mathrm{~kg}(12.66 \mathrm{lb})$. These values correspond to 0.09 and $0.45 \mathrm{psi}$, respectively. As seen in the Figure 9, the material with the $1.12 \mathrm{~kg}$ surcharge swelled to about $25 \%$ of the original volume in approximately 140 days. The swell then reversed and stabilized around $17 \%$. The sample with a $5.74 \mathrm{~kg}$ surcharge gradually reached a maximum swell of about $17 \%$ in 535 days. It appears that the small surcharge $(1.12 \mathrm{~kg})$ weight was moved upward by crystal growth in pore spaces between aggregate particles. Long cylindrical, needlelike crystals (possibly gypsum or ettringite) of about $2.5 \mathrm{~cm}(1 ")$ in length were observed in FBC waste aggregate samples exposed to water. The needle-like structure may have eventually collapsed and caused the surcharge weight to move downward. Therefore, a decrease in the swell measurements occurred. Final swell values of both samples were about $17 \%$ after a 995 day soaking period. Because of the large magnitudes of swelling observed from long-term tests, the $100 \%$ FBC waste aggregates could not be used as a highway base or subbase. 
Two additional synthetic waste aggregate samples were tested. The samples were identified as FBC/FA-1 (made from 90\% FBC waste and 10\% fly ash) and FBC MS-1 (made from $95 \%$ FBC waste and $5 \%$ microsilica). Microsilica is a by-product from the glass industry. Los Angeles abrasion, sodium sulfate soundness, and specific gravity and absorption tests were performed on these samples to evaluate durability. No particle size analyses were performed because the samples were graded by size before testing. Results of tests performed on the aggregates made with FBC waste blended with $10 \%$ fly ash and $5 \%$ microsilica are shown in Tables 12 and 13. Both the soundness and LA abrasion indices improved with the addition of fly ash and microsilica. Properties of these aggregates will be discussed further in the section on "Improved Product Development" (Task 2, Subtask 2.3).

Specific gravity tests (AASHTO T 84) were performed on the FBC/FA1 (3/8" to 4 mesh) sample in an oven-dried (standard test method) state and in an air-dried (or as-is) condition. The specific gravity of the oven-dried aggregate, measured by the standard test method, was 2.53 , and the specific gravity of the air-dried (as-is) aggregate was 2.19. The absorption of the oven-dried aggregate was $17.89 \%$. The absorption of the air-dried aggregate absorption was $6.38 \%$. Drying the $\mathrm{FBC}$ waste aggregate at $110^{\circ} \mathrm{C}\left(230^{\circ} \mathrm{F}\right)$ in an oven increases the ability of the aggregate to absorb water.

\section{Preparations for Field Demonstration}

The Ohio Department of Transportation (ODOT) was contacted for assistance in locating a site suitable for consideration of using FBC waste as a chemical stabilizer in a highway subgrade. The Onio DOT does not routinely stabilize subgrades with chemical additives; however, discussions were held concerning using FBC waste as a subgrade stabilizer on portions of 1-275 in the Cincinnati area. The pavement on this route was scheduled to be removed, the subgrade modified, and a new pavement was to be placed. A decision was made not to use this site as an experimental field demonstration due to the uncertainty of some of the engineering properties of the FBC waste (mainly its potential to swell) and the high traffic volume. Traffic control for followup testing would be very costly on a high traffic volume interstate route.

A potential site was located by the Ohio Coal Development Office in Belmont County, Ohio, for a possible demonstration of the use of $\mathrm{FBC}$ waste aggregate in a bituminous concrete surface application. Aggregate samples (crushed river gravel, AASHTO number 8 , and natural sand) were obtained from Lash Excavating and Paving, an asphalt paving contractor in Belmont 
County. Gradation tests were performed on the coarse aggregate (AASHTO No. 8) and the fine aggregate (natural sand) to determine if the aggregate met Ohio DOT specification number 404 for aggregates in a bituminous concrete surface. Results of gradation tests show the aggregate meets grain size specifications as seen in Figures 10 and 11. A control paving mix was made using the Marshall Mix Design method for low volume roads. Aggregate used in the control mix was blended to proportions normally used ( $52 \%$ coarse; AASHTO number 8 and $48 \%$ fine; natural sand). Results of the Marshall Mix Design for the control mix are presented in Figures 12 through 16 . The Marshall stability of this mix was $2,068 \mathrm{~N}(465 \mathrm{lb})$, which did not meet the minimum criteria of $3,336 \mathrm{~N}(750 \mathrm{lb})$. Voids in mineral aggregate ranged from 19.8 to $20.4 \%$, which meets the ODOT specification of $16 \%$ minimum. The lowest percent air voids (at $6.0 \%$ asphalt cement) was $5.8 \%$, slightly more than the $5 \%$ maximum allowed by ODOT specifications. Values of flow did not meet ODOT requirements.

An additional mix was made using $25 \%$ coarse aggregate, $25 \%$ synthetic aggregate, $46 \%$ fine aggregate, and $4 \%$ fly ash, which was acquired from the Ohio Edison Bruce Mansfield power plant and used as a mineral filler. Synthetic aggregate was made with $90 \% \mathrm{FBC}$ waste and $10 \%$ fly ash. Results are shown in Figures 17 through 21 . The stability of this mix met the minimum stability requirement of $3,336 \mathrm{~N}(750 \mathrm{lb})$ at $6.5 \%$ asphalt cement. Voids in mineral aggregates and flow were within ODOT specifications; however, the percent of air voids was higher than the $5 \%$ maximum allowed by ODOT specifications. The Belmont County site was not selected because it would have required a pilot plant-scale facility for aggregate production. It was calculated that 20 tons of synthetic aggregates would have been required to do the Belmont demonstration project. The capacity to manufacture the necessary tonnage was beyond the scope of this study. An alternative field demonstration was conducted at an alternative site in West Mifflin, Pennsylvania, which will be discussed later in Task 2, Subtask 2.4 "Large Sample Production and Field Testing".

\section{Task 2. Waste Pelletization Research with Coolside, LIMB, and FBC Wastes}

\section{Task 2.1 Sample Acquisition}

The objective of this subtask was to obtain Coolside, LIMB and FBC waste samples generated under different process conditions. These samples will have distinctively different chemical compositions and properties, representing a range of wastes produced from each process. 
Coolside wastes used in this task include samples from the Ohio Edison Edgewater Station Coolside Demonstration and from the CONSOL Coolside pilot plant. The Edgewater Station Coolside waste sample (Sample No. 2) was collected by the University of Kentucky from the ESP hopper on February 13,1990, during the Coolside process demonstration program (see the Phase 1 Milestone Report). Three Coolside waste samples of about $500 \mathrm{lb}$ each were collected from the CONSOL Coolside pilot plant operating in the once-through mode (January 7-9, 1992), a recycle mode (January 10-15, 1992) and in an advanced mode (January 23,1992 ), respectively. These samples were produced at conditions that simulate operation of the Coolside process as installed on a unit burning medium-sulfur Ohio coal (see the Phase 3 Milestone report). Analyses of Edgewater and CONSOL Coolside waste samples are presented in Table 14. Three samples of LIMB wastes were taken from the ESP hopper at the Ohio Edison Edgewater Station LIMB Demonstration on May 25, 1989, July 10, 1991, and July 25, 1991 . One pulverized coal (p.c.) fly ash sample was taken from the ESP hopper at the Ohio Edison Bruce Mansfield Station on October 12, 1992. Analyses of Edgewater LIMB waste samples and the Bruce Mansfield fly ash sample are presented in Table 15.

All Coolside and LIMB waste samples were used in the baseline pelletization task (Task 2, Subtask 2.2) and in the improved product development task (Task 2, Subtask 2.3) to prepare synthetic aggregates for evaluation. The p.c. fly ash was used in Subtasks 2.3 and 2.4 .

Four FBC wastes were taken from the ash silo of a coal-fired circulating fluidized-bed combustor (FBC) at P. H. Glatfelter Co. (Spring Grove, PA) on February 18, 1992, September 2, 1993, March 14, 1994, and October 22, 1994. The FBC unit fired a Pittsburgh seam coal (the same seam as Ohio 8). The FBC waste samples included fly ash and bottom ash generated by the unit. The February 18, 1992, and September 2, 1993, waste samples were used in the baseline pelletization study (Task 2.2) and the improved product development study (Task 2.3). The March 14, 1994, and October 22, 1994, waste samples were used in the large sample production and field testing program (Task 2.4). Waste from a second FBC also was tested. It was planned originally that the waste from the second FBC would be collected from a coal-fired bubbling fluidized-bed combustor at the Quaker State Congo Refinery (Newell, West Virginia). However, continuing plant operating problems prevented the collection of this material. Therefore the waste was collected from a coal-fired circulating fluidized-bed combustor at the GM Pontiac Plant near Detroit on September 16, 1988. The GM FBC waste was tested in the baseline pelletization study. Analyses of Glatfelter and GM FBC waste samples are presented in Table 16. 
Tasks 2.2 and 2.3 Baseline Pelletization and Improved Product Development

The objective of Task 2.2 was to determine the effects of pelletizing, mixing, and curing process variables on waste pellet properties and to determine the relationship between chemical, physical, and engineering properties of pelletized LIMB, Coolside, and AFBC wastes. The objective of Task 2.3 was to evaluate the feasibility of producing lightweight aggregates for concrete application, and to optimize the mix formulation for producing synthetic aggregate for road construction.

The Baseline Pelletization study included evaluating the effect of process variables on the preparation of pellets from Coolside, LIMB and FBC wastes and determining the properties of pellets for use as synthetic aggregates in construction. Pellets were made using a three-step process that includes hydration (or densification), disk pelletization, and curing. This process takes advantage of the cementitious properties of wetted Coolside, LIMB, and FBC wastes to make a product with enlarged particle size and adequate strength for construction uses. The pellets were evaluated for use in construction, according to Ohio, ASTM and other standard specifications. Four Coolside wastes, three LIMB wastes, and two FBC wastes were used in the baseline pelletization study (see Phase 2, Task 2, Subtask 2.1, Sample Acquisition). The dry waste was first mixed with the appropriate amount of water to hydrate the LIMB or FBC wastes or densify the Coolside waste. The wetted material was pelletized with additional water in a rotary disk pelletizer to enlarge the particle size. The pelletized products were cured under high humidity at elevated temperature to produce a synthetic aggregate with the required aggregate properties. Considerable effort was required to optimize the hydration conditions and the curing steps to prepare high-strength pellets. Certain factors were identified as being important for producing pellets with adequate properties for construction use: the proper amount of water addition; high conversion of $\mathrm{CaO}$ to $\mathrm{Ca}(\mathrm{OH})_{2}$ during hydration; and avoidance of steam condensation on the pellet surface during curing. Based on the laboratory test results, a practical method was developed and high strength pellets were made. A 55-gallon curing drum, equipped with steam injection for heating and humidity control, was designed and constructed. The curing drum was designed as a prototype that could be scaled up for a large-scale operation.

In a typical baseline pelletization test, a batch of about $70 \mathrm{lb}$ of pellets was produced for evaluation from each of the LIMB, Coolside, and FBC wastes. A Littleford Brothers LM-130 batch mixer was used for hydration (or densification), and a 36" i.d. rotary disk pelletizer was used for pelletization. The pelletized products were cured in the $55 \mathrm{gal}$ drum at about $180^{\circ} \mathrm{F}$ and 90 - 
$100 \%$ relative humidity for $24 \mathrm{hr}$. The pellet properties that were evaluated included: unit weight, grain size distribution, specific gravity, water absorption, pellet compressive strength, LA abrasion index, and soundness index. All of these properties are important for use of aggregate in road construction. Properties of Coolside, LIMB, and FBC waste pellets from the baseline pelletization study are discussed in the next sections.

\section{Coolside Waste Pellets}

Table 17 compares the properties of pellets prepared from the Ohio Edison Edgewater Station and the CONSOL pilot plant Coolside wastes with the Ohio coarse aggregate specifications. Coolside wastes from the CONSOL pilot plant included those generated in once-through, recycle and advanced operation modes. Tests $A$ and $B$ were conducted to evaluate the effect of water addition during the mixing step on the pellet properties (Table 17). Both tests used Coolside waste from the CONSOL pilot plant operated in the once-through mode. In Test A, 22\% water (dry ash basis) was added during the mixing step. In Test $B, 25 \%$ water (the optimum amount of water) was added during the mixing step. As indicated in Table 17, the pellet compressive strength increased from $39 \mathrm{lb}$ to $70 \mathrm{lb}$ and the LA abrasion index decreased from $50.1 \%$ to $34.2 \%$ when the optimum amount of water was added in Test $B$. All latter tests were conducted with the addition of the optimum amount of water during the mixing step. The properties of waste pellets produced with optimum water addition during the mixing step depended on the Coolside operating conditions (once-through, recycle, or advanced) under which the waste was generated. Pellets made from the waste generated in the recycle mode have higher compressive strength (145 lb) and lower LA abrasion index (23.7\%) than those produced in the once-through or advanced mode. The pellets from the recycle mode also have higher bulk specific gravity (1.73) and lower water absorption (2.6\%) than the others. When compared with the Ohio specifications for use as aggregates in road construction (Table 17), Coolside waste pellets meet the requirement for LA abrasion index ( $50 \%$ max. weight loss in aggregate base use, $40 \%$ max. weight loss in asphalt and portland cement concrete use). The pellets fail to meet specifications for unit weight and soundness index. Most of the Coolside waste pellets were between $3 / 4^{\prime \prime}$ and 4 mesh. The particle size distribution of the Coolside waste pellets meets the Ohio requirements for use as coarse aggregates in road construction. The particle size distribution is related to pelletization operation. A specific size specification could be met by adjusting the conditions during disk pelletization and crushing. 
As discussed above, Coolside waste pellets fail to meet the Ohio specifications for unit weight and soundness index for use as road construction aggregates. However, the dry unit weights of pellets from all of the Coolside waste materials meet the ASTM C331 unit weight specification ( $55 \mathrm{lb} / \mathrm{ft}^{3}$ max, dry basis) for coarse lightweight aggregates in concrete masonry units. For this use, there is no soundness index specification. As shown in Table 17, Coolside waste pellets also have high compressive strength and low specific gravity. Thus, the baseline pelletization program indicated that pellets made from Coolside wastes may have potential for use as lightweight coarse aggregates in concrete masonry units. The Improved Product Development Program (Phase 2, Task 2, Subtask 2.3), discussed below, was focused in that direction.

In the baseline pelletization study, it was shown that Coolside waste pellets have potential for use as lightweight coarse aggregates in concrete masonry units. The properties of pellets made from the Coolside pilot plant recycle mode waste were examined and compared with the ASTM lightweight aggregate specifications (Table 18). Included in the table for comparison are the properties of waste pellets made from the Coolside recycle mode waste blended with $10 \%$ fly ash. These were made to evaluate the effect of fly ash addition on pellet properties. As shown in Table 18, pellets made from the Coolside waste blended with $10 \%$ fly ash have higher pellet compressive strength, unit weight, bulk specific gravity, and lower water absorption than those generated from neat Coolside waste. The former has a slightly coarser size distribution than the latter. However, as discussed previously, the pellet size distribution is more related to pelletization operations than to sample characteristics. The LA abrasion indices of the pellets prepared from the neat Coolside waste and the 90/10 Coolside waste/fly ash blend are $23.7 \%$ and $20.5 \%$, respectively. Both are well within the AASHTO Class A and Ohio specifications ( $40 \mathrm{wt} \% \mathrm{max}$ ) for use as aggregates in road construction. There is no abrasion index requirement for lightweight aggregate. Soundness indices of both pellets ( $99.0 \%$ and $76.7 \mathrm{wt} \%)$ are too high to meet the specification (12\% max.) for use as aggregates in road construction. However, as shown in Table 18, the dry unit weights of these pellets are $50.8 \%$ and $57.6 \mathrm{lb} / \mathrm{ft}^{3}$, within or close to the ASTM C331 specification ( $55 \mathrm{lb} / \mathrm{ft}^{3}$, max.) for use as coarse lightweight aggregates in concrete masonry units. There is no soundness index requirements for lightweight aggregates. As shown in Table 18, the percent of clay lumps and friable particles decreased from $2.6 \%$ for neat Coolside waste pellets to $1.7 \%$ for the $10 \%$ fly ash addition case. The ASTM C331 specification for the clay lumps and friable particles is $2 \%$ (max.). Both pellets meet the staining material (ASTM C142) and organic impurity (ASTM C40) specifications of ASTM C331 for use of lightweight aggregates in concrete masonry units. These results demonstrate that high 
strength lightweight pellets with a low abrasion index can be prepared from Coolside waste with blended fly ash. The pellets meet the specifications for lightweight aggregates and could be used as coarse lightweight aggregates in concrète masoniry units.

\section{LIMB Waste Pellets}

Properties of pellets from LIMB wastes generated under different process operating conditions are presented in Table 19. The July 10,1991, sample was obtained at the following conditions: $3.8 \%$ sulfur coal, Ca/S mol ratio of $1.4 ; \mathrm{SO}_{2}$ removal of $40 \%$. The July 25,1991 , sample was obtained at the following conditions: $1.6 \%$ sulfur coal, $\mathrm{Ca} / \mathrm{S} \mathrm{mol}$ ratio of 1.5 , and $45 \% \mathrm{SO}_{2}$ removal. On May 25, 1991, sample was obtained at the following conditions: $3.0 \%$ sulfur coal, $\mathrm{Ca} / \mathrm{S} \mathrm{mol}$ ratio of 2.0 , and $50 \% \mathrm{SO}_{2}$ removal. Pellets made with the July 25 waste have a higher pellet compressive strength (104 vs $44 \mathrm{lb}$ ) and a lower LA abrasion index ( $38.2 \%$ vs $54.5 \%$ weight loss) than the pellets made with the July 10 waste. Pellets made with the July 25 waste meet the maximum requirement for the LA abrasion index ( $50 \%$ for aggregate base and $40 \%$ for asphalt and portland cement concrete) for use as coarse aggregate in road construction, according to the Ohio specifications. The lower pellet strength and higher LA abrasion index of the pellets from the July 10 waste probably are associated with higher $\mathrm{CaSO}_{4}$ content and lower fly ash content (as indicated by the $\mathrm{SiO}_{2}, \mathrm{Al}_{2} \mathrm{O}_{3}$ and $\mathrm{Fe}_{2} \mathrm{O}_{3}$ contents). As shown in Table 19, pellets from the July 25 waste have higher bulk specific gravity (1.60 vs 1.51) and lower water absorption $(6.11 \%$ vs $11.60 \%)$. Most of the LIMB waste pellets are in the size range of $3 / 4 " \times 4$ mesh, similar to the Coolside waste pellets reported previously. The pellets from the LIMB waste generated on May 25, 1991, are strong, coarse, have a low LA abrasion index and a low water absorption capacity, but are too dense to meet lightweight aggregate specifications.

Similar to Coolside waste pellets, the pellets made from the July 10 and 25 LIMB waste samples fail to meet the Ohio specifications for unit weight and soundness index. However, the dry unit weight of the pellets meet the minimum ASTM C331 unit weight specification ( $55 \mathrm{lb} / \mathrm{ft}^{3}$, dry basis) for use as coarse lightweight aggregates in concrete masonry units. This application for LIMB waste pellets was pursued in the Improved Product Development Program (Subtask 2.3), discussed below.

The properties of pellets made from the Edgewater LIMB waste (7/10/91) were compared with the ASTM lightweight aggregate specifications in Table 20. Also included in the table are properties of waste pellets made from the LIMB waste $(7 / 10 / 91)$ blended with $10 \%$ fly ash. These 
were made to evaluate the effect of fly ash addition on pellet properties. As shown in Table 20, pellets made from LIMB waste blended with $10 \%$ fly ash have much higher pellet compressive strength, unit weight and bulk specific gravity, and a lower water absorption than those generated from the neat LIMB waste. The $10 \%$ blend product has a coarser size distribution than the neat pellets. As discussed previously, the size distribution is more related to pelletization operations than to pellet characteristics. The LA abrasion index of the pellets prepared from the 90/10 LIMB wasteffly ash blend is $22.1 \mathrm{wt} \%$, which is substantially lower than the abrasion index of the pellets made from the neat LIMB waste (54.5\%). The 90/10 LIMB waste/fly ash blend pellets meet the AASHTO Class $A$ and Ohio LA abrasion index specifications ( $40 \mathrm{wt} \%$, max.) for use as aggregates in road construction. The pellets soundness index $(42.7 \mathrm{wt} \%)$ is too high to meet the specification $(12 \%, \max )$ for use as aggregates in road construction. However, as shown in Table 20, the dry unit weight is $55.3 \mathrm{lb} / \mathrm{ft}^{3}$, which is close to the ASTM C331 specification (55 lb/ft', max) for use as coarse lightweight aggregates in concrete masonry units. There is no soundness index requirements for lightweight aggregate in this application. As shown in Table 20, the percent of clay lumps and friable particles decreased from $2.4 \%$ (neat LIMB waste pellets) to $0.7 \%$ with $10 \%$ fly ash addition. The ASTM C331 specification for the content of clay lumps and friable particles is $2 \%$ max. Tests of staining material (ASTM C142) and organic impurity (ASTM C40) in the 90/10 LIMB waste/fly ash pellets meet the ASTM C331 specification for lightweight aggregates. These results demonstrate that high-strength lightweight aggregates with a low abrasion index can be prepared from LIMB waste with blended fly ash. Similar to the aggregates prepared from Coolside waste with blended fly ash, these aggregates (although failing to meet the soundness index specification of road aggregate) pass the specifications for lightweight aggregates and have potential for use as coarse lightweight aggregates in concrete masonry units.

\section{FBC Waste Pellets}

Properties of pellets produced from Glatfelter FBC wastes obtained on two sampling dates (February 28, 1992 and September 2, 1993) are presented in Table 21. The waste samples were generated at the Pyropower circulating fluidized-bed combustor at the P. H. Glatfelter Co.. Operating conditions were: $2.3 \%$ sulfur coal, $\mathrm{Ca} / \mathrm{S} \mathrm{mol}$ ratio of about 2.9 , and $90 \% \mathrm{SO}_{2}$ removal. Also presented in Table 21 are the American Association of State Highway and Transportation Officials (AASHTO) specifications for Class A coarse aggregate. AASHTO Class A aggregate is a premium road aggregate that meets or exceeds all Ohio specifications (Table 17) for road construction aggregate. The effects of mixing time and the amount of water added during 
hydration on pellet properties were examined in Tests $A, B$, and $C$. The pellets were made with the February 28, 1992 waste sample. The mixing times for the hydration step in Tests $A$ and $B$ were $12 \mathrm{~min}$ and $20 \mathrm{~min}$, respectively. As shown in Table 21, the cured pellets from Test $A$ have a compressive strength of about $40 \mathrm{lb}$ and an $\mathrm{LA}$ abrasion index of $51.5 \%$. As the mixing time increased to $20 \mathrm{~min}$, the compressive strength of the cured pellets increased to about $90 \mathrm{lb}$ and the LA abrasion index decreased to $37.4 \%$. Pellets produced in Test $B$ have higher unit weight (72.1 vs $\left.69.8 \mathrm{lb} / \mathrm{ft}^{3}\right)$ and bulk specific gravity $(2.00 \mathrm{vs} 1.90)$ than pellets produced in Test $A$. Thermogravimetric analysis (TGA) data indicate that the conversion of $\mathrm{CaO}$ to $\mathrm{Ca}(\mathrm{OH})_{2}$ increased from $60 \%$ (Test $A$ ) to $80 \%$ (Test $B$ ) as the mixing time increased. This indicates that high $\mathrm{CaO}$ conversion during the hydration step is important for preparation of $F B C$ waste pellets with high strength and low abrasion index. The amount of water added also is important for improving pellet properties. Test $C$ of the February 28,1992 , sample was conducted with the same mixing time as Test $B$ (high $\mathrm{CaO}$ conversion), but the optimum amount of water was added during the hydration step. As shown in Table 21, the compressive strength of the cured pellets increased to about $190 \mathrm{lb}$ and the LA abrasion index decreased to $26.8 \%$. The sample taken September 2 , 1993, also was pelletized with the longer mixing time (high $\mathrm{CaO}$ conversion) and optimum water addition. As indicated in Table 21, the two batches of FBC waste pellets have similar bulk specific gravity (2.02 vs 2.03 ), water absorption ( $4.1 \%$ vs $3.9 \%$ ) and soundness index ( $6.9 \%$ vs $6.6 \%)$, even though these two wastes have different sulfur contents $(7.88 \%$ vs $6.00 \%)$ and $\mathrm{Ca} / \mathrm{S}$ mol ratios (3.47 vs 4.43), as shown in Table 16. Both batches of FBC waste pellets easily meet the AASHTO Class A specifications for use as coarse aggregate in road construction (Table 21). The difference in compressive strength (190 lb vs $140 \mathrm{lb}$ ) could be related to pellets characteristics or sizes of particles used for strength determination. The difference in unit weight ( $\left.71.8 \mathrm{vs} 74.6 \mathrm{lb} / \mathrm{ft}^{3}\right)$ is most likely caused by size distribution. Size distribution is more related to pelletizer operation than to sample characteristics. The difference in size distribution should not affect the pellet characteristics, such as LA abrasion and soundness indices, specific gravity and water absorption. In contrast to LIMB and Coolside waste pellets, pellets from Glatfelter FBC wastes sampled on two dates meet all AASHTO Class A and the Ohio specifications for use as aggregates in road construction.

FBC waste from a second Pyropower circulating fluidized-bed combustor, the GM Pontiac Plant, also was used to prepare FBC waste pellets (GM FBC waste) for evaluation. Table 22 compares the properties of pellets produced from the GM FBC waste and the Glatfelter FBC waste (taken February 28,1992 ) with the AASHTO specifications for Class A coarse aggregates. As indicated 
in Table 16, GM FBC waste has higher "unburned" carbon content (10.78\% vs $4.93 \%$ ), lower $\mathrm{Ca} / \mathrm{S}$ mol ratio (1.66 vs 3.47 ) and lower lime index ( 6.4 vs 26.3 , based on $\mathrm{CaO}$ ) than the July 28 , 1992, Glatfelter FBC waste. As shown in Table 22, the pellets made with the GM and Glatfelter FBC wastes have similar pellet compressive strength (183 vs $190 \mathrm{lb}$ ), unit weight ( 75.3 vs 71.8 $\left.\mathrm{lb} / \mathrm{ft}^{3}\right)$, bulk specific gravity ( 1.98 vs 2.03 ) and water absorption ( 3.0 vs $3.9 \%$ ). Both batches meet the AASHTO Class A unit weight requirement $\left(70 \mathrm{lb} / \mathrm{ft}^{3}, \mathrm{~min}\right)$. The main differences in the pellet properties are the LA abrasion index (17.7 vs $26.8 \%$ ), soundness index ( 71.8 vs $6.6 \%)$ and particle size distribution. The pellets from the GM FBC waste have a lower $L A$ abrasion index and an higher soundness index. Both batches meet the AASHTO Class A LA abrasion index requirement $(40 \%$ max). However, the soundness index $(71.8 \%)$ of the GM FBC waste pellets is too high to meet the AASHTO Class A soundness index requirement $(12 \%, \max$.) and the Ohio soundness index specifications (15\%, max. in aggregate base and asphalt concrete and $12 \%$, max. in portland cement concrete) for use as aggregates in road construction. The high soundness index of the GM FBC waste pellets may be associated with its high "unburned" carbon content $(10.78 \%)$ and low lime index $(6.4 \% \mathrm{CaO})$. The difference in particle size distribution of pellets from GM and Glatfelter FBC wastes is more related to pelletization operations than to sample characteristics and should not affect the pellet characteristics discussed above, except possibly the unit weight. Since the GM FBC waste pellets fail to meet the specifications for use as aggregate in road construction, Glatfelter FBC waste was selected for further evaluation.

The test results described above indicate that pellets prepared from $100 \%$ Glatfelter FBC waste meet the AASHTO specifications for the Class A coarse aggregates for use in road construction. Long-term durability is a concern for use of synthetic aggregates in construction. Therefore, FBC waste pellets aged for 197 days also were evaluated. Table 23 compares the properties of fresh and aged Glatfelter FBC waste pellets with those of AASHTO Class A gravel and crushed limestone, both of which are naturally occurring aggregates used commercially in highway surface wearing course. As indicated in Table 23, fresh FBC waste pellets and natural aggregates have similar LA abrasion and soundness indices. The fresh FBC waste pellets have lower specific gravity and unit weight, and a higher water absorption than gravel and crushed limestone. After aging for 197 days, there is little change in LA abrasion index (25.2\% vs $26.9 \%$ weight loss), but a substantial increase in soundness index ( $51 \%$ vs $6.6 \%)$. The soundness index of the aggregate made from $100 \% \mathrm{FBC}$ waste pellets degraded with aging and became too high to meet the specifications for use as aggregate in road construction. 
The degradation of FBC waste pellets with aging can be reduced by blending FBC waste with an additive during pellet preparation. As an example, the effect of $10 \%$ blended pulverized coal (p.c.) fly ash on the properties of fresh and aged FBC waste pellets is shown in Table 24. As indicated, there are few substantial changes in LA abrasion index (29.1\% vs $22.0 \%$ ), soundness index $(11.1 \%$ vs $10.9 \%$ ) and compressive strength (199 vs $191 \mathrm{lb}$ ) of the pellets made from the $90 / 10$ FBC waste/fly ash blend before and after aging for 207 days. The aged pellets meet the AASHTO Class A aggregate for use in road construction. In contrast, pellets from $100 \%$ FBC waste degraded and do not meet the specification. The effects of blended fly ash and other additives on the properties of FBC waste pellets were examined further in the Improved Product Development Program (Subtask 2.3), discussed below.

As discussed previously, pellets prepared from Glatfelter FBC waste meet all AASHTO Class A coarse aggregate specifications. However, as shown in Table 23, the soundness index of FBC waste pellets increased from $7 \%$ to $51 \%$ after storing (or aging) for 197 days. Degradation of FBC waste pellets caused by aging could be eliminated by adding fly ash (Table 24). Microsilica was tested as another additive. Tests were made with processed microsilica and waste microsilica. Properties of the pellets made from the February 28,1992 , Glatfelter FBC waste with the addition of $5 \%$ and $10 \%$ processed microsilica were evaluated to study the effect of this additive on the properties of FBC waste pellets. Properties of the pellets made with blended microsilica are presented in Table 25. Also included are the properties of the pellets made from the neat FBC waste and the AASHTO Class A coarse aggregate specifications. The processed microsilica is a by-product of glass manufacturing. It could lower the pellet soundness index and water absorption by decreasing the pellet void volume. As shown in Table 25, pellets made from FBC waste with $5 \%$ and $10 \%$ processed microsilica have similar pellet compressive strength, unit weight, and bulk specific gravity, but lower water absorption and soundness index than the neat FBC waste pellets. No measurable weight loss was observed in the soundness test on pellets made from the FBC waste/processed microsilica blend. Although processed microsilica can improve the pellet soundness index, it may be too expensive (about $\$ 200 /$ ton) for this application.

Waste microsilica, a less expensive waste stream, also was obtained for evaluation. The properties of pellets prepared with $5 \%$ blended waste microsilica (based on total weight) are listed in Table 26. Included in the table for comparison are the properties of pellets produced from FBC waste blended with $5 \%$ processed microsilica. Pellet compressive strength, LA abrasion index, 
unit weight, bulk specific gravity and water absorption are similar to pellets made from FBC waste with $5 \%$ blended waste microsilica and those produced with $5 \%$ blended processed microsilica. The major difference between the two pellets is the increase in soundness index. The waste microsilica product soundness index is $11.6 \%$. The soundness index of FBC waste pellets blended with $5 \%$ waste microsilica also is higher than that of the neat FBC waste pellets (11.6\% vs $6.6 \%)$.

As discussed previously, the soundness index of pellets prepared with $100 \%$ FBC waste increased with aging. The effect of aging on the soundness index of pellets prepared with $5 \%$ and $10 \%$ blended process microsilica was examined. Test results are presented in Table 27. As shown in the table, the soundness indices of pellets made with $10 \%$ and $5 \%$ blended processed microsilica increased (14.0\% vs $0 \%$ and $7.9 \%$ vs $0 \%$ ) after aging for about 110 days. The pellets prepared with the $90 / 10$ blend of FBC waste and p.c. fly ash did not exhibit this problem (see Table 24). Fly ash generally is inexpensive. Later work, described below, was concentrated on pellets made from FBC waste/fly ash blends.

In addition to aging during storage, freeze/thaw and wet/dry cycle durability is a concern for use of synthetic aggregate in road construction. A study was conducted to determine the effect of natural weathering on the properties of FBC waste pellets made with $10 \%$ blended fly ash. FBC waste pellets were placed in two types of open-topped containers exposed to the natural freeze/thaw and wet/dry conditions throughout the winter of 1993/94. Precipitation was allowed to drain from one container (the drained weathering test) but not from the other (the undrained weathering test). In the undrained test, $4.4 \mathrm{lb}$ of FBC waste pellets were placed outside in an open-topped stainless steel pan (20" $\left.L \times 12^{\prime \prime} W \times 21 / 2 " D\right)$ from November 30, 1993, to April 4, 1994 , to determine the changes in pellet properties under undrained winter weathering conditions. The properties of pellets before and after natural weathering are listed in Table 28. Properties determined include compressive strength, soundness index, and particle size distribution. The pellets had an initial compressive strength of $180 \mathrm{lb}$, a soundness index of $3.1 \%$ and equal amount of two size fractions of $1 / 2 " \times 3 / 8^{\prime \prime}$ and $3 / 8^{\prime \prime} \times 4$ mesh. The FBC waste pellets used in the test were prepared on November 16, 1993 with $10 \%$ blended fly ash in a baseline pelletization test.

In the drained test, about $100 \mathrm{lb}$ of FBC waste pellets were placed in an open-topped wooden box (24" $\left.L \times 14^{\prime \prime} W \times 8^{\prime \prime} D\right)$ from December 30,1993, to April 4, 1994, to determine pellet property 
changes under winter weathering conditions. The properties of pellets before and after the drained weathering test are given in Table 29. Properties determined include pellet compressive strength, LA abrasion index, soundness index, and particle size distribution. The pellets had an initial compressive strength of $146 \mathrm{lb}$, an abrasion index of $31.6 \%$, a soundness index of $0.5 \%$ and over $90 \%$ of the particles were between $3 / 4$ " $\times 4$ mesh. The FBC waste pellets used in the test were prepared from a semi-continuous waste pelletization scale-up test, which will be discussed in the section on the large sample production and field testing study (Subtask 2.4).

Test results from both drained and undrained tests indicate that $\mathrm{FBC}$ waste pellets made with $10 \%$ blended fly ash degraded after exposure to natural weathering throughout the winter. In the undrained test, the compressive strength of the air-dried pellets decreased from $180 \mathrm{lb}$ to 63.2 $\mathrm{lb}$, the soundness index increased from $3.1 \%$ to $22.5 \%$, the -8 mesh fines increased from 0 to $10.6 \%$, and the coarse $\left(3 / 4^{\prime \prime} \times 1 / 2\right.$ ") particles increased from 0 to $9.3 \%$ after 114 days natural weathering (Table 28). The decrease in compressive strength and the increases in soundness index and fines indicate pellet degradation. In the drained test, the compressive strength of the air-dried pellets decreased from $146 \mathrm{lb}$ to $132 \mathrm{lb}$, the LA abrasion index increased from $31.6 \%$ to $58.4 \%$, the soundness index increased from $0.5 \%$ to $46.7 \%$, and there was only a slight difference in the size distribution of the pellets after 85 days natural weathering (Table 29). Thus, pellet degradation is promoted more by undrained conditions than by drained conditions.

The cause for the changes of pellet properties in the drained and undrained weathering tests was examined by thermogravimetric analysis (TGA) of pellet samples taken during and after the natural weathering tests. Characterization results indicate that the slow hydration of anhydrite $\left(\mathrm{CaSO}_{4}\right)$ to gypsum $\left(\mathrm{CaSO}_{4} \cdot 2 \mathrm{H}_{2} \mathrm{O}\right)$ is the major cause for the loss in pellet durability. Analyses by $X$-ray diffraction (XRD) and scanning electron microscopy (SEM) support this conclusion. With this information, it was possible to devise two strategies to improve the pellet durability: diluting the anhydrite, or water-proofing the pellets. The anhydrite can be diluted by admixing FBC waste with fly ash during pellet preparation. The added fly ash also provides alumina, which may consume some of the anhydrite by reaction to produce ettringite. The pellets can be waterproofed by incorporating small quantities of asphalt emulsion in the pellet formulation, or by coating the pellets with asphalt after pellet production, as would be done during actual use in asphalt concrete during road construction. 
The effects of blending FBC waste with fly ash on pellet properties were evaluated. Pellets were produced from five blends of FBC waste and fly ash that ranged from $90 / 10$ to $50 / 50$ FBC waste/fly ash in the semi-continuous scale-up tests (discussed later). The properties of aggregates made from FBC waste and the blends of FBC waste/fly ash are shown in Table 30. All batches meet the AASHTO grade A coarse aggregate specifications for $L A$ abrasion index and unit weight. The $90 / 10,75 / 25$, and $65 / 35$ blends have pellet compressive strengths of $200 \mathrm{lb}$ or greater, and a soundness index meeting the Class $A$ coarse aggregate soundness index specification ( $12 \%$ or below). The $50 / 50$ blend has a relatively high soundness index $(28 \%)$, and fails to meet the specifications. All batches have similar water absorption and specific gravity. The undrained weathering tests (Figure 37) indicate that the durability of FBC waste pellets improves as fly ash addition is increased from $10 \%$ to $25 \%$ to $35 \%$.

An accelerated freeze/thaw test was devised to reduce the time required to evaluate pellet durability. In this test, the pellets are immersed in water for 24 hours and then subjected to at least 50 continuous freeze/thaw cycles $\left(\sim 0^{\circ} \mathrm{F} / \sim 50^{\circ} \mathrm{F}\right)$ over the course of at least seven days. Changes in pellet characteristics and particle size distribution caused by freeze/thaw conditions are examined following the treatment. The freeze/thaw durability of pellets produced with $90 / 10$, $75 / 25,65 / 35$, and $50 / 50$ blends of FBC ash and fly ash were compared using this method. Substantial degradation was observed with pellets made with $90 / 10$ blend of FBC waste and fly ash. $86 \%$ of the $1 / 2 " \times 3 / 8^{\prime \prime}$ pellets and $71 \%$ of the $3 / 8^{\prime \prime} \times 4$ mesh pellets were reduced to smaller sizes after the freeze/thaw treatment. In comparison, $54 \%$ and $29 \%$ of the two respective sizes from the pellets prepared with the $75 / 25$ blend of FBC waste and fly ash were reduced to smaller sizes. $23 \%$ and $18 \%$ of the two respective sizes from the pellets prepared with the $65 / 35$ blend of FBC waste and fly ash were reduced to smaller sizes after the treatment. More degradation (37\% and $17 \%$, respectively) occurred with pellets made from the $50 / 50$ blend of FBC waste and fly ash. Durability of the FBC waste pellets was improved in both the undrained weathering test and the freeze/thaw test as fly ash addition was increased from $10 \%$ to $25 \%$ to $35 \%$. The pellets made from the $65 / 35$ blend of FBC waste and fly ash have the best freeze/thaw durability. On the basis of durability and the other properties, the $65 / 35$ blend of FBC waste and fly ash was selected for further development.

The effects of adding asphalt emulsion and wax emulsion to the pellet formulation on pellet properties were evaluated. Pellets were produced from the $65 / 35$ blends of FBC waste and fly ash with the addition of $1.5 \%, 3 \%$, and $6 \%$ asphalt emulsion in the semi-continuous scale-up 
tests (discussed later). The properties of pellets made with and without asphalt emulsion addition are shown in Table 31 . The properties of pellets made with $5 \%$ wax emulsion addition are included for comparison. Pellet properties compared include LA abrasion and soundness index, compressive strength, water absorption, bulk specific gravity, and particle size. As indicated in Table 31, pellets made with asphalt emulsion addition meet the AASHTO grade A coarse aggregate specifications for $L A$ abrasion and soundness indices, unit weight and size distribution. Pellets made with $5 \%$ wax emulsion addition have high soundness index $(16 \%)$, and fail to meet the soundness index specification (12\%, max.). In addition to soundness index, the freeze/thaw durability of pellets made with asphalt emulsion addition were evaluated. $42.1 \%, 14.4 \%$, and $54.4 \%$ of the $1 / 2$ " $\times 3 / 8$ " pellets and $27.7 \%, 16.8 \%$, and $18.7 \%$ of the $3 / 8$ " $\times 4$ mesh pellets were reduced to small sizes with $1.5 \%, 3 \%$, and $6 \%$ asphalt emulsion addition, respectively, after about 50 freeze/thaw cycles. In comparison, $23.1 \%$ and $18.1 \%$ of the two respective sizes from the pellets made without asphalt emulsion addition were reduced to smaller sizes after treatment. The pellets made with $3 \%$ asphalt emulsion addition have the best freeze/thaw durability.

The freeze/thaw durability of pellets made from the $65 / 35$ blend of FBC waste and fly ash can be further improved by coating the aggregate with asphalt ( $A C 20$ ). Coating the aggregate with asphalt simulates the environment that would be experienced by the aggregates in the asphalt concrete in road construction. The $65 / 35$ blend pellets were coated with asphalt ( $5 \%$ by weight) and subjected to freeze/thaw testing. The pellets produced with a formulation containing asphalt emulsion were likewise coated with asphalt and tested. As shown in Table 32, after 54 freeze/ thaw cycles, $7.5 \%$ of the $1 / 2$ " $\times 3 / 8$ " coated pellets and $4.1 \%$ of the $3 / 8$ " $\times 4$-mesh coated pellets were reduced to smaller pieces. The percent of degradation after freeze/thaw treatment was reduced to zero for the pellets made with $3 \%$ asphalt emulsion addition during preparation and coated with $5 \%$ asphalt before the freeze/thaw treatment. This demonstrates that the combination of appropriate fly ash addition and water-proofing greatly improves the durability of the FBC waste pellets.

\section{Leaching Test of FBC Waste Aggregates}

At the recommendation of Ohio EPA, the leaching characteristics of the synthetic aggregate was determined using de-ionized water as the leaching medium, in accordance with the procedures of ASTM D3987. The allowable limits of the leachable species are 30 times the Maximum Concentration Limits (MCL) set by the primary and secondary water standards of the Safe Drinking Water Act (SDWA) of January 30, 1991. The synthetic aggregate tested was produced 
from $90 \%$ Glatfelter FBC waste and 10\% Ohio Edison Bruce Mansfield Station fly ash as part of the original field demonstration plan. A portion of the synthetic aggregate ( $40 \%$ by weight) was crushed to meet the Ohio requirement for use of coarse aggregate in highway construction. The leaching results are presented in Table 33. As shown, the concentrations of all species are much less than 30 times the MCL set by the primary and secondary water standards. Test results were submitted to Ohio EPA for approval on February 4,1994. Ohio EPA concluded on March 8, 1994, that the synthetic aggregate meet the criteria for non-toxic material, as defined in Policy 4.07. Subsequently, the Division of Surface Water determined that the use of synthetic aggregate made from FBC ash and fly ash in road construction is a beneficial use and does not require a plan approval or permit-to-install from the Ohio EPA.

\section{Asphalt Mix Design for FBC Waste Aggregates}

One of the most frequently used tests to design asphalt and aggregate mixes for use as asphalt concrete in highway construction is the Marshall Stability Test. Trumbull Corporation conducted the Marshall Stability Test with mixtures of synthetic aggregates and limestone to determine the properties of asphalt concrete specimens made with synthetic aggregate as a partial replacement for crushed limestone. The synthetic aggregate used was produced from $90 \%$ Glatfelter FBC waste and $10 \%$ Ohio Edison Bruce Mansfield fly ash that was partially crushed (80\%) to meet AASHTO No. 8 coarse aggregate size requirements. The crushed limestone used was a Class A AASHTO No. 8 coarse aggregate used commercially in the pavement of surface wearing course. Manufactured limestone and sand were the fine aggregates. Results of the Marshall Stability Test using various asphalt mix designs with synthetic aggregate as part of the coarse aggregates are shown in Table 34. FBC waste aggregate was substituted for AASHTO No. 8 limestone coarse aggregates at different ratios: $25 \%$ substitution in mix A, $50 \%$ substitution in mix $B$, and $100 \%$ substitution in mix $C, D$, and $E$, with asphalt cement contents of $6.0 \%, 6.0 \%, 5.5 \%$, $6.0 \%$ and $6.5 \%$, respectively.

As shown in Table 34, the various asphalt cement mix designs exhibit high Marshall stability $(>2200 \mathrm{lb})$. In the cases in which FBC waste pellets made up the entire coarse aggregate (mixes $C, D$, and E), Marshall stability increases with decreasing asphalt cement addition in the mix. Mix C, with $5.5 \%$ asphalt cement content, has the highest Marshall stability $(2875 \mathrm{lb})$. The Marshall flow increases from 11 to 15.3 , bulk specific gravity decreases from 2.236 to 1.927 and voids in mineral aggregates increase from $17.4 \%$ to $22.8 \%$ as the mix design changes from $A$ to E. Both FBC waste aggregate substitution and asphalt cement addition affect air voids. As 
indicated in Table 34, air voids increase from $7.4 \%$ to $15.5 \%$ with increasing substitution of FBC waste aggregate for No. 8 limestone from $25 \%$ (mix A), to $50 \%$ (mix B) and to $100 \%$ (mix D), all at $6.0 \%$ asphalt cement addition. Air voids decrease from $16.1 \%$ to $15.4 \%$ with increasing asphalt cement addition from $5.5 \%(\mathrm{mix} \mathrm{C}$ ), to $6.0 \%$ (mix D) and to $6.5 \%(\mathrm{mix} E$ ), all at $100 \%$ substitution. As a point of reference, Trumbull Corp. reported that a similar mix design with $100 \%$ natural aggregates has a Marshall stability of $2167 \mathrm{lb}$, a flow of 13, a bulk specific gravity of 2.356, air voids of $3.0 \%$, and voids in mineral aggregates of $15.5 \%$. Although each of the mixes with synthetic aggregates exhibits high Marshall stability, the high percent of air voids may be a concern.

A test patch of asphalt concrete with $\mathrm{FBC}$ waste aggregate as part of coarse aggregate was planned to evaluate the effect of weathering on the durability of asphalt concrete produced with FBC waste pellets under heavy traffic conditions.

Task 2.4 Large Sample Production and Field Testing

The objective of this subtask was to produce a large quantity of synthetic aggregate for a field trial as a road construction material.

The batch waste pelletization method was modified for semi-continuous operation to produce a large quantity of synthetic aggregate for use in the field trial and to obtain data for designing a large continuous pilot-plant. Semi-continuous pelletization tests were used to produce the large sample used in the field trial and to produce pellets to evaluate the effect of fly ash and additives on pellet properties in the Improved Product Development Program (Task 2, Subtask 2.3). As in the batch pelletization procedure, the semi-continuous pelletization procedure includes hydration, disk pelletization, and curing. Hydration was conducted with three batches each of $48.7 \mathrm{lb} \mathrm{FBC}$ waste and $26.3 \mathrm{lb}$ fly ash by mixing both with an appropriate amount of water in a Littleford Brothers LM-130 batch mixer. A hydration of temperature of about $190^{\circ} \mathrm{F}$ was maintained from the heat of hydration after the first batch was hydrated. The disk pelletization was conducted by adding hydrated waste semi-continuously to a $36^{\prime \prime}$ i.d. disk pelletizer at a feed rate of about $400 \mathrm{lb} / \mathrm{hr}$ continuously. The curing was conducted by feeding the pelletized products to a $55 \mathrm{gal}$ curing drum at a feed rate of $750 \mathrm{lb} / \mathrm{hr}$. The pelletized products were cured in the curing drum at $\sim 180^{\circ} \mathrm{F}$ and $\sim 90 \%$ relative humidity for $24 \mathrm{hr}$. About $250 \mathrm{lb}$ pellets was produced in each test. 
Twelve scale-up pelletization tests were conducted with a mix of $65 \%$ Glatfelter FBC waste and $35 \%$ Ohio Edison Bruce Mansfield fly ash from October to December 1995 to produce about $3,000 \mathrm{lb}$ of synthetic aggregate for use in the field demonstration. The pellets had compressive strengths ranging from $210 \mathrm{lb}$ to $310 \mathrm{lb}$. $80 \mathrm{wt} \%$ of the pellets were greater than $3 / 8$ ". Most of the $+3 / 8$ " pellets $(70 \%$ of the total weight) were crushed in a Denver jaw crusher and combined with the uncrushed portion (the native $-3 / 8^{\prime \prime}$ fraction plus the remaining uncrushed $+3 / 8$ " fraction) to produce a $3000 \mathrm{lb}$ batch of synthetic aggregates for use in the field trial. Properties of the final blend of aggregates produced are presented in Table 35.

As shown in Table 35, the synthetic aggregates produced had an LA abrasion index of $19 \%$, a soundness index of $11 \%$, a unit weight of $75.0 \mathrm{lb} / \mathrm{ft}^{3}$, fractured pieces of $70 \%$, and a particle size distribution that complies with AASHTO No. 8 coarse aggregate specifications. The aggregate properties meet the AASHTO Class A aggregate specifications. The aggregates were delivered to Trumbull Corp. on December 20,1994, for use in the field demonstration.

Trumbull Cop. constructed a bituminous test patch in which $3000 \mathrm{lb}$ of FBC waste aggregates served as $50 \%$ of the coarse aggregates. The test patch was located on a truck road (Noble Drive) near the Trumbull Maintenance facility in West Mifflin, PA, on April 28, 1995. A blend of $50 / 50 \mathrm{FBC}$ waste aggregate and crushed limestone aggregate $(3,000 \mathrm{lb}$ each) was mixed with asphalt emulsion ( $6.5 \% \mathrm{AC}-20$ asphalt added to the aggregates) in a pug mill. The crushed limestone aggregate is a Class $A$ aggregate with an LA abrasion index of $21 \%$, a soundness index of $7.8 \%$, a unit weight of $103 \mathrm{lb} / \mathrm{ft}^{3}$ and a AASHTO No.8 coarse aggregate size. The limestone served two purposes: 1) as an internal standard, and 2) as a filler to increase the size of the test patch. The mix was transferred to a paver, laid on the road, and compacted with a 20 ton steel roller. The test patch was about $70 \mathrm{ft}$ long and about $12 \mathrm{ft}$ wide, tapering down to $7 \mathrm{ft}$ wide. The first $35 \mathrm{ft}$ of the test patch had a thickness of 1.5-2" and the second (tapered) $35 \mathrm{ft}$ had a thickness of less than 1.5". Several open spots in the test patch were caused by uneven spreading of the small batch of bituminous mix by a high-capacity paver. There was no visible degradation of the aggregate during paving and rolling. The aggregates coated well with the asphalt. Heavy trucks began traveling over the test patch $24 \mathrm{hr}$ after construction.

The mix used in this test was a flexible bituminous surface formulation that contains open graded coarse aggregates and no fine aggregates. This mix is designed to be relatively permeable to water. The heavy truck traffic and the permeability of the open-graded mix design should make 
this a severe test of the aggregate durability under natural weathering conditions. Periodic core samples were taken to evaluate aggregate durability.

The asphalt-coated aggregates used in the test patch construction on 4/28/95 (sampled from the pug mill prior to paving) were immersed in water, treated for 50 freeze/thaw cycles $\left(\sim 50^{\circ} \mathrm{F} / \sim 0{ }^{\circ} \mathrm{F}\right)$ and then extracted with tetrahydrofuran (THF) to remove the asphalt coating. The THF-extracted aggregates then were screened to determine size distribution for evaluation of the freeze/thaw effect on aggregate durability. The size distribution of the THF-extracted aggregate from the pug mill before and after this freeze and thaw treatment are presented in Table 36. As shown in Table 36, the yields of the $4 \times 8$ mesh, $8 \times 50$ mesh and -50 mesh fractions of the THF-extracted aggregates increased from $12 \%, 4 \%$, and $3 \%$ to $19 \%, 12 \%$, and $6 \%$, respectively. Therefore, only a slight fraction of asphalt coated aggregates degraded after the freeze/thaw treatment while immersed in water.

\section{Task 2.5 Monitoring and Report Writing}

The bituminous test patch was inspected, and drill core samples were taken periodically from April 28, 1995, to May 30,1996, to determine aggregate durability under heavy traffic and natural weathering conditions. The winter of $1995 / 1996$ was particularly harsh. The last inspection made under the Grant Agreement was May 30, 1996. The patch surface remained uniformly hard, with no soft spots. The visual inspections revealed no apparent degradation of the patch or the aggregate. The test patch maintained its integrity 13 months after construction.

A circular coring tool ( 3 "i.d.) was fabricated and used to collect monthly core samples from the test patch. Each month, three core samples were collected from the test patch. The core samples represented the full thickness of the test paving layer $\left(\sim 1.5^{\prime \prime}\right)$. The test patch core samples were combined and extracted with tetrahydrofuran (THF) to remove the asphalt coating from the aggregates. The THF-extracted aggregates then were screened to determine size distribution for evaluation of aggregate durability. The size distribution of the core samples after THF extraction are compared in Table 37. The size distribution of the THF-extracted aggregates used in the test patch construction on $4 / 28 / 95$ is included in the table for comparison. The $4 / 28 / 95$ sample is not from a pavement core; it was collected at the exit of the pug mill. No core samples were taken between $12 / 4 / 95$ and $4 / 18 / 96$, because the surface became too hard to obtain intact cores with the available equipment. The drill rig was modified by the addition of a 
diamond bit of to permit the collection of intact core samples from the test patch on $4 / 18 / 96$ and $5 / 30 / 96$.

As shown in Table 37, the 4/28/95 pug mill sample collected before construction has a coarser grain size than the pavement core samples. The increases in fines ( $8 \times 50$ mesh and -50 mesh size fractions) in the 6/1/95 core sample, relative to the pug mill sample, may indicate that there was some degradation of the aggregates during paving or in the first month of services. However, at least some of the fines (and maybe all of them) were generated by the coring operation. Most of the aggregates on the perimeter of each $3^{\prime \prime}$ core were fractured by the coring tool. There are no consistent differences in the fines ( $8 \times 50$ and -50 mesh) of the core samples taken from the period of $6 / 1 / 95$ to $5 / 30 / 96$. It appears that the properties of the $4 / 18 / 96$ core sample is anomalous. Characterization of the aggregate components of the 4/18/96 core indicates that the sample was associated with less representative sampling, which will be discussed later in this section. The size distribution of the core samples indicates that the aggregates maintained their integrity through the test period from 6/1/95 to 5/30/96.

The $8 \times 50$ mesh and -50 mesh fractions of the THF extracted core samples taken from the test patch were submitted for elemental analysis to determine the amount of extraneous material and to evaluate the relative degradation of the FBC pellets and limestone in the sized fractions. The elemental analysis was used to estimate the composition of the aggregates as FBC waste pellet, calcium carbonate, or other (extraneous) material. The content of FBC waste pellets was estimated based on the sulfur analysis, assuming that the sulfur concentration is derived entirely from FBC waste pellets. The calcium carbonate content was estimated based on the difference between the total calcium in each size fraction and the calcium derived from the FBC waste pellets. Calcium carbonate represents the limestone aggregate. The remaining material not categorized as FBC waste pellets or calcium carbonate is categorized as "others" (extraneous material) in Table 38. The "others" category includes impurities in the limestone aggregate and other stones, sand, and dirt carried in the test patch by traffic. The aggregate components (FBC waste pellets, calcium carbonate and others) in the $8 \times 50$ mesh and -50 mesh fractions of the core samples are compared in Table 38.

As shown in the Table 38, there is no obvious trend with time in the estimated composition of the aggregates in the two size fractions. The 4/18/96 core sample has anomalously lower contents of FBC waste pellets and calcium carbonate, and higher content of "others" $(\sim 6 \%)$ in the $8 \times 50$ 
mesh and -50 mesh fractions. The elemental analyses indicate that "others" have high silicon contents $\left(\sim 50 \%\right.$, based on $\left.\mathrm{SiO}_{2}\right)$ and that it is composed mainly of siliceous materials, which are probably from sand and dirt. It appears that non-representative core samples were collected on $4 / 18 / 96$. This is consistent with the observation that the $4 / 18 / 96$ core sample has a size distribution that is quite different from other core samples (Table 38). With the exception of the anomalous sample, the relative contents of $\mathrm{FBC}$ waste pellets and limestone aggregate in the fines ( $8 \times 50$ mesh and - 50 mesh fractions) remained nearly constant through the test period. This result is consistent with the hypothesis that the fines were generated primarily by the coring operation, and not by natural degradation. It is concluded that the FBC waste aggregates maintained their integrity as well as the Class $A$ limestone. 
Table 1. Index Properties of Hydrated FBC Waste and Soil Used For Subgrade Stabilization

\begin{tabular}{|c|c|c|c|c|c|c|c|c|c|}
\hline \multirow{2}{*}{ Sample } & \multirow{2}{*}{ LL } & \multirow{2}{*}{ PL } & \multirow{2}{*}{ PI } & \multirow{2}{*}{$\begin{array}{l}\text { Specific } \\
\text { Gravity }\end{array}$} & \multicolumn{3}{|c|}{$\%$ Passing } & \multirow{2}{*}{\multicolumn{2}{|c|}{$\begin{array}{l}\text { Classification } \\
\text { AASHTO UNIFIED }\end{array}$}} \\
\hline & & & & & $2 \mathrm{~mm}$ & $.075 \mathrm{~mm}$ & $.002 \mathrm{~mm}$ & & \\
\hline Clay & 45 & 20 & 25 & 2.66 & 99.4 & 85.9 & 42.0 & A-7-6 (22) & $C L$ \\
\hline FBC Waste & \multicolumn{3}{|c|}{ Non Plastic } & 2.73 & 99.4 & 50.5 & 5.5 & $A-4(0)$ & $S M$ \\
\hline
\end{tabular}

Table 2. Results of CBR Tests on Clay Soil-Hydrated FBC Waste Mixtures

\begin{tabular}{||l|c|c|c|c|c||}
\hline \multirow{2}{*}{ Penetration, Inches } & \multicolumn{5}{|c|}{ CBR } \\
\cline { 2 - 6 } & 0.1 & 0.2 & 0.3 & 0.4 & 0.5 \\
\hline Clay Soil & 2.07 & 1.64 & 1.46 & 1.33 & 1.28 \\
\hline $8 \%$ FBC Waste Mixture & 5.20 & 5.07 & 4.88 & 4.72 & 4.74 \\
\hline
\end{tabular}

Table 3. Results of CBR Tests, Coolside Waste Generated in Recycle Mode

\begin{tabular}{|c|c|c|c|}
\hline $\begin{array}{c}\text { Age Time } \\
\text { (Days) }\end{array}$ & $\begin{array}{c}\text { Surcharge Weight } \\
\text { kg (lb) }\end{array}$ & $\begin{array}{c}\text { Soaking Time } \\
\text { (Days) }\end{array}$ & CBR \\
\hline 0 & $5.64(12.42)$ & 115 & 36.3 \\
\hline 0 & $1.03(2.27)$ & 220 & 6.0 \\
\hline 7 & $1.13(2.50)$ & 100 & 50.0 \\
\hline 7 & $5.71(12.57)$ & 99 & 143.8 \\
\hline
\end{tabular}


Table 4. CBR Values at $2.54 \mathrm{~mm}$ (0.1 in) Penetration from Coolside Waste Samples Obtained from the Edgewater Power Plant, Demonstration Run Nos. 1 and 3

\begin{tabular}{||c|c|c|c||}
\hline \multirow{2}{*}{ Relative Compaction, \% } & \multicolumn{3}{|c|}{ CBR at 2.54 mm (0.1 in) } \\
\cline { 2 - 4 } & Low Energy & Standard & Modified \\
\hline 90 & 1.4 & 4.8 & 6.4 \\
\hline 95 & 3.7 & 10.5 & 7.9 \\
\hline 100 & 9.3 & 7.1 & 6.4 \\
\hline
\end{tabular}

Table 5. Index Properties of Wormser FBC Residue and P.C. Ash

\begin{tabular}{|c|c|c|c|c|c|c|c|c|c|}
\hline \multirow{3}{*}{$\frac{\text { Sample }}{\text { FBC Residue }}$} & \multirow{2}{*}{ LL } & \multirow{2}{*}{ PL } & \multirow{2}{*}{$\mathrm{PI}$} & \multirow{2}{*}{$\begin{array}{l}\text { Specific } \\
\text { Gravity }\end{array}$} & \multicolumn{3}{|c|}{ Percent Passing } & \multirow{2}{*}{\multicolumn{2}{|c|}{$\begin{array}{c}\text { Classification } \\
\text { AASHTO UNIFIED }\end{array}$}} \\
\hline & & & & & $2 \mathrm{~mm}$ & $.075 \mathrm{~mm}$ & $.002 \mathrm{~mm}$ & & \\
\hline & \multicolumn{3}{|c|}{ Non Plastic } & 3.34 & 98.2 & 49.4 & 2.0 & $A-4(0)$ & $S M$ \\
\hline PC Ash & \multicolumn{3}{|c|}{ Non Plastic } & 2.17 & 100.0 & 86.4 & 10.0 & $A-4(0)$ & ML \\
\hline
\end{tabular}

Table 6. Results of Moisture-Density Tests, Wormser FBC Residue and P.C. Ash

\begin{tabular}{||l|c|c|}
\hline \multicolumn{1}{|c|}{ Sample ID } & $\begin{array}{c}\text { Maximum Dry Density } \\
\mathrm{kg} / \mathrm{m}^{3}\left(\mathrm{lb} . / \mathrm{ft} .^{3}\right)\end{array}$ & $\begin{array}{c}\text { Optimum Moisture } \\
\text { Content, \% }\end{array}$ \\
\hline FBC Residue & $1,427(89.1)$ & 26.4 \\
\hline PC Ash & $1,094(68.3)$ & 35.3 \\
\hline
\end{tabular}

Table 7. Results of Freeze Thaw Test (AASHTO T-103) on Aggregates Made With $100 \%$ FBC Waste

\begin{tabular}{|c|c|}
\hline Sieve Designation & Percent Loss After 10 cycles \\
\hline $12^{\prime \prime}$ & 67.6 \\
\hline $3 / 8^{\prime \prime}$ & 25.2 \\
\hline No. 4 & 55.6 \\
\hline
\end{tabular}


Table 8. Results of Particle Size Analysis (AASHTO T-27) on Aggregates Made with $100 \%$ FBC Waste

\begin{tabular}{|c|c|}
\hline Sieve Designation & Percent Passing \\
\hline $1^{\prime \prime}$ & 100.0 \\
$3 / 4^{\prime \prime}$ & 99.6 \\
$1 /{ }^{\prime \prime}$ & 97.4 \\
$3 / 8^{\prime \prime}$ & 86.3 \\
No. 4 & 3.5 \\
No. 8 & 2.3 \\
\hline
\end{tabular}

Table 9. Specific Gravity and Absorption (AASHTO T-85) Test Results on Aggregates Made with $100 \%$ FBC Waste

\begin{tabular}{||c|c|c|}
\hline Sieve Designation & Specific Gravity, Apparent & Absorption, $\%$ \\
\hline $3 / 4^{\prime \prime}-1 / 2 "$ & 2.55 & 17.75 \\
$1 / 2 "$ - 3/8" & 2.57 & 17.73 \\
$3 / 8^{\prime \prime}-$ No. 4 & 2.58 & 18.03 \\
\hline
\end{tabular}

Table 10. Results of the Los Angeles Abrasion Test (AASHTO T-96) on Aggregates Made with $100 \%$ FBC Waste

\begin{tabular}{|l|c|c|}
\hline \multicolumn{1}{|c|}{ Size Designation } & Percent Wear & $\begin{array}{c}\text { Ohio DOT Specification, } \\
\% \text { Wear }\end{array}$ \\
\hline Grading B (3/4 In. $-1 / 2 \ln )$. & 27.3 & $40, \max$ \\
Grading C (3/8 In.-1/4 In.) & 21.8 & $40, \max$ \\
\hline
\end{tabular}

Table 11. Sodium Sulfate Soundness Test (AASHTO T-104) on Aggregates Made with $100 \%$ FBC Waste

\begin{tabular}{||l|c|c|}
\hline \multicolumn{1}{|c|}{ Sieve Designation } & Percent Loss & $\begin{array}{c}\text { Ohio DOT Specification, } \\
\% \text { Loss Max }\end{array}$ \\
\hline 3/4" - $1 / 2 "$ & 91.9 & 12 \\
$3 / 8^{\prime \prime}-$ No. 4 & 76.0 & 12 \\
No. 4 - No. 8 & 71.0 & 12 \\
\hline
\end{tabular}


Table 12. Results of Sodium Sulfate Soundness Tests on Synthetic Aggregate Produced from FBC Waste Blended with $10 \%$ Fly Ash or $5 \%$ Microsilica

\begin{tabular}{||l|c|c|}
\hline \multirow{2}{*}{ Aggregate, Sieve Size } & \multicolumn{2}{c|}{ Percent loss } \\
\cline { 2 - 3 } & Uncrushed & Crushed \\
\hline FBC/FA1, 3/8" to 4 mesh $(9.5$ to $4.75 \mathrm{~mm})$ & 17.2 & \\
\hline FBC/MS1, 3/8" to 4 mesh $(6.3$ to $4.75 \mathrm{~mm})$ & 44.7 & 55.9 \\
\hline FBC/MS1, 4 to 8 mesh $(4.75$ to $2.36 \mathrm{~mm})$ & & 38.7 \\
\hline
\end{tabular}

Table 13. Results of Los Angeles Abrasion Tests on Synthetic Aggregate Produced from FBC Waste Blended with $10 \%$ Fly Ash or $5 \%$ Microsilica

\begin{tabular}{||c|c|c|}
\hline \multirow{2}{*}{ Aggregate, Grading, Sieve Size } & \multicolumn{2}{|c|}{ Percent loss (500 revolutions) } \\
\cline { 2 - 3 } & Uncrushed & Crushed \\
\hline $\begin{array}{l}\text { FBC/FA1, Grading C, } \\
\text { 3/8" to 4 mesh (9.5 to } 4.75 \mathrm{~mm})\end{array}$ & 18.4 & 17.3 \\
\hline $\begin{array}{l}\text { FBC/MS1, Grading C, } \\
\text { 3/8" to 4 mesh, (9.5 to } 4.75 \mathrm{~mm})\end{array}$ & 18.3 & 19.4 \\
\hline
\end{tabular}


Table 14. Analyses of Coolside Waste Samples

\begin{tabular}{|c|c|c|c|c|}
\hline & \multirow{2}{*}{$\begin{array}{c}\text { Edgewater } \\
\text { Coolside } \\
\text { Sample }\end{array}$} & \multicolumn{3}{|c|}{ Coolside Pilot Plant Waste Samples } \\
\hline & & Once-Through & Recycle & Advanced \\
\hline $\begin{array}{l}\text { Moisture, wt \% (as rec.) } \\
\text { Ultimate Analysis. wt \% (dry) }\end{array}$ & 0.83 & 4.42 & 1.85 & 2.95 \\
\hline Carbon & 6.36 & 1.66 & 1.31 & 1.20 \\
\hline Hydrogen & 0.59 & 0.94 & 0.63 & 0.66 \\
\hline Nitrogen & 0.14 & 0.10 & 0.08 & 0.07 \\
\hline Sulfur (total) & 2.10 & 7.46 & 7.97 & 9.31 \\
\hline Sulfate sulfur & - & 3.83 & 2.85 & 3.13 \\
\hline Sulfite sulfur & - & 3.88 & 4.79 & 6.28 \\
\hline Carbonate $\left(\mathrm{CO}_{3}\right)$ & 1.20 & 6.23 & 4.54 & 4.55 \\
\hline Ash, $750^{\circ} \mathrm{C}$ & 88.93 & 89.94 & 93.61 & 93.83 \\
\hline Maior Element, wt \% (dry) & & & & \\
\hline $\mathrm{SiO}_{2}$ & 35.38 & 17.75 & 23.36 & 19.92 \\
\hline $\mathrm{Al}_{2} \mathrm{O}_{3}$ & 17.03 & 8.07 & 10.89 & 9.92 \\
\hline $\mathrm{TiO}_{2}$ & 0.79 & 0.37 & 0.48 & 0.45 \\
\hline $\mathrm{Fe}_{2} \mathrm{O}_{3}$ & 10.52 & 5.22 & 6.78 & 6.55 \\
\hline $\mathrm{CaO}$ & 14.06 & 35.01 & 26.68 & 31.39 \\
\hline MgO & 1.00 & 1.26 & 1.18 & 0.64 \\
\hline $\mathrm{Na}_{2} \mathrm{O}$ & 1.79 & 2.80 & 2.72 & 0.24 \\
\hline $\mathrm{K}_{2} \mathrm{O}$ & 1.69 & 0.77 & 1.00 & 0.88 \\
\hline $\mathrm{P}_{2} \mathrm{O}_{5}$ & 0.22 & 0.14 & 0.18 & 0.16 \\
\hline $\mathrm{SO}_{3}$ & 5.81 & 20.50 & 20.86 & 23.02 \\
\hline Unaccounted & 11.71 & 8.11 & 5.87 & 6.83 \\
\hline $\mathrm{Ca} / \mathrm{S}$ mol.. Ratio & 3.46 & 2.44 & 1.83 & 1.95 \\
\hline
\end{tabular}

Table 15. Analyses of LIMB Waste Samples from Ohio Edison Edgewater Station and Fly Ash from Ohio Edison Bruce Mansfield Station

\begin{tabular}{|c|c|c|c|c|}
\hline & \multicolumn{3}{|c|}{ Edgewater LIMB Samples } & \multirow{2}{*}{$\begin{array}{c}\text { Mansfield Fly Ash } \\
10 / 12 / 92 \\
\end{array}$} \\
\hline & $5 / 25 / 89$ & $7 / 10 / 91$ & $7 / 25 / 91$ & \\
\hline $\begin{array}{l}\text { Moisture, wt \% (as rec.) } \\
\text { Ulitimate Analysis, wt \% (dry) }\end{array}$ & 0.06 & 0.15 & 0.14 & 0.22 \\
\hline Carbon & 2.84 & 4.13 & 5.42 & 1.7 \\
\hline Hydrogen & 0.09 & 0.11 & 0.13 & 0.0 \\
\hline Nitrogen & 0.09 & 0.10 & 0.09 & 0.0 \\
\hline Sulfur (total) & 4.80 & 5.14 & 2.73 & 0.41 \\
\hline Sulfate sulfur & 4.80 & 5.14 & 2.73 & - \\
\hline Carbonate $\left(\mathrm{CO}_{3}\right)$ & 3.07 & 2.50 & 3.01 & - \\
\hline Ash, $750^{\circ} \mathrm{C}$ & 95.94 & 94.31 & 93.01 & 97.2 \\
\hline \multicolumn{5}{|l|}{ Major Element, wt \% (dry) } \\
\hline $\mathrm{SiO}_{2}$ & 26.02 & 21.98 & 34.44 & 47.4 \\
\hline $\mathrm{Al}_{2} \mathrm{O}_{3}$ & 11.11 & 10.17 & 15.83 & 21.4 \\
\hline $\mathrm{TiO}_{2}$ & 0.50 & 0.48 & 0.72 & 0.27 \\
\hline $\mathrm{Fe}_{2} \mathrm{O}_{3}$ & 12.72 & 13.18 & 9.69 & 21.4 \\
\hline $\mathrm{CaO}$ & 30.65 & $\cdot 31.92$ & 24.53 & 2.9 \\
\hline MgO & 0.74 & 0.86 & 0.95 & 0.89 \\
\hline $\mathrm{Na}_{2} \mathrm{O}$ & 0.12 & 0.17 & 0.22 & 0.40 \\
\hline $\mathrm{K}_{2} \mathrm{O}$ & 1.05 & 0.89 & 1.63 & 2.0 \\
\hline $\mathrm{P}_{2} \mathrm{O}_{5}$ & 0.21 & 0.05 & 0.26 & 0.37 \\
\hline $\mathrm{SO}_{3}$ & 12.29 & 13.26 & 7.33 & 1.06 \\
\hline Unaccounted & 4.58 & 7.04 & 4.40 & 1.9 \\
\hline Ca/S Mol.. Ratio & 3.56 & 3.44 & 4.78 & - \\
\hline Lime Index, wt \% of $\mathrm{CaO}$ & 19.7 & 14.4 & 17.4 & - \\
\hline
\end{tabular}


Table 16. Analyses of FBC Waste Samples from Glatfelter and GM

\begin{tabular}{|c|c|c|c|c|c|}
\hline & \multicolumn{4}{|c|}{ Glatfelter FBC Waste } & \multirow{2}{*}{$\begin{array}{c}\text { GM FBC Waste } \\
9 / 16 / 88\end{array}$} \\
\hline & 2/18/92 & 9/2/93 & $3 / 14 / 94$ & $10 / 22 / 94$ & \\
\hline $\begin{array}{l}\text { Moisture, wt \% as rec'd } \\
\text { Ultimate Analysis, wt \% dry }\end{array}$ & 0.10 & 0.08 & 0.08 & 0.19 & 0.10 \\
\hline Carbon & 4.93 & 3.48 & 3.45 & 3.33 & 10.78 \\
\hline Hydrogen & 0.05 & 0.07 & 0.09 & 0.00 & 0.09 \\
\hline Nitrogen & 0.11 & 0.05 & 0.02 & 0.06 & 0.20 \\
\hline Sulfur, total & 7.88 & 6.00 & 5.58 & 6.05 & 7.45 \\
\hline Sulfate Sulfur & 7.88 & 6.00 & - & - & - \\
\hline Carbonate $\left(\mathrm{CO}_{3}\right)$ & 1.59 & 3.77 & - & - & - \\
\hline Ash @ $750^{\circ} \mathrm{C}$ & 93.84 & 94.18 & 90.2 & 94.52 & 85.04 \\
\hline $\mathrm{SiO}_{2}$ & 12.30 & 12.03 & 13.85 & 14.10 & 18.51 \\
\hline $\mathrm{Al}_{2} \mathrm{O}_{3}$ & 5.96 & 5.30 & 5.75 & 6.52 & 9.70 \\
\hline $\mathrm{TiO}_{2}$ & 0.26 & 0.22 & 0.22 & 0.27 & 0.40 \\
\hline $\mathrm{Fe}_{2} \mathrm{O}_{3}$ & 4.06 & 4.36 & 4.48 & 5.19 & 11.49 \\
\hline $\mathrm{CaO}$ & 47.79 & 47.81 & 45.08 & 41.02 & 21.06 \\
\hline MgO & 3.57 & 7.19 & 8.85 & 9.09 & 3.19 \\
\hline $\mathrm{Na}_{2} \mathrm{O}$ & 0.22 & 0.24 & 0.25 & 0.38 & 0.11 \\
\hline $\mathrm{K}_{2} \mathrm{O}$ & 0.48 & 0.69 & 0.88 & 1.15 & 0.78 \\
\hline $\mathrm{P}_{2} \mathrm{O}_{5}$ & 0.19 & 0.13 & 0.15 & 0.15 & 0.06 \\
\hline $\mathrm{SO}_{3}$ & 19.69 & 15.41 & 14.48 & 17.14 & 18.13 \\
\hline Unaccounted & 5.48 & 6.62 & 6.01 & 4.99 & 16.57 \\
\hline Ca/S Mol.. Ratio & 3.47 & 4.43 & 4.45 & 3.87 & 1.66 \\
\hline Lime Index, wt $\%$ of $\mathrm{CaO}$ & 26.3 & 28.0 & 25.7 & 21.3 & 6.4 \\
\hline
\end{tabular}


Table 17. Properties of Coolside Waste Pellets and the
Ohio Specifications for Road Aggregates

\begin{tabular}{|c|c|c|c|c|c|c|c|c|}
\hline & \multirow{3}{*}{$\begin{array}{c}\text { Edgewater } \\
\text { Coolside } \\
\text { Waste } \\
\text { Pellets }\end{array}$} & \multicolumn{4}{|c|}{ Coolside Pilot Plant Waste Pellets } & \multicolumn{3}{|c|}{$\begin{array}{l}\text { Ohio Specifications for Aggregates } \\
\text { in Highway Construction }\end{array}$} \\
\hline & & \multicolumn{2}{|c|}{$\begin{array}{l}\text { Once- } \\
\text { Through }\end{array}$} & \multirow[t]{2}{*}{$\begin{array}{l}\text { Re- } \\
\text { cycle }\end{array}$} & \multirow[t]{2}{*}{ Advanced } & \multirow{2}{*}{$\begin{array}{c}\text { Aggre- } \\
\text { gate } \\
\text { Base }\end{array}$} & \multirow[t]{2}{*}{$\begin{array}{l}\text { Asphalt } \\
\text { Concrete }\end{array}$} & \multirow{2}{*}{$\begin{array}{l}\text { Portland } \\
\text { Cement } \\
\text { Concrete }\end{array}$} \\
\hline & & $A(c)$ & $B(d)$ & & & & & \\
\hline 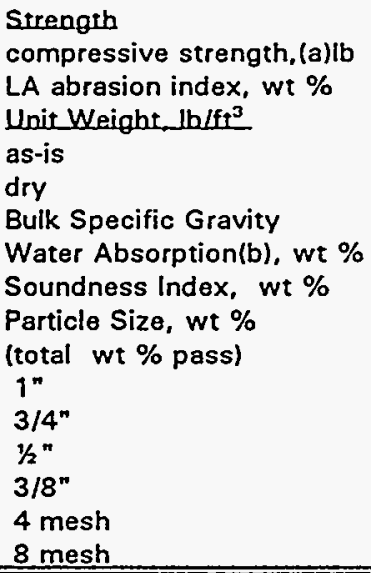 & $\begin{array}{r}99.6 \\
34.0 \\
61.6 \\
49.9 \\
1.58 \\
5.10 \\
\cdots\end{array}$ & $\begin{array}{r}39.3 \\
50.1 \\
60.8 \\
44.7 \\
1.54 \\
4.64 \\
\ldots .-\end{array}$ & $\begin{array}{r}70.1 \\
34.2 \\
\\
60.2 \\
44.2 \\
1.63 \\
3.67 \\
\ldots .\end{array}$ & $\begin{array}{r}145.2 \\
23.7 \\
\\
63.6 \\
50.8 \\
1.73 \\
2.60 \\
99.0\end{array}$ & $\begin{array}{r}39.2 \\
\cdots \\
59.6 \\
46.0 \\
1.66 \\
4.63 \\
\ldots . .\end{array}$ & \begin{tabular}{r}
50 \\
$(\max )$. \\
65 \\
$(\min )$. \\
$\cdots-$. \\
\hdashline 15 \\
1 (max.) \\
Coarse
\end{tabular} & \begin{tabular}{r}
40 \\
$(\max )$. \\
70 \\
$(\mathrm{~min})$ \\
$\cdots$. \\
\hdashline 15 \\
$(\max )$. \\
Coarse
\end{tabular} & $\begin{array}{r}40 \\
(\max .) \\
70 \\
(\min .) \\
\ldots . . \\
\cdots \\
12 \\
(\max .) \\
\text { Coarse }\end{array}$ \\
\hline
\end{tabular}

(a) Average of ten measurements

(b) Measured with "as is" pellets

(c) $22 \%$ added water (dry basis) in densification during pellet preparation

(d) $25 \%$ added water (dry basis), the optimum amount of added water, in densification during pellet preparation

\section{Table 18. Properties of Coolside Waste Pellets With and Without Blended Fly Ash}

\begin{tabular}{|c|c|c|c|}
\hline & $\begin{array}{c}\text { Pellets from } 100 \% \text { CONSOL } \\
\text { Pilot Plant Test No. } 2 \\
\text { (recycle mode) }\end{array}$ & $\begin{array}{c}\text { Pellets from } 90 / 10 \\
\text { Coolside Waste/Fly Ash } \\
\text { Blend }\end{array}$ & $\begin{array}{l}\text { Lightweight Aggregate } \\
\text { Specification (ASTM C 331) }\end{array}$ \\
\hline \multicolumn{4}{|l|}{ Strength } \\
\hline Compressive Strength, Ib & 145 & 185 & $\cdots$ \\
\hline LA Abrasion Index, wt \% & 23.7 & 20.5 & $\cdots$ \\
\hline \multicolumn{4}{|l|}{ Unit Weight,/b/fts } \\
\hline As Is & 63.5 & 71.0 & $\cdots$ \\
\hline Dry & 50.8 & 57.6 & 55 (max.) \\
\hline Bulk Specific Gravity & 1.73 & 1.98 & $\cdots$ \\
\hline Water Absorption, wt \% & 2.7 & 2.0 & --- \\
\hline Soundness Index, wt \% & 99 & 77 & -- \\
\hline Clay Limps, wt \% & 2.6 & 1.7 & 2.0 \\
\hline Stain Materials & negative & negative & negative \\
\hline Organic Impurity & negative & negative & negative \\
\hline \multirow{2}{*}{\multicolumn{4}{|c|}{$\begin{array}{l}\text { Particle Size, wt \% } \\
\text { (total wt \% pass) }\end{array}$}} \\
\hline & & & \\
\hline $1^{\prime \prime}$ & 91.8 & 98.2 & Coarse \\
\hline $1 / 2 "$ & 29.0 & 18.1 & \\
\hline 4 mesh & 5.1 & 5.6 & \\
\hline 8 mesh & 3.0 & 3.1 & \\
\hline
\end{tabular}

(a) ASTM specification for lightweight aggregate for concrete masonry units 
Table 19. Properties of LIMB Waste Pellets

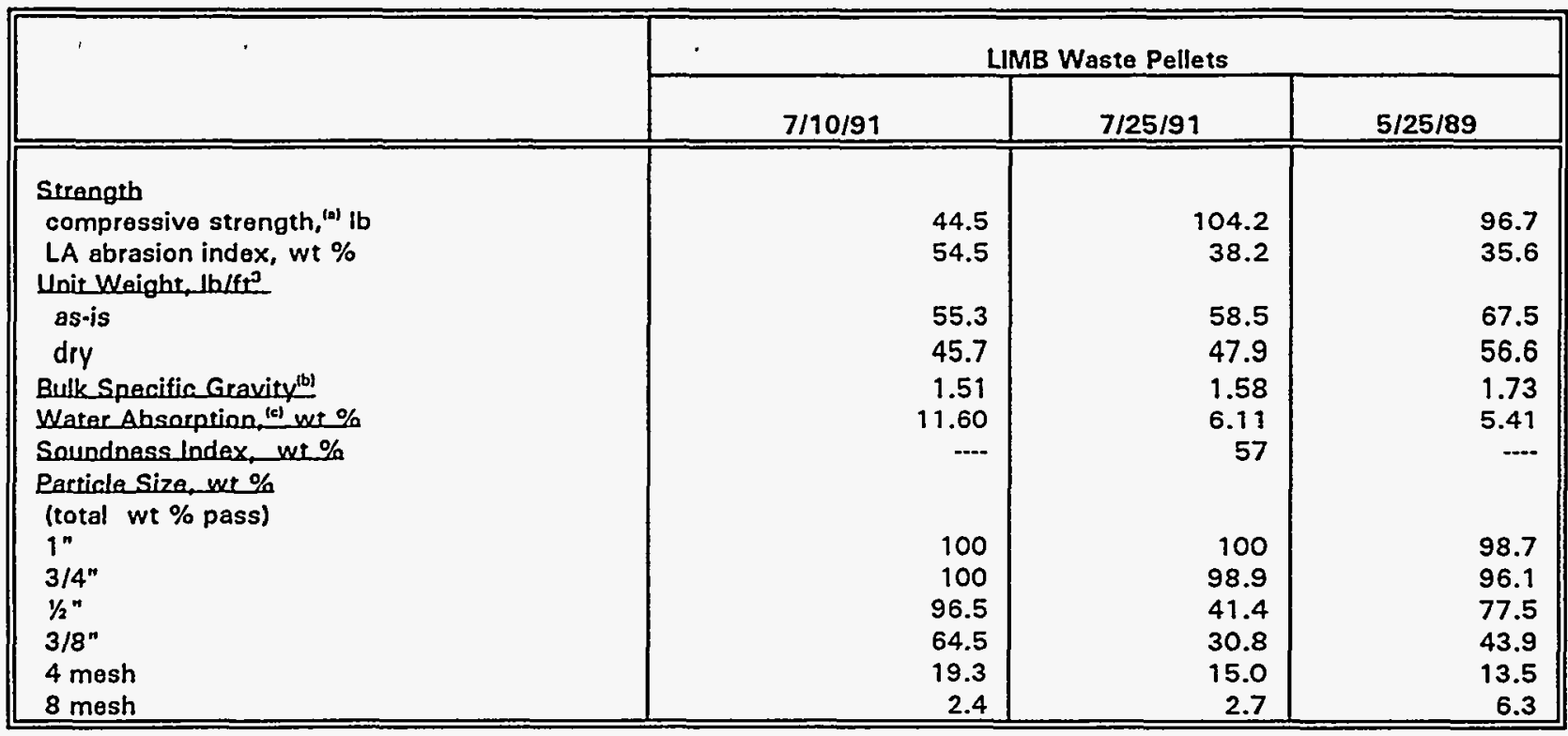

(a) The pellet compressive strength values are each the average of ten measurements on $-1 / 4 \times 1 / 2$ " pellets with a Soiltest compression tester.

(b) Includes the permeable and impermeable voids in the particles, but not the voids between particles

(c) Includes water in the pores of the material, but not water adhering to the outside surface of the particles. FBC, Coolside

Table 20. Properties of LIMB Waste Pellets With and Without Blended Fly Ash

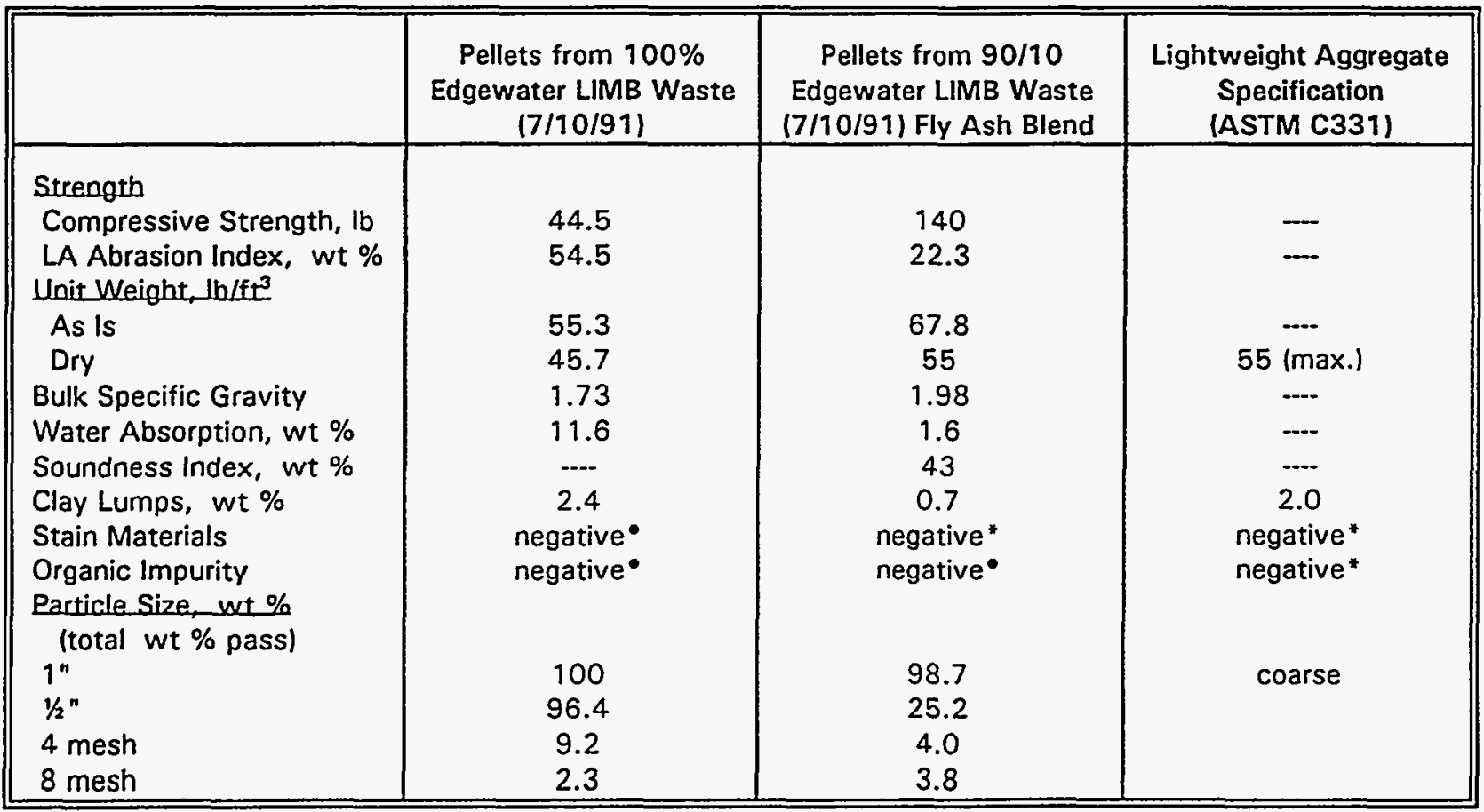

"Negative, as required to meet the ASTM C331 specification for lightweight aggregates. 
Table 21. Properties of FBC Waste Pellets

\begin{tabular}{|c|c|c|c|c|c|}
\hline & \multicolumn{3}{|c|}{ 2/28/92 FBC Waste } & \multirow{2}{*}{$\begin{array}{c}\text { The } 9 / 2 / 93 \text { FBC } \\
\text { Waste(d) }\end{array}$} & \multirow{2}{*}{$\begin{array}{c}\text { Class A Coarse } \\
\text { Aggregate(e) }\end{array}$} \\
\hline & $A(a)$ & $B(b)$ & $C(c)$ & & \\
\hline $\begin{array}{l}\text { Compressive strength (a), lb } \\
\text { LA abrasion index, wt \% }\end{array}$ & $\begin{array}{l}43.0 \\
51.5\end{array}$ & $\begin{array}{l}90.4 \\
37.4\end{array}$ & $\begin{array}{r}190 \\
26.8\end{array}$ & $\begin{array}{r}140 \\
22.6\end{array}$ & $40 \overline{(\max .)}$ \\
\hline \multicolumn{6}{|l|}{ Unit Weight lb/ft ${ }^{3}$} \\
\hline as-is & 69.8 & 72.1 & 71.8 & 74.6 & 70 (min.) \\
\hline dry & 61.5 & 64.9 & 62.7 & 65.9 & - \\
\hline Bulk Specific Gravity (b) & 1.90 & 2.00 & 2.03 & 2.02 & - \\
\hline Water Absorption (c), wt \% & 6.3 & 4.3 & 3.9 & 4.1 & - \\
\hline Soundness, wt \% & - & - & 7 & 7 & 12 (max.) \\
\hline \multicolumn{6}{|l|}{$\frac{\text { Particle Size, wt } \% \text { (total }}{\text { wt } \% \text { pass) }}$} \\
\hline $1^{11}$ & 99.5 & 100 & 98.6 & 100 & Coarse \\
\hline $3 / 4^{\prime \prime}$ & 97.0 & 99.5 & - & - & \\
\hline $1 / 2 "$ & 87.2 & 91.9 & 53.3 & 96.0 & \\
\hline $3 / 8^{\prime \prime}$ & 72.3 & 69.6 & 21.2 & 78.2 & \\
\hline 4 mesh & 12.9 & 16.8 & 3.0 & 9.3 & \\
\hline 8 mesh & 3.4 & 2.5 & 1.8 & 1.8 & \\
\hline
\end{tabular}

(a) Mixing for 12 min. during hydration

(b) Mixing for $20 \mathrm{~min}$. during hydration

(c) Mixing for $20 \mathrm{~min}$. with optimum water addition.

(d) Mixing for $20 \mathrm{~min}$. with optimum water addition

(e) AASHTO Standard Specification M-283

Table 22. Properties of Pellets Prepared with GM and Glatfelter FBC Wastes

\begin{tabular}{|c|c|c|c|}
\hline & $\begin{array}{l}\text { GM FBC } \\
\text { Waste }\end{array}$ & $\begin{array}{c}\text { Glatfelter FBC } \\
\text { Wastela) }\end{array}$ & Class A Coarse Aggregate \\
\hline $\begin{array}{l}\text { Compressive Strength, lb } \\
\text { LA abrasion index, wt \% } \\
\text { Unit Weight_lb/ft }\end{array}$ & $\begin{array}{r}183 \\
17.7\end{array}$ & $\begin{array}{r}190 \\
26.8\end{array}$ & 40(max.) \\
\hline As is & 75.3 & 71.8 & 70 (min.) \\
\hline Dry & 61.0 & 62.7 & $\ldots$ \\
\hline Bulk_Specific_Gravity & 2.00 & 2.03 & -.-- \\
\hline Water Absorption, wa \% & 3.0 & 3.9 & --- \\
\hline Saundness lodex, wt \% & 72 & 7 & 12 (max.) \\
\hline $\begin{array}{l}\text { Particle Size, wt \% } \\
\text { (total wt \% nass) }\end{array}$ & & & \\
\hline $11 / 2 n$ & 100 & 100 & Coarse \\
\hline $1^{n}$ & 100 & 98.6 & \\
\hline $1 / 2 "$ & 86.9 & 53.3 & \\
\hline $3 / 8^{\prime \prime}$ & 57.5 & 21.2 & \\
\hline 4 mesh & 4.8 & 3.0 & \\
\hline 8 mesh & 1.5 & 1.8 & \\
\hline
\end{tabular}

(a) Prepared from the $2 / 28 / 92$ FBC waste 
Table 23. Comparison of Properties of Fresh and Aged Pellets Made with $100 \%$ FBC Waste with Natural Aggregates

\begin{tabular}{||l|r|r|r|r|}
\hline & \multicolumn{2}{|c|}{ FBC Waste Pellets (a) } & \multirow{2}{*}{ Gravel } & \multicolumn{1}{c|}{$\begin{array}{c}\text { Crushed } \\
\text { Limestone }\end{array}$} \\
\cline { 2 - 3 } & \multicolumn{2}{|c|}{ Fresh } & Aged for 197 days & \\
\hline LA Abrasion Index, wt \% & 26.8 & 25.2 & 28.1 & 21.0 \\
Soundness, wt \% & 7 & 51 & 8 & 8 \\
Unit Weight (d), lb/ft & 71.8 & 71.8 & 96.3 & 103 \\
Grain Size & Coarse & Coarse & Coarse & Coarse \\
Compressive Strength, lb & 190 & 143 & 310 & 203 \\
Water Absorption (b), wt \% & 3.9 & - & 1.7 & 0.5 \\
Specific Gravity & 2.03 & -52 & 2.67 \\
\hline
\end{tabular}

(a) from the September 2, 1992, Glatfelter FBC waste (b) as-is pellets

Table 24. The Effect of Aging on the Properties of Aggregates Made from $100 \%$ FBC Waste and the 90/10 FBC Waste and Fly Ash Blend

\begin{tabular}{|l|r|r|r|r|}
\hline & \multicolumn{2}{|c|}{ Pellets from 100\% FBC Waste } & \multicolumn{2}{|c|}{$\begin{array}{l}\text { Pellets from 90/10 FBC Wastel } \\
\text { Fly Ash Blend }\end{array}$} \\
\hline \hline Aging Time, days & 0 & 197 & 0 & 207 \\
\hline LAAbrasion Index, wt \% & 26.8 & 25.2 & 29.1 & 11 \\
Soundness, wt \% & 7 & 51 & 11 \\
Compressive Strength, wt \% & 190 & 143 & 199 & 191 \\
\hline
\end{tabular}

Table 25. Properties of FBC Waste Pellets With and Without Blended Microsilica (Processed)

\begin{tabular}{|c|c|c|c|c|}
\hline & $\begin{array}{c}100 \% \text { FBC } \\
\text { Waste }\end{array}$ & $\begin{array}{c}95 / 5 \text { FBC } \\
\text { Waste/Microsilica } \\
\text { Blend } \\
\end{array}$ & $\begin{array}{c}90 / 10 \text { FBC } \\
\text { Waste/Microsilica } \\
\text { Blend }\end{array}$ & $\begin{array}{l}\text { AASHTO Class A } \\
\text { Coarse Aggregate }\end{array}$ \\
\hline$\frac{\text { Wear }}{\text { LA Abrasion Index, wt\% }}$ & 26.8 & 24.0 & 25.0 & $40(\max )$ \\
\hline $\begin{array}{l}\text { Unit Weight, } 1 \mathrm{~b} / \mathrm{ft}^{\mathrm{S}} \\
\text { moist (as is) } \\
\text { dry }\end{array}$ & $\begin{array}{l}71.8 \\
62.7\end{array}$ & $\begin{array}{l}73.6 \\
63.4\end{array}$ & $\begin{array}{l}70.4 \\
59.3\end{array}$ & 70 (min) \\
\hline Soundness, wt\% & 6.6 & 0 & 0 & $12(\max )$ \\
\hline Grain Size & coarse & coarse & coarse & coarse \\
\hline Compressive Strength, Ib & 190 & 190 & 178 & - \\
\hline Water Absorption, wt\% & 3.9 & 3.0 & 2.5 & - \\
\hline
\end{tabular}


Table 26. Properties of FBC Waste Pellets Blended with $5 \%$

Processed and Waste Microsilica

\begin{tabular}{|l|c|c|c||}
\hline & $\begin{array}{c}\text { Processed } \\
\text { Microsilica }\end{array}$ & $\begin{array}{c}\text { Waste } \\
\text { Microsilica }\end{array}$ & $\begin{array}{c}\text { AASHTO Class A } \\
\text { Coarse Aggregate }\end{array}$ \\
\hline Wear & 24.0 & 25.0 & 40 (max) \\
Unit Weight, Ib/ft & 73.6 & 73.2 & 70 (min) \\
moist (as is) & 63.4 & 61.9 & - \\
dry & 0 & 11.6 & 12 (max) \\
Soundness, wt\% & $c 0 a r s e$ & $c 0 a r s e$ & coarse \\
Grain Size & 190 & 191 & - \\
Compressive Strength, lb & 2.05 & 2.03 & - \\
Bulk Specific Gravity & 3.0 & 2.5 & - \\
Water Absorption, wt\% & &
\end{tabular}

Table 27. The Effect of Aging on the Properties of FBC Waste Pellets Made With 5\% and 10\% Blended Processed Microsilica

\begin{tabular}{||l|c|c|c|c||}
\hline & \multicolumn{2}{|c|}{$90 / 10$ Blend } & \multicolumn{2}{c||}{$95 / 5$ Blend } \\
\hline Storage Time, Days & 0 & 110 & 0 & 112 \\
\hline LA Abrasion Index, wt \% & 25.0 & - & 24.0 & - \\
Soundness Index, wt \% & 0 & 14 & 0 & 8 \\
Compressive Strength, lb & 178 & -- & 190 & - \\
\hline
\end{tabular}


Table 28. Properties of 90/10 FBC Waste/Fly Ash Pellets from the Undrained Weathering Test (a)

\begin{tabular}{||l|c|c||}
\hline & $\begin{array}{c}\text { Pre-Test } \\
\text { November 30, 1993 }\end{array}$ & $\begin{array}{c}\text { Post-Test } \\
\text { April 4, 1994 } \\
\text { (Air-Dried) }\end{array}$ \\
\hline Compressive Strength, lb & 180 & 63.2 \\
Soundness Index, wt \% & 3.1 & 22.5 \\
Particle Size, wt \% & & \\
\hline $1^{\prime \prime} \times 3 / 4^{\prime \prime}$ & 0 & 0 \\
$3 / 4^{\prime \prime} \times 1 / 2 "$ & 0 & 9.3 \\
$1 / 2^{\prime \prime} \times 3 / 8^{\prime \prime}$ & 50 & 37.0 \\
$3 / 8^{\prime \prime} \times 4$ mesh & 50 & 42.7 \\
4 mesh $\times 8$ mesh & 0 & 0.4 \\
-8 mesh & 0 & 10.6 \\
\hline
\end{tabular}

(a) Prepared in baseline pelletization test

Table 29. Properties of $90 / 10$ FBC Waste/Fly Ash Pellets from the Drained Weathering Test (a)

\begin{tabular}{|l|c|c|}
\hline & December 30, 1993 & $\begin{array}{c}\text { April 4, 1994 } \\
\text { (Air-Dried) }\end{array}$ \\
\hline $\begin{array}{l}\text { Compressive Strength, lb } \\
\text { Abrasion index, wt \% }\end{array}$ & 146 & 132 \\
& 31.6 & 58.4 \\
Soundness Index(b), wt \% & 0.5 & 46.7 \\
& & \\
Particle Size, wt \% & 1.8 & 2.7 \\
\hline $1^{\prime \prime} \times 3 / 4^{\prime \prime}$ & 20.4 & 22.2 \\
$3 / 4^{\prime \prime} \times 1 / 2$ & 22.2 & 27.2 \\
$1 / 2^{\prime \prime} \times 3 / 8^{\prime \prime}$ & 50.1 & 42.5 \\
$3 / 8^{\prime \prime} \times 4$ mesh & 3.1 & 2.7 \\
4 mesh $\times 8$ mesh & 2.4 & 2.7 \\
-8 mesh & & \\
\hline
\end{tabular}

(a) Prepared in a semi-continuous scale-up pelletization run 
Table 30. Properties of FBC Waste Pellets with Blended Fly Ash

\begin{tabular}{|c|c|c|c|c|c|c|}
\hline & $\begin{array}{l}100 \% \\
\text { FBC }\end{array}$ & $\begin{array}{c}90 / 10 \\
\text { FBC/Fly } \\
\text { Ash }\end{array}$ & $\begin{array}{l}75 / 25 \\
\text { FBC/Fly } \\
\text { Ash }\end{array}$ & $\begin{array}{c}\text { 65/35 } \\
\text { FBC/Fly } \\
\text { Ash }\end{array}$ & $\begin{array}{c}50 / 50 \\
\text { FBC/Fly } \\
\text { Ash } \\
\end{array}$ & $\begin{array}{c}\text { Class A } \\
\text { Coarse } \\
\text { Aggregate(a) }\end{array}$ \\
\hline LA Abrasion Index, wt \% & 27 & 23 & 25 & 19 & 22 & 40 (max.) \\
\hline Soundness, wt \%(b) & 7 & 0 & 6 & 12 & 28 & 12 (max.) \\
\hline Unit Weight, lb/ft ${ }^{3}$ & 71.8 & 71.4 & 77.4 & - & - & 70 (min.) \\
\hline Compressive Strength, lb & 190 & 210 & 208 & 200 & 162 & - \\
\hline Water Absorption, wt \%(c) & 3.9 & 4.2 & 3.8 & 3.7 & 3.6 & - \\
\hline Specific Gravity & 1.98 & 2.01 & 2.04 & 2.00 & 2.02 & - \\
\hline Particle Size & coarse & coarse & coarse & coarse & coarse & coarse \\
\hline $\begin{array}{l}\text { Freeze/Thaw Degrad.\%(d) } \\
1 / 2 " \times 3 / 8^{\prime \prime} \\
3 / 8^{\prime \prime} \times 4 \text { mesh }\end{array}$ & - & $\begin{array}{l}86 \\
71\end{array}$ & $\begin{array}{l}54 \\
29\end{array}$ & $\begin{array}{l}23 \\
18\end{array}$ & $\begin{array}{l}37 \\
17\end{array}$ & - \\
\hline
\end{tabular}

(a) AASHTO Standard Specification M-283

(b) After five cycle treatment using sodium sulfate solution (ASTM C88-83)

(c) As-is pellets, not pre-dried

(d) About 50 continuous freeze/thaw cycles $\left(\sim 0^{\circ} \mathrm{F} / \sim 50^{\circ} \mathrm{F}\right)$ over about seven days.

\section{Table 31. Properties of Pellets Made from the $65 / 35$ Blends of FBC Waste and Fly Ash with Additives}

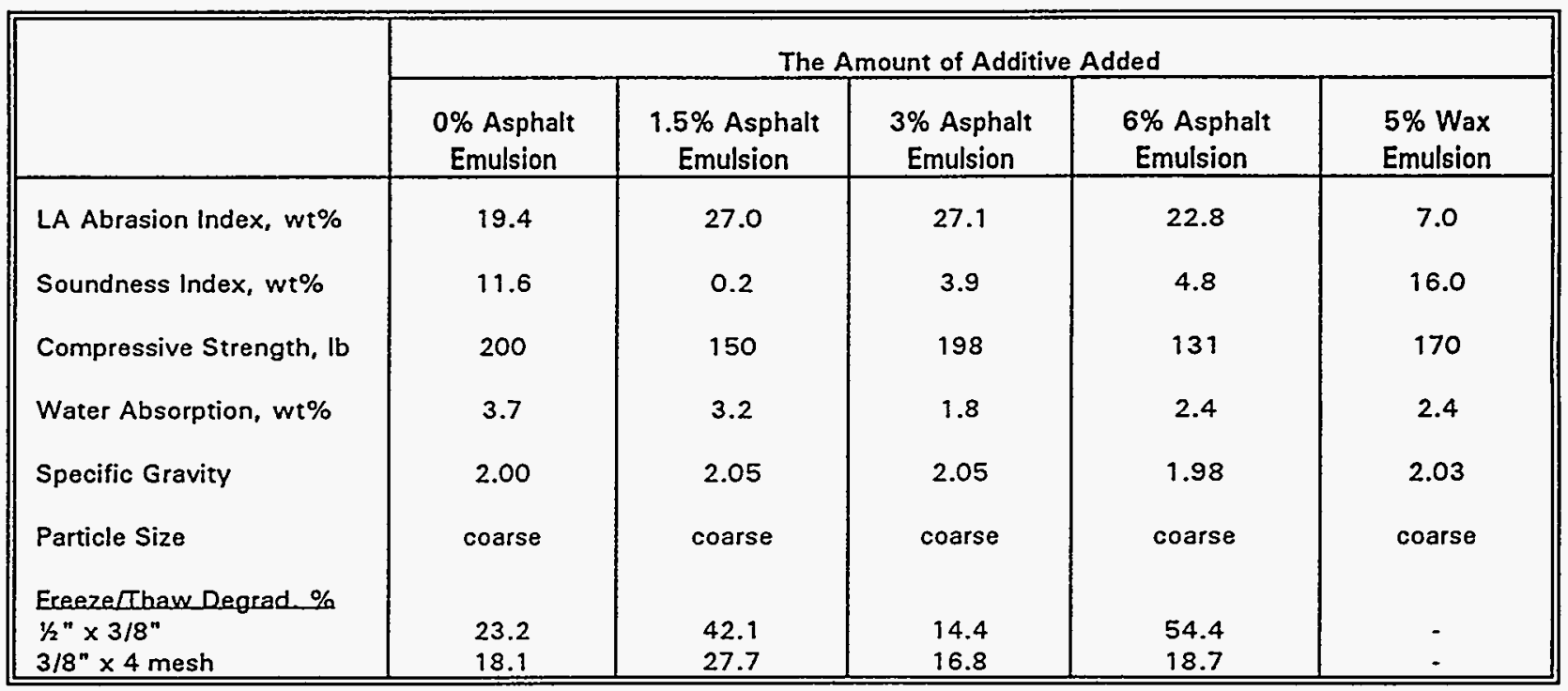


Table 32. Degradation of 65/35 FBC Waste/Fly Ash Pellets with Asphalt Emulsion Addition and Asphalt Coating

after 54 Cycles in the Freeze/Thaw Test

\begin{tabular}{||l|c|c|c||}
\hline \multirow{2}{*}{ Particle Size } & \multicolumn{3}{|c|}{ Percent of Degradation (\%) } \\
\cline { 2 - 4 } & $\begin{array}{c}\text { 3\% Asphalt } \\
\text { Emulsion(a) }\end{array}$ & $\begin{array}{c}\text { 5\% Asphalt } \\
\text { Coating(b) }\end{array}$ & $\begin{array}{c}\text { 3\% Asphalt Emulsion and } \\
5 \% \text { Asphalt Coating }\end{array}$ \\
\hline $1 / 2 " \times 3 / 8 "$ & 14.4 & 7.5 & 0 \\
\hline $3 / 8^{\prime \prime} \times 4$ mesh & 16.8 & 4.1 & 0 \\
\hline
\end{tabular}

(a) Asphalt Emulsion was added during hydration

(b) Asphalt coating was done before the freeze/thaw test

Table 33. Analysis of ASTM Leachate of 90/10 FBC Waste/Fly Ash Pellets and Comparison with Relevant SDWA Standards

\begin{tabular}{||l|r|r||}
\hline \multicolumn{1}{|c|}{ Species } & \multicolumn{1}{c|}{$\begin{array}{c}\text { ASTM } \\
\text { Leachate }\end{array}$} & $\begin{array}{c}\text { 30 Times SDWA } \\
\text { Maximum Concentration } \\
\text { Limits (1/30/91) }\end{array}$ \\
\hline Chloride, $\mathrm{mg} / \mathrm{L}$ & 10 & 7,500 \\
Fluoride, $\mathrm{mg} / \mathrm{L}$ & $<1$ & 60 \\
Sulfate, $\mathrm{mg} / \mathrm{L}$ & 1,780 & 7,500 \\
$\mathrm{Fe}, \mathrm{mg} / \mathrm{L}$ & $<0.06$ & 9 \\
$\mathrm{TDS}, \mathrm{mg} / \mathrm{L}$ & 4,170 & 15,000 \\
Acidity, as $\mathrm{CaCO}, \mathrm{mg} / \mathrm{L}$ & $-1,196$ & \\
Alkalinity, as $\mathrm{CaCO}_{3}, \mathrm{mg} / \mathrm{L}$ & 2,018 & \\
\hline $\mathrm{pH}$ & 12.36 & 1,500 \\
\hline $\mathrm{As}, \mu \mathrm{g} / \mathrm{L}$ & $<12.8$ & 60,000 \\
$\mathrm{Ba}, \mu \mathrm{g} / \mathrm{L}$ & 196 & 150 \\
$\mathrm{Cd}, \mu \mathrm{g} / \mathrm{L}$ & $<0.60$ & 3,000 \\
$\mathrm{Cr}, \mu \mathrm{h} / \mathrm{L}$ & 10.2 & 1,500 \\
$\mathrm{~Pb}, \mu \mathrm{g} / \mathrm{L}$ & 4.19 & 60 \\
$\mathrm{Hg}, \mu \mathrm{g} / \mathrm{L}$ & $<1$ & 1,500 \\
$\mathrm{Se}, \mu \mathrm{g} / \mathrm{L}$ & $<14.8$ & 30,000 \\
$\mathrm{Cu}, \mu \mathrm{g} / \mathrm{L}$ & 1.48 & 1,500 \\
$\mathrm{Mn}, \mu \mathrm{g} / \mathrm{L}$ & 1.15 & 150,000 \\
$\mathrm{Zn}, \mu \mathrm{g} / \mathrm{L}$ & 7.91 & \\
\hline
\end{tabular}

- No SDWA primary or secondary standard.

** The pH standard is 6.5 to 8.5 , not appropriate to multiply by 30 . 
Table 34. Comparison of Marshall Test Results in Asphalt Mix Design

\begin{tabular}{||l|r|r|r|r|r||}
\hline \multicolumn{1}{|c|}{ Mix } & \multicolumn{1}{c|}{ A } & \multicolumn{1}{c|}{ B } & \multicolumn{1}{c|}{ C } & \multicolumn{1}{c|}{ D } & \multicolumn{1}{c||}{ E } \\
\hline Mix Composition & & & & & \\
90/10 FBC Waste/Fly Ash Pellets, wt \% & 7.9 & 16.7 & 38.5 & 38.0 & 37.5 \\
Mfd. Limestone Sand, wt \% & 62.5 & 60.6 & 56.0 & 56.0 & 56.0 \\
No.8 Limestone, wt \% & 23.6 & 16.7 & 0.0 & 0.0 & 0.0 \\
Asphalt Cement, wt \% & 6.0 & 6.0 & 5.5 & 6.0 & 6.5 \\
Test Results & & & & \\
Marshall Stability, Ib & 2250 & 2325 & 2875 & 2305 & 2275 \\
Flow, 10-2 in & 11 & 12 & 14.5 & 15 & 15.3 \\
Bulk Specific Gravity & 2.236 & 2.154 & 1.934 & 1.936 & 1.927 \\
Air Voids, \% & 7.4 & 10.7 & 16.1 & 15.5 & 15.4 \\
Voids Mineral Aggregate, \% & 17.4 & 19.6 & 21.8 & 22.1 & 22.8 \\
\hline
\end{tabular}

Table 35. Properties of Synthetic Aggregates Used in Field Trial

\begin{tabular}{||l|c|}
\hline & Synthetic Aggregate \\
\hline LA Abrasion Index, wt\% & 19 \\
Soundness Index, wt\% & 11 \\
Unit Weight, ib/t' & 75 \\
Fractured Pieces, wt\% & 70 \\
Particle Size, wt \% & \\
\hline $1 / 2 "$ & 100 \\
$3 / 8^{\prime \prime}$ & 89.5 \\
4 mesh & 12.9 \\
8 mesh & 0 \\
\hline
\end{tabular}

Table 36. Size Distribution of the THF-Extracted Aggregate from the Pug Mill Before and After Freeze/Thaw Treatment

\begin{tabular}{|l|r|r|}
\hline \multirow{2}{*}{ Size Fraction } & \multicolumn{2}{|c|}{ Weight Percent, \% } \\
\cline { 2 - 3 } & Before Treatment & After Treatment \\
\hline $3 / 8^{\prime \prime}$ & 13 & 6 \\
$3 / 8^{\prime \prime} \times 4$ mesh & 68 & 57 \\
$4 \times 8$ mesh & 12 & 19 \\
$8 \times 50$ mesh & 4 & 12 \\
-50 mesh & 3 & 6 \\
\hline
\end{tabular}


Table 37. Size Distribution of the THF-Extracted Aggregates from the Pug Mill and Test Patch Cores

\begin{tabular}{|c|c|c|c|c|c|c|c|c|c|c|}
\hline $\begin{array}{c}\text { Size } \\
\text { Distribution, wt\% }\end{array}$ & $\begin{array}{l}\text { 4/28/95 } \\
\text { pug mill }\end{array}$ & $\begin{array}{l}6 / 1 \\
\text { core } \\
\end{array}$ & $\begin{array}{l}6 / 27 \\
\text { core } \\
\end{array}$ & $\begin{array}{l}8 / 3 \\
\text { core } \\
\end{array}$ & $\begin{array}{l}9 / 1 \\
\text { core }\end{array}$ & $\begin{array}{l}10 / 2 \\
\text { core } \\
\end{array}$ & $\begin{array}{l}11 / 1 \\
\text { core }\end{array}$ & $\begin{array}{c}12 / 4 / 95 \\
\text { core }\end{array}$ & $\begin{array}{c}4 / 18 / 96 \\
\text { core }^{*}\end{array}$ & $\begin{array}{c}5 / 30 / 96 \\
\text { core } \\
\end{array}$ \\
\hline$+3 / 8 "$ & 13 & 4 & 13 & 9 & 11 & 9 & 6 & 12 & 4 & 7 \\
\hline $3 / 8^{\prime \prime} \times 4$ mesh & 68 & 45 & 36 & 43 & 42 & 42 & 44 & 31 & 23 & 36 \\
\hline $4 \times 8$ mesh & 12 & 17 & 16 & 15 & 13 & 15 & 16 & 17 & 19 & 21 \\
\hline $8 \times 50$ mesh & 4 & 18 & 19 & 15 & 13 & 13 & 14 & 17 & 34 & 20 \\
\hline-50 mesh & 3 & 16 & 17 & 18 & 21 & 21 & 20 & 23 & 20 & 16 \\
\hline
\end{tabular}

*Non-representative core sample, c.f., test.

Table 38. Estimated Composition of Fines in the THF-Extracted Test Patch Cores after Freeze/Thaw Treatment

\begin{tabular}{||l|l|l|l|l|l|l|l|l||}
\hline $\begin{array}{c}\text { Aggregate } \\
\text { Components, wt \% }\end{array}$ & $\begin{array}{c}6 / 27 / 95 \\
\text { core }\end{array}$ & $\begin{array}{c}8 / 3 / 95 \\
\text { core }\end{array}$ & $\begin{array}{c}9 / 1 / 95 \\
\text { core }\end{array}$ & $\begin{array}{c}10 / 2 / 95 \\
\text { core }\end{array}$ & $\begin{array}{c}11 / 9 / 95 \\
\text { core }\end{array}$ & $\begin{array}{c}12 / 4 / 95 \\
\text { core }\end{array}$ & $\begin{array}{c}4 / 18 / 96 \\
\text { core* }\end{array}$ & $\begin{array}{c}5 / 30 / 96 \\
\text { core }\end{array}$ \\
\hline $\begin{array}{l}\text { 8x 50mash fraction } \\
\text { FBC Waste Pellets }\end{array}$ & 35 & 33 & 30 & 36 & 31 & 31 & 0.3 & 31 \\
Calcium Carbonate & 48 & 50 & 53 & 50 & 51 & 51 & 39 & 49 \\
Others & 17 & 17 & 17 & 14 & 18 & 18 & 60.7 & 20 \\
& & & & & & & & \\
-50 mesh fraction & & & & & & & & \\
FBC Waste Pellets & 33 & 31 & 29 & 33 & 35 & 9 & 5 & 27 \\
Calcium Carbonate & 41 & 40 & 42 & 42 & 38 & 42 & 37 & 42 \\
Others & 26 & 29 & 29 & 25 & 27 & 29 & 58 & 31 \\
\hline
\end{tabular}

*Non-representative core sample, c.f., test. 


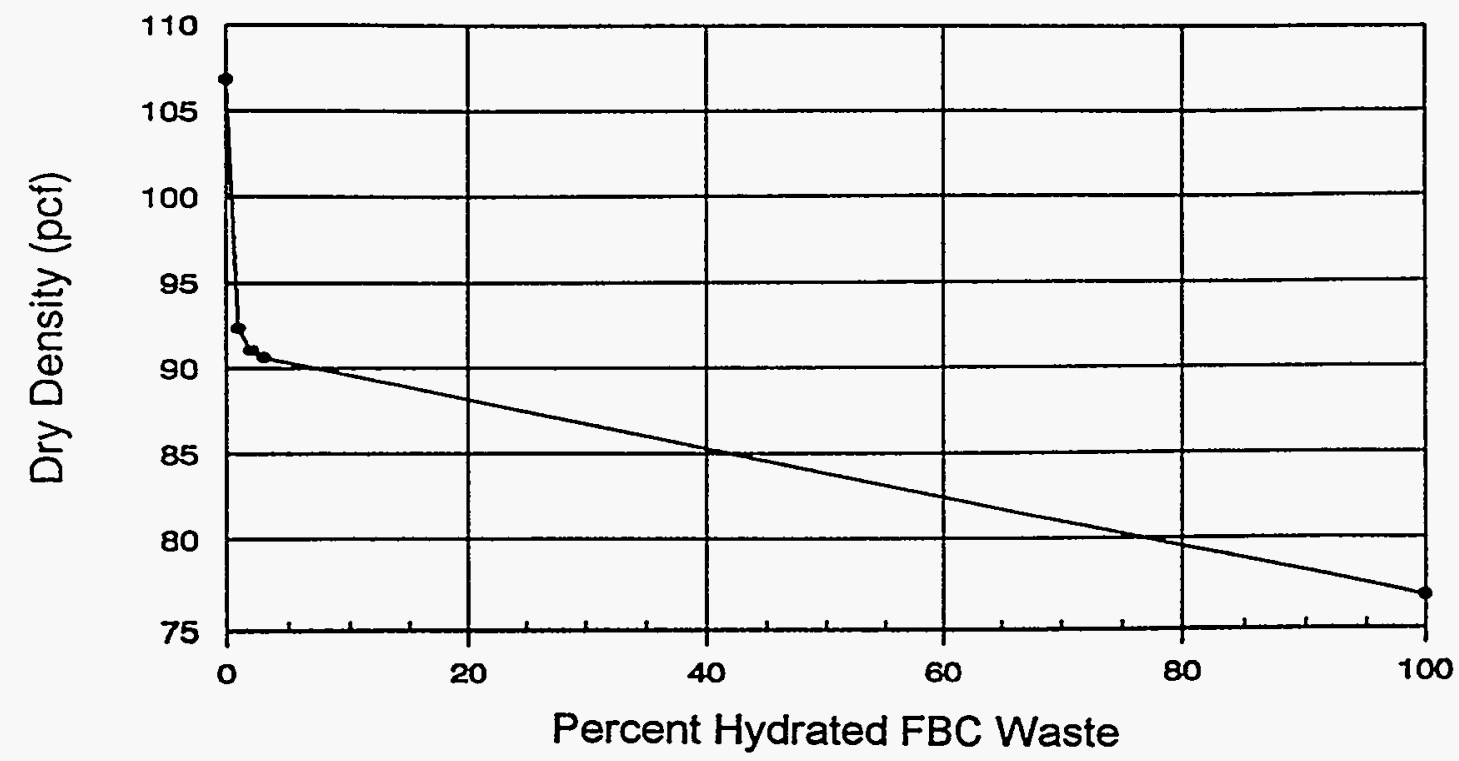

Figure 1. Maximum Dry Density vs Percent of Hydrated FBC Waste in Clay Soil-FBC Waste Mixture.

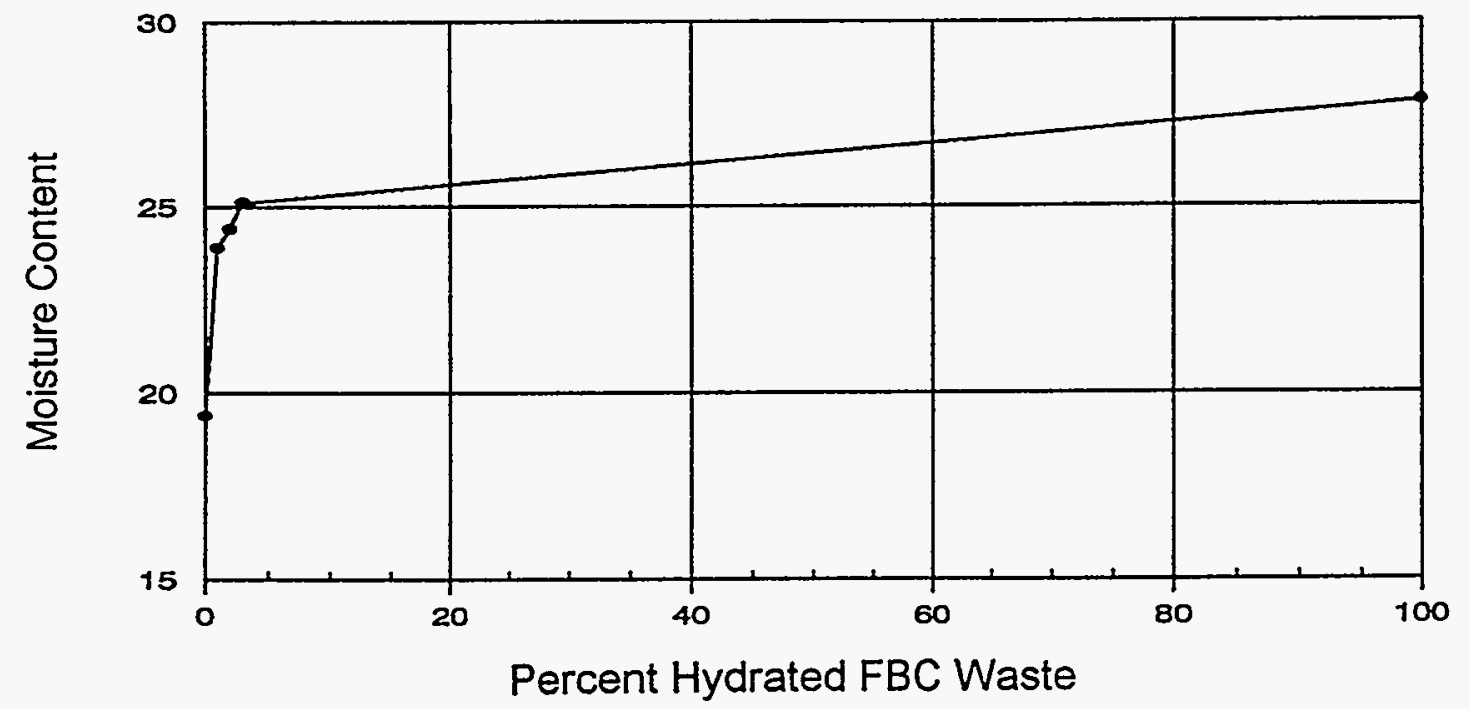

Figure 2. Optimum Moisture Content vs Percent of Hydrated FBC Waste in Clay Soil Waste Mixture. 


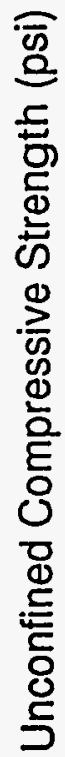

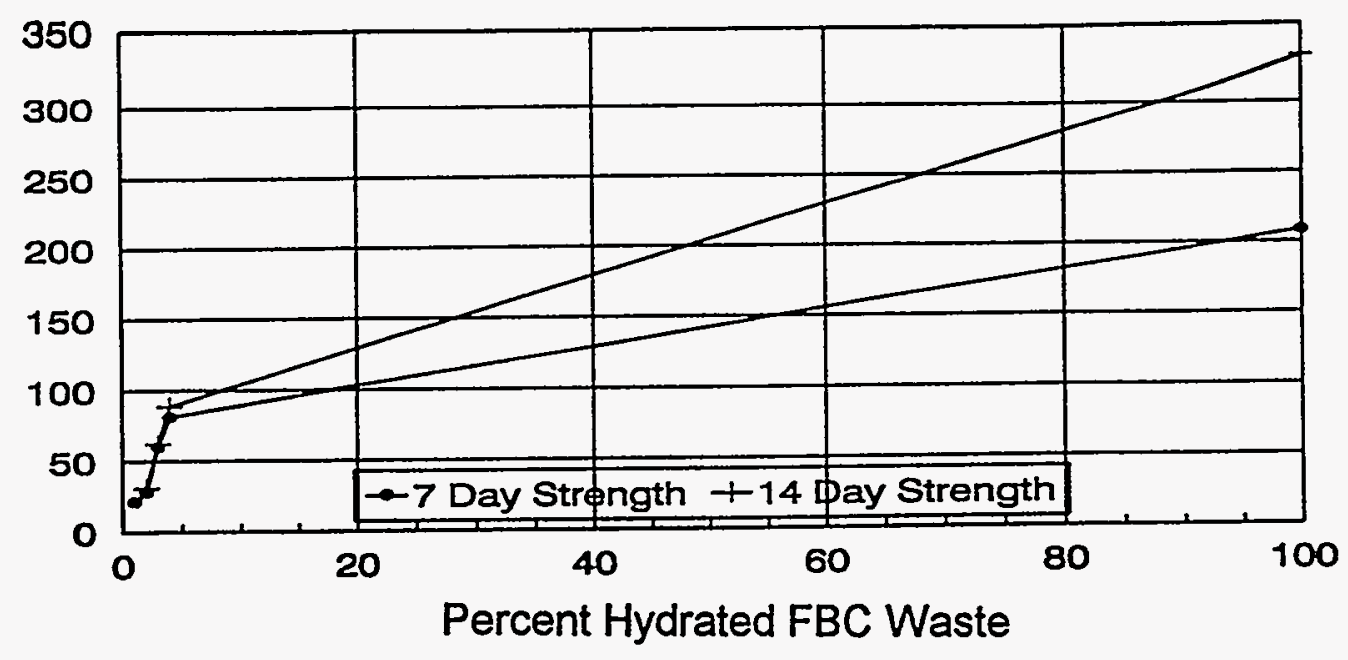

Figure 3. Unconfined Compressive Strength as a Function of Percent of Hydrated FBC Waste in Clay Soil-FBC Waste Mixture.

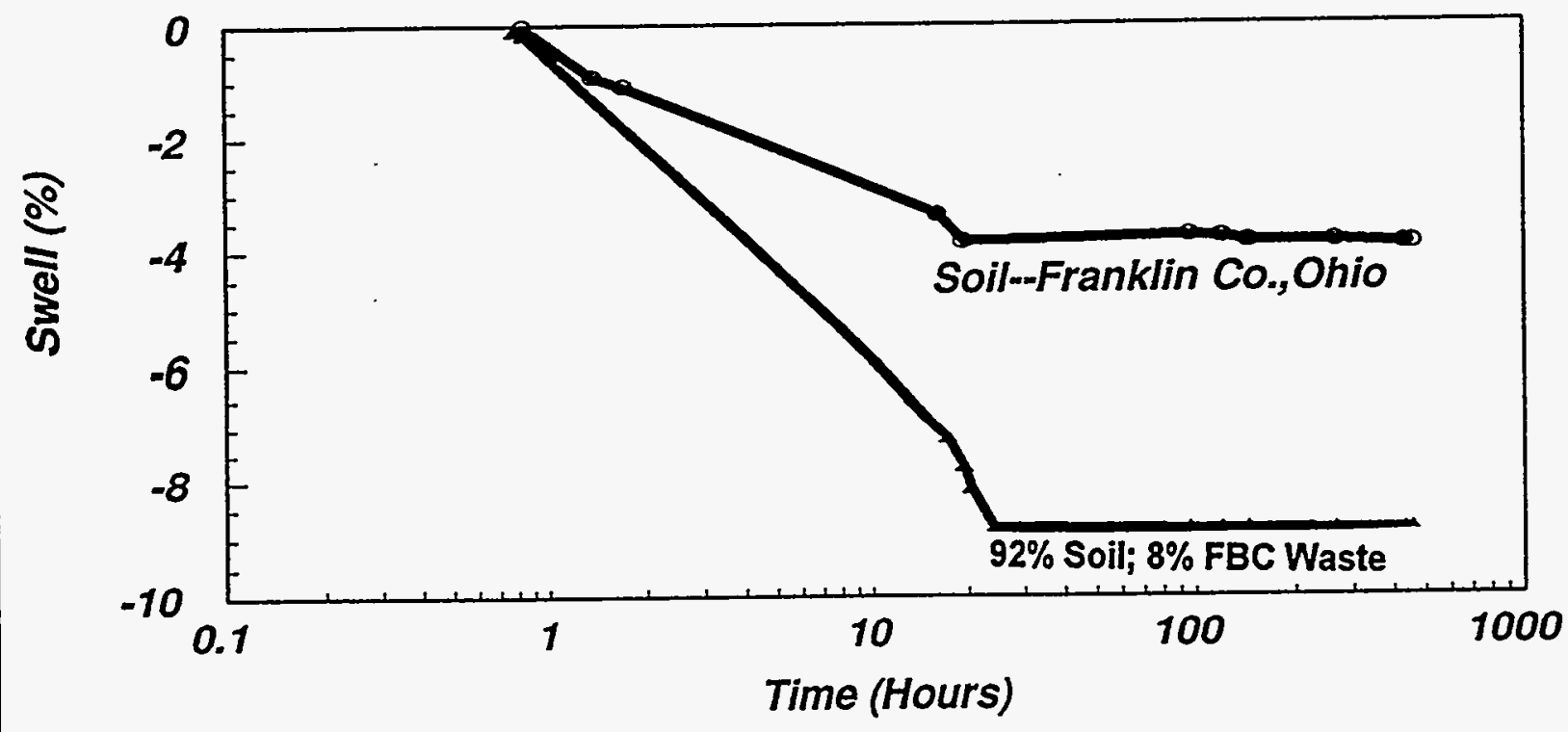

Figure 4. Swell of Clay Soil and Soil-FBC Waste Mix During 19-Day Soak-CBR Samples. 


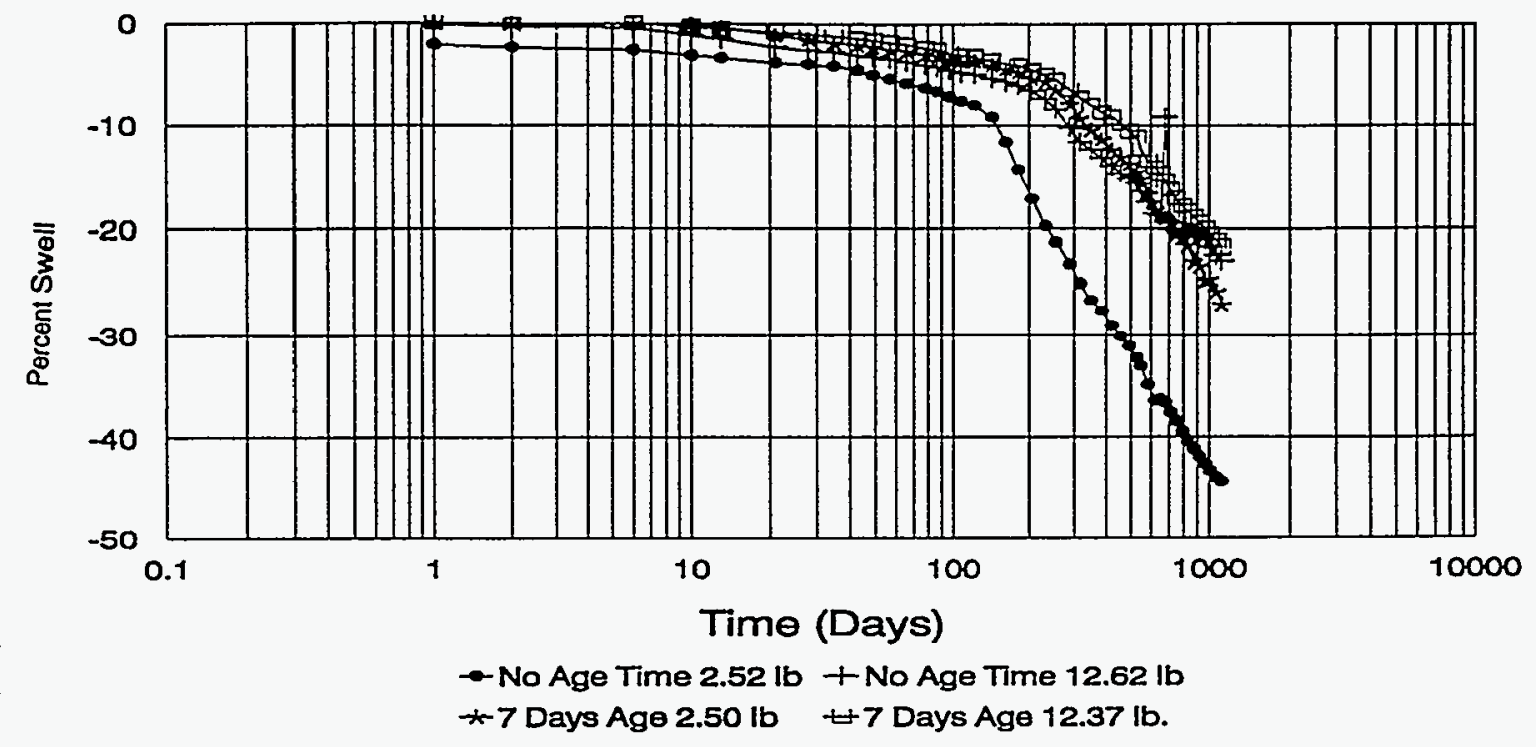

Figure 5. Long-Term Swell of Non-Hydrated FBC Waste.

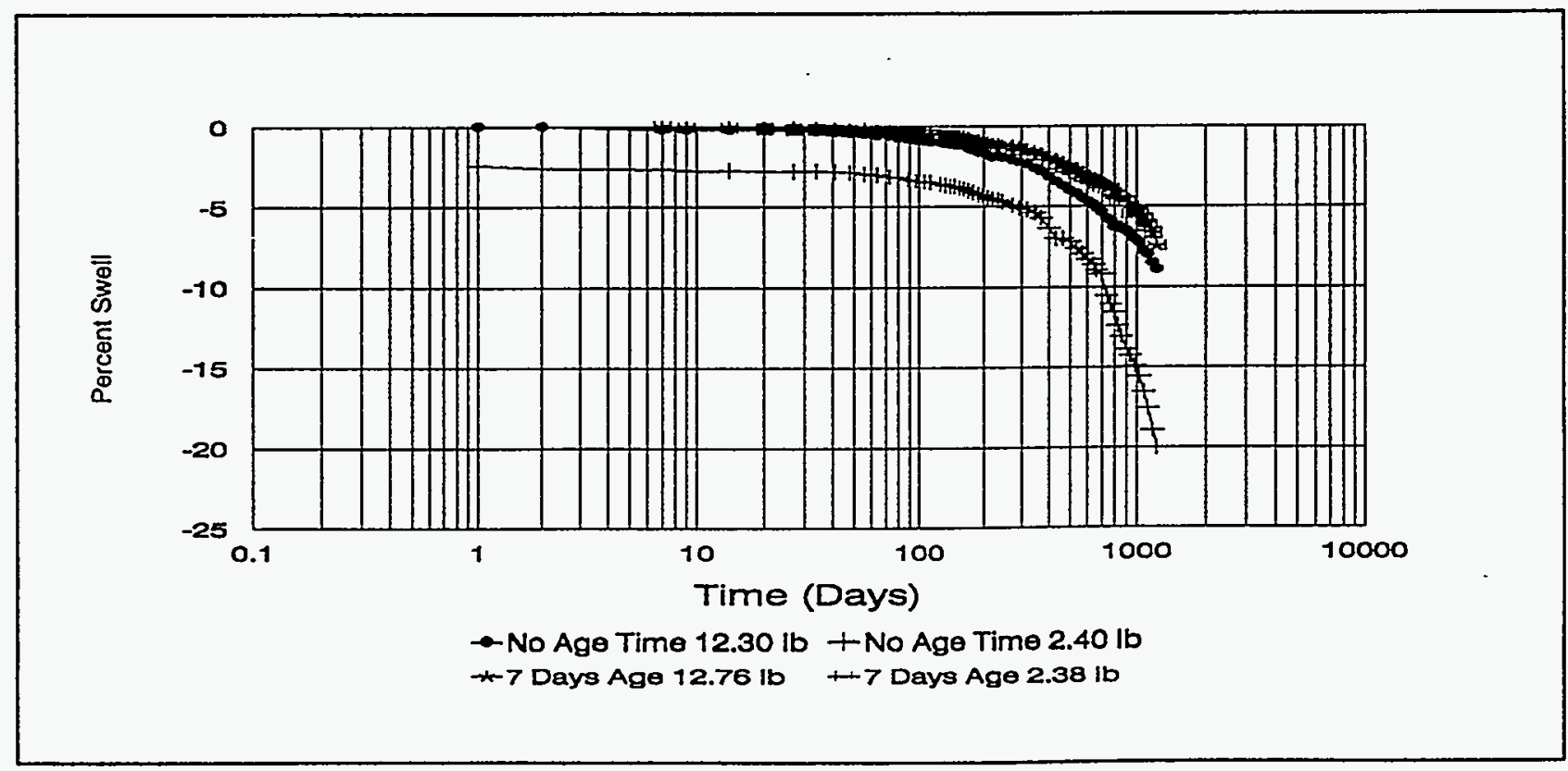

Figure 6. Long-Term Swell of Hydrated FBC Waste. 


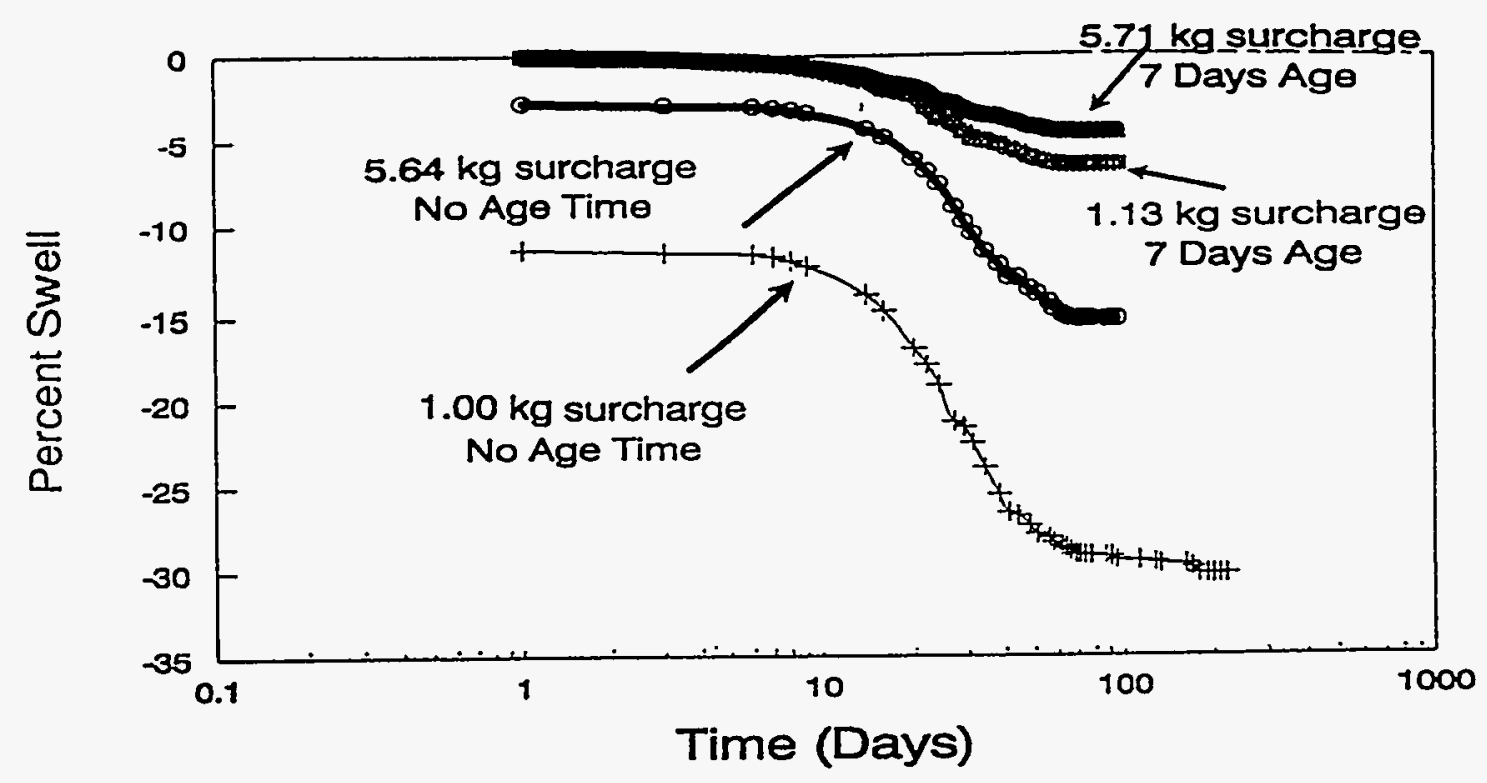

Figure 7. Swell of CBR Samples from Coolside Waste Generated in Recycle Mode.

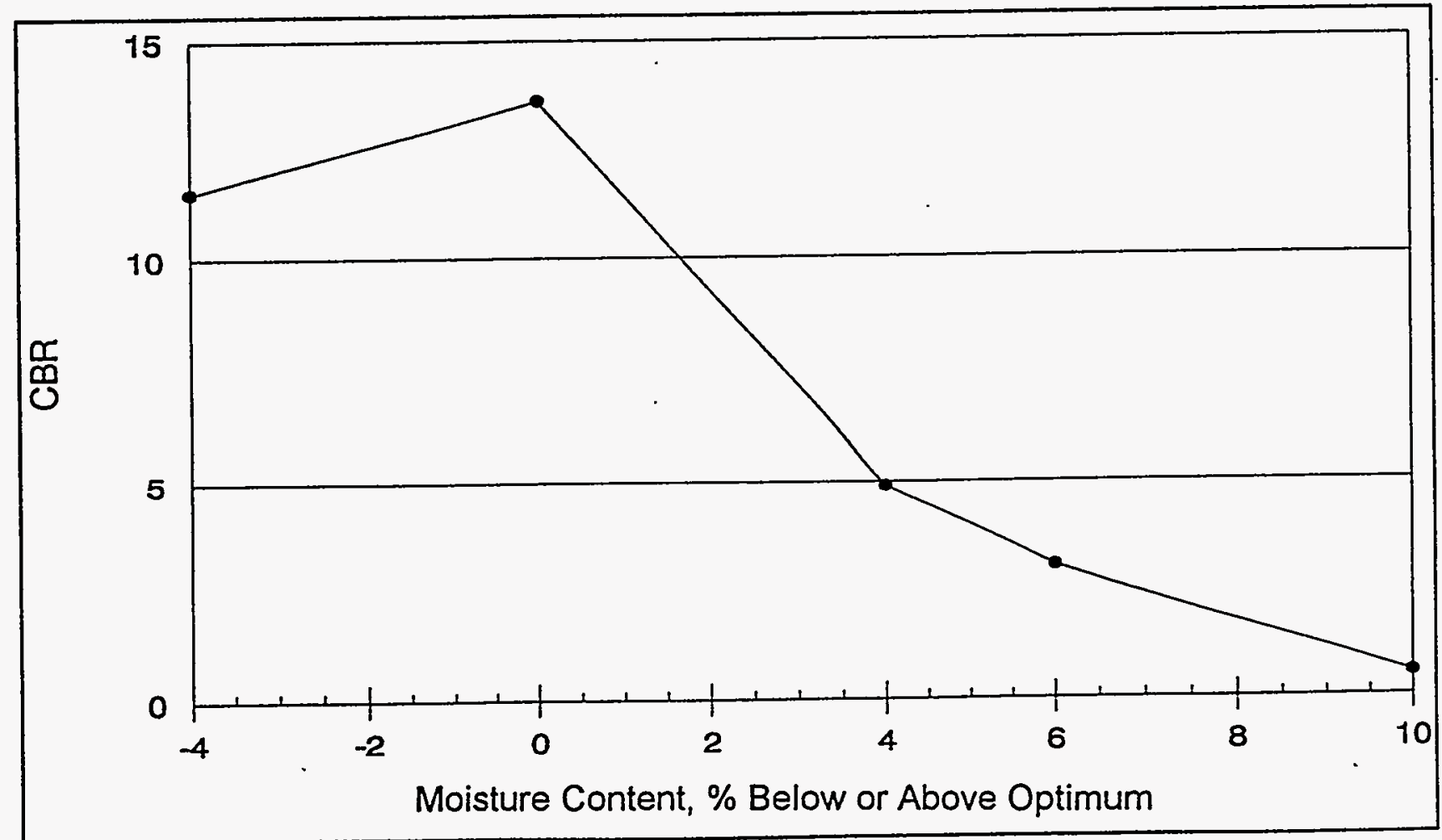

Figure 8. Effect of Moisture Content on CBR Specimens from Coolside Demonstration Run No. 1, Barrel No. 1050. 


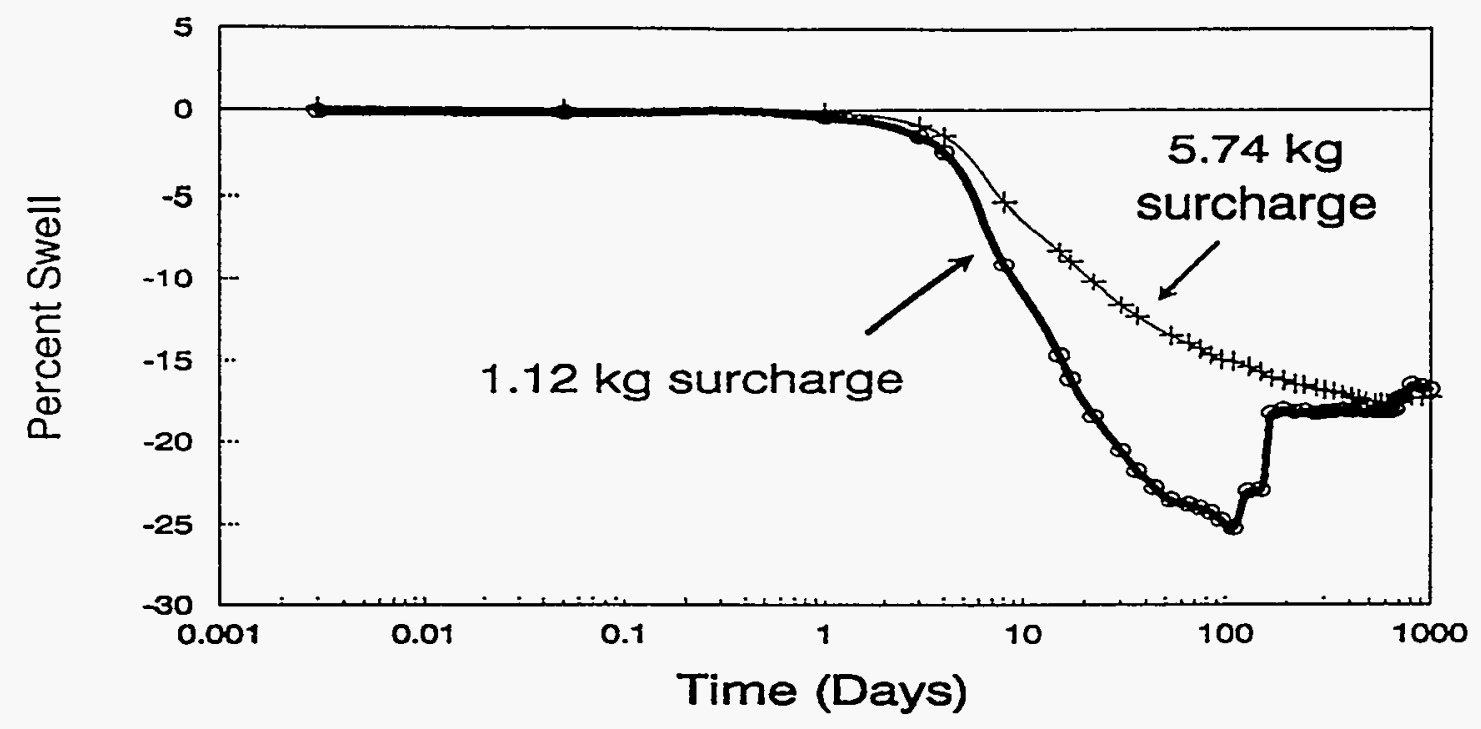

Figure 9. Long-Term Swell of 100\% FBC Waste Synthetic Aggregate.

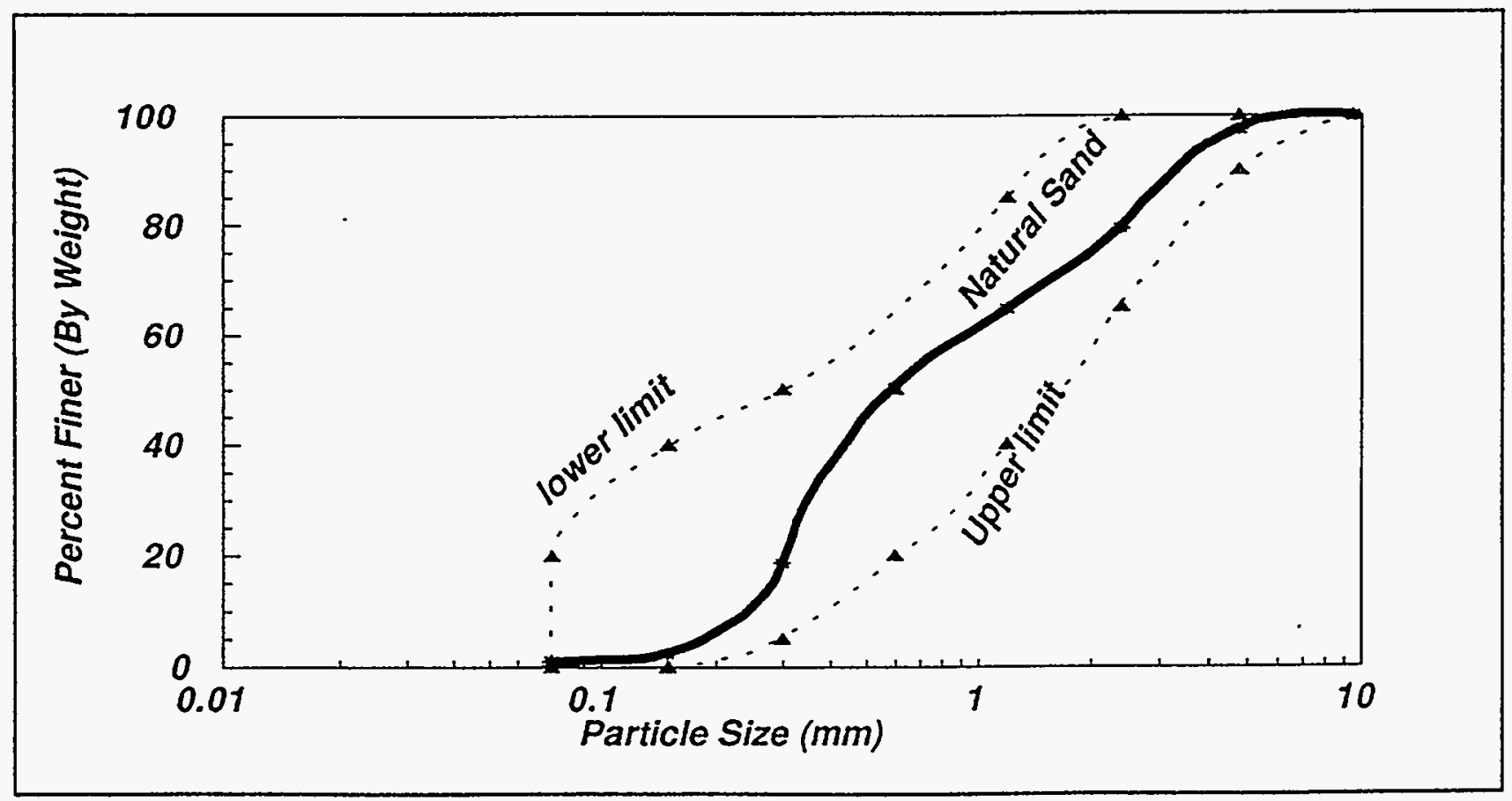

Figure 10. Grain Size of Natural Sand and Specification Limits. 


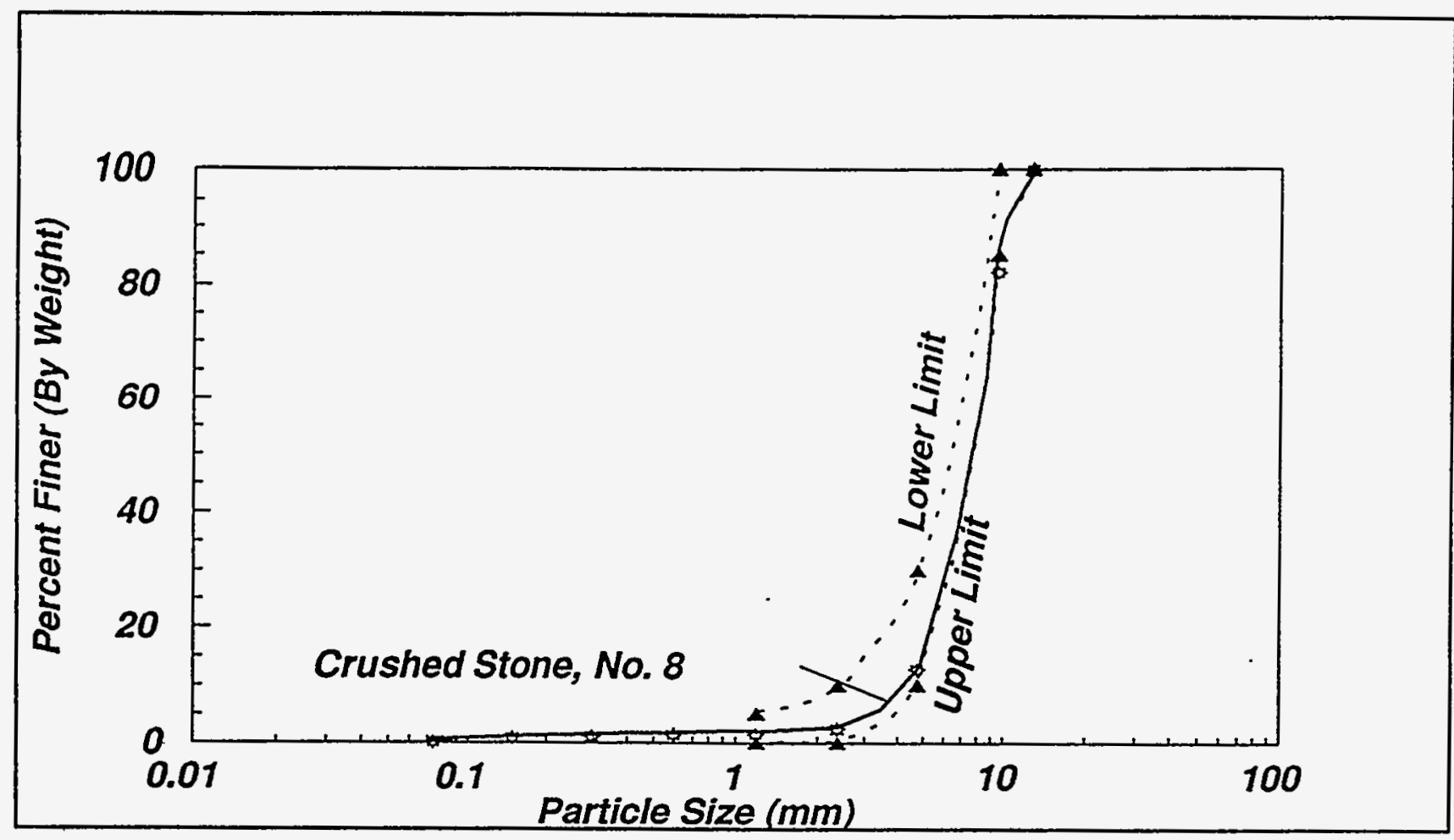

Figure 11. Grain Size of Crushed Stone and Specification Limits.

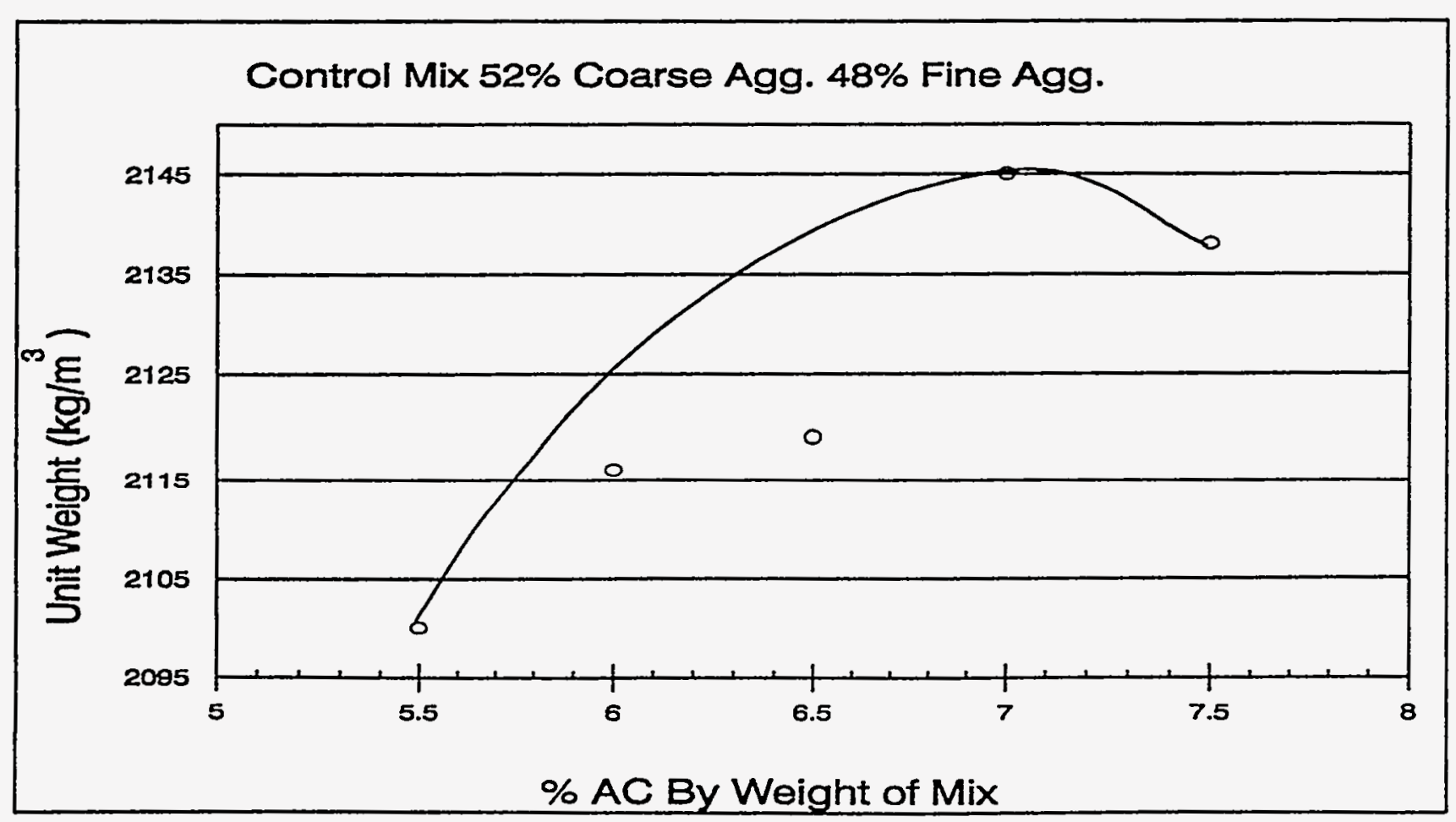

Figure 12. Unit Weight vs Asphalt Content -- Control Mix. 


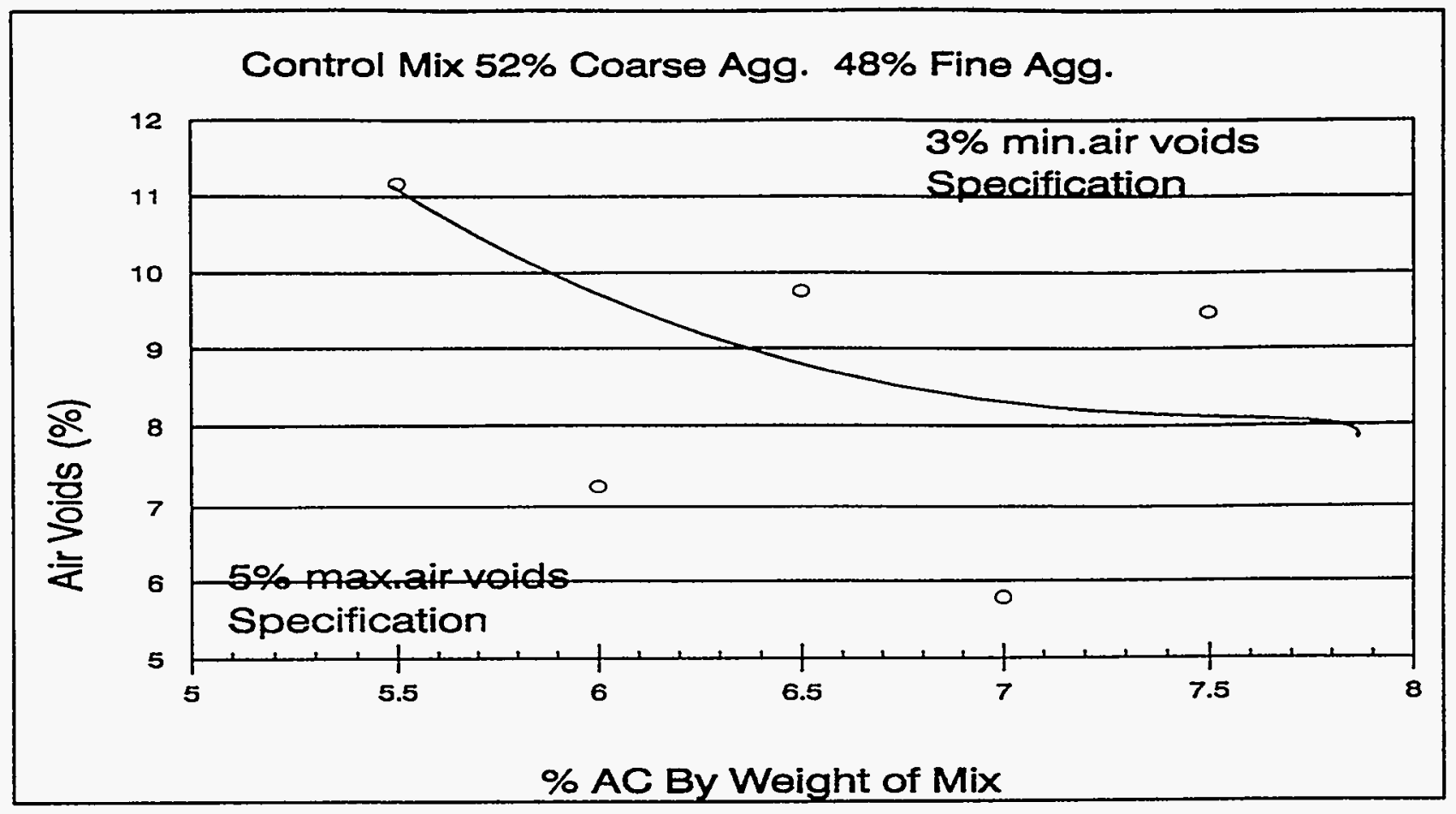

Figure 13. Percent Air Voids vs Asphalt Content -- Control Mix.

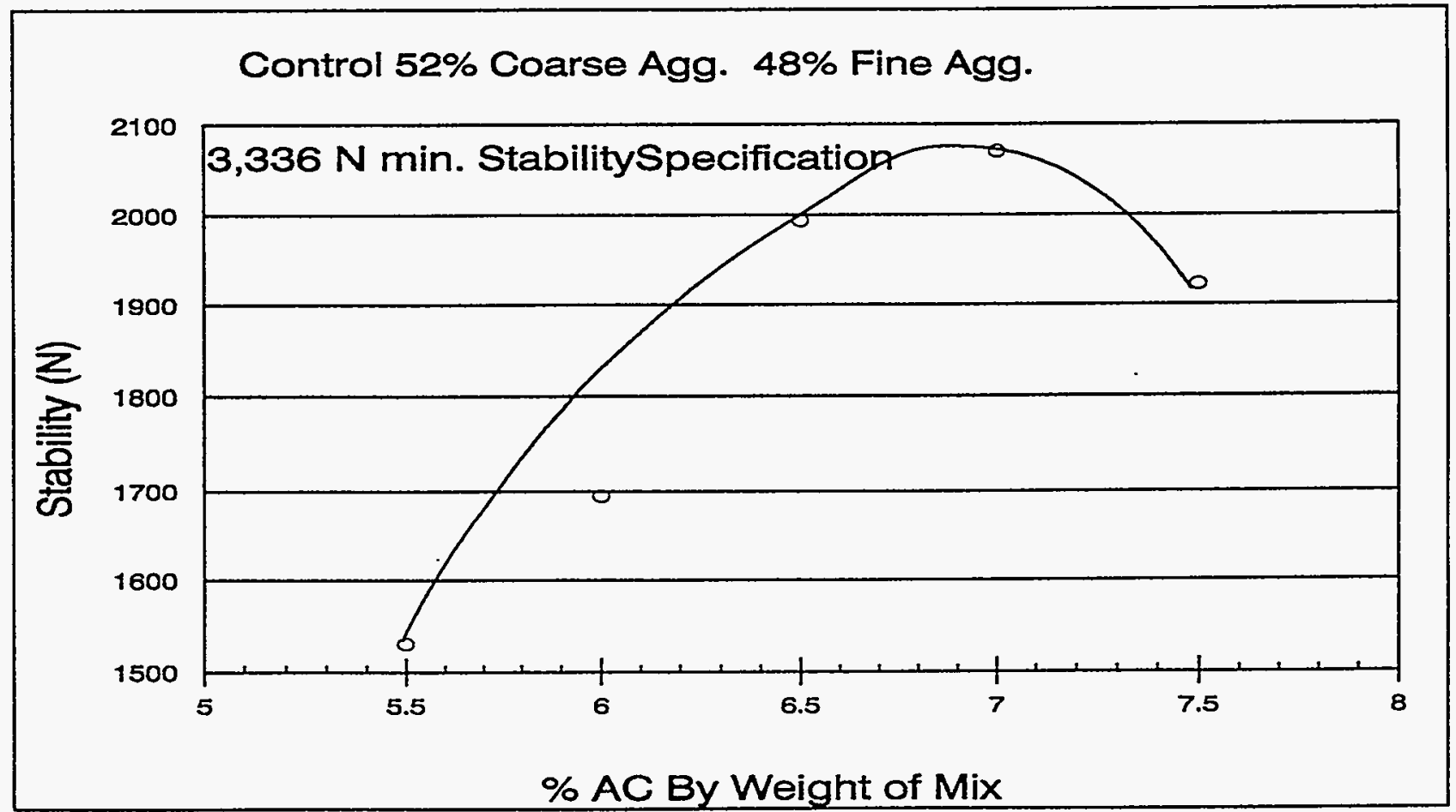

Figure 14. Stability vs Asphalt Content -- Control Mix. 


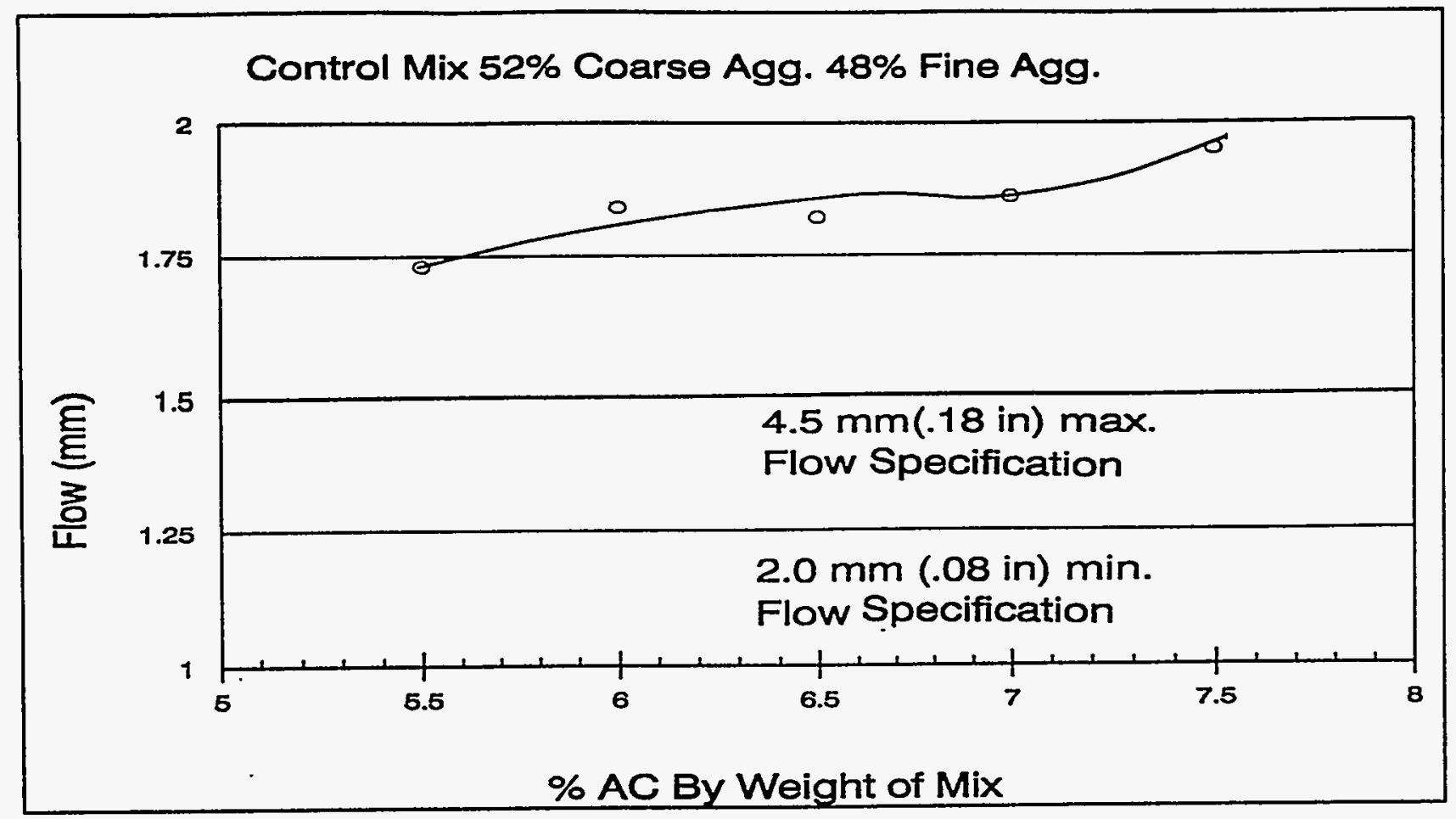

Figure 15. Flow vs Asphalt Content - Control Mix.

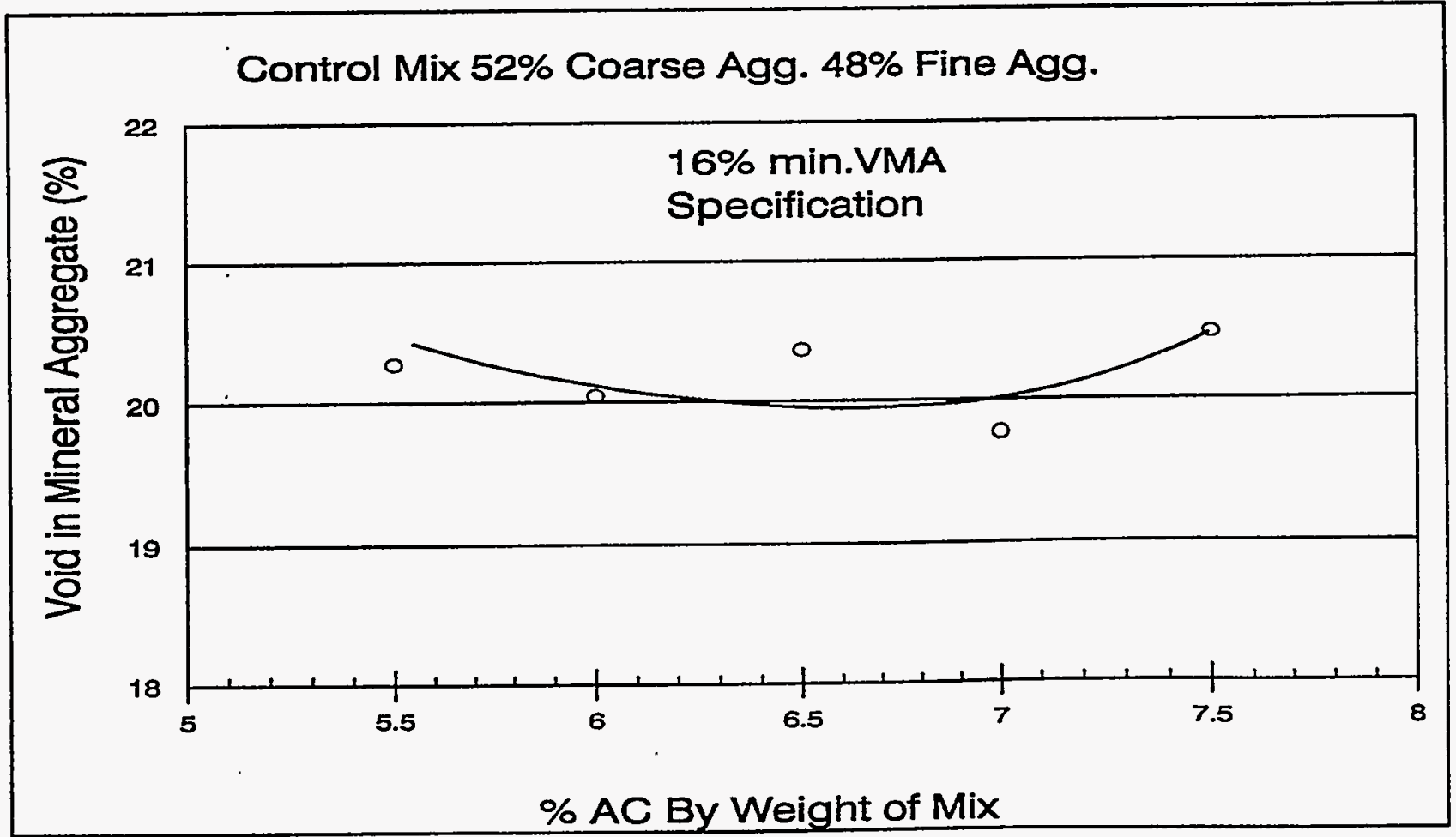

Figure 16. Voids in Mineral Aggregate vs Asphalt Content -- Control Mix. 


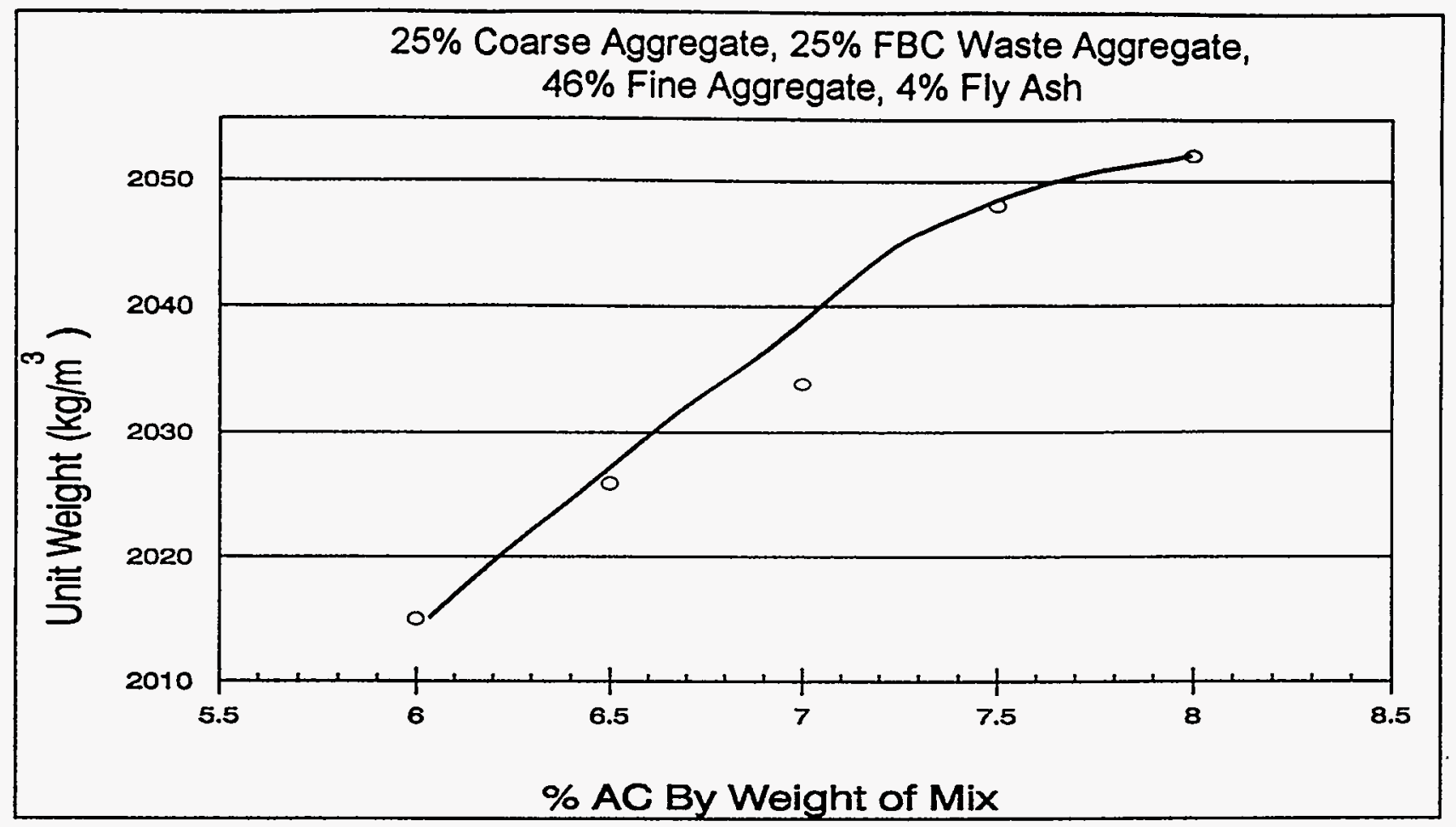

Figure 17. Unit Weight vs Asphalt Content.

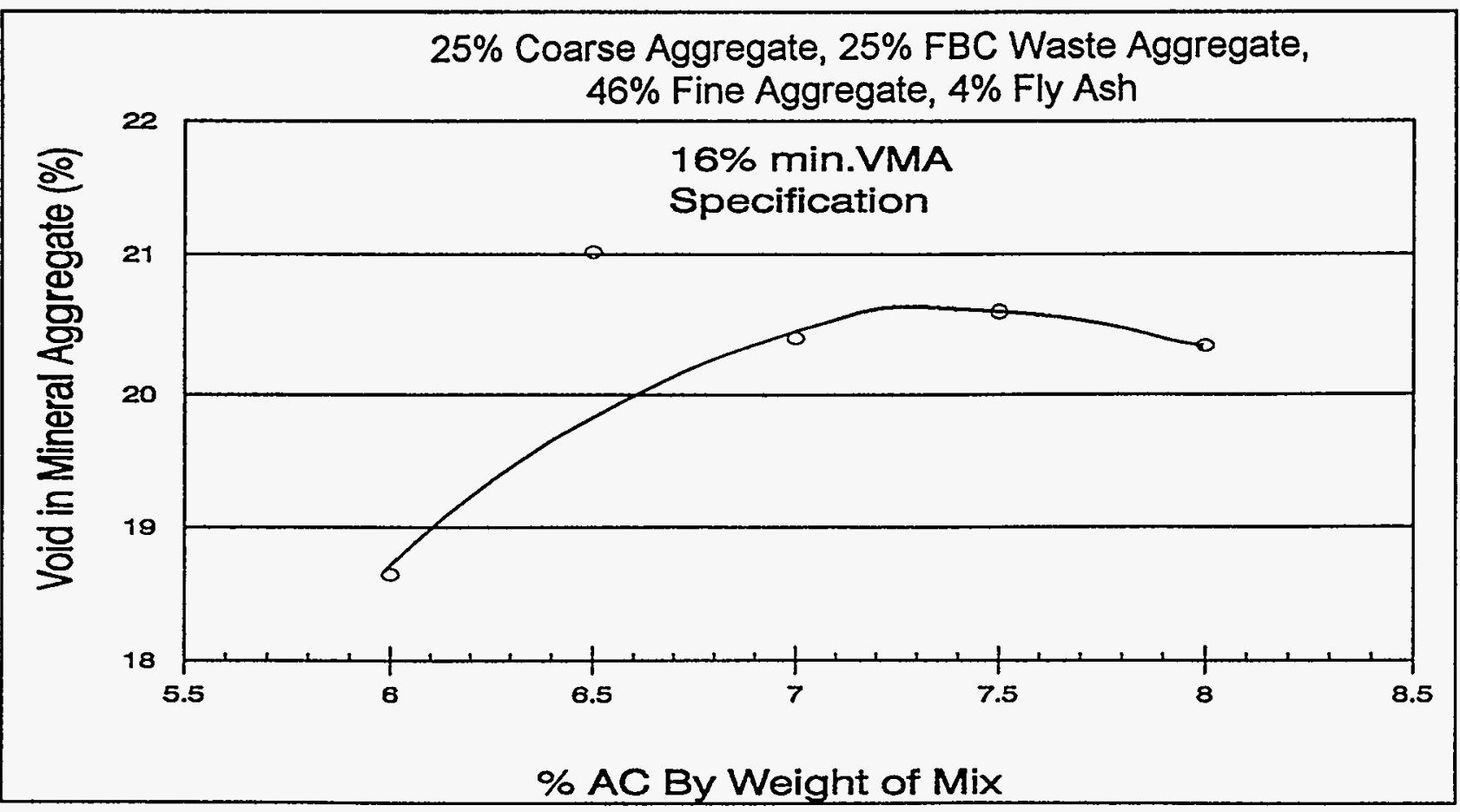

Figure 18. Voids in Mineral Aggregate vs Asphalt Content. 


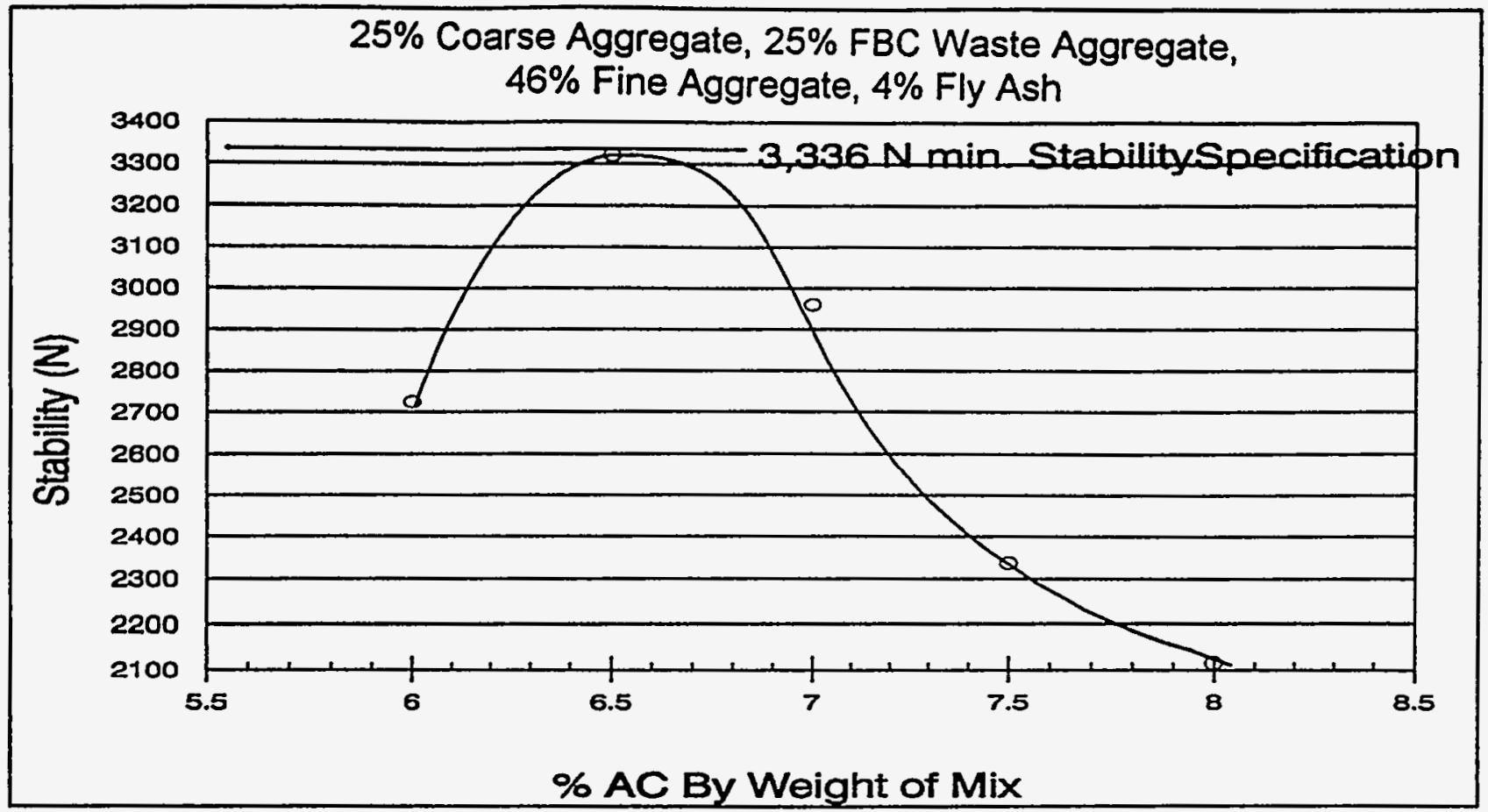

Figure 19. Stability vs Asphalt Content.

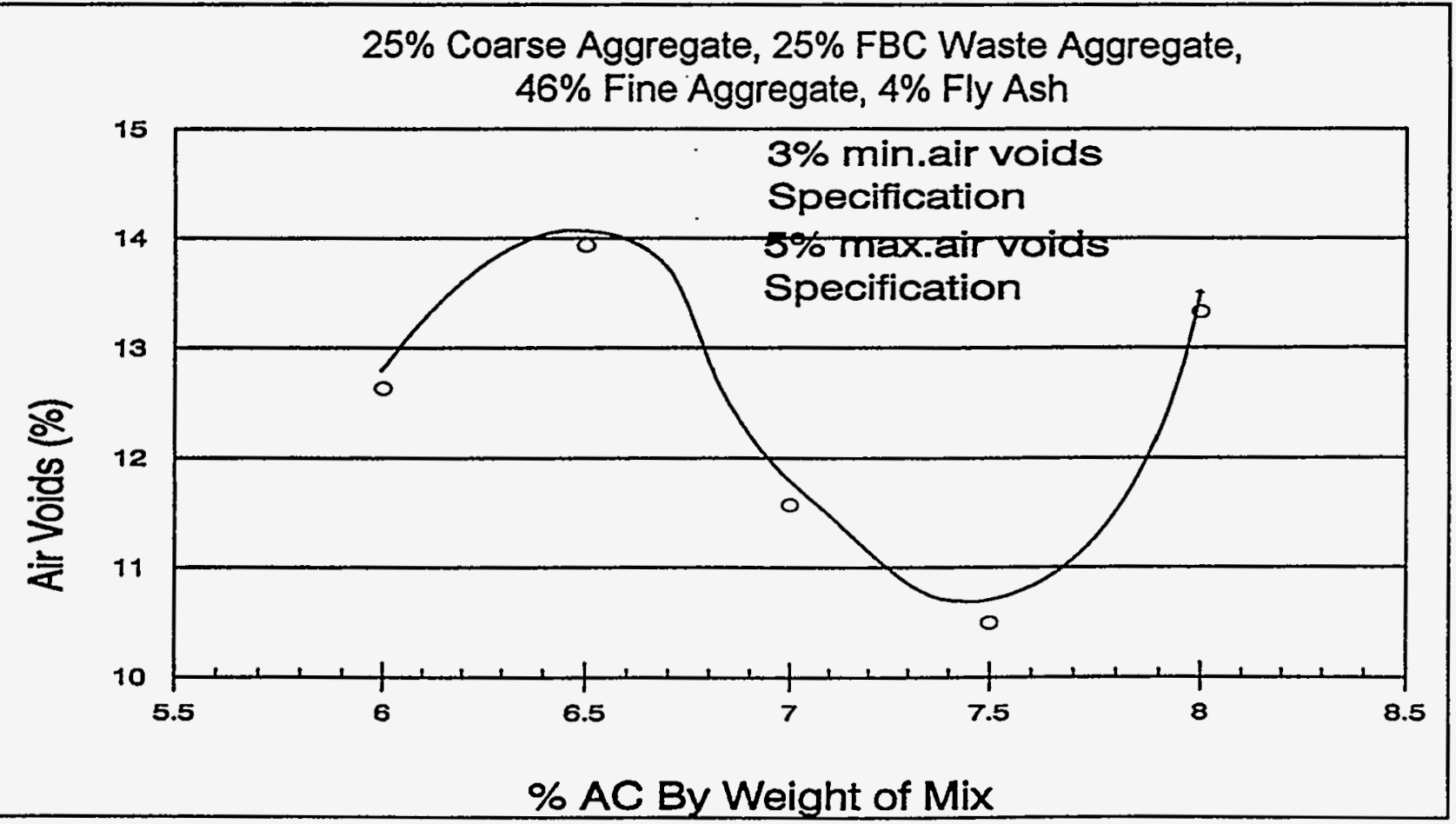

Figure 20. Percent Air Voids vs Asphalt Content. 


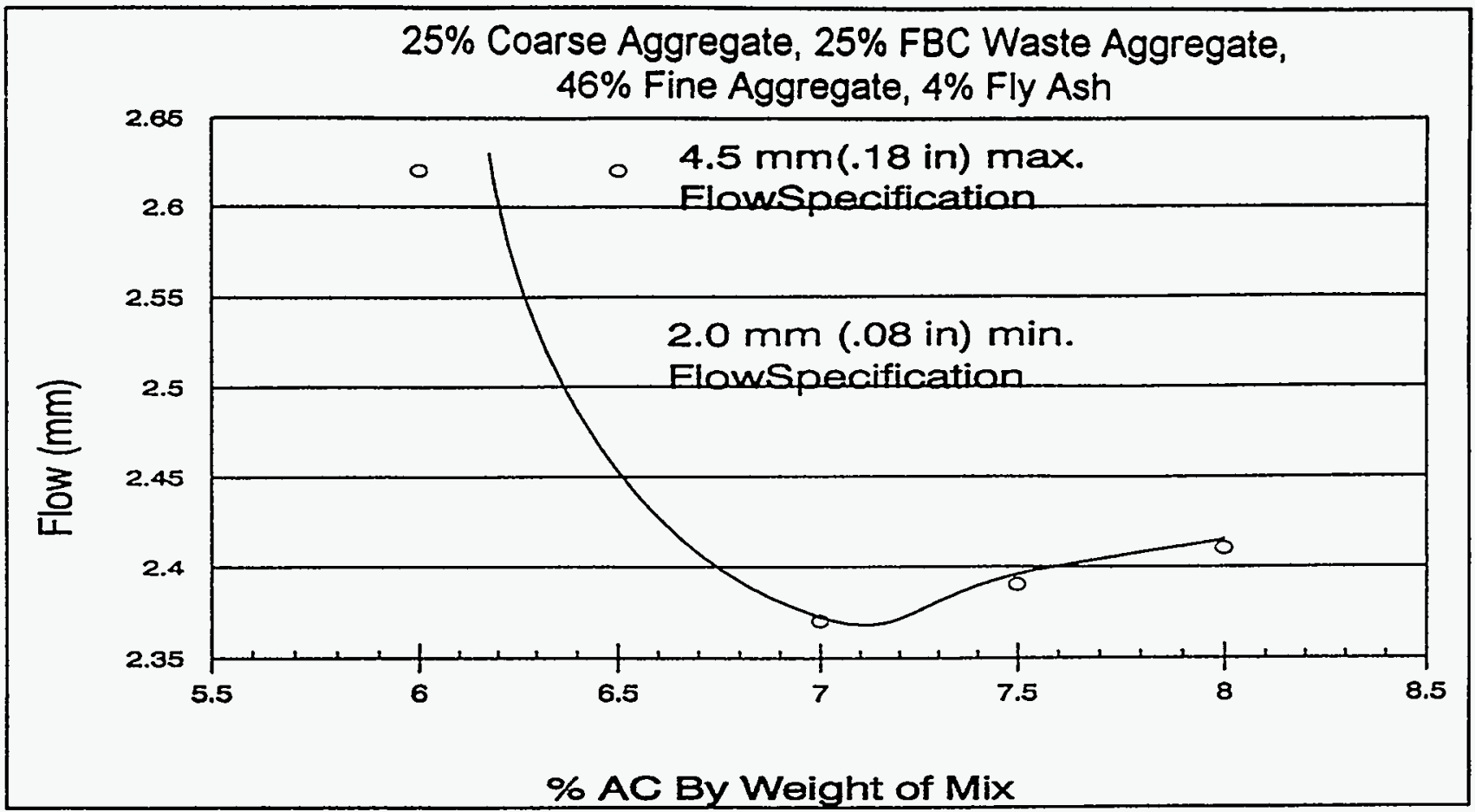

Figure 21. Flow vs Asphalt Content.

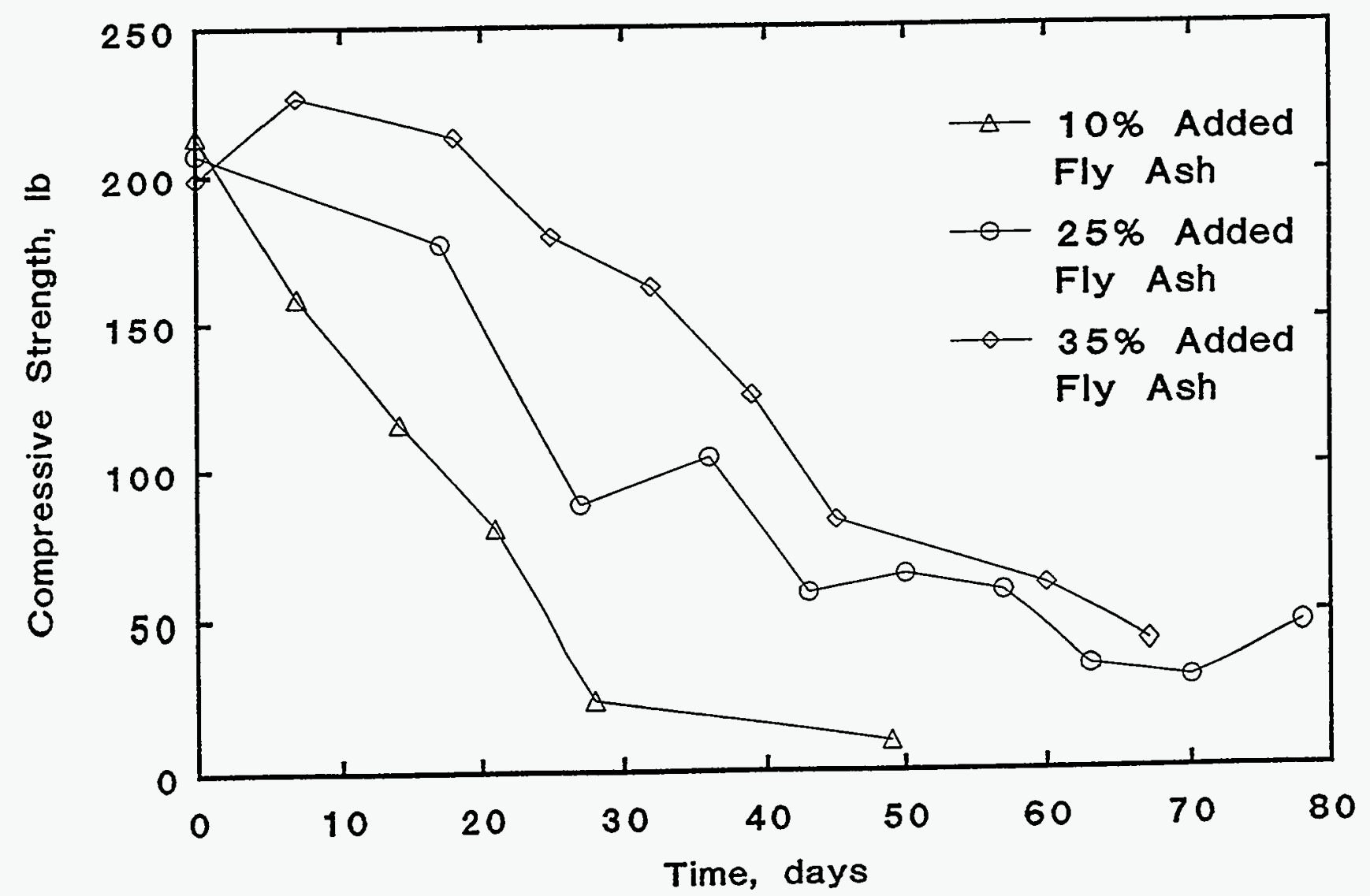

Figure 22. Compressive Strength of FBC Waste Aggregate Blended with Different Amounts of Fly Ash as a Function of Time in the Undrained Weathering Test. 
APPENDIX 3 


\title{
COOLSIDE WASTE MANAGEMENT DEMONSTRATION OCDO GRANT AGREEMENT NO. CDO/D-902-9
}

\author{
Milestone Report - Phase 3: \\ Pilot Plant Production of Coolside Waste Samples
}

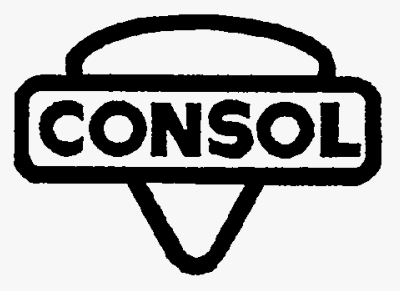

CONSOL Inc.

Research \& Development

4000 Brownsville Road

Library, PA 15129

M. R. Stouffer (412-854-6639)

M. M. Wu (412-854-6708)

H. A. Rosenhoover (412-854-6698) 
TABLE OF CONTENTS

SUMMARY

Page

BACKGROUND AND OBJECTIVE

PROCESS DESCRIPTIONS

Coolside Desulfurization Process

Advanced Coolside Desulfurization

EXPERIMENTAL

Coolside Pilot Plant

Coolside Pilot Plant Operation

Fixed Operating Conditions

Test Fly Ash

Test Hydrated Lime

Test Conditions

Pilot Plant Operating Procedures

Advanced Coolside Operation

Waste Sample Preparation

4

4

4

6

7

7

8

8

8

9

10

10

RESULTS AND DISCUSSION 12

Waste Samples 12

Coolside Desulfurization Performance 12

Test Data 12

Data Reliability 13

Coolside Pilot Plant Operability 14

Advanced Coolside Desulfurization Performance 15

$\begin{array}{ll}\text { REFERENCES } & 17\end{array}$

Table

\section{LIST OF TABLES}

$1 \quad$ Coolside Tests: Fixed Conditions 18

2 Analyses of Grab Samples of Fly Ash From CEI Avon Lake Plant Unit No. 9

3 Analyses of Dravo Black River Hydrated Lime Samples 20

$4 \quad$ Analyses of Mississippi Hydrated Lime 21

5 Analyses of Composite Waste Samples 22

6A Coolside Process Data for Test $1 \quad 23$

6B Coolside Process Data for Test $2 \quad 25$

6C Coolside Process Data for Test $4 \quad 27$

Figure

\section{LIST OF FIGURES}

1 Coolside Desulfurization Process
$2 \quad$ Schematic of Coolside Pilot Unit

Page 


\section{Milestone Report - Phase 3 \\ Pilot Plant Production of Coolside Waste Samples}

\section{SUNAARY}

Four Coolside waste samples were generated in CONSOL's 0.1 MHe Coolside pilot plant. These samples will be used to determine the impact of process variables on waste properties and on disposal and utilization options in Phases 1 and 2. Process operations spanned a range that is of interest for application of the Coolside technology to Ohio coals. Two samples were generated without sorbent recycle, one each with and without sodium additive promotion. One sample was generated with sorbent recycle and with additive promotion. Another sample was generated through the use of a proprietary second-generation sorbent injection process (Advanced Coolside process), which currently is being developed by CONSOL for achieving higher $\mathrm{SO}_{2}$ removals and higher sorbent utilizations than the Coolside process. No additive was used in this process. For all samples, the pilot plant flue gas composition (fly ash and $\mathrm{SO}_{2}$ ) was controlled to simulate that which would be produced by burning a medium-sulfur Ohio coal. For the Coolside process testing, the sorbent was Black River hydrated lime. This 1 ime is produced in Carntown, Kentucky, which is located on the Ohio River; it is sold into the Ohio market. The sorbent for the Advanced Coolside tests was from Mississippi Lime Co. Both of these limes were used extensively in the Coolside process demonstration at the Ohio Edison Edgewater Station. The fly ash was from a medium-sulfur Ohio coal and was produced at the Cleveland Electric Illuminating Company (CEI) Avon Lake Plant Unit No. 9. For the four process conditions, three $500 \mathrm{lb}$ samples and one $90 \mathrm{lb}$ sample were generated. Desulfurization performance was measured in all the tests. Under Coolside process conditions, up to $75 \% \mathrm{SO}_{2}$ removal was achieved. Performance was consistent with that observed in previous pilot and field tests at the Ohio Edison Coolside demonstration. 
Pilot plant operability and data reliability were very good. Therefore, the wastes produced are representative of the two processes and are reliable samples for waste management studies. The waste samples were divided between the University of Kentucky and CONSOL for use in Phases 1 and 2 of the project. 


\section{BACKGROUND AND OBJECTIVE}

The Coolside process can be run in different operation modes to achieve various performance targets (e.g., sorbent utilization and $\mathrm{SO}_{2}$ removal). Different operating modes will result in differences in the composition of the waste. These differences can affect the physical, chemical and engineering properties of the waste. The properties are relevant to disposal and utilization. The objective of the Phase 3 study is to provide additional Coolside waste samples for use in Phases 1 and 2 . The additional samples will promote the general applicability of project results and will indicate the effect of differences in waste composition on disposal and utilization options. The CONSOL Coolside pilot plant was used to generate these waste samples. In accordance with the OCDO Grant Agreement, sample generation conditions were discussed and agreed upon by the project participants (OCDO, UK/CAER, DOE/METC and CONSOL) at the Second Advisory Committee meeting on January 7, 1992, at Lexington, Kentucky. ${ }^{1}$ It was agreed that about $500 \mathrm{1b}$ each of coolside waste samples would be generated from the once-through mode with sodium additive (Test 1) and the recycle mode with sodium additive (Test 2) of the Coolside process, and by blending fly ash with waste samples generated in pilot plant runs in the Advanced Coolside mode (Test 3 ).

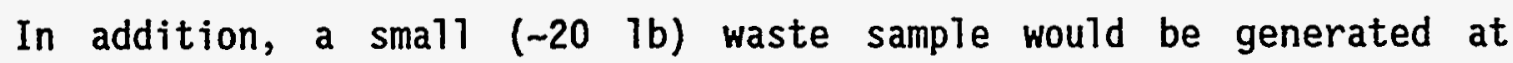
conditions similar to those used in as Test 1 , except that no sodium additive would be used (Test 4 ). These samples would be produced at process conditions that simulate the operation of the Coolside process as installed on a unit burning a medium-sulfur Ohio coal. 


\section{PROCESS DESCRIPTIONS}

\section{COOLSIDE DESULFURIZATION PROCESS}

The Coolside desulfurization process (Figure 1) is a low capital cost technology for $\mathrm{SO}_{2}$ control on coal-fired boilers. It involves injection of hydrated lime into flue gas downstream of the air preheater, with humidification by water sprays to close approach $\left(20-25^{\circ} \mathrm{F}\right)$ to adiabatic saturation. Sorbent activity can be improved by injection of a watersoluble additive, such as $\mathrm{NaOH}$ or $\mathrm{Na}_{2} \mathrm{CO}_{3}$, with the humidification water. $\mathrm{SO}_{2}$ is removed by entrained sorbent particles during humidification and by the sorbent bed in the existing particulate collector. The solid waste from the process is dry and is removed using the existing ash handling equipment. The spent sorbent can be recycled to improve sorbent utilization efficiency.

The Coolside process has been developed by CONSOL through laboratory, pilot (0.1 MWe), and field (1 MWe) testing (References 2-5). The process was demonstrated at the 104 MWe Ohio Edison Edgewater Station Unit 4 in Lorain, Ohio, as part of the U.S. Department of Energy's Clean Coal Technology program (CCT 1). ${ }^{6}$. The Edgewater demonstration showed that the process can achieve up to $70 \% \mathrm{SO}_{2}$ removal and up to $35 \%$ sorbent utilization. CONSOL conducted pilot-scale testing in support of the demonstration $;^{7}$ this work included waste management studies in support of the demonstration to determine the various properties of Coolside waste that are relevant to handling and disposal issues. ${ }^{8,9}$

\section{ADVANCED COOLSIDE DESULFURIZATION}

The Advanced Coolside process is a second-generation duct sorbent injection technology developed by CONSOL. The process utilizes the improved performance of hydrated 1 ime at closer approaches to adiabatic saturation and improvements in sorbent recycle to achieve higher $\mathrm{SO}_{2}$ removal and higher sorbent utilization than the coolside process. The ongoing development of this process is being funded, in part, by the U.S. Department of Energy. ${ }^{10}$ The goal of the development program is to make 
the process more attractive as a compliance option for the 1990 Clean Air Act amendments. Specific performance goals are to achieve $90 \% \mathrm{SO}_{2}$ removal and $60 \%$ sorbent utilization, while retaining the low capital cost advantages of the Coolside process.

In the Advanced Coolside process, most of the fly ash is removed before the point of sorbent injection. The process allows the option of disposing of the spent sorbent separately or disposing of spent sorbent and fly ash together. 


\section{EXPERIMENTAL}

\section{COOLSIDE PILOT PLANT}

The 0.1 Whe pilot test facility (Figure 2) is designed to study Coolside desulfurization performance over a wide range of process conditions. The unit is described in detail in Reference 4 . It allows site-specific simulation of flue gas conditions, including gas composition, temperature, fly ash loading, and residence time. The unit uses a commercial two-fluid nozzle for water spray humidification as required for the Coolside process simulation. The unit allows measurements of $\mathrm{SO}_{2}$ removal across the humidifier alone and across the entire system, which includes both the humidifier and particulate collector (baghouse).

The pilot plant consists of a flue gas generation system, Coolside process system (including lime injector and humidifier), a baghouse, and continuous flue gas sampling and analysis systems. The flue gas leaving the baghouse is recycled after removal of excess moisture by a condenser/separator, providing over $80 \%$ of the flue gas requirement. The recycle gas is mixed with the flue gas introduced from a natural gas burner. By injecting $\mathrm{CO}_{2}, \mathrm{H}_{2} \mathrm{O}, \mathrm{SO}_{2}$ and fly ash into this combined gas, the inlet flue gas conditions can be matched to the flue gas conditions downstream of the air preheater in a coal-fired boiler. The pilot humidifier is a vertical, 8.3-inch ID, cylindrical duct and provides a 20-foot long down-flow humidification zone. The flue gas velocity is variable from 8 to $25 \mathrm{ft} / \mathrm{sec}$, giving $0.8-2.5 \mathrm{sec}$ residence times. The humidifier is installed with thermocouples and observation ports every two feet along its length. Hydrated lime is injected into the flue gas at the top of the humidifier. The amount of water spray controls the humidifier exit gas temperature for the desired approach to saturation. A water-soluble additive such as $\mathrm{NaOH}$ can be fed as an aqueous solution into the humidification water stream. The pilot baghouse is a pulse-jet unit with a total cloth area of $115 \mathrm{ft}^{2}$. The pilot unit includes three continuous $\mathrm{SO}_{2} / \mathrm{O}_{2}$ flue gas analys is systems to measure $\mathrm{SO}_{2}$ removal. The $\mathrm{O}_{2}$ analyses are used to correct for air in-leakage. The $\mathrm{SO}_{2}$ removal can be measured across the humidifier only and across the system; i.e., 
across both the humidifier and baghouse. The humidifier exit gas sampling system was specialiy designed to prevent further $\mathrm{SO}_{2}$ reaction with reactive solids in the sample system.

The Coolside pilot $\mathrm{plant}$ has been used for Coolside process variable tests, ${ }^{4}$ studies of Cool side desulfurization mechanisms, ${ }^{5}$ support tests for the Edgewater demonstration, ${ }^{7,8}$ and ongoing process improvement studies. Through this testing, the operability of the pilot plant and the reliability of the test data have been well established. Operating conditions have been identified which allow operation with minimal humidifier wall wetting and minimal solids deposition. Spent sorbent analyses confirm the desulfurization data, which are based on flue gas analyzers. Solid elemental material balances close to within $\pm 10 \%$, indicating good process data reliability. Desulfurization performance data have been consistent with results of the 1 MWe field tests and the 104 MHe Edgewater demonstration.

\section{COOLSIDE PILOT PLANT OPERATION}

\section{Fixed Operating Conditions}

Table 1 shows the fixed operating conditions for the Coolside pilot testing.

All tests were conducted with an inlet $\mathrm{SO}_{2}$ content of $2000 \mathrm{ppmv}$ (dry basis), simulating the use of a medium-sulfur Ohio coal. Fly ash (vida infra) from an Ohio coal was added to the flue gas to provide for a fly $\mathrm{ash} / \mathrm{S}$ ratio of $4.0 \mathrm{lb} / 1 \mathrm{~b}$. This is about the same ash/s ratio as in the coal from which the fly ash was obtained. The inlet flue gas temperature was fixed at $300^{\circ} \mathrm{F}$, a typical temperature downstream of the air preheater in a coal-fired plant.

All Coolside testing was conducted at $25^{\circ} \mathrm{F}$ approach to adiabatic saturation. This is about the minimum approach for good operability in the pilot plant. (In the 104 MWe Edgewater demonstration, the minimum practical approach was about $20^{\circ} \mathrm{F}$.) 
Pilot plant operating conditions shown in Table 1 were selected to give good operability on the basis of previous experience.

\section{Test Fly Ash}

The fly ash used in the pilot testing was collected from the Cleveland Electric Illuminating Company (CEI) Avon Lake Plant Unit No. 9 on January 7, 1992. Analyses of two grab samples of fly ash used in the testing are given in Table 2. The Avon Lake plant burns a medium-sulfur Ohio coal.

\section{Test Hydrated Lime}

The sorbent used in the Coolside pilot plant operation was Dravo Lime Company Black River hydrated lime. This material serves the Ohio Market; it is produced in Carntown, Kentucky, on the Ohio River. This 1 ime was one of two test sorbents used in the Edgewater Coolside demonstration. of 12 commercial hydrated 7 imes evaluated as candidate sorbents for the demonstration, Black River had the lowest delivered cost to the Edgewater site in Lorain, Ohio. It also showed a moderately high activity level in pilot tests. Table 3 gives analyses of grab samples of lime used in this test program; these samples are typical of Black River 7 ime samples tested in previous CONSOL pilot tests.

\section{Test Conditions}

The following Coolside tests were conducted in the waste generation program:

\begin{tabular}{|c|c|c|c|}
\hline Test No. & $\begin{array}{c}\text { Fresh Ca/S } \\
\text { mol Ratio }\end{array}$ & $\begin{array}{c}\text { Fresh NaOH/Ca } \\
\text { mol Ratio }\end{array}$ & $\begin{array}{c}\text { Recycle Ratio } \\
\text { lb recycle/lb hydrated lime }\end{array}$ \\
\hline 1 & 2.0 & 0.20 & 0 \\
2 & 1.3 & 0.20 & 3.0 \\
4 & 2.5 & 0 & 0 \\
\hline
\end{tabular}


These tests allow the effects on waste properties of recycie and of sodium additive use to be investigated. Sodium hydroxide $(\mathrm{NaOH})$ was used as the additive, because it is easier to handle than $\mathrm{Na}_{2} \mathrm{CO}_{3}$ in pilot tests.

\section{Pilot Plant Operating Procedures}

Pilot plant operating procedures were much the same as those used in previous Coolside test programs. These procedures are detailed in other references. $4,5,7,8$ Procedures to ensure data reliability included regular gas analyzer calibration, periodic flue gas flow meter calibration, and periodic solids sampling and elemental analyses to confirm measured desulfurization performance.

One difference between testing in this program and that in other programs was that these test periods were longer, with operation and maintenance proceeding continuously, 24 hours per day. The table below shows operating durations for the Coolside tests:

Test No.

1

2

4
On-Stream Hours

35.3

55.1

5.9

During each test period, there were shutdowns for routine maintenance and shutdowns due to minor operating problems. Over $500 \mathrm{lb}$ of waste sample was generated in each of Tests 1 and 2. As planned, Test 4 was shorter and produced about $90 \mathrm{~Tb}$ of waste sample.

For the recycle test, baghouse solids were recycled on a semi-continuous basis. That is, the baghouse ash was collected and returned periodically to the recycle feeder in batches. The maximum inventory in the recycle feeder hopper was kept constant and the excess baghouse material was discarded (or collected) as waste. This mode of operation allowed closer simulation of steady-state continuous recycle than in previous test programs. The initial waste produced in the recycle test was not at its 
steady-state composition; therefore, it was not included in the waste sample. Also, data from the early periods of the test were not included in the data analysis. The time at which steady-state conditions were established was defined as the time at which sulfur and calcium concentrations in the waste became reasonably constant.

\section{ADVANCED COOLSIDE OPERATION}

Test 3 was conducted in the Advanced Coolside mode. The Test 3 spent sorbent sample was produced in the CONSOL Advanced Coolside pilot plant. Fly ash from the Avon Lake plant (the same as previously described) was mixed with the spent sorbent to simulate one possible option for waste disposal for the process. The spent sorbent was a composite of waste produced in five runs conducted under generally similar conditions. The mixing of spent sorbent and fly ash gave a waste sample of about $500 \mathrm{lb}$.

A11 five of the Advanced Coolside runs were conducted with sorbent recycle. The average fresh $\mathrm{Ca} / \mathrm{S}$ mol ratio was about 1.2. Additives are not currently employed in the Advanced Coolside process. Therefore, the Test 3 waste sample can be compared to those from the Coolside process to study the effect of sodium on waste characteristics.

For the Test 3 waste sample, the $\mathrm{fly}$ ash $/ \mathrm{S}$ ratio is $4.0 \mathrm{~Tb} / \mathrm{lb}$; the same as in the Coolside wastes. The same CEI Avon Lake fly ash was used to produce the Test 3 waste sample.

A different hydrated Time, Mississippi hydrated Time, which is produced in St. Genevieve, Missouri, was used in the Advanced Coolside runs. Mississippi lime was the other test sorbent in the Edgewater Coolside demonstration $^{6}$ and the most active of 12 sorbents screened in the pilot support tests for the demonstration. 7,8 Table 4 gives a typical analysis of this lime.

\section{HASTE SAMPLE PREPARATION}

The waste sampies from Tests 1 and 2 were prepared as follows: 
1. Divided waste sample $(-500 \mathrm{lb})$ into two separate 55 -gal uncoated drums.

2. Tumbled each drum, end over end, for at least two hours.

3. Divided the waste sample in each drum into two halves.

4. Transferred half of the waste from each drum to the other drum.

5. Tumbled each drum for at least two hours.

6. Transferred the waste sample to a coated drum equipped with a plastic liner.

The waste sample from Test 3 was prepared as follows:

1. Divided waste samples (300 ib total) into two separate 55-gal uncoated drums.

2. Tumbled each drum, end over end, for at least two hours.

3. Divided the waste sample in each drum into two halves.

4. Transferred half of the waste from each drum to the other drum.

5. Added $97.6 \mathrm{tb}$ of f7y ash to each drum and tumbled each drum for at least two hours.

6. Transferred the waste sample to a coated drum equipped with a plastic liner.

The waste sample from Test 4 was taken as received. 


\section{RESULTS AND DISCUSSION}

\section{WASTE SAMPLES}

Table 5 lists the waste samples generated in this program and their chemical analyses. For Coolside Tests 1 and 2, $500 \mathrm{lb}$ of sample was generated; for Test 4, $90 \mathrm{lb}$ was generated. For the Advanced Coolside waste (sample from Test 3 ), about $500 \mathrm{lb}$ was generated. Analyses of duplicate batches from Test 2 confirm that the blending procedure produced waste samples which are acceptably homogeneous. The analytical data shown in Table 5 reflect the different conditions used to generate the wastes.

\section{COOLSIDE DESULFURIZATION PERFORMANCE}

\section{Test Data}

The following table summarizes the average coolside performance data from the three test periods:

\begin{tabular}{|c|c|c|c|c|c|c|c|}
\hline \multirow{2}{*}{$\begin{array}{l}\text { Test } \\
\text { No. }\end{array}$} & \multirow{2}{*}{$\begin{array}{l}\mathrm{Ca} / \mathrm{S} \text {, } \\
\text { mol } \\
\text { Ratio }\end{array}$} & \multirow{2}{*}{$\begin{array}{c}\mathrm{Na} / \mathrm{Ca} \text {, } \\
\text { mol } \\
\text { Ratio }\end{array}$} & \multirow{2}{*}{$\begin{array}{c}\text { Recycle } \\
\text { Ratio, } \\
\text { Ib recycle/ } \\
\text { lb lime }\end{array}$} & \multicolumn{2}{|c|}{$\mathrm{SO}_{2} \underset{\%}{\text { Removal, }}$} & \multicolumn{2}{|c|}{$\begin{array}{c}\text { Sorbent } \\
\text { Utilization, } \\
\%\end{array}$} \\
\hline & & & & Duct & System & Duct & System \\
\hline 1 & 2.08 & 0.19 & 0 & 64 & 78 & 28 & 34 \\
\hline 2 & 1.25 & 0.21 & 2.9 & 53 & 72 & 38 & 52 \\
\hline 4 & 2.42 & 0 & 0 & 41 & 60 & 17 & 25 \\
\hline
\end{tabular}

Sorbent utilization $\left(U_{1}\right)$ is defined as follows:

$U_{1}=\Delta \mathrm{SO}_{2} /(\mathrm{C}+1 / 2 \mathrm{~N}) \times 100$

where,

$\Delta \mathrm{SO}_{2}=1 \mathrm{~b} \mathrm{~mol} / \mathrm{hr} \mathrm{SO}_{2}$ removed 
C = $1 \mathrm{~b} \mathrm{~mol} / \mathrm{hr}$ total fresh calcium fed

$N=1 \mathrm{~b} \mathrm{~mol} / \mathrm{hr}$ total fresh sodium fed

These desulfurization data are generally consistent with results of pilot plant support tests for the Edgewater demonstration ${ }^{7,8}$ which were also conducted with Black River hydrated lime. The results shown above are averages over those periods of the entire test where operation was stable and where process instrumentation was on-line and accurate. Table 6 lists averaged data for each of these periods used in computing the overall average performance for the tests. The data in Table 6 were obtained from the VANTAGE historical database, which stores real time pilot data in the VAX computer system. Table 6 also lists averages of additional test conditions not inlcuded in the above table.

\section{Data Reliability}

Data reliability was confirmed by comparing sorbent utilizations calculated from average flue gas analyzer data and average sorbent/additive feed rate data (1isted above) with those calculated from waste solids analyses. As shown in the following table, agreement was very good.

\begin{tabular}{|c|c|c|}
\hline \multirow{2}{*}{ Test No. } & \multicolumn{2}{|c|}{ System Sorbent Utilization, $\%$} \\
\cline { 2 - 3 } & By Gas AnaTyses & By Solids Analyses \\
\hline 1 & 34 & 36 \\
2 & 52 & 49 \\
4 & 25 & 26 \\
\hline
\end{tabular}

The sorbent utilizations based on solids analyses $\left(U_{2}\right)$ were calculated from the analyses of composited waste samples (Table 5) as follows:

$U_{2}=S /(C+1 / 2 N) \times 100$ 
where,

$S=16$ mol of sulfur per lb waste

$C=1 \mathrm{~b}$ mol of calcium per $1 \mathrm{~b}$ waste

$N=1 \mathrm{~b}$ mol of sodium per $1 \mathrm{~b}$ waste

The sulfur concentration used in the calculation was the average of sulfur concentrations as determined by LECO analysis (total sulfur in Table 5) and by ICP analysis (ash $\mathrm{SO}_{3}$ in Table 5). It should be noted that the utilization calculated from solids analyses (Equation 2) is approximate, since the sulfur and calcium in the fly ash component of the waste sample slightly affects this calculation. (The $S / C a$ mol ratio in the fly ash was 0.19.) Therefore, utilizations calculated from gas analysis (Equation 1) are reported.

\section{COOLSIDE PILOT PLANT OPERABILITY}

Coolside pilot plant operability was good during the waste generation program. Normal operating problems associated with the pilot plant occurred during some of the testing; however, these did not affect waste properties or desulfurization performance data.

In general, long-term plant operability was better than in previous pilot programs. Continuous, stable operating periods of up to 15.5 hours were attained. Since the pilot plant was not designed for around-the-clock continuous operation, the run length in previous programs has typically been limited to about six hours.

One source of operating problems was deposition on the humidifier walls. In most cases, the deposit formed was a thin, dry coating on the wall. These deposits were loose and sloughed off on their own, or could easily be removed by rapping the walls or by air lancing. In some cases when a larger volume of material fell from the walls, the $120^{\circ}$ bend at the bottom of the vertical humidifier and/or the transition from 8-inch to 4-inch pipe was restricted, requiring shutdown for cleaning. In a few instances, the thickness of the deposit increased to a point where it was 
wetted by the nozzle spray, which then lead to accelerated deposition. Therefore, it was important to assure that the deposit layer remained thin and dry. This was assured by regular rapping of the walls and air lancing.

Another operating problem was nozzle failure. After relatively short periods of operation, nozzles had to be replaced because they either began to wet the walls or showed reduced drying performance. Sometimes, nozzle failure was evidenced by an increase in feed water pressure at a constant water flow rate. This problem has been observed previousty; however, the cause has not been identified. The operating time before nozzle replacement is required typically ranges from 8 to 40 hours. In some cases, new nozzles do not perform acceptably.

\section{ADVANCED COOLSIDE DESULFURIZATION PERFORMANCE}

For the five Advanced Coolside runs from which the waste sample (Test 3 ) was composited, duct $\mathrm{SO}_{2}$ removals ranged from 60 to $85 \%$ and system $\mathrm{SO}_{2}$ removals (including the baghouse) ranged 73 to $97 \%$. Sorbent utilizations based on solids analyses ranged 49 to $61 \%$. The results of these runs indicated that the performance objectives of $90 \% \quad \mathrm{SO}_{2}$ removal and $60 \%$ sorbent utilization are achievable. Based on chemical analyses of the composite waste sample for these Advanced Coolside Runs (Table 5), the utilization of the waste is about $52 \%$ (Equation 2). As noted above, this is an approximate utilization value, since the sulfur and calcium in the fly ash component slightly affects this calculation.

In the Advanced Coolside runs, conditions deviated somewhat from those for steady-state, continuous recycle. This is obvious from the observation that system $\mathrm{SO}_{2}$ removals were higher than the product of fresh $\mathrm{Ca} / \mathrm{S}$ mol ratio (1.2, in most runs) and sorbent utilization based on solids analyses. Accuracy of $\mathrm{SO}_{2}$ removal data and of spent sorbent analyses was confirmed by the good closure of elemental material balances. Had steady-state, continuous recycle been attained, $\mathrm{SO}_{2}$ removals at the same fresh $\mathrm{Ca} / \mathrm{S}$ ratio would have been lower, while sorbent utilizations would have been higher. This was confirmed in later 
tests, in which steady state was more closely simulated by using longer run durations and smaller solids inventories. 


\section{REFERENCES}

1. Robl, T. L. "Quarterly Coolside Project TAC Meeting Notes", letter to J. Harness, H. Johnson, M. M. Wu, February 6, 1992.

2. Yoon, H.; Stouffer, M. R.; Rosenhoover, W. A.; Statnick, R. M. "Laboratory and Field Development of Coolside $\mathrm{SO}_{2}$ Abatement Technology", Proceedings of the Second Annual Pittsburgh Coal Conference, Pittsburgh, PA, September 1985.

3. Yoon, H.; Ring, P. A.; Burke, F. P. "Coolside $\mathrm{SO}_{2}$ Abatement Technology: $1 \mathrm{MW}$ Field Tests", Proceedings of the Coat Technology ' 85 Conference, Pittsburgh, PA, November 1985.

4. Yoon, H.; Stouffer, M. R.; Rosenhoover, W. A.; Withum, J. A.; Burke, F. P. "Pilot Process Variable Study of Coolside Desulfurization", Environ. Prog. 1988, 7, 2, 104-111.

5. Stouffer, M. R.; Yoon, H.; Burke F. P. "An Investigation of the Mechanisms of Flue Gas Desulfurization by In-Duct Dry Sorbent Injection", I\&EC Research, 1989, 28, 20.

6. Yoon, H.; Statnick, R. M.; Withum, J. A.; McCoy, D. C. "Coolside Desulfurization Process Demonstration at Ohio Edison Edgewater Power Station", Proceedings of the 84th Annual Meeting of the Air and Waste Management Association, Vancouver, British Columbia, June 1991.

7. Stouffer, M. R.; Rosenhoover, W. A.; Yoon, H. "Pilot Plant and Sorbent Evaluation Studies for 104 MHe Coolside Process Demonstration", Processing and Utilization of High-Sulfur Coals III, Elsevier Science Publishers, Amsterdam, 1990.

8. Stouffer, M. R.; Wu, M. M.; Yoon, H.; WinscheT, R. A. "Support Activities for Edgewater Coolside Process Demonstration" Proceedings of the 1988 Fall International Symposium of the American Flame Research Committee, Pittsburgh, PA, October 1988.

9. Wu, M. M.; Winschel, R. A.; Withum, J. A. "Properties of Sol id Waste from the Coolside $\mathrm{SO}_{2}$ Abatement Process", Proceedings of the 81st Air Pollution Control Association Annual Meeting and Exhibition, Dal1as, TX, June 1988.

10. Yoon, H.; Burke, F. P. "Advanced Duct Sorbent Injection for $\mathrm{SO}_{2}$ Control", Proceedings of the Seventh Annual Coal Preparation, Utilization, and Environmental Control Contractors' Conference, Pittsburgh, PA, July 1991. 
TABLE 1

COOLSIDE TESTS: FIXED CONDITIONS

\begin{tabular}{|c|c|}
\hline Flue Gas Conditions & \\
\hline $\begin{array}{l}\text { Flue Gas } \mathrm{SO}_{2} \text { Content } \\
\text { Fly Ash Load (Fly Ash/S Ratio) } \\
\text { Fly Ash } \\
\text { Flue Gas Inlet Temperature } \\
\text { Adiabatic Saturation Temperature }\end{array}$ & $\begin{array}{l}2000 \mathrm{ppmv} \text { (dry basis) } \\
4.0 \mathrm{lb} / \mathrm{lb} \\
\text { CEI Avon Lake Unit } 9 \text { Fly Ash } \\
300^{\circ} \mathrm{F} \\
125^{\circ} \mathrm{F}\end{array}$ \\
\hline Coolside Process Conditions & \\
\hline $\begin{array}{l}\text { Approach to Adiabatic Saturation } \\
\text { Hydrated Lime }\end{array}$ & $\begin{array}{l}25^{\circ} \mathrm{F} \\
\text { Dravo Lime Company Black } \\
\text { River Lime }\end{array}$ \\
\hline Pilot Plant Operating Conditions & \\
\hline $\begin{array}{l}\text { Flue Gas Flow } \\
\text { Inlet Velocity } \\
\text { Humidifier Residence Time } \\
\text { Nozzle } \\
\text { Nozzle Operation }\end{array}$ & $\begin{array}{l}175 \mathrm{scfm} \\
11 \mathrm{ft} / \mathrm{sec} \text { at } 300^{\circ} \mathrm{F} \\
2.0 \mathrm{sec} \\
\text { Spraying Systems } 1 / 8 \mathrm{JJ}-\mathrm{J} 12 \\
100-105 \mathrm{psig} \text { Atomizing Air } \\
\text { Pressure }(-50 \mathrm{scf} / \mathrm{gal})\end{array}$ \\
\hline
\end{tabular}

$11 \mathrm{~s}$ 
TABLE 2

ANALYSES OF GRAB SAMPLES OF FLY ASH FROM

CEI AVON LAKE PLANT UNIT No. 9

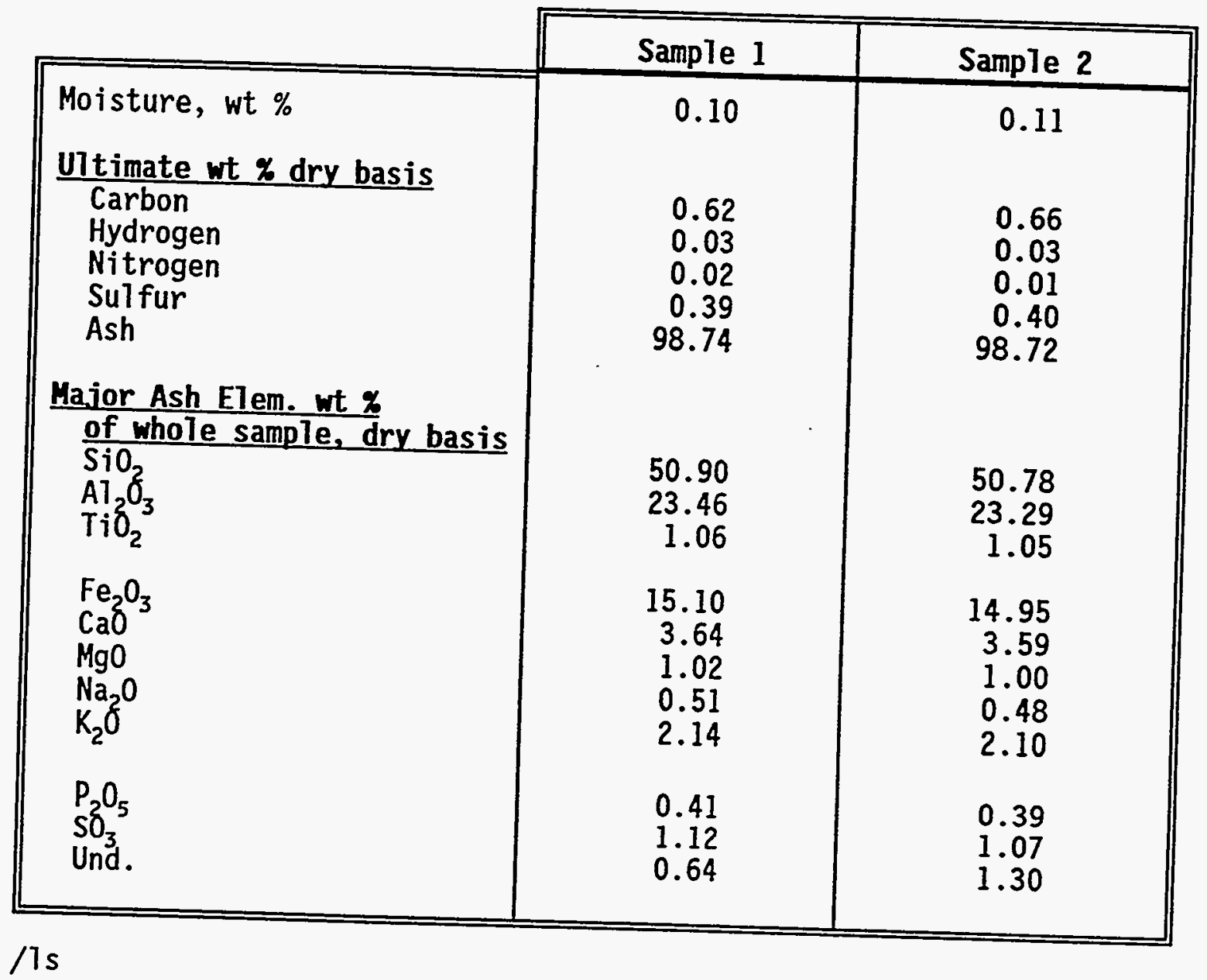


TABLE 3

ANALYSES OF DRAVO BLACK RIVER HYDRATED LIME SAYPLES

\begin{tabular}{|c|c|c|c|c|}
\hline & $\begin{array}{r}\text { Sample } \\
\text { No. } 1 \\
\end{array}$ & $\begin{array}{r}\text { Sample } \\
\text { No. } 2 \\
\end{array}$ & $\begin{array}{r}\text { Sample } \\
\text { No. } 3 \\
\end{array}$ & $\begin{array}{r}\text { Sample } \\
\text { No. } 4 \\
\end{array}$ \\
\hline $\begin{array}{l}\text { BET Surface Area, } \mathrm{m}^{2} / \mathrm{g} \\
\text { Lime Index as } \mathrm{Ca}(\mathrm{OH})_{2}\end{array}$ & $\begin{array}{l}16.9 \\
92.1\end{array}$ & $\begin{array}{l}15.9 \\
92.4\end{array}$ & $\begin{array}{l}16.5 \\
91.5\end{array}$ & $\begin{array}{l}15.2 \\
92.0\end{array}$ \\
\hline $\begin{array}{l}\text { wt } \% \text { of Hydrated Lime, } \\
\text { as received } \\
\text { Moisture } \\
\text { Ash }\left(925^{\circ} \mathrm{C}\right) \\
\text { Carbonate }\left(\mathrm{CO}_{3}{ }^{2-}\right) \\
\mathrm{Ca}(\mathrm{OH})_{2}(\mathrm{TGA})^{-1}\end{array}$ & $\begin{array}{r}0.4 \\
76.5 \\
0.7 \\
88.6\end{array}$ & $\begin{array}{r}0.5 \\
76.5 \\
0.7 \\
89.5\end{array}$ & $\begin{array}{r}0.4 \\
76.6 \\
0.6 \\
89.6\end{array}$ & $\begin{array}{r}0.6 \\
76.4 \\
0.4 \\
89.4\end{array}$ \\
\hline $\begin{array}{l}\text { Ash Elements, wt \% of dry } \\
\text { Hydrated Lime } \\
\mathrm{SiO}_{2} \\
\mathrm{Al}_{2} \mathrm{O}_{3} \\
\mathrm{TiO}_{2} \\
\mathrm{Fe}_{2} \mathrm{O}_{3} \\
\mathrm{CaO} \\
\mathrm{MgO} \\
\mathrm{Na}_{2} \mathrm{O} \\
\mathrm{K}_{2} \mathrm{O} \\
\mathrm{P}_{2} \mathrm{O}_{5} \\
\mathrm{SO}_{3}\end{array}$ & $\begin{array}{r}1.2 \\
0.4 \\
<0.1 \\
0.2 \\
74.6 \\
2.0 \\
<0.1 \\
<0.1 \\
<0.1 \\
0.1\end{array}$ & $\begin{array}{r}1.2 \\
0.4 \\
<0.1 \\
0.2 \\
75.2 \\
2.1 \\
<0.1 \\
<0.1 \\
<0.1 \\
<0.1\end{array}$ & $\begin{array}{r}1.2 \\
0.4 \\
<0.1 \\
0.2 \\
75.7 \\
2.1 \\
<0.1 \\
<0.1 \\
<0.1 \\
0.1\end{array}$ & $\begin{array}{r}1.4 \\
0.5 \\
0.1 \\
0.2 \\
72.6 \\
2.1 \\
<0.1 \\
<0.1 \\
<0.1 \\
0.1\end{array}$ \\
\hline $\begin{array}{l}\text { Malvern Particle Size, } \text { um } \\
+42.9 \\
42.9 \times 20.5 \\
20.5 \times 11.4 \\
11.45 .4 \\
5.4 \times 1.9 \\
-1.9 \\
\text { Mean Particle diameter, } \mu \mathrm{m}\end{array}$ & & & $\begin{array}{r}0 \\
4.5 \\
18.5 \\
35.3 \\
33.5 \\
8.2 \\
6.1\end{array}$ & \\
\hline
\end{tabular}


TABLE 4

ANALYSES OF MISSISSIPPI HYDRATED LIME

\begin{tabular}{|c|c|c|}
\hline & $\begin{array}{r}\text { Sample } \\
\text { No. } 1 \\
\end{array}$ & $\begin{array}{r}\text { Sample } \\
\text { No. } 2 \\
\end{array}$ \\
\hline $\begin{array}{l}\text { BET Surface Area, } \mathrm{m}^{2} / \mathrm{g} \\
\text { Lime Index as } \mathrm{Ca}(\mathrm{OH})_{2}\end{array}$ & $\begin{array}{l}22.6 \\
93.2\end{array}$ & $\begin{array}{l}24.4 \\
93.9\end{array}$ \\
\hline $\begin{array}{l}\text { wt } \% \text { of Hydrated Lime, as received } \\
\text { Moisture } \\
\text { Ash }\left(925^{\circ} \mathrm{C}\right) \\
\text { Carbonate }\left(\mathrm{CO}_{3}{ }^{2-}\right) \\
\mathrm{Ca}(\mathrm{OH})_{2}(\mathrm{TGA})\end{array}$ & $\begin{array}{r}0.6 \\
- \\
2.3 \\
90.8 \\
\end{array}$ & $\begin{array}{r}0.5 \\
75.1 \\
2.2 \\
90.9\end{array}$ \\
\hline $\begin{array}{l}\text { Ash Elements, wt } \% \text { of dry Hydrated Lime } \\
\mathrm{SiO}_{2} \\
\mathrm{AT}_{2} \mathrm{O}_{3} \\
\mathrm{TiO}_{2} \\
\mathrm{Fe}_{2} \mathrm{O}_{3} \\
\mathrm{CaO} \\
\mathrm{MgO} \mathrm{O} \\
\mathrm{Na}_{2} \mathrm{O} \\
\mathrm{K}_{2} \mathrm{O} \\
\mathrm{P}_{2} \mathrm{O}_{5} \\
\mathrm{SO}_{3}\end{array}$ & $\begin{array}{r}- \\
- \\
73 . \\
- \\
- \\
- \\
-\end{array}$ & $\begin{array}{r}0.8 \\
0.1 \\
<0.1 \\
0.1 \\
74.7 \\
0.6 \\
<0.1 \\
<0.1 \\
<0.1 \\
0.2\end{array}$ \\
\hline $\begin{array}{l}\text { Malvern Particle Size, } \text {,m } \\
66.9 \\
66.9 \times 42.9 \\
42.9 \times 20.5 \\
20.5 \times 11.4 \\
11.4 \times 5.4 \\
5.4 \times 1.9 \\
-1.9\end{array}$ & $\begin{array}{l}- \\
- \\
- \\
- \\
-\end{array}$ & $\begin{array}{r}0 \\
1.1 \\
6.3 \\
9.3 \\
31.3 \\
40.9 \\
11.1\end{array}$ \\
\hline Mean Particle diameter, $\mu \mathrm{m}$ & - & 5.3 \\
\hline
\end{tabular}

$/ 1 \mathrm{~s}$ 
TABLE 5

ANALYSES OF COMPOSITE WASTE SAMPLES

\begin{tabular}{|c|c|c|c|c|c|}
\hline & \multirow[b]{2}{*}{ Test 1} & \multicolumn{2}{|c|}{ Test 2} & \multirow[b]{2}{*}{ Test 3} & \multirow[b]{2}{*}{ Test 4} \\
\hline & & Drum 1 & Drum 2 & & \\
\hline Moisture & 4.42 & 1.85 & 1.92 & 2.95 & 3.42 \\
\hline $\begin{array}{l}\frac{\text { Ultimate, }}{\text { wt \% dry basis }} \\
\text { Carbon } \\
\text { Hydrogen } \\
\text { Nitrogen } \\
\text { Sulfur } \\
\text { Ash }\end{array}$ & $\begin{array}{r}1.66 \\
0.94 \\
0.10 \\
7.46 \\
89.46\end{array}$ & $\begin{array}{r}1.31 \\
0.63 \\
0.08 \\
7.96 \\
93.61\end{array}$ & $\begin{array}{r}1.37 \\
0.61 \\
0.10 \\
8.02 \\
93.67\end{array}$ & $\begin{array}{r}1.20 \\
0.66 \\
0.07 \\
9.31 \\
93.83\end{array}$ & $\begin{array}{r}1.68 \\
1.05 \\
0.12 \\
5.82 \\
87.72\end{array}$ \\
\hline $\begin{array}{l}\text { 0thers, wt \% } \\
\text { as determined } \\
\text { Sulfate } \\
\text { Sulfite } \\
\mathrm{CO}_{3}\end{array}$ & $\begin{array}{l}3.83 \\
3.88 \\
6.23\end{array}$ & $\begin{array}{l}2.86 \\
4.79 \\
4.54\end{array}$ & $\begin{array}{l}2.59 \\
4.89 \\
4.23\end{array}$ & $\begin{array}{l}3.13 \\
6.28 \\
4.55\end{array}$ & $\begin{array}{l}2.27 \\
2.79 \\
5.73\end{array}$ \\
\hline $\begin{array}{l}\text { Major Ash Elem., wt \% } \\
\text { of dry waste } \\
\mathrm{SiO}_{2} \\
\mathrm{Al}_{2} \mathrm{O}_{3} \\
\mathrm{TiO}_{2} \\
\mathrm{Fe}_{2} \mathrm{O}_{3} \\
\mathrm{CaO} \\
\mathrm{MgO} \\
\mathrm{Na}_{2} \mathrm{O} \\
\mathrm{K}_{2} \mathrm{O} \\
\mathrm{P}_{2} \mathrm{O}_{5} \\
\mathrm{SO}_{3} \\
\text { Und }\end{array}$ & $\begin{array}{r}17.75 \\
8.07 \\
0.37 \\
5.22 \\
35.01 \\
1.26 \\
2.80 \\
0.77 \\
0.14 \\
20.50 \\
8.11\end{array}$ & $\begin{array}{r}23.36 \\
10.89 \\
0.48 \\
6.78 \\
26.68 \\
1.18 \\
2.72 \\
1.00 \\
0.18 \\
20.86 \\
5.87\end{array}$ & $\begin{array}{r}23.70 \\
1.03 \\
0.48 \\
6.87 \\
26.90 \\
1.17 \\
2.71 \\
1.02 \\
0.18 \\
20.90 \\
5.04\end{array}$ & $\begin{array}{r}19.92 \\
9.92 \\
0.45 \\
6.55 \\
31.39 \\
0.64 \\
0.24 \\
0.88 \\
0.16 \\
23.02 \\
6.83\end{array}$ & $\begin{array}{r}18.45 \\
8.39 \\
0.37 \\
5.33 \\
40.03 \\
1.42 \\
0.26 \\
0.77 \\
0.13 \\
15.08 \\
9.77\end{array}$ \\
\hline
\end{tabular}

$/ 7 \mathrm{~s}$ 
TABLE 6A

COOLSIDE PROCESS DATA FOR TEST 1

\begin{tabular}{|c|c|c|c|c|c|c|c|c|}
\hline $\begin{array}{l}\text { Date, } 1992 \\
\text { Time }\end{array}$ & $\begin{array}{c}1 / 7 \\
2320 \\
0200\end{array}$ & $\begin{array}{l}1 / 7 \\
0620 \\
0900\end{array}$ & $\begin{array}{c}1 / 7 \\
1200 \\
1400\end{array}$ & $\begin{array}{c}1 / 7 \\
2300 \\
0050\end{array}$ & $\begin{array}{l}1 / 8 \\
0400 \\
0740\end{array}$ & $\begin{array}{c}1 / 8 \\
1500 \\
1620\end{array}$ & $\begin{array}{c}1 / 8 \\
1800 \\
1940\end{array}$ & $\begin{array}{l}1 / 8 \\
2230 \\
2330\end{array}$ \\
\hline $\begin{array}{l}\text { Proceses Varlablos : } \\
\text { Approach, }{ }^{\circ} \mathrm{F} \\
\text { Approach (baghouse), }{ }^{\circ} \mathrm{F} \\
\text { Fresh Ca/S, mol } \\
\text { Recycle Ratio } \\
\text { Additive/Fresh Lime Mass Ratio } \\
\text { Additive/Fresh Ca mol Ratio }\end{array}$ & $\begin{array}{r}24 \\
25 \\
2.05 \\
0.00 \\
0.1145 \\
0.2254\end{array}$ & $\begin{array}{r}25 \\
25 \\
2.02 \\
0.00 \\
0.0992 \\
0.1952\end{array}$ & $\begin{array}{r}24 \\
26 \\
2.06 \\
0.00 \\
0.0992 \\
0.1952\end{array}$ & $\begin{array}{r}25 \\
23 \\
2.09 \\
0.00 \\
0.0956 \\
0.1882\end{array}$ & $\begin{array}{r}25 \\
25 \\
2.09 \\
0.00 \\
0.0950 \\
0.1871\end{array}$ & $\begin{array}{r}27 \\
27 \\
2.04 \\
0.00 \\
0.0919 \\
0.1809\end{array}$ & $\begin{array}{r}25 \\
24 \\
2.08 \\
0.00 \\
0.1032 \\
0.2032\end{array}$ & $\begin{array}{r}25 \\
24 \\
2.07 \\
0.00 \\
0.0942 \\
0.1854\end{array}$ \\
\hline $\begin{array}{l}\text { Flue Gas Condhione } \\
\text { Inlet Temp., oF } \\
\text { Humidifier Exit Temp., }{ }^{\circ} \mathrm{F} \\
\text { Wot Bulb, of } \\
\text { Flow, scim } \\
\text { SO }_{2} \text { Content, ppm dry } \\
\text { Humidifier Residence, sec } \\
\text { Fly Ash Load, gr/scf }\end{array}$ & $\begin{array}{r}299 \\
150 \\
126 \\
173 \\
2032 \\
1.92 \\
4.42 \\
\end{array}$ & $\begin{array}{r}300 \\
150 \\
125 \\
172 \\
2055 \\
1.92 \\
3.39\end{array}$ & $\begin{array}{r}300 \\
149 \\
125 \\
172 \\
1988 \\
1.93 \\
4.53 \\
\end{array}$ & $\begin{array}{r}294 \\
151 \\
126 \\
172 \\
1997 \\
1.94 \\
4.46\end{array}$ & $\begin{array}{r}293 \\
150 \\
125 \\
172 \\
2000 \\
1.93 \\
4.45\end{array}$ & $\begin{array}{r}300 \\
151 \\
124 \\
172 \\
1996 \\
1.93 \\
4.46\end{array}$ & $\begin{array}{r}299 \\
140 \\
125 \\
172 \\
1995 \\
1.93 \\
4.46\end{array}$ & $\begin{array}{r}300 \\
150 \\
125 \\
172 \\
1997 \\
1.93 \\
4.48\end{array}$ \\
\hline $\begin{array}{l}\text { Nozale Condhions } \\
\mathrm{H}_{2} \mathrm{O} \text { Pressure, psig } \\
\mathrm{H}_{2} \mathrm{O} \text { Flow, gph } \\
\text { Air Pressure, psig } \\
\text { Air Flow, scfm } \\
\text { Air/ } \mathrm{H}_{2} \mathrm{O} \text {, scf/gal }\end{array}$ & $\begin{array}{r}97 \\
4.50 \\
103 \\
2.9 \\
38.04\end{array}$ & $\begin{array}{r}93 \\
4.66 \\
103 \\
3.1 \\
39.33\end{array}$ & $\begin{array}{r}90 \\
4.37 \\
103 \\
3.1 \\
42.03\end{array}$ & $\begin{array}{r}99 \\
4.44 \\
103 \\
3.1 \\
42.03\end{array}$ & $\begin{array}{r}95 \\
4.31 \\
103 \\
3.0 \\
41.94\end{array}$ & $\begin{array}{r}97 \\
4.28 \\
102 \\
3.1 \\
43.96\end{array}$ & $\begin{array}{r}97 \\
4.39 \\
103 \\
3.1 \\
42.42\end{array}$ & $\begin{array}{r}96 \\
4.34 \\
103 \\
3.0 \\
41.41\end{array}$ \\
\hline $\begin{array}{l}\mathrm{SO}_{2} \text { Removal } \\
\text { Humidifier, \% } \\
\text { System, \% }\end{array}$ & $\begin{array}{l}53.2 \\
66.4\end{array}$ & $\begin{array}{l}57.0 \\
71.8\end{array}$ & $\begin{array}{l}61.7 \\
79.7\end{array}$ & $\begin{array}{l}60.3 \\
79.5\end{array}$ & $\begin{array}{l}63.0 \\
80.5\end{array}$ & $\begin{array}{l}60.3 \\
72.9\end{array}$ & $\begin{array}{l}59.6 \\
75.9\end{array}$ & $\begin{array}{l}59.0 \\
76.0\end{array}$ \\
\hline $\begin{array}{l}\text { Sorbent Utilizatton } \\
\text { (by Gas Analysis) } \\
\text { Humidifier, } \% \\
\text { System, \% }\end{array}$ & $\begin{array}{l}23.3 \\
29.1\end{array}$ & $\begin{array}{r}25.7 \\
32.4\end{array}$ & $\begin{array}{l}27.3 \\
35.2\end{array}$ & $\begin{array}{l}26.3 \\
34.7\end{array}$ & $\begin{array}{l}27.6 \\
35.3\end{array}$ & $\begin{array}{l}32.8 \\
27.1\end{array}$ & $\begin{array}{l}26.0 \\
33.2\end{array}$ & $\begin{array}{l}26.0 \\
33.5\end{array}$ \\
\hline $\begin{array}{l}\text { Flow Rates } \\
\text { Lime, Ib/hr } \\
\text { Fly Ash, } \mathrm{lb} / \mathrm{hr} \\
\text { Recycle, } \mathrm{lb} / \mathrm{hr} \\
\text { Anhydrous Additive, Ib/hr }\end{array}$ & $\begin{array}{l}7.90 \\
6.54 \\
0.00 \\
0.90\end{array}$ & $\begin{array}{l}7.95 \\
5.00 \\
0.00 \\
0.79\end{array}$ & $\begin{array}{l}7.97 \\
6.66 \\
0.00 \\
0.80\end{array}$ & $\begin{array}{l}7.91 \\
6.56 \\
0.00 \\
0.76\end{array}$ & $\begin{array}{l}7.89 \\
6.57 \\
0.00 \\
0.75\end{array}$ & $\begin{array}{l}7.90 \\
6.56 \\
0.00 \\
0.73\end{array}$ & $\begin{array}{l}7.91 \\
6.58 \\
0.00 \\
0.82\end{array}$ & $\begin{array}{l}7.91 \\
6.61 \\
0.00 \\
0.74\end{array}$ \\
\hline $\begin{array}{l}\text { Humidification Water Balance } \\
\mathrm{H}_{2} \mathrm{O} \text { Feed, gph } \\
\text { Theoretical } \mathrm{H}_{2} \mathrm{O}, \mathrm{gph} \\
\text { Actual/Theoretical }\end{array}$ & $\begin{array}{l}4.50 \\
3.94 \\
1.14\end{array}$ & $\begin{array}{l}4.66 \\
3.94 \\
1.18\end{array}$ & $\begin{array}{l}4.37 \\
4.02 \\
1.09\end{array}$ & $\begin{array}{l}4.44 \\
3.81 \\
1.16\end{array}$ & $\begin{array}{l}4.31 \\
3.84 \\
1.12\end{array}$ & $\begin{array}{l}4.28 \\
4.02 \\
1.07\end{array}$ & $\begin{array}{l}4.39 \\
3.95 \\
1.11\end{array}$ & $\begin{array}{l}4.34 \\
3.98 \\
1.09\end{array}$ \\
\hline Run Hours & 2.67 & 2.67 & 2.00 & 1.83 & 3.67 & 1.33 & 1.67 & 1.00 \\
\hline
\end{tabular}

/7s 
TABLE 6A (Continued)

COOLSIDE PROCESS DATA FOR TEST 1

\begin{tabular}{|c|c|c|c|c|c|}
\hline $\begin{array}{l}\text { Date, } 1992 \\
\text { Tlme }\end{array}$ & $\begin{array}{c}1 / 9 \\
0000 \\
0240\end{array}$ & $\begin{array}{l}1 / 9 \\
0720 \\
0920\end{array}$ & $\begin{array}{c}1 / 9 \\
0930 \\
1240\end{array}$ & $\begin{array}{c}1 / 9 \\
1300 \\
1850\end{array}$ & $\begin{array}{c}1 / 7-1 / 9 \\
\text { Average }\end{array}$ \\
\hline $\begin{array}{l}\text { Process Variables } \\
\text { Approach, }{ }^{\circ} \mathrm{F} \\
\text { Approach (baghouse), }{ }^{\circ} \mathrm{F} \\
\text { Fresh Ca/S, mol } \\
\text { Recycle Ratio } \\
\text { Additive/Fresh Lime Mass Ratio } \\
\text { Additive/Fresh Ca mol Ratio }\end{array}$ & $\begin{array}{r}25 \\
26 \\
2.07 \\
0.00 \\
0.0838 \\
0.1650 \\
\end{array}$ & $\begin{array}{r}25 \\
25 \\
2.06 \\
0.00 \\
0.1070 \\
0.2106 \\
\end{array}$ & $\begin{array}{r}25 \\
26 \\
2.04 \\
0.00 \\
0.0845 \\
0.1663 \\
\end{array}$ & $\begin{array}{r}24 \\
27 \\
2.06 \\
0.00 \\
0.0979 \\
0.1926 \\
\end{array}$ & $\begin{array}{r}25 \\
26 \\
2.08 \\
0.00 \\
0.0970 \\
0.1910\end{array}$ \\
\hline $\begin{array}{l}\text { Flue Gas Condhions } \\
\text { Inlet Temp., }{ }^{\circ} \mathrm{F} \\
\text { Humidifier Exit Temp., }{ }^{\circ} \mathrm{F} \\
\text { Wet Bulb, }{ }^{\circ} \mathrm{F} \\
\text { Flow, scfm } \\
\mathrm{SO}_{2} \text { Content, ppm dry } \\
\text { Humidifier Residence, sec } \\
\text { Fy Ash Load, gr/scf }\end{array}$ & $\begin{array}{r}299 \\
150 \\
125 \\
172 \\
1996 \\
1.93 \\
4.48 \\
\end{array}$ & $\begin{array}{r}300 \\
150 \\
125 \\
172 \\
1998 \\
1.93 \\
4.49 \\
\end{array}$ & $\begin{array}{r}299 \\
150 \\
125 \\
172 \\
2000 \\
1.93 \\
4.49\end{array}$ & $\begin{array}{r}299 \\
149 \\
125 \\
172 \\
2000 \\
1.93 \\
4.48\end{array}$ & $\begin{array}{r}298 \\
150 \\
125 \\
172 \\
2006 \\
1.93 \\
4.38\end{array}$ \\
\hline $\begin{array}{l}\text { Nozzde Conditions } \\
\mathrm{H}_{2} \mathrm{O} \text { Pressure, psig } \\
\mathrm{H}_{2} \mathrm{O} \text { Flow, gph } \\
\text { Air Pressure, psig } \\
\text { Air Flow, scfm } \\
\text { Air/ } \mathrm{H}_{2} \mathrm{O}, \text { sct/gal }\end{array}$ & $\begin{array}{r}99 \\
4.40 \\
103 \\
3.1 \\
42.43\end{array}$ & $\begin{array}{r}93 \\
4.27 \\
103 \\
2.9 \\
41.27\end{array}$ & $\begin{array}{r}93 \\
4.13 \\
102 \\
2.9 \\
42.48\end{array}$ & $\begin{array}{r}93 \\
3.67 \\
103 \\
2.9 \\
48.08\end{array}$ & $\begin{array}{r}94 \\
4.31 \\
103 \\
3.0 \\
41.49\end{array}$ \\
\hline $\begin{array}{l}\mathrm{SO}_{2} \text { Removal } \\
\text { Humidifier, } \% \\
\text { System, } \%\end{array}$ & $\begin{array}{l}63.4 \\
78.5\end{array}$ & $\begin{array}{l}69.2 \\
84.6\end{array}$ & $\begin{array}{l}69.4 \\
83.3\end{array}$ & $\begin{array}{l}70.8 \\
83.4\end{array}$ & $\begin{array}{l}64.1 \\
78.0\end{array}$ \\
\hline $\begin{array}{l}\text { Sorbent Utillization } \\
\text { (by Gas Analysis) } \\
\text { Humidifier, } \% \\
\text { System, } \% \\
\end{array}$ & $\begin{array}{l}28.2 \\
35.0 \\
\end{array}$ & $\begin{array}{l}30.4 \\
37.1 \\
\end{array}$ & $\begin{array}{l}31.4 \\
37.6 \\
\end{array}$ & $\begin{array}{l}31.3 \\
36.9\end{array}$ & $\begin{array}{l}28.1 \\
34.2\end{array}$ \\
\hline $\begin{array}{l}\text { Flow Rates } \\
\text { Lime, lb/hr } \\
\text { Fly Ash, lb/hr } \\
\text { Recycle, lb/hr } \\
\text { Anhydrous Additive, lb/hr }\end{array}$ & $\begin{array}{l}7.88 \\
6.61 \\
0.00 \\
0.66\end{array}$ & $\begin{array}{l}7.89 \\
6.60 \\
0.00 \\
0.84 \\
\end{array}$ & $\begin{array}{l}7.88 \\
6.60 \\
0.00 \\
0.67\end{array}$ & $\begin{array}{l}7.85 \\
6.60 \\
0.00 \\
0.77 \\
\end{array}$ & $\begin{array}{l}7.89 \\
6.45 \\
0.00 \\
0.77\end{array}$ \\
\hline $\begin{array}{l}\text { Humidification Water Balance } \\
\mathrm{H}_{2} \mathrm{O} \text { Feed, gph } \\
\text { Theoretical } \mathrm{H}_{2} \mathrm{O} \text {, gph } \\
\text { Actual/Theoretical }\end{array}$ & $\begin{array}{l}4.40 \\
3.97 \\
1.11 \\
\end{array}$ & $\begin{array}{l}4.27 \\
4.01 \\
1.06\end{array}$ & $\begin{array}{l}4.13 \\
4.00 \\
1.03\end{array}$ & $\begin{array}{l}3.67 \\
4.04 \\
0.91\end{array}$ & $\begin{array}{l}4.31 \\
3.96 \\
1.09\end{array}$ \\
\hline Run Hours & 2.67 & 2.00 & 3.17 & 5.83 & 30.50 \\
\hline
\end{tabular}

/1s 
TABLE 6B

COOLSIDE PROCESS DATA FOR TEST 2

\begin{tabular}{|c|c|c|c|c|c|c|c|c|}
\hline $\begin{array}{l}\text { Date, } 1992 \\
\text { TIme }\end{array}$ & $\begin{array}{l}1 / 10 \\
1800 \\
1910\end{array}$ & $\begin{array}{l}1 / 10 \\
1930 \\
2050\end{array}$ & $\begin{array}{l}1 / 13 \\
0920 \\
0650\end{array}$ & $\begin{array}{l}1 / 14 \\
0650 \\
1110\end{array}$ & $\begin{array}{c}1 / 14 \\
1110 \\
1400\end{array}$ & $\begin{array}{l}1 / 14 \\
1500 \\
1600\end{array}$ & $\begin{array}{l}1 / 15 \\
2330 \\
0250\end{array}$ & $\begin{array}{l}1 / 15 \\
0310 \\
0520\end{array}$ \\
\hline $\begin{array}{l}\text { Process Variables } \\
\text { Approach, of } \\
\text { Approach (baghouse), }{ }^{\circ} \\
\text { Fresh Ca/S, mol } \\
\text { Recycle Ratio } \\
\text { Additive/Fresh Lime Mess Ratio } \\
\text { Additive/Fresh Ca mol Ratio }\end{array}$ & $\begin{array}{r}24 \\
24 \\
1,28 \\
2,76 \\
0.1141 \\
0.2247\end{array}$ & $\begin{array}{r}24 \\
25 \\
1.29 \\
2.67 \\
0.1025 \\
0.2018\end{array}$ & $\begin{array}{r}24 \\
26 \\
1.28 \\
2.98 \\
0.1104 \\
0.2173 \\
\end{array}$ & $\begin{array}{r}25 \\
23 \\
1.22 \\
2.86 \\
0.1059 \\
0.2084\end{array}$ & $\begin{array}{r}24 \\
23 \\
1.17 \\
3.19 \\
0.1139 \\
0.2242\end{array}$ & $\begin{array}{r}24 \\
23 \\
1.28 \\
2.77 \\
0.1212 \\
0.2386\end{array}$ & $\begin{array}{r}25 \\
25 \\
1.11 \\
2.93 \\
0.0056 \\
0.1882\end{array}$ & $\begin{array}{r}25 \\
25 \\
1.28 \\
2.91 \\
0.1052 \\
0.2071\end{array}$ \\
\hline $\begin{array}{l}\text { Flue Gas Condhions } \\
\text { Inlet Temp., oF } \\
\text { Humidifier Exit Temp., oF } \\
\text { Wet Bulb, }{ }^{\circ} \text { F } \\
\text { Flow, scfm } \\
\text { SO, Content, ppm dry }_{2} \text { Comidifier Residence, sec } \\
\text { Fly Ash Load, gr/scf }\end{array}$ & $\begin{array}{r}301 \\
149 \\
125 \\
172 \\
2008 \\
1.93 \\
5.29\end{array}$ & $\begin{array}{r}304 \\
149 \\
125 \\
172 \\
2009 \\
1.92 \\
4.68\end{array}$ & $\begin{array}{r}296 \\
149 \\
126 \\
170 \\
2002 \\
1.96 \\
4.49\end{array}$ & $\begin{array}{r}300 \\
150 \\
125 \\
170 \\
2000 \\
1.95 \\
4.26\end{array}$ & $\begin{array}{r}301 \\
149 \\
125 \\
170 \\
2036 \\
1.95 \\
4.19\end{array}$ & $\begin{array}{r}302 \\
149 \\
126 \\
170 \\
2001 \\
1.94 \\
4.46\end{array}$ & $\begin{array}{r}300 \\
150 \\
125 \\
170 \\
2023 \\
1.95 \\
3.56\end{array}$ & $\begin{array}{r}301 \\
149 \\
124 \\
170 \\
1999 \\
1.95 \\
4.06\end{array}$ \\
\hline $\begin{array}{l}\text { Nozte Conditions } \\
\mathrm{H}_{2} \mathrm{O} \text { Pressure, psig } \\
\mathrm{H}_{2} \mathrm{O} \text { Flow, gph } \\
\text { Air Pressure, psig } \\
\text { Air Flow, scfm } \\
\text { Air// } \mathrm{H}_{2} \mathrm{O} \text {, sct/gal }\end{array}$ & $\begin{array}{r}95 \\
4.37 \\
103 \\
3.1 \\
42.62\end{array}$ & $\begin{array}{r}95 \\
433 \\
103 \\
3.1 \\
42.75\end{array}$ & $\begin{array}{r}97 \\
4.34 \\
102 \\
3.8 \\
51.97\end{array}$ & $\begin{array}{r}100 \\
4.54 \\
102 \\
2.9 \\
38.92\end{array}$ & $\begin{array}{r}92 \\
4.33 \\
102 \\
2.9 \\
40.06\end{array}$ & $\begin{array}{r}95 \\
4.38 \\
102 \\
2.8 \\
38.79\end{array}$ & $\begin{array}{r}90 \\
4.26 \\
99 \\
2.9 \\
40.52\end{array}$ & $\begin{array}{r}91 \\
4.33 \\
101 \\
2.8 \\
38.45\end{array}$ \\
\hline $\begin{array}{l}\mathrm{SO}_{2} \text { Removal } \\
\text { Humidifier, } \% \\
\text { System, } \%\end{array}$ & $\begin{array}{l}67.9 \\
74.3\end{array}$ & $\begin{array}{l}63.6 \\
78.6\end{array}$ & $\begin{array}{l}50.2 \\
66.7\end{array}$ & $\begin{array}{l}60.3 \\
79.5\end{array}$ & $\begin{array}{l}56.5 \\
76.7\end{array}$ & $\begin{array}{l}61.6 \\
75.6\end{array}$ & $\begin{array}{l}44.2 \\
61.8\end{array}$ & $\begin{array}{l}55.0 \\
74.6\end{array}$ \\
\hline $\begin{array}{l}\text { Sorbent Utilization } \\
\text { (by Gas Analysis) } \\
\text { Humidifier, } \% \\
\text { System, } \%\end{array}$ & $\begin{array}{l}47.2 \\
51.6\end{array}$ & $\begin{array}{l}44.8 \\
55.4\end{array}$ & $\begin{array}{l}35.5 \\
47.1\end{array}$ & $\begin{array}{l}44.5 \\
58.8\end{array}$ & $\begin{array}{l}43.2 \\
58.8\end{array}$ & $\begin{array}{l}43.1 \\
52.9\end{array}$ & $\begin{array}{l}36.4 \\
50.9\end{array}$ & $\begin{array}{l}38.8 \\
52.7\end{array}$ \\
\hline $\begin{array}{l}\text { Flow Rates } \\
\text { Lime, lb/hr } \\
\text { Fly Ash, lb/hr } \\
\text { Recycle, lb } / \mathrm{hr} \\
\text { Anhydrous Additive, lb/hr }\end{array}$ & $\begin{array}{r}4.92 \\
7.80 \\
14.14 \\
0.56 \\
\end{array}$ & $\begin{array}{r}4.92 \\
6.90 \\
13.14 \\
0.50 \\
\end{array}$ & $\begin{array}{r}4.75 \\
6.53 \\
14.16 \\
0.52 \\
\end{array}$ & $\begin{array}{r}4.90 \\
6.21 \\
14.01 \\
0.52 \\
\end{array}$ & $\begin{array}{r}4.51 \\
6.11 \\
14.39 \\
0.51 \\
\end{array}$ & $\begin{array}{r}4.84 \\
6.50 \\
13.44 \\
0.59 \\
\end{array}$ & $\begin{array}{r}4.21 \\
5.19 \\
12.35 \\
0.40\end{array}$ & $\begin{array}{r}4.87 \\
5.91 \\
14.17 \\
0.51\end{array}$ \\
\hline $\begin{array}{l}\text { Humidification Water Balance } \\
\mathrm{H}_{2} \mathrm{O} \text { Feed, } \mathrm{gph} \\
\text { Theoretical } \mathrm{H}_{2} \mathrm{O}, \mathrm{gph} \\
\text { Actual/Theoretical }\end{array}$ & $\begin{array}{l}4.37 \\
4.38 \\
1.00\end{array}$ & $\begin{array}{l}4.33 \\
4.39 \\
0.99\end{array}$ & $\begin{array}{l}4.34 \\
4.12 \\
1.05\end{array}$ & $\begin{array}{l}4.54 \\
4.24 \\
1.07\end{array}$ & $\begin{array}{l}4.33 \\
4.27 \\
1.01\end{array}$ & $\begin{array}{l}4.38 \\
4.30 \\
1.02\end{array}$ & $\begin{array}{l}4.26 \\
4.09 \\
1.04\end{array}$ & $\begin{array}{l}4.33 \\
4.25 \\
1.02\end{array}$ \\
\hline Run Hours & 1.17 & 1.33 & 2.83 & 4.33 & 2.83 & 1.00 & 3.33 & 2.17 \\
\hline
\end{tabular}

$/ 1 \mathrm{~s}$ 
TABLE 6B (Continued)

COOLSIDE PROCESS DATA FOR TEST 2

\begin{tabular}{|c|c|c|c|c|c|c|c|}
\hline $\begin{array}{l}\text { Dinte, } 1992 \\
\text { Time }\end{array}$ & $\begin{array}{r}1 / 15 \\
0610 \\
1050\end{array}$ & $\begin{array}{l}1 / 15 \\
1120 \\
1450\end{array}$ & $\begin{array}{c}1 / 21 \\
0725 \\
081\end{array}$ & $\begin{array}{c}1 / 21 \\
0830 \\
092\end{array}$ & $\begin{array}{c}1 / 21 \\
0935 \\
095\end{array}$ & $\begin{array}{c}1 / 21 \\
1010 \\
111\end{array}$ & $\begin{array}{c}1 / 15 \\
\text { Average }\end{array}$ \\
\hline $\begin{array}{l}\text { Proceas Varlables } \\
\text { Approach, }{ }^{\circ} \\
\text { Approach (baghouse), }{ }^{\circ} \mathrm{F} \\
\text { Fresh } \mathrm{Ca} / \mathrm{S}, \text { mol } \\
\text { Recycle Ratio } \\
\text { Additive/Fresh Lime Mass Ratio } \\
\text { Additive/Fresh Ca mol Ratio }\end{array}$ & $\begin{array}{r}24 \\
24 \\
1.29 \\
2.97 \\
0.1120 \\
0.2205 \\
\end{array}$ & $\begin{array}{r}24 \\
24 \\
1.29 \\
2.97 \\
0.1064 \\
0.2094\end{array}$ & $\begin{array}{r}26 \\
29 \\
1.32 \\
2.96 \\
0.1185 \\
0.2334 \\
\end{array}$ & $\begin{array}{r}26 \\
31 \\
1.32 \\
2.96 \\
0.1107 \\
0.2179 \\
\end{array}$ & $\begin{array}{r}25 \\
31 \\
1.31 \\
2.95 \\
0.1116 \\
0.2197 \\
\end{array}$ & $\begin{array}{r}25 \\
30 \\
1.29 \\
2.93 \\
0.1128 \\
0.2221 \\
\end{array}$ & $\begin{array}{r}25 \\
25 \\
1.25 \\
2.94 \\
0.1082 \\
0.2129\end{array}$ \\
\hline $\begin{array}{l}\text { Flue Gas Conditions } \\
\text { Inlet Temp., 'F } \\
\text { Humidifier Exit Temp., ' } \mathrm{F} \\
\text { Wet Bulb, oF } \\
\text { Flow, sctm } \\
\mathrm{SO}_{2} \text { Content, ppm dry } \\
\text { Humidifier Residence, sec } \\
\text { Fly Ash Load, gr/scf }\end{array}$ & $\begin{array}{r}300 \\
149 \\
125 \\
170 \\
1999 \\
1.95 \\
3.97\end{array}$ & $\begin{array}{r}302 \\
149 \\
125 \\
170 \\
2000 \\
1.95 \\
3.69\end{array}$ & $\begin{array}{r}299 \\
151 \\
125 \\
170 \\
1982 \\
1.95 \\
4.78\end{array}$ & $\begin{array}{r}301 \\
150 \\
124 \\
170 \\
1973 \\
1.94 \\
4.65\end{array}$ & $\begin{array}{r}299 \\
150 \\
125 \\
170 \\
2006 \\
1.95 \\
4.28\end{array}$ & $\begin{array}{r}300 \\
150 \\
125 \\
170 \\
2019 \\
1.95 \\
4.60\end{array}$ & $\begin{array}{r}301 \\
150 \\
125 \\
170 \\
2003 \\
1.95 \\
3.99\end{array}$ \\
\hline $\begin{array}{l}\text { Nozzle Conditions } \\
\mathrm{H}_{2} \mathrm{O} \text { Pressure, psig } \\
\mathrm{H}_{2} \mathrm{O} \text { Flow, gph } \\
\text { Air Pressure, psig } \\
\text { Air Flow, scfm } \\
\text { Air/ } / \mathrm{H}_{2} \mathrm{O} \text {, scf/gal }\end{array}$ & $\begin{array}{r}91 \\
4.35 \\
101 \\
2.8 \\
38.61 \\
\end{array}$ & $\begin{array}{r}94 \\
4.34 \\
103 \\
2.8 \\
39.28\end{array}$ & $\begin{array}{r}98 \\
3.98 \\
103 \\
3.2 \\
48.74\end{array}$ & $\begin{array}{r}97 \\
3.95 \\
104 \\
3.3 \\
50.02\end{array}$ & $\begin{array}{r}94 \\
3.96 \\
104 \\
3.2 \\
48.71\end{array}$ & $\begin{array}{r}92 \\
3.96 \\
104 \\
3.2 \\
48.00\end{array}$ & $\begin{array}{r}92 \\
4.33 \\
102 \\
2.9 \\
40.07\end{array}$ \\
\hline $\begin{array}{l}\mathrm{SO}_{2} \text { Removal } \\
\text { Humidifier, } \% \\
\text { System, } \%\end{array}$ & $\begin{array}{l}57.4 \\
76.0 \\
\end{array}$ & $\begin{array}{l}58.8 \\
77.1\end{array}$ & $\begin{array}{l}44.2 \\
61.6\end{array}$ & $\begin{array}{l}45.0 \\
69.2\end{array}$ & $\begin{array}{l}46.4 \\
72.2\end{array}$ & $\begin{array}{l}52.4 \\
73.0\end{array}$ & $\begin{array}{l}53.4 \\
72.1\end{array}$ \\
\hline $\begin{array}{l}\text { Sorbent Utilization } \\
\text { (by Gas Analysis) } \\
\text { Humidifier, } \% \\
\text { System, } \%\end{array}$ & $\begin{array}{l}40.1 \\
53.0\end{array}$ & $\begin{array}{l}41.3 \\
54.2\end{array}$ & $\begin{array}{l}29.9 \\
41.7\end{array}$ & $\begin{array}{l}30.7 \\
47.2\end{array}$ & $\begin{array}{l}31.9 \\
49.7\end{array}$ & $\begin{array}{l}36.5 \\
50.8\end{array}$ & $\begin{array}{l}38.5 \\
52.0\end{array}$ \\
\hline $\begin{array}{l}\text { Flow Rates } \\
\text { Lime, lb/hr } \\
\text { Fly Ash, lb/hr } \\
\text { Recycle, lb/hr } \\
\text { Anhydrous Additive, lb/hr }\end{array}$ & $\begin{array}{r}4.85 \\
5.79 \\
14.38 \\
0.54\end{array}$ & $\begin{array}{r}4.85 \\
5.37 \\
14.38 \\
0.52\end{array}$ & $\begin{array}{r}4.88 \\
6.96 \\
14.44 \\
0.58\end{array}$ & $\begin{array}{r}4.87 \\
6.78 \\
14.42 \\
0.54\end{array}$ & $\begin{array}{r}4.89 \\
6.24 \\
14.46 \\
0.55\end{array}$ & $\begin{array}{r}4.87 \\
6.70 \\
14.26 \\
0.55\end{array}$ & $\begin{array}{r}4.73 \\
5.81 \\
13.92 \\
0.51\end{array}$ \\
\hline $\begin{array}{l}\text { Humidification Water Balance } \\
\mathrm{H}_{2} \mathrm{O} \text { Feed, gph } \\
\text { Theoretical } \mathrm{H}_{2} \mathrm{O} \text {, gph } \\
\text { Actual/Theoretical }\end{array}$ & $\begin{array}{l}4.35 \\
4.23 \\
1.03\end{array}$ & $\begin{array}{l}4.34 \\
4.28 \\
1.01\end{array}$ & $\begin{array}{l}3.98 \\
4.14 \\
0.96\end{array}$ & $\begin{array}{l}3.95 \\
4.22 \\
0.94\end{array}$ & $\begin{array}{l}3.96 \\
4.17 \\
0.95\end{array}$ & $\begin{array}{l}3.96 \\
4.22 \\
0.94\end{array}$ & $\begin{array}{l}4.33 \\
4.21 \\
1.03\end{array}$ \\
\hline Run Hours & 4.67 & 3.50 & 0.83 & 0.83 & 0.33 & $1 . \infty$ & 17.67 \\
\hline
\end{tabular}

$/ 1 \mathrm{~s}$ 
TABLE 6C

COOLSIDE PROCESS DATA FOR TEST 4

\begin{tabular}{|c|c|c|c|c|c|}
\hline $\begin{array}{l}\text { Datte, } 1992 \\
\text { Time }\end{array}$ & $\begin{array}{c}1 / 23 \\
0435-0615\end{array}$ & $\begin{array}{c}1 / 23 \\
0635-0710\end{array}$ & $\begin{array}{c}1 / 23 \\
0005-0950\end{array}$ & $\begin{array}{c}1 / 23 \\
1010-1045\end{array}$ & $\begin{array}{c}\text { 1/23 } \\
\text { Average }\end{array}$ \\
\hline $\begin{array}{l}\text { Procese Vartables } \\
\text { Approach, of } \\
\text { Approach (baghouse), of } \\
\text { Fresh Ca/S, mol } \\
\text { Recycle Ratio } \\
\text { Additive/Fresh Lime Masss Ratio } \\
\text { Additive/Fresh Ca mol Ratio }\end{array}$ & $\begin{array}{r}25 \\
28 \\
2.42 \\
0.00 \\
0.0000 \\
0.0000\end{array}$ & $\begin{array}{r}26 \\
27 \\
2.42 \\
0.00 \\
0.0000 \\
0.0000\end{array}$ & $\begin{array}{r}26 \\
28 \\
2.43 \\
0.00 \\
0.0000 \\
0.0000\end{array}$ & $\begin{array}{r}25 \\
30 \\
2.47 \\
0.00 \\
0.0000 \\
0.0000\end{array}$ & $\begin{array}{r}25 \\
28 \\
2.42 \\
0.00 \\
0.0000 \\
0.0000\end{array}$ \\
\hline $\begin{array}{l}\text { Flue Gas Condittons } \\
\text { Inlot Temp., }{ }^{\circ} \mathrm{F} \\
\text { Humidifier Exit Tomp., }{ }^{\circ} \mathrm{F} \\
\text { Wot Bulb, }{ }^{\circ} \mathrm{F} \\
\text { Flow, sclm } \\
\mathrm{sO}_{2} \text { Content, ppm dry } \\
\text { Humidifier Residence, sec } \\
\text { Fly Ash Load, gr/scf }\end{array}$ & $\begin{array}{r}300 \\
150 \\
125 \\
175 \\
1977 \\
1.90 \\
4.50 \\
\end{array}$ & $\begin{array}{r}300 \\
151 \\
125 \\
175 \\
1972 \\
1.89 \\
4.46 \\
\end{array}$ & $\begin{array}{r}300 \\
151 \\
125 \\
174 \\
1985 \\
1.90 \\
4.46 \\
\end{array}$ & $\begin{array}{r}299 \\
150 \\
125 \\
175 \\
1967 \\
1.89 \\
4.32\end{array}$ & $\begin{array}{r}300 \\
150 \\
125 \\
175 \\
1976 \\
1.89 \\
4.45\end{array}$ \\
\hline $\begin{array}{l}\text { Nozzle Conditions } \\
\mathrm{H}_{2} \mathrm{O} \text { Pressure, psig } \\
\mathrm{H}_{2} \mathrm{O} \text { Flow, gph } \\
\text { Air Pressure, psig } \\
\text { Air Flow, scfm } \\
\text { Air/ } \mathrm{H}_{2} \mathrm{O}, \mathrm{sct} / \mathrm{gal} \\
\end{array}$ & $\begin{array}{r}91 \\
4.07 \\
104 \\
3.4 \\
49.57 \\
\end{array}$ & $\begin{array}{r}93 \\
4.31 \\
104 \\
3.3 \\
46.15 \\
\end{array}$ & $\begin{array}{r}93 \\
4.32 \\
104 \\
3.3 \\
46.22 \\
\end{array}$ & $\begin{array}{r}93 \\
4.08 \\
104 \\
3.4 \\
49.26 \\
\end{array}$ & $\begin{array}{r}92 \\
4.20 \\
104 \\
3.4 \\
47.91\end{array}$ \\
\hline $\begin{array}{l}\mathrm{SO}_{2} \text { Removal } \\
\text { Humidifier, \% } \\
\text { System, \% }\end{array}$ & $\begin{array}{l}39.9 \\
61.1\end{array}$ & $\begin{array}{l}43.7 \\
57.5\end{array}$ & $\begin{array}{l}37.9 \\
53.0\end{array}$ & $\begin{array}{l}44.5 \\
68.4\end{array}$ & $\begin{array}{l}40.9 \\
60.0\end{array}$ \\
\hline $\begin{array}{l}\text { Sorbent Utllization } \\
\text { (by Gas Analysis) } \\
\text { Humidifier, } \% \\
\text { System, } \%\end{array}$ & $\begin{array}{l}16.5 \\
25.3\end{array}$ & $\begin{array}{l}18.1 \\
23.8\end{array}$ & $\begin{array}{l}15.6 \\
21.8\end{array}$ & $\begin{array}{l}18.0 \\
27.6\end{array}$ & $\begin{array}{l}16.9 \\
24.7\end{array}$ \\
\hline $\begin{array}{l}\text { Flow Rates } \\
\text { Lime, lb/hr } \\
\text { Fyy Ash, } \mathrm{lb} / \mathrm{hr} \\
\text { Recycle, lb/hr } \\
\text { Anhydrous Additive, lb/hr }\end{array}$ & $\begin{array}{l}9.42 \\
6.74 \\
0.00 \\
0.00\end{array}$ & $\begin{array}{l}9.43 \\
6.69 \\
0.00 \\
0.00\end{array}$ & $\begin{array}{l}9.47 \\
6.67 \\
0.00 \\
0.00\end{array}$ & $\begin{array}{l}9.46 \\
6.48 \\
0.00 \\
0.00\end{array}$ & $\begin{array}{l}9.44 \\
6.67 \\
0.00 \\
0.00\end{array}$ \\
\hline $\begin{array}{l}\text { Humidification Water Balance } \\
\mathrm{H}_{2} \mathrm{O} \text { Feed, gph } \\
\text { Theoretical } \mathrm{H}_{2} \mathrm{O}, \mathrm{gph} \\
\text { Actual/Theoretical }\end{array}$ & $\begin{array}{l}4.07 \\
4.00 \\
1.02\end{array}$ & $\begin{array}{l}4.31 \\
3.98 \\
1.08\end{array}$ & $\begin{array}{l}4.32 \\
3.95 \\
1.09\end{array}$ & $\begin{array}{l}4.08 \\
3.99 \\
1.02\end{array}$ & $\begin{array}{l}4.20 \\
4.00 \\
1.05\end{array}$ \\
\hline Run Hours & 1.67 & 0.58 & 0.75 & 0.58 & 3.58 \\
\hline
\end{tabular}

/1s 


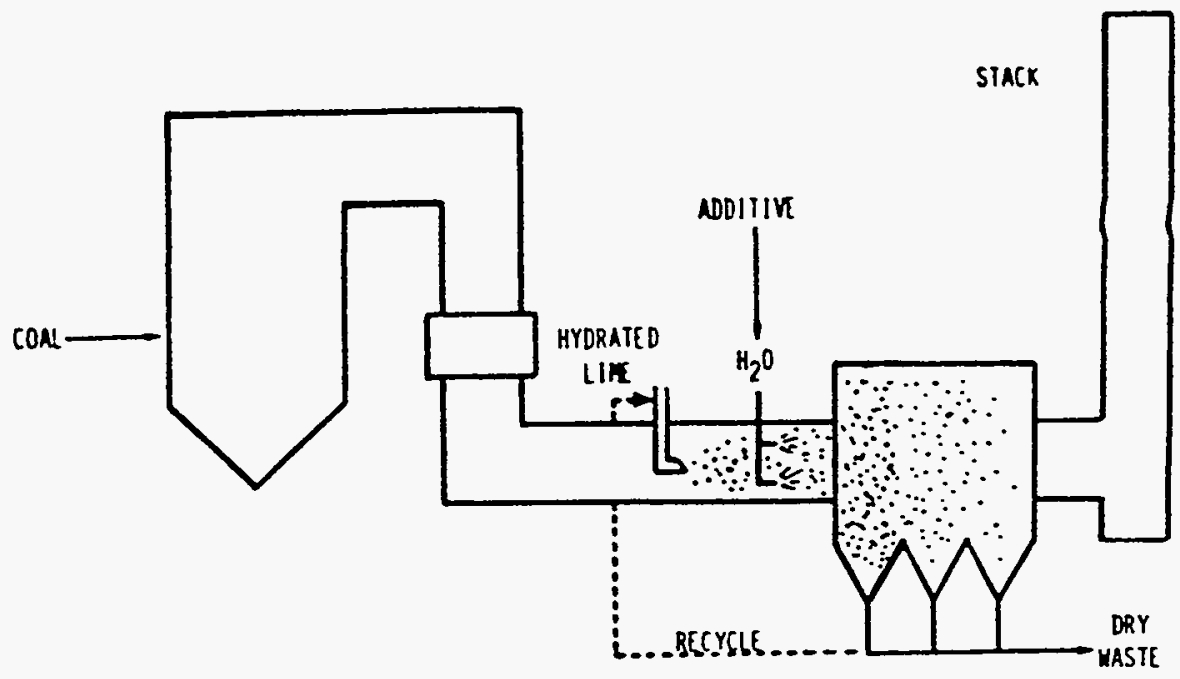

Figure 1. Coolside Desulfurization Process.

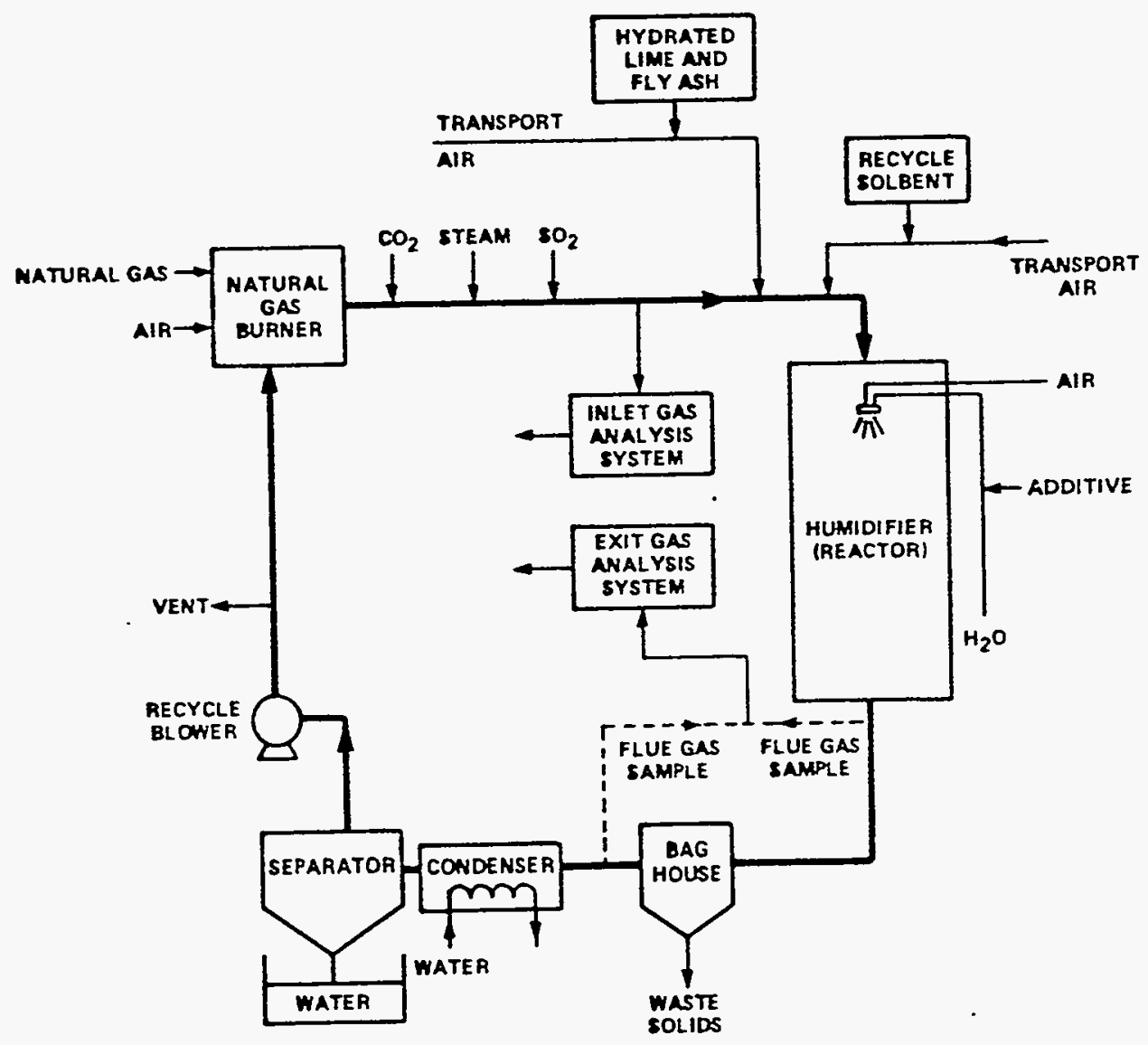

Figure 2. Schematic of Coolside Pilot Unit. 